JHU/APL

Enclosure (1) to: TL-97-033

ANSP-M-24

DoE/NE/32170-24

December 1996

PECFIVEO

MAY 081998

OSTI
United States Department of Energy

19901 Germantown Road

Germantown, Md 20874-1290

Aerospace Nuclear Safety Program

\title{
REENTRY RESPONSE OF THE \\ LIGHTWEIGHT RADIOISOTOPE HEATER \\ UNIT RESULTING FROM A CASSINI \\ VENUS-VENUS-EARTH-JUPITER \\ GRAVITY ASSIST MANEUVER ACCIDENT
}

\section{MASTER}

DISTRIBUTIOA

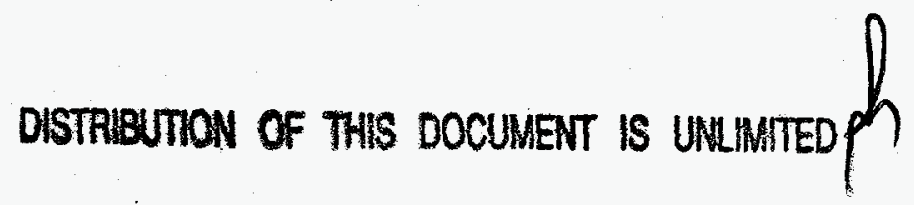

THE JOHNS HOPKINS UNIVERSITY - APPLIED PHYSICS LABORATORY Johns Hopkins Road, Laurel, Maryland 20723-6099

Contract Number: N00024-97-C-8119; Task ACA 


\section{DISCLAIMER}

This report was prepared as an account of work sponsored by an agency of the United States Government. Neither the United States Government nor any agency thereof, nor any of their employees, makes any warranty, express or implied, or assumes any legal liability or responsibility for the accuracy, completeness, or usefulness of any information, apparatus, product, or process disclosed, or represents that its use would not infringe privately owned rights. Reference herein to any specific commercial product, process, or service by trade name, trademark, manufacturer, or otherwise does not necessarily constitute or imply its endorsement, recommendation, or favoring by the United States Government or any agency thereof. The views and opinions of authors expressed herein do not necessarily state or reflect those of the United States Government or any agency thereof. 


\section{DISCLAIMER}

Portions of this document may be illegible electronic image products. Images are produced from the best available original document. 


\title{
REENTRY RESPONSE \\ OF THE LIGHTWEIGHT RADIOISOTOPE HEATER UNIT \\ RESULTING FROM A CASSINI VENUS-VENUS-EARTH-JUPITER GRAVITY ASSIST MANEUVER ACCIDENT
}

\author{
Sponsored by the United States Department of Energy \\ NE-50, 19901 Germantown Road \\ Germantown, MD 20874-1290
}

Approved by

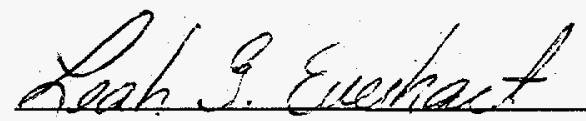

Leah G. Everhart, Assistant Program Manager Aerospace Nuclear Safety Program, JHU/APL

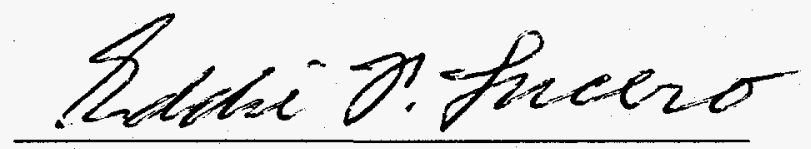

Eddie F. Lucero, Program Manager

Aerospace Nuclear Safety Program, JHU/APL

The Johns Hopkins University Applied Physics Laboratory

Johns Hopkins Road, Laurel, MD 20723-6099

Contract No. N00024-97-C-8119 


\section{Cassini/VVEJGA LWRHU Reentry Response \\ Executive Summary}

Reentry analyses consisting of ablation response, thermal response and thermal stress response have been conducted on the Lightweight Radioisotope Heater Unit (LWRHU) for Cassini/VenusVenus-Earth-Jupiter-Gravity-Assist (VVEJGA) reentry conditions. Sequential ablation analyses of the LWRHU aeroshell, and the fuel pellet have been conducted in reentry regimes where the aeroshell has been deemed to fail. The failure criterion for ablation is generally assumed to be recession corresponding to $75 \%$ and $100 \%$ of the wall thickness. The $75 \%$ recession failure criteria allows for uncertainties that result mainly because of the high energies involved in the VVEJGA reentries compared to orbital decay reentries. Risk evaluations should consider the fact that for shallow flight paths the unit may disassemble at high-altitude as a result of ablation or may remain intact with a clad that had been molten. indicate that:

Within the limitations of the methodologies and assumptions of the analyses, the results

1. For a side-on stable LWRHU reentry, aeroshell ablation failures occur for all reentry angles. For the steep reentries, failure criteria are met shortly after the time of peak heating. For shallow reentry angles, failure occurs prior to the time of peak heating. In all cases, the fuel pellet containment would be released at altitudes greater than approximately $140,000 \mathrm{ft}$. For all cases, the released fuel pellet will melt, resulting in atmospheric source terms.

2. For a side-on spinning LWRHU reentry, aeroshell ablation failures are minimal. This orientation results in the least severe environment with only the capture angle, $\gamma=-5.2^{\circ}$, indicating aeroshell failure. Following aeroshell failure, the released fuel pellet melts. The reentries that indicate aeroshell survival through the hypersonic flight could show incremental ablation leading to failure during subsonic flight. Additionally, the $\gamma=-7^{\circ}$ reentry indicates the presence of melted clad and fuel within the aeroshell. Resolidification occurs following hypersonic flight; this reentry could result in a ground source term.

3. For the tumbling LWRHU reentry, the aeroshell survives for most angles. Only the shallow cases (at less than $\gamma=-15^{\circ}$ ), show ablation failure. For the tumbling cases, the ablation is most severe in the end cap region resulting in a breach in the threaded area. The fuel pellet released for the shallowest angles $\left(\gamma=-7^{\circ}\right.$, and $\left.\gamma=-5.2^{\circ}\right)$ completely melt. Steeper angles $\left(\gamma=\left|-10^{\circ}\right|\right.$ and greater) do not show melt. For the shallower angles, resolidification occurs following hypersonic flight.

4. For the thermostructural analyses, using both a $1 \%$ and $5 \%$ allowable strain, all reentry angles and orientations examined resulted in small localized failures, but aeroshell breach is not predicted for any case.

The analyses included in this report concentrate on VVEJGA reentry scenarios. Analyses reported previously have demonstrated that the LWRHU has adequate design margin to survive reentry from orbital decay scenarios and most injection scenarios at speeds up to escape speeds. The exception is a narrow range of flight path angles that produce multiple skip trajectories which may have excessive ablation. 


\section{TABLE OF CONTENTS}

ABSTRACT i

LIST OF TABLES vi

LIST OF FIGURES viii

ACRONYMS XV

I. INTRODUCTION 1

II. MODULE DESCRIPTION 2

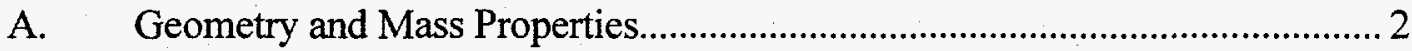

B. LWRHU Reentry Design Features............................................................ 2

1. Component Design.......................................................................... 2

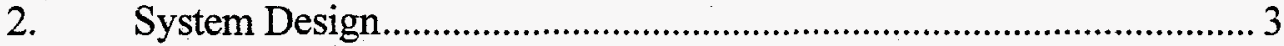

III. REENTRY CONDITIONS

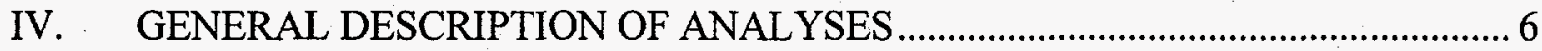

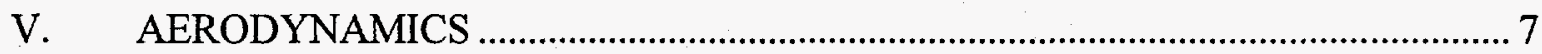

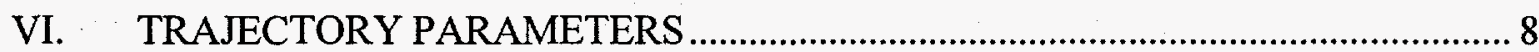

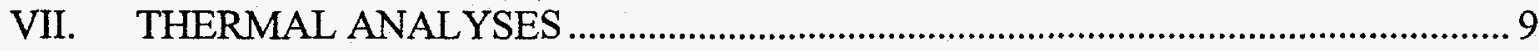

A. General Discussion and Summary .......................................................

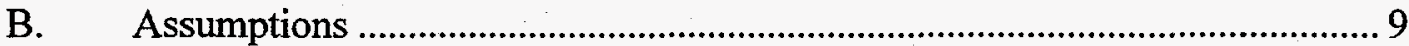

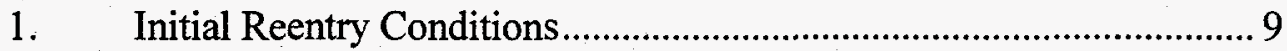

2. Reentry Configuration.............................................................. 10

3. Reentry Orientation.......................................................................... 10

4. Failure Criteria for Ablation and Thermal Response .......................... 10

- Ablation Failure Criterion.......................................................... 11

- Melt Failure Criterion ..................................................................... 11

- Fuel Melt Disposition .................................................................... 12

5. LWRHU Sequential Breakup Scenario ........................................ 12 
C. Thermal Models.

1. Reentry Thermal Analysis Program Limitations............................... 13

2. Thermal and Ablation Response Considerations .............................. 13

- LWRHU Thermal Models .............................................................. 13

- Interface Modeling ....................................................... 14

3. Initial Value and Boundary Conditions ............................................ 14

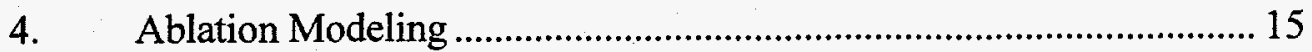

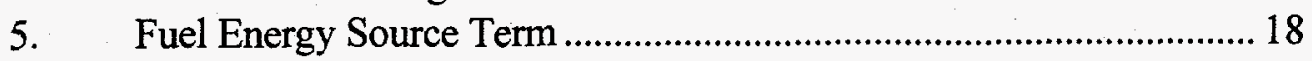

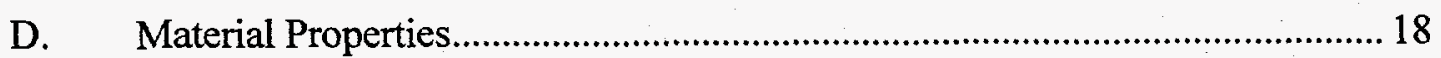

1. Thermal Diffusion Properties ......................................................... 19

- Fine Weave Pierced Fabric 3-D Carbon-Carbon ............................20

- Thermal Conductivity ..................................................... 20

- Thermal Capacitance........................................................... 20

- Pyrolytic Graphite ........................................................................ 21

- Thermal Conductivity ...................................................... 21

- Thermal Capacitance...................................................... 22

- Platinum-30\% Rhodium Alloy .................................................. 23

- Thermal Conductivity ........................................................23

- Thermal Capacitance ........................................................ 23

- Plutonium Dioxide ...................................................................... 23

- Thermal Conductivity ....................................................... 23

- Thermal Capacitance........................................................... 24

2. Thermal Radiative Properties ............................................................ 24

- Fine Weave Pierced Fabric 3-D Carbon-Carbon .......................... 25

- Pyrolytic Graphite...................................................................... 25

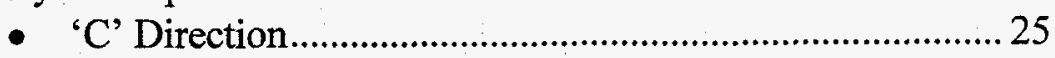

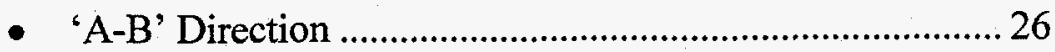

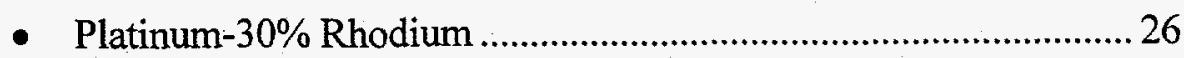

- Plutonium Dioxide .......................................................................... 26

3. Phase Change Properties .................................................................. 26

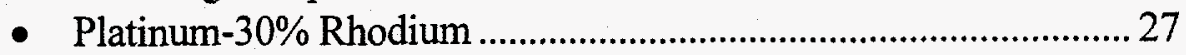

- Carbon Eutectic Temperature ........................................... 27

- Latent Heat of Fusion ......................................................... 27

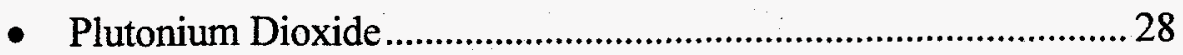

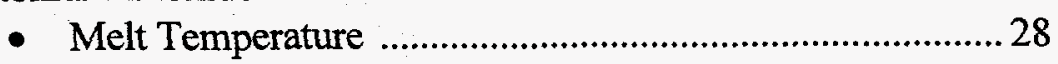

- Latent Heat of Fusion ...................................................... 28

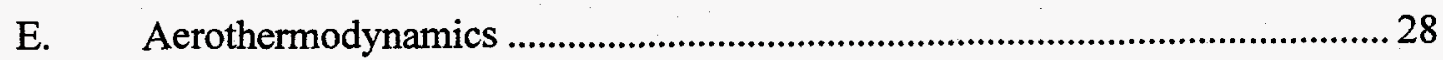

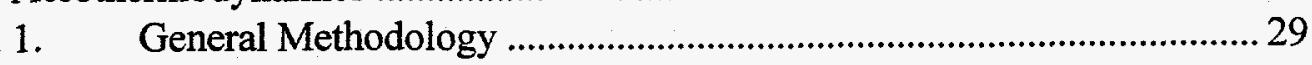

2. Reference Convective and Free Molecule Heating ............................. 30

3. Reference (and Body Reference) Radiative Heating............................ 30

4. Body Reference Convective Heating................................................. 31 
5. Adjustments to Body Reference Heating Rates ................................ 32

- Precursor Heating...................................................................... 32

- Radiation Coupling ................................................................... 32

- Catalytic Surface Effects................................................................ 33

6. Other Body Reference Variables .................................................... 33

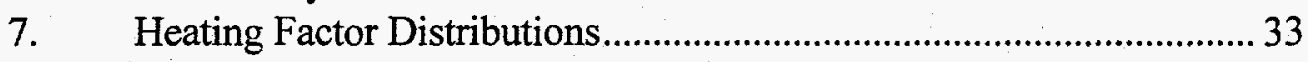

- Side-on Stable Orientation ........................................................... 34

- Cylindrical Surface ............................................................ 34

- End Face .......................................................................... 36

- Side-on Spinning Orientation .......................................................... 36

- Cylindrical Surface......................................................... 36

- End Face .................................................................... 37

- Random Tumbling Orientation................................................... 37

- Cylindrical Surface........................................................... 38

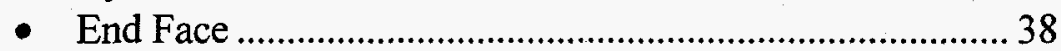

8. Corrections to Local Heating Rates .................................................. 40

- Low Density Effects .......................................................................40 40

- Surface Mass Transfer Effects, Convective Heating ..................... 40

- Surface Mass Transfer Effects, Radiative Heating ........................ 40

9. Fuel Pellet Aerothermodynamics ................................................ 41

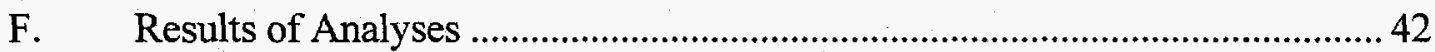

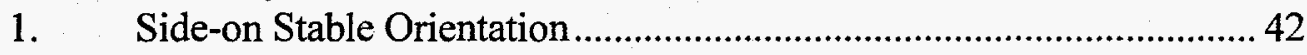

2. Side-on Spinning Orientation ...................................................... 43

3. Tumbling Approximations Orientation ............................................. 44

- Quasi-Tumbling LWRHU ....................................................... 44

- Random Tumbling LWRHU .........................................................4 44

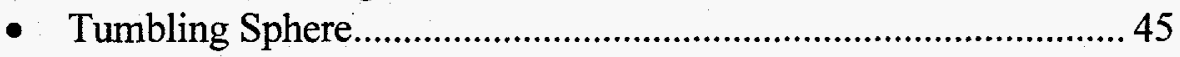

4. Component Temperature and Aeroshell Recession ............................. 45

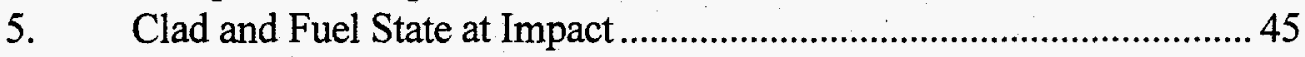

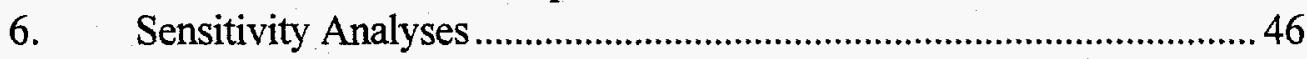

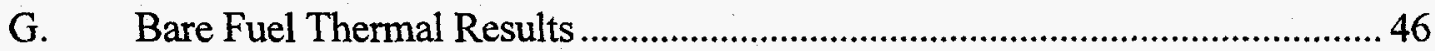

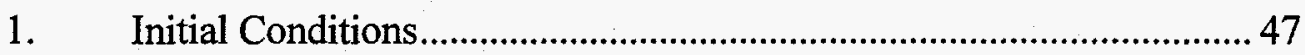

2. Trajectory Results and Discussion.............................................. 48

3. Side-on Stable LWRHU Release................................................... 49

4. Side-on Spinning LWRHU Release ................................................. 50

5. Random Tumbling LWRHU Release............................................... 51

6. Summary of Melt Fractions .............................................................. 51 


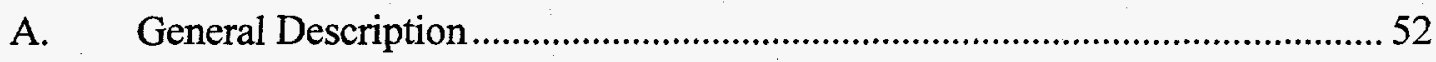

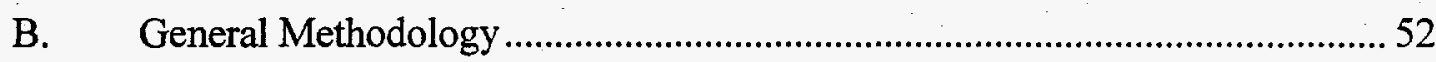

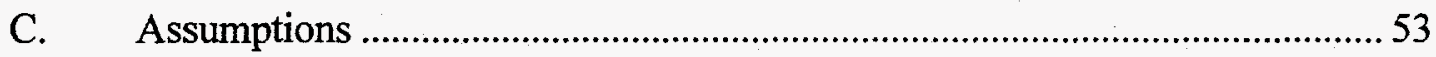

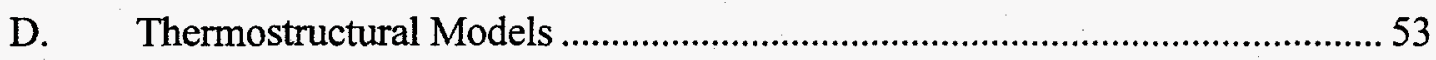

1. Thermoelastic Models.......................................................................5 53

2. Thermoplastic Models..................................................................... 54

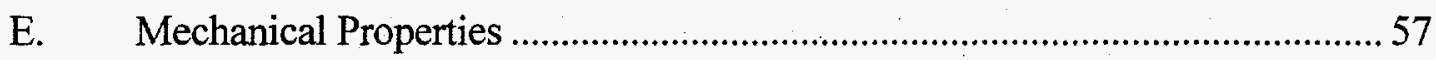

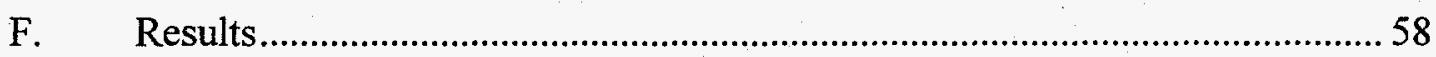

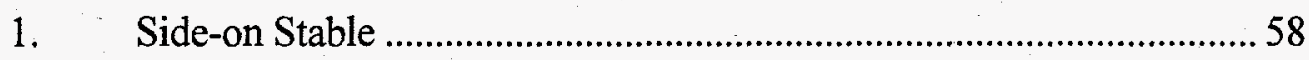

- Side-on Stable Mechanical and Temperature Loads..................... 58

- Side-on Stable Runs....................................................................... 59

- Side-on Stable Results .................................................................. 59

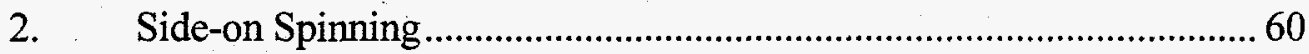

- Side-on Spinning Mechanical and Temperature Loads .................6 60

- Side-on Spinning Runs ....................................................................6 60

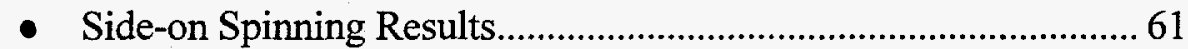

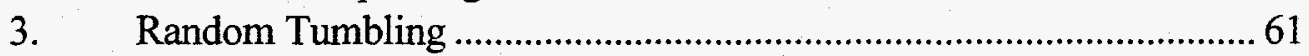

- Random Tumbling Mechanical and Temperature Loads .............. 61

- Random Tumbling Runs............................................................... 61

- Random Tumbling Results ........................................................62

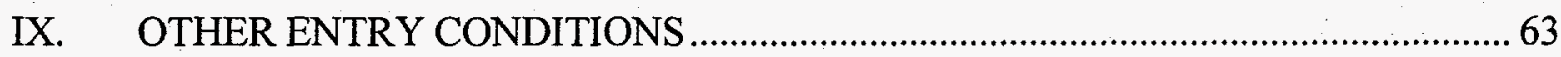

- Orbital decay, powered entry, etc.......................................................................6 63

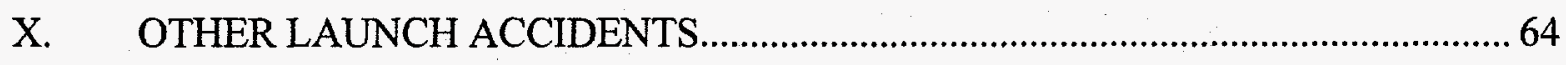

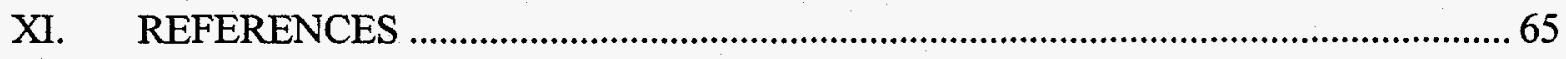


List of Tables

Table 1 General Description of the LWRHU Components

Table 2 Estimated Drag Coefficients for LWRHU Side-on Orientation

Table 3 Estimated Drag Coefficients for LWRHU Tumbling Orientation

Table 4 Estimated Drag Coefficients and Terminal Velocity for the LWRHU

Table $5 \quad$ TDOF Trajectory Results

Table 6 LWRHU Thermal Response Modeling

Table 7 Initial Reentry Temperature Distribution for the LWRHU

Table 8 Cassini/VVEJGA Reentry Aerothermodynamic Engineering Methods for the LWRHU Configuration

Table 9 Side-on Stable LWRHU 3D Thermal Analyses Results

Table 10 Side-on Spinning LWRHU 3D Thermal Analyses Results

Table 11 Quasi-Tumbling LWRHU 3D Thermal Analyses Results

Table 12 Random Tumbling LWRHU 3D Thermal Analyses Results

Table 13 Tumbling Sphere LWRHU 3D Thermal Analyses Results

Table 14 Side-on Stable LWRHU Component Temperatures at $75 \%$ and $100 \%$ (burnthrough) Failure

Table 15 Side-on Spinning LWRHU Component Temperatures at $75 \%$ and $100 \%$ (burnthrough) Failure

Table 16 Quasi-Tumbling LWRHU Component Temperatures at $75 \%$ and $100 \%$ (burnthrough) Failure

Table 17 Tumbling Sphere LWRHU Component Temperatures at $75 \%$ and $100 \%$ (burnthrough) Failure

Table 18 Random Tumbling LWRHU Component Temperatures at $75 \%$ and $100 \%$ (burnthrough) Failure 


\section{List of Tables (Continued)}

Table 19 Side-on Spinning LWRHU 3D Clad and Fuel Temperature. Results for Cases not demonstrating $100 \%$ Ablation Failure.

Table 20 Quasi-Tumbling LWRHU 3D Clad and Fuel Temperature. Results for Cases not demonstrating $100 \%$ Ablation Failure.

Table 21 Tumbling Sphere LWRHU 3D Clad and Fuel Temperature. Results for Cases not demonstrating $100 \%$ Ablation Failure.

Table 22 Random Tumbling LWRHU 3D Clad and Fuel Temperature. Results for Cases not demonstrating $100 \%$ Ablation Failure.

Table 23 Aeroshell and End Cap Recession

Table $24 \quad$ Initial Trajectory Conditions for Fuel Pellet Release

Table 25 Estimated Drag Coefficients and Bridging Parameters for Released Fuel Pellet

Table 26 Fuel Release Atmospheric Source Terms

Table 27 Side-on Stable LWRHU Thermostructural Analysis Results

Table 28 Side-on Spinning LWRHU Thermostructural Analysis Cases

Table 29 Side-on Spinning LWRHU Thermostructural Analysis Results

Table 30 Random Tumbling LWRHU Thermostructural Analysis Cases

Table 31 Random Tumbling LWRHU Thermostructural Analysis Results 
The Johns Hopkins University

Applied Physics Laboratory

Laurel, Maryland 20723.6099

\section{List of Figures}

Figure 1

Figure 2

Figure 3

Figure 4

Figure 5

Figure 6

Figure 7

Figure 8

Figure 9

Figure 10

Figure 11

Figure 12

Figure 13

Figure 14

Figure 15

Figure 16

Figure 17

Figure 18
Cross-Sectional View of LWRHU Assembly

LWRHU Aeroshell Body

LWRHU Clad Body

LWRHU Fuel Pellet

Variation of Initial Altitude Verses Flight Path Angle for Early Release of GPHS Module and LWRHU Entries; Cassini-VVEJGA Mission Profile

TDOF Results: LWRHU Side-on Representative VVEJGA Trajectory Profiles for Hypersonic Flight

TDOF Results: LWRHU Side-on Representative VVEJGA Reentry Altitude Histories

TDOF Results: LWRHU Tumbling Cylinder Representative VVEJGA Trajectory Profiles for Hypersonic Flight

TDOF Results: LWRHU Tumbling Cylinder Reentry Representative VVEJGA Altitude Histories

TDOF Results: LWRHU Tumbling Sphere Representative VVEJGA Trajectory Profiles for Hypersonic Flight

TDOF Results: LWRHU Tumbling Sphere Representative VVEJGA Altitude Histories

TDOF Results: LWRHU Side-on Stable and Spinning Representative Reentry Deceleration Histories

TDOF Results: LWRHU Tumbling Cylinder Reentry Representative Deceleration Histories

TDOF Results: LWRHU Tumbling Sphere Representative Reentry Deceleration Histories

TDOF Results: LWRHU Side-on Stable and Spinning Reference Convective Heating Histories

TDOF Results: LWRHU Tumbling Cylinder Reference Convective Heating Histories

TDOF Results: LWRHU Tumbling Sphere Reference Convective Heating Histories

TDOF Results: LWRHU Side-on Stable and Spinning Radiative Heating Histories 


\section{List of Figures (Continued)}

Figure 19

Figure 20

Figure 21

Figure 22

Figure 23

Figure 24

Figure 25

Figure 26

Figure 27

Figure 28

Figure 29

Figure 30

Figure 31

Figure 32

Figure 33

Figure 34

Figure 35

Figure 36

Figure 37

Figure 38
TDOF Results: LWRHU Tumbling Cylinder Radiative Heating Histories

TDOF Results: LWRHU Tumbling Sphere Radiative Reference Heating Histories

TDOF Results: LWRHU $-90^{\circ}$ Reentry Sample Steep Trajectory for Different Orientations

TDOF Results: LWRHU $-7^{\circ}$ Reentry Sample Shallow Trajectory for Different Orientations

TDOF Results: LWRHU Sample Reentry Deceleration Forces for Different Orientations

TDOF Results: LWRHU $-90^{\circ}$ Reentry Sample Steep Heating Histories for Different Orientations

TDOF Results: LWRHU $-7^{\circ}$ Reentry Sample Shallow Heating Histories for Different Orientations

TDOF Results: LWRHU Side-on Total Pressure Behind Normal Shock

TDOF Results: LWRHU Side-on Total Enthalpy Histories

TDOF Results: LWRHU Tumbling Cylinder Total Pressure Behind Normal Shock

TDOF Results: LWRHU Tumbling Cylinder Total Enthalpy Histories

TDOF Results: LWRHU Tumbling Sphere Total Pressure Behind Normal Shock

TDOF Results: LWRHU Tumbling Sphere Total Enthalpy Histories

TDOF Results: LWRHU $-90^{\circ}$ Comparison of Total Enthalpy for Different Orientations

TDOF Results: LWRHU $-90^{\circ}$ Comparison of Total Pressure for Different Orientations

LWRHU Thermal Model: Quarter Section

Fuel Pellet 2-D Side-on and 2-D Tumbling Thermal Models

Thermal Conductivity Schedules for LWRHU Interface Gas Fills

Surface Energy Balance - Reentry Thermal Analysis

Surface Mass Balance - Reentry Thermal Analysis 
The Johns Hopkins University

Applied Physics Laboratory

Laurel, Maryland 20723-6099

List of Figures (Continued)

Figure 39

Figure 40

Figure 41

Figure 42

Figure 43

Figure 44

Figure 45

Figure 46

Figure 47

Figure 48

Figure 49

Figure 50

Figure 51

Figure 52

Figure 53

Figure 54

Figure 55

Figure 56

Figure 57

Figure 58

Figure 59
Thermochemical Ablation Regimes for Graphite

Thermal Conductivity Schedules for FWPF ${ }^{\text {tm }}$

Specific Heat Schedule for FWPF ${ }^{\text {tm }}$

Thermal Conductivity Properties for Pyrolytic Graphite

Specific Heat Schedule for Pyrolytic Graphite

Thermal Conductivity Schedules for Pt-30 Rh

Specific Heat Schedule for Pt-30 Rh

Thermal Conductivity Schedules for $\mathrm{PuO}_{2}$

Specific Heat Schedule for $\mathrm{PuO}_{2}$

Thermal Radiative Properties for LWRHU Constituent Materials

Aerothermodynamics Regimes for the Cassini / VVEJGA Reentries; Side-on Stable LWRHU Configuration

Composite Plot Comparing Experimental Data with the Detra, Kemp, and Riddell Correlation

Stagnation Velocity Gradient Correction on the Side-on LWRHU

Stagnation Convective Heating Rate (Without Geometry Scaling)

Hypersonic, Windward Circumferential Convective Heating Factor Distributions

Hypersonic, Stagnation Line Convective Heating Factor Distributions

Hypersonic, Windward Circumferential Radiative Heating Factor Distributions

Hypersonic, Stagnation line Radiative Heating Factor Distributions

Hypersonic, Circumferential Static Pressure Factor Distribution as a Function of Axial Location; Side-On Orientation

Hypersonic, Angle of Attack Convective Heating Factor, Stagnation Line of LWRHU's Cylindrical Surface; Tumbling Flight Orientation

Local Convective Heating Ratio on LWRHU's End Face, Zero Angle of Attack; Random Tumbling Flight Orientation

Average Convective Heating Ratio to LWRHU's End Face as a Function of Angle of Attack; Random Tumbling Flight Orientation 
List of Figures (Continued)

Figure 60 Comparison of Correlated and Experimental Static Pressure Distributions over LWRHU's End Face, Zero Angle of Attack; Random Tumbling Flight Orientation

Figure 61 Blockage of Convective Heating Rate as a Function of the Mass Transfer Parameter

Figure 62 Blockage of Stagnation Radiative Heating Rates Vs Injectant Mass Flux and Chemistry Constituency

Figure 63

Continuum and Kinetic Flow Regimes with Threshold Values for Radiation Blockage

Figure 64

RETAP Results: Comparison of Fuel Release Conditions with Sandia Melt Boundaries and FOILS EIS Studies

Figure 65

RETAP Results: LWRHU $-90^{\circ}$ Side-on Stable VVEJGA Reentry

Figure 66

RETAP Results: LWRHU $-70^{\circ}$ Side-on Stable VVEJGA Reentry

Figure 67

RETAP Results: LWRHU $-50^{\circ}$ Side-on Stable VVEJGA Reentry

Figure 68

RETAP Results: LWRHU $-30^{\circ}$ Side-on Stable VVEJGA Reentry

Figure 69

RETAP Results: LWRHU $-15^{\circ}$ Side-on Stable VVEJGA Reentry

Figure 70

RETAP Results: LWRHU $-10^{\circ}$ Side-on Stable VVEJGA Reentry

Figure 71

RETAP Results: LWRHU $-7^{\circ}$ Side-on Stable VVEJGA Reentry

Figure 72

RETAP Results: LWRHU $-5.2^{\circ}$ Side-on Stable VVEJGA Reentry

Figure 73

Figure 74

RETAP Results: Side-on Stable LWRHU 3D -90 VVEJGA Reentry Ablation Failure Relative to Reference Heat Pulse

Figure 75

RETAP Results: Side-on Stable LWRHU 3D -70 VVEJGA Reentry Ablation Failure Relative to Reference Heat Pulse

Figure 76 RETAP Results: Side-on Stable LWRHU 3D -30 ${ }^{\circ}$ VVEJGA Reentry Ablation Failure Relative to Reference Heat Pulse

Figure 77 RETAP Results: Side-on Stable LWRHU 3D -15 VVEJGA Reentry Ablation Failure Relative to Reference Heat Pulse

Figure 78 RETAP Results: Side-on Stable LWRHU 3D -10 $0^{\circ}$ VVEJGA Reentry Ablation Failure Relative to Reference Heat Pulse 


\section{List of Figures (Continued)}

Figure 79

Figure 80

Figure 81

Figure 82

Figure 83

Figure 84

Figure 85

Figure 86

Figure 87

Figure 88

Figure 89

Figure 90

Figure 91

Figure 92

Figure 93

Figure 94

Figure 95

Figure 96
RETAP Results: Side-on Stable LWRHU 3D - $7^{\circ}$ VVEJGA Reentry Ablation Failure Relative to Reference Heat Pulse

RETAP Results: Side-on Stable LWRHU 3D -5.2० VVEJGA Reentry Ablation Failure Relative to Reference Heat Pulse

RETAP Results: Side-on Spinning LWRHU 3D-5.2 ${ }^{\circ}$ VVEJGA Reentry Recession Failure at Modeled Surface Nodes

RETAP Results: Side-on Spinning LWRHU 3D $-5.2^{\circ}$ VVEJGA Reentry Ablation Failure Relative to Reference Heat Pulse

RETAP Results: LWRHU $-7^{\circ}$ Side-on Spinning VVEJGA Reentry

RETAP Results: Quasi-Tumbling Cylinder VVEJGA Reentry

Recession Failure at Modeled Surface Nodes

RETAP Results: Quasi-Tumbling VVEJGA Reentry Ablation Failure

Relative to Reference Heat Pulse

RETAP Results: Random Tumbling 3D LWRHU VVEJGA Reentry

Recession Failure at Modeled Surface Nodes

RETAP Results: Random Tumbling 3D LWRHU VVEJGA Reentry

Ablation Failure Relative to Reference Heat Pulse

RETAP Results: Tumbling Sphere VVEJGA Reentry Recession Failure at Modeled Surface Nodes

RETAP Results: Tumbling Sphere VVEJGA Reentry Ablation Failure Relative to Reference Heat Pulse

RETAP Results: Side-on Stable 3D LWRHU VVEJGA Recession Failure Comparison: JANNAF 89 and Lawrence Livermore Tables

RETAP Results: Side-on Stable 3D LWRHU VVEJGA Comparison of Ablation Failure Relative to Reference Heat Pulse: JANNAF 89 and Lawrence Livermore Tables

RETAP Results: Side-on Stable 3D LWRHU VVEJGA Failure Comparison: Gas-Radiation Cooling Effect

Summary of Released Fuel Pellet Orientations Examined

TDOF Fuel Pellet Results: Side-on Stable Velocity v. Altitude Profiles

TDOF Fuel Pellet Results: Side-on Stable Altitude Histories

TDOF Fuel Pellet Results: Reference Heating Rate Histories for Side-on Stable Breach Releasing Side-on Pellet 
List of Figures (Continued)

Figure 97

Figure 98

Figure 99

Figure 100

Figure 101

Figure 102

Figure 103

Figure 104

Figure 105

Figure 106

Figure 107

Figure 108

Figure 109

Figure 110

Figure 111

Figure 112
TDOF Fuel Pellet Results: Reference Heating Rate Histories for Side-on Stable Breach Releasing Tumbling Pellet

TDOF Fuel Pellet Results: Reference Heating Rate History for Side-on Spinning Breach Releasing Side-on Pellet

TDOF Fuel Pellet Results: Reference Heating Rate History for Side-on Spinning Breach Releasing Tumbling Pellet

TDOF Fuel Pellet Results: Reference Heating Rate History for Tumbling Breach Releasing Side-on Pellet

TDOF Fuel Pellet Results: Reference Heating Rate History for Tumbling Breach Releasing Tumbling Pellet

RETAP Results: $-5.2^{\circ}$ Side-on Stable LWRHU (100\% Ablated) Releasing Side-on Fuel Pellet; Thermal Response Through Centerline with Reference Convective Heating

LWRHU Fuel Results: Comparison of $-5.2^{\circ}$ Stable LWRHU Releasing Stable Fuel Response at $1 \mathrm{X}$ and $3 \mathrm{X}$ Reference Convective Heating LWRHU Fuel Results: Comparison of $-7^{\circ}$ Stable LWRHU Releasing Stable Fuel Response at $1 \mathrm{X}$ and $3 \mathrm{X}$ Reference Convective Heating LWRHU Fuel Results: Comparison of $-10^{\circ}$ Stable LWRHU Releasing Stable Fuel Response at $1 \mathrm{X}$ and $3 \mathrm{X}$ Reference Convective Heating LWRHU Fuel Results: Comparison of $-15^{\circ}$ Stable LWRHU Releasing Stable Fuel Response at $1 \mathrm{X}$ and $3 \mathrm{X}$ Reference Convective Heating LWRHU Fuel Results: Comparison of $-30^{\circ}$ Stable LWRHU Releasing Stable Fuel Response at $1 \mathrm{X}$ and $3 \mathrm{X}$ Reference Convective Heating LWRHU Fuel Results: Comparison of $-50^{\circ}$ Stable LWRHU Releasing Stable Fuel Response at $1 \mathrm{X}$ and $3 \mathrm{X}$ Reference Convective Heating LWRHU Fuel Results: Comparison of $-70^{\circ}$ Stable LWRHU Releasing Stable Fuel Response at $1 \mathrm{X}$ and $3 \mathrm{X}$ Reference Convective Heating LWRHU Fuel Results: Comparison of $-90^{\circ}$ Stable LWRHU Releasing Stable Fuel Response at $1 \mathrm{X}$ and $3 \mathrm{X}$ Reference Convective Heating LWRHU Fuel Results: 75\% Ablated Stable LWRHU Releasing Spinning Fuel Response at Reference Convective Heating LWRHU Fuel Results: $100 \%$ Ablated Stable LWRHU Releasing Spinning Fuel Response at Reference Convective Heating 
List of Figures (Continued)

Figure 113 LWRHU Fuel Results: $75 \%$ Ablated Stable LWRHU Releasing Tumbling Fuel Response at Reference Convective Heating

Figure 114 LWRHU Fuel Results: $100 \%$ Ablated Stable LWRHU Releasing Tumbling Fuel Response at Reference Convective Heating

Figure 115 LWRHU Fuel Results: 75\% Ablated Spinning LWRHU Releasing Sideon Stable Fuel, Response at Full Convective Heating

Figure 116 LWRHU Fuel Results: 75\% Ablated Tumbling LWRHU Releasing Side-on Stable Fuel, Response at Full Convective Heating

Figure 117 LWRHU Fuel Results: 75\% Ablated Tumbling LWRHU Releasing Spinning Fuel, Response at Full Convective Heating

Figure 118 LWRHU Fuel Results: 75\% Ablated Tumbling LWRHU Releasing Tumbling Fuel, Response at Full Convective Heating

Figure 119 Thermostructural Model for the LWRHU Aeroshell

Figure 120 Temperature Loads on LWRHU Aeroshell Model

Figure 121 Thermoplastic Stress-Strain Material Properties of FWPF ${ }^{\text {th }}$

Figure 122 Thermoelastic Stress-Strain Material Properties of FWPF ${ }^{\text {th }}$

Figure 123 Normal Strains of FWPF Unit Cell Model with Thermoelastic Material Properties under Hydrostatic Compressive Loads

Figure 124 LWRHU Aeroshell Free-Body Diagram

Figure 125 LWRHU Side-on Stable, $\gamma=-90^{\circ}$, Strain Factors of Safety

Figure 126 LWRHU Side-on Spinning, $\gamma=-90^{\circ}$, Strain Factors of Safety

Figure 127 LWRHU Side-on Spinning, $\gamma=-50^{\circ}$, Strain Factors of Safety

Figure 128 LWRHU Side-on Spinning, $\gamma=-7^{\circ}$, Strain Factors of Safety

Figure 129 LWRHU Random Tumbling, $\gamma=-90^{\circ}$, Strain Factors of Safety

Figure 130 LWRHU Random Tumbling, $\gamma=-50^{\circ}$, Strain Factors of Safety

Figure 131 LWRHU Random Tumbling, $\gamma=-7^{\circ}$, Strain Factors of Safety 
List of Acronyms

\begin{tabular}{|c|c|}
\hline ABACUS & Not an Acronym \\
\hline $\mathrm{ACE}$ & Aerotherm Chemical Equilibrium Code \\
\hline ANSP & Aerospace Nuclear Safety Program \\
\hline ANSPNL & ANSP Non-linear Code \\
\hline CFD & Computational Fluid Dynamics \\
\hline CMA & Charring Material Thermal Response \\
\hline DEIS & Draft Environmental Impact Statement \\
\hline DoE & U.S. Department of Energy \\
\hline FEA & Finite Element Analysis \\
\hline FEM & Finite Element Model \\
\hline FEMAP & Finite Element Modeling and Post Processing \\
\hline $\mathrm{FS}$ & Factor of Safety \\
\hline FSAR & Final Safety Analysis Report \\
\hline FWPF & Fine Weave Pierced Fabric \\
\hline GPHS & General Purpose Heat Source \\
\hline HNUS & Haliburton NUS \\
\hline INT3-D & 3-D Interpolation Code \\
\hline JANNAF & Joint Army Navy NASA Air Force \\
\hline JHU/APL & The Johns Hopkins University Applied Physics Laboratory \\
\hline JPL & Jet Propulsion Laboratory \\
\hline LANL & Los Alamos National Laboratory \\
\hline LASL & Los Alamos Scientific Laboratory \\
\hline $\mathrm{LL}$ & Lawrence Livermore \\
\hline LMMS & Lockheed Martin Missiles and Space \\
\hline LORAN-C & Langley Optimized Radiative Non-Equilibrium \\
\hline LRAD-3 & Louisiana State University Radiation Transport Model \\
\hline LWRHU & Lightweight Radioisotope Heater Unit \\
\hline MHW & Multihundred Watt \\
\hline MSC & MacNeal-Schwendler Corporation \\
\hline NASA & National Aeronautics and Space Administration \\
\hline NASTRAN & NASA Structural Analysis \\
\hline ORNL & Oak Ridge National Laboratory \\
\hline PG & Pyrolytic Graphite \\
\hline RACER & LMMS Flowfield Solver Code \\
\hline RADICAL & Aerotherm's Radiation Transport Code \\
\hline RAD/EQUIL & Aerotherm's Radiation/Equilibrium Transfer Code \\
\hline RASLE & Radiation Shock Layer Equilibrium Code \\
\hline RETAP & Reentry Thermal Analysis Program \\
\hline RIFSP & NASA's Radiating, Inviscid Flow, Stagnation Point Code \\
\hline SAR & Safety Analysis Report \\
\hline
\end{tabular}


Applied Physics Laboratory

Laurel, Maryland 20723-6099

\section{List of Acronyms (Continued)}

SoRI

SRMU

TDOF

VEEGA

VVEJGA

1-D

2-D

3-D
Southern Research Institute

Solid Rocket Motor Upgrade

Three-Degree-of-Freedom Code

Venus-Earth-Earth Gravity Assist

Venus-Venus-Earth-Jupiter-Gravity Assist

One-dimensional

Two-dimensional

Three-dimensional 


\section{I. $\quad$ NTRODUCTION}

In the Aerospace Nuclear Safety Program (ANSP) effort associated with the Cassini Mission, The Johns Hopkins University Applied Physics Laboratory (JHU/APL) has been tasked by the U.S. Department of Energy (DoE) with the primary responsibility of assessing the survivability of the Lightweight Radioisotope Heater Unit (LWRHU) in an inadvertent entry into the Earth's atmosphere. This report constitutes the JHU/APL contribution to the Final Safety Analysis Report (FSAR) for the LWRHU. It includes methodology, assumptions, and results of thermal, ablation response, and thermostructural (thermal and mechanical stress) analyses.

The key personnel and their roles in this study are as follows: E. F. Lucero is the Program Manager for the JHU/APL Aerospace Nuclear Safety Program; L. G. Everhart is the Assistant Program Manager; P. T. Brenza: Solid Rocket Motor Upgrade (SRMU) Fire/LWRHU Thermal Response (under separate cover); L. G. Everhart: Thermal and Ablation Response Predictions; Thermal Analyses Code Development; D. W. Conn: Aerothermodynamic Methodology; LWRHU and Bare Fuel Thermal Models; Material Thermal Properties; Y. Chang: Thermoplastic Methodology; J. A. Ecker: Thermal Stress Analyses; E. F. Lucero and R. C. Sharbaugh: Aerodynamics and Motion Studies; and A. Combs: Program Secretary. 


\section{MODULE DESCRIPTION}

\section{A. Geometry and Mass Properties}

The LWRHU is a thermal energy system operating from the radioactive decay of 238 plutonium dioxide to deliver approximately one thermal watt of power and is employed aboard spacecraft to locally condition temperature sensitive structure and equipment over the spacecraft's mission lifetime. The LWRHU design shown in Figure 1 represents the Galileo configuration and remains unchanged for the Cassini mission. The LWRHU material constituency is defined in this figure and indicates a high temperature capability through extensive use of carbon materials. The geometric parameters and mass properties that are pertinent to the aerodynamic, thermal, and thermostructural analyses of the LWRHU and components, Figures 1-4, are described here for easy reference.

Figure 2 details the aeroshell geometry and shows the aerodynamic envelope of the primary reentry body (i.e., the full LWRHU assembly) to be a right cylinder with nominal dimensions of $25.95 \mathrm{~mm}$ in diameter and $31.95 \mathrm{~mm}$ in length. Figure 3 shows the clad and fuel assembly. The other reentry body of interest is the integral fuel pellet shown in Figure 4. For purposes of reentry analyses, this geometry was approximated as a right cylinder based on the pellet's total volume and maximum diameter of $6.00 \mathrm{~mm}$. This approximation permitted double use of the aerothermodynamics defined for the analyses as discussed in Chapter VII.

Reference drawings providing detailed dimensional descriptions for all components are provided in Appendix B.1 of Reference 1. A detailed weight distribution (also taken from Reference 1) of the system's components is provided in Table 1. Total assembly weight is approximately 40.0 grams.

\section{B. LWRHU Reentry Design Features}

\section{Component Design}

At the component level, the primary reentry design elements for the LWRHU are the heat shield component (aeroshell); the thermal insulator components; and the fuel's structural containment (clad) component. The aeroshell is constructed from Fine Weave Pierced Fabric, FWPF ${ }^{\mathrm{tm}}$ (TEXTRON Specialty Materials) three-dimensional (3-D) Cartesian weave carbon-carbon, which provides comparable energy accommodation and, depending on densification, comparable ablation performance to the predecessor graphites (e.g., POCO grades) used in earlier heat source designs. FWPF ${ }^{\text {to }}$ was the material of choice for the aeroshell due to its superior fracture mechanics attributes. Its ability to arrest crack propagation (strain energy) will likely result in a potential thermostructural failure remaining a localized event as opposed to a catastrophic event as identified with bulk graphites. 
Thermal insulation of the fuel containment assembly is provided by three pyrolytic graphite (PG) nested insulator sleeves and two $P G$ insulator plugs. The direction perpendicular to the plane of deposition (the ' $c$ ' direction) is aligned in the radial direction for the cylindrical sleeves to provide low conductivity heat transmission for flight configurations represented by a side-on stable, side-on spinning or tumbling orientation. Similarly, the 'c' direction for the plugs is aligned in the axial direction to provide protection for end-on, end-overend or tumbling flight orientations. Standoffs have been machined on the sleeves and the containment member to maintain open interface gaps to further increase thermal resistance to heat flow directed to the containment assembly. To prevent the formation of critical thermal paths to the containment component via the high thermal conductivity directions of the pyrolytic graphite insulators (i.e., parallel to the deposition plane or ' $a-b$ ' plane), the sleeves and plugs are mated in a stepped fashion. The stepped interface also prevents radiation transmission directly from the aeroshell's interior surface to the containment and provides a tortuous pathway for gas infiltration.

The LWRHU's remaining design element, the fuel's structural containment component, is fabricated from the noble alloy, $\mathrm{Pt} 30 \mathrm{Rh}$. This material meets all design requirements such as fuel compatibility, high melting point, high temperature strength and excellent oxidation resistance. Its key attribute, however, resides in its excellent ductility behavior in combination with a very low ductile to brittle transition temperature zone. These characteristics permit significant deformation of the containment shell in absorbing hard target impact energies and thereby reduces the likelihood of a breach upon reentry impact.

\section{System Design:}

Reentry design considerations at the systems level can generally be related to design strategies involving size, shape and weight. Taken collectively, they determine a reentry body's ballistic coefficient $(\beta)$ :

where

$$
\beta=\frac{W}{C_{D} S_{r e f}}
$$

$$
\begin{aligned}
& W=\text { weight of the reentry body, } \\
& C_{D}=\text { drag coefficient, and } \\
& S_{\text {ref }}=\text { reference area for the drag coefficient. }
\end{aligned}
$$

A low $\beta$ heat source design is desirable for several reasons including rapid deceleration with resulting lowered convective and radiative heating rates and reduced heat pulse flight periods; increased likelihood of sustained laminar flow; prolonged subsonic cooling; and, reduced impact velocities. Upon survival of the reentry heat pulse, the latter two considerations become important factors in determining fuel containment impact performance. The LWRHU's reduced weight and blunt geometry (i.e., high $\mathrm{C}_{\mathrm{D}}$ ) meets the design criteria for a low ballistic coefficient reentry body. 
One of the system's design parameters, size, provides several positive reentry benefits. A small reentry body such as the LWRHU can inhibit boundary layer transition and the advent of turbulent heating; reduce radiative heating due to a thinner shock layer; and increase the likelihood for full scale ground testing to assist design validation. This last factor was instrumental in validating the LWRHU's end-on ablation design for the high probability orbital decay reentry environment via full scale arc-jet testing (Reference 2). Negative aspects of a small reentry body include increased convective stagnation and downstream heating and limited system thermal capacitance to accommodate the reentry heat load. This thermal capacitance limitation can be countered through judicious material selection, configuration and arrangement at the component level. This was illustrated earlier by the internal thermal design of the PG insulators. 


\section{III. $\quad$ REENTRY CONDITIONS}

The mission profile for Cassini includes launch by the Titan IV/Centaur launch vehicle with injection into a VVEJGA trajectory. The VVEJGA trajectory involves one pass by the Earth with some small probability of accidental reentry. This mission profile results in a broad spectrum of possible reentry scenarios, ranging in severity from orbital decay to the very high speed VVEJGA reentries. Detailed analyses of typical reentry scenarios, other than VVEJGA, were provided in the Galileo FSAR for the LWRHU, Reference 1. The Cassini VVEJGA reentries are the primary subject of this report.

The Cassini/VVEJGA Earth reentry breakup analysis conducted by the Jet Propulsion Laboratory (JPL), References 3 and 4, provided the basis for the initial velocity and altitude conditions selected for the LWRHU analysis. A summary of these results is given in Reference 5. Note that JHU/APL chose to conduct the analyses for early release and upwind entry conditions, Figure 5. The initial velocity is $63,812 \mathrm{ft} / \mathrm{s}$. The initial altitudes vary from $323 \mathrm{kft}$ to $236 \mathrm{kft}$ for $-5.2^{\circ}$ to $-90^{\circ}$ reentry angles, respectively.

Initial flight path angles $(\gamma)$ of $-90,-70,-50,-30,-15,-10,-7$, and the capture angle of -5.2 degrees were selected for analysis. The baseline 3-D model generated for the analysis is based on a side-on stable flight orientation. Four additional orientations are also used: side-on spinning, quasi-tumbling cylinder, tumbling sphere, and random tumbling cylinder, the last three being various approximations to a tumbling orientation for the LWRHU. The rationale and necessity for these selections are discussed in Section VII. The sequential analyses for the fuel pellet consider side-on stable, side-on spinning, and random tumbling orientations. 


\section{GENERAL DESCRIPTION OF ANALYSES}

The purpose of the LWRHU reentry analyses is to assess the survivability of the LWRHU under orbital, super-orbital, and VVEJGA reentry modes. Computer simulations are used to calculate trajectory parameters including radiative and convective heating rates. These parameters are used in further simulations to generate multi-dimensional ablation, thermal response, and thermal stress response of the LWRHU and sequential configurations, e.g., the fuel pellet.

The orbital and super-orbital (escape velocity conditions) reentry modes were addressed in the 1985 Galileo FSAR, Reference 6. For the present analyses, the VVEJGA entry modes are emphasized. A discussion of the other scenarios is given in Sections IX and X.

The reentry trajectory of the LWRHU was simulated at JHU/APL using a three-degree-offreedom (TDOF) simulation. This TDOF program was used to generate, in addition to trajectory parameters, the heating rate, $\dot{q}$, stagnation pressure, $\mathrm{P}_{\mathrm{t}}$, and stagnation enthalpy, $\mathrm{h}_{t}$, histories that are used as inputs to one-dimensional (1-D) and multi-dimensional thermal analysis programs. The 1-D Charring Material Thermal Response and Ablation (CMA) program generates preliminary thermal and ablation (recession) response information while the multi-dimensional Reentry Thermal Analysis Program (RETAP) generates detailed recession and temperature distributions. The distributions are used as inputs to programs that are used to evaluate the structural integrity of the module due to thermal and mechanical stresses - the McNeal Schwendler Corporation/National Aeronautics and Space Administration (NASA) Structural Analysis (MSC/NASTRAN) and Chang's Thermoplastic programs. These computer programs are more fully described in the pertinent sections on thermal and stress analyses, see Sections VII and VIII. 


\section{V. $\quad$ AERODYNAMICS}

The LWRHU and LWRHU component aerodynamics used as inputs to the TDOF trajectory simulation consist of the drag coefficient,

where

$$
C_{D}=\frac{\operatorname{drag}(l b s)}{1 / 2 \rho V^{2} S_{r e f}}
$$

$$
\begin{aligned}
& \rho=\text { density in slugs } / \mathrm{ft}^{3} \\
& \mathrm{~V}=\text { velocity in } \mathrm{ft} / \mathrm{sec} \\
& \mathrm{S}_{\text {ref }}=\text { reference area for the drag coefficient }
\end{aligned}
$$

These are given as either a function of Mach number and altitude or as a function of Mach number in the continuum flow regime and a bridging function for interpolating between continuum and free molecule flow. A constant value of angle of attack is assumed. Side-on stable, side-on spinning, and tumbling orientations have been selected for LWRHU thermal ablation, and thermal stress analysis. The aerodynamic estimates used as inputs to the TDOF simulation, References 7 and 8 , are given in Tables 2 and 3 for these orientations. Since aerodynamic data for cylinders of these low fineness ratios $(\approx 1.2)$ are very sparse (especially in the free molecule regime), these aerodynamic estimates are based on a variety of data found on blunt bodies - disks, plates and some cylinders.

The drag coefficient at terminal velocity conditions of the LWRHU, and the terminal velocity were estimated, Reference 9, in support of impact tests performed by Los Alamos National Laboratory, Table 4 . The cross-flow drag (and consequently terminal velocity) is highly dependent on the wall temperature. The transition from cold wall drag to hot wall drag is dependent on Reynolds' number and surface roughness. The expected ranges in these parameters for the LWRHU are such that either cold or hot values are possible, Table 4. The average is representative of a tumbling LWRHU.

For comparison, the drag coefficient for an ablated LWRHU approximating a spherical shape is given in Table 3. This value also applies to spherical fuel particles except that the reference area is the cross-sectional area of the sphere and the reference length is its diameter. The drag coefficient for the side-on and tumbling fuel pellets are also those of References 7 and 8 (Tables 2 and 3) but the reference area and length are those of the fuel pellet, i.e., for the side-on orientation $\mathrm{S}_{\mathrm{ref}}=D x l=0.000629 \mathrm{ft}^{2}$ and $\mathrm{D}_{\mathrm{ref}}=0.0205 \mathrm{ft}$ and for the tumbling orientation $\mathrm{S}_{\mathrm{ref}}=\pi \mathrm{D}^{2} / 4=0.0354 \mathrm{ft}^{2}$. 


\section{TRAJECTORY PARAMETERS}

The output from the TDOF trajectory simulation is used as inputs to the thermal and thermostructural analysis codes. This output includes velocity, altitude and deceleration versus time as well as reference heating rates and other aerotherodynamic parameters.

The results of the TDOF simulations are presented in Table 5 and in Figures 6 through 25 . The simulations are run through the hypersonic portion of flight for each angle. A Mach number of 5 is selected to indicate the end of hypersonic flight. Figures 6 and 7 show the representative trajectories developed for the side-on stable and side-on spinning orientations. Figure 6 is the velocity verses altitude profiles. Figure 7 is the time history of the altitudes. Similar diagrams are supplied in Figures 8 and 9, and 10 and 11 for the quasi and random tumbling cylinder, and the tumbling sphere orientations, respectively. The resulting deceleration profiles are shown in Figures 12 through 14. The extreme steep reentry angles result in $\mathrm{g}$ forces in excess of forty times those experienced by the extreme shallow reentry angles. The maximum peak deceleration, approximately $1050 \mathrm{gs}$, occurs for the $-90^{\circ}$ tumbling sphere reentry. The $-5.2^{\circ}$ side-on reentry will experience a peak deceleration of approximately 25 gs.

The heating rate histories are given in Figures 15 through 20. The reference convection and radiation portions are shown separately. Figure 15 is the convective heating for the side-on stable and spinning orientations. In this case the shallow reentry maximum heating rate is about four times less than that of the steep reentry. The conditions for reference convective heating are 1) a 1-ft radius sphere, 2) stagnation flow, 3) cold wall heating, 4) zero mass transfer, and 5) no radiation coupling. Similar results are shown for the tumbling cases in Figures 16 and 17. The reference radiative heating, Figures 18 through 20, shows a difference of about a factor of seven between the reentry extremes with the tumbling sphere experiencing the highest values. In each orientation, the maximum radiation heating dominates the heat pulse for steep flight path angles while peak convective heating dominates the shallow angles.

Figures 21 through 25 are provided to give a quick frame of reference for the trajectory difference induced by the LWRHU flight orientations. A sample steep $\left(-90^{\circ}\right)$ and shallow $\left(-7^{\circ}\right)$ reentry diagram is supplied for the altitude and velocity histories, deceleration profiles, and the convective and radiative heating histories. Figures 26 through 33 illustrate the stagnation pressure and total enthalpy histories. 


\section{THERMAL ANALYSES}

\section{A. General Discussion/Summary}

The ablation and thermal response analyses examine the reentry angles $-5.2^{\circ}$ (approximate capture angle), $-7.0^{\circ},-10^{\circ}$, and $-15^{\circ}$ for the shallow cases and $-30^{\circ},-50^{\circ},-70^{\circ}$, and $-90^{\circ}$ for steep cases. The following cases were investigated:

- $\quad$ Side-on Stable 3-D LWRHU.

- $\quad$ Side-on Spinning 3-D LWRHU.

- Tumbling Spherical 3-D LWRHU.

- Quasi-Tumbling Cylindrical 3-D LWRHU.

- $\quad$ Full Random Tumbling Cylindrical 3-D LWRHU.

- Effect of Carbon/Air Thermochemistry Table Selection examined at $\gamma=-15^{\circ}$, $-30^{\circ},-50^{\circ},-70^{\circ}$, and $-90^{\circ}$.

- Effects of gas dynamic/radiation coupling on convective heating examined for $\gamma=-90^{\circ}$.

The three tumbling orientations noted are various approximations to a LWRHU tumbling about its major axes.

B. Assumptions

\section{Initial Reentry Conditions}

The initial conditions for the generation of LWRHU thermal analysis is taken from the TDOF output listed in Table 5. The JPL early release zero wind conditions for the General Purpose Heat Source (GPHS) and LWRHU (References 3 and 4) were used as initial trajectory conditions. The JHU/APL selected entry, based on JPL communications, is for an upwind entry. These release conditions are illustrated in Figure 5. Note that although zero wind conditions from References 3 and 4 are used, the model simulations in TDOF involve upwind reentries into the Earth's atmosphere. JPL did not generate upwind release conditions but noted that the difference between upwind and zero wind were small. The upwind Earth reentry results in an increase in the initial velocity of approximately $3000 \mathrm{ft} / \mathrm{s}$ at the equator relative to a downwind entry. The increase in radiative and convective heating for this increased velocity profile causes possible failures to occur earlier than would occur for a downwind case; therefore, the upwind modeling provides the more conservative analysis approach.

The drag coefficient data used in developing appropriate reentry trajectories for the orientations examined are listed in Tables 2 and 3. The side-on stable and side-on spinning orientations utilize the same drag data, and the quasi-tumbling and random tumbling cylindrical orientations utilize the same drag data. Independent drag data are used of the tumbling sphere 
orientation. Therefore, three different sets of trajectories are necessary to cover the selected model orientations.

\section{Reentry Configurations}

This study considered a simplified sequential breakup scenario consisting of (a) a full LWRHU assembly followed by (b) instantaneous release of the integral fuel pellet should failure events occur during the course of reentry. The fuel pellet is considered to be integral according to inputs from Dr. E. W. Johnson, Reference 10.

\section{Reentry Orientation}

The primary orientations assumed for the LWRHU assembly and subsequent breakup configurations was a sustained side-on stable attitude, a side-on spinning, and several approximations to a tumbling orientation. A side-on stable reentry orientation is a hotter condition than a dynamic, spinning or a tumbling mode and, of the three idealistic attitudes, previous studies indicate that a side-on stable orientation will more likely produce a clad melt event. Of all the in-flight failure modes, the clad melt event bears the greatest significance since it nearly always results in fuel release (if not during flight, then ultimately at impact).

It is judged that the tumbling orientation is the most likely of the three analyzed because of the low aspect ratio of the cylinder. The side-on stable is judged to be the least likely of the three (end-on which is probably even less likely was not considered for thermal analysis). The conditional probabilities suggested for these orientations are:

random tumbling: 0.6

side-on spinning: 0.3

side-on stable: 0.1

Two other flight orientations were examined that are termed 'quasi-tumbling cylinder' and 'tumbling sphere'. These orientations are fictitious artifices that were intended to provide qualitative indications of shape change effects on aeroshell ablation for a (random) tumbling LWRHU. The 'quasi-tumbling cylinder' represents the case where the side-on spinning aerothermodynamic factors (i.e., convective, radiative and free-molecule heating, pressure and recovery enthalpy) were applied to both the cylindrical surface and end face of the LWRHU thermal model and provides a lower bound estimate. As an upper bound, the 'tumbling sphere' case applied spinning sphere heating rates to both the cyindrical surface and end face. However, they represent extreme bounds on shape change effects since both orientations were examined over a reentry's entire heat pulse. It is expected that a LWRHU initially in a (random) tumbling orientation would likely degenerate into a shape and motion resembling a 'tumbling sphere' for shallow reentries possessing an extended heat pulse. Results reported for these two orientation cases should be viewed in a qualitative sense. 
The fuel particle analyses conducted where failure is indicated for the LWRHU assembly assumes integral fuel pellets for all considered flight orientations: side-on stable, side-on spinning, and random tumbling. Conditional probabilities for fuel pellet orientations are not necessary since full fuel pellet melt is predicted for most cases.

\section{Failure Criteria for Ablation and Thermal Response}

The failure criteria for the ablation and melt failure modes were conservatively assigned in these studies in recognition of the uncertainties associated with the high energy Cassini/VVEJGA reentry thermal environments. It is noted that these uncertainty provisions are not the end result of sensitivity studies and accompanying statistical analyses but rather are subjectively selected on the basis of engineering judgment. In most cases, statistics are simply not available for the numerous environmental and response variables that determine these failure modes. Sensitivity analyses would have to be conducted to provide the effect of uncertainties on the LWRHU reentry response parameters and related failure conditions.

\section{a. Ablation Failure Criterion}

The ablation failure criterion for the prior Galileo/VEEGA reentry analyses was assigned at $50 \%$ recession of the wall thickness of the aeroshell member in its side-on stable flight orientation. This criterion was based, in part, on the use of a Joint Army, Navy, NASA, Air Force (JANNAF) $\mathrm{C}_{1}-\mathrm{C}_{5}$ thermochemical equilibrium model and restricted to those immediate aeroshell regions overlaying the insulator sleeves and the fuel containment assembly. Further conservatism was imposed by assuming failure to occur when the stagnation array of aeroshell nodes met the $50 \%$ threshold. Therefore, aeroshell failure and release of the internals were considered to occur when the failure site was limited to a central angle, $\Delta \theta= \pm 15^{\circ}$ about the aeroshell's stagnation point.

The failure criteria assigned to the Cassini/VVEJGA analyses varied from the Galileo criteria in two regards. First, the recession threshold was increased to $75 \%$ of the wall thickness to account for a more severe thermochemical ablation model. The VVEJGA studies considered the Lawrence Livermore $\mathrm{C}_{1}-\mathrm{C}_{7}$ equilibrium model which results in significantly greater total vapor pressures (and therefore recession rates) than the JANNAF model at equivalent surface pressures and 'elevated' temperatures. Second, since the VVEJGA studies were expanded to include a random tumbling flight orientation, the $75 \%$ criterion not only applied to the aeroshell wall overlaying the insulators and fuel containment but also to the wall thickness at the aeroshell/aeroshell end cap threaded interface.

For material ablation, the $75 \%$ wall thickness recession criterion discussed previously is deemed to denote a conservative point of probable aeroshell failure, but the $100 \%$ wall thickness recession is also of note. A $25 \%$ nonconservative approach illustrates a range 
of uncertainty and could allow the aeroshell material to survive through the hypersonic flight region. The ablation failure results are only valid to the point of structural failure due to material loss or mechanical overloading.

\section{b. Melt Failure Criterion}

The melt failure criterion for the VVEJGA studies remains basically the same as assigned for the prior Galileo/Venus-Earth-Earth Gravity Assist (VEEGA) studies. The two components subject to melting are the fuel containment member (clad) and the fuel itself. The VVEJGA reentries result in localized pressures low enough to dismiss the possibility of a melt event for the carbon-carbon aeroshell and the PG insulators.

Two criteria are specified for the melt failure mode depending on the reentry configuration under consideration. For a full LWRHU reentry configuration, the sensitivity of a melt event to environmental uncertainties are somewhat damped by the presence of the PG insulators. For this situation, the failure threshold is assigned the true melt temperature minus the uncertainty allowance of $300^{\circ} \mathrm{F}$. The clad's threshold value becomes $2790^{\circ} \mathrm{F}$ based on a carbon eutectic melt temperature of $3090^{\circ} \mathrm{F}\left(3550^{\circ} \mathrm{R}\right)$. This failure threshold is lower than the Galileo assignment (i.e., $2900^{\circ} \mathrm{F}$ ) by conservatively choosing the lower bound of $\mathrm{Pt} 30 \mathrm{Rh}$ 's carbon eutectic range reported in the literature. The melt threshold for the fuel is $4812^{\circ} \mathrm{R}$. For the current VVEJGA studies, the second criterion applies to the bare fuel pellet reentry configuration. The uncertainty allowance for this condition is assigned at $500^{\circ} \mathrm{F}$ since the sensitivity of unprotected fuel to environmental uncertainties will be greater than for the full LWRHU configuration. This assignment is obviously very subjective, being strongly dependent upon the time of release of the fuel within the reentry heat pulse. The bare fuel allowance results in a melt threshold of $4312^{\circ} \mathrm{F}$.

Regarding the uncertainty band formed by the failure threshold (e.g., the 300 or $500^{\circ} \mathrm{F}$ temperature band on melt failure), the farther a response variable such as 'aeroshell recession' or 'clad temperature' is predicted to penetrate this uncertainty band, the greater the probability of occurrence for this failure event. Here probability is definable only in a qualitative sense (high, low, etc.)

\section{c. $\quad$ Fuel Melt Disposition}

A further conservatism contained in the VVEJGA analyses relates to the disposition of the fuel melt during reentry. The formation of a melt front on the reentering fuel pellet is assumed to be immediately stripped aerodynamically. The stripped molten material is assumed to further breakup in a cascading manner into a final aerosol state containing inhalable particulates. Therefore, the total mass fraction of the fuel pellet predicted to melt during reentry is equated to an atmospheric source term. 


\section{LWRHU Sequential Breakup Scenario}

The sequential breakup scenario assumed for the side-on stable Galileo/VEEGA studies was basically the release of the fuel containment assembly (clad and fuel pellet) upon incipient failure of the aeroshell due to ablation. This was followed by complete release of the integral fuel pellet upon incipient failure of the containment due to melt. Incipient failure is defined as the initiation of a failure site at the immediate stagnation region of the respective aeroshell and containment thermal models. It was observed from the Galileo studies, however, that the containment shell melted almost immediately upon release from the aeroshell and provided very little time delay within the heat pulse for subsequent fuel release. Thus in the Cassini/VVEJGA studies complete release of the fuel pellet is assumed upon incipient failure of the aeroshell and/or the aeroshell end cap.

The 'full LWRHU assembly to fuel pellet reentry sequence' may be a highly conservative approach to the Cassini/VVEJGA reentry analyses. The qualification is that arc jet tests at convective heating rates $1 / 3$ to $1 / 10$ the predicted VVEJGA rates, and at much lower stagnation pressures, indicated catastrophic failure of the LWRHU in about twice the heat pulse interval. The sequence provides a bounding condition on early fuel release within the reentry heat pulse and subsequent generation of atmospheric source terms due to fuel melt. It recognizes no benefit from the internal insulators in delaying release of the fuel upon incipient failure of the aeroshell (re: Figure 1). Unfortunately, the dilemma in selecting a more realistic breakup scenario arises from the large uncertainties in defining local environments upon initial breach of the aeroshell. These uncertainties include augmentation of the convective heating at the local failure site, mechanical erosion effects at the failure site due to internal ram pressure and convective heating from the shock layer gases infiltrating throughout the assembly's open interfaces. The disposition of the PG insulators is another important factor in terms of either remaining integral to maintain thermal protection or fragmenting due to thermostructural loading. It should be clear that qualitatively, fuel release may be delayed beyond the release scenario selected for these studies but time of fuel release cannot be answered reliably in quantitative terms.

\section{Thermal Models}

\section{Reentry Thermal Analysis Program Limitations}

The principal demand in modeling the three-dimensional LWRHU assembly for this study was one in which generic classes of reentry flight motions could be adequately simulated by a single model. A side-on orientation was selected to represent the class of motions where the longitudinal axis (generatrix) is mostly perpendicular to the wind (including oscillatory motion) and a tumbling orientation was selected to portray the more dynamic class of motions. A 3-D model representing a quarter section of the LWRHU assembly was selected for these studies. Similarly, two separate two-dimensional fuel pellet thermal models are implemented. 
The numerical solutions of the thermal diffusion equation were performed by the RETAP. This code has the capability to handle both nonlinear thermal properties and boundary conditions in multi-dimensional space; all the appropriate ablation models for a carbonair system; multiple heat transfer modes for 'open' and 'shut' internal interfaces; and, aerothermodynamics at all velocity regimes either through a data input stream or by internal algorithms (e.g., low density and surface mass transfer corrections).

One principal shortcoming of RETAP is its inability to shrink a structure's thermal network in accordance with the code's surface ablation calculations. For the LWRHU simulations, a dimensionally stable network impacts the thermal and ablation response by increasing the aeroshell's thermal gradients (i.e., thermal stresses) while maintaining a large section modulus; increasing the aeroshell's computed ablation loss; decreasing peak clad temperatures; and, increasing clad impact temperatures. The effects influencing the thermostructural failure mode will be compensating while the effect on computed ablation loss introduces conservatism in assessing the ablation failure mode. The latter clad effects underestimate the evaluation of the clad melt and impact failure modes. The extent of these effects on clad failure assessments, while thought to be small, represents an uncertainty in the analyses that is countered by allowances in the failure criterion.

A second shortcoming involving the TDOF/RETAP code combination is the absence of ablation shape change feedback on flight dynamics and resulting aerothermodynamic input (via the TDOF code) on RETAP's computed response modes. Shape change is believed to have little effect for either the side-on stable or spinning flight orientations since increases in convective (or radiative) heating will be compensated by decreases in radiative (or convective) heating as well as a lower reentry ballistic coefficient due to changes in geometry (i.e., the LWRHU's effective leading radius), weight and aerodynamics. However, the absence of shape change effects for the random tumbling studies may lead to underestimated response predictions for shallow reentries (i.e., extended heat pulse periods) since the LWRHU would likely degenerate into a geometry resembling a sphere. This effect is an uncertainty in the analyses; the sensitivity to this shape has been investigated by using a spherical approximation.

The model development contained several simplifications for the common purpose of preserving RETAP's numerical stability at a reasonable computational time step. These simplifications included:

a. The mass of the stand-offs machined on the insulator sleeves, clad, threaded end cap and the circumferential weld bead of the clad end cap were lumped into the proximate grid node of the stand-off's component. However, the gaps forced by these stand-offs were preserved in the model. 
b. The threaded interface formed by the aeroshell and the end cap was assumed to be a homogeneous material rather than attempting to detail the thread form. This was accomplished by assuming the interface to be closed at a very high thermal contact conductance.

c. The spanner holes in the threaded end cap were omitted, resulting in a slight over specification of the end cap's mass (i.e., thermal capacitance) and omission of localized cavity heating effects. The effects of this omission on clad response was thought to be minor for the tumbling study and negligible for the side-on studies.

d. The fuel was modeled as an integral pellet rather than in some arbitrary orientation of fragments that might arise from the fuel's internal thermal stresses or from launch vibration environment(s).

e. The platinum-rhodium alloy shim was lumped into the near proximity fuel nodes rather than being modeled as a separate component. This resulted in a composite nodal structure in which the overall thermal capacitance was based on the participating materials thermal capacitance weighted by their volume fraction. The thermal conductivity and total hemispherical emittance properties of the composite assumed the fuel's schedule.

f. The modeling assumed the clad cap closures were identical rather than attempting to discriminate on the vented closure with attending frit.

\section{Thermal and Ablation Response Considerations}

There are two basic modeling requirements to be considered in addressing reentry environments. The first requirement is one of modeling the LWRHU's structural configuration in variables appropriate for solving the general thermal diffusion partial differential equation. This model is generally referred to as a thermal model or network since the thermal variables possess electrical analogies. The second modeling requirement is one of simulating the ablation processes operating at the LWRHU aeroshell's external surface. The ablation modeling is obviously coupled directly to the thermal environment as well as to the structural thermal network. These modeling requirements apply to the sequential breakup configurations as well.

\section{a. $\quad$ LWRHU Thermal Models}

Solutions to the thermal diffusion equation must be done numerically since, for reentry problems such as the VVEJGA scenarios, the equation itself is highly nonlinear and the required boundary conditions are nonlinear in both time and space. A numerical approach requires subdividing the various structural components into discrete elements or nodes with each of their center of mass being a spatial location for a calculated temperature history as the solution marches in time along the reentry trajectory. Each node is characterized by a thermal 
capacitance and thermal conductance (connecting adjacent nodes) wherein the total composite is termed the thermal network. The 3-D thermal network for the LWRHU assembly considered in the side-on analyses is shown in Figure 34 and represents a cross-sectional view in cylindrical coordinates $(R, \theta)$ taken at the mid-plane of the assembly. The network is described by a $12 \mathrm{R} \times 7 \theta$ notation for a total nodal count of 84 . The second thermal model is shown in Figure 35. Figure 35.a shows the two-dimensional (2-D) side-on fuel pellet reentry configuration that would result from either an ablation or thermal stress failure of the LWRHU aeroshell. This model shows an enhanced spatial grid fineness for the pellet from that shown in Figure 34 to improve on the resolutions of melt mass fraction during reentry. The third model considered (Figure 35.b) was the 2-D tumbling fuel pellet configuration.

\section{(1) Three-Dimensional LWRHU Assembly Model}

The genesis for the quarter section 3-D LWRHU model was a model constructed during the development phases of the LWRHU to validate the assembly's reentry thermal design (circa: 1979/1980). A good portion of this model had to be reworked based on the Table 1 reference drawings, particularly regarding the insulator components. A typical longitudinal (i.e., radial, r - axial, z) cross-section of the LWRHU is shown in Table 6.a and provides regional representations of the thermal model simulation. The thermal model response regions are provided in Table 6.a and the thermal model interface regions in Table 6.b.

The resulting thermal model for the LWRHU assembly is shown in Figure 34. Figure 34.a presents the $r-z$ cross-section for the stagnation segment (i.e., $\Delta \theta$ $=0^{\circ}$ to $15^{\circ}$ ) of the model and depicts the capacitance (lumped mass) and surface nodal locations and the corresponding nodal indices. It is noted that these are the nodes of interest for the side-on orientation studies. Figure $34 . \mathrm{b}$ presents the $\mathrm{r}-\theta$ cross-section at the model's mid-span location $(\mathrm{z} / \mathrm{L}=0)$. This cross-section closely parallels the 2-D thermal model considered in the Galileo studies except for a coarser nodal grid in the aeroshell and finer grids in the middle and outer sleeves. It is neither required nor practical to precisely model the LWRHU assembly in order to capture its thermal response behavior. Table 6.c outlines the simplifications considered in the construction of the model. The model is characterized by 331 capacitance nodes and approximately 475 surface nodes. The computational (stability) time step was typically in the range of 0.001 to 0.002 second which was more than adequate to capture the nonlinearites of the VVEJGA reentry problem. Material thermal properties used in computing nodal thermal capacitances and conductances are discussed in Section VII.D.

\section{(2) Two-Dimensional Fuel Pellet Thermal Models}

Following discussions with Dr. Emest W. Johnson, EG\&G/Mound, the release of fuel subsequent to incipient failure of the aeroshell was assumed to be in an integral pellet form rather than fragments. The fuel pellet shown in Figure 5 is essentially a right cylinder with chamfered ends. The pellet was modeled as a right cylinder based on its 
maximum diameter and its total volume. This permitted use of the aerothermodynamics developed for the LWRHU assembly due to similarity in geometries. Upon release from the failed LWRHU, the pellet assumed the same basic flight orientations as considered for the LWRHU. The side-on stable and spinning studies adopted the Galileo's r- $\theta$, mid-span model which is shown in Figure 35.a. For the random tumbling orientation, a simple $r-z$ model was constructed which is shown in Figure 35.b. The basic objective for these models was to determine the mass fraction of the simulated pellet that melted during bare fuel reentry.

\section{b. Interface Modeling}

Table 6.a and Table 6.b indicate there are numerous interfaces in the interior of the LWRHU which will influence thermal energy transport to the critical clad components. The modes of heat transfer considered at an internal interface are dependent upon the local state of the interface. For 'open' interfaces (i.e., a gap between opposing components), the modes of heat transfer are typically (a) thermal radiation and (b) gaseous conduction if the intervening gap is gas filled. For 'shut' interfaces, solid conduction becomes the dominant heat transfer mode.

\section{Thermal Radiation Heat Transfer}

In determining interface thermal radiation between opposing surface nodes, total hemispherical emittance data followed by total normal data were given top priority. These data are presented as a function of temperature in Section VII.D which discusses material properties. Based on these data, the radiation exchange factors for the various interfaces were determined on the basis of closely spaced parallel plates. This is a reasonable approximation for determining heat transfer for 'open' interfaces and provides a conservative estimate for 'shut' interfaces. Radiative heat transfer is assumed to occur only between the pair of opposing surface nodes at an interface. Heat transfer from a surface node to other nodes at the opposing surface of an interface is negligible due to the small gap sizes existing at the interfaces.

\section{(2) Gaseous Conduction Heat Transfer}

Open interface gaseous conduction is determined from the Fourier heat conduction law and therefore is sensitive to both the gas fill's thermal conductivity and the interface gap size. The remaining consideration for this heat transfer mode is the gap fill history during reentry.

Typically in past analyses, gaseous conduction was either neglected by assuming the open interfaces to be evacuated or determined on the basis of either a helium or air gas fill. This study developed interface thermal data for all three possibilities. The thermal conductivity schedules selected for the helium and air gas fills as a function of temperature are presented in Figure 36. These schedules were developed (from data from References 11 
through 16) for a one atmosphere pressure rather than introducing bivariate dependency on both pressure and temperature. It is noted that over the anticipated temperature range of the LWRHU interfaces, pressure corrections on thermal conductivity are negligible (Reference 11). As a further justification, when interface temperatures are near maximum, radiation becomes the dominant heat transfer mode.

Determination of gap distributions for the interfaces represented an uncertainty in the analyses due to possible tolerance build-up permutations during assembly, internal component thermal expansion effects, etc. As a result, distributions were established for three hypothetical interface states: (a) 'perfect alignment', (b) 'translational displacement' and (c) 'thermal expansion'. These states and the gap specifications for the radial and axial interfaces in a side-on stable orientation are defined in Table 6.d. The assignment of interface states for each of the flight orientation cases is provided in Table 6.e.

Early LWRHU reentry design studies for parking orbit accident scenarios considered evacuated gaps during initial reentry that subsequently became filled with helium discharged from the plutonium dioxide fuel. Helium release was arbitrarily related to threshold temperature levels specified for the clad member (Reference 6). A more sophisticated helium release model as well as the modeling of air influx and efflux through the aeroshell's permeable wall resulted in a dilution of the helium effect over most of the heat pulse examined for a shallow reentry from parking orbit (i.e., a $\gamma_{\min }$ reentry). It was demonstrated that the thermal response of the clad using this sophisticated mixing (helium-air) model could be closely approximated by a one atmosphere air fill in the gaps from initial reentry to impact (Reference 6). For steeper reentries, the dilution of released helium becomes even greater due to greater mass flux of air through the permeable aeroshell at the higher windward pressure distributions. As the case for the Galileo studies, the simpler air fill model (from initial reentry) was chosen for all the VVEJGA flight orientation studies.

\section{(3) Solid Conduction Heat Transfer}

Solid conduction at 'shut' interfaces or localized segments such as the designed stand-offs is governed by the acting thermal contact conductance at the closure. Contact conductance is dependent upon numerous conditions including the material pair in contact, temperature, pressure, surface finish, gas fill, contact geometry, etc. Unfortunately, engineering data addressing these variables for the LWRHU material configuration are acutely absent. Table 6.e presents subjective interface thermal contact conductance schedules considered in prior LWRHU side-on stable analyses. For the VVEJGA study, a literature survey was conducted in an attempt to obtain data representative of the material pairs comprising the LWRHU's interfaces (References 17 to 23). This effort resulted in the schedules presented in Table 6.f for the indicated states for the side-on stable flight orientation. The contact conductances were valued according to qualitative compressive stress states (i.e., 'high' or 'low' contact pressures) with the high stress value being approximately three times the low stress value based on some limited FWPF carbon- 
carbon conductance data (References 19 and 20). Due to lack of data, the contact conductances were held constant with temperature. Since interface closures involve engineering surfaces, contact will not be perfect but will be limited to surface asperites in direct contact. Consequently, there will be local voids at the interface that will also permit radiative and gaseous conduction transfer. Radiative transfer is computed according to Section VII.C.2.b.(1) and gaseous conduction is assumed to be a contributing component in the values presented in Table 6.f. The notes contained in Table 6.f indicate how these data were used for the side-on spinning and random tumbling flight orientations.

\section{Initial Value and Boundary Conditions}

The numerical solution of the thermal diffusion equation via the RETAP code requires an initial value of the LWRHU's temperature field and two boundary conditions for each spatial variable.

\section{a. Initial Value}

The initial temperature field for the LWRHU assembly remained the same as considered in the early LWRHU design studies and is presented in Table 7. This distribution represents the steady state operating temperature in a space environment. Use of this distribution implies the open interfaces within the assembly are in vacuum up to the time of release of the LWRHU from the parent spacecraft. The initial fuel temperature for bare fuel reentry scenarios equate to the pellet distributions at the time of incipient failure of the LWRHU's aeroshell.

\section{b. Boundary Conditions}

One of the required boundary conditions is met through specification of adiabatic surfaces during construction of a thermal model based on the structure's symmetry. The use of adiabatic surfaces are implied in the use of a quarter section thermal model for the LWRHU assembly shown in Figure 34. Similar use of adiabatic surfaces are contained in the 2-D models for the fuel pellet shown in Figure 35. The adiabatic surfaces contained in the $r-\theta$ fuel model do not reflect on the symmetry of the problem but were imposed to expedite the analyses through use of an existing Galileo model.

The second boundary condition is provided by the specification of energy balances specified at all internal surfaces of the LWRHU components and the aerodynamic (i.e., external) surface of the aeroshell. Whether the internal interfaces are represented by 'open' or 'shut' interfaces, the total thermal energy flux into a surface equals the total thermal energy flux away from the surface. The total energy flux is comprised of solid conduction, gaseous conduction and thermal radiation heat transfer modes in various combinations pending the particular configuration of the interface. 
The energy balance at aerodynamic surfaces is arguably the prime relationship in the solution of a reentry problem in that it couples the thermal environment to the thermal and ablation response modes of the structure. The surface energy balance conducted at every surface node in the LWRHU or fuel pellet thermal models for the VVEJGA analyses is shown in Figure 37. The solution of the surface energy balance for the LWRHU's carbon-carbon aeroshell is simplified by the unity Lewis number analogy equating the local mass transfer coefficient to the local heat transfer coefficient. The assumption of a unity Lewis number for a carbon-air system represents a good approximation based on the multi-component reacting boundary layer solutions with ablation by Putz and Bartlett (Reference 24).

\section{4. $\quad$ Ablation Modeling}

\section{a. Carbon-Carbon (Aeroshell, Aeroshell End Cap)}

Figure 38 illustrates that the surface mass balance for an ablating carbon surface comprises a mass flux convected into a surface control volume in its solid phase equated to a mass flux convected (and diffused) out of the surface volume in its vapor phase (thermochemical ablation) as well as ejected in its solid phase (mechanical ablation). For the VVEJGA analyses, mechanical ablation was not explicitly considered but represents an uncertainty contained in the allowance specified by the ablation failure criterion (i.e., $75 \%$ aeroshell wall thickness). While an approximate mechanical ablation model has been developed at APL for bulk graphites (specifically, ATJ grade, Reference 25) based on the data of Lundell and Dickey (Reference 26), this model was not considered representative of 3-D carbon-carbon. The FWPF should have stronger resistance to mechanical effects due to the orthogonal weave construction, particularly for relatively low stagnation pressures characteristic of low ballistic coefficient reentry bodies such as the LWRHU.

Figure 39 illustrates the thermochemical ablation regimes for a carbon (or graphite)-air system and shows a sampling of the JANNAF ' 71 sublimation model and the diffusion-limited and moderate rate-limited oxidation models used in the Galileo/VEEGA analyses. Since the VVEJGA analyses were truncated to only the heat pulse flight period rather than full flight to impact, ablation considerations were limited to carbon's diffusion-limited oxidation and sublimation models. The diffusion-limited oxidation plateau in Figure 39 is based on a carbon monoxide reaction product and represents, in theory, the limiting solid carbon consumption rate for an oxidation process. The carbon monoxide based model was used in both the VEEGA and the VVEJGA analyses. As the name implies, this limit is reached when reaction rates become so rapid that the consumption rate of the reactant, oxygen, becomes limited to oxygen's ability to diffuse across the boundary layer to the reacting surface.

Two sublimation models were considered for these studies. The Lawrence Livermore (LL) carbon thermodynamics (characterized by vapor species $\mathrm{C}_{1}$ through $\mathrm{C}_{7}$, References 27 and 28) and the 1986 JANNAF thermodynamics $\left(\mathrm{C}_{1}\right.$ through $\mathrm{C}_{5}$ vapor species, Reference 29) were provided as input to the Aerotherm Chemical Equilibrium (ACE) code 
(Reference 30) to develop equilibrium ablation models in the CMA code format (mass transfer parameter and wall gaseous enthalpy as a function of pressure and temperature, References 31 and 32). For a given set of carbon vapor thermodynamics, surface pressure, and surface temperature, the use of an equilibrium versus a nonequilibrium ablation model provides an upper bound on sublimation loss of carbon. It is noted that nonequilibrium ablation is a more realistic mode for the shallow low density reentry corridor but will progress, in the limit, toward equilibrium ablation rates at higher pressures associated with steeper reentries.

The LL model's total vapor pressure is significantly greater than the JANNAF model and was used to evaluate the LWRHU's aeroshell ablation failure mode. Use of the LL model was the basis for increasing the failure criterion to the $75 \%$ wall thickness value from the $50 \%$ value considered in the Galileo studies with the ' 71 JANNAF model. While an equilibrium model is more critical in evaluating the ablation failure mode, a nonequilibrium ablation model is more critical in evaluating the thermostructural failure mode due to higher surface temperatures and temperature gradients. The ' 86 JANNAF model was used as a substitute for a LL nonequilibrium model in the determination of aeroshell temperature distributions for thermostructural failure evaluation. The resulting ' 86 JANNAF model showed negligible differences in ablation parameters from the prior ' $71 \mathrm{JANNAF}$ model used in the VEEGA analyses. Additional information on APL's sublimation and oxidation models can be found in References 25 , 33 and 34.

The analyses did not consider melting as an ablation process due to the high pressures measured at carbon's triple point from early investigations (References 35 to 38 ) and more recently by Cezairliyan and Miller (Reference 39). These pressures range from 100 to 110 atmospheres which dwarfs the maximum stagnation pressures experienced across the VVEJGA reentry spectrum.

\section{b. $\quad$ Plutonium Dioxide (Fuel Pellet)}

Ablation modeling of the fuel pellet is much more complicated than the modeling of a carbon aeroshell due to the addition of a melting event. Fuel modeling is a moving boundary problem involving three layers consisting of a solid core topped by a melt layer topped by a vaporization/air mixture layer. Further modeling complications arise in describing the hydrodynamic behavior of the melt layer in reaction to aerodynamic loading (e.g, stripping due to shear loads, ballooning due to normal loads, etc).

The VVEJGA analyses circumvented these complications by taking a (presumably) conservative, simplistic approach to the fuel reentry problem. The same surface energy balance was used as indicated in Figure 37 but with substitution of plutonium dioxide thermochemistry to determine vaporization rates. However, at fuel melt temperatures, vapor pressure is still at low levels and does not represent a significant loss mechanism. The approach consisted of a thermal response analyses without inclusion of moving boundaries or shape change effects to determine the mass fraction of the pellet in the melt phase. This melt mass fraction was 
equated as an atmospheric source term implying that the formation of a melt layer is immediately stripped from the pellet core into fine droplets with further breakup due to aerodynamic forces and heating into an inhalable mist. The fuel's heat of fusion effect was incorporated into the analyses through alteration of the material's thermal capacitance (i.e., density $\mathrm{x}$ specific heat product) schedule at the melt failure criterion's temperature (i.e., $\mathrm{T}_{\text {fail }}=\mathrm{T}_{\text {melt }}-500^{\circ} \mathrm{R}$ ).

References 40 and 41 .

Further perspectives on the fuel reentry problem are available in

\section{Fuel Energy Source Term}

The energy source term of the plutonium dioxide fuel was included in RETAP's thermal analyses despite its limited influence on clad thermal response during the VVEJGA heat pulse flight periods. Its principal influence resides in the evaluation of the LWRHU's impact failure mode by partially governing the clad's thermal response during subsonic flight to impact. The VVEJGA studies assumed the fuel's one thermal watt of energy was uniformly distributed over the entire pellet.

\section{Material Properties}

Determination of the thermal response of a reentry structure requires specification of the thermodynamic property (specific heat) and the transport property (thermal conductivity) for all materials involved in the design. The need to obtain numerical solutions to the heat diffusion equation is caused, in part, by the problem's nonlinearity resulting from the strong dependence on temperature of the above properties. In addition to these properties, the solution of the surface energy balance (Figure 37) requires information on the thermal radiation properties, absorptivity and emissivity, to determine absorbed shock layer radiation and surface re-radiation. Additionally, these properties are required to determine radiation transfer at the internal interfaces.

The constituent materials of the LWRHU are shown in Figure 1. Briefly, they are aeroshell body and aeroshell end cap: Fine Weave Pierced Fabric (FWPF ${ }^{\mathrm{tm}}$ ) 3-D carbon-carbon; insulator bodies (sleeves) and insulator cap (end plug): pyrolytic graphite; clad body assembly (fuel containment) including end closures, shim and vent frit: platinum-30\% rhodium alloy (Pt-30Rh); and, fuel pellet: plutonium dioxide $\left(\mathrm{PuO}_{2}\right)$.

The following schedules of thermal diffusion properties, thermal radiative properties and phase change properties defined herein for the LWRHU constituent materials were the product of a literature survey and evaluation in support of the Cassini/ VVEJGA reentry safety studies. In some instances, this resulted in a departure from the schedules considered for the prior LWRHU Galileo/VEEGA reentry studies (Reference 42). 
Gaseous conduction at the interface gaps was based on the thermal conductivity of air (at 1 atmosphere) using the National Bureau of Standard's schedule for temperature at below $2500^{\circ} \mathrm{R}$ and the Yos model for temperatures greater than $2500^{\circ} \mathrm{R}$ (Reference 43).

High temperature problems such as reentry are not only difficult to address due to the involved physics but also due to the uncertainty of physical property data required for such evaluations. The thermophysical data used in the Cassini studies are not exceptional in that the high temperature entries are based on extrapolations, or engineering judgment, from a lower temperature data base and represent a major source of uncertainty in studies of this type. This is particularly true for the fuel reentry problem where the focus is on the behavior of a high temperature melt layer. Due to a void of data in the melt phase, all thermal properties at or beyond the melt temperature of $\mathrm{Pt}-30 \mathrm{Rh}$ and $\mathrm{PuO}_{2}$ were maintained at constant values. It is also noted that data indicated in the form of symbols in the material property presented in the succeeding figures are not necessarily experimental values. They may represent selected points from curves that have been faired or fitted to data from theoretical or experimental investigations. The cited references should be explored if further clarification of such data is desired.

\section{Thermal Diffusion Properties}

Reentry thermal response calculations for the LWRHU require numerical solutions of the thermal diffusion equation and, thereby, definition of the density, specific heat and thermal conductivity properties of the constituent materials are required. Further, the thermal conductivity properties must be defined on a directional basis when necessary to account for material processing effects.

\section{a. Fine Weave Pierced Fabric 3-D Carbon-Carbon}

\section{(1) Thermal Conductivity (directional property)}

The directional thermal conductivity schedules for $\mathrm{FWPF}^{\mathrm{th}}$ carbon-carbon material are shown in Figures 40 (a), (b) and (c) for the $X(=Y)$ axis, $45^{\circ}$ off $X-Y$ axes and $\mathrm{Z}$ axis respectively. Comparison of the Air Force $\left(\mathrm{P}^{4}\right)$ and Department of Energy Southern Research Institute (SoRI) data References 44 through 46 are presented along with the variability band for the Air Force data. The X (and Y) axis thermal conductivity schedule for the Cassini studies (Figure 40) remains the same as defined for the Galileo studies and represents the mid-range of the variability band. Published Textron data (Reference 47) are consistent with the principal data sources.

The $45^{\circ}$ off $\mathrm{X}-\mathrm{Y}$ axes location should provide the maximum off axes effect in transforming directional Cartesian data to the LWRHU's cylindrical geometry. Figure 40 (b) shows that available $45^{\circ}$ data (Reference 48 ) is very close to the recommended X-axis data. As a consequence, all baseline studies utilized the $\mathrm{X}$-axis schedule to represent radial and 
circumferential conductivities rather than introducing the complexity of transforming available conductivity data to a cylindrical coordinate system. The $\mathrm{X}$-axis schedule was also used in this manner in the Galileo studies.

The recommended Z-axis conductivity schedule represents the mid-range of the principal data sources as well as values considered in early design studies of the LWRHU (Reference 49) and the GPHS (Reference 50). The recommended schedule falls on the upper bound of the Air Force data. The Galileo/VEEGA analyses did not consider Z-axis data since 2-D modeling of the LWRHU was restricted to the $r-\theta$ plane.

\section{(2) Thermal Capacitance (density $\mathrm{x}$ specific heat)}

The density considered in the Cassini studies is $124.1 \mathrm{lb} / \mathrm{ft}^{3}$ and represents the average density of certification data for eight FWPF ${ }^{\text {tm }}$ billets used in the manufacture of GPHS flight quality modules for the Cassini mission (Reference 51). This value remains very close to the density of $124.24 \mathrm{lb}_{\mathrm{m}} / \mathrm{ft}^{3}$ considered for the Galileo studies.

The specific heat schedule is shown in Figure 41 and departs from the schedule considered for Galileo. The basis for the Cassini schedule is the JANNAF data for solid carbon (Reference 52). For comparable heating conditions, the aeroshell thermal response at elevated temperatures will be greater using the Cassini schedule and thereby represents a conservative change from the Galileo studies.

\section{b. $\quad$ Pyrolytic Graphite}

\section{(1) Thermal Conductivity (directional property)}

The directional thermal conductivity schedules for PG are shown in Figures 42 (a) and (b) for the direction perpendicular to the deposition plane (i.e., the 'c' axis direction) and the direction parallel to the deposition plane (i.e., the ' $a-b$ ' alias basal plane direction) respectively. This material is highly anisotropic due to its highly ordered crystallite orientation in the ' $a-b$ ' directions but with variation in the stacking (i.e., spacing and tilt angle) of the crystallite layers in the ' $c$ ' direction. The degree of anisotropy is very sensitive to the parameters of the carbon vapor deposition and annealing processes.

The ' $c$ ' direction thermal conductivity for PG is the critical thermal property in defining the insulation effectiveness of the LWRHU's thermal design. This direction is aligned with the radial direction of the insulator bodies (i.e., the nested insulator sleeves) and the axial direction of the insulator caps to arrest heat flow to the cladded fuel assembly. Definition of the ' $c$ ' direction property is differentiated between the insulator sleeves (for a cylindrical geometry) and insulator caps (a plate geometry). Justification for this differentiation is based on conformance test data for these manufactured parts (References 53 and 54) as well as 
similar behavior demonstrated for these geometries in an early Los Alamos Scientific Laboratory (LASL) investigation of PG thermal conductivity (Reference 55). The Morrison data were based on processing parameters similar to those specified for the manufacture of the Cassini insulators.

The solid curve in Figure 42 (a) is the recommended ' $c$ ' direction schedule for the insulator sleeves and is the product of a task force investigation during the early design phases of the GPHS (References 56 and 57). The variability of this schedule is $\pm 25 \%$ as indicated by the cross-hatched area The curve's low temperature peak has been validated by other experimental investigations (Reference 58). The recommended constant at the high temperature end (Reference 57) infers an absence of delaminations consistent with the requirements contained in the sleeve's acceptance specification (Reference 59). With delaminations, the conductivity schedule would demonstrate a nonlinear dependency on temperature (i.e., $\approx \mathrm{T}^{3}$ ) due to radiative transmission. It was noted that the task force data (Reference 57) agreed well with other PG investigations having comparable carbon vapor deposition parameters (References 60 and 61). The recommended schedule, however, is lower than the aforementioned Morrison data at moderate temperature levels. It is noted that the conformance datum for the sleeve falls below the insulator acceptance range (a favorable condition). The recommenced ' $c$ ' schedule was also used in the Galileo studies for the insulator sleeve components.

Figure 42 (a) also contains the Cassini 'c' schedule defined for the insulator caps. This schedule is guided by Morrison's plate data and follows the trend established by the insulator sleeve schedule by fairing into a plateau value at elevated temperatures. The conformance datum for the insulator caps is consistent with the recommended schedule and is located near the upper bound of the acceptance range. The Morrison data for both cylinder and plate geometries suggest the presence of delaminations in the test specimens as indicated by the rapid increase of conductivity as temperatures approach $4000^{\circ} \mathrm{R}$. These data were discounted in defining the ' $c$ ' schedule. Differentiation of the ' $c$ ' direction conductivity for the insulator caps was not an issue in the Galileo studies since thermal modeling was restricted to the LWRHU's midspan $\mathbf{r}-\theta$ plane.

Availability of thermal conductivity data for PG in the ' $a-b$ ' directions was more restrictive than the ' $c$ ' direction data base. Figure 42 (b) indicates that the recommended schedule for the Cassini studies is based on the tabular schedule reported in Reference 49. This schedule conforms with the curve reported by Super-Temp Company (Reference 58) with both curves converging to a common plateau value at elevated temperatures. As in the ' $c$ ' direction data base, experimental data above $5000^{\circ} \mathrm{F}$ in the parallel direction are unavailable. The conformance data for both the insulator sleeves and caps are at or above the upper bound of the acceptance specification for the Cassini mission. No attempt was made to adjust the selected conductivity schedule to these higher data. In maintaining a lower estimate for the ' $a-b$ ' conductivity, the effect on clad assembly thermal response is conservative by reducing the amount of heat flow shunted away from the design's critical thermal paths in the 'c' direction. The recommended ' $a-b$ ' schedule was also used in the Galileo studies. 
(2) Thermal Capacitance (density $\mathrm{x}$ specific heat)

The PG's density and specific heat values used in reducing the GPHS task force's thermal diffusion data to obtain ' $c$ ' direction thermal conductivity (Reference 57) was selected for the Cassini studies. The density was defined at $137.3 \quad \mathrm{lb}_{\mathrm{m}} / \mathrm{ft}^{3}$ $\left(2.2 \mathrm{~g} / \mathrm{cm}^{3}\right)$ and conforms with the Cassini acceptance specification for the insulator parts. This value was also used in the Galileo studies.

The specific heat schedule is shown in Figure 43 by the solid line curve extending to approximately $4000^{\circ} \mathrm{R}$. The schedule represents a polynomial fit of average specific heat data published at the Purdue Thermophysical Research Center (Reference 62). These data were also used in the Galileo studies. The figure also contains specific heat data published by Super-Temp (Reference 58) and by JANNAF (Reference 52) which show good agreement over this temperature range. The Cassini schedule departs from the Galileo schedule at $4000^{\circ} \mathrm{R}$. This schedule is guided by Super-Temp and JANNAF (theoretical) data to a plateau value of $0.520 \mathrm{BTU} /\left(\mathrm{lb}_{\mathrm{m}}{ }^{\circ} \mathrm{R}\right)$. The Galileo curve continues to increase at a constant slope. For comparable heat loads, the lower thermal capacitance defined for the Cassini study at elevated temperatures will result in a higher thermal response of the insulator parts and, therefore, greater heat flow to the cladded assembly.

\section{c. Platinum-30\% Rhodium Alloy}

\section{Thermal Conductivity}

Figure 44 presents the $\mathrm{Pt}-30 \mathrm{Rh}$ thermal conductivity schedule for the Cassini studies. Oak Ridge National Laboratory (ORNL) recommended this schedule in support of early LWRHU design studies (Reference 63). Also contained in this figure are conductivity values for other platinum alloys including Pt 3008 (References 64 and 65), Pt20Rh, the clad material used in the SNAP 19 heat source (Reference 66), and Pt-40Rh (Reference 67). The Cassini schedule remains the same as used in the Galileo studies up to a temperature of approximately $2500^{\circ} \mathrm{R}$. At this temperature, the Cassini schedule's departure from Galileo is based on similar conductivity/temperature trends demonstrated by the sibling alloys, $\mathrm{Pt}-20 \mathrm{Rh}$ and $\mathrm{Pt}$ $40 \mathrm{Rh}$. No attempt was made to integrate into the Cassini conductivity schedule the effects of selfannealing and resulting grain growth due to the clad's temperature response during reentry. The Cassini schedule is not increased beyond the carbon eutectic melting point of Pt-30Rh as indicated by the cross hatched region in this figure. For comparable heating conditions, the slight departure of the Cassini schedule to lower conductivity values at elevated temperatures will not significantly affect the thermal response (i.e., radial gradients) of the clad due to metals' intrinsically high conductivity properties. The end result on heat transmission to the fuel is minor. 


\section{(2) Thermal Capacitance (density $\mathrm{x}$ specific heat)}

Quoted values for Pt-30Rh alloy's density range from 1093.8 (Reference 49) to $1100.7 \mathrm{lb}_{\mathrm{m}} / \mathrm{ft}^{3}$ (Reference 68). The lower value was considered in both the Cassini and Galileo studies.

Figure 45 presents the specific heat schedule considered in both the Cassini and Galileo studies as recommended by ORNL (Reference 63). It is clear from this figure that the recommended schedule for Pt-30Rh is represented by the specific heat property for Pt 3008 . This appears to be a reasonable substitute given the slightly higher values of this property for the sibling alloy, Pt-20Rh.

\section{d. Plutonium Dioxide}

\section{(1) Thermal Conductivity}

Figure 46 presents the thermal conductivity of $\mathrm{Pu}_{2}$ considered for the Cassini and Galileo studies. The Cassini study uses the conductivity schedule proposed by Haliburton NUS Corporation (Reference 69) during the GPHS Draft Environmental Impact Statement (DEIS) effort. In the temperature region of interest, the principal difference between the two schedules is Cassini's increase in conductivity at elevated temperatures due to radiation transmission. This increase is consistent with the fuel pellet's porosity at approximately $87 \%$ theoretical density. Other fuel conductivity data (References 70 through 73 ) are included in this figure and generally indicate good agreement over the temperature range of 1000 to $3000^{\circ} \mathrm{R}$

The effect of the change in schedules for the reentry of a full LWRHU assembly is likely minor by slightly increasing the fuel's internal thermal response for comparable heat loads. The consequence, however, will be more visible for bare fuel reentry involving formation of a melt front. This front will now travel somewhat further into the interior of the fuel pellet (or fragment) and thereby increase estimated source terms.

\section{(2) Thermal Capacitance (density $\mathrm{x}$ specific heat)}

The thermal power of the Galileo LWRHU fuel pellets was 1.1 thermal watts corresponding to a nominal density of $87.4 \%$ theoretical (or $625.3 \mathrm{lb}_{\mathrm{m}} / \mathrm{ft}^{3}$, Reference 74). The same fuel density was assumed for the Cassini study and has since been substantiated by reported values in the LWRHU FSAR (draft) for the Cassini mission (Reference 75).

The specific heat schedule for the Cassini studies is shown in Figure 47. It is slightly altered from the schedule considered for the Galileo effort. The principal difference occurs at low temperatures and is of little consequence in governing the thermal response 
of fuel for reentry conditions. The change to the General Electric recommended schedule (Reference 70) for the Cassini studies was based solely on the fact it provided lower values of specific heat than for Galileo in the high temperature region of interest. The differences are small though $(\leq 5 \%)$, and should provide only a minor increase in fuel thermal response for comparable heat loads.

\section{Thermal Radiative Properties}

In defining thermal radiative properties for the LWRHU's constituent materials, all components were considered opaque including such non-metallic solids as the FWPF $^{\text {th }}$ aeroshell parts and the PG insulator parts. The resulting restriction on radiation processes to solely a surface effect required that only emittance $(\varepsilon)$ and absorptance $(\alpha)$ properties be defined for the LWRHU materials. Due to a lack of absorptance data, Kirchoff's law (Reference 76) was invoked (as an expedient) such that $\alpha=\varepsilon$ for all materials. Whenever possible, emittance properties were defined as a function of temperature with priority given to data in the following descending categories: total hemispherical, total normal, low wavelength $(\lambda)$ normal, or any $\lambda$ normal. The latter categories imply gray body radiation.

\section{a. Fine Weave Pierced Fabric 3-D Carbon-Carbon (directional property)}

Total hemispherical emittance of outgassed FWPF ${ }^{\text {tm }}$ carbon-carbon was measured at Purdue University with the resulting data fitted for the $X(=Y)$ axis and for the 'average' of the data in the $X$ and $Z$ axes (Reference 77). The schedules presented in Figure 48 (a) are based on these FWPF ${ }^{\mathrm{tm}}$ curve fits with the off-axis schedule being equated to the 'average' data.

It is indicated from this figure that the expected maximum temperature region for the FWPF ${ }^{\mathrm{tm}}$ aeroshell during reentry exceeds the range of applicability for the FWPF ${ }^{\text {th }}$ curve fits. The Z-axis schedule (inferred from the available curve fits) is shown to exceed the limiting black body value in the neighborhood of $7000^{\circ} \mathrm{R}$ with the 'off-axis' schedule proceeding in that direction as well. As a result, the schedules for the $\mathrm{Z}$ and the 'off-axis' directions were revised to arbitrarily approach 0.95 at $8000^{\circ} \mathrm{R}$ as shown in this figure's caption.

The Galileo studies used the $\mathrm{X}$-axis emittance schedule to determine radiative transmission in the radial direction. The recommended schedule was changed to the 'offaxis' emittance curve for the Cassini studies and will result in a slightly higher radial heat flux from the aeroshell to the outer insulator sleeve 'for comparable temperature conditions'. 


\section{b. Pyrolytic Graphite (directional property)}

\section{'C'Direction}

The recommended total hemispherical emittance schedules in the ' $c$ ' direction are shown in Figure 48 (b) to be differentiated on the basis of surface finish. The 'as-deposited' surface schedules labeled 'early GPHS design' dates back to earlier mission safety analyses (e.g., Pioneer SNAP-19, Reference 66). These normal emittance data were reported by Super-Temp (Reference 58) and are based on SORI spectral data (Reference 78) integrated over the wavelength range of 0.20 to 0.35 microns $(\mu)$. The GPHS task force took issue with this schedule and recommended a total hemispherical schedule based on the 'Kingery' slope as shown in this figure (References 49 and 56). This schedule was endorsed for the Galileo studies for all PG surfaces and selected for the Cassini studies for 'as-deposited' surfaces. A departure in the Cassini schedule occurs at $6000^{\circ} \mathrm{R}$ by continuing on to 0.95 at $8000^{\circ} \mathrm{R}$ rather than being arbitrarily truncated at 0.8 as indicated for the Galileo schedule. At these temperatures, this change will have a minor effect on radiative transmission between the outer insulator sleeve/aeroshell body and insulator cap/aeroshell end cap components.

The Cassini schedule for 'machined' PG surfaces is guided by the experimental total hemispherical data from a Russian investigation of surface conditions on emittance (Reference 79). This departure from the Galileo studies will noticeably increase radiative transmission involving machined PG components. The machining process acts to agitate crystallite orientations, resulting in a higher degree of emittance as indicated in this figure. The Cassini curve was extended out to the reference 0.95 value at $8000^{\circ} \mathrm{R}$. It is noted that this investigation's 'asdeposited' data agree reasonably well with the Kingery curve.

$$
\text { 'A-B' Direction }
$$

There are very little data available in the open literature for pyrolytic graphite's ' $a-b$ ' directional emittance. The curve shown in Figure 48 (c) is total normal data integrated from the spectral measurements of SORI and reported by Super-Temp (References 58 and 78). These data were used in the early LWRHU design studies (Reference 49) and selected for both the Cassini and Galileo studies.

\section{c. Platinum-30\%Rhodium}

The literature survey did not reveal any emittance data for $\mathrm{Pt}-30 \mathrm{Rh}$ but did provide total hemispherical emittance data for a sibling alloy, Pt-13Rh (Reference 80), the platinum alloy, Pt3008 (Reference 49), and pure platinum (References 62, 79 and 81). This group of data is presented in Figure 48 (d) along with the arbitrary 'constant emittance' schedule of 0.2 used in the Galileo studies (References 49). The recommended Cassini schedule is shown to be a composite guided by the Pt3008 data at low to moderate temperatures, the Pt-13Rh data at the 
higher temperatures and extended at constant slope to the carbon eutectic temperature. This change from the Galileo schedule will result in a minor increase in radiative transmission from the clad assembly to the fuel pellet during a full LWRHU assembly reentry.

\section{d. $\quad$ Plutonium Dioxide}

The total hemispherical emittance schedule presented in Figure 48(e) is based on the curve fit provided by LASL (Reference 71) and linearly extended beyond its applicable range to values nearing the black body limit at $\mathrm{PuO}_{2}$ 's melt temperature. The Cassini's schedule arbitrarily departs from the Galileo schedule to fair into the reference 0.95 value at the melting point to remain consistent with the emittance extrapolations considered for the FWPF and PG materials. This departure from the Galileo schedule will result in a minor reduction in radiation relief during bare fuel reentry and thereby sustain the melt front slightly longer for critical reentries.

\section{Phase Change Properties}

The remaining thermophysical properties requiring definition for the LWRHU reentry studies are associated with phase changes for the $\mathrm{Pt}-30 \mathrm{Rh}$ and $\mathrm{PuO}_{2}$ materials and include solid/liquid latent heats and associated melting temperatures. In the case of Pt-30Rh, the melt condition is defined as the carbon eutectic temperature resulting from contact of the clad body and clad caps with their PG neighbors. Additionally, the latent heat of vaporization for $\mathrm{PuO}_{2}$ for the liquid/vapor phase change becomes a requirement if a 'stable' melt layer is formed on a reentering fuel pellet or fragment.

Solid/vapor phase change considerations for the FWPF ${ }^{\mathrm{m}}$ and PG materials are provided by the carbon-air thermochemistry models discussed in Section VII C. Liquefaction of these materials is not considered in these studies since LWRHU reentry surface pressures will be considerably lower than carbon's (graphite's) equilibrium triple point values (i.e., approximately 100 atmospheres).

\section{a. Platinum-30\%Rhodium}

\section{(1) Carbon Eutectic Temperature}

Figures 44 and 45 show a carbon eutectic temperature band (the cross-hatched region) that ranges from 3550 to $3660^{\circ} \mathrm{R}$. The upper bound of this eutectic range is the temperature typically quoted for this alloy (Reference 49) and considered in the Galileo studies. The lower bound temperature is an estimated value of $\mathrm{Pt}-30 \mathrm{Rh}$ in contact with a POCO grade of graphite (Reference 65). Since the alloy's carbon eutectic temperature is sensitive to the particular type of graphite, and since the graphite partner associated with the upper bound value could not be identified, the lower temperature was considered for the Cassini studies. This 
represents a conservative change from the Galileo framework by slightly lowering the clad melt failure criterion and thereby resulting in a slightly earlier failure occurrence in a critical reentry heat pulse.

\section{(2) Latent Heat of Fusion $\left(\Delta \mathrm{H}_{\mathrm{f}}\right)$}

The literature search did not reveal direct values for $\Delta \mathrm{H}_{\mathrm{f}, \mathrm{P} \mathrm{t}-30 \mathrm{Rh}}$. Alternatively, a composite value was determined as the product of the weight fractions of the participating elements and their respective $\Delta \mathrm{H}_{\mathrm{f}}$ values. The latent heats of fusion for platinum and rhodium (Reference 81 ) are given as:

$$
\begin{aligned}
& \Delta \mathrm{H}_{\mathrm{f}, \mathrm{Pt}}=43.2 \mathrm{BTU} / \mathrm{lb}_{\mathrm{m}} \text { and } \\
& \Delta \mathrm{H}_{\mathrm{f}, \mathrm{Rh}}=99.5 \mathrm{BTU} / \mathrm{b}_{\mathrm{m}}
\end{aligned}
$$

with the resulting value for $\mathrm{Pt}-30 \mathrm{Rh}$ determined as:

$$
\Delta \mathrm{H}_{\mathrm{f}, \mathrm{P}-30 \mathrm{Rh}}=60.1 \mathrm{BTU} / \mathrm{lb}_{\mathrm{m}} .
$$

This value is slightly higher than the value considered for the Galileo studies at $57.5 \mathrm{BTU} / \mathrm{lb}_{\mathrm{m}}$ and will slightly inhibit the progression of a melt front for bare fuel reentries.

\section{b. $\quad$ Plutonium Dioxide}

\section{(1) Melt Temperature}

The melt temperature of $\mathrm{PuO}_{2}$ for use in the GPHS Cassini/VVEJGA studies was recommended by Martin Marietta AstroSpace (Reference 51) at:

$$
\mathrm{T}_{\text {melt,PuO2 }}=4812^{\circ} \mathrm{R} \text {. }
$$

The recommendation is based on cited temperatures in several references (References 83 through 85). This value was also used in the LWRHU Galileo and Cassini studies. Eutectic formation of $\mathrm{PuO}_{2}$ with Pt-30Rh alloy was not considered in either study.

\section{(2) Latent Heat of Fusion $\left(\Delta \mathrm{H}_{\mathrm{f}}\right)$}

$\mathrm{PuO}_{2}$ including:

There are several values quoted for the heat of fusion for

$\Delta \mathrm{H}_{\mathrm{fPuO2}}=112.0 \mathrm{BTU} / \mathrm{lb}_{\mathrm{m}}$ (General Electric, Reference 85),

$\Delta \mathrm{H}_{\mathrm{fPUO2}}=117.0 \mathrm{BTU} / \mathrm{lb}_{\mathrm{m}}(\mathrm{LASL}$, Reference 70$)$ and

$\Delta \mathrm{H}_{\mathrm{f}, \mathrm{PuO} 2}=101.0 \mathrm{BTU} / \mathrm{lb}_{\mathrm{m}}$ (Aerotherm Corporation, Reference 88). 
The Cassini study considered an average of the above values:

$$
\Delta \mathrm{H}_{\mathrm{fPuO2}}(\text { ave })=110.0 \mathrm{BTU} / \mathrm{lb}_{\mathrm{m}}
$$

which is slightly lower than the above General Electric value used in the Galileo studies.

\section{E. Aerothermodynamics}

Due to the high energies of the VVEJGA reentries, the aerothermodynamics for the LWRHU are complicated by the addition of shock layer radiation as a mode of heat transfer. Furthermore, both the radiative and convective modes are influenced by chemistry effects that further complicate the reentry environments. This is illustrated in Figure 49 which shows thresholds for both the dissociation and ionization processes as well as the kinetic threshold as overlays on the trajectory tracks for the various reentry angles of the Cassini-VVEJGA reentry map. These thresholds are very approximate but serve to illustrate the various aerothermodynamic regimes encountered by a LWRHU reentry. Also, it is noted that the kinetic threshold shown here is for a one-foot radius-sphere; however, for the smaller LWRHU leading edge, this threshold will be translated to lower altitudes since, for this geometry, the forward (stagnation) shock layer thickness is considerably smaller relative to required kinetic relaxation distances. This figure indicates that the high energy VVEJGA reentries result in a highly ionized (and therefore radiating) shock layer in the stagnation region. It also indicates that nonequilibrium chemistry is probably a consideration in the maximum heating region for most of the reentry zones. This has implications regarding both the radiative and convective heat transfer modes as will be discussed later.

LWRHU aerothermodynamics occurring during the hypersonic heat pulse for the Cassini/VVEJGA class of reentries can be characterized by a multicomponent, reactive flow field with strong gas dynamic/radiation and ablation coupling. Additionally, for certain regions in the VVEJGA reentry corridor, the LWRHU will experience chemical and thermodynamic nonequilibrium flow fields. The high energy states and complexity of the VVEJGA reentry flow fields require numerical solutions for surface aerothermodynamics using advanced computational fluid dynamics (CFD) techniques such as the Lockheed Martin Missiles and Space (LMMS) flow field solver (RACER) and the Langley Optimized Radiative Non-Equilibrium (LORAN-C) codes employed in the GPHS/VVEJGA analyses (Reference 89). The LWRHU/VVEJGA reentry analyses were not supported by such CFD facilities. The available means for this study were engineering methods used in prior reentry safety studies, model/method syntheses from available experimental and numerical data and engineering judgment. Recognizing the broad range of uncertainties associated with this approach, the VVEJGA reentry analyses were steered in a conservative direction through (a) specification of failure mode criteria, (b) imposed structural breakup sequence of the LWRHU, (c) selection of thermophysical data and (d) selection of environment/response models. 
The following discussion along with Table 8 provides a brief commentary on the considerable LWRHU aerothermodynamics defined for the (a) side-on stable, (b) side-on spinning and (c) random tumbling flight orientations. Table 8 contains quick look summaries of the computational methods. Also included in this section is an extensive list of references providing further background material. For the VVEJGA studies, aerothermodynamics were defined only for the hypersonic flow regime $\left(M_{\infty} \geq 5.0\right)$ encompassing the reentry heat pulse period.

\section{General Methodology,Full LWRHU Assembly}

The development of VVEJGA aerothermodynamics for the LWRHU reentry simulations follows the same general computational flow as employed in the Galileo/VEEGA studies. This flow is described by four sequential steps:

a. The initial step requires determination of reference convective and radiative heating rates as a function of reentry flight time using JHU/APL's TDOF trajectory code (Reference 90).

b. The reference convective and radiative heating rate histories from the TDOF results are transformed to corresponding body reference heating rates on the LWRHU.

c. Local heating rate distributions are determined from heating factor (i.e., local to body reference heating rate ratio) distributions times the body reference heating rate determined in (ii).

d. Local heating rate distributions are corrected for low density and surface mass transfer effects due to thermochemical ablation.

The TDOF reference heating rates provide the required trajectory time dependency while the heating rate distributions are typically held constant with time in the hypersonic regime and provide spatial dependency.

\section{Reference Convective and Free Molecule Heating. Full LWRHU Assembly}

Convective heating is considered in the generic sense to represent surface energy transport due to both 'particle' conduction and mass diffusion for a multicomponent flow field in the continuum flow regime and transitional heating (including the above processes) in the kinetic (low density) flow regime. For continuum flow, reference convective heating represents a laminar, stagnation heating rate to a 1 -foot radius, smooth, cold wall sphere in absence of surface mass transfer or radiation coupling effects. Predictions are based on the Detra, Kemp and Riddell correlation (Reference 91) which was developed from numerical and experimental data largely representative of a dissociated shock layer (References 92, 93). As indicated from Figure 50, the 
correlation performs well against high energy shock tube data that extend well into the ionization regime. It has been demonstrated that this correlation matches the data aggregate's least squares fit and is superior to a number of the early ionization stagnation theories (Reference 94).

The reference heating rate in the free molecule flow regime is represented by the total available flow energy, $\left(\rho_{\infty} \mathrm{V}_{\infty}\right)^{3} / 2$, to a surface perpendicular to the particle stream with a thermal accommodation coefficient of unity (Reference 95). This reference value also equates to the body reference condition represented by the LWRHU's mid-span stagnation condition in a sideon stable orientation.

\section{Reference (and Body Reference) Radiative Heating, Full LWRHU Assembly}

The TDOF reference and body reference radiative heating rates are equivalent in the VVEJGA study and represent the same stagnation conditions discussed above for the free molecule heating condition. The mid-span location was selected to isolate the heating rates from flow expansion effects occurring at the cylindrical end stations (end effects). The radiative heating rate tables developed by Sutton and Hartung (Reference 96) represent numerical solutions from NASA's Radiating, Inviscid Flow, Stagnation Point (RIFSP) code (Reference 97) for a broad matrix of velocity and altitude conditions over a spherical nose radii range from 0.05 to 10.0 meters. The code utilizes the radiation equilibrium transport model (RAD/EQUIL) (References 98 and 99) which provides for both atomic and continuum radiation transfer mechanisms in air and has demonstrated reasonable performance against numerous ground-based and flight experimental test data (Reference 100). This matrix of data has been incorporated into the TDOF code and is accessed by a user supplied effective spherical radius for the reference LWRHU geometry. The equivalent spherical radius for the side-on LWRHU configuration was based on equating the LWRHU's stagnation shock layer thickness (i.e., shock standoff for adiabatic flow) with the stagnation shock standoff for a spherical geometry with nose radius $R$ (Reference 101). Downstream heating distributions were determined from a radiating flow field code for transverse cylinders which uses an approximate radiation transport model. This code, obtained from the NASA-Langley Research Center (Olstad, Reference 102) is felt to provide a good engineering approximation for downstream heating histories in the form of the ratio: $\dot{q}($ local $) / \dot{q}$ (stag). These ratios are subsequently reduced using the stagnation values obtained from Aerotherm's Radiation/Equilibrium Transfer Code (RAD/EQUIL).

The neglect of the shock layer's radiation cooling for VVEJGA's high energy flight conditions leads to an overestimation of the effective spherical radius and thereby a conservative estimate of radiative heating at the body reference location. For added conservatism, it is noted that the data are products of inviscid flow field solutions and are not adjusted in the VVEJGA studies for the shock layer's cooler viscous sublayer (presumably a self-absorption effect). It has been argued that this effect can noticeably reduce surface radiation (Reference 103) 
based on comparisons of the Sutton-Hartung data with solutions obtained with the Radiation Shock Layer Equilibrium (RASLE) code (Reference 104). It is further argued that an equilibrium radiation transport model (i.e., the RAD/EQUIL code) likely overestimates radiative heating for the VVEJGA 'shallow' reentries where nonequilibrium flow phenomena are present.

\section{Body Reference Convective Heating, Full LWRHU Assembly}

As previously indicated for the radiative heating mode, the body reference condition was the mid-span location of the LWRHU in a side-on stable orientation. Here again, the mid-span convective heating rates are assumed unaffected by end effects. Transformation of the TDOF reference heating rates to the body reference location accounts for flow dimensionality (axisymmetric vs two-dimensional flows), stagnation velocity gradients (sphere vs transverse cylinder) and scaling effects (body radii, reference sphere to LWRHU leading edge). The stagnation velocity gradient ratio of a transverse cylinder to a sphere of equal radii is very close to unity in the hypersonic regime as indicated in Figure 51 (Reference 105). The full conversion factor for velocity gradient and scaling effects are described by

$$
\frac{q_{G E O, c}}{q_{\text {ref }, c}}=M F *\left(\frac{\beta_{G E O}}{\beta_{S}}\right)^{0.5} *\left(\frac{1}{\sqrt{2}}\right)^{\delta} *\left(\frac{R_{S}}{b / 2.0}\right)^{0.5} *\left[\sqrt{\left.1+\left(\frac{b}{1}\right) 0.5\right]^{\delta^{\prime}}}\right.
$$

where $\frac{q_{G E O, c}}{q_{\text {ref,c }}}=$ geometry specific stagnation convective heat flux ratio

$\mathrm{b}, 1=$ width, length of reentry body

$\mathrm{R}_{\mathrm{S}} \quad$ = reference sphere radius (=12")

$\mathrm{q}_{\text {ref,c }}=$ reference sphere stagnation heating rate

$\beta_{\mathrm{S}} \quad=$ stagnation velocity gradient parameter for sphere

$\beta_{\mathrm{GEO}}=$ stagnation velocity gradient parameter for specific geometry

$\delta \quad=$ geometric constant

0 for axisymmetric flow,

1 for 2-D planar flow (cylinder) or 3-D flat plate

$\delta^{\prime} \quad=$ geometric constant

0 for planar flow (transverse flow), or axisymmetric

1 for 3-D flat plate normal flow.

MF = weighting factor for supersonic or subsonic heating, usually at unity

\section{5. $\quad$ Adjustments to Body Reference Heating Rates}

\section{a. $\quad$ Precursor Heating}

The body reference radiative heating rates were adjusted for precursor effects. This effect essentially represents a feedback of energy into the shock layer and an incremental increase in surface radiation due to absorption of radiation emitted from the hot shock layer by the cool ambient air upstream of the leading shock wave. The compression of this higher 
enthalpy ambient stream leads to higher shock layer enthalpies and a fractional recovery of the shock layer energy lost by radiation cooling. Data on this effect are scattered and highly dependent upon flight velocities. Based on some early investigations (References 106 to 110), an approximate model was formulated in which stagnation radiative heating rates were increased by $15 \%$ for velocities at or greater than $65 \mathrm{~K}$ fps and linearly decreased from this value at $65 \mathrm{~K}$ to $0 \%$ at $40 \mathrm{~K}$ fps. This effect is second order compared to the uncertainties associated with radiative heating at VVEJGA level energies but has been included in the simulations to acknowledge its presence during VVEJGA reentries.

\section{b. $\quad$ Radiation Coupling}

Another effect attributed to high intensity shock layer radiation is its coupling effect on convective heating. The coupling effect identifies the changes in the enthalpy and concentration surface gradients defining convective heating due to radiation cooling of the shock layer and self-absorption in the viscous sublayer. The data from the GPHS/VVEJGA studies did not provide insight on this interaction due to the overlying presence of reactive and radiating ablation products. Literature (References 110 to 113) revealed conflicting data on this effect for non-ablating surfaces but, in general, suggested that coupling increased convective heating to large radii bodies and decreased heating to small radii bodies such as the LWRHU. Based on this observation, the VVEJGA studies neglected any coupling adjustments to body reference convective heating rates which suggests further conservatism in the analyses. This effect becomes muted for massive blowing of ablating surfaces that blocks surface convective heating to diminished levels (i.e., at or near zero surface gradients in the ablation layer).

\section{c. $\quad$ Catalytic Surface Effects}

Catalytic recombination effects on body reference convective heating was not considered in these studies due to the absence of recombination coefficient data for carbon-carbons in the open literature. The use of the Detra, Kemp and Riddell equation to predict reference heating rates infers thermochemical equilibrium surface conditions via its excellent correlation of numerical equilibria stagnation data (Reference 92). The assumption of a thermochemical equilibrium surface boundary condition provides a close approximation to a fully catalytic surface (Reference 114) and hence, maximum convective heating rates relative to catalytic surface reactions.

\section{Other Body Reference Variables:}

Two other aerothermodynamic body reference variables required in the studies are the total enthalpy (based on the stream's total kinetic energy) and stagnation pressure (aft of the leading shock wave). Neither of these body reference variables are considered to be influenced by end effects and both equate to the reference histories provided by the TDOF code. Similar to the body reference heating rate data, these body reference variables are reduced by 
recovery enthalpy and static pressure factors (again, local to reference ratios) specific to the LWRHU's local values as a function of time. Local recovery enthalpy allows adjustments to convective heating for 'hot wall' effects and to reduce convective heating to a Stanton Number in evaluating the numerical solution's surface energy boundary condition. Local static pressure assists in evaluating local thermochemical ablation mass fluxes due to the ablation model's bivariate dependency on surface temperature and pressure.

\section{Heating Factor Distributions}

orientations:

Heating factor distributions were developed for three general flight

- side-on stable,

- side-on spinning and

- random tumbling.

From a heating viewpoint, the side-on stable orientation was an expedient model to represent the class of motions where the longitudinal axis (generatrix) is mostly perpendicular to the wind, $90^{\circ}$ angle of attack, $\alpha$. The expediency relates to the ability to estimate aerothermodynamics for the side-on orientation with available computational means while generally providing a conservative estimate of the response parameters for the VVEJGA environment. The possible exception is a psuedo-steady angle of attack orientation; however, the means to estimate shock layer radiation at angle of attack were not available for these studies. Furthermore, ablation shape change effects on flight trajectory, flight motion and aerothermodynamics for such an orientation are first order and cannot be estimated with JHU/APL's current arsenal of codes. Also, the end-on stable case was not examined specifically since it proved to be less critical than the side-on stable orientation in the Galileo/VEEGA studies (Reference 115) and is not a likely flight orientation due to the LWRHU's small fineness ratio.

Again, from a heating viewpoint, the LWRHU random tumbling orientation represents flight motions which possess angular rotation about the longitudinal axis. Given the small fineness ratio of the LWRHU and chaotic release conditions from the Cassini spacecraft, this is a highly likely flight orientation. It inherently contains greater uncertainty in estimating heating factor distributions. The side-on spinning orientation has full rotation about the LWRHU's longitudinal axis.

\section{a. Side-On Stable Orientation, Full LWRHU Assembly}

Since the LWRHU's ablation failure criterion is declared as an incipient breach of the aeroshell in the stagnation region, construction of heating factor distributions for this orientation are essentially focused on the windward flow field region of the LWRHU. The 
VVEJGA baseline studies considered only the Option 1 heating factor distributions presented in the following figures.

\section{Cylindrical Surface}

Figure 52 compares theoretical (References 116 to 118) versus experimental (References 118 to 120) circumferential convective (continuum) heating rates for a transverse cylinder over a central angle $(\theta)$ range of $90^{\circ}$. The VVEJGA studies use factor distribution based on the Kemp/Rose/Detra theory instead of the Galileo/VEEGA distribution (i.e., Lees $M_{\infty}=3.0$ distribution) since it bounded most of the downstream experimental data. Figure 52 indicates the VVEJGA model is slightly conservative relative to the VEEGA model at the downstream locations. The free molecule heating factor distribution $(\cos (\theta)$ function) remained the same as considered in the Galileo/VEEGA studies.

Figure 53 compares an early LWRHU design curve for axial heating distributions along the stagnation line (Reference 121) versus experimental data measured for the MultiHundred Watt (MHW) heat source, a right cylinder configuration (Reference 119). The experimental data were chosen as the better axial stagnation heating distribution model for the VVEJGA studies since the LWRHU design distribution was largely based on conservative engineering judgment. The local circumferential factor distribution as a function of axial distance from the mid-span location becomes the product of Figures 52 and 53 Option 1 distributions. Figure 53 also indicates the free molecule axial heating factor distribution along the stagnation line is absent of end effects.

Circumferential radiative heating factor distributions were estimated from a radiating inviscid flow field code using an approximate (step) radiation transport model (References 102 and 122). Three trajectories, representative of the shallow, moderate and steep class of VVEJGA reentries were examined. To account for uncertainty in the radiation transport step model, the distribution selected for the VVEJGA studies was a trigonometric function $\left(\cos ^{3 / 2}(\theta)+B \sin ^{2}(\theta)\right)$ that bounded the numerical data. This model not only introduced conservatism in the forward windward region of the LWRHU but also eliminated the time dependency of the numerical data. The VVEJGA distribution model and associated numerical data for a flight path angle of $-90^{\circ}$ is presented in Figure 54 .

The only data source providing a suggestion of axial radiative heating distributions was the end face radial heating distributions generated by LMMS for the right cylinder simulation of a broadface stable GPHS module. The end effects (i.e., flow expansion) for the right cylinder's axisymmetric flow will be more exaggerated than the threedimensional flow for the side-on LWRHU in reducing radiative heating rates. Figure 55 presents distributions determined by LMMS for the GPHS simulation with and without ablation (Reference 123). Using the guidance provided by LMMS on zero ablation distribution, the LWRHU's VVEJGA model was defined to provide a reduced influence from end effects. Here 
again, the circumferential radiative heating factor distributions as a function of axial location were assumed to be the product of the VVEJGA distributions recommended in Figures 54 and 55.

Several sources of static pressure factor distributions for transverse cylinders (References 124 to 126 ) were examined during this study with all demonstrating the expected Newtonian behavior in the near stagnation region. The curve fit distributions of Miller (Reference 124) were selected for the VVEJGA model since they provided both circumferential and axial variations and correspondingly lower pressures at the mid-span location $(z / L=0)$ than the Galileo/VEEGA model (Gregorek and Korkan, Reference 125). For a fixed surface temperature, a lower static (surface) pressure will translate to a higher aeroshell ablation mass flux for the VVEJGA studies. The VVEJGA, VEEGA and the modified Newtonian pressure distributions are compared in Figure 56. To expedite data input, the windward recovery enthalpy factor distribution remained the same as the Galileo/VEEGA model. This distribution was based on an isentropic, perfect gas (calorically and thermally perfect) expansion using the Gregorek and Korkan static pressure distribution.

Leeward side aerothermodynamic distributions $\left(90^{\circ} \leq \theta\right.$ $\leq 180^{\circ}$ ) were approximated by setting the free molecule and radiation distribution factors to zero, the convective heating factor at $6 \%$ to $8 \%$ and the static pressure at about $2 \%$ to $4 \%$. The recovery enthalpy factor was arbitrarily set at unity which acts to underestimate ablation losses; however, the wake region is well beyond the 'region of influence' for aeroshell ablation failures for this orientation.

\section{(2) End Face}

Similar to the leeward side of the cylindrical surface, aerothermodynamic parameters for the end face were approximated since this face is not influential in the side-on stable orientation. The radiative and free molecule factor distributions were set to zero and the convective heating distribution at a constant value of $10 \%$ to provide a conservative value to reduce end losses. The recovery enthalpy and static pressures were set to the leeward values which will affect prediction accuracy of ablation losses but in a non-critical ablation region.

\section{b. $\quad$ Side-On Spinning Orientation. Full LWRHU Assembly}

\section{(1) Cylindrical Surface}

For this flight orientation, the spin rates were assumed high enough to permit smearing (i.e., integration over a $\theta$ range of $0^{\circ}$ to $180^{\circ}$ ) of the distributions determined for the side-on stable case. Consequently, for any given axial location, all aerothermodynamic 'spin' factors were considered circumferentially uniform on the cylindrical surface. No adjustments were made to the side-on stable free molecule factor distributions in obtaining the free molecule spin factor. Similarly, no adjustments were made to the convective 
heating factor distributions developed as a function of axial location; however, the radiative heating factor distributions were adjusted on the leeward side from the prior zero heating specification to a range varying from $10 \%$ at $\theta=120^{\circ}$ to $5 \%$ at $180^{\circ}$. This introduces a slight conservatism in the radiative spin factor and is based on the rationale that a low velocity wake structure may raise base temperatures to sufficient levels to emit accountable levels of radiation.

The leeward factor distributions for recovery enthalpy and pressure become significantly more influential for the spinning case since they now contribute directly to the uniform spinning factors used to determine thermochemical ablation. A study was conducted that examined hypersonic wake flow field literature to establish more realistic recovery enthalpy distribution in the wake region. Upon determining that the LWRHU's near wake flow field structure was laminar (References 127, 128), the spherical, low density temperature data of Dogra, et al. (Reference 129) were selected for the VVEJGA shallow reentry class (i.e., for $\gamma \leq$ $\left.15^{\circ}\right)$. For the moderate to steep reentry classes $\left(\gamma \geq 15^{\circ}\right)$, the only high density data that appeared useful were the blunt cone data of Bulmer (Reference 130). For low velocity, recirculation flow in the wake, the ratio of local recovery to stagnation temperature data (as determined from Bulmer's local static to stagnation temperate ratios) were equated to the local recovery enthalpy ratio. The windward distribution remained the same as indicated for the side-on stable case.

A second study was conducted to more accurately describe static pressures in the leeward flow field. Literature revealed that the leeward separation point for supersonic, two-dimensional (2-D) flow for transverse cylinders was approximately $120^{\circ}$ (References 131 to 133 ) followed by a constant static pressure field in the wake. In determining the pressure spin factors, the pressure distributions for the side-on stable case were adjusted in the wake region using Gregorek and Korkan's static pressure factor (i.e., $2 \%$ ).

\section{(2) End Face}

All the aerothermodynamic factors remained the same as assigned for the side-on stable case.

\section{c. Random Tumbling Orientation, Full LWRHU Assembly}

The random tumbling flight orientation represents a likely class of motion for the LWRHU. For purposes of analyses, this motion was depicted as a spinning right cylinder that was also rotating over a total angle of attack sweep of $90^{\circ}$. As an expedient for conducting the study, it was assumed that rotational rates were sufficiently large to permit smearing of the heating factor distributions as a function of $\theta$ and $\alpha$. Clearly, to define aerothermodynamics for this motion, free molecule, convective and radiative heating factor distributions would need to be determined as a function of angular displacement for the LWRHU's body axis system. Program constraints did not permit development of this bank of data via CFD technology. Alternatively, the approximate methodology of Klett (Reference 134) was followed closely to define the free 
molecule and convective heating random tumbling factors. Klett's study developed methods for determining drag coefficients and convective heating to a right cylinder for both the free molecule and laminar continuum flow regimes at orientations of end-on, side-on, end-over-end and random tumbling. The random tumbling factor for radiative heating essentially came down to use of engineering judgment in determining angle of attack dependency.

For all aerothermodynamic parameters, the random tumbling factor was assumed to be the product of the local spin factor times the stagnation line angle of attack factor. As with the other flight orientation cases, the random tumbling factors were ratios in which the denominator represented the parameter's body reference value. All spin factors were based on the results of the prior side-on spinning study.

\section{(1) Cylindrical Surface}

A study was conducted that examined stagnation line convective heating theory and experimental data for yawed (or swept), circular cylinders (References $118,120,135$ to 140 ) to obtain an engineering relationship for $\alpha$ dependency. Defining $\alpha=0^{\circ}$ as the LWRHU's end-on orientation along the wind velocity vector, and yaw $(\lambda)=0^{\circ}$ as flow perpendicular to a transverse cylinder, then $\alpha$ becomes the complement of $\lambda$ (i.e., $\alpha=90^{\circ}-\lambda$ ). The engineering relationship of Sayano and Greenwald (Reference 139) was chosen due to its simplistic form and its good agreement with the experimental data of Bergquaum (Reference 140). Bergquaum measured convective heating as a function of yaw angle at various axial stations along a 1.25-inch diameter right circular cylinder. End effects could only be qualitatively assessed. The data indicate that end effects on convective heating rates to the cylindrical surface occur more noticeably at low angles of attack and therefore do not greatly affect the integrated average. Sayano and Greenwald's convective heating factor distribution is shown in Figure 57 with the data at $0^{\circ}$ representing Klett's flat plate data at the LWRHU's thermal model nodal stations. The random tumbling free molecule heating factor was taken directly from Klett.

Applicable data or theories for stagnation line shock layer radiation on cylindrical surfaces at angle of attack were not available for this study. Consequently, shock layer radiation dependency on angle of attack was assumed to take a similar functional form as demonstrated for $\theta$ (i.e., the side-on stable distribution). The function used a $\cos (\alpha)$ dependency rather than $\cos (\alpha)^{3 / 2}$ to allow for uncertainties in developing the random tumbling factor.

The product of the spin factor times the stagnation line angle of attack factor was also considered for the static pressure factor and is believed to result in conservatively low pressure values (relative to thermochemical ablation). Carroll's curve fit of midspan experimental data for cylinders (Reference 118) was used to express stagnation line pressure as a function of $\alpha$. The recovery enthalpy factor utilized Feller's expression for stagnation line recovery temperature (Reference 135) as a function of sweep angle and, once again, implies perfect gas flow theory. 


\section{(2) End Face}

The convective and radiative heating factor distributions were integrated over an $\alpha$ sweep of $180^{\circ}$ and normalized to the stagnation heating value at $\alpha=0^{\circ}$ (the end-on orientation). The normalization parameter was subsequently transformed to the body reference heating rates. For convective heating, this transformation involved considerations of changes in flow dimensionality (i.e., axisymmetric vs 2-D flow) and stagnation velocity gradient (flat face cylinder vs. transverse cylinder, References 105, 141). For radiative heating, the transformation assumed a ratio of the effective spherical radii (disc to transverse cylinder) raised to a power $n$. The effective spherical radii were based on relationships for adiabatic shock layers (References 101, 142) and the power $n$ was determined from the tabular data of Sutton and Hartung (Reference 78).

Klett has curve fitted the convective heating distribution on the end face at $\alpha=0^{\circ}$ based on available flat face cylinder experimental data (References 117,143 and 144). This heating factor distribution is shown in Figure 58. Klett has reported the average heating to a disc at angle of attack relative to the average heating at the $\alpha=0^{\circ}$ position based on a composite of experimental data (References 144, 145), laminar flat plate theory at $\alpha=90^{\circ}$ and representative values of base heating at $\alpha=180^{\circ}$ (References 146, 147). This average heating distribution is shown in Figure 59. Rather than applying uniform heating over the end face based on the integrated average heating over the $180^{\circ} \alpha$ sweep, this study redistributed the heating over radial lengths of the LWRHU thermal model's end face nodes, $(\Delta r / R)_{i=1,2, \text { etc }}$ in proportion to the local integrated heating over the $(\Delta \mathrm{r} / \mathrm{R})_{\mathrm{i}=1,2, \text { etc }}$ to the integrated average over the entire face using the $\alpha=0^{\circ}$ heating factor distribution of Figure 58. Use of the data in Figures 58 and 59 provided the angle of attack average per end face node in the proper form prior to transformation to the body reference condition. The angle of attack factor for free molecule heating was taken directly from Klett which was based on a reduced form of Oppenheim's theory for flat plates at $\alpha$ for windward, uniform heating (Reference 148) and zero leeward side heating $\left(90^{\circ} \leq \alpha \leq 180^{\circ}\right)$.

Literature did not provide guidance on shock layer radiative heating distributions for discs at angle of attack; however, the flow field of a disc at angle of attack is three-dimensional which will act to reduce radiative heating levels due to flow expansion effects. This 3-D effect was assumed to be related to the disc's local sweep angle. Due to lack of data, this study was simplified by assuming uniform heating at the stagnation value over the disc at $\alpha=0^{\circ}$ whereas the average windward heating $\left(0^{\circ} \leq \alpha \leq 90^{\circ}\right)$ was the integrated product of the heating variation with angle of attack and disc leading edge sweep angle. Both of the variations assumed the general trigonometric functional form as discussed for the angle of attack and spin angle for the cylindrical section. On the leeward side, the average heating factor (relative to the $\alpha=0^{\circ}$ stagnation value) was conservatively assumed to be $10 \%$. 
Similar to the radiative heating dilemma, static pressure and recovery enthalpy data for a disc at angle of attack were also unavailable for this study. Time constraints did not permit investigation of flat plate literature. Consequently, engineering judgement was implemented commencing with the requirement that the disc be treated as a constant (average) pressure surface. The average static pressure factor at $\alpha=0^{\circ}$ was based on the integration of Love's correlation for a flat face cylinder (Reference 149). This distribution along with experimental data for this configuration (References 141, 150), is shown in Figure 60. The average disc pressure at angle of attack (relative to the average at $\alpha=0^{\circ}$ ) was assumed to have the same functional form as considered for the cylindrical surface. The resulting integrated average was further reduced by an arbitrary factor of 0.75 to account for 3-D flow effects. Recovery enthalpies at windward angles of attack were determined from isentropic flow theory based on the average static pressure ratio as a function of $\alpha$. For the leeward $\alpha$ range, recovery enthalpies were guided by recovery temperature data in cylindrical wakes as a function of yaw angle (Reference 136). This resulted in assuming a constant recovery enthalpy factor of 0.8 .

\section{Corrections to Local Heating Rates}

\section{a. Low Density Effects}

The local convective heating rates are corrected for low density effects identified with the kinetic flow regimes (Reference 151) using the bridging relationship of Matting (Reference 152). This bridging relationship is a simple exponential function based on the current, local ratio of the limiting free molecule to thin shear layer (high density) convective heating rates with the convective heating rate corrected for vorticity effects. This density correction is applied to the heating rates associated with the spinning and random tumbling orientations, and to the windward regions for stable orientation. Once the heating ratio exceeds a value of ten, the bridging value has essentially merged to the high density continuum value and this correction is zero.

\section{b. $\quad$ Surface Mass Transfer Effects, Convective Heating}

The blowing (or blockage) effect on convective heating due to thermochemical ablation is based on the correlation of Putz and Bartlett (Reference 153). The correlation is based on a matrix of multicomponent, reacting boundary layer solutions for a carbon/air system in thermochemical equilibria. This correlation represents both similar and nonsimilar (i.e., downstream) solutions and accounts for boundary layer mass addition and homogeneous reactions on local surface enthalpy gradients. Unfortunately, the Putz and Bartlett correlations did not permit stable solutions for the high energy VVEJGA reentries in regions producing high ablation rates. This instability was also experienced in the Galileo studies and the corrective action (Reference 154) involving the substitution of the generic Mickley-Spalding blowing correlation (Reference 155) was also adopted for the VVEJGA studies. As indicated in 
Figure 61, the Mickley-Spalding correlation provides an upper bound on convective heating blockage relative to the preferred Putz and Bartlett expression.

\section{c. Surface Mass Transfer Effects, Radiative Heating}

The general effect of carbon thermochemical ablation on shock layer radiation is to reduce surface radiation by self-absorption in a carbon vapor laden viscous sublayer. This form of blockage was modeled for the VVEJGA studies from the early investigations of Moss and others (References 97, 156, and 157) and the more recent studies by Gupta, et al. (Reference 111). Regarding the investigations of Moss and Gupta, radiation/ablation coupled solutions were performed using a viscous shock layer technique for multicomponent, reacting gas mixtures in thermochemical equilibria with radiation transport models, the Louisiana State University Radiation Transport model (LRAD-3) (Moss) and Aerotherm's Radiation Transport code (RADICAL) (Gupta, et al.), and injectants of air and carbon phenolic. The high carbon yield of carbon phenolic ablation species was assumed representative of the FWPF ${ }^{\text {tm }}$ carbon-carbon ablation products. Figure 62 presents the numerical data and the VVEJGA model for continuum, stagnation flow and indicates that carbon vapor blockage can account for approximately $40 \%$ of incident radiation at high ablation rates. The VVEJGA model was assumed to be valid at downstream locations and to linearly decrease from the continuum value at a rarefaction threshold (Cheng's parameter, Figure 63) to zero at the free molecule threshold (Reference 158, 159). The rarefaction threshold is representative of a fully merged shock layer, and the molecular threshold is to account for low density effects in the kinetic flow regimes. Cheng's rare faction parameter, $\mathrm{K}_{\mathrm{r}}$, is defined as $\mathrm{K}_{\mathrm{T}}^{2}=0.6 /\left(\mathrm{K}_{\mathrm{n}} \mathrm{M}_{\infty}^{0.5}\right)$ where $\mathrm{K}_{\mathrm{n}}$ is Knudsen's number.

Radiative and ablative coupled flow solutions in the kinetic regimes are neither available nor well understood at this time. However, a rational conjecture is that increased rarefaction of the shock layer (and more specifically, the ablation sublayer) should reduce the effectiveness of the sublayer blockage due to the reduced number densities and absorption cross sections of the injectant species.

\section{9. $\quad$ Fuel Pellet Aerothermodynamics}

By modeling the fuel pellet as a right cylinder, the aerothermodynamics of the full LWRHU assembly could be directly employed to define the aerothermodynamics of the pellet. The general methodology and the determination of body reference heating rates remained the same except for geometry scaling effects (i.e., change in the cylindrical radius). Furthermore, the heating factor distributions developed for the LWRHU were assumed valid for the pellet by adjusting the LWRHU data for the various flight orientations according to the pellet thermal model's $r / R$ (local nodal to end face radii) location of radial surface nodes and, likewise, the $\mathrm{z} / \mathrm{L}$ (local nodal to mid-span axial length) location of the axial nodes. No adjustments were made to the circumferential distributions used to define the side-on stable and spinning orientations. 


\section{F. Result of Analyses}

The RETAP simulations are used to generate the thermal and ablation response of the LWRHU model exposed to the previously discussed trajectory environments. The overall material ablation failure results are illustrated in Figure 64. This diagram shows the altitude and velocity at which each reentry analysis resulted in a $75 \%$ wall thickness recession. As indicated, all reentry angles for the side-on stable orientation will result in failure. This represents the most extreme reentry orientation. Also shown in Figure 64 is a representation of the fuel $\left(\mathrm{PuO}_{2}\right)$ melt boundary developed at Sandia National Laboratory for a $250-\mu \mathrm{m}$ sphere in a stable orientation, Reference 161. The altitude and velocity of expected fuel release conditions for the Cassini mission LWRHU are well in excess of the melt/no melt boundary shown in the diagram.

\section{1. $\quad$ Side-on Stable Orientation, LWRHU}

The most severe recession case examined for the side-on stable orientation is the $-90^{\circ}$ reentry flight path. As indicated in Figure $65-\mathrm{A}$, The surface temperature nears $8000^{\circ} \mathrm{R}$ in approximately 2 seconds. The heat pulse is of such short duration, though, that the internal components, specifically the clad and fuel, do not show significant thermal response. Figure 65-B shows the mass transfer parameter from the Lawrence Livermore thermochemistry tables for the surface response. The diagram shows that the surface material is well into the sublimation region within 0.25 seconds and rapidly drops back down into the diffusion limited regime once the heat pulse is removed. The wall recession (see Figure 65-C) associated with the mass transfer parameter demonstrates failure in less than 2 seconds and complete burn through within 2.27 seconds. For the surface nodes that indicated ablation failure, Figure 65-D contains information when each of the failures occurred. As indicated, all of the surface nodes within the modeled stagnation line exhibit wall thickness failure at about the same time. The combination of nodes 85 and 64 failing meet the fuel release criteria, and this is the point where aeroshell failure is assumed.

Figures 66 through 72 contain similar information for the remaining side-on stable reentry angles. Only slight differences from the $-90^{\circ}$ case are seen for the $-70^{\circ}$ reentry. For the shallower angles, as the trajectory flight time is increased, a decrease in mass transfer parameter and an increased amount of surface recession (due to lower pressures and longer exposure) is seen. Also, as the flight time lengthens the internal components show significant thermal response. Failure, however, occurs before the internal components encroach on their melt temperatures.

The location within the trajectory at the point of aeroshell breach is of significant importance for determining the end state disposition of fuel released into the atmosphere. Figures 73 through 80 show the point of ablation failure relative to the reentry heat pulse for each reentry angle. For the steep reentries, the $75 \%$ failure criteria are met shortly after the time of peak heating. For decreasing reentry angles, the relative location of ablation failure to the heat pulse is shifted towards the ascent side of the heat pulse. For the shallow reentry angles, full wall burn-through occurs by the time peak heating is reached. Since it is assumed that the clad 
and fuel assemblies will be released when the aeroshell is breached, with immediate removal of the clad material, sufficient amount of the heat pulse remains to cause vaporization of the fuel prior to ground impact.

The side-on stable reentry results are summarized in Table 9. Peak heating data and both $75 \%$ and $100 \%$ recession data are shown for each angle. As shown in this table, aeroshell burn through will occur well within the hypersonic portion of flight. As a matter of reference, several pertinent response temperatures at time of failure are noted in Table 9. These data further indicate that although the internal components show greater response for shallow angles, no melting occurs at the time of clad release.

\section{Side-on Spinning Orientation, LWRHU}

The side-on spinning orientation results in the least severe environment for the LWRHU model. As summarized in Table 10 , only one case, the $-5.2^{\circ}$ reentry, indicates aeroshell failure. While this case meets the necessary conservative failure criteria, it does not indicate $100 \%$ burn through and it could survive the hypersonic flight and be intact at the time it impacts the ground. Figure 81 shows the recession failure time of the modeled surface nodes for the $-5.2^{\circ}$ reentry. Figure 82 shows the point of ablation failure relative to the reentry heat pulse. As indicated in both of these diagrams, the aeroshell does not fail due to ablation until about 112 seconds into the trajectory.

Although the remaining $\mathrm{V}-\gamma$ selections survive the hypersonic flight, the long trajectory allows significant thermal response of the internal components, especially for the shallow reentry angles. The component thermal response for the $-7^{\circ}$ reentry case is shown in Figure 83. It represents the intensity of the internal heating for an LWRHU that survives the Cassini VVEJGA reentry. Melting of the clad will occur for reentry by the end of hypersonic flight. Also, the fuel rapidly approaches the point of melting.

Therefore, under certain flight conditions, clad and fuel melt within an unbreached aeroshell are distinct possibilities. The effects of melted clad and fuel material on the internal heating and aeroshell surface response have not been addressed in this analysis. Reference 162 indicates that fuel capsule temperatures can be significantly higher in flight than in a test environment. This effect is due to inertial loading which induces greater thermal contact conductance at the interface standoffs.

The current analyses are conducted with air filled gaps. References 163 and 164 discuss the possibilities of various gases or a vacuum occupying the gap spaces prior to material melt. These references demonstrate how contact resistance can be altered by various flight conditions. It is unknown what effect melted material could have on the gap conductance; therefore, molten materials from the clad and fuel could force the thermal conductance to be significantly different than the current results indicate. Due to inertial effects, the conductance in 
the stagnation region would increase. The appropriate material properties and variations in contact resistance during melting are not sufficiently understood to allow consideration at this time. The occurrence of clad melt is therefore registered as a melt failure and registered as a source term.

\section{Tumbling Approximations, LWRHU}

\section{a. Quasi-Tumbling Cylindrical LWRHU Orientation}

The quasi-tumbling cylindrical orientation of the LWRHU is only slightly more severe than the side-on spinning orientation. Table 11 summarizes the responses for the quasi-tumbling orientation. Only the extreme shallow angles, $-5.2^{\circ}$ and $-7^{\circ}$ reentries, show aeroshell failure. The $-5.2^{\circ}$ reentry shows $100 \%$ aeroshell burn through just prior to the end of the hypersonic flight. The $-7^{\circ}$ reentry indicates that $75 \%$ wall recession will occur at about half way through the hypersonic flight. Figure 84 shows the times of recession failure of the modeled surface nodes and Figure 85 shows the point of ablation failures relative to the reentry heat pulses. Again, the aeroshell breach releases molten material, and an aeroshell impacting at ground level could release material registered as a ground source term.

\section{b. $\quad$ Random Tumbling Cylindrical LWRHU Orientation}

Much like the quasi-tumbling cylindrical orientation of the LWRHU, the random tumbling orientation shows aeroshell survival for most orientation reentries. Only the shallow cases, less than $-15^{\circ}$ reentries, show a $75 \%$ aeroshell wall thickness failure. All reentries indicate that $100 \%$ aeroshell burn through will not occur (see Table 12). Figures 86 and 87 show the times of recession failure of the modeled surface nodes, and the point of ablation failures relative to the reentry heat pulses, respectively. Again, the aeroshell breach releases molten material.

While the other orientations indicate that the aeroshell provides the critical path for ablation failure, this orientation shows that the ablation is most severe in the end cap region. Failure would occur by breaking out the end cap to release the internal components. This orientation was expected to represent the lower boundary of material ablation for all of the non-stable cases. Indeed, the aeroshell tends to survive, but it also represents the most severe conditions for end cap ablation. The lower boundary is somewhere between the quasi and random tumbling orientations.

\section{c. Tumbling Sphere Orientation, LWRHU}

The most severe approximation to the tumbling orientation examined is that of the tumbling sphere. This orientation was expected to represent the upper boundary of material ablation for all of the non-stable orientations. Burn-through failure of $100 \%$ occurs for the convectively dominated shallow reentry heat pulses. Only the steepest, radiatively. 
dominated heat pulses do not show $100 \%$ failure. All cases, though, show at least $75 \%$ wall recession. Figure 88 shows the times of recession failure of the modeled surface nodes. The timing of the failures, relative to the heat pulse, oppose the trend established by the side-on stable case. Shallow angles show failure significantly after the peak heating while the steeper cases fail close to the time of peak heating. This is illustrated in Figure 89 and summarized in Table 13.

\section{Component Temperature and Aeroshell Recession}

Since the condition of the LWRHU components at the point of ablation failure or impact are important in determining the fuel source terms, additional temperature and recession data are supplied in table format. Tables 14 through 18 contain the average, maximum and minimum component temperatures for each orientation and flight path that exhibit ablation failure. Separate tables are supplied for the $75 \%$ and the $100 \%$ failure criteria; for example, Tables 14-A and 14-B contain the $75 \%$ and $100 \%$ failure data, respectively, for the side-on stable orientation. Listed at the bottom of these tables are the melt temperatures for the clad and fuel materials.

For the conditions that do not indicate $100 \%$ burn-through failure, the clad and fuel temperature histories over the heat pulse are important. (Note that the end of the trajectory is Mach 5.0 and not impact.) Maximum temperature data are supplied in Tables 19-A through 22$\mathrm{B}$, with the $\mathrm{A}$ providing clad data and $\mathrm{B}$ providing fuel data. For the conditions that indicate melting of the pertinent material, the trajectory flight period during the melt phase is supplied. Sometimes the material will resolidify prior to the end of the trajectory. For the cases that do not demonstrate resolidification, the end of the hypersonic flight time is used as the end of the melt temperatures.

Also, for the conditions that do not indicate $100 \%$ burn-through failure, the amount of recession to which the aeroshell and the end cap are subjected is listed in Tables $23-\mathrm{A}$ through 23-D. These recession data are supplied as a percentage of the original modeled wall thickness at the modeled points. Table $23-\mathrm{D}$ is the recession percentage for the random tumbling cylinder orientation. One data point within that table (for $-5.2^{\circ}$ reentry) indicates that $100 \%$ recession occurred, but this reentry angle is listed as not showing a condition of $100 \%$ burn through. In this case, only one of the aeroshell's extreme edge surface nodes (node 85 , refer to Figure 26 for node positions) shows full ablation which is deemed insufficient to allow break out of the end cap, and therefore no burn-through failure.

\section{Clad and Fuel State at Impact}

Additional analyses were performed for representative cases that indicated clad/fuel melt during hypersonic flight to determine the thermal condition of the clad and fuel at impact. For the spinning cases, RETAP runs conducted at $\gamma=-7^{\circ}$ and $-15^{\circ}$ well into subsonic flight show that both clad and fuel cool down to approximately $1100^{\circ}$ R. Similar results were obtained for 
the tumbling cases at $\gamma=-7^{\circ}$ and $-10^{\circ}$. Therefore, any clad and fuel contained within the aeroshell sufficiently resolidifies prior to ground impact. There was insufficient ablation during the supersonic and subsonic flight to induce further aeroshell failure.

\section{Sensitivity Analyses}

A brief examination of the sensitivity to the thermochemistry data used (JANNAF or Lawrence Livermore) is conducted using representative side-on stable LWRHU trajectories $\left(-15^{\circ},-30^{\circ}\right.$, and $\left.-90^{\circ}\right)$. Figure 90 shows the time of $75 \%$ and $100 \%$ surface recession results for both thermochemistry tables. As expected, the JANNAF thermochemistry data obtains later failure (with higher temperatures) than the Lawrence Livermore data, but only by a maximum of approximately 0.2 seconds for each case. Figure 91 shows the time of failure relative to the heat pulse. Only the $-90^{\circ}, 100 \%$ ablation failure delays burn through until near the end of the heat pulse. The $-30^{\circ}$ reentry shows burn through near the peak of the radiative heat pulse, and the $-15^{\circ}$ reentry shows failure at the peak of the convective heat pulse.

The effect of the gas dynamic-radiation coupling is examined, also. This effect is examined for the $-90^{\circ}$ side-on stable LWRHU only. Additionally, the JANNAF data are used in this analysis. Figure 92 shows the time at $75 \%$ and $100 \%$ surface recession and the time of failure relative to the heat pulse for the condition of full blockage and no blockage (a factor of 1.3, and 1.0, respectively, on the convective heat pulse). As indicated, the gas dynamic-radiation coupling factor has a more significant effect on the failure time than the different thermochemistry data discussed previously. The $75 \%$ ablation failure is shifted to about $1.8 \mathrm{~s}$ from $2.1 \mathrm{~s}$ when this effect is included in the analyses. Without coupling, the $100 \%$ ablation failure occurs near the end of the heat pulse; with coupling, the $100 \%$ ablation failure occurs near the peak of the heat pulse. While the differences in burn-through times are only between $0.3 \mathrm{~s}$ to $0.4 \mathrm{~s}$ in the trajectory, it is a very critical time in the trajectory because the heat load remaining impacts the fuel state.

\section{G. Bare Fuel. Thermal and Ablation Results}

The fuel pellet, as illustrated in Figure 4, is a cylinder of dimensions $8.06 \mathrm{~mm}$ by $6.25 \mathrm{~mm}$ capped with small $50^{\circ}$ conical frustrums $0.643 \mathrm{~mm}$ long with a maximum diameter of 6.0 $\mathrm{mm}$. The resultant fuel volume is $275.271 \mathrm{~mm}^{3}$. The plutonium oxide density and weight are $10.017 \mathrm{~g} / \mathrm{cm}^{3}$ and $2.664 \mathrm{~g}$, respectively.

The fuel pellet thermal analysis requires appropriate surface thermochemistry data for the $\mathrm{PuO}_{2}$. Temperature dependent enthalpy data, in the form of input data, from the Aerotherm Chemical Equilibrium (ACE) code were obtained from Mr. B. Laub, of Aerotherm (Reference 165). These data were used to run $A C E$ for the desired pressure and mass transfer parameter values. The resulting thermochemistry tables were converted to the form required for use in RETAP. The maximum mass transfer parameter data generated by ACE was 3.5. 


\section{Initial Conditions}

A thermal analysis is performed on an intact fuel pellet for every condition of accidental LWRHU reentry that indicates ablation failure. The initial conditions for generating reentry trajectories for these released fuel pellets are listed in Table 24 . The time of modeled wall section failure does not necessarily indicate the time at which the fuel pellet would break free from the remaining aeroshell. For purposes of trajectory generation, the release time is determined by averaging the time of failure for vital sections of the aeroshell. The release times are determined based on the occurrence of one of two assumed conditions that would allow release of an intact fuel containment assembly. The conditions are the failure of the aeroshell region overlaying the insulator sleeves for a central angle of $\pm 15^{\circ}$, and the failure of the aeroshell and end cap region that would degrade the integrity of the end cap threads for a central angle of $30^{\circ}$ thereby allowing disengagement of the end cap. For example, referring to Figure 34, the average time of ablation failure for nodes 1,22 , and 43 signifies time of pellet release. Alternatively, the average time of ablation in the end cap region leading to failure at nodes 64 and 85 signifies pellet release.

The motion behavior of a released pellet is not known apriori. When a LWRHU breach occurs, the pellet could slip out and orient side-on into the flow stream in a stable manner or in a spinning manner. More likely, it would be flipped out and either tumble or trim to a preferred oscillation about an edge. Without full six degree of freedom modeling and full understanding of the initial forces on a pellet breaking free of an aeroshell, it cannot be determined what is the expected orientation of a released fuel pellet. For this reason, limiting cases are examined: full random tumbling condition (representative of most severe oscillation motions); sideon stable or spinning (representative of least severe oscillation motions). Figure 93 is a flow diagram that illustrates all conditions of release and orientation for the current TDOF trajectory generations. A total of forty trajectories are generated to cover all expected conditions for a fuel pellet released from a LWRHU exhibiting ablation failure. The analyses encompass the following cases.

a. Side-on stable 2-D fuel pellet released from a breached side-on stable LWRHU at $\gamma=-5.2^{\circ},-7.0^{\circ},-10^{\circ},-15^{\circ},-30^{\circ},-50^{\circ},-70^{\circ}$, and $-90^{\circ}$ for upwind entries. Released conditions generated for both $75 \%$ and $100 \%$ wall recession leading to LWRHU breach.

b. Side-on spinning 2-D fuel pellet released from a breached side-on stable LWRHU at $\gamma=-5.2^{\circ},-7.0^{\circ},-10^{\circ},-15^{\circ},-30^{\circ},-50^{\circ},-70^{\circ}$, and $-90^{\circ}$ for upwind entries. Released conditions generated for both $75 \%$ and $100 \%$ wall recession leading to LWRHU breach.

c. Full random tumbling cylindrical (R-Z model) 2-D fuel pellet released from a breached side-on stable LWRHU at $\gamma=-5.2^{\circ},-7.0^{\circ}$, $-10^{\circ},-15^{\circ},-30^{\circ},-50^{\circ},-70^{\circ}$, and $-90^{\circ}$ for upwind entries. Released conditions generated for both $75 \%$ and $100 \%$ wall recession leading to LWRHU breach. 
d. Side-on stable 2-D fuel pellet released from a breached side-on spinning LWRHU at $\gamma=-5.2^{\circ}$ for upwind entries. Released conditions generated for $75 \%$ wall recession leading to LWRHU breach.

e. Side-on spinning 2-D fuel pellet released from a breached side-on spinning LWRHU at $\gamma=-5.2^{\circ}$ for upwind entries. Released conditions generated for $75 \%$ wall recession leading to LWRHU breach.

f. Full random tumbling cylindrical (R-Z model) 2-D fuel pellet released from a breached side-on spinning LWRHU at $\gamma=-5.2^{\circ}$ for upwind entries. Released conditions generated for $75 \%$ wall recession leading to $L W R H U$ breach.

g. Side-on stable 2-D fuel pellet released from a breached full random tumbling LWRHU at $\gamma=-5.2^{\circ},-7.0^{\circ}$, and $-10^{\circ}$ for upwind entries. Released conditions generated for $75 \%$ wall recession leading to LWRHU breach.

h. Side-on spinning 2-D fuel pellet released from a breached full random tumbling LWRHU at $\gamma=-5.2^{\circ},-7.0^{\circ}$, and $-10^{\circ}$ for upwind entries. Released conditions generated for $75 \%$ wall recession leading to LWRHU breach.

i. Full random tumbling cylindrical ( $R-Z$ model) 2-D fuel pellet released from a breached full random tumbling LWRHU at $\gamma=-5.2^{\circ}$, $-7.0^{\circ}$, and $-10^{\circ}$ for upwind entries. Released conditions generated for $75 \%$ wall recession leading to LWRHU breach.

The drag coefficient data for side-on and tumbling pellets are listed in Table 25. The side-on stable and side-on spinning orientations utilize the same drag data. The initial velocity of the fuel pellets at the time of release varies between $59.5 \mathrm{kft} / \mathrm{s}$ and $20.9 \mathrm{kft} / \mathrm{s}$ with the lowest velocity at release noted for the $-5.2^{\circ}$ reentry of a spinning LWRHU. The initial altitudes vary from $245 \mathrm{kft}$ and $126 \mathrm{kft}$.

\section{Trajectory Results and Discussion}

The results of the TDOF simulations are presented in Figures 94 through 101. Figure 94 contains the velocity verses altitude profiles for each LWRHU orientation releasing a side-on fuel pellet. Figure 95 gives the altitude histories. For alternate fuel orientations at release, only minor deviations in the trajectory data were noted. The reference heating rate histories are contained in Figures 96 through 101. Figure 96 illustrates (a) the convective and (b) the radiative heating rates for the side-on stable and spinning fuel orientations after release from breached sideon stable LWRHUs. Figure 97 illustrates the same information for a tumbling fuel pellet. Similar data for fuel pellets released from breached side-on spinning LWRHUs (Figures 98 and 99), and from breached tumbling LWRHUs (Figures 100 and 101) are shown. Of particular note is the very 
low heating levels demonstrated for the pellet released from the spinning LWRHU. This is due to breach occurring very late in the LWRHU trajectory, well past the heat pulse.

\section{Side-on Stable LWRHU Release of Fuel Pellet}

For all fuel pellets released from a side-on stable LWRHU, the heating rates were too severe to allow proper software convergence when the full geometry conversion effect on convective heating is incorporated. The full correct geometry influence is a factor of about 7.25. The severe heat pulses introduce rapidly changing conditions and lead to solution oscillations beginning sometime after the material temperatures reach approximately $6500^{\circ} \mathrm{R}-7000^{\circ} \mathrm{R}$. The model generally shows convergence using the reference stagnation heating for a $1-\mathrm{ft}$ sphere, and for a geometry influence on the convective heating of around 3. An exception typically occurs for the surface stagnation point which usually exceeds $7000^{\circ} \mathrm{R}$ under reference heating conditions for a side-on stable pellet, because oscillations occur for any node that exceeds $6500-7000^{\circ} \mathrm{R}$. This occurs in part due to tabulated thermochemistry data ending in this temperature region, and in part due to numerical limitations within the software.

The code response is to force divergent oscillations about the true value until the heat load begins to decrease. The average values between consecutive computational time steps show a smooth rise in thermal response with respect to the rising heat input. On the cooling portion, the solution shows a convergent oscillation until the thermal response is within tabulated values. The repercussion is that the hot surface (i.e. stagnation point) behaves alternately as a large heat source and heat sink. It is expected that the net result allows a reasonable approximation to appropriate internal conduction, but not exact solutions. Since the melt temperature of the $\mathrm{PuO}_{2}$ material is well below the oscillation range, it is not reasonable to expect accurate solutions over $7000^{\circ} \mathrm{R}$ due to uncertainties in material properties above melting point.

The problem is in determining when sufficient heat is conducted to the pellet center to induce full pellet melting. As illustrated in Figure 102, the leeward surface of the side-on stable fuel pellet (node 7) shows the lowest thermal response to the environment. Melting in this leeward region, therefore, will be used to indicate full pellet melt. Although the uncertainty band on the melting of $\mathrm{PuO}_{2}$ has been noted to be $300^{\circ}-500^{\circ} \mathrm{F}, 4812^{\circ} \mathrm{R}$ is marked on each thermal response diagram. Also of note in Figure 102 is the thermal dwell illustrated by several nodes at approximately $4200^{\circ} \mathrm{R}$. This anomaly is due to the heat of fusion consideration and a limiting condition within the enthalpy data. The dwell shows up for every node that heats up or cools (relatively) slowly through the $4000-4400^{\circ} \mathrm{R}$ region.

\section{a. $\quad$ Side-on Stable Fuel Pellet}

The thermal responses of node 7 for a side-on stable pellet released from a side-on stable LWRHU are shown in Figures 103 through 110. Each Figure contains the response diagrams for release from both a $75 \%$ and $100 \%$ ablation failed LWRHU. In all cases, the 
pellet does not show full melting when subjected to reference convective heating, indicated as $1 \mathrm{X}$. Full melting does occur in all cases, though, when the pellet is subjected to three times ( $3 \mathrm{X})$ the referenced heating. Furthermore, the melt temperature is exceeded almost immediately upon release from the LWRHU. All fuel pellets, then, become radiological source terms a very brief time after release from the LWRHU. Results indicating full pellet melt $\left(4812^{\circ} \mathrm{R}\right)$ with $1 \mathrm{X}$ or $3 \mathrm{X}$ reference heating imply that it is logical to assume full pellet melt with a heating factor of 7.25.

\section{b. Side-on Spinning Fuel Pellet}

Figures 111 and 112 show the thermal responses of a side-on spinning pellet released from a side-on stable LWRHU under representative reentry angles $\left(-7^{\circ}\right.$, $15^{\circ},-90^{\circ}$ ). Since all surface locations are subjected to identical heat loads, these figures illustrate the temperatures from the surface to the center of the fuel pellet (noted as nodes 1, 8, 9, 10 for surface to center). Figure 111 contains the response diagrams for release from $75 \%$ ablation failed LWRHU, and Figure 112 shows the same for $100 \%$ ablation release. In most instances, only the reference convective heating environment was required to induce full pellet melting. The steep reentry cases show an exception. At release from a $75 \%$ ablated LWRHU, the center portion did not melt, but when subjected to $3 \mathrm{X}$ the convective heating environment, it showed complete melt. Only for the later release ( $100 \%$ ablation of the LWRHU) did the pellet show partial survival. Furthermore, only a small difference in center node response was indicated between the $1 \mathrm{X}$ and $3 \mathrm{X}$ environments. It is possible, then, that only the outer part of the late released, steep reentry pellet would vaporize.

The surface nodes in Figures 111 and 112 for the steeper angles illustrated oscillatory behavior discussed previously. For $-15^{\circ}$ in Figure 111, the oscillation was not severe, and it is shown by the data in the graph. For $-90^{\circ}$, however, the oscillation was severe, and was removed, as indicated by the plotted response beginning later than the other nodes. For all remaining diagrams, if the node response plot starts at a later release time, it is due to removal of oscillating data that first converged in excess of $7000^{\circ} \mathrm{R}$. For cases where the node first converged at lower temperatures, then heated to greater than $6500-7000^{\circ} \mathrm{R}$, the oscillating data was removed but a line connecting the beginning and ending of oscillation will be shown, as in the $-15^{\circ}$ case in Figure 112.

\section{c. Tumbling Fuel Pellet}

The release of a tumbling pellet results in much the same behavior as that noted for the spinning pellet. Figures 113 and 114 for a pellet released under the same representative reentry angles. For the tumbling pellet, a different model (refer to Figure 36) was used, and the representative nodes are tracked in the diagrams: two surface symmetry nodes (1, and 15 ), and the center node (4). Again, under $1 \mathrm{X}$ reference convective heating environments, the shallow angles show full pellet melt, and only surface melting is shown for the steeper angles. $3 \mathrm{X}$ 
heating was not sufficient to raise the center thermal response to a melting level. It is possible that only the outer part of the steep reentry pellet would vaporize.

\section{Side-on Spinning LWRHU Release of Fuel Pellet}

The side-on spinning LWRHU demonstrated only one condition of failure: $75 \%$ ablation at $-5.2^{\circ}$ reentry. The stable, spinning, and tumbling pellet results are shown in Figure 115. Prior to failure, the internal fuel pellet reached approximately $4800^{\circ} \mathrm{R}$ before cooling down to about $4300^{\circ} \mathrm{R}$ at the time of release. It is unlikely that the integrity of the pellet structure would be intact at the time of release. Assuming the pellet to be undamaged, though, the analyses indicate that only release of a side-on stable pellet would result in remelting (see Figure 115). The test cases run for a pellet release from a spinning LWRHU are conducted with full geometry conversion effect on the convective heating input; approximately $7.25 \mathrm{X}$.

\section{5. $\quad$ Random Tumbling LWRHU Release of Fuel Pellet}

The random tumbling LWRHU fuel pellet release cases are conducted with a full geometry conversion effect also. The stable, spinning, and tumbling pellet results are shown in Figures 116,117 , and 118, respectively. Under these three conditions, only the shallowest angles $\left(-5.2^{\circ}\right.$ and $\left.-7^{\circ}\right)$ indicate full pellet melt. The $-10^{\circ}$ reentry conditions show that no melting would occur. For any pellet released from a tumbling LWRHU (the most probable orientation), any reentry angle of $10^{\circ}$ or greater will result in no high altitude vaporization based on current available response data.

\section{Summary of Melt Fractions}

A survey of the temperature states of the nodes modeled in the thermal analysis of the fuel pellet was made to obtain an indication of the melt volume (mass). The results are tabulated in Table 26. Table 26 illustrates the volume percentage of the fuel pellet that will melt and be counted as atmospheric source terms. Two melt temperatures are listed: $4812^{\circ} \mathrm{R}$ and $4312^{\circ} \mathrm{R}$ to illustrate the uncertainty. In general for the side-on stable LWRHU where release due to ablation was obtained at all entry angles, $100 \%$ fuel melt was obtained. For a spinning LWRHU at $\gamma=-5.2^{\circ}$, and for cases of a tumbling LWRHU orientation at $\gamma=-5.2^{\circ}$ and $-7^{\circ}$, the melt fraction was also $100 \%$. These releases occur at altitudes greater than about 125,000 feet. For a random tumbling LWRHU reentering at $\gamma \geq 10^{\circ}$, no atmospheric source terms occur. Instead, these are considered ground source terms. 


\section{THERMOSTRUCTURAL ANALYSIS}

\section{A. General Description}

The thermostructural response of the LWRHU for inadvertent reentry is analyzed with two primary analysis tools: MSC/NASTRAN, and a thermoplastic (temperature-dependent plastic) analysis program called ANSP Non-linear (ANSPNL). A third program Finite Element Modeling and Post Processing (FEMAP) is used for graphical pre- and post-processing. MSC/NASTRAN is a commercial general-purpose finite element analysis (FEA) program that performs structural and thermal analyses, and is used for thermoelastic (temperature-dependent elastic) analysis here. The thermoplastic analysis program ANSPNL is a custom code written by Mr. Y. Chang of JHU/APL specifically for this problem [References 166-171]. It performs additional analyses on stresses calculated by MSCANASTRAN, and accounts for the different nonlinear material behaviors of the FWPF at different elevated temperatures and loadings.

Thermostructural analyses were conducted for those LWRHU orientations where ablation failure was not indicated by the thermal analysis, as discussed in Section VII. The exception is the side-on stable orientation where ablation failure was indicated at all reentry angles. For this orientation, thermostructural analyses were conducted to check out the procedures. The general methodology, assumptions, thermostructural models, mechanical properties, loadings, and results of thermostructural analysis of the LWRHU are described in more detail in following sections.

\section{B. General Methodology}

Assuming LWRHU release, three reentry orientations are considered: side-on stable, side-on spinning, and random tumbling. For each reentry orientation, significant trajectory points are chosen for analysis. These are gencrally points of highest mechanical loadings, heat fluxes, temperatures, gradients, material burn-through, or combinations of these. Thermal loads are obtained for these points, and the temperatures from the thermal analysis are mapped onto a finite element model (FEM) of the LWRHU with a 3-D Interpolation program (INT3D), written by Mr. J. Ecker of JHU/APL. The deceleration loads are obtained from the TDOF analysis, and the pressure loads are obtained from the thermal analyses using RETAP. These loads are translated into the FEM. The thermoelastic material properties of the FWPF are used in the finite element analysis, and elemental thermoelastic stresses are generated. These are then input directly into the thermoplastic analysis program ANSPNL, where the temperature-dependent plastic material properties of the FWPF are considered. The thermoplastic strains are obtained iteratively. The final converged strains are compared to strain allowables at temperature for each strain component, and the thermostructural integrity of the LWRHU is assessed. More detailed descriptions of these steps are provided in following sections. 


\section{Assumptions}

There are various assumptions used in the thermostructural analysis of the LWRHU. Material properties used are the best available, and some material properties are extrapolated at higher temperatures where test data are not available. The analysis occurs at points in the trajectories where maximum mechanical and thermal loadings occur, and assumes a linear, not a timestepping analysis. That is, each loaded state is assumed to be path-independent. There are assumptions inherent to the thermoplastic analysis method itself. The thermoplastic analysis method assumes that a finite element is equivalent to a unit cell; this assumption becomes more reasonable as the stress gradients within a finite element approach zero. Also, strain continuity between elements is not assured, as the thermoplastic analysis method decouples each element from its neighbors in performing the iterations. Certain material behaviors are not modeled, such as: strain rate effects, creep, dynamic effects, stress relaxation, and ablation. Future analyses will consider the reduction in wall thickness caused by ablation.

Only compression material properties are used for compression and tension behavior, for both thermoelastic and thermoplastic analyses. For thermoelastic material behavior, test data show that compression and tension material properties are similar. For thermoplastic material behavior, test data show that the compression stress-strain behavior is more nonlinear (i.e., more strain deformation) than for tension. Since the analysis uses factors of safety (FS), where FS $=$ material allowable strain or strength $\div$ calculated strain or stress, and FS $<1.0$ indicates failure, use of the thermoplastic compression material properties for both compression and tension behavior is conservative.

It is assumed that in order for a breach of the LWRHU aeroshell to occur, failure must be extensive. This is because the material can still carry load when the strain allowable is exceeded, as described in Section VIII E, Mechanical Properties. Since there is no well-defined measure of the extent of failure over the surface of the aeroshell, perhaps a better measure would be failure through the thickness of the aeroshell. Also, overall failure of a composite generally requires interaction of all components of the composite material. Thus this criterion for breach will be used: breach is indicated when a factor of safety less than 1.0 is seen through the entire thickness of the aeroshell for all three normal directions $\mathrm{x}, \mathrm{y}$, and $\mathrm{z}$ at any point on the aeroshell.

\section{Thermostructural Models}

\section{Thermoelastic Models}

The thermostructural models are the MSC/NASTRAN finite element models for thermoelastic analysis and the thermoplastic analysis models. The thermoelastic finite element models are described first. A full LWRHU is shown in Figure 1, and the portion that is modeled is shown in Figure 119. Only the outer aeroshell, which is fabricated of a threedimensional weave carbon-carbon composite, FWPF, is modeled. The FEM covers $180^{\circ}$ and half 
the length of the LWRHU. This quarter-model considers the relationship between the LWRHU geometry and the FWPF material, and the symmetries of the loadings. The geometry of the model is described in cylindrical coordinates, while the Cartesian weave FWPF material is described in Cartesian coordinates. This material orientation causes the material properties to vary sinusoidally over the circumference of the LWRHU. A full cycle of material property variation occurs over a $90^{\circ}$ segment of the structure, so that modeling a $180^{\circ}$ segment allows for two full cycles of material property variation to be covered, while also allowing for consideration of the windward and leeward sides of the aerodynamic loads, the temperature loads, and the deceleration loads. Axisymmetric boundary conditions are applied at the $\theta=0^{\circ}$ and the $180^{\circ}$ interfaces of the model, and symmetric boundary conditions are applied at the $z=L / 2$ interface. The MSC/NASTRAN model of the LWRHU was used for all three configurations, each with its own loadset. The mechanical loads on the model are the internal and external pressures on the aeroshell, the decelerations on the aeroshell, and the forces of the internal components on the aeroshell caused by deceleration. The temperature loads on the model for all three configurations are shown in Figure 120.

There are some modeling techniques which have to be observed for thermostructural analysis of the LWRHU with MSCNASTRAN, Version 68, in order to obtain correct results. These techniques and the errors which they avoid are detailed in Reference 166.

\section{Thermoplastic Models}

The material behavior of FWPF is nonlinear, and this nonlinear behavior becomes more pronounced with elevated temperatures (Figure 121), hence the term "thermoplastic." Note that the thermoelastic material properties (Figure 122) are the initial tangent moduli of the thermoplastic material properties of Figure 121. Since the LWRHU is expected to encounter temperatures well above $5000^{\circ} \mathrm{F}$ during an inadvertent reentry, it is paramount that this nonlinear behavior be modeled. But this modeling is complex. FWPF has six strain components ( 3 normal and 3 shear), and each of these six strain components exhibits its own unique thermoplastic behavior. Also, the six strain components are coupled, not independent.

A number of strategies to model the thermoplastic behavior of FWPF were investigated. MSC/NASTRAN can perform thermoelastic analysis of orthotropic composite materials, but not thermoplastic analysis. MSCNNASTRAN has a capability called initial anisotropy, wherein the material initially deforms according to the anisotropic constitutive equation, but subsequent nonlinear deformation is calculated assuming that the material is isotropic. This clearly is insufficient for accurate representation of heat sources fabricated of FWPF. The finite element program ABAQUS was explored, but it would require user-written modifications to incorporate the thermoplastic material properties. It was ultimately decided to write a postprocessor program to MSC/NASTRAN thermoelastic analysis. 
The development of the thermoplastic analysis program ANSPNL is documented in References 166 through 171. The thermoplastic analysis method has as its basic goal an extension of the finite element codes' capability for thermoelastic analysis into the thermoplastic region. The method for thermoplastic analysis of carbon-carbon composites consists of three basic parts, which are described next.

First, the nonlinear stress-strain data of each strain component at each temperature are used to develop material property vs. strain energy density equations of the form

$$
M P=A\left[1-B\left(\frac{U}{U_{o}}\right)^{c}\right]
$$

where

$\mathrm{MP}=$ material property $\mathrm{E}$ or $\mathrm{G}, \mathrm{psi}$

$A=$ initial value of material property, psi

$\mathrm{B}=$ initial curvature

$\mathrm{C}=$ rate of change of curvature

$\mathrm{U}_{\mathrm{o}}=$ unit strain energy density, $1 \mathrm{psi}$

$\mathrm{U}=$ strain energy density, psi

according to the strategy of Jones [Reference 172]. The stress, strain, and strain energy density are highly interdependent. Curve-fit coefficients are set up for each nonlinear strain component at each temperature. These coefficients are interpolated for temperatures intermediate to those at which the data are defined. The moduli are assumed to become negligible above $8000^{\circ} \mathrm{F}$, and the coefficients are extrapolated with that constraint, for temperatures above those for which test data exist.

The second part begins with the elemental stresses in the material coordinate system from a thermoelastic finite element analysis using MSC/NASTRAN, described earlier in Section VIII. D1. These stresses are applied to the stress-strain constitutive Equation (2), 


$$
\left\{\begin{array}{c}
\varepsilon_{x} \\
\varepsilon_{y} \\
\varepsilon_{z} \\
\gamma_{x y} \\
\gamma_{y z} \\
\gamma_{z x}
\end{array}\right\}=\left[\begin{array}{cccccc}
\frac{1}{E_{x}} & \frac{-v_{x y}}{E_{x}} & \frac{-v_{z x}}{E_{z}} & 0 & 0 & 0 \\
& \frac{1}{E_{x}} & \frac{-v_{z x}}{E_{z}} & 0 & 0 & 0 \\
& \frac{1}{E_{z}} & 0 & 0 & 0 \\
& & \frac{1}{G_{x y}} & 0 & 0 \\
& & & \frac{1}{G_{z x}} & 0 \\
\sigma_{z} & & & & \frac{1}{G_{z x}}
\end{array}\right]+\Delta T\left\{\begin{array}{c}
\sigma_{x} \\
\sigma_{y} \\
\sigma_{x y} \\
\tau_{y z} \\
\tau_{z x}
\end{array}\right\}\left\{\begin{array}{c}
\alpha_{x} \\
\alpha_{x} \\
\alpha_{z} \\
0 \\
0
\end{array}\right\}
$$

where

$\sigma=$ normal stress, $\mathrm{psi}$

$\varepsilon=$ normal strain, in $/$ in

$\tau=$ shear stress, psi

$\gamma=$ shear strain, in $/$ in

$\mathrm{E}=$ modulus, $\mathrm{psi}$

$$
\mathrm{E}_{\mathrm{X}}=\mathrm{E}_{\mathrm{Y}} \neq \mathrm{E}_{\mathrm{Z}}
$$

$\mathrm{G}=$ shear modulus, $\mathrm{psi}$

$v=$ Poisson's ratio

$\Delta \mathrm{T}=$ change in temperature, ${ }^{\circ} \mathrm{F}$

$\alpha=$ coefficient of thermal expansion, in $/$ in $/{ }^{\circ} \mathrm{F}$

Equation (2) is modified to allow iteration on strains based on the dependence of the thermoplastic material properties on strain energy density and temperature developed in the first part above:

$$
\left(M_{x}\right)_{i}=f\left[\left(U_{x}\right)_{i}\right]=f\left(\frac{\sigma_{x} \cdot\left(\varepsilon_{x}\right)_{i}}{2}\right) \text { or } f\left(\frac{\tau_{x} \cdot\left(\gamma_{x}\right)_{i}}{2}\right)
$$

where

$\mathrm{MP}=\mathrm{E}$ or $\mathrm{G}$

$\mathrm{x}=$ direction: $\mathrm{x}, \mathrm{y}, \mathrm{z}, \mathrm{xy}, \mathrm{yz}$, or $\mathrm{zx}$

$\mathrm{i}=$ index on iteration

The material properties in Equation (2) are replaced by terms of the form of Equation (3) to allow for iteration. The iteration is done on an element-by-element basis. Since the thermoelastic moduli are the initial tangent moduli of the thermoplastic stress-strain curves, the thermoelastic stresses can be used as initial values in the thermoplastic analysis. The finite element mesh must be fine enough 
so that each finite element represents a unit cell. A unit cell is traditionally defined as the smallest portion of a composite weave which still retains all the essential characteristics of the composite. In finite element analysis, the largest elements (i.e., coarsest mesh size) which can accurately represent the strain distribution in the structure are desirable to minimize model size and cost. Consequently, the definition of a unit cell is modified here to be the largest volume represented by a finite element where the stress gradients are small. The stress-strain constitutive equation can then be applied to the stresses of each finite element individually. Iteration on the stress-strain constitutive equation continues until convergence (successive values of strain energy density within $0.5 \%(0.005)$ for each strain component).

The third part is a variation on the second. The majority of the elements converge readily, but for those elements which do not, and which oscillate about an apparent value, Gauss-Seidel iteration with successive under-relaxation is used to accelerate convergence. This simple strategy anticipates that the next iteration will overshoot the desired value, so a multiplier less than 1 is imposed on the increment. In equation form,

$$
u_{i, j}^{k+1^{\prime}}=u_{i, j}^{k^{\prime}}+\omega \cdot\left(u_{i, j}^{k+1}-u_{i, j}^{k^{\prime}}\right)
$$

where

$\mathrm{u}=$ value of the iterant

$\mathrm{k}=$ iteration level

$\omega=$ relaxation parameter

$\mathrm{i}, \mathrm{j}=$ indices on iterant variants

'= replaced value

Here, the iterants are the strain vectors described by Equation (2) as modified by Equation (3). The three parts of the thermoplastic analysis method have been coded into computer programs: ANSPNL3, ANSPNL4, and ANSPNL5, respectively. Different loadings of the same structure, and different structures of the same material, require the execution of just the last two parts.

The thermoplastic analysis program ANSPNL can be used for axisymmetric structures with material whose principal axes are aligned with the Cartesian coordinate system, such as the LWRHU or the GIS. This was verified in Reference 170. The finite element formulation of an axisymmetric structure maintains the proper boundary conditions and strain conditions so that the postprocessing thermoplastic analysis program ANSPNL does not have to make modifications to consider these aspects.

\section{E. Mechanical Properties}

Test data for mechanical properties of FWPF over the temperatures and loadings of interest for reentry analysis are limited. Two sources of data are the Southern Research Institute 
(SoRI) and the Los Alamos National Laboratory (LANL) [References 173, 174]. (The references contain technical data whose export is restricted by the Arms Export Control Act, and therefore the data are not included in this report.) The formats of the data from the two sources differ. The LANL data essentially reports ultimate strength only. The SoRI data come in the form of stressstrain plots. These are much more useful in terms of supplying nonlinear data for nonlinear analysis, or in this case, for thermoplastic analysis. SoRI also supplied ultimate strength data. It should be noted that the LANL ultimate strength data were approximately $20 \%$ higher than the SoRI data. These differences may be attributable to different test methods or to natural variation of test samples. We shall focus here on the SoRI data.

Fine Weave Pierced Fabric consists of xy weave fabric pierced in the $\mathrm{z}$-direction by pultruded rods, forming an orthogonal weave composite, which is then infiltrated and impregnated with organic precursor, pyrolyzed under pressure, and graphitized. The material properties in the $\mathrm{x}$ and $y$ directions are identical, while the material properties in the $z$ direction are different. In addition, the tension properties in any direction are different from the compression properties in that same direction. Compression and shear data of FWPF at various elevated temperatures up to $5500^{\circ}$ $\mathrm{F}$ from SoRI are used in the analysis here (Figure 121).

A strain allowable of $1 \%(0.01 \mathrm{in} / \mathrm{in})$ is used in the thermostructural analysis to assess factors of safety (FS), where FS = material allowable strain or strength $\div$ calculated strain or stress. A factor of safety $=1.0$ indicates that calculated strain is equal to the allowable strain. Stress at $1 \%$ strain is arbitrarily chosen by SoRI in reporting the ultimate compressive strength, but SoRI reported that FWPF will continue to support load beyond the $1 \%$ value, probably beyond $5 \%$ strain [Reference 175]. Note that the 1\% strain should be considered a lower bound compressive strength, indicating not catastrophic failure, but a point of increased plastic deformation. Thus the factors of safety on strains reported here based on $1 \%$ strain allowables should be viewed as very conservative. Since the thermostructural analysis here considers plastic deformation, a 5\% strain allowable could probably be used. For reference, the factors of safety are also calculated using a $5 \%$ strain allowable for each of the three reentry orientations.

Composite materials can have properties and behaviors which are different from those of isotropic materials. Two classic examples are: the Poisson's ratio $v$ for a composite can be greater than 0.5 , and a composite can have a negative coefficient of thermal expansion $\alpha$. For the FWPF composite material under the mechanical and thermal loadings of interest here, compressive (i.e., negative) stresses can result in tensile (i.e., positive) strains. Thus a body such as the LWRHU aeroshell fabricated of FWPF under the pressure and acceleration forces and the temperature loads of reentry, can result in compressive stresses at a point on the body for some or all material directions, but concurrently result in tensile strains for some or all material directions at the same point on the body. This phenomenon can be explained by examining the stress-strain constitutive equation for FWPF, Equation (2), where each strain term is coupled with all stress terms and with the $\Delta \mathrm{T}$ term. 
To demonstrate, a study was conducted on a unit cell model using thermoelastic material properties for FWPF, arbitrary compressive stresses of $\sigma_{x}=\sigma_{y}=\sigma_{z}=-10,000$ psi, and temperatures ranging from 70 to $7000^{\circ} \mathrm{F}$. The resulting strains are plotted vs. temperature in Figure 123. In the plots of Figure 123, note that some strains are tensile (positive) at certain temperatures, and around $5000^{\circ} \mathrm{F}$, all the strains are tensile, even though the stresses are all compressive (negative). Other combinations of compressive stresses also show some or all tensile strains. The study is documented in Reference 176. This material behavior was also observed for the thermoplastic analysis of the LWRHU, reported in following sections.

\section{F. Results of Analysis}

\section{1. $\quad$ Side-on Stable}

\section{(a) Side-on Stable Mechanical and Temperature Loads}

The pressures, decelerations, and temperatures for various reentry angles and times were obtained from the RETAP code. These had to be translated to MSC/NASTRAN format and to the LWRHU finite element model. The temperatures were mapped from the thermal model (Figure 119) to the finer mesh of the finite element model (Figure 34) with the INT3D code. The derivation of the mechanical loads for the FEM of the LWRHU side-on stable configuration follows. A free-body diagram of the aeroshell is shown in Figure 124. The mechanical loads on the aeroshell in the $\mathrm{x}$-direction are given by

$$
P_{x}=p_{x}+a_{x}+c_{x}
$$

where $\mathrm{P}=$ integrated pressure force, external

$\mathrm{p}=$ integrated pressure force, internal

$\mathrm{a}=$ acceleration force on aeroshell

$c=$ contact force of internal components on aeroshell

The RETAP code does not necessarily balance the integrated pressure force with the force caused by the deceleration, so the decelerations were calculated separately for input to the FEM. First, the external pressures only from RETAP were applied on the model. Then the pressures were integrated over the areas upon which they act to get a net force in the x-direction. Then the deceleration force was set equal to this force from the pressures, and a deceleration was calculated from $\mathrm{F}=\mathrm{ma}$, where $\mathrm{m}=$ mass of the LWRHU aeroshell and its internal components, and $\mathrm{a}=$ acceleration or deceleration. Then in the model, acceleration was applied on the aeroshell alone. The contact forces of the internal components on the aeroshell were obtained by multiplying the mass of the internal components by the acceleration, then distributing these forces as pressures over the contact area as a cosine distribution. Finally the internal and external pressures were applied to the model. 
(b) Side-on Stable Runs

The reentry angle and times for which thermostructural analyses were performed are $\gamma=-90^{\circ}$ at $0.10,0.40,0.60,0.80,1.00,1.20,1.40,1.60,1.80,2.00,2.15,2.35$, 2.50 , and 2.75 seconds. For the side-on stable orientation, only the reentry angle of $\gamma=-90^{\circ}$ was analyzed for thermostructural effects, since the thermal analysis showed ablation failure at all reentry angles (see Table 9), and further thermostructural analysis was not deemed necessary. The results of thermostructural analyses are discussed in Section VIII. F.1.c.

The mechanical and temperature loads described in Section VIII. F.1.a were applied to the LWRHU finite element model with thermoelastic material properties. The elemental stresses in the material coordinate system were then read into the thermoplastic analysis program ANSPNL4. Those elements which did not converge readily were run through the iteration program ANSPNL5 with the same algorithm, but with a successive under relaxation iteration scheme. The thermoplastic strains were iterated until convergence $(\mathrm{dU}<0.005)$. Most elements converged within $0.5 \%$, but some converged to higher values of $1 \%$. For those in the latter situation, the factors of safety on strain were high, so no further effort was expended to get finer convergence.

\section{(c) Side-on Stable Results}

Table 27 shows the time of initial indicated failure using a strain allowable of $0.01 \mathrm{in} / \mathrm{in}$, and the time with the lowest factor of safety during each trajectory. Also shown for reference is the time of initial indicated failure using a strain allowable of $0.05 \mathrm{in} / \mathrm{in}$, if failure is predicted. For each of these times, the element number, the factor of safety, and the material direction are provided. Figure 125 shows the contour plots for factors of safety on strains, where a strain allowable of $0.01 \mathrm{in} / \mathrm{in}$ is used, for the timestep at which the lowest factor of safety is indicated for the steep reentry angle. The location of the element with the lowest factor of safety is shown in Figure 125. Almost all of the normal strains are tensile. From the criterion for breach described in Section VIII. C (Assumptions), breach is not indicated from a thermostructural standpoint. Note that the contour plots of factors of safety with a strain allowable of 0.05 in/in can be obtained by multiplying the FS levels by 5 .

The significance of considering thermoplastic material behavior is demonstrated by comparison to thermoelastic analysis results for the $\gamma=-90^{\circ}$ side-on stable configuration. Thermoelastic analysis results are based on comparisons of predicted stresses to stress allowables. Thermoelastic analysis predicts failure starting at $0.40 \mathrm{sec}$, whereas thermoplastic analysis predicts failure starting at $1.00 \mathrm{sec}$.

\section{Side-on Spinning}

(a) Side-on Spinning Mechanical and Temperature Loads 
The pressures, decelerations, and temperatures for various reentry angles and times were obtained from the Reentry Thermal Analysis Program (RETAP) code. The methodology for converting temperatures from RETAP to the LWRHU FEM for the side-on spinning configurations is similar to that for the side-on stable configuration, but the methodology for obtaining decelerations is different. For side-on spinning, the thermal analysis model spreads the pressure loads over the circumference of the LWRHU model to simulate spinning. Following the same strategy as for the pressures loads, the derivation of the decelerations also assumes an equal distribution over the circumference. To determine the decelerations for the $180^{\circ}$ NASTRAN model, the RETAP pressures were integrated in the direction normal to the symmetry plane to obtain forces, and decelerations were calculated from the forces. Since the decelerations were assumed equal in all radial directions, these decelerations on the aeroshell were then radially distributed evenly over $180^{\circ}$, as were the contact forces calculated from decelerations of the internal components. Finally the internal and external pressures were applied to the model.

\section{(b) Side-on Spinning Runs}

A listing of the reentry angles and times for which thermostructural analyses for side-on spinning were performed is shown in Table 28. For the side-on spinning orientation, reentry angles of $\gamma=-7^{\circ},-10^{\circ},-15^{\circ},-30^{\circ},-50^{\circ},-70^{\circ}$, and $-90^{\circ}$ were analyzed for thermostructural effects, since the thermal analysis did not show ablation failure at these reentry angles (see Table 10). The results of thermostructural analyses are discussed in Section VIII. F.2.c.

The mechanical and temperature loads described in Section VIII. F.2.a were applied to the LWRHU finite element model with thermoelastic material properties. The elemental stresses in the material coordinate system were then read into the thermoplastic analysis program ANSPNL4. Those elements which did not converge readily were run through the iteration program ANSPNL5 with the same algorithm, but with a successive under relaxation iteration scheme. The thermoplastic strains were iterated until convergence $(\mathrm{dU}<0.005)$. Most elements converged within $0.5 \%$, but some converged to higher values of $7 \%$. For those in the latter situation, the factors of safety on strain were high, so no further effort was expended to get finer convergence.

\section{(c) Side-on Spinning Results}

Table 29 shows the time of initial indicated failure using a strain allowable of $0.01 \mathrm{in} / \mathrm{in}$, and the time with the lowest factor of safety during each trajectory. Also shown for reference is the time of initial indicated failure using a strain allowable of $0.05 \mathrm{in} / \mathrm{in}$, if failure is predicted. For each of these times, the element number, the factor of safety, and the material direction are provided. Figures 126 through 128 show the contour plots for factors of safety on strains, where a strain allowable of $0.01 \mathrm{in} / \mathrm{in}$ is used, for the timestep at which the lowest factor of safety is indicated for the shallow, intermediate, and steep reentry angles, respectively. 
The locations of the elements with the lowest factors of safety are shown in Figures 126 through 128. Almost all of the normal strains are tensile. From the criterion for breach described in Section VIII. C, breach is not indicated from a thermostructural standpoint. Note that the contour plots of factors of safety with a strain allowable of 0.05 in/in can be obtained by multiplying the FS levels by 5 .

\section{3. $\quad$ Random Tumbling}

(a) Random Tumbling Mechanical and Temperature Loads

The random tumbling configuration is similar to the side-on spinning, but adds an additional pressure load in the longitudinal direction. Determination of the decelerations in the longitudinal direction is straightforward, similar to the methodology described in Section VIII. F.1.a.

\section{(b) Random Tumbling Runs}

A listing of the reentry angles and times for which thermostructural analyses for random tumbling were performed is shown in Table 30 . For the random tumbling orientation, reentry angles of $\gamma=-15^{\circ},-30^{\circ},-50^{\circ},-70^{\circ}$, and $-90^{\circ}$ were analyzed for thermostructural effects, since the thermal analysis did not show ablation failure at these reentry angles (see Table 12). The results of thermostructural analyses are discussed in Section VIII. F.3.c.

The mechanical and temperature loads described in Section F.3.a were applied to the LWRHU finite element model with thermoelastic material properties. The elemental stresses in the material coordinate system were then read into the thermoplastic analysis program ANSPNL4. Those elements which did not converge readily were run through the iteration program ANSPNLS with the same algorithm, but with a successive under relaxation iteration scheme. The thermoplastic strains were iterated until convergence $(\mathrm{dU}<0.005)$. Most elements converged within $0.5 \%$, but some converged to higher values of $6 \%$. For those in the latter situation, the factors of safety on strain were high, so no further effort was expended to get finer convergence.

\section{(c) Random Tumbling Results}

Table 31 shows the time of initial indicated failure using a strain allowable of $0.01 \mathrm{in} / \mathrm{in}$, and the time with the lowest factor of safety during each trajectory. Also shown for reference is the time of initial indicated failure using a strain allowable of $0.05 \mathrm{in} / \mathrm{in}$, if failure is predicted. For each of these times, the element number, the factor of safety, and the material direction are provided. Figures 129 through 131 show the contour plots for factors of safety on strains, where a strain allowable of $0.01 \mathrm{in} / \mathrm{in}$ is used, for the timestep at which the lowest factor of safety is indicated for the shallow, intermediate, and steep reentry angles, respectively. 
The locations of the elements with the lowest factors of safety are shown in Figures 129 through 131. Almost all of the normal strains are tensile. From the criterion for breach described in Section VIII. C, breach is not indicated from a thermostructural standpoint. Note that the contour plots of factors of safety with a strain allowable of $0.05 \mathrm{in} / \mathrm{in}$ can be obtained by multiplying the FS levels by 5 . 


\section{OTHER REENTRY CONDITIONS}

Reentry analysis for other reentry scenarious including parking orbits, minimum gamma entries, and explosions were addressed in Appendix G of Reference 1. Except for the explosion scenario, the analyses conducted for this Safety Analysis Report (SAR) include those conditions both in scope and in modelling the problem. The results, documented in Reference 1 showed that no ablation failure of the LWRHU nor fuel melt is expected of the orbital decay or minimum gamma entries based on the assumptions used therein (multiple skips are not included). The previous SAR (Reference 1) examined an orbital and ascent explosion. This previous study showed that for an assumed bare clad entry, clad melt, but not fuel melt, would occur. 


\section{OTHER LAUNCH ACCIDENTS}

Another Cassini accident scenario analyzed by JHU/APL is a ground launch accident in which a LWRHU module lays on Titan SRMU burning propellant. The predicted thermal response of the LWRHU to this accident is documented by P. T. Brenza in References 177 and 178. Because it is not a reentry accident scenario it is not included in this report but is mentioned here for completeness. 
Applied Physics Laboratory

Laurel, Maryland 20723-60199
Enclosure (2) to:

A1C-97-095

Page 5

\section{REFERENCES}

\section{SECTION II}

1. Johnson, E. W., Light Weight Radioisotope Heater Unit Safety Analysis Report (LWRHUFSAR), Vol. I, Reference Design Document, Monsanto Research Corporation Report MLM-3293, Miamisburg, OH, October 1985.

2. Lutz, S. A., "Lightweight Radioisotope Heater Unit Module Ablation Response Testing at the NASA Ames 20 MW Aerodynamic Heating Facility: Final Test Report," BFD-2-90-011, The Johns Hopkins University Applied Physics Laboratory, 15 May 1990.

\section{SECTION III}

3. McRonald, A., "RTG Module Release Conditions for Inadvertent Earth Reentry of the Cassini Spacecraft During the VVEJGA Earth Flyby on 8-17-99, Revisited," JPL Interoffice Memorandum 312/94.6-759, 27 May 1994.

4. FAX from Angus McRonald, JPL, to Eddie Lucero, JHU/APL, dated 30 November 1994, Re: LWRHU Release Conditions.

5. Brenza, P. T., "Release Conditions for GPHS and LWRHU Reentry Thermal Analysis for the Cassini-VVEJGA FSAR," JHU/APL AM-94-D0035, BFD-2-94-027, October 11, 1994.

\section{SECTION IV}

6. Johnson, E. W., Light Weight Radioisotope Heater Unit Safety Analysis Report (LWRHUSAR), Vol. II, Accident Model Document, Appendix G., Monsanto Research Corporation Report MLM-3293, Miamisburg, OH, October 1995.

7. Lucero, E. F., "Estimated Continuum and Free Molecualr Aerodynamics and Bridging Parameters for the LWRHU," JHU/APL BFD-2-88-004, 2 March 1988.

8. Lucero, E. F., "Estimated Drag for GPHS at Angles of Attack, $\gamma_{R}$, of $30^{\circ}$ and $90^{\circ}$, for GIS at $\gamma_{\mathrm{R}}=27^{\circ}$ and for a Tumbling LWRHU Module for Mach Numbers Subsonic to Hypersonic," JHU/APL AM-91-D0005 (BFD-2-91-004), 28 January 1991.

9. Lucero, E. F., “ Estimated Subsonic Aerodynamics and Terminal Velocity for the RTG, GPHS Module, GIS, Fuel Clad, and LWRHU," BFD-2-87-023, 10 December 1987. 


\section{SECTION VII. B. 2.}

10. Communications of 24 June 1996, 10 July 1996, and 12 July 1996 from Dr. E. W. Johnson, Lamb Assoc., on Using Fuel Integral Fuel Pellet for Reentry Response Analysis.

\section{SECTION VII. C.}

11. Vargaftik, N. B., "Heat Conductivity of Gases and Liquids," FTD-MT-24-135-71, Foreign Technology Division, Wright-Patterson Air Force Base, Ohio, November 1971.

12. Collins, D. I. and Grief, R., International Journal of Heat and Mass Transfer, Volume 1, page 56, 1966.

13. Thermodynamics and Transport Properties of Gases, National Bureau of Standards Circular $564,1955$.

14. Yos, J. M., "Transport Properties of Nitrogen, Hydrogen, Oxygen and Air to $33000^{\circ} \mathrm{K}$," RAD-TM-63-7, Avco Corporation, Wilmington, MA, March 1963.

15. Gardiner, G. K., "A Critical Survey of Six Thermodynamic and Transport Properties of Air,” NWC TP 4997, Naval Weapons Center, February 1971.

16. Bade, W. L., et al., American Rocket Society Journal, Volume 31, page 1151, 1961.

17. Eck, M. B., "Final Report on Earth Swingby Reentry," FOILS ENGINEERING, under NASA-JPL contract 959658, 22 November 1993.

18. "Final Report for the General Purpose Heat Source - Radioisotope Thermoelectric Generator Program," GESP-7209, General Electric Company - Astro Space Division, 1 February 1991.

19. Williams, R. K., "Review and Interpretation of Contact Conductance Measurements on FWPF Interfaces," Intra-Laboratory Correspondence, Oak Ridge National Laboratory, 5 May 1980.

20. Letter to R. Hartman, General Electric - Astro Space Division from R. L. Heestrand, Oak Ridge National Laboratory, Subject: "Contact Conductance Data," 4 February 1980.

21. Cross, R. W. and Fletcher, L. S., "Thermal Contact Conductance of Uranium Dioxide Zircalloy Interfaces," AIAA Paper 78-85, AIAA 16th Aerospace Sciences Meeting, January 1978. 
22. Minges, M. L., "Thermal Contact Resistance - Volume 1: A Review of the Literature," AFML-TR-65-375, Air Force Systems Command, Wright-Patterson Air Force Base, Ohio, April 1966.

23. Letter to R. Hartman, General Electric - Astro Space Division, from C. Alexander, Battelle Columbus Laboratories, discusses the contact conductance measured at the chamfered FWPF $^{\text {th }} /$ pyrolytic graphite interface of the General Purpose Heat Source aeroshell, 31 March 1981.

24. Putz, R. E. and Bartlett, E. P., "Heat Transfer and Ablation Rate Correlations for Reentry Heat Shield and Nosetip Applications," AIAA Paper No. 72-91, AIAA $10^{\text {th }}$ Aerospace Sciences Meeting, San Diego, CA, January 1972.

25. Perini, L. L., "Review of Graphite Ablation Theory and Experimental Data," ANSP-M-1, JHU/APL, Laurel, MD, December 1976.

26. Lundell, J. H. and Dickey, R. R., "Graphite Ablation at High Temperatures," AIAA Paper No. 71-418, AIAA 6th Thermophysics Conference, Tullahoma, TN, April 1971.

27. Leider, H. R., et. al., "Thermodynamic Properties of Carbon Up to the Critical Point," Carbon, Vol. 11, pp 555-563, 1973.

28. Lee, E. L. and Sanborn, R. H., "Extended and Improved Thermal Functions for the Gaseous Carbon Species $\mathrm{C}_{1}-\mathrm{C}_{7}$ from 298 to $10000^{\circ} \mathrm{K}$," High Temperature, Sciences, Vol. 5, pp $438-453,1973$.

29. JANNAF Thermochemical Tables, 3rd Edition, National Bureau of Standards, 1986.

30. "User's Manual - Aerotherm Chemical Equilibrium (ACE) Surface Thermochemistry Program, Version 2," Aerotherm Corporation, June 1966.

31. "User's Manual - Aerotherm Charring Material Thermal Response and Ablation Program, Version 3, Volume 1: Program Description and Sample Problems," AFRPL-TR-70-92, Air Force Rocket Propulsion Laboratory, April 1970.

32. "An Analysis of the Coupled Chemical Reacting Boundary Layer and Charring Ablator Parts I through VI," Reports NASA CR-1060 to NASA CR-1065, Aerotherm Corporation, June 1968.

33. Chan, C. C., "Upgrade of Graphite Surface Thermochemistry Tables," AM-93-E018, JHU/APL, Laurel, MD, 5 February 1993. 
34. Hunter, L. W., "The Ablation Rate of Burning Carbon and In-Depth Heat Flux," ANSP-260 (also BBP-84-153), JHU/APL, Laurel, MD, 27 July 271984.

35. Jones, M. T., "The Phase Diagram of Carbon," National Carbon Research Laboratories, Report No. PRC-3, January 1958.

36. Palmer, H. B. and Shelef, M., Chemistry and Physics of Carbon, Volume 1, Marcel-Dekker, New York, 1965.

37. Bundy, F. P., "Melting of Graphite at Very High Pressure," Journal of Chemical Physics, Vol. 36, No.2, p 618, February 1963.

38. Schoessow, G. J., "Graphite Triple Point and Solidus-Liquidus Interface Experimentally Determined Up to 1000 Atmospheres," Physics Review Letter, Vol. 21, p.738, 1968.

39. Cezairliyan, A. and Miller, A. P., "Measurement of the Radiance Temperature (at $655 \mathrm{~nm}$ ) of Melting Graphite Near Its Triple Point by a Pulse-Heating Technique," International Journal of Thermophysics, Vol. 11, No. 4, p. 643, 1990.

40. Conn, D. W., "Perspectives of the Fuel Reentry Problems - ANSP Planning," ANSP-282, JHU/APL, Laurel, MD, 14 March 1986.

41. March, E. B., "Thermal and Ablation Response of Small Spherical Particles for Reentry Summary Report," SC-RR-68-547, Sandia Laboratories, Albuquerque, New Mexico, October 1968.

\section{SECTION VII. D}

42. Aerospace Nuclear Safety Program, "Reentry Response of the Light Weight Radioisotope Heater Unit Resulting From a Venus-Earth-Earth Gravity Assist Maneuver Accident," JHU/APL-M-19, Johns Hopkins University/Applied Physics Laboratory, October 1988.

43. Gardiner, G., A Critical Survey of Six Thermodynamic and Transport Properties of Air, NWC TP 4997, Naval Weapons Center, China Lake, CA, February 1971.

44. Waeber, K. R., "Comparison of DoE Sponsored FWPF" Data with that from the Air Force $\mathrm{P}^{4}$ Program," ANSP-167, Johns Hopkins University/Applied Physics Laboratory, 18 September 1979.

45. Waeber, K. R., "Summary of Mechanical and Thermal Property Information for AVCO's Fineweave Pierced Fabric," ANSP-187, Johns Hopkins University/Applied Physics Laboratory, 16 July 1980. 
46. Letter to F. Schumann (Teledyne Isotopes) from G. F. Fornaro (Southern Research Institute), Subject: "Thermal Expansion Test Data on GE 3DCC and FWPF"," 14 June 1979.

47. O'Connor, T. and Mazzola, J. H., Textron Specialty Materials Data Sheet, "Representative Properties, 3-D Fineweave Pierced Fabric ${ }^{\mathrm{m}}$ Block".

48. Letter, "Preliminary Data Package for $\mathrm{P}^{4}$ FWPF Material Properties" with Enclosure: "PAN Carbon-Carbon Fine Weave Pierced Fabric Preliminary Data Package," Southern Research Institute, 24 August 1978.

49. J. C. Uzzell, "Handbook of Thermal Properties for Nuclear Power System Reentry Analysis," ANSP-169, The Johns Hopkins University Applied Physics Laboratory, October 1979.

50. Technical Information Exchange Meeting between Applied Physics Laboratory and General Electric Company, Purpose: "Comparison of the GPHS Thermal Properties" with principals P. T. Brenza (APL) and J. Loffreda (GE Astro-Space).

51. Letter to W.D. Owings (U.S. Department of Energy) from R.J. Hemler (Martin Marietta Astro Space), Subject: "Material Properties for the GPHS," 22 July 1994.

52. D. R. Stull and H. Prophet (Project Directors), JANAF Thermochemical Tables (Second Edition), National Bureau of Standards, June 1971.

53. Y. Shah (Manager, Quality Assurance), "Certificate of Conformance" (for Insulator Caps), Certificate Number 94202, BF Goodrich, Aerospace Super-Temp, 25 November 1994.

54. Fax to T. C. Craft (EG\&G Mound Applied Technologies) from Y. Shah, (BF Goodrich, Aerospace Carbon Products), containing: "Certificate of Test" (for Insulator Tubes), 26 January 1995.

55. B. H. Morrison, et al., "Thermal Diffusivity of Pyrolytic Graphite From 23 to $1900^{\circ}$ C," LADC-8307, Los Alamos Scientific Laboratory, 1966.

56. Memo to 'GPHS Task Force' from R. N. R. Mulford (Los Alamos Scientific Laboratory), Subject: "Pyrolytic Graphite Thermal Conductivity and Emittance," 27 July 1979.

57. Memo to 'GPHS Task Force' from R. N. R. Mulford (Los Alamos Scientific Laboratory), Subject: "Thermal Conductivity of Pyrolytic Graphite," 24 September 1979. 
58. W. H. Smith and D. H. Leeds, "Pyrolytic Graphite," Modern Materials, Volume 7, Academic Press, Inc., New York and London, 1970.

59. E. Howell, "Acceptance Specification, Pyrolytic Graphite, Plate and Cylinder," SPA790171-Issue D (U), EG\&G Mound Applied Technologies, 17 March 1994.

60. Y. S. Touloukian and D. P. DeWitt (Ed), Curve 30/Table 7 in Thermophysical Properties of Matter, Volume 8 - Thermal Radiative Properties, Nonmetallic Solids, Thermophysical Properties Research Center, Plenum Press, New York, 1972.

61. J. Roque', High Temperature - High Pressure, Volume 9, pp.207-210, 1977.

62. Y. S. Touloukian (Editor), Thermophysical Properties of High Temperature Solid Materials, Volume I: Elements, pages 782, 783, Thermophysical Properties Research Center, Purdue University, The MacMillan Company, New York, 1967.

63. Letter to J. C. Hagan (JHU/APL) from A. C. Schaffhauser, (Oak Ridge National Laboratory), Subject: "Transmittal of Pt-30\% Rh Thermal Data," February 1979.

64. Letter to J. C. Hagan (JHU/APL) from J. R. Keiser (Oak Ridge National Laboratory), Subject: "Transmittal of Properties of Pt-3008," ORNL Letter No. 0815-26-78, 15 August 1978.

65. "SNAP 19/Pioneer F Safety Analysis Report; Volume II: Accident Model Document," INSD-2873-42-2, Teledyne Isotopes, December 1970.

66. Y. S. Touloukian and D. P. DeWitt (Ed), Thermophysical Properties of Matter, Volume 2, Part I: Nonferrous Binary Alloys, pages 403, 404, Thermophysical Properties Research Center, Plenum Press, New York, 1972.

67. Letter to S. E. Bronisz (Los Alamos Scientific Laboratory) from A. C. Schaffhauser (Oak Ridge National Laboratory), Subject: "Data on Pt-3008 for RHU Application," 4 December 1978.

68. ASM Handbook, Volume 2: Properties and Selection: Nonferrous Alloys and SpecialPurpose Materials, Formerly 10th Edition, Metals Handbook.

69. Firstenberg, H., "Proposed High Temperature Thermal Conductivity of $\mathrm{PuO}_{2}$ (DRAFT)," Halliburton NUS Corporation, October 1993. 
70. Letter to Messrs. S. E. Bronisz (LASL), M. T. Goldstone (SR) and E. W. Johnson MRC) from R. Hartman (General Electric), Enclosure: "Fuel Thermal Properties Used in Analysis," 29 January 1980.

71. T. K. Keenan, et al., "Data Sheets for PPO Radioisotope Fuel," LA-5160-MS, Revision 1, Los Alamos Scientific Laboratory, December 1975.

72. Conn, D. W., "PuO ${ }_{2}$ Microsphere Reentry Studies: Thermal Properties," ANSP-275, The Johns Hopkins University Applied Physics Laboratory, 16 August 1985.

73. "Final Safety Analysis Report for the Ulysses Mission - Volume 1: Reference Design Document," UL-FSAR-002, General Electric Astro-Space, March 1990.

74. Johnson, E. W., "Light-Weight Radioisotope Heater Unit Safety Analysis Report (LWRHU-SAR), Volume 1: A. Introduction and Executive Summary, B. Reference Design Document (RDD)," Mound Report MCM-3293, October 1985.

75. Johnson, E. W., "Light-Weight Radioisotope Heater Unit (LWRHU) Draft of Final Safety Analysis Report (FSAR) for the Cassini Mission," Lamb Associates, Inc., 30 June 1996.

76. Touloukian, Y. S. and Ho, C. H., Thermophysical Properties of Selected Aerospace Materials, Part I: Thermal Radiation Properties, Thermophysical and Electronic Properties Information Center, University of Purdue, 1976.

77. Letter to A. Schock (Fairchild Industries) from G. Wei (Oak Ridge National Laboratory), Subject: "Total Hemispherical Emittance of FWPF" C-C," 8 January 1980.

78. Plunkett, J. D., et al., "The Spectral and Integrated Emissivity of Carbon and Graphite" In Proceedings of the Fourth Conference on Carbon, pp 457-481, Pergamon, Oxford, 1960.

79. Dmitriev, A. A. and Lutkov, A. I., "Integral Hemispherical Degree of Blackness of Graphite," High Temperature, (volume unknown, refer to D. Conn, APL).

80. Glawe, G. E. and Shepard, C. E., "Some Effects of Exposure to Exhaust-Gas Streams on Emittance and Thermoelectric Power of Bare-Wire Platinum Rhodium-Platinum Thermocouples," NACA TN 3253, August 1954.

81. Davisson, C. and Weeks, J. R. Jr., "The Relation Between the Total Thermal Emissive Power of a Metal and its Electrical Resistivity," Journal Optical Society America and Review of Scientific Instruments, Volume 8, Number 5, May 1924. 
82. Hampel, C. A. (Ed.), Rare Metals Handbook, 2nd Edition, Robert Krieger Publishing Company, Inc., Huntington, N.Y., 1971.

83. Aitken, E. A. and Evans, S. K., "Liquid/Solid Transformations in the U-Pu-O System," General Electric Company Vallecitos Nuclear Center, Pleasanton, California, January 1968.

84. Grave, G. R., et al., "Plutonium-238 Isotopic Power Sources: A summary Report," MLM1270 AEC R\&D Report, Mound Laboratory, Miamisburg, Ohio, 19 August 1965.

85. Laube, M., Plutonium, Pergamon Press, Oxford, England, (The MacMillan Company, New York, N.Y.), 1964 (pp 8-29, 47-62 and174-187).

86. Loffreda, J., "Plutonium Dioxide Particle Re-Entry," Program Information Request/Release No. 6624, 13 January 1986.

87. Lundberg, R. E., et al., "A Study of the Behavior of Small Spheres During Reentry," Sandia Report SC-CR-67-635, Aerotherm Corporation, June 1967.

88. 'Pears, C. D., "Some Problems in Emittance Measurements at the Higher Temperatures and Surface Characterization", Southern Research Institute,' received from A. Schaffhauser, Oak Ridge National Laboratory, circa 1978.

\section{SECTION VII E}

89. Bhutta, B. A., et al., "New Technique for the Computation of Severe Reentry Environments," AIAA Paper 96-1861, 31st AIAA Themophysics Conference, June 1996.

90. Perini, L. L., "User's Manual for the 3DOF Trajectory Computer Program," ANSP-M-6, The Johns Hopkins University Applied Physics Laboratory (JHU/APL), Laurel, MD, September 1973.

91. Detra, R. W., et al., "Addendum to "Heat Transfer to Satellite Vehicles Re-entering the Atmosphere," Jet Propulsion, Vol. 27, No. 12, December 1957.

92. Fay, J. A. and Riddell, F. R., "Stagnation Point Heat Transfer in Dissociated Air," Journal of the Aeronautical Sciences, Vol. 25, No. 2, February 1958.

93. Rose, R. H. and Starks, W. L., "Stagnation Point Heat Transfer Measurements in Dissociated Air," Journal of the Aeronautical Sciences, Vol. 25, No. 2, February 1958.

94. Perini, L. L., "Compilation and Correlation of Experimental, Hypersonic, Stagnation Point Convective Heating Rates," ANSP-M-4, JHU/APL, Laurel, MD, July 1972. 
95. Probstein, R. F., "Heat Transfer in Rarefied Gas Flow," in Theory and Fundamental Research in Heat Transfer, Proceedings of ASME Annual Meeting, November 1960", Pergamon Press, New York City, NY, 1963.

96. Sutton, K. and Hartung, L. C., "Equilibrium Radiative Heating Tables for Earth Entry," NASA Technical Memorandum 102652, Langley Research Center, May 1990.

97. Sutton, K., "Characteristics of Coupled Nongray Radiating Gas Flows with Ablation Products Effects About Blunt Bodies During Planetary Entries," PhD Thesis, North Carolina State University, Raleigh, North Carolina, 1973.

98. Nicolet, W. E., "Advanced Methods for Calculating Radiation Transport in AblationProduct Contaminated Boundary Layers," NASA CR-1656, Aerotherm Corporation, September 1970.

99. Nicolet, W. E., "User's Manual for the Generalized Radiation Transfer Code (RAD/EQUIL)," Report to UM-69-9, Aerotherm Corporation, Mountain View, CA, October 1969.

100. Sutton, K., "Air Radiation Revisited," AIAA Paper 84-1733, AIAA 19th Thermophysics Conference, Snowmass, CO, June 1984; also in Progress in Astronautics and Aeronautics: Thermal Design of Aeroassisted Orbital Transfer Vehicles, ed. H. F. Nelson, Vol. 96, AIAA, New York, 1985.

101. Ambrosio, A.and Wortman, A., "Stagnation Point Shock Detachment Distance for Flow Around Spheres and Cylinders," Journal of the Aeronautical Sciences, Vol.29, No. 2, February 1962.

102. Olstad, W., "Program to Compute the Inviscid Radiating Flow About a Circular Cylinder," Informal transmittal from W. Olstad, NASA-Langley Research Center, Hampton, VA, 13 December 1973.

103. Eck, M. B., 'References 9 and 10' contained in the report "Final Report on Earth Swingby Reentry," FOILS Engineering, prepared under NASA-JPL Contract 959658, November 1993.

104. Nicolet, W. E. and Balakrishnan, A., "Radiating Shock Layer Environment (RASLE) User's Manual," Aerotherm Report UM-79-10/AS, Acurex Corporation/Aerotherm Aerospace Systems Division, Mountain View, CA, July 1979. 
105. Perini, L. L., "Compilation of Experimental Stagnation Point Velocity Gradients and Heat Transfer Data in Subsonic and Supersonic Flow," ANSP-068, The Johns Hopkins University Applied Physics Laboratory, Laurel, MD, August 1975.

106. Lasher, L. E. and Wilson, K. H., "Effect of Shock Precursor Heating on Radiative Flux to Blunt Bodies", NASA CR-1265, Lockheed Missile and Space Company, Palo Alto, CA, February 1969.

107. Anderson, J. D., “An Engineering Survey of Radiating Shock Layers", AIAA Journal, Vol. 7, No.9, September 1969.

108. Liu, J. T. C., "Influence of Upstream Absorption on the Stagnation Region Shock Layer Radiation," AIAA Journal, Vol. 8, No. 10, October 1970.

109. Coleman, W. D., et. al., "Effects of Environmental and Ablator Performance Uncertainties on Heat Shielding Requirements for Hyperbolic Entry Vehicles," Journal of Spacecraft and Rockets, Vol. 5, No. 11, November 1968.

110. Rigdon, W. S., et. al., "Stagnation Point Heat Transfer During Hypervelocity Atmospheric Entry," NASA CR-1462, McDonnell Douglas Corporation, February 1970.

111. Gupta, R., et. al., "Viscous-Shock-Layer Solutions with Coupled Radiation and Ablation Injection for Earth Entry," AIAA Paper 90-1697, AIAA/ASME 5th Joint Thermophysics and Heat Transfer Conference, June 1990.

112. Howe, J. and Viegas, J., "Solutions of the Ionized Radiating Shock Layer Including Reabsorption and Foreign Species Effects and Stagnation Region Heat Transfer," NASA TR R-159, NASA Ames Research Center, 1963.

113. Hoshizaki, H. and Wilson, K., "Convective and Radiative Heat Transfer During Superorbital Entry," NASA CR-584, Lockheed Missile and Space Company, Palo Alto, CA, September 1966.

114. Scott, C. D., "Wall Catalytic Recombination and Boundary Conditions In Nonequilibrium Hypersonic Flows - With Applications," in Advances in Hypersonics, Volume II: Modeling Hypersonic Flows, (eds) J. Bertin, J. Periaux, J Ballman, Birkhauser Boston Press, Cambridge, MA, 1992.

115. JHU/APL ANSP-M-19, "Reentry Response of the Light Weight Radioisotope Heater Unit Resulting From a Venus-Earth-Earth Gravity Assist Maneuver Accident," Laurel, MD, October 1988. 
116. Lees, L., "Laminar Heat Transfer Over Blunt-Nosed Bodies at Hypersonic Flight Speeds," Jet Propulsion, Vol. 26, No. 4, April 1956.

117. Kemp, N. H., et. al., "Laminar Heat Transfer Around Blunt Bodies in Dissociated Air," Journal of the Aeronautical Sciences, Vol. 26, No. 7, July 1959.

118. Carroll, H. R., "General Heating Distributions on the Tumbling Infinite Cylinder," AIAA Paper 78-42, AIAA 16th Aerospace Sciences Meeting, Huntsville, Alabama, January 1978.

119. Knight, D., "MHW Aerodynamic Heating Test Final Report," PIR 4665, General Electric Missile and Space Division, Valley Forge, PA, 5 October 1973.

120. Bertin, J. J., et al., "Flow Field Measurements for Space-Shuttle-Related Cylindrical Configuration in Hypersonic Streams," AIAA Paper 72-294, AIAA 7th Thermophysics Conference, San Antonio, TX, April 1972.

121. Schock, A., "Light-Weight Radioisotope Heater Unit," Report FSEC-ESD-217-81/181, Fairchild Space and Electronics Company, Germantown, MD 20767, 3 April 1981.

122. Olstad, W., "Nongray Radiating Flow about Smooth Symmetrical Bodies," AIAA Journal, Vol. 9, No. 1, January 1971.

123. "Monthly Technical Report for the Period 27 June through 31 July 1994," Martin Marietta Astro Space, King of Prussia, PA, Figure 3-4, Document No. RR16, Cassini RTG Program, 24 February 1995.

124. Miller, W. H., "Pressure Distributions on Single and Tandem Cylinders Mounted on Flat Plate for Mach Number 5.0 Flow," Defense Research Laboratory, DRL-538, 1 June 1966.

125. Gregorek, G. M. and Korkan, K. D., "An Experimental Observation of the Mach and Reynolds Number Independence of Cylinders in Hypersonic Flow," AIAA Journal, Vol. 1, No. 1, January 1963.

126. Bertram, M. H. and Everhart, P. E., Figure 6 in "An Experimental Study of the Pressure and Heat-Transfer Distribution on a 70 Degree Sweep Slab Delta Wing in Hypersonic Flow," NASA TR R-153, December 1963.

127. Webb, W. L., et al., "Hypersonic Wake Transition," AIAA Journal, Vol. 1, No. 3, March 1963. 
128. Slattery, R. and Clary, W., "Experimental Measurement of Turbulent Transition, Motion, Statistics and Gross Radial Growth Behind Hypervelocity Objects," Physics of Fluids, Vol. 5, pp. 849-855.

129. Dogra, V. K., et. al., "Hypersonic Rarefied Flow Past Spheres Including Wake Structure," Journal of Spacecraft and Rockets, Vol. 31, No. 5, September-October .1994.

130. Bulmer, B. M., "Heat-Transfer Measurements in a Separated Laminar Base Flow," Journal of Spacecraft and Rockets, Vol. 14, No. 11, November 1977.

131. Sykes, D. M., "The Supersonic and Low Speed Flows Past Circular Cylinders of Finite Length Supported at One End,"Journal of Fluid Mechanics, Vol. 12, Part 3.

132. Gowen, F. E. and Perkins, E. W., "Drag of Circular Cylinders for Wide Range of Reynolds Number and Mach Number," NACA TN 2960, June 1953.

133. Token and Oguro, "Experimental Investigation of Hypersonic Flow Around 2-D Circular Cylinders," ARL 65-212, Aeronautical Research Laboratories, October 1965.

134. Klett, R. D., "Drag Coefficients and Heating Ratios for Right Circular Cylinders in FreeMolecular and Continuum Flow From Mach 10 to 30," SC-RR-64-2141, Sandia Laboratory, Albuquerque, NM, December 1964.

135. Feller, W. V., "Investigation of Equlibrium Temperatures and Average Laminar HeatTransfer Coefficients for the Front Half of Swept Circular Cylinders at a Mach Number of 6.9," NACA RM L55F08C, Langley Aeronautical Laboratory, August 1955.

136. Beckwith, I. E. and Gallagher, J. J., "Local Heat Transfer and Recovery Temperatures on Yawed Cylinders at Mach Number of 4.15 and High Reynolds Numbers," NASA TR R104, Langley Research Center, 1961.

137. Beckwith, I. E., "Similar Solutions for the Compressible Boundary Layer on a Yawed Cylinder with Transpiration Cooling," NASA TR R-42, 1959.

138. Amirkabirian, I. and Bertin, J. J., "Aerothermodynamic Environment About a Highly Swept Wing Leading Edge," Journal of Spacecraft and Rockets, Vol. 24, No. 3, May-June 1967.

139. Sayano, S. and Greenwald, G. F., "Approximate Method for Calculating Heat Transfer to Yawed Cylinders in Laminar Flow," Journal of Spacecraft and Rockets, Vol. 10, No. 2, February 1973. 
140. Bergquaum, J. E., "Aerodynamic Heating Investigation on Cylindrical Configuration in AEDC Tunnel (R-128)," Lockheed Rpt LMSC805538, Lockheed Missile and Space Company, Sunnyvale, CA, November 1965.

141. Trimmer, L. L. and Clark, E. L., "Stagnation-Point Velocity Gradients for Spherical Segments in Hypersonic Flow," AIAA Journal, Vol. 7, No.10, October 1969.

142. Serbin, H., "Supersonic Flow Around Blunt Bodies," Journal of the Aeronautical Sciences, Vol. 25, No. 1, January 1958.

143. Stoney, W. E., Jr. and Swanson, A. G., "Heat Transfer Measured on a Flat-Face Cylinder in Free Flight at Mach Numbers up to 13.9," NACA RM-L-57E13, 17 June 1957.

144. Klett, R. D. (9312) to Clark, A. J., Jr., (9312), Internal Memo "RFD-2 Aeroheating Hot Shot Test Conducted at Rhodes and Bloxsom, October 8-12, 1963," Unclassified, Sandia Laboratories, 21 November 1963.

145. "Investigation of Re-entry Destruction of Nuclear Auxiliary Powerplants," Report AVCORAD-TR-61-28, Unclassified, October 1961.

146. Larson, H. K., "Heat Transfer in Separated Flows," Journal of the Aerospace Sciences, Vol. 26, No. 11, November 1959.

147. Powers, E. W., et al., "A Shock Tube Investigation of Heat Transfer in the Wake of a Hemisphere-Cylinder, with Application to Hypersonic Flight," Journal of the Aerospace Sciences, Vol. 26, No. 1, January 1959.

148. Oppenheim, A. K., "Generalized Theory of Convective Heat Transfer in a Free-Molecular Flow," Journal of the Aeronautical Sciences, Vol. 23, No. 1, January 1953.

149. Love, E. S., et al., "Some Topics in Hypersonic Body Shaping," AIAA Paper No. 69-181, AIAA 7th Aerospace Sciences Meeting, New York City, NY, January 1969.

150. Matthews, R. K. and Eaves Jr., R. H., "Comparison of Theoretical and Experimental Pressure and Heat Transfer Distributions on Three Blunt Nosed Cylinders In Hypersonic Flow," AEDC-TR-67-148, Arnold Engineering Development Center, Tullahoma, TN, September 1967.

151. Probstein, R. F., "Shock Wave and Flow Field Development in Hypersonic Re-Entry," ARS Journal, February 1961. 
152. Matting, F. W., "Approximate Bridging Relations in the Transitional Regime Between Continuum and Free-Molecule Flows," Journal of Spacecraft and Rockets, Vol. 8, No. 1, January 1971.

153. Putz, K. E. and Bartlett, E. P., "Heat-Transfer and Ablation-Rate Correlations for Reentry Heatshield and Nosetip Applications," AIAA Paper 72-91, AIAA 10th Aerospace Sciences Meeting, San Diego, CA, January 1972.

154. Chan, C. C., "Oscillation and Non-convergence of CMA Results During High Velocity Galileo-VEEGA Reentries," Internal Memo BFD-2-90-025, JHU/APL, 28 September 1990.

155. Spalding, D. B., "A Standard Formulation of the Steady Convective Mass-Transfer Problem," International Journal of Heat and Mass Transfer, Vol.1, 1960.

156. Moss, J. N., "Radiative Viscous-Shock-Layer Solutions with Coupled Ablation Injection," AIAA Journal, Vol. 14, No. 9, September 1976.

157. Garrett, L. B., et al., "An Implicit Finite-Difference Solution to the Viscous Shock Layer Including the Effects of Radiation and Strong Blowing," NASA TR R-388, NASA Langley Research Center, November 1972.

158. Conn, D. W., "Heat Source Ablation Blockage of Shock Layer Radiation," Internal Memo BFD-2-92-016, JHU/APL, 31 August 1992.

159. Everhart, L. G., "TDOF and RETAP Code Modifications to Include Mass Flux Blockage and Cheng's Rarefaction Parameter Calculations," Internal Memo BFD-2-92-020, JHU/APL, 14 December 1992.

160. Perini, L. L., "Heat and Mass Transfer Correlation Equations for Subliming Graphite in High Speed Flow," ANSP-M-11, JHU/APL, August 1974.

\section{SECTION VII. F.}

161. Minges, M. L., "Thermal Contact Resistance - Volume 1: A Review of the Literature," AFML-TR-65-375, Air Force Systems Command, Wright-Patterson Air Force Base, Ohio, April 1966.

162. Conn, D. W., Reentry Safety Evaluation of Radioisotope Heater Unit (RHU)-Voyager Mission, Vol. I: Steep Angle Superorbital Reentry Thermal Response and Shallow Angle Reentry Ablation Response, JHU/APL, April 1978, Unpublished. 
163. Perini, L. L., "Impact Temperature of the Light Weight Radioisotope Heater Unit (LWRHU)," JHU/APL, ANSP-225, BFD-4-82-016, 3 November 1982.

164. Perini, L. L., "Air Infiltration into the General Purpose Heat Source (GPHS) During Reentry," JHU/APL, ANSP-238, BFD-4-83-025, 3 August 1983.

165. Facsmile communication from Dr. B. Laub, Aerotherm Corporation to L. G. Everhart, on Thermochemical Data for $\mathrm{PuO}_{2}$, 25 July 1996.

\section{SECTION VIII}

166. Chang, Y., "Errors in MSC/NASTRAN for Thermostructural Analysis," JHU/APL Memorandum AM-96-E087, 28 August 1996.

167. Chang, Y., "Extensions to the Approximate Thermoplastic Analysis Method Using Fine Weave Pierced Fabric Material Properties," JHU/APL Memorandum AM-95-E150/BFD-295-014, 5 July 1995.

168. Chang, Y., "Updated Material Properties Data for Fine Weave Pierced Fabric in the Thermoplastic Analysis Program," JHU/APL Memorandum AM-96-E026, 15 March 1996.

169. Chang, Y., "A Limitation of the SAAS III Finite Element Analysis Program, and a Proposed Solution for Cassini Thermostructural Analysis," JHU/APL Memorandum AM96-E045, 15 April 1996.

170. Chang, Y., "Thermoplastic Analysis of Axisymmetric Structures Fabricated of Cartesian Weave Fine Weave Pierced Fabric," JHU/APL Memorandum AM-96-E053, 23 May 1996.

171. Chang, Y., "A Thermoplastic Analysis Method for Three-Dimensional Weave Carboncarbon Composites," Proceedings of the Space Technology Applications International Forum (STAIF-97), Albuquerque, NM, 26-30 January 1997.

172. Jones, R. M., "Modeling Nonlinear Deformations of Carbon-Carbon Composite Materials," AIAA Journal, Vol. 18, No. 8, August 1980.

173. "Evaluation of Carbon-Carbon Composites for The Pan Pilot Production Program $\left(\mathrm{P}^{4}\right)$," Southern Research Institute, AFML-TR-78-2, Volume II, May 1979.

174. Cull, T. A., "Status Report: FWPF Graphite Mechanical Properties Tests," Los Alamos National Laboratory NMT-9:ADM90-C026, 2 May 1990. 
175. Waeber, K. R., "Fine Weave Pierced Fabric Compressive Failure Response: Consultation with Southern Research Institute," JHU/APL Memorandum ANSP-272, BFD-4-85-006, 7 May 1985.

176. Chang, Y., "Checkout of MSC/NASTRAN for Thermoelastic Analysis of Carbon-Carbon Composite Materials," JHU/APL Memorandum AM-94-E239/BFD-2-94-035, 12 December 1994.

\section{SECTION X}

177. Brenza, P. T., "Thermal Response of a LWRHU to Titan SRMU Fuel Fragment Fires for the Cassini Mission," JHU/APL AM-96-D015, 13 August 1996.

178. Facsimile to Dr. E. W. Johnson, from P. T. Brenza, "One Dimensional Predictions of LWRHU Thermal Response to Titan SRMU Propellant Fires," 27 November 1996. 


\section{Additional References for Table 8}

8.1 NASA Ames Research Staff, "Equations, Tables and Charts for Compressible Flow," NASA Report 1135, 1953.

8.2 Wittliff, C. E. and Curtis, J. T., "Normal Shock Wave Parameters in Equilibrium Air," Report CAL-111, Cornell Aeronautical Laboratory, August 1962.

8.3 Marrone, P. V., "Normal Shock Waves in Air: Equilibrium Composition and Flow Parameters for Velocitites from 26,000 to 50,000 Ft/Sec," Report AG-1729-A-2, Cornell Aeronautical Laboratories, August 1962.

8.4 "SNAP-27 Safety Report, Volume II: Accident Model Document, Supplement No. 1, Appendix P - Reentry Evaluation of GLFC and LM," DIN: 6300-300PRI, Isotope Power Systems Operation/Missile and Space Division," General Electric Co., September 1968. 
Table 1

General Description of the LWRHU Components

\section{Component}

Fuel pellet

Frit Vent

Clad Cap, Vent End

Clad Cap, Closure End

$\infty$
Material

$\mathrm{PuO}_{2}$

Pt

Pt-30Rh

Pt-30Rh

Pt-30Rh

Pt-30Rh

PG

$P G$

PG

PG

FUPF

FWPF
Material

Specffication

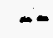

SPA-790605

SPA-790139

SPA-790139

SPA-790139

SPA-790139

SPA-790171

SPA-790171

SPA-790171

SPA-790171

SPA-790053

SPA-790053
Jrawing

Number

AYC-790106

AYC -790098

AYC-790099

AYC-790105

AYC-790100

AYC-790101

AYC-790381

AYC-790382

AYC -790383

AYC-790384

AYC-790385

AYC -790380

TOTAL WEIGHT
Typical

We ight, $g$

2.664

5.702

2.532

3.166

1.486

0.624

2.543

21.070

39.787

"Two Required 


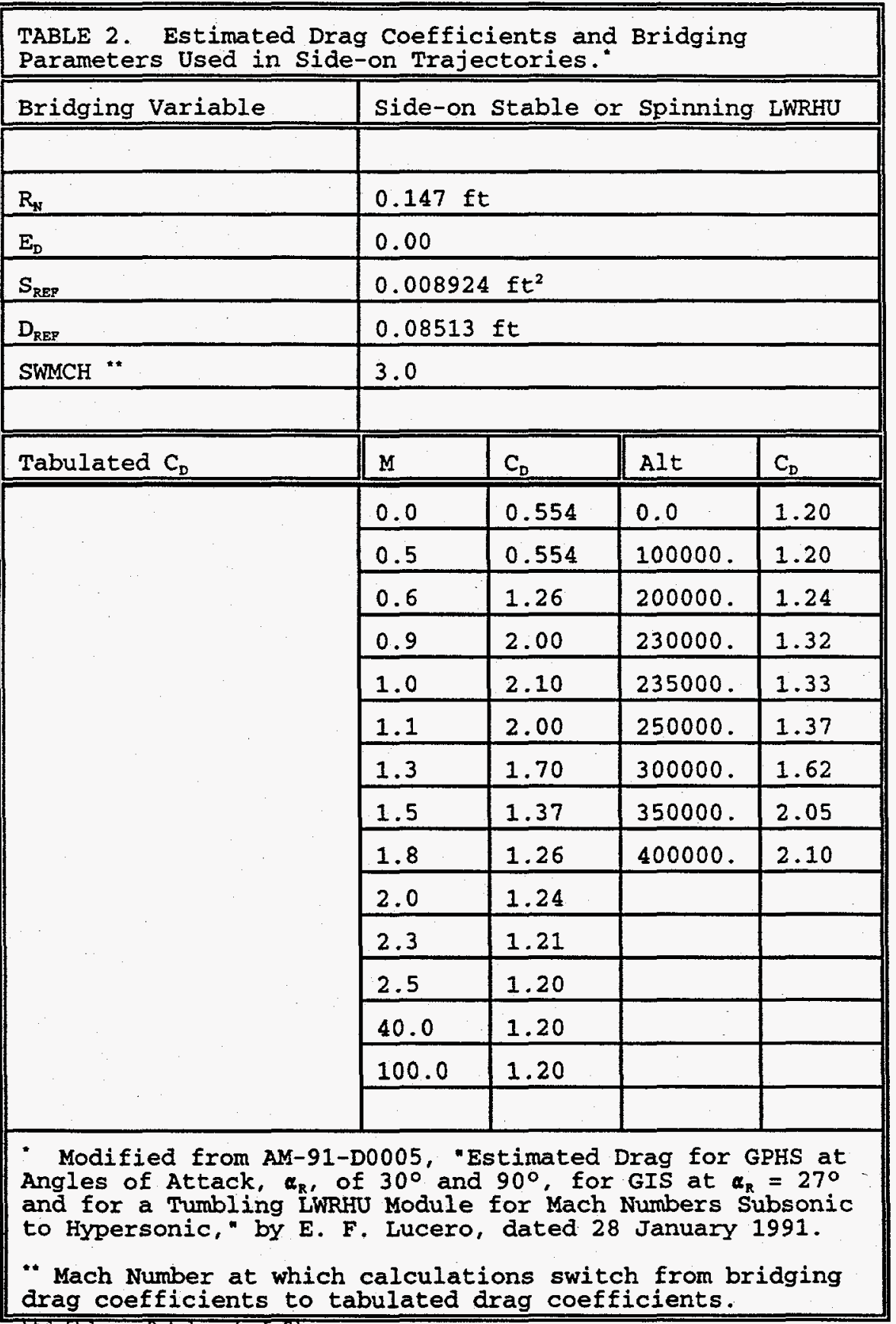


TABLE 3. Estimated Drag Coefficients and Bridging Parameters Used in Tumbling Trajectories.

\begin{tabular}{|c|c|c|c|c|c|c|}
\hline Bridging Variable & \multicolumn{2}{|c|}{$\begin{array}{l}\text { Random Tumbling } \\
\text { Cylinder }\end{array}$} & \multicolumn{2}{|c|}{ Tumbling Sphere } & \multicolumn{2}{|c|}{$\begin{array}{l}\text { Quasi-Tumbling } \\
\text { Cylinder }\end{array}$} \\
\hline$C_{D C}$ & \multicolumn{2}{|l|}{0.00} & \multicolumn{2}{|l|}{1.02} & \multicolumn{2}{|l|}{1.90} \\
\hline$C_{D F M}$ & \multicolumn{2}{|l|}{0.00} & \multicolumn{2}{|l|}{2.04} & \multicolumn{2}{|l|}{2.95} \\
\hline $\mathbf{R}_{\mathrm{N}}$ & \multicolumn{2}{|c|}{$0.147 \mathrm{ft}$} & \multicolumn{2}{|c|}{$0.0477 \mathrm{ft}$} & \multicolumn{2}{|c|}{$0.147 \mathrm{ft}$} \\
\hline$E_{D}$ & \multicolumn{2}{|c|}{0.00} & \multicolumn{2}{|l|}{0.0} & \multicolumn{2}{|c|}{2.20} \\
\hline$S_{\text {REP }}$ & \multicolumn{2}{|c|}{$0.008924 \mathrm{ft}^{2}$} & \multicolumn{2}{|c|}{$0.00714 \mathrm{ft}^{2}$} & \multicolumn{2}{|c|}{$0.00569 \mathrm{ft}^{2}$} \\
\hline$D_{\text {REF }}$ & \multicolumn{2}{|c|}{$0.08514 \mathrm{ft}$} & \multicolumn{2}{|c|}{$0.0954 \mathrm{ft}$} & \multicolumn{2}{|c|}{$0.08513 \mathrm{ft}$} \\
\hline SWMCH ** & \multicolumn{2}{|c|}{0.9} & \multicolumn{2}{|c|}{3.0} & \multicolumn{2}{|c|}{3.0} \\
\hline \multirow[t]{16}{*}{ Tabulated $C_{D}$} & $\mathrm{M}$ & $C_{D}$ & $\mathbf{M}$ & $C_{D}$ & $M$ & $\mathrm{C}_{\mathrm{D}}$ \\
\hline & 0.0 & 0.554 & 0.0 & 0.55 & 0.0 & 0.94 \\
\hline & 0.5 & .554 & 0.2 & 0.57 & 0.2 & 0.94 \\
\hline & 0.6 & 1.26 & 0.4 & 0.58 & 0.5 & 0.96 \\
\hline & 0.9 & 2.00 & 0.6 & 0.62 & 0.6 & 1.53 \\
\hline & 1.0 & 2.10 & 0.8 & 0.69 & 0.8 & 2.04 \\
\hline & 1.1 & 2.0 & 0.9 & 0.91 & 1.0 & 2.35 \\
\hline & 1.3 & 1.60 & 1.0 & 1.04 & 1.2 & 2.28 \\
\hline & 1.5 & 1.37 & 1.2 & 1.11 & 1.4 & 2.02 \\
\hline & 1.8 & 1.26 & 1.4 & 1.13 & 1.6 & 1.95 \\
\hline & 2.0 & 1.24 & 1.6 & 1.12 & 1.8 & 1.92 \\
\hline & 2.3 & 1.21 & 2.0 & 1.11 & 2.0 & 1.90 \\
\hline & 2.5 & 1.20 & 3.0 & 1.06 & 3.0 & 1.90 \\
\hline & 40.0 & 1.20 & 4.0 & 1.02 & 10.0 & 1.90 \\
\hline & & & 50.0 & 1.02 & 100.0 & 1.90 \\
\hline & & & 100.0 & 1.02 & & \\
\hline
\end{tabular}

- Modified from AM-91-D0005, "Estimated Drag for GPHS at Angles of Attack, $\alpha_{R}$, of $30^{\circ}$ and $90^{\circ}$, for GIS at $\alpha_{R}=27^{\circ}$ and for a Tumbling LWRHU Module for Mach Numbers Subsonic to Hypersonic," by E. F. Lucero, dated 28 January 1991.

* Mach Number at which calculations switch from bridging drag coefficients to tabulated drag coefficients.

c:Itoflaragco.tab (wp5I) 
Table 4

Estimated Drag Coefficients and Terminal Velocity for the LWRHU.

Axial

A. Drag Coefficient

$S=0.00567 \rho t^{2}$
1.0

0.7865

1.121
A verage

Crosselow

0.89 Cold

1.06 Hot

B. $v_{\text {term }}: \mathrm{ft} / \mathrm{sec} /(\mathrm{m} / \mathrm{sec})$

Axial

$\begin{array}{ll}\text { Sea } & 10 \mathrm{~K} \\ \text { Level ft } & \mathrm{ft}\end{array}$

Level $\underline{f t}$

$W=0.08886$ $\begin{array}{ll}115 & 134 \\ (35) & (41)\end{array}$
Crossflow

A verage
Sea $\quad 10 K$

Level ft Level ft

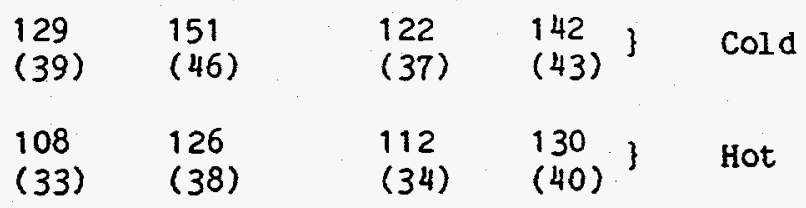


Applied Physics Laboratory

Laurel. Maryland 20723-60)99

\begin{tabular}{|c|c|c|c|c|c|c|c|c|}
\hline Reentry Angle $\left({ }^{\circ}\right)$ & $5.2^{\circ}$ & $7.0^{\circ}$ & $10.0^{\circ}$ & $15^{\circ}$ & $30^{\circ}$ & $50^{\circ}$ & $70^{\circ}$ & $90^{\circ}$ \\
\hline \multicolumn{9}{|l|}{ SIDE-ON } \\
\hline $\operatorname{Max} Q_{\text {rad }}\left(B t u / f^{2}{ }^{2} s\right)$ & 147.91 & 559.44 & 1300.822 & 2709.51 & 7022.15 & 11061.55 & 13139.76 & 13649 \\
\hline $\operatorname{Max} Q_{\mathrm{com}}\left(\mathrm{Btu} / \mathrm{ft}^{2} \mathrm{~s}\right)$ & 1527.66 & 2133.25 & 2714.57 & 3408.54 & 4752.14 & 5843.53 & 6379.77 & 6463.98 \\
\hline $\operatorname{Max} \mathbf{M}_{-}$ & 73.66 & 73.74 & 73.86 & 73.88 & 73.71 & 73.32 & 72.105 & 71.172 \\
\hline $\operatorname{Max} P_{L_{2}}$ (atm) & 0.2653 & 0.5396 & 0.9643 & 1.5882 & 3.4019 & 5.4631 & 6.8072 & 7.2403 \\
\hline Max Decel (Gs) & 38.34 & 74.55 & 132.48 & 217.09 & 461.8 & 738.83 & 919.12 & 977.09 \\
\hline $\begin{array}{l}\text { Time of Max Qrad } \\
(\mathrm{sec})\end{array}$ & 28.77 & 18.78 & 12.03 & 7.47 & 3.75 & 2.38 & 2.03 & 1.92 \\
\hline $\begin{array}{l}\text { Time of Max } Q_{\infty} \\
\text { (sec) }\end{array}$ & 23.45 & 15.61 & 10.00 & 6.30 & 3.20 & 2.18 & 1.88 & 1.77 \\
\hline $\begin{array}{l}\text { Time of Max M_ } \\
(\mathrm{sec})\end{array}$ & 7.00 & 4.00 & 1.00 & 0.00 & 0.00 & 0.00 & 0.00 & 0.00 \\
\hline $\begin{array}{l}\text { Time of Max } P_{t, 2} \\
(\mathrm{sec})\end{array}$ & 30.50 & 22.60 & 14.23 & 9.02 & 4.65 & 3.08 & 2.53 & 2.37 \\
\hline $\begin{array}{l}\text { Time of Max Decel } \\
\text { (sec) }\end{array}$ & 29.67 & 22.40 & 14.13 & 9.02 & 4.65 & 3.08 & 2.53 & 2.37 \\
\hline $\begin{array}{l}\text { Time at End of Heat } \\
\text { Pulse (sec) }\end{array}$ & 69.20 & 38.20 & 23.23 & 14.59 & 7.40 & 4.93 & 4.04 & 3.79 \\
\hline Time at $M_{-}=5(\mathrm{sec})$ & 218.40 & 59.00 & 33.10 & 19.80 & 9.70 & 6.20 & 5.10 & 4.70 \\
\hline Reentry Angle $\left({ }^{\circ}\right)$ & $5.2^{\circ}$ & $7.0^{\circ}$ & $10.0^{\circ}$ & $15^{\circ}$ & $30^{\circ}$ & $50^{\circ}$ & $70^{\circ}$ & $90^{\circ}$ \\
\hline \multicolumn{9}{|c|}{ TUMBLING CYLINDER } \\
\hline Max Q Q & 162.42 & 613.13 & 1398.42 & 2858.31 & 7266.43 & 11279.45 & 13351.78 & 13861.8 \\
\hline Max $Q_{\operatorname{cow}}\left(B t u / f^{2} s\right)$ & 1589.89 & 2220.79 & 2807.32 & 349.43 & 4832.44 & 5919.34 & 6452.9 & 6535.79 \\
\hline $\operatorname{Max} \mathbf{M}$ & 73.63 & 73.78 & 73.86 & 73.88 & 73.71 & 73.32 & 72.105 & 71.172 \\
\hline $\operatorname{Max} P_{t_{2}}(\mathbf{a t m})$ & 0.2134 & 0.5645 & 0.9974 & 1.631 & 3.4596 & 5.5254 & 6.8695 & 7.2969 \\
\hline Max Decel (Gs) & 28.86 & 76.34 & 134.89 & 220.57 & 467.86 & 747.23 & 929 & 986.81 \\
\hline $\begin{array}{l}\text { Time of Max Q-as } \\
\text { (sec) }\end{array}$ & 29.61 & 19.15 & 12.19 & 7.51 & 3.75 & 2.38 & 2.03 & 1.92 \\
\hline $\begin{array}{l}\text { Time of Max Qooer } \\
(\mathrm{sec})\end{array}$ & 24.08 & 15.70 & 10.02 & 6.37 & 3.20 & 2.18 & 1.88 & 1.72 \\
\hline $\begin{array}{l}\text { Time of Max M_ } \\
(\mathrm{sec})\end{array}$ & 8.00 & 3.50 & 1.00 & 0.00 & 0.00 & 0.00 & 0.00 & 0.00 \\
\hline $\begin{array}{l}\text { Time of Max } P_{L 2} \\
(s e c)\end{array}$ & 32.01 & 22.31 & 14.13 & 8.99 & 4.65 & 3.03 & 2.53 & 2.37 \\
\hline Time of Max Deq & 32.01 & 22.31 & 14.14 & 8.99 & 4.65 & 3.03 & 2.53 & 2.37 \\
\hline $\begin{array}{l}\text { Time at End of Heat } \\
\text { Pulse (sec) }\end{array}$ & 96.90 & 38.21 & 23.24 & 14.54 & 7.40 & 4.88 & 4.28 & 4.07 \\
\hline Time at $M_{-}=5(\mathrm{sec})$ & 280.20 & 59.15 & 33.10 & 19.60 & 9.65 & 6.80 & 5.51 & 5.27 \\
\hline & & & & & & & & \\
\hline
\end{tabular}


Applied Physics Laboratory

Laurel, Maryland 20723-60999

\begin{tabular}{|c|c|c|c|c|c|c|c|c|}
\hline \multicolumn{9}{|c|}{ TABLE 5. TDOF Trajectory Results. (cont.) } \\
\hline Reentry Angle ( ${ }^{\circ}$ ) & $5.2^{\circ}$ & $7.0^{\circ}$ & $10.0^{\circ}$ & $15^{\circ}$ & $30^{\circ}$ & $50^{\circ}$ & $70^{\circ}$ & $90^{\circ}$ \\
\hline \multicolumn{9}{|l|}{ TUMBLING SPHERE } \\
\hline $\operatorname{Max} Q_{r a d}\left(B t u / f^{2} s\right)$ & 226.72 & 966.49 & 2398.57 & 4777.05 & 11237.55 & 16943.79 & 20902.36 & 21963.9 \\
\hline $\operatorname{Max} Q_{\cos }\left(B t u / f^{2} s\right)$ & 1656.94 & 2411.81 & 3194.79 & 4080.49 & 5797.59 & 7222.54 & 7941.81 & 8048.32 \\
\hline $\operatorname{Max} \mathbf{M}_{-}$ & 73.61 & 73.79 & 73.86 & 73.88 & 73.71 & 73.32 & 72.105 & 71.172 \\
\hline $\operatorname{Max} P_{L_{2}}(\mathrm{~atm})$ & 0.2541 & 0.7447 & 1.4054 & 2.4073 & 5.2545 & 8.4305 & 10.4947 & 11.1568 \\
\hline Max Decel (Gs) & 25.39 & 67.84 & 128.04 & 219.29 & 478.69 & 768.03 & 956.08 & 1016.4 \\
\hline $\begin{array}{l}\text { Time of Max Qred } \\
\text { (sec) }\end{array}$ & 30.51 & 21.19 & 13.12 & 8.11 & 3.90 & 2.80 & 2.27 & 2.16 \\
\hline $\begin{array}{l}\text { Time of Max Qour } \\
(\mathrm{sec})\end{array}$ & 24.58 & 17.33 & 11.35 & 6.99 & 3.61 & 2.45 & 2.02 & 12.91 \\
\hline $\begin{array}{l}\text { Time of Max M_ } \\
\text { (sec) }\end{array}$ & 8.00 & 3.50 & 1.00 & 0.00 & 0.00 & 0.00 & 0.00 & 0.00 \\
\hline Time of Max $P_{L 2}(\mathrm{sec})$ & 33.83 & 24.83 & 15.76 & 10.00 & 4.95 & 3.25 & 2.67 & 2.51 \\
\hline $\begin{array}{l}\text { Time of Max Decel } \\
\text { (sec) }\end{array}$ & 31.86 & 24.83 & 15.76 & 9.94 & 4.95 & 3.25 & 2.67 & 2.51 \\
\hline $\begin{array}{l}\text { Time at End of Heat } \\
\text { Pulse (sec) }\end{array}$ & 104.03 & 42.73 & 25.00 & 15.50 & 7.80 & 5.02 & 4.12 & 3.83 \\
\hline Time at $M_{-}=5$ (sec) & 324.51 & 63.80 & 34.51 & 20.55 & 9.65 & 6.14 & 4.99 & 4.67 \\
\hline \multicolumn{9}{|c|}{$P_{42}$ - Total pressure behind a normal shock wave } \\
\hline \multicolumn{9}{|c|}{\begin{tabular}{|l|l|l} 
M_ - Free stream Mach number & & \\
\end{tabular}} \\
\hline \multicolumn{9}{|c|}{ Qre - Reference radiative heat flux; RAD/EQUIL, inviscid stagnation streamline flow field, effective spherical radius } \\
\hline \multicolumn{9}{|c|}{\begin{tabular}{|l|l|}
$Q_{0 m}$ - Reference convective heat flux; 1 ft radius sphere, stagnation how, cold wall, zero mass transfer & \\
\end{tabular}} \\
\hline \multicolumn{9}{|c|}{\begin{tabular}{|l|l|l|l|l|} 
End of Heat Pulse - $Q_{\text {na }}>0$ and $Q_{\text {and }}->100\left(B t u / f^{2} s\right)$ & & & & \\
\end{tabular}} \\
\hline & & $\cdot+1$ & & & & & & \\
\hline c:ludof lintab.xis & & & & & & & & \\
\hline
\end{tabular}


Table 6

LWRHU Thermal Response Modeling

a.) LWRHU Thermal Model Response Regions

Region Number

1

2

3

4

5

6

7

8

9

10

11

12

13
Component

aeroshell body

aeroshell end cap

outer insulator body

middle insulator body

inner insulator body

clad body

clad end cap

fuel pellet (with shim)

insulator cap-layer 1

insulator cap-layer 2

insulator cap-layer 3

insulator cap-layer 4

insulator cap-layer 5
Material

FWPF carbon-carbon

FWPF carbon-carbon

pyrolytic graphite

pyrolytic graphite

pyrolytic graphite

Pt-30Rh alloy

Pt-30Rh alloy

$238 \mathrm{PuO}_{2}$

pyrolytic graphite pyrolytic graphite pyrolytic graphite pyrolytic graphite pyrolytic graphite

REGIONAL REPRESENTATIONS OF THE LWRHU THERMAL MODEL

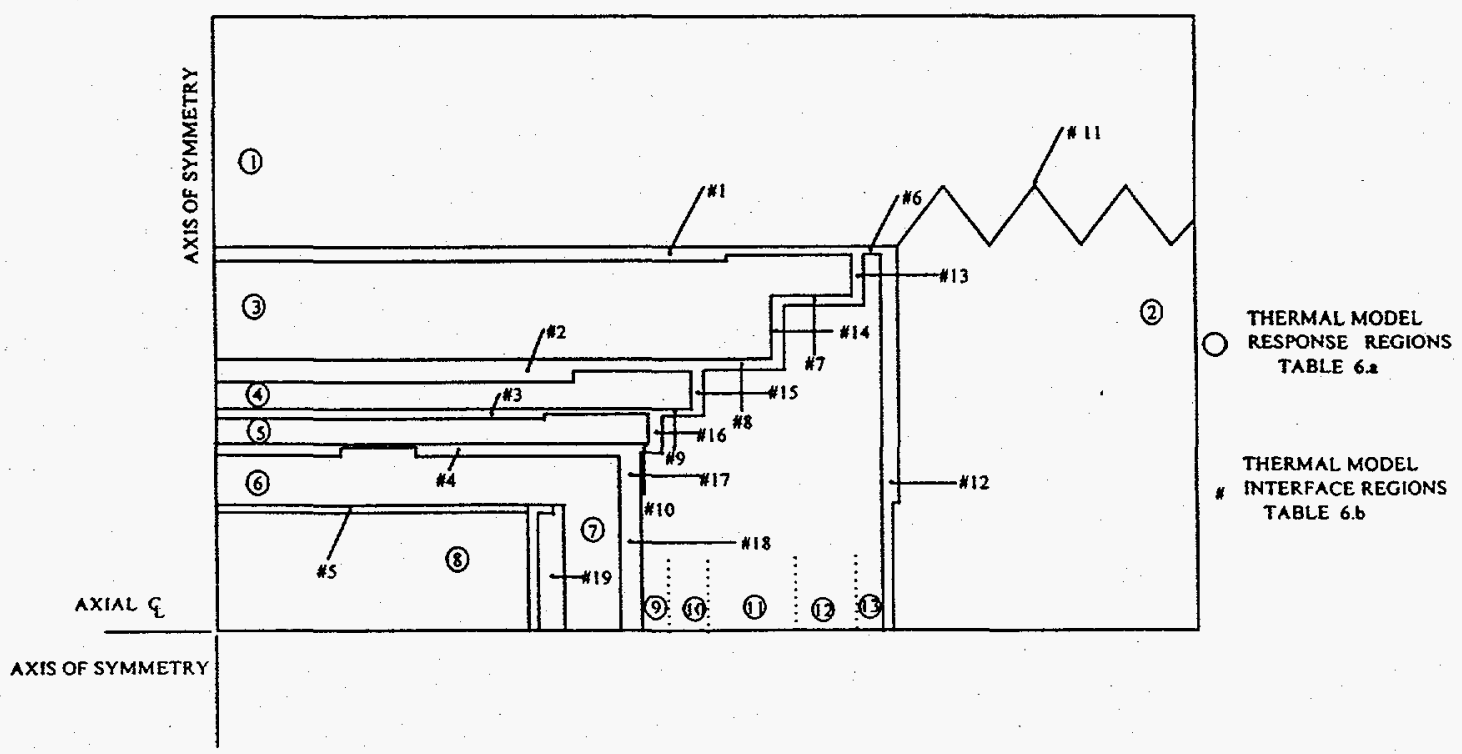


Table 6 (Continued)

LWRHU Thermal Response Modeling

b.) LWRHU Interface Regions, Side-On Orientation

A. Radial

Region
1
2
3

4
5
6
7
7
9

10
11

B. Axial

\section{Circumferential}

Interface Components aeroshell body/outer insulator body outer insulator body/middle insulator body middle insulator body/inner insulator body

inner insulator body/clad body clad body/fuel pellet aeroshell body/insulator cap-layer 5

outer insulator body/insulator cap-layer 4 outer insulator body/insulator cap-layer 3 middle insulator body/insulator cap-layer 2

inner insulator body/insulator cap-layer 1 aeroshell body/aeroshell end cap

insulator cap-layer 5/aeroshell end cap outer insulator body/insulator cap-layer 5 outer insulator body/insulator cap-layer 4

middle insulator body/insulator cap-layer 3 inner insulator body/insulator cap-layer 2 clad body/insulator cap-layer 1

clad end cap/insulator cap-layer 1 fuel pellet (with shim)/clad end cap

None 
The Johns Hopkins University

Applied Physics Laboratory

Laurel, Maryland 20723-6099

Table 6 (Continued)

LWRHU Thermal Response Modeling

c.) Representative Interface States for the Side-on Stable LWRHU Assembly

\section{Radial Interfaces}

\begin{tabular}{|c|c|c|c|c|c|c|}
\hline \multirow{3}{*}{ Interface Regions } & \multicolumn{6}{|c|}{ Interface States } \\
\hline & \multicolumn{2}{|c|}{$\begin{array}{l}\text { A. Perfect } \\
\text { alignment }^{(i)}\end{array}$} & \multicolumn{2}{|c|}{$\begin{array}{l}\text { B. Translational } \\
\text { displacement } t^{(\mathrm{i})}\end{array}$} & \multicolumn{2}{|c|}{$\begin{array}{l}\text { C. Thermal } \\
\text { expansion }^{\text {(iii) }}\end{array}$} \\
\hline & state & $\begin{array}{l}\text { gap } \\
\text { (mils) }\end{array}$ & $\begin{array}{c}\text { state } \\
\left(\theta=45^{\circ}\right)\end{array}$ & $\begin{array}{l}\text { gap } \\
\text { (mils) }\end{array}$ & state & $\begin{array}{c}\text { gap } \\
\text { (mils) }\end{array}$ \\
\hline $\begin{array}{l}\text { 1. Aeroshell body outer } \\
\text { insulator body (\#1) } \\
\text { - local stand-off(iv) } \\
\text { - main(iv) }\end{array}$ & $\begin{array}{l}\text { open } \\
\text { open }\end{array}$ & $\begin{array}{l}3.0 \\
6.1 \\
\end{array}$ & $\begin{array}{l}\text { open } \\
\text { open }\end{array}$ & $\begin{array}{l}1.0 \\
4.0 \\
\end{array}$ & $\begin{array}{l}\text { shut } \\
\text { open }\end{array}$ & $\begin{array}{l}0.0 \\
3.1 \\
\end{array}$ \\
\hline $\begin{array}{l}\text { 2. Outer insulator body } \\
\text { middle insulator } \\
\text { body ( } \# 2) \\
\text { - local stand-off } \\
\text { - main }\end{array}$ & $\begin{array}{l}\text { open } \\
\text { open }\end{array}$ & $\begin{array}{l}2.7 \\
5.8\end{array}$ & $\begin{array}{l}\text { open } \\
\text { open }\end{array}$ & $\begin{array}{l}1.3 \\
4.4\end{array}$ & $\begin{array}{l}\text { shut } \\
\text { open }\end{array}$ & $\begin{array}{l}0.0 \\
3.1\end{array}$ \\
\hline $\begin{array}{l}\text { 3. Middle insulator body } \\
\text { inner insulator body }(\sharp 3) \\
\text { - local stand-off } \\
\text { - main }\end{array}$ & $\begin{array}{l}\text { open } \\
\text { open }\end{array}$ & $\begin{array}{l}2.9 \\
5.8\end{array}$ & $\begin{array}{l}\text { open } \\
\text { open }\end{array}$ & $\begin{array}{l}1.1 \\
6.3\end{array}$ & $\begin{array}{l}\text { shut } \\
\text { open }\end{array}$ & $\begin{array}{l}0.0 \\
2.9\end{array}$ \\
\hline $\begin{array}{l}\text { 4. Inner insulator body } \\
\text { clad body (\#4) } \\
\text { - local stand-off } \\
\text { - main }\end{array}$ & $\begin{array}{l}\text { open } \\
\text { open }\end{array}$ & $\begin{array}{c}5.1 \\
10.1 \\
\end{array}$ & $\begin{array}{l}\text { open } \\
\text { open }\end{array}$ & $\begin{array}{l}1.6 \\
6.6 \\
\end{array}$ & $\begin{array}{l}\text { shut } \\
\text { open }\end{array}$ & $\begin{array}{l}0.0 \\
5.0\end{array}$ \\
\hline $\begin{array}{l}\text { 5. Clad body fuel pellet } \\
\text { main }(v)(\# 5)\end{array}$ & open & 6.9 & open & 2.4 & shut & 0.0 \\
\hline $\begin{array}{l}\text { 6. Aeroshell body } \\
\text { insulator cap (\#6) } \\
\text { main }\end{array}$ & open & 2.5 & open & 1.0 & shut & 0.0 \\
\hline $\begin{array}{l}\text { 7. Outer insulator body } \\
\text { insulator cap (\#7) } \\
\text { main }\end{array}$ & open & 2.0 & open & 2.3 & shut & 0.0 \\
\hline $\begin{array}{l}\text { 8. Outer insulator body } \\
\text { insulator cap (\#8) } \\
\text { - main }\end{array}$ & open & 2.4 & open & 2.7 & shut & 0.0 \\
\hline $\begin{array}{l}\text { 9. Middle insulator body } \\
\text { insulator cap (\#9) } \\
\text { - main }\end{array}$ & open & 2.5 & open & 4.2 & shut & 0.0 \\
\hline $\begin{array}{l}\text { 10. Inner insulator body } \\
\text { insulator cap ( } \# 10) \\
\text { main }\end{array}$ & open & 1.8 & open & 3.0 & shut & 0.0 \\
\hline $\begin{array}{l}\text { 11. Aeroshell body } \\
\text { aeroshell end cap (\#11) } \\
\text { main }\end{array}$ & shut & 0.0 & shut & 0.0 & shut & 0.0 \\
\hline
\end{tabular}


Table 6 (Continued)

LWRHU Thermal Response Modeling

c.) Representative Interface States for the Side-on Stable LWRHU Assembly

\section{Axial Interfaces}

\begin{tabular}{|c|c|c|c|c|}
\hline \multirow{3}{*}{ Interface Regions } & \multicolumn{4}{|c|}{ Interface States } \\
\hline & \multicolumn{2}{|c|}{$\begin{array}{l}\text { A. Perfect } \\
\text { alignment }^{(i)}\end{array}$} & \multicolumn{2}{|c|}{$\begin{array}{l}\text { B. Thermal } \\
\text { expansion }^{(i)}\end{array}$} \\
\hline & state & $\begin{array}{l}\text { gap } \\
\text { (mils) }\end{array}$ & $\begin{array}{c}\text { state } \\
\left(\theta=45^{\circ}\right)\end{array}$ & $\begin{array}{l}\text { gap } \\
\text { (mils) }\end{array}$ \\
\hline $\begin{array}{l}\text { 12. Aeroshell end cap } \\
\text { insulator cap (\#12) } \\
\text { - local stand-off } \\
\text { - main }\end{array}$ & $\begin{array}{l}\text { shut } \\
\text { open }\end{array}$ & $\begin{array}{l}0.0 \\
4.9\end{array}$ & $\begin{array}{l}\text { shut } \\
\text { open }\end{array}$ & $\begin{array}{l}0.0 \\
4.9\end{array}$ \\
\hline $\begin{array}{l}\text { 13. Outer insulator body } \\
\text { insulator cap (\#13) } \\
\text { - main }\end{array}$ & open & 3.0 & shut & 0.0 \\
\hline $\begin{array}{l}\text { 14. Outer insulator body } \\
\text { insulator cap (\#14) } \\
\text { - main }\end{array}$ & open & 3.0 & shut & 0.0 \\
\hline $\begin{array}{l}\text { 15. Middle insulator body } \\
\text { insulator cap (\#15) } \\
\text { - main }\end{array}$ & shut & 0.0 & shut & 0.0 \\
\hline $\begin{array}{l}\text { 16. Inner insulator body } \\
\text { insulator cap (\#16) } \\
\text { - main }\end{array}$ & open & 3.0 & shut & 0.0 \\
\hline $\begin{array}{l}\text { 17. Clad body insulator cap } \\
\text { (\#17) } \\
\text { - local stand-off } \\
\text { - main }\end{array}$ & $\begin{array}{l}\text { open } \\
\text { open }\end{array}$ & $\begin{array}{l}5.0 \\
8.0\end{array}$ & $\begin{array}{l}\text { shut } \\
\text { open }\end{array}$ & $\begin{array}{l}0.0 \\
3.0\end{array}$ \\
\hline $\begin{array}{l}\text { 18. Clad end cap_insulated } \\
\text { cap (\#18) } \\
\text { - local stand-off } \\
\text { - main }\end{array}$ & $\begin{array}{l}\text { open } \\
\text { open }\end{array}$ & $\begin{array}{l}5.0 \\
8.0\end{array}$ & $\begin{array}{l}\text { shut } \\
\text { open }\end{array}$ & $\begin{array}{l}0.0 \\
3.0\end{array}$ \\
\hline $\begin{array}{l}\text { 19. Fuel-shim clad end cap } \\
\text { - main }(\# 19)\end{array}$ & open & 5.0 & open & 5.0 \\
\hline
\end{tabular}


The Johns Hopkins University

Applied Physics Laboratory

Laurel, Maryland 20723.6099

Table 6 (Continued)

LWRHU Thermal Response Modeling

\section{Notes:}

(i) Perfect alignment state

radial: all internal components co-aligned along the aeroshell's longitudinal axis.

axial: all internal component's play uniformly distributed fore and aft; assembly pre-loaded to remove minimum play (i.e., middle insulator body/insulator cap).

gap size: room temperature, nominal dimensions.

(ii) Translational displacement state

radial: - forward (i.e., windward displacement of all internal components along the forward stagnation line $\left(\theta=0^{\circ}\right)$ due to flight deceleration.

gap size: room temperature, nominal dimensions; represented by gap at an aeroshell body central angle, $\theta=45^{\circ}$; modeled by law of cosines.

(iii) Thermal expansion state

gap size: hypothetical representation in which heated state of the components results in closure of all radial and axis interfaces except where constrained by stand-offs.

(iv) Translational displacement state:

For the initial segment of an interface $\left(0^{\circ} \leq \theta \leq 15^{\circ}\right.$ Figure 34), the contact area of the segment is assumed to be over an area of $0^{\circ} \leq \theta \leq 5^{\circ}$ to distinguish between an engineering surface and an ideally smooth surface (point contact).

(v) Exception to rate (iv):

The initial segment of the clad body/fuel pellet interface is assumed to be fully in contact. 
Table 6 (Continued)

\section{LWRHU Thermal Response Modeling}

d.) Assignment of Interface States

\section{Flight Orientation}

1. Side-on stable $e^{(a)}$

- radial interfaces

- axial interfaces

2. Side-on spinning ${ }^{(b)}$

- radial interfaces

- axial interfaces

3. Random tumbling ${ }^{(c)}$

- radial interfaces

- axial interfaces

\author{
Shallow Reentries \\ $\left(\gamma \leq 15^{\circ}\right)$
}

te

te

te

te
Steep Reentries

$\left(\gamma>15^{\circ}\right)$

td

pa

te

$\mathrm{pa}$

legend:

te: 'thermal expansion' interface state

td: 'translational displacement' interface state

pa: 'perfect alignment' interface state

notes:

(a) for shallow reentries, both radial and axial interfaces are assumed to thermally expand to closure (except where constrained by stand-offs) due to durated heat pulse; for steep reentries, high inertia loading will shift interior components forward with radial interfaces closing in the stagnation region but axial interfaces affected neither by thermal expansion or inertia effects.

(b) same assignments as side-on stable except now radial interfaces for all reentries are closed (except where constrained by stand-offs) to further simulate simultaneous closure at all circumferential locations due to high spin rates.

(c) same assignments as side-on spinning except now all radial and axial interfaces for all reentries are closed (except where constrained by stand-offs) to further simulate simultaneous closure at all locations due to high rotational rates about all three axes. 
Table 6 (Continued)

LWRHU Thermal Response Modeling

e.) Thermal Contact Conductances Prior Side-on Stable LWRHU Analyses

\begin{tabular}{|c|c|c|c|}
\hline \multirow{2}{*}{\multicolumn{2}{|c|}{ Interface Region }} & \multicolumn{2}{|c|}{ 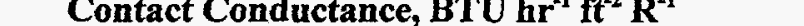 } \\
\hline & & 3-D Design ${ }^{(a)}$ & 2-D Galileo/VEEGA ${ }^{(b)}$ \\
\hline \multirow[t]{11}{*}{ Radial: } & 1 & 1000 & $10^{5}$ (perfect) \\
\hline & 2 & 500 & $10^{5}$ (perfect) \\
\hline & 3 & 500 & $10^{5}$ (perfect) \\
\hline & 4 & 500 & $10^{5}$ (perfect) \\
\hline & 5 & 2000 & $10^{5}$ (perfect) \\
\hline & 6 & 1000 & \\
\hline & 7 & 0 (open) & \\
\hline & 8 & 0 (open) & \\
\hline & 9 & 0 (open) & \\
\hline & 10 & 0 (open) & \\
\hline & 11 & $10^{7}$ (perfect) & \\
\hline \multirow[t]{8}{*}{ Axial: } & 12 & 0 (open) & \\
\hline & 13 & 0 (open) & \\
\hline & 14 & 0 (open) & \\
\hline & 15 & 0 (open) & \\
\hline & 16 & 0 (open) & \\
\hline & 17 & 0 (open) & \\
\hline & 18 & 0 (open) & \\
\hline & 19 & 0 (open) & \\
\hline
\end{tabular}

Notes:

(a) All axial interface regions were arbitrarily kept 'open' in this study to channel heat flow into the claded fuel assembly.

(b) This study modeled the mid-span section of the LWRHU; for conservatism, assumed perfect thermal contact at all interfaces. 
Table 6 (Continued)

LWRHU Thermal Response Modeling

f.) Thermal Contact Conductances

Based on Interface States and Compressive Stress States, Side-on Stable Flight Orientation

I. Radial Interfaces

Stress State

region materials

\#1 FWPF/PG

\#2 PG/PG

\#3 PG/PG

\#4 PG/Pt30Rh

\#5 $\mathrm{Pt} 30 \mathrm{Rh} / \mathrm{PuO}_{2}$

\#6 PWPF/PG

\#7 PG/PG

\#8 PG/PG

\#9 PG/PG

\#10 PG/PG

\#11 FWPF/FWPF

II. Axial Interfaces region material

$\# 12^{(\mathrm{a})} \mathrm{FWPF} / \mathrm{PG}$

\#13 PG/PG

\#14 PG/PG

\#15 PG/PG

\#16 PG/PG

\#17 Pt30Rh/PG

\#18 Pt30Rh/PG

\#19 Pt30Rh/Pt30Rh

Interface States

$\begin{array}{cccc}\text { Thermal Expansion } & \text { Translational } & \text { Displacement } \\ \text { high } & \text { low } & \text { high } & \text { low }\end{array}$ Contact Conductances

3500 (BTU hr-1 $\mathrm{ft}^{-2} \mathrm{R}^{-1)}$

1500

3500

1500

1500

1500

2250

2250

150

3500

Thermal Expansion

3500

1500

1500

1500

500

500

500

500

$10^{7}$

150

not applicable

not applicable

not applicable

not applicable

$10^{7}$

1000

$$
\begin{aligned}
& \text { Perfect Alignment } \\
& 3500 \\
& \text { not applicable } \\
& \text { not applicable } \\
& 1500 \\
& \text { not applicable } \\
& \text { not applicable } \\
& \text { not applicable } \\
& \text { not applicable }
\end{aligned}
$$

500

750

750

not applicable

Notes: (a) The aeroshell end cap insulator cap interface (\#12) and the insulator cap/middle insulator body (\#15) were assumed in a high compression state due to pre-loading during assembly.

(b) For the side-on spinning flight orientation, all radial interfaces for the 'thermal expansion' and the 'translational displacement' interface states were assumed to be in a high compression state due to high rotational rates about the longitudinal axis.

(c) For the random tumbling flight orientation all radial and axial interfaces were assumed to be in a high compression state for the 'thermal expansion' interface state due to high rotational rates about all three axes. 
The Johns Hopkins University

Applied Physics Laboratory

Laurel, Maryland 20723-6099

Table 7

Iniatial Reentry Temperature Distribution

LWRHU Assembly

$\begin{array}{llll}\text { Component } & \text { Initial Node } & \text { Final Node } & \text { Temperature, }\end{array}{ }^{\circ} R$

Aeroshell Body 1

Aeroshell End Cap $\quad 127$

126

560,0

210

560.0

Outer Insulator Sleeve

211

231

780.0

(upper layer)

Outer Insulator Sleeve $\quad 435$

462

780.0

(lower layer)

Middle Insulator Sleeve

232

252

875.0

(upper layer)

Middle Insulator Sleeve 463

(lower layer)

Inner Insulator Sleeve $\quad 253$

483

875.0

266

1105.0

Clad

267

281

280

308

322

1405.0

Fuel Pellet

309

1410.0

Clad End Cap

323

End Insulator-Region $12 \quad 351$

350

1405.0

End Insulator-Region $11 \quad 379$

378

870.0

399

880.0

End Insulator-Region $10 \quad 400$

420

890.0

End Insulator-Region 9421

434

900.0

910.0 
TABLE 8

Summary of LWRHU Assembly Engineering Models

Cassini/VEEJGA Aerothermodynamics ${ }^{(\mathbf{a})}$

\section{Aerothermodynamics}

Engineering Models ${ }^{(\mathbf{b})}$

Features/Discussion

\section{Reference Parameters ${ }^{(c)}$}

\section{Convective heat flux \\ - reference conditions}

- model...q $q_{c, \text { ref }}$

- documentation ${ }^{(d)}$

(i) Detra, Kemp and Riddell, ref. 91

(ii) Fay and Riddell, ref. 92

2. Shock layer radiative heat flux

- reference conditions

- models.... $\mathrm{q}_{\mathrm{r}, \mathrm{ref}}$

(i) radiation transport

(ii) flow field

(iii) effective spherical radius ${ }^{(e)}$, side-on LWRHU

- documentation

(i) Nicolet, ref. 99

(ii) Sutton \& Hartung, ref. 96

(iii) Ambrosio \& Wortman, ref. 101
1. 'particle' conduction and mass diffusion heat flux, BTU $\mathrm{ft}^{-2} \mathrm{sec}^{-1}$ - $1 \mathrm{ft}$ sphere; continuum, laminar stagnation heating; cold wall; zero mass transfer and radiation coupling.

- $\left[17,600 /(\mathrm{R})^{0.5}\right]\left(\rho_{\infty} / \rho_{s 1}\right)^{0.5}\left(\mathrm{~V}_{\infty} / \mathrm{V}_{\mathrm{c}}\right)^{3.15}$, $\mathrm{Btu} / \mathrm{ft}^{-2} \mathrm{sec}^{-1}$; Figure 50

- good agreement in dissociation/ionization regimes; based on Fay $\&$ Riddell theory and shock tube heat transfer data.

(i) correlation and experimental data.

(ii) background and underlying theory.

\section{Btu $\mathrm{ft}^{-2} \sec ^{-1}$}

- stagnation streamline heat flux based on inviscid, equilibrium flow; effective spherical radius for side-on LWRHU at mid-span. (i) RAD/EQUIL code

(ii) Radiating Inviscid Flow Stagnation

(iii) $R_{\text {eff sph }}=2.70 R_{\text {LwRHU }}$ Point (RIFSP) code.

(i) detailed spectral absorption coef; continuum and atomic line transport, themodynamic equilibria; self-absorption.

(ii) radiation coupled, inviscid, stagnation streamline flow field.

(iii) conservative estimate of effective radius based on adiabatic shock standoff correlations.

(i) user's manual, RAD/EQUIL code

(ii) radiation tables based on RAD/EQUIL - RIFSP solutions.

(iii) adiabatic stagnation shock stand-off correlations 
Table 8, Page 2

Aerothermodynamics

I. (continued)

3. Free molecule heat flux - reference condition

- model...qqim, ref

- documentation

(i) Probstein, ref. 95

S

\section{Pressure}

- reference conditions

- model...pa, ref

- documentation

(i) Wittliff \& Curtis, ref. 8.2

(ii) Marrone, ref. 8.3

\section{Enthalpy}

- reference conditions

- model... $\mathrm{H}_{\mathrm{t} \text {,ef }}$

- documentation

(i) NASA 1135, ref. 8.1

\section{Body Reference Parameters ${ }^{(\text {() }}$}

\section{Convective heat flux - reference conditions \\ - model... $q_{e, \text { bref }} / q_{c, \text { ref }}$ \\ - documentation}

(i) Perini, ref. 105
Engineering Models ${ }^{\left({ }^{()}\right.}$

Features/Discussion
3. Btu $\mathrm{ft}^{-2} \mathrm{sec}^{-1}$
- unit thermal accommodation coefficient.

- $\left(\rho_{\infty} \mathrm{V}_{\infty}\right)^{3} / 2 \mathrm{~J}$

(i) background and theory.

4. stagnation pressure aft of normal shock, atmospheres. - normal shock jump conditions; thermodynamic equilibrium air.

$\gamma \approx 1.35$ to correlate 'real gas' normal shock data.

(i) normal shock tables; thermodynamic equilibrium air.

(ii) same as (i)

5. stagnation enthalpy, Btu $\mathrm{lbm}^{-1}$

- 1-D inviscid, adiabatic flow.

- $\mathrm{h}_{\infty}+\left(\mathrm{V}_{\infty}\right)^{2 / 2 \mathrm{~g} J}$

(i) compressible flow and shock wave tables, perfect air.

1. Btu $\mathrm{ft}^{-2} \sec ^{-1}$

- see I.1 discussion; mid-span of side-on LWRHU.

- $1 /(2)^{0.5}\left(\beta_{\text {so, LWRHU }} / \beta_{\text {ref }}\right)^{0.5}\left(\mathrm{R}_{\text {Tef }} / \mathrm{R}_{\mathrm{LWRHU}}\right)^{0.5}$ - transformation from ref sphere to side-on LWRHU at mid-span. Figure 51

(i) stagnation velocity gradient $(\beta)$ theory and exp data for various geometries. 


\section{II. (continued)}

2. Shock layer radiative heat flux

3. Free molecule heat flux

4. Pressure

5. Enthalpy
2. see 1.2 discussion

3. see 1.3 discussion

4. see $\mathrm{I} .4$ discussion

5. see I.5 discussion

\section{Adjustments, Body Reference Parameters}

1. Precursor heating factor

- model...F $F_{r, \infty}$

- documentation

(i) Lasher \& Wilson, ref. 106

2. Radiation coupling factor

- model...F $F_{c, m}$

$\approx 1.0-8.57 \mathrm{E}-6(65000-30000)$
1. multiplier on body reference radiative heat flux; pre-shock heating effect

$\bullet \approx 1.0+6.0 \mathrm{E}-6(65000-40000)$

- documentation

(i) Howe \& Viegas, ref. 112

(ii) Hoshizaki \& Wilson, ref. 113
- $15 \%$ increase at $65,000 \mathrm{fps}$; engineering judgement based on limited numerical precursor data.

(i) background theory; numerical data.

2. multiplier on body reference convective heat flux; modification of $\mathrm{q}_{\mathrm{a}, \text { bref }}$ surface gradients due to shock layer radiation effects.

- $30 \%$ decrease at $65,000 \mathrm{fps}$; engineering judgement based on limited small radius body coupling data; analyses considered no adjustment due to uncertainty in data; conservative.

(i) numerical data

(ii) numerical data. 
Table 8, Page 4

\section{Aerothermodynamics}

Engineering Models ${ }^{(b)}$

Features/Discussion

\section{Local Parameter Factors ${ }^{(\mathrm{g})}$, Side-On Stable (SOS) Orientation,}

A. Cylindrical Surface SOS Factors, $F(\theta, \alpha, z / L))_{x, 00, c \infty}$

1. General Method, $F(\theta, \alpha=90, z / L))_{x, \text { sos, } \alpha} \quad$ 1. $[F(\theta, 90,0) F(0,90, z / L)]_{x, s 0 s, c s}$

\section{2.a Convective factor, $\theta$-direction}

$$
\text { - model }
$$

(i) $\mathrm{F}(\theta, 90,0)_{c, s 00, c \infty}, \theta \leq 90^{\circ}$

(ii) $\mathrm{F}(\theta, 90,0)_{c, 00,, \infty}, \theta \geq 90^{\circ}$

- documentation

(i) Kemp, Rose and Detra, ref. 117

(ii) Lees, ref. 116

(iii) SNAP-27, ref. 8.4

2.b Convective factor, $z /$-direction

- model...F $\mathrm{F}(0,90, z \Omega \mathrm{L})_{\mathrm{c}, 504 . \infty}$

- documentation

(i) Knight, ref. 119
- Figure 53, (Multi-Hundred Watt Heat Source experimental data).

A. angle of attack, $\alpha={ }^{\circ}$ for a side-on orientation.

1. Local parameter ( $x$ ) factor is the product of the local $\theta$ value at midspan times the local axial value along the stagnation line.

(i) thermodynamic equilibrium theory; slightly more conservative than the Galileo/VEEGA model (Lees).

(ii) engineering judgement based on SNAP-27 base heating survey; values not influential on aeroshell stagnation response.

(i) VEEJGA theoretical model; experimental data.

(ii) VEEGA theoretical model.

(iii) theoretical and experimental base heating schedules.

- stagnation line heating distributions for right cylinder geometry.

(i) Multi-Hundred Watt experimental heating distributions. 
Table 8, Page 5

\section{Aerothermodynamics}

\section{IV. (continued)}

3.a Shock layer radiative factor, $\theta$-direction - model

(i) $\mathrm{F}(\theta, 90,0)_{r, \text { sol.ces }}, \theta \leq 90^{\circ}$

(i) $\approx \cos ^{3 / 2} \theta+B \sin ^{2} \theta$ $B$ fitted graphically, Figure 54

(ii) $F(\theta, 90,0)_{r, s o s, c s}, \theta \geq 90^{\circ}$

(ii) zero base heating

$\dot{0}$

- documentation
(i) Olstad, ref. 102

(ii) Olstad, ref. 112

3.b Shock layer radiative factor, $\mathrm{z} / \mathrm{L}$-direction

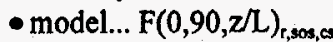

- Figure 55

- documentation

(i) Cassini RTG Program MTPR,

$$
\text { ref. } 123
$$

4.a Free molecule heating factor, $\theta$-direction - model
(i) $\mathrm{F}(\theta, 90,0)_{\mathrm{fm}, \mathrm{sos}, \mathrm{cs}}, \theta \leq 50^{\circ}$
(ii) $\mathrm{F}(\theta, 90,0)_{\mathrm{fm}, 00, \mathrm{sa}}, \theta \geq 90^{\circ}$
(i) Figure $52, \cos (\theta)$

- documentation

(i) Probstein, ref. 95
-

(i) function bounds NASA-LRC (Olstad) inviscid, radiating flow field numerical data; 6 step model for absorption coefficient; continuum and atomic line transport; thermodynamic equilibria; self-absorption; radiation cooling.

(ii) engineering judgement based on Olstad code's data; values not influential on aeroshell stagnation response.

(i) user's guide for the Olstad code.

(ii) background theory; uv spectra contribution to absorption coefficient updated in the code.

- engineering judgement based on CFD radial distributions for a flat faced cylinder (GPHS-Lockheed Martin) with and without ablation.

(i) CFD heating distributions for the broadface stable GPHS module.

(ii) values not influential on aeroshell stagnation response.

(i) background and theory. 
Table 8, Page 6

Aerothermodynamics

Engineering Models $^{(\mathbf{b})}$

Features/Discussion

\section{IV. (continued)}

4.b Free molecule heating factor, $\mathrm{z} /$-direction - $\mathrm{F}(0,90, \mathrm{z} / \mathrm{L})_{\mathrm{m}, \mathrm{cos}, \mathrm{ar}}$

- no variation along stagnation line due to end effects.

5. Static pressure factor, $\theta \& z / L$ directions - model

(i) $F(\theta, 90, z / L)_{p, s o s, c s}, \theta \leq 90^{\circ}$

- Figure 56

(i)

(i) circumferential pressure factors as a function of axial distance. $C_{p}(\theta) / C_{p, b r e f}=0.261+0.441 \cos \theta+0.217 \cos 2 \theta+0.064 \cos 3 \theta+0.016 \cos 4 \theta+0.005 \cos 5 \theta-0.001 \cos 6 \theta, z / L=0.0$ $C_{r}(\theta) / C_{p b}=0.250+0.431 \cos \theta+0.230 \cos 2 \theta+0.079 \cos 3 \theta+0.018 \cos 4 \theta+0.005 \cos 5 \theta-0.001 \cos 6 \theta, z / L=0.5$. $\mathrm{C}_{\mathrm{p}}(\theta) / \mathrm{C}_{\mathrm{p}, \text { bref }}=0.219+0.401 \cos \theta+0.242 \cos 2 \theta+0.106 \cos 3 \theta+0.035 \cos 4 \theta+0.014 \cos 5 \theta-0.003 \cos 6 \theta, z / \mathrm{L}=1.0$

(ii) $F(\theta, 90, z / L))_{p, c o s, a, c}, \theta \geq 90^{\circ}$

- documentation

(i) Miller, ref. 124

(ii) Gregorek and Korkan, ref. 125

(ii) 2 to $4 \%$

(ii) based on experimental data base.

(i) correlations and experimental data.

(ii) correlation and experimental data; Galileo/VEEGA

6. Recovery enthalpy factor, $\theta \& \mathrm{z} / \mathrm{L}$ directions - model

(i) $\mathrm{F}(\theta, 90, \mathrm{z} / \mathrm{L})_{\mathrm{Hr}, \mathrm{sos}, \mathrm{cs}}, \theta \leq 90^{\circ}$

(ii) $\mathrm{F}(\theta, 90, \mathrm{z} / \mathrm{L})_{\mathrm{Hr}, \mathrm{sos}, \mathrm{cs}}, \theta \geq 90^{\circ}$

- documentation

(i) NASA Report 1135, ref. 8.1

(ii) Gregorek and Korkan, ref. 125

- Galileo/VEEGA model for windward and leeward factors.

(i) isentropic expansion to Gregorek's pressure distribution; uniform axial distribution; thermodynamic equilibrium air; recovery enthalpy ratio equated to recovery temperature ratio (infers perfect gas).

(ii) Galileo/VEEGA model; arbitrarily set to $100 \%$ which underestimates leeward ablation; not influential on aeroshell stagnation response.

(i) compressible gas dynamics tables.

(ii) static pressure distribution driving isentropic expansion. 
Aerothermodynamics

Engineering Models ${ }^{(\mathbf{b})}$

Features/Discussion

\section{IV. (continued)}

B. End Face SOS Factors, $F(\delta, \alpha, r / R)_{X, \infty 0, e f}$

1. Convective heating factor - model...F $(\delta, 90, \mathrm{r} / \mathrm{R})_{c, \text { sos, ef }}$

- $10 \%$, uniform distribution over face
B. Factors approximated since this surface not influential on aeroshell stagnation response for this flight orientation.

- conservative estimate in strong expansion flow region; act to reduce energy losses from end face.

\section{Radiative \& free molecule heating factors}

- models... $F(\delta, 90, r / R)_{r, \text { sos, ef }}$,

$$
\text { ... } F(\delta, 90, r / R)_{\text {fm,sos, ef }}
$$

3. Static pressure \& recovery enthalpy factors

- models... $F(\delta, 90, r / R)_{p, s o s, e f}$

$$
\text { ... } F(\delta, 90, r / R)_{1 \mathrm{Hr}, 00 \mathrm{~s}, \mathrm{ef}}
$$

$\bullet 0 \%$

\section{Parameter Factors, Side-On Spinning (SOSP)Orientation}

A. assumes high spin rates to permit smearing via integration of local parameter factors established for the side-on stable orientation. Local leeward factors are now influential on aeroshell response and have been adjusted as noted.

\section{General Method}

1. $F(\theta, 90, z / L)_{\mathrm{X}, \text { sosp,cs }}=\int F(\theta, 90, z / L)_{\mathrm{X}, \text { sos,cs }} d \theta, 0^{\circ} \leq \theta \leq 180^{\circ}$

2. Convective, free molecule heating spin factors

3. Shock layer radiative heating local factor

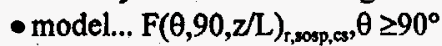

- $10 \%$ at $\theta=120^{\circ}$ to $5 \%$ at $\theta=180^{\circ}$.
2. no leeward adjustments on local factor models presented and discussed in IV.A.2,4

3. adjusted leeward local radiation factors.

- engineering judgement; conservative estimate. 
Table 8, Page 8

Aerothermodynamics

\section{V.A. (continued)}

4. Static pressure spin factor

- model... $F(\theta, 90, z / \mathrm{L})_{p, \text { sosp }, \boldsymbol{a}}$

- $2 \%$ at $\theta \geq 120^{\circ}$

- documentation

(i) Gregorek and Korkan, ref. 125

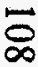

\section{Recovery enthalpy spin factor}

- model... $F(\theta, 90, \mathrm{z} / \mathrm{L})_{\mathrm{Hr}, \text { onp }, \mathrm{cs}}$

- $\mathrm{H}_{\mathrm{r}}(\theta) / \mathrm{H}_{\mathrm{r}}(0) \approx \mathrm{T}_{\mathrm{r}}(\theta) / \mathrm{T}_{\mathrm{r}}(0)$

(i) shallow reentries, $\gamma \leq 15^{\circ}$

(ii) steep reentries, $\gamma \geq 15^{\circ}$

- documentation

(i) Dogra, ref. 129

(ii) Bulmer, ref. 130

B. End Face SOSP Factors, $F(\delta, \alpha, r / R)_{x, s o s p, e f}$

\section{Parameter Factors, Random Tumbling (RT) Orientation}

\section{Features/Discussion}

- adjusted base separated flow according to experimental data of Gregorek and Korkan.

(i) experimental data and correlation.

5. adjusted leeward local recovery enthalpy factors.

- identity based on adiabatic, perfect air; assumes base

Mach \# $\approx 0.5$; recovery factor, $r=0.85$.

(i) numerical temperature data of Dogra, et. al. for laminar base flow.

(ii) experimental temperature data of Bulmer for laminar base flow.

B. same models as IV.B; not influential on aeroshell stagnation response.

VI. random tumbling involves 3-axis rotation wherein both surfaces are influential on aeroshell response; assumes high rotational rates about axes to permit smearing via integration on $\theta$ and $\alpha$. 


\section{A. (continued)}

A. Cylindrical Surface RT Factors

1. General Method

1. random tumbling factor $\mathrm{X}$ is the product of the of the spin factor for parameter $X$ (from

Section V.A.) times the angle of attack factor of parameter X's stagnation value at midspan.

- model... $F(\theta, \alpha, z / L))_{\mathrm{X}, \mathrm{r}, \boldsymbol{}}$

$=\left[F(\theta, 90, z / \mathrm{L})_{\mathrm{X}, \text { sosp, }, \mathrm{se}} F(0, \alpha, 0)_{\mathrm{X}, \mathrm{rns}}\right]$ $F(0, \alpha, 0)_{\mathrm{x}, \pi, \omega}=(1 / 2 \pi \sin \alpha d \alpha) \int F(0, \alpha, 0)_{\mathrm{X}, \pi, \omega} 2 \pi \sin \alpha d \alpha, 0^{\circ} \leq \theta \leq 90^{\circ}$

- documentation

(i) Klett, ref. 134

2. Convective heting local $\alpha$ factor

- model...F $(0, \alpha, 0)_{c, r, c s}$

$\bullet=\cos ^{1.1} \alpha+0.05 \sin ^{2} \alpha$, Figure 57

- documentation

(i) Sayano \& Greenwald, ref. 139

(ii) Berquaum, ref. 140

3. Shock layer radiative heating local $\alpha$ factor

- model... $F(0, \alpha, 0)_{r, n, a}$

$\bullet=\cos \alpha+0.10 \sin ^{2} \alpha$

4. Free molecule heating random

tumbling factor... $F(\theta, \alpha, \mathrm{Z} / \mathrm{L})_{\mathrm{fm}, n, \mathrm{~m}}$
- modification of Klett's method for random tumbling right cylinder.

(i) drag coefficient and convective heating; right cylinder geometry; variety of flight attitudes.

2. convective heating at stagnation line's midspan location as a function of $\alpha$.

- conservative estimate; bounds experimental data in low $\alpha$ range.

(i) background and correlation.

(ii) experimental stagnation data at $\alpha$.

3. no available numerical or experimental data for cylinders at $\alpha$; model based on engineering judgement.

- guided by side-on stable convective and radiative heating local factor distributions (refer to IV.A.2a,3a).

4. taken directly from Klett. 
Table 8, Page 10

Aerothermodynamics

Engineering Models ${ }^{(b)}$

Features/Discussion

VI. A. (continued)

5. Static pressure local $\alpha$ factor - model... $\mathrm{F}(0, \alpha, 0)_{p, n, a}$

- correlation based on shuttle's external tank experimental

- documentation

(i) Carroll, ref.118 test data at $\alpha$.

B. End Face RT Factors

1. Convective heating local $\alpha$ factor - model

(i) $\mathrm{F}(--, \alpha, r / \mathrm{R})_{\mathrm{c}, \mathrm{r}, \mathrm{ef}}, \alpha \leq 90^{\circ}$

(i)

$\left[\mathrm{q}_{c}(\mathrm{r} / \mathrm{R}) / \mathrm{q}_{\mathrm{c}}(0)\right]_{\alpha=0} \approx 1.0+0.6(\mathrm{r} / \mathrm{R})^{3.3}$, Figure $58 \ldots$

Figure 59 schedule...

$1 /(2)^{0.5}\left(\beta_{\text {ef,LWRHU }} / \beta_{\text {so,LWRHU }}\right)^{0.5}$ (i) correlation, experimental data (external tank)

- assumes recovery temperature ratio equates to recovery

enthalpy ratio; implies perfect air gas dynamics; no end effects.

(i) background, correlation, experimental data.

(ii) experimental data.

(i) sweep angle effects not considered; factor comprised of end face heating distribution at $\alpha=0^{\circ}$, average end face heating as $f\left(\alpha / \alpha=0^{\circ}\right)$ and transformation of normalization variable back to body reference value.

end face heating distribution at $\alpha=0^{\circ}$,

average heating to end face at $\alpha / \alpha=0^{\circ}$.

normalization to body reference condition. 
Table 8, Page 11

\section{Aerothermodynamics}

\section{B. (continued)}

\section{1. (continued)}

- model
(ii) $\mathrm{F}(--, \alpha, \mathrm{r} / \mathrm{R})_{c, r, f,}, \alpha \geq 90^{\circ}$

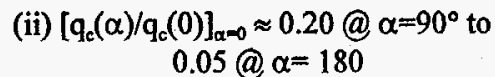

- documentation
(i) Klett, ref. 134
(ii) Stoney, ref. 143
(iii) Kemp, et. al., ref. 117
(iv) Trimmer, ref. 141

2. Shock layer radiative heating local factor

- model

(i) $\mathrm{F}(\delta, \alpha, \mathrm{r} / \mathrm{R})_{\mathrm{r}, \mathrm{t} \text { ef }}, \alpha \leq 90^{\circ}$

(i) $R_{\text {eff sph,ef }}=1.545(\mathrm{~K}-1)^{0.5} \mathrm{R}_{\mathrm{LWRHU}} \ldots$

$$
R_{\text {eff sph,bref }}=2.70 R_{L W R H U} \cdots
$$$$
F(0,0, r / R)_{r, r, e f}=\left(R_{\text {eff sph,ef }} / R_{\text {eff sph,bref }}\right)^{n}, n=0.40 \text { steep ... }
$$$$
\mathrm{q}(\delta, 0, \mathrm{r} / \mathrm{R})_{\mathrm{r}, \mathrm{ef}} / \mathrm{q}(0,0, \mathrm{r} / \mathrm{R})_{\mathrm{r}, \mathrm{ef}}=\cos ^{1.1} \delta, 0 \leq \delta \leq 90^{\circ} \ldots
$$

$$
\mathrm{n}=0.65 \text { shallow ... }
$$

$\mathrm{q}(0, \alpha, \mathrm{r} / \mathrm{R})_{\mathrm{r}, \mathrm{ef}} / \mathrm{q}(0,0, \mathrm{r} / \mathrm{R})_{\mathrm{r}, \mathrm{ef}}=\cos ^{1.5} \alpha+0.10 \sin ^{2} \alpha, \ldots$

(ii) $\mathrm{F}(\delta, \alpha, \mathrm{r} / \mathrm{R})_{r, n, f}, \alpha \geq 90^{\circ} \quad$ (ii) $10 \%, 90^{\circ} \leq \alpha \leq 180^{\circ}$ (ii) engineering judgement; conservative, uniform heating on leeward end face as a $f(\alpha)$.

(i) background, theory, heating at $\alpha=0^{\circ}$, Fig.D. 10 .

(ii) experimental data at $\alpha=0^{\circ}$.

(iii) same as (ii).

(iv) stagnation velocity gradients, experimental data, correlation.

2. uniform windward heating at $\alpha=0^{\circ}$; average heating is integrated product of sweep $(\delta)$ and angle of attack $(\alpha)$ dependency.

effective spherical radius by equating stagnation shock stand-offs for sphere and disc; adiabatic shock layer.

effective spherical radius, body reference condition. transformation of $\alpha=0^{\circ}$ end face heating to body reference condition as a normalization factor. engineering judgement; dependency on end face local sweep angle based on cylindrical surface $\alpha$ behavior. engineering judgement; dependency on end face $\alpha$ based on cylindrical surface $\theta$ behavior.

(ii) engineering judgement; uniform heating. 
Table 8, Page 12

\section{Aerothermodynamics}

VI.B. (continued)

\section{2. (continued)}

- documentation

(i) Serbin, ref. 142

(ii) Sutton \& Hartung, ref. 96

\section{Free molecule heating local $\alpha$ factor} - model

(i) $\mathrm{F}(--, \alpha, r / R)_{\mathrm{fm}, \mathrm{n}, \text { of }}, 0^{\circ} \leq \alpha \leq 90^{\circ}$

(ii) $\mathrm{F}(--, \alpha, \mathrm{r} / \mathrm{R})_{\text {fm, th } \text { ef }}, 90^{\circ} \leq \alpha \leq 180^{\circ}$

(i) 0.255

- documentation

(i) Klett, ref. 134

Engineering Models ${ }^{(b)}$

Features/Discussion

4. Static pressure local $\alpha$ factor

- model

(i) $\mathrm{F}(--, \alpha, \mathrm{r} / \mathrm{R})_{\mathrm{p}, \mathrm{th}, \text { ef }}, 0^{\circ} \leq \alpha \leq 90^{\circ}$

(i)

Figure 60 schedule ...

$\mathrm{F}_{3-\mathrm{p}}\left(\sin ^{2} \alpha+0.015 \cos ^{2} \alpha\right) \ldots$

$$
F_{3-D} \approx 0.75
$$

(ii) $\mathrm{F}(--, \alpha, \mathrm{r} / \mathrm{R})_{\mathrm{p}, \mathrm{rt} \text {, of }}, 90^{\circ} \leq \alpha 180^{\circ}$

(ii) $\approx 0.015$ (i) stagnation shock stand-off correlations, sphere and disc.

(ii) radiation tables; used to determine exponent ' $n$ ' in IV.B.2(i).

(i) directly from Klett.

(i) background and theory.

4. assumed end face to be a constant (average) pressure surface but attenuated by a 3-D flow factor $\left(\mathrm{F}_{3-\mathrm{D}}\right)$ to account for flow expansion effects.

(i)

static pressure distribution at $\alpha=0^{\circ}$.

engineering judgement; static pressure dependency on $\alpha$ based on stagnation line $\alpha$ dependency for cylindrical surface.

engineering judgement; attempt to account for flow expansion effects due to disc edge effects.

(ii) engineering judgement; constant (average) value 
Table 8, Page 13

Aerothermodynamics

\section{VI.B. (continued)}

4. (continued)

- documentation

(i) Love, et. al., ref. 149

(ii) Carroll, ref. 118

5. Recovery enthalpy

\section{- model}
(ii) $F(--, \alpha, r / R)_{H, r n, ~ e f}, 90^{\circ} \leq \alpha \leq 180^{\circ}$
(ii) $\approx 0.80$

- documentation

(i) Beckwith \& Gallagher, ref. 136

\section{Corrections to Local Heating Rates}

1. Low density correction, convective heating

- model... $F_{\text {alow } p}$

\section{Features/Discussion}

(i) end face pressure distribution at $\alpha=0^{\circ}$.

(ii) stagnation line pressure distribution at $\alpha$.

5. constant (average) recovery enthalpy at $\alpha$ based on isentropic expansion to static pressure ratio determined in 4. as $f(\alpha)$.

-

(i) engineering judgement; isentropic expansion to end face's static pressure as $f(\alpha)$; used $\gamma \approx 1.2$ to represent real gas expansion.

(ii) engineering judgement; conservative estimate based on leeward recovery temperature experimental data.

-

(i) experimental recovery temperature data in the wake of yawed cylinders.

1. correcting convective heating based on continuum (i.e., high density) theory or data for rarefied flow effects during early reentry.

- semi-empirical engineering bridging relationship based on kinetic theory; assumed to be valid at all locations for sos,rt orientations and windward locations for so. 
Table 8, Page 14
Aerothermodynamics
Engineering Models ${ }^{(\mathrm{b})}$
Features/Discussion

\section{VII. (continued)}

1. (continued)

- documentation

(i) Matting, ref. 152

(i) theory and experimental data.

2. Surface mass transfer (ablation), convective heating

- model

$\pm$ (i) Putz-Bartlett correlation,

(ii) Mickley-Spalding correlation, (refer to Figure 61 schedules)

- documentation

(i) Putz and Bartlett, ref. 153

(ii) Spalding, ref. 155

3. Surface mass transfer (ablation), radiative heating

- model

- documentation

(i) Gupta, et.al., ref. 111

(ii) Moss, ref. 156
- synthesized from data of Gupta, Moss, etc; refer to Figure 62 schedule
- assumed valid at all locations and to linearly decrease from continuum value at merged shock layer threshold to zero at the free molecule threshold

(i) numerical data.

(ii) numerical data

(i) Putz and Bartlett provides blowing effects including localized combustion, injectant chemistry on surface gradients; produced unstable solutions.

(ii) classical blowing relationship; implemented in analyses to provide stable solutions; smaller reduction in convective heating that Putz and Bartlett; assumed valid at all locations.

(i) theory, experimental data and correlations

ii) foundation for Mickley-Spalding correlation. 
Table 8, Page 15

Notes:

(a) Aerothermodynamics refers to convective (i.e., conduction and mass diffusion) heating, shock layer radiative heating, free molecule heating, static pressure and recovery enthalpy. Pressure is included since it is one of the independent variables governing ablation which, in turn, effects

convective heating. Recovery enthalpy is required to correct convective heating for 'hot' wall effects and also to reduce convective heating to a

Stanton number in the surface energy balance boundary conditon.

(b) The prescribed engineering models are, in most instances, (1) either correlations or syntheses of applicable experimental or numerical data or (2) of attack.

engineering judgement based on such data. Use of engineering judgement was particularly the case in accounting for shock layer radiation at angle

(c) The reference parameters are the five aerothermodynamic variables presented in note (a). The trajectory histories of these parameters are determined in the 3DOF code and provided as fundamental input to the RETAP code.

(d) Only the salient references are provided in the table. Further references are provided in the text of the report

(e) The effective spherical radius is determined for the LWRHU in its various considered orientations by equating the stagnation shock stand-off of a right cylinder in its particular orientation (e.g., side-on) to the shock stand-off of a sphere. The resulting effective spherical radius is used to enter the Sutton-Hartung radiation tables (ref. 96) which is based on a radiating, stagnation streamline flowfield for a hemisphere.

(f) The body reference condition for the aerothermodynamic parameters is defined as the mid-span stagnation value for a side-on stable LWRHU.

(g) The local parameter factor is the ratio of the value of the parameter at a local 'nodal' station (on either the cylindrical surface or the end face) by the parameter's body reference value.

Convention:

$\mathbf{F}(,,)_{a, b, c}$ :

$\mathrm{F}(,,$,$) ...the local parameter factor as a function of flight or geometry variables.$

subscript a...aerothermodynamic parameter; c convective heating

$r \quad$ shock layer radiative heating

fm free molecule heating

p. static pressure

$\mathrm{Hr}$ recovery enthalpy

subscript b...flight orientation

sos side-on stable

sosp side-on spinning

it random tumbling

subscript c...LWRHU surface

cs cylindrical surface

ef end face

$\mathbf{F}(-,,)_{a, b, c}:$

-...indicates variable is not in use in determining the factor. 


\section{Nomenclature:}

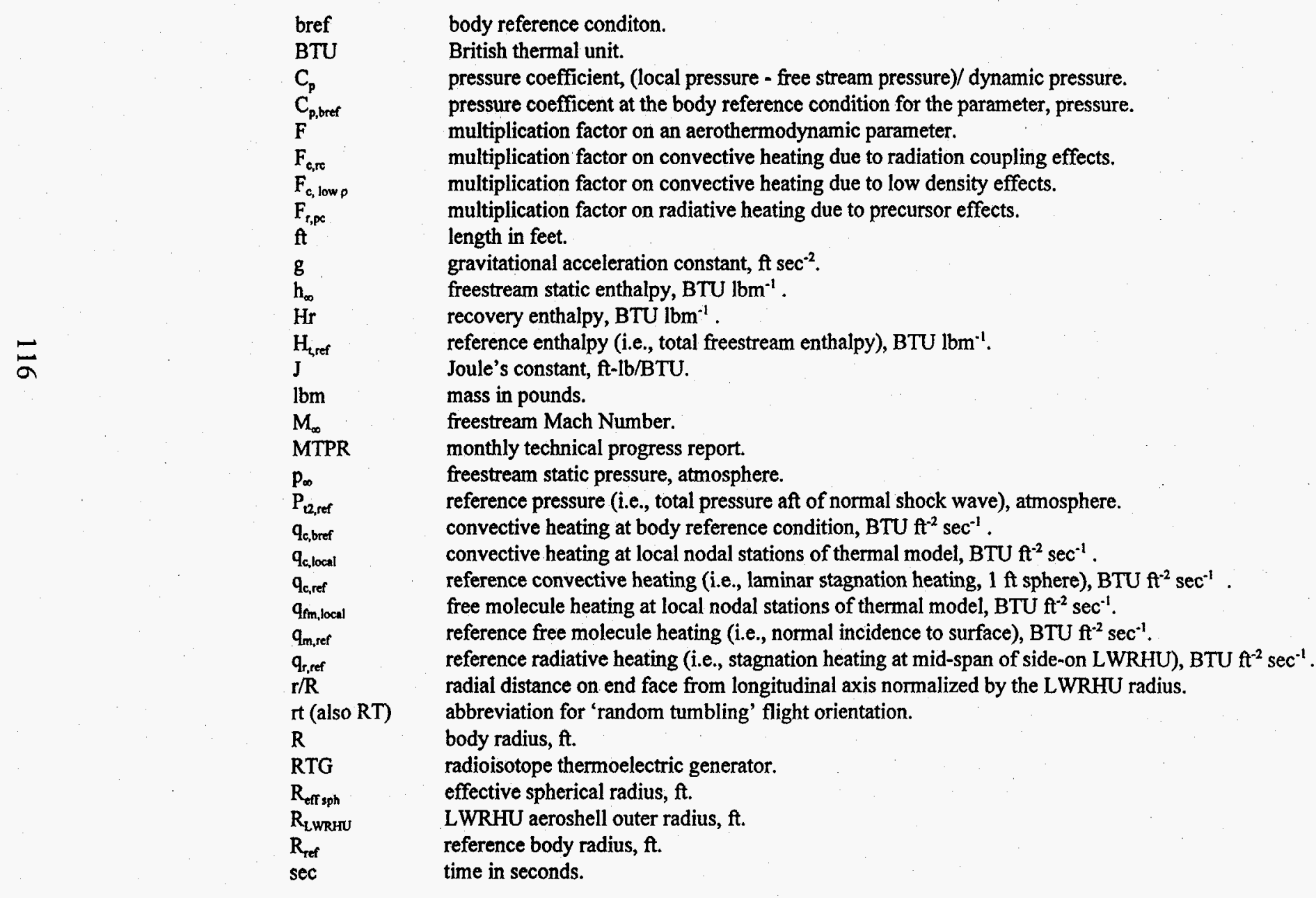

convective heating at local nodal stations of thermal model, BTU $\mathrm{ft}^{-2} \mathrm{sec}^{-1}$

free molecule heating at local nodal stations of thermal model, BTU $\mathrm{ft}^{-2} \mathrm{sec}^{-1}$.

eference free molecule heating (i.e., normal incidence to surface), BTU $\mathrm{ft}^{-2} \mathrm{sec}^{-1}$

(

WRH aeroshell outer radius,

time in seconds. 
Table 8 , Page 17

Nomenclature (continued):

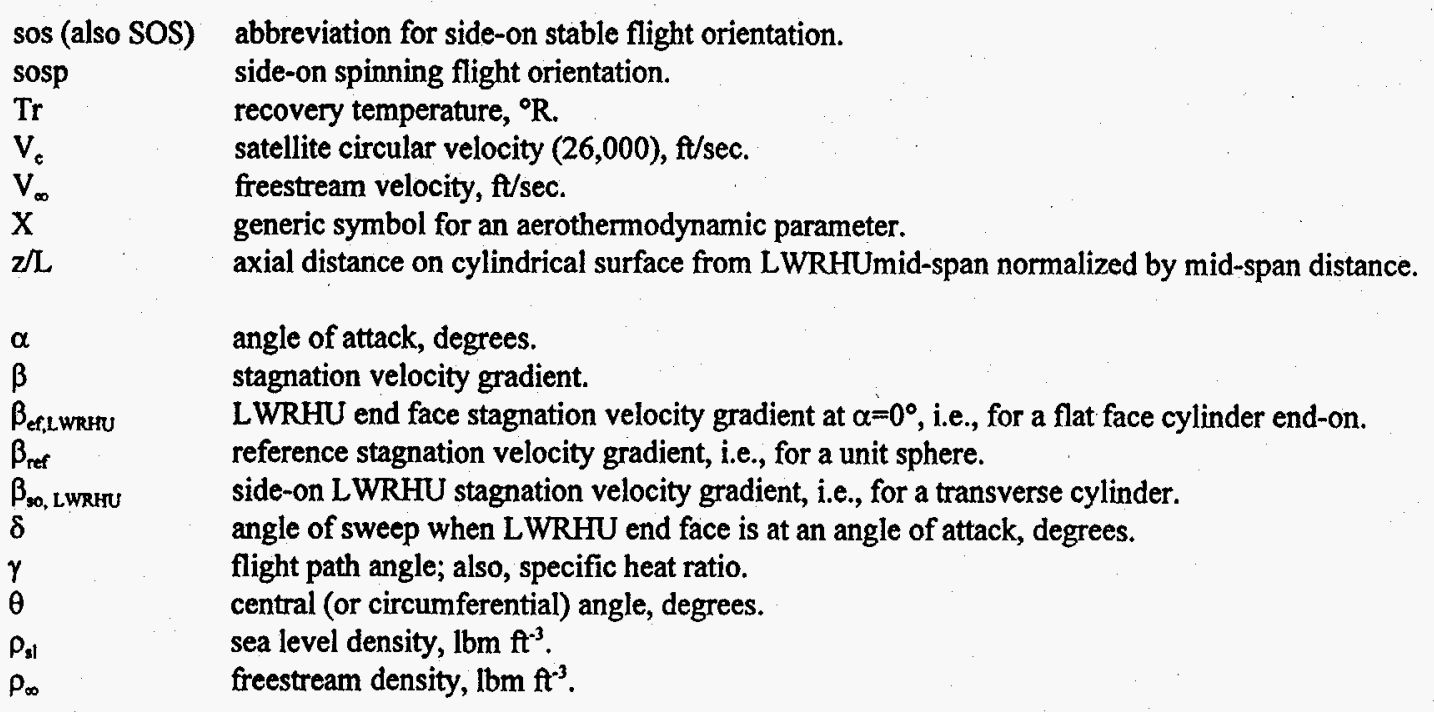




\begin{tabular}{|c|c|c|c|c|c|c|c|c|}
\hline \multicolumn{2}{|r|}{ TABLE 9.} & \multicolumn{7}{|c|}{ Side-On Stable LWRHU 3D Thermal Analyses Results } \\
\hline Reentry Angle & $-5.2^{\circ}$ & $-7.0^{\circ}$ & $-10^{\circ}$ & $-15^{\circ}$ & $-30^{\circ}$ & \begin{tabular}{|c|}
$-50^{\circ}$ \\
\end{tabular} & $-70^{\circ}$ & $-90^{\circ}$ \\
\hline Time of $M=5$ & 218.4 & 59.0 & 33.1 & 19.8 & 9.7 & 6.2 & 5.1 & 4.7 \\
\hline $\begin{array}{l}\text { Time of Peak } \\
\text { gconv }\end{array}$ & 23.45 & 15.61 & 10.00 & 6.30 & 3.20 & 2.18 & 1.88 & 1.77 \\
\hline $\begin{array}{l}\text { Peak Ref } \\
\text { Qconv }\end{array}$ & 1527.66 & 2133.25 & 2714.57 & 3408.54 & 4752.14 & 5843.53 & 6379.77 & 6463.98 \\
\hline $\begin{array}{l}\text { Time of Peak } \\
\text { Qrad }\end{array}$ & 28.77 & 18.78 & 12.03 & 7.47 & 3.75 & 2.38 & 2.03 & 1.92 \\
\hline Peak Ref Qrad & 147.91 & 559.44 & 1300.82 & 2709.51 & 7022.15 & 11061.55 & 13139.76 & 13649.0 \\
\hline $\begin{array}{l}\text { Time at } 758 \\
\text { Ablation }\end{array}$ & $\begin{array}{c}22.61 \\
\text { Node } 1\end{array}$ & $\begin{array}{r}14.41 \\
\text { Node } 1\end{array}$ & $\begin{array}{c}9.39 \\
\text { Node } 1\end{array}$ & $\begin{array}{c}6.10 \\
\text { Node } 1\end{array}$ & $\begin{array}{c}3.36 \\
\text { Node } 64\end{array}$ & $\begin{array}{c}2.40 \\
\text { Node } 64\end{array}$ & $\begin{array}{c}2.06 \\
\text { Node } 64\end{array}$ & $\begin{array}{c}1.97 \\
\text { Node } 64\end{array}$ \\
\hline $\begin{array}{l}\text { Altitude at } \\
758 \text { Ablation }\end{array}$ & $\begin{array}{c}242813.0 \\
\text { Node } i^{\circ}\end{array}$ & $\begin{array}{c}226161.7 \\
\text { Node }{ }^{2}\end{array}$ & $\begin{array}{l}211150.0 \\
\text { Node } i\end{array}$ & $\begin{array}{c}195188.7 \\
\text { Node }{ }^{7}\end{array}$ & $\begin{array}{l}169057.5 \\
\text { Node } 64 \\
\end{array}$ & \begin{tabular}{|c|}
151804.9 \\
Node 64
\end{tabular} & \begin{tabular}{|c|}
143076.2 \\
Node 64
\end{tabular} & $\begin{array}{c}139728.6 \\
\text { Node } 64\end{array}$ \\
\hline $\begin{array}{l}\text { Velocity at } \\
758 \text { Ablation }\end{array}$ & $\begin{array}{l}59462.9 \\
\text { Node } 1\end{array}$ & $\begin{array}{l}58959.2 \\
\text { Node } 1\end{array}$ & $\begin{array}{c}58145.9 \\
\text { Node } 1 \\
\end{array}$ & $\begin{array}{l}56990.2 \\
\text { Node } 1\end{array}$ & $\begin{array}{l}54339.6 \\
\text { Node } 64 \\
\end{array}$ & $\begin{array}{l}51865.0 \\
\text { Node } 64\end{array}$ & $\begin{array}{l}50022.1 \\
\text { Node } 64\end{array}$ & $\begin{array}{l}48846.3 \\
\text { Node } 64 \\
\end{array}$ \\
\hline $\begin{array}{l}\text { Surface Temp } \\
\text { at Failure }\end{array}$ & $\begin{array}{l}6033.6 \\
\text { Node } 1\end{array}$ & $\begin{array}{l}6206.4 \\
\text { Node } 1 \\
\end{array}$ & $\begin{array}{l}6347.5 \\
\text { Node } 1 \\
\end{array}$ & $\begin{array}{l}6504.0 \\
\text { Node } 1 \\
\end{array}$ & $\begin{array}{c}6752.0 \\
\text { Node } 64\end{array}$ & $\begin{array}{c}6926.4 \\
\text { Node } 64 \\
\end{array}$ & $\begin{array}{c}7012.8 \\
\text { Node } 64\end{array}$ & $\begin{array}{c}7041.4 \\
\text { Node } 64\end{array}$ \\
\hline $\begin{array}{l}\text { Clad Temp at } \\
\text { Failure }\end{array}$ & $\begin{array}{c}1635.9 \\
\text { Node } 267\end{array}$ & $\begin{array}{c}1213.8 \\
\text { Node } 267\end{array}$ & $\begin{array}{c}959.0 \\
\text { Node } 267\end{array}$ & $\begin{array}{c}804.2 \\
\text { Node } 267\end{array}$ & \begin{tabular}{|c|}
753.5 \\
Node 267
\end{tabular} & $\begin{array}{c}761.4 \\
\text { Node } 267\end{array}$ & $\begin{array}{c}770.5 \\
\text { Node } 267\end{array}$ & $\begin{array}{c}773.6 \\
\text { Node } 267\end{array}$ \\
\hline $\begin{array}{l}\text { Fuel Temp at } \\
\text { Failure }\end{array}$ & $\begin{array}{c}1223.8 \\
\text { Node } 281\end{array}$ & $\begin{array}{c}921.9 \\
\text { Node } 281\end{array}$ & $\begin{array}{c}821.2 \\
\text { Node } 281 \\
\end{array}$ & $\begin{array}{c}816.9 \\
\text { Node } 281 \\
\end{array}$ & \begin{tabular}{|c|}
858.9 \\
Node 281 \\
\end{tabular} & $\begin{array}{c}891.0 \\
\text { Node } 281 \\
\end{array}$ & $\begin{array}{c}903.0 \\
\text { Node } 281 \\
\end{array}$ & $\begin{array}{c}906.2 \\
\text { Node } 281\end{array}$ \\
\hline $\begin{array}{l}\text { Time at } 100 \% \\
\text { Ablation }\end{array}$ & $\begin{array}{l}25.05 \\
\text { Node } 1\end{array}$ & $\begin{array}{l}15.94 \\
\text { Node } 1\end{array}$ & $\begin{array}{c}10.49 \\
\text { Node } 1\end{array}$ & $\begin{array}{c}6.89 \\
\text { Node } 1\end{array}$ & $\begin{array}{c}3.81 \\
\text { Node } 64\end{array}$ & $\begin{array}{c}2.73 \\
\text { Node } 64\end{array}$ & $\begin{array}{c}2.35 \\
\text { Node } 64\end{array}$ & $\begin{array}{c}2.27 \\
\text { Node } 64\end{array}$ \\
\hline $\begin{array}{l}\text { Altitude } 1008 \\
\text { Ablation } \\
\end{array}$ & $\begin{array}{l}238193.6 \\
\text { Node } i^{6}\end{array}$ & $\begin{array}{c}218778 i^{7} \\
\text { Node } i\end{array}$ & $\begin{array}{c}202042.7 \\
\text { Node } i^{7}\end{array}$ & $\begin{array}{c}184649.3 \\
\text { Node } i^{3}\end{array}$ & $\begin{array}{l}157780.0 \\
\text { Node } 64\end{array}$ & $\begin{array}{l}139596.5 \\
\text { Node } 64\end{array}$ & $\begin{array}{l}130280.9 \\
\text { Node } 64\end{array}$ & $\begin{array}{l}126327.4 \\
\text { Node } 64\end{array}$ \\
\hline $\begin{array}{l}\text { Velocity } 1008 \\
\text { Ablation }\end{array}$ & $\begin{array}{l}57483.3 \\
\text { Node } 1 \\
\end{array}$ & $\begin{array}{l}56495.5 \\
\text { Node } 1 \\
\end{array}$ & $\begin{array}{l}55144.7 \\
\text { Node } 1 \\
\end{array}$ & $\begin{array}{l}53193.4 \\
\text { Node } 1 \\
\end{array}$ & $\begin{array}{r}49367.3 \\
\text { Node } 64 \\
\end{array}$ & $\begin{array}{l}45299.1 \\
\text { Node } 64 \\
\end{array}$ & $\begin{array}{l}42253.7 \\
\text { Node } 64\end{array}$ & \begin{tabular}{|l|}
40259.1 \\
Node 64 \\
\end{tabular} \\
\hline $\begin{array}{l}\text { Surface Temp } \\
\text { at Failure }\end{array}$ & $\begin{array}{l}6060.9 \\
\text { Node } 1 \\
\end{array}$ & $\begin{array}{l}6255.6 \\
\text { Node } 1 \\
\end{array}$ & $\begin{array}{r}6408.0 \\
\text { Node } 1 \\
\end{array}$ & $\begin{array}{l}6567.2 \\
\text { Node } 1 \\
\end{array}$ & $\begin{array}{l}6814.5 \\
\text { Node } 64\end{array}$ & $\begin{array}{r}6981.4 \\
\text { Node } 64\end{array}$ & $\begin{array}{c}7053.3 \\
\text { Node } 64\end{array}$ & \begin{tabular}{|c|}
7063.9 \\
Node 64
\end{tabular} \\
\hline $\begin{array}{l}\text { Clad Temp at } \\
\text { Failure }\end{array}$ & $\begin{array}{c}1849.9 \\
\text { Node } 267\end{array}$ & $\begin{array}{c}1352.8 \\
\text { Node } 267\end{array}$ & $\begin{array}{c}1064.7 \\
\text { Node } 267\end{array}$ & \begin{tabular}{|c|}
868.1 \\
Node 267 \\
\end{tabular} & $\begin{array}{c}761.0 \\
\text { Node } 267 \\
\end{array}$ & \begin{tabular}{|c|}
757.4 \\
Node 267 \\
\end{tabular} & \begin{tabular}{|cc}
763.2 \\
Node 267 \\
\end{tabular} & \begin{tabular}{|c|}
765.1 \\
Node 267 \\
\end{tabular} \\
\hline $\begin{array}{l}\text { Fuel Temp at } \\
\text { Failure }\end{array}$ & $\begin{array}{c}1394.1 \\
\text { Node } 281 \\
\end{array}$ & \begin{tabular}{|c|}
1004.6 \\
Node 281 \\
\end{tabular} & \begin{tabular}{|c|}
857.4 \\
Node 281 \\
\end{tabular} & $\begin{array}{c}821.1 \\
\text { Node } 281\end{array}$ & $\begin{array}{c}846.9 \\
\text { Node } 281 \\
\end{array}$ & \begin{tabular}{|c|}
879.7 \\
Node 281 \\
\end{tabular} & $\begin{array}{c}892.6 \\
\text { Node } 281 \\
\end{array}$ & $\begin{array}{c}895.6 \\
\text { Node } 281 \\
\end{array}$ \\
\hline $\begin{array}{l}\text { For Model Node } \\
758 \text { Ablation } \\
1008 \text { Ablation } \\
\text { Reference Qrad } \\
\text { Seference Qconv } \\
\text { Reference } \\
\text { All Reentry A } \\
\text { All Times are } \\
\text { All Temperatur } \\
\text { All Altitudes } \\
\text { All Velocities } \\
\text { All heating ra }\end{array}$ & $\begin{array}{l}\text { implies } \\
\text { is for } \\
\text { ical rad } \\
\text { is for } \\
\text { gles uti } \\
\text { given in } \\
\text { e are gi } \\
\text { are give } \\
\text { are giv } \\
\text { te data }\end{array}$ & $\begin{array}{l}\text { S refer } t \\
133 \text { inches } \\
177 \text { inche } \\
\text { /EQuIL, i } \\
\text { 1s, mid-sp } \\
\text { ft radius } \\
\text { ize Lawren } \\
\text { seconds } \\
\text { en in of } \\
\text { in feet } \\
\text { in ft/s } \\
\text { re given i }\end{array}$ & $\begin{array}{l}\text { an LWRHU } \\
\text { sphere, } \\
\text { ace Liverm } \\
\text { in Btu/ft }\end{array}$ & $\begin{array}{l}\text { stagn } \\
\text { ore? }\end{array}$ & $\begin{array}{l}\text { flow, } c \\
\text { hemist: }\end{array}$ & $\begin{array}{l}\text { 1 wall, } \\
\text { Tables, }\end{array}$ & ero $\mathrm{m}$ & . \\
\hline
\end{tabular}




\begin{tabular}{|c|c|c|c|c|c|c|c|c|}
\hline \multicolumn{2}{|c|}{ TABLE 10} & \multicolumn{7}{|c|}{ Side-On Spinning LWRHU 3D Thermal Analyses Results } \\
\hline Reentry Angle & $-5.2^{\circ}$ & $-7.0^{\circ}$ & $-10^{\circ}$ & $-15^{\circ}$ & $-30^{\circ}$ & $-50^{\circ}$ & $-70^{\circ}$ & $-90^{\circ}$ \\
\hline Time of $M=5$ & 218.4 & 59.0 & 33.1 & 19.8 & 9.7 & 6.2 & 5.1 & 4.7 \\
\hline $\begin{array}{l}\text { Time of Peak } \\
\text { Qconv }\end{array}$ & 23.45 & 15.61 & 10.00 & 6.30 & 3.20 & 2.18 & 1.88 & 1.77 \\
\hline $\begin{array}{l}\text { Peak Ref } \\
\text { Qconv }\end{array}$ & 1527.66 & 2133.25 & 2714.57 & 3408.54 & 4752.14 & 5843.53 & 6379.77 & 6463.98 \\
\hline $\begin{array}{l}\text { Time of Peak } \\
\text { Qrad }\end{array}$ & 28.77 & 18.78 & 12.03 & 7.47 & 3.75 & 2.38 & 2.03 & 1.92 \\
\hline Peak Ref Qrad & 147.91 & 559.44 & 1300.82 & 2709.51 & 7022.15 & 11061.55 & 13139.76 & 13649.0 \\
\hline $\begin{array}{l}\text { Time at } 758 \\
\text { Ablation }\end{array}$ & $\begin{array}{r}112.80 \\
\text { Node } 34 \\
\end{array}$ & $\mathrm{NF}$ & NF & $\mathrm{NF}$ & NF & NF & $\mathrm{NF}$ & NF \\
\hline $\begin{array}{l}\text { Altitude at } \\
758 \text { Ablation }\end{array}$ & $\begin{array}{l}237633.0 \\
\text { Node } 34\end{array}$ & NF & $\mathrm{NF}$ & NF & NF & NF & NF & NF \\
\hline $\begin{array}{l}\text { Velocity at } \\
758 \text { Ablation }\end{array}$ & $\begin{array}{l}20851.0 \\
\text { Node } 34\end{array}$ & $\mathrm{NF}$ & $\mathrm{NF}$ & NF & NF & NF & NF & NF \\
\hline $\begin{array}{l}\text { Surface Temp } \\
\text { at Failure }\end{array}$ & $\begin{array}{c}3291.4 \\
\text { Node } 34 \\
\end{array}$ & NF & $\mathrm{NF}$ & NF & NF & NF & NF & NF \\
\hline $\begin{array}{l}\text { Clad Temp at } \\
\text { Failure }\end{array}$ & $\begin{array}{r}3756.5 \\
\text { Node } 278 \\
\end{array}$ & NF & $\mathrm{NF}$ & $\mathrm{NF}$ & NF & NF & NF & NF \\
\hline $\begin{array}{l}\text { Fuel Temp at } \\
\text { Failure }\end{array}$ & $\begin{array}{r}3824.23 \\
\text { Node } 304 \\
\end{array}$ & $\mathrm{NF}$ & $\mathrm{NF}$ & NF & NF & NF & NF & NF \\
\hline $\begin{array}{l}\text { Time at } 1008 \\
\text { Ablation }\end{array}$ & NF & NF & NF & $\mathrm{NF}$ & NF & NF & NF & NF \\
\hline $\begin{array}{l}\text { Altitude } 1008 \\
\text { Ablation }\end{array}$ & NF & NF & NF & $\mathrm{NF}$ & NF & NF & NE & NE \\
\hline $\begin{array}{l}\text { Velocity } 1008 \\
\text { Ablation }\end{array}$ & NF & NF & NF & NF & NF & NF & NF & NF \\
\hline $\begin{array}{l}\text { Surface Temp } \\
\text { at Failure }\end{array}$ & NF & NE & NF & NF & NF & NF & NF & NF \\
\hline $\begin{array}{l}\text { Clad Temp at } \\
\text { Failure }\end{array}$ & NF & NF & NF & $\mathrm{NF}$ & $\mathrm{NF}$ & $\mathrm{NF}$ & NF & NF \\
\hline $\begin{array}{l}\text { Fuel Temp at } \\
\text { Failure }\end{array}$ & $\mathbf{N F}$ & $\mathrm{NF}$ & NF & NF & NF & NF & NF & NF \\
\hline $\begin{array}{l}\text { For Model Node } \\
75 \% \text { Ablation } \\
1008 \text { Ablation } \\
\text { Reference } Q_{\text {rad }} \\
\text { spher } \\
\text { Reference } Q_{\text {conv }} \\
\text { All Reentry Ar } \\
\text { Al1 Times are } \\
\text { Al1 Temperatur } \\
\text { All Altitudes } \\
\text { All Velocities } \\
\text { All heating }\end{array}$ & $\begin{array}{l}\text { Location } \\
\text { mplies } 0 \\
\text { implies } 0 \\
\text { is for RA } \\
\text { ical radi } \\
\text { is for } 1 \\
\text { gles util } \\
\text { given in } \\
\text { e are giv } \\
\text { are given } \\
\text { are give } \\
\text { te data a }\end{array}$ & $\begin{array}{l}33 \text { refer } \\
37 \text { inche } \\
177 \text { inch } \\
\text { /EQuIL, } \\
\text { Is, mid-s } \\
\text { Et radius } \\
\text { ze Lawre } \\
\text { econds } \\
\text { in in } \mathrm{F} \\
\text { in feet } \\
\text { in ft/s } \\
\text { given }\end{array}$ & $\begin{array}{l}\text { - Figure } \\
\text { s } \\
\text { nviscid } \\
\text { an LWRHU } \\
\text { sphere, } \\
\text { ce Liver } \\
\text { n Btu/ft }\end{array}$ & $\begin{array}{l}\text { agnation } \\
\text { tagnatior } \\
\text { re Therm }\end{array}$ & $\begin{array}{l}\text { streamli } \\
\text { flow, ce } \\
\text { chemistr }\end{array}$ & $\begin{array}{l}\text { e flow fi } \\
\text { d wall, } \\
\text { Tables, }\end{array}$ & $\begin{array}{l}\text { ld, effec } \\
\text { ero mass }\end{array}$ & tive \\
\hline
\end{tabular}

\section{c: \wp51/leahmem|rhufail2.tab}


The Johns Hopkins University

Applied Physics Laboratory

Laurel, Maryland 20723-6099

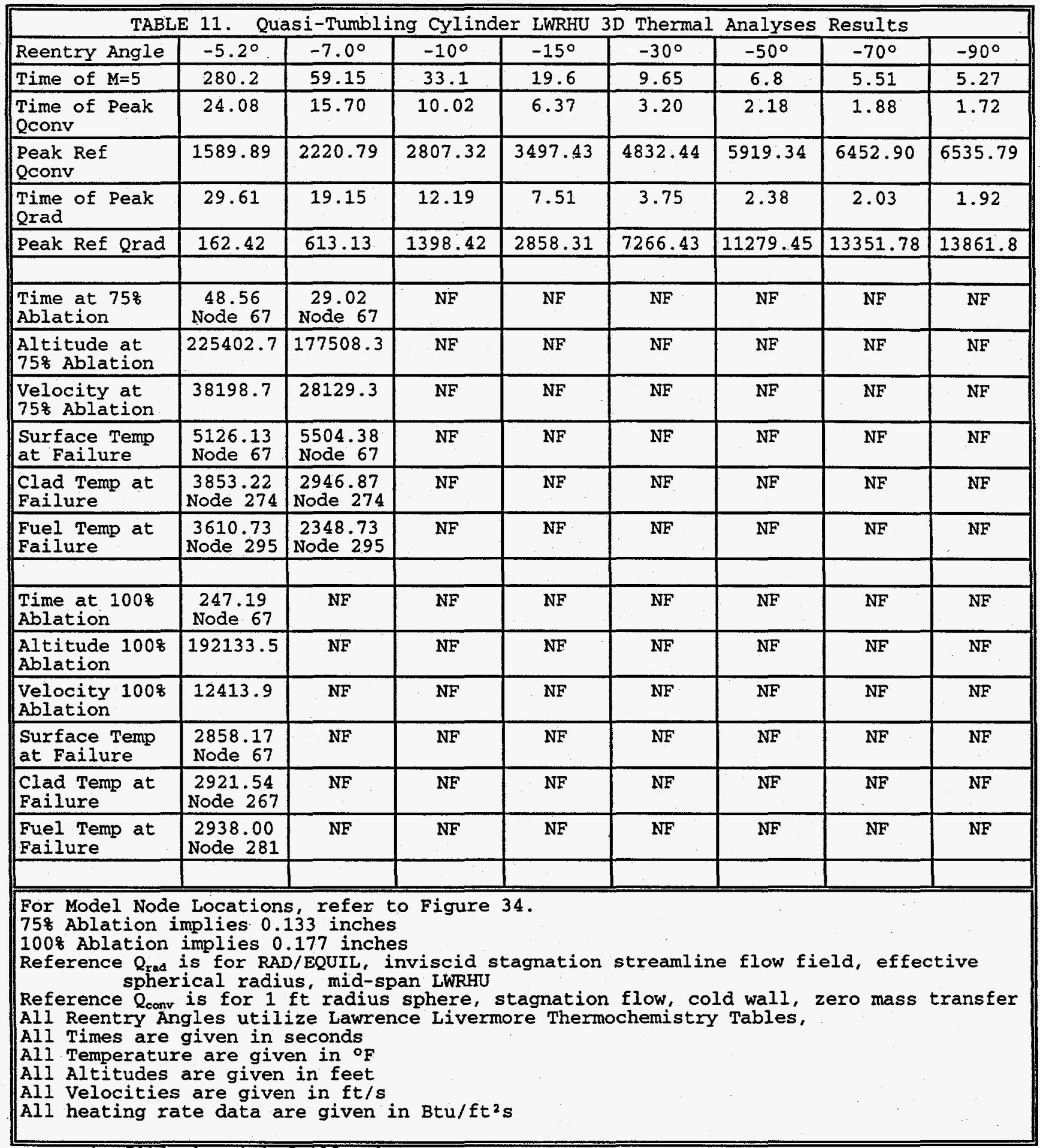

c: |wp5T/leahmem|rhufail2.tab 


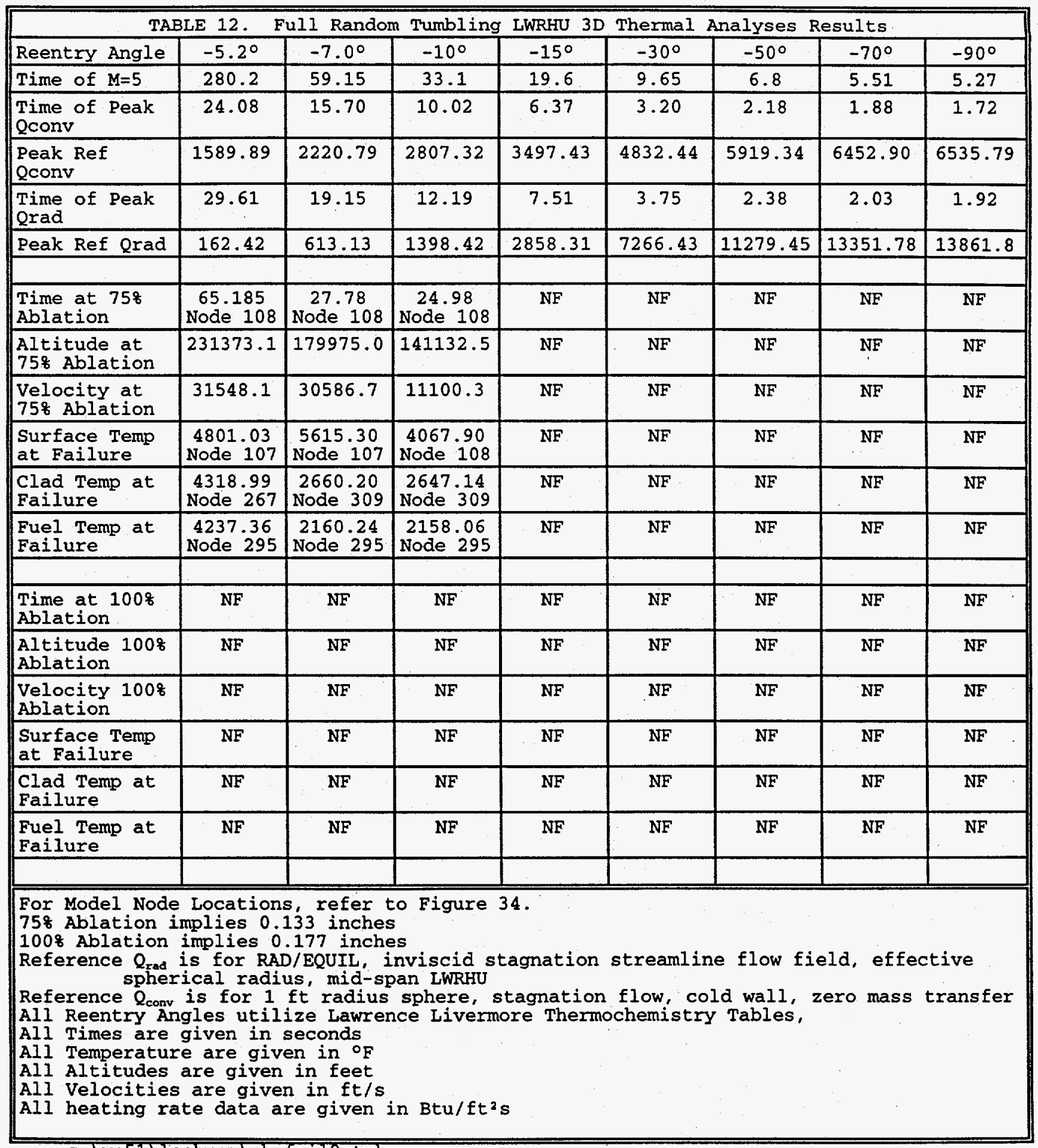


The Johns Hopkins University

Applied Physics Laboratory

Laurel, Maryland 20723.6099

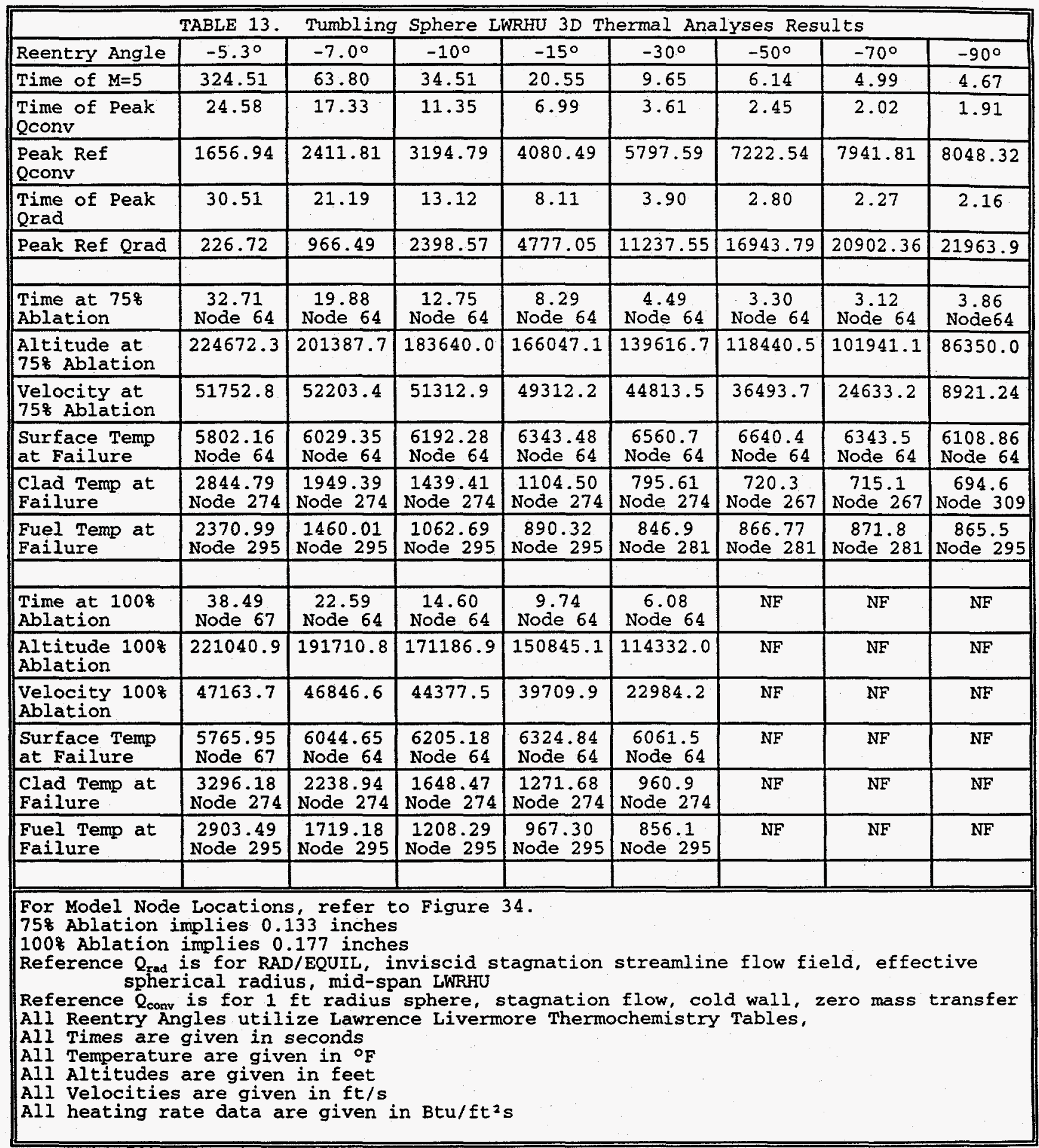

c: \wp51/leahmem\rhufail2.tab 


\begin{tabular}{|c|c|c|c|c|c|c|c|c|}
\hline & & & & & & & & \\
\hline & $-\infty \quad(2) \quad 1$ & & & & & & & $-0.2 \quad(2)$ \\
\hline 75\% Fail Time & 1.96616 & 2.05516 & 2.39577 & 3.36169 & 6.09997 & 9.39213 & 14.41483 & 22.61389 \\
\hline Avg AeroShell & 5452.0193 & 5478.2588 & 5530.6356 & 5595.1521 & 5597.7893 & 5541.1770 & 5448.8789 & 5285.1187 \\
\hline Max Acroshell & 7045.9102 & 7017.3086 & 6930.8984 & 6755.9102 & 6503.9766 & 6351.5742 & 6206.3867 & 6033.5703 \\
\hline Min Aeroshell & 3328.4753 & 3409.6121 & 3629.8784 & 4061.1343 & 4614.1328 & 4819.3594 & 4889.8984 & 4812.6641 \\
\hline Avg End Cap & 4434.3628 & 4500.1078 & 4656.7568 & 4915.8691 & 5127.4048 & 5153.1960 & 5110.9134 & 4983.2692 \\
\hline Max End Cap & 5666.1563 & 5678.6797 & 5657.8945 & 5584.6406 & 5475.7734 & 5492.0352 & 5459.0586 & 5364.1289 \\
\hline Min End Cap & 3112.5569 & 3200.1338 & 3466.8687 & 4005.3608 & 4631.2344 & 4840.3125 & 4908.9727 & 4835.3242 \\
\hline Avg Out Sleeve & 2518.0308 & 2575.2455 & 2758.1268 & 3106.6879 & 3582.3098 & 3810.3045 & 4015.9680 & 4216.8582 \\
\hline Max Out Sleeve & 5258.7813 & 5331.3242 & 5553.8828 & 5852.5703 & 5991.8359 & 5950.7813 & 5881.3320 & 5775.1719 \\
\hline Min Out Sleeve & 747.8887 & 767.6475 & 839.6711 & 1027.9900 & 1460.9282 & 1831.6091 & 2279.3157 & 2848.6450 \\
\hline Avg Mid Sleeve & 662.8381 & 677.6812 & 733.8875 & 886.2973 & 1246.4917 & 1560.8896 & 1953.7409 & 2501.0115 \\
\hline Max Mid Sleeve & 760.0847 & 781.2122 & 859.7593 & 1065.6262 & 1548.5032 & 1933.3140 & 2392.3347 & 2972.2124 \\
\hline Min Mid Sleeve & 590.1060 & 599.4023 & 636.2686 & 743.0627 & 994.5774 & 1238.5000 & 1560.4260 & 2066.0557 \\
\hline Avg In Sleeve & 593.9981 & 599.2815 & 621.9705 & 698.4615 & 901.3259 & 1115.1557 & 1406.1136 & 1869.5860 \\
\hline Max In Sleeve & 596.1294 & 601.3823 & 623.9844 & 700.7617 & 904.8298 & 1118.3967 & 1410.0156 & 1876.2700 \\
\hline Min In Sleeve & 591.9036 & 597.2200 & 620.0042 & 696.3699 & 898.0251 & 1111.9028 & 1401.7407 & 1862.3264 \\
\hline Avg Clad & 774.9070 & 771.7232 & 762.1307 & 753.1737 & 806.7236 & 971.7393 & 1222.3169 & 1643.8018 \\
\hline Max Clad & 781.4653 & 778.2971 & 768.6082 & 758.4150 & 810.6221 & 978.0496 & 1229.9531 & 1651.8779 \\
\hline Min Clad & 766.0762 & 763.0959 & 754.3909 & 748.4192 & 802.6941 & 963.8508 & 1212.3428 & 1634.2603 \\
\hline Avg Fuel & 896.8124 & 893.4793 & 880.9162 & 848.9968 & 814.5463 & 824.2805 & 927.8196 & 1235.3806 \\
\hline Max Fuel & 929.1697 & 926.8726 & 917.3643 & 886.9546 & 830.8838 & 830.2512 & 947.0793 & 1271.0479 \\
\hline Min Fuel & 886.8303 & 883.1592 & 869.5510 & 836.7656 & 808.6201 & 808.2751 & 876.0017 & 1139.7498 \\
\hline Avg End Plug & 1423.6846 & 1464.8266 & 1607.6382 & 1934.8764 & 2394.5532 & 2745.2142 & 3083.3926 & 3449.5940 \\
\hline Max End Plug & 3815.5601 & 3898.6655 & 4164.3359 & 4627.3477 & 4950.4258 & 5105.5781 & 5177.0313 & 5175.7422 \\
\hline Min End Plug & 587.6721 & 597.3330 & 623.6125 & 696.6714 & 872.9519 & 1090.1831 & 1391.2522 & 1861.0664 \\
\hline & & & & & & & & \\
\hline \multicolumn{9}{|c|}{ All reentry angles utilize Lawrence Livermore Thermochemistry tables to obtain conservative ablation response. } \\
\hline \multicolumn{9}{|c|}{\begin{tabular}{|l|l|l|l|l|} 
All Time values are given in seconds. & & & & \\
\end{tabular}} \\
\hline \multicolumn{9}{|c|}{ All Temperature values are given in ${ }^{\circ} \mathrm{F}$. } \\
\hline \multicolumn{9}{|c|}{ Clad (Pt30Rh) melt temperature (eutectic $-300^{\circ}$ ): $2790^{\circ} \mathrm{F}$} \\
\hline \multicolumn{9}{|c|}{ Fuel (238PuO2) melt temperature: $4350^{\circ} \mathrm{F}$ (uncertainty allowance $500^{\circ}: 3850^{\circ} \mathrm{F}$ ) } \\
\hline \multicolumn{9}{|c|}{ (1): Failure criteria met at stagnation point node 1.} \\
\hline \multicolumn{9}{|c|}{ (2): Failure criteria met by burn through of outer aeroshell edge. } \\
\hline (3): Failure crit & by burn throug & at end cap and & outer aeroshel & edge. & & & & \\
\hline
\end{tabular}




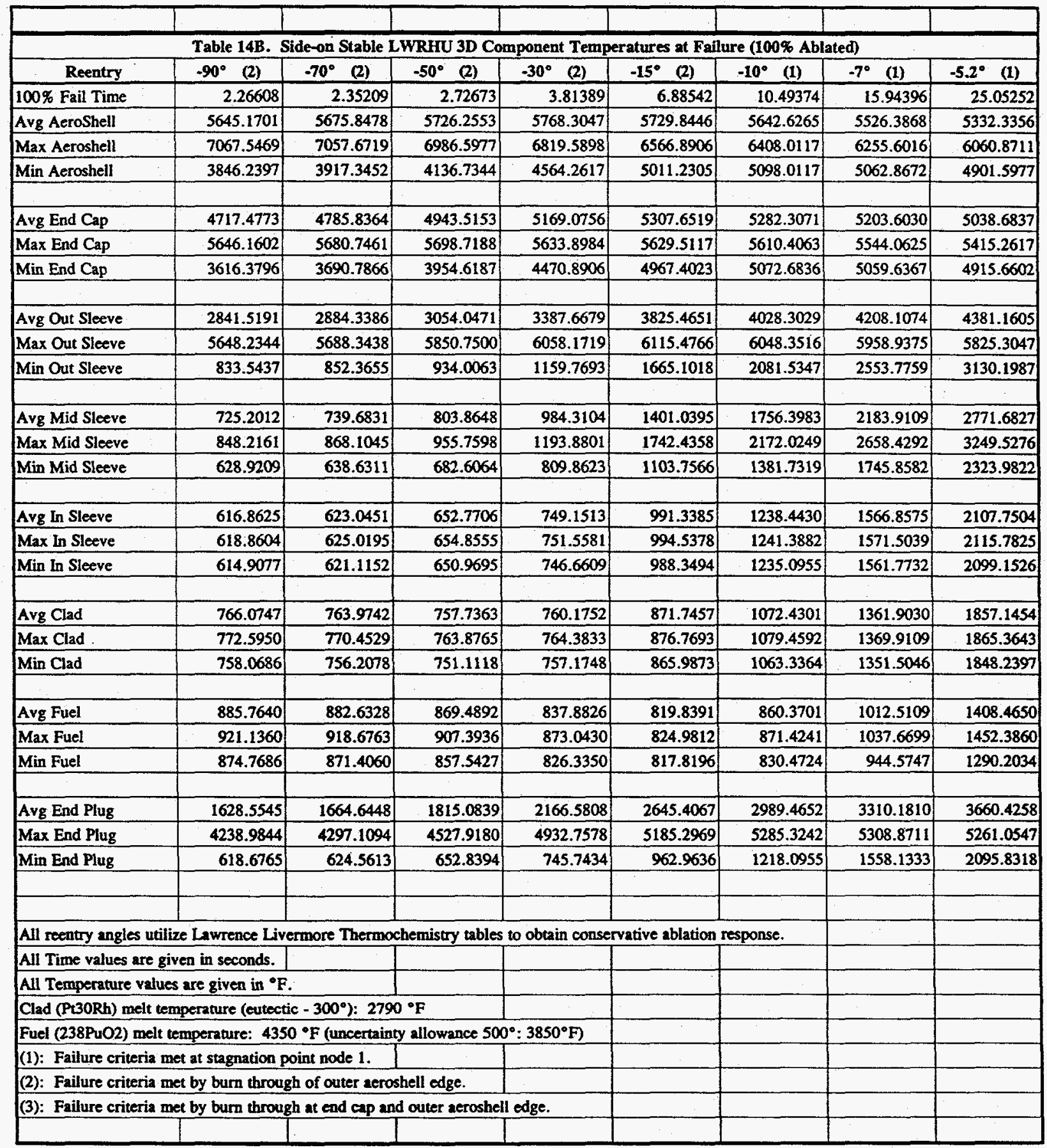




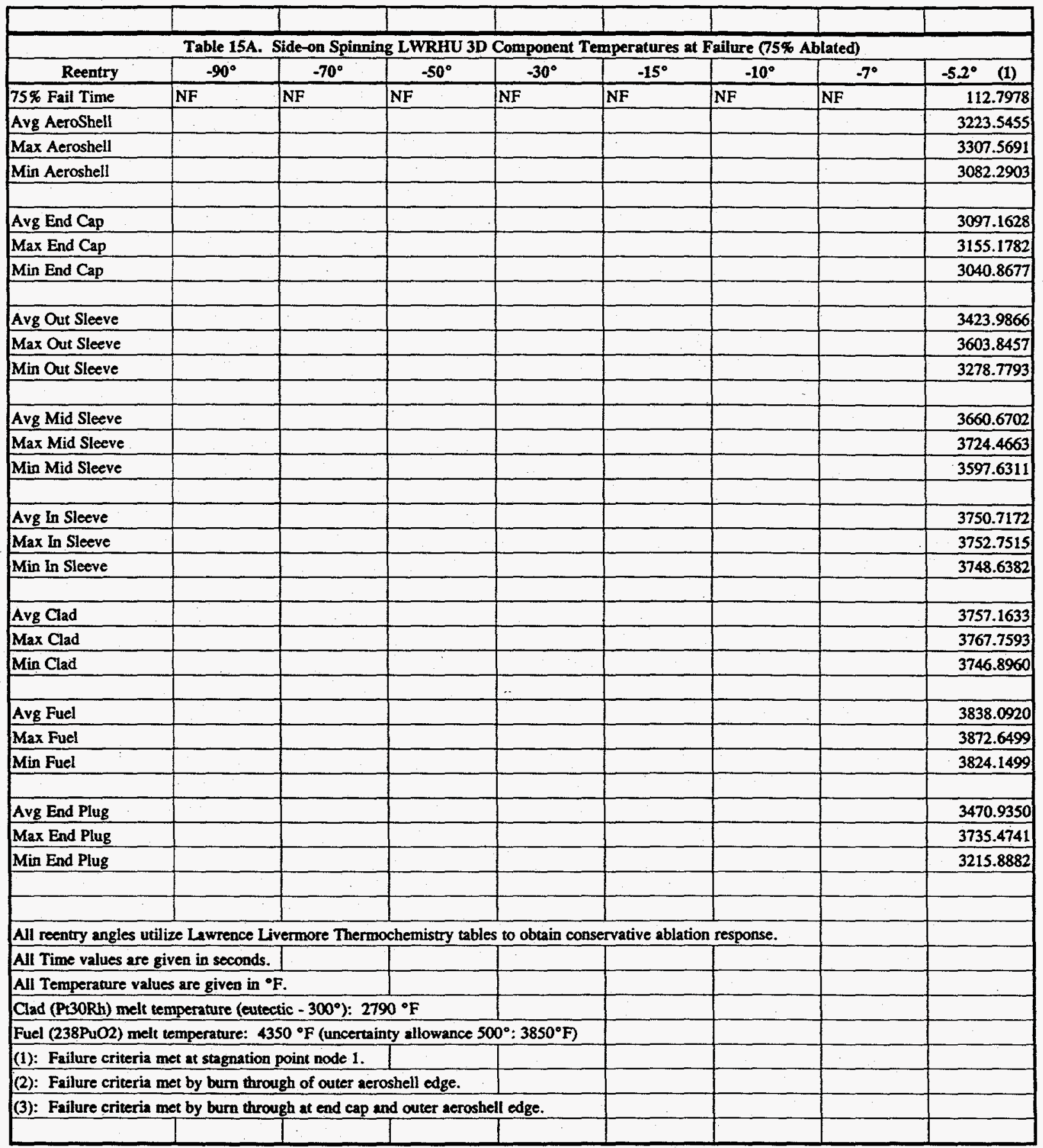




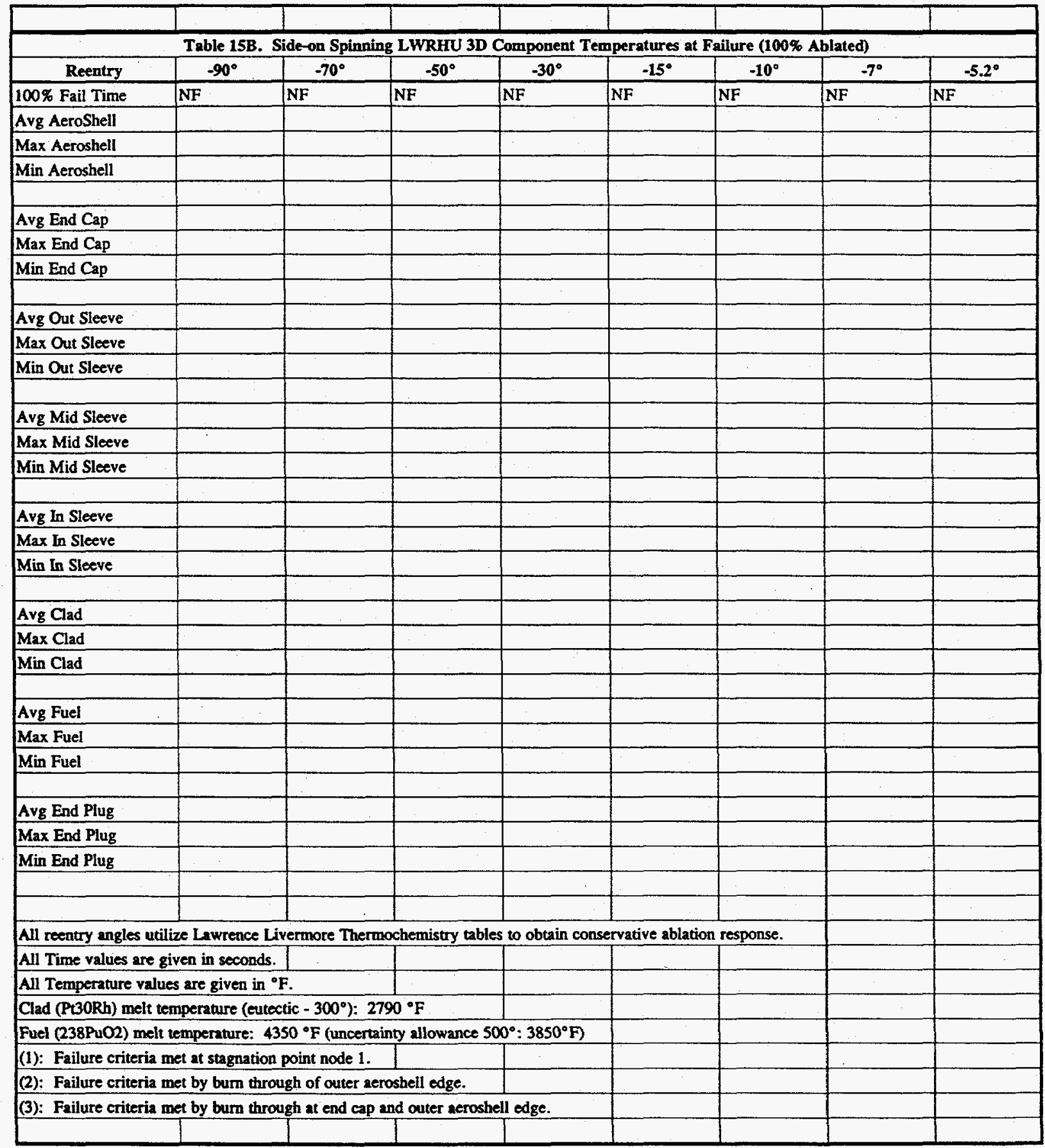




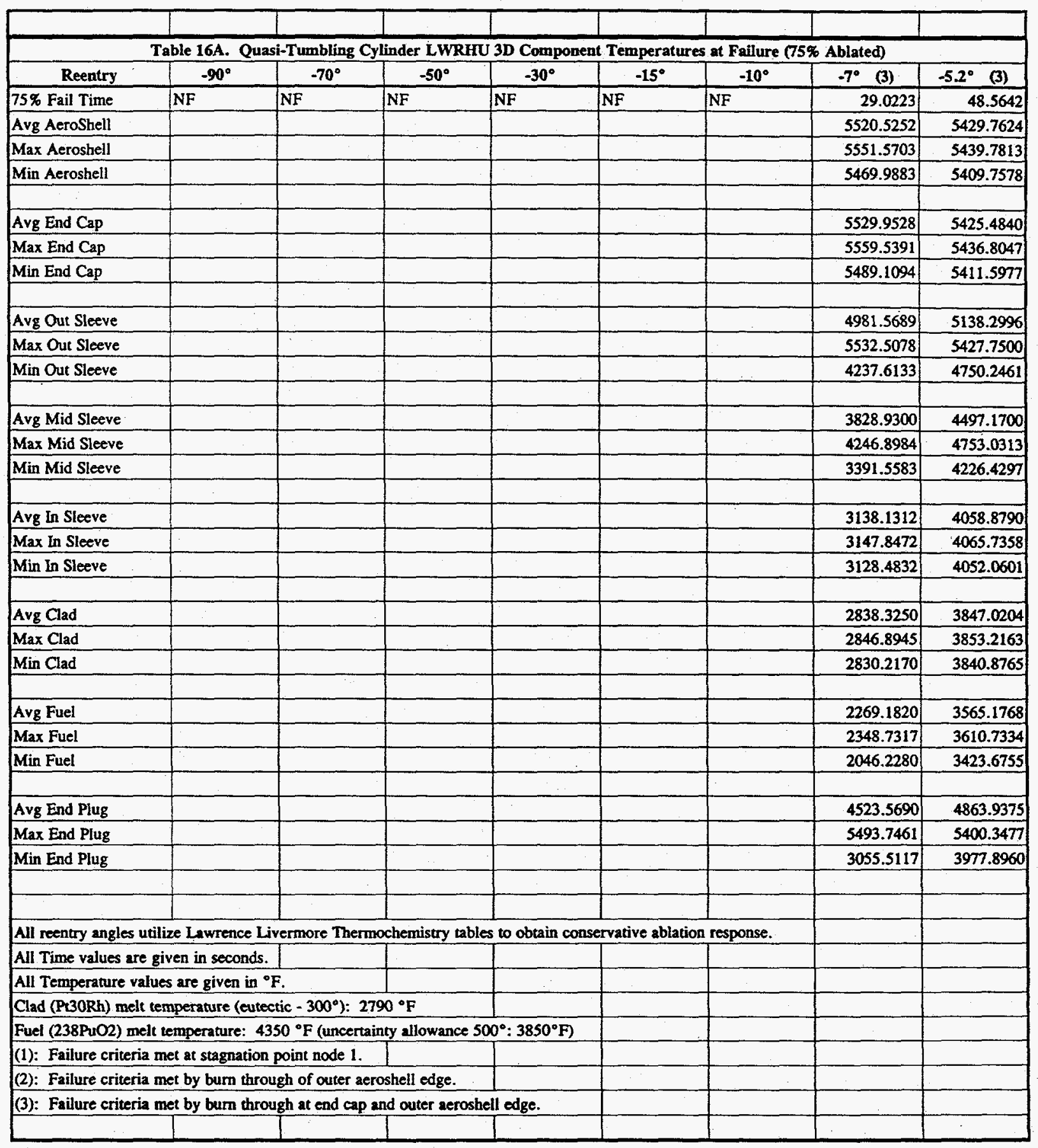




\begin{tabular}{|c|c|c|c|c|c|c|c|c|}
\hline \multicolumn{9}{|c|}{ Table 16B. Quasi-Tumbling Cylinder LWRHU 3D Component Temperatures at Failure (100\% Ablated) } \\
\hline Reentry & $.90^{\circ}$ & $.70^{\circ}$ & $-50^{\circ}$ & $-30^{\circ}$ & $-15^{\circ}$ & $-10^{\circ}$ & $-7^{\circ}$ & $-5.2^{\circ} \quad(3)$ \\
\hline 100\% Fail Time & NF & $\mathrm{NF}$ & NF & $\mathrm{NF}$ & $\mathrm{NF}$ & NF & NF & 247.18666 \\
\hline Avg AeroShell & & & & & & & & 2864.4654 \\
\hline Max Aeroshell & & & & & & & & 2877.0173 \\
\hline Min Aeroshell & & & & & & & & 2848.4414 \\
\hline \\
\hline Avg End Cap & & & & & & & & 2867.84804 \\
\hline Max End Cap & & & & & & & & 2878.5427 \\
\hline \multirow{2}{*}{\multicolumn{9}{|c|}{ Min End Cap }} \\
\hline & & & & & & & & \\
\hline Avg Out Sleeve & & & & & & & & 2910.80594 \\
\hline Max Out Sleeve & & & & & & & & 2950.604 \\
\hline \multirow{2}{*}{\multicolumn{9}{|c|}{ Min Out Sleeve }} \\
\hline & & & & & & & & \\
\hline Avg Mid Sleeve & & & & & & & & 2948.08125 \\
\hline Max Mid Sleeve & & & & & & & & 2951.1235 \\
\hline \multirow{2}{*}{\multicolumn{9}{|c|}{ Min Mid Sleeve }} \\
\hline & & & & & & & & \\
\hline Avg In Sleeve & & & & & & & & 2937.98219 \\
\hline Max In Sleeve & & & & & & & & 2938.2349 \\
\hline \multicolumn{9}{|l|}{ Min In Sleeve } \\
\hline & & & & & & & & \\
\hline Avg Clad & & & & & & & & 2915.96777 \\
\hline Max Clad & & & & & & & & 2921.542 \\
\hline \multirow{2}{*}{\multicolumn{9}{|c|}{ Min Clad }} \\
\hline & & & & & & & & \\
\hline Avg Fuel & & & & & & & & 2935.42832 \\
\hline Max Fuel & & & & & & & & 2943.5115 \\
\hline \multirow{2}{*}{\multicolumn{9}{|c|}{2931.2773}} \\
\hline & & & & & & & & \\
\hline Avg End Plug & & & & & & & & 2922.00642 \\
\hline Max End Plug & & & & & & & & 2947.4216 \\
\hline \multirow[t]{3}{*}{ Min End Plug } & & & & & & & & 2884.907 \\
\hline & & & & & & & & \\
\hline & & & & & & & & \\
\hline \multicolumn{9}{|c|}{ All reentry angles utilize Lawrence Livermore Thermochemistry tables to obtain conservative ablation response. } \\
\hline \multicolumn{9}{|c|}{\begin{tabular}{|l|l|l|l|} 
All Time values are given in seconds. & \\
\end{tabular}} \\
\hline \multicolumn{9}{|c|}{\begin{tabular}{|l|l|} 
All Temperature values are given in ${ }^{\circ} \mathrm{F}$. & \\
\end{tabular}} \\
\hline \multicolumn{9}{|c|}{\begin{tabular}{|l|l|l} 
Clad (Pt30Rh) melt temperature (eutectic $-300^{\circ}$ ): $2790^{\circ} \mathrm{F}$ & \\
\end{tabular}} \\
\hline \multicolumn{9}{|c|}{ Fuel $\left(238 \mathrm{Pu} 02\right.$ ) melt temperature: $4350^{\circ} \mathrm{F}$ (uncertainty allowance $500^{\circ}: 3850^{\circ} \mathrm{F}$ ) } \\
\hline \multicolumn{9}{|c|}{\begin{tabular}{|l|l|l|l|} 
(1): Failure criteria met at stagnation point node 1. & & \\
\end{tabular}} \\
\hline \multicolumn{9}{|c|}{ (2): Failure criteria met by burn through of outer aeroshell edge. } \\
\hline \multicolumn{9}{|c|}{ (3): Failure criteria met by burn through at end cap and outer aeroshell edge. } \\
\hline & & & & & & & & \\
\hline
\end{tabular}


Applied Physics Laboratory

Laurel, Maryland 20723-6099

\begin{tabular}{|c|c|c|c|c|c|c|c|c|}
\hline \multicolumn{9}{|c|}{ Table 17A. } \\
\hline Reentry & $-90^{\circ} \quad(3)$ & $-70^{\circ} \quad(3)$ & $-50^{\circ} \quad(3)$ & $-30^{\circ} \quad(3)$ & $-15^{\circ} \quad(3)$ & $-10^{\circ} \quad(3)$ & $-7^{\circ} \quad(3)$ & $-5.3^{\circ} \quad(3)$ \\
\hline $75 \%$ Fail Time & 3.86365 & 3.11726 & 3.29719 & 4.48906 & 8.28594 & 12.74505 & 19.88202 & 32.70866 \\
\hline Avg AeroShell & 5558.7222 & 6341.0996 & 6504.5803 & 6449.9597 & 6287.9409 & 6153.8247 & 6004.8872 & 5790.9483 \\
\hline Max Aeroshell & 5890.1289 & 6458.4805 & 6644.1328 & 6563.2891 & 6345.1289 & 6193.2422 & 6030.0859 & 5802.8945 \\
\hline Min Aeroshell & 5136.3906 & 6215.3086 & 6216.3164 & 6237.4258 & 6177.5508 & 6075.1289 & 5952.3125 & 5765.0820 \\
\hline Avg End Cap & 5624.6251 & 6189.9015 & 6309.4880 & 6317.2033 & 6227.2821 & 6115.6509 & 5982.1629 & 5779.2953 \\
\hline Max End Cap & 5865.7852 & 6365.0391 & 6638.3867 & 6560.5898 & 6343.1992 & 6191.6875 & 6028.4570 & 5799.6641 \\
\hline Min End Cap & 5303.8789 & 5759.9648 & 5758.5664 & 5945.3828 & 6054.0859 & 5998.9961 & 5906.4688 & 5742.2227 \\
\hline Avg Out Sleeve & 3983.2040 & 3947.3818 & 3945.1258 & 4131.6771 & 4466.0288 & 4667.3001 & 4871.2021 & 5078.5189 \\
\hline Max Out Sleeve & 5873.4336 & 6060.9688 & 6033.4297 & 6105.8438 & 6088.5703 & 6002.5313 & 5897.1445 & 5731.2852 \\
\hline Min Out Sleeve & 1539.6396 & 1286.9590 & 1317.3655 & 1611.7285 & 2345.9707 & 2904.8994 & 3508.0454 & 4208.7500 \\
\hline Avg Mid Sleeve & 1217.8190 & 1021.0766 & 1046.0740 & 1278.2316 & 1891.9074 & 2402.1556 & 3015.7131 & 3802.9595 \\
\hline Max Mid Sleeve & 1488.2434 & 1224.5898 & 1255.9844 & 1561.5559 & 2332.8423 & 2909.2185 & 3519.6824 & 4216.7070 \\
\hline Min Mid Sleeve & 947.6833 & 816.8040 & 834.9231 & 995.2656 & 1453.9788 & 1892.3882 & 2501.3877 & 3371.1113 \\
\hline Avg In Sleeve & 844.6361 & 744.0649 & 758.0465 & 885.0367 & 1281.4778 & 1668.1779 & 2233.0526 & 3125.0709 \\
\hline Max In Sleeve & 845.0247 & 744.3894 & 758.3599 & 885.4604 & 1283.2642 & 1672.2324 & 2240.0754 & 3134.4211 \\
\hline Min In Sleeve & 844.2444 & 743.7385 & 757.7310 & 884.6091 & 1279.6831 & 1664.1938 & 2226.1050 & 3115.7803 \\
\hline Avg Clad & 766.9660 & 713.7166 & 719.5846 & 792.6063 & 1097.3669 & 1431.0064 & 1940.8461 & 2836.5791 \\
\hline Max Clad & 769.1343 & 715.1174 & 720.3379 & 795.6130 & 1104.5242 & 1439.3525 & 1949.4131 & 2844.8101 \\
\hline Min Clad & 764.6570 & 712.6626 & 718.7402 & 788.9343 & 1086.7610 & 1418.4167 & 1929.1174 & 2829.0022 \\
\hline Avg Fuel & 854.9799 & 867.6332 & 863.1073 & 844.0947 & 880.4851 & 1034.8048 & 1408.8282 & 2294.9405 \\
\hline Max Fuel & 885.9080 & 904.4661 & 899.5007 & 870.0623 & 890.3223 & 1062.6917 & 1460.0942 & 2370.9878 \\
\hline Min Fuel & 844.4392 & 854.8735 & 850.5464 & 835.2742 & 853.7139 & 959.4138 & 1271.6101 & 2081.1045 \\
\hline Avg End Plug & 2780.8820 & 2483.9395 & 2509.4254 & 2844.2383 & 3439.9568 & 3782.9444 & 4135.6807 & 4553.7282 \\
\hline Max End Plug & 5463.3828 & 5347.4063 & 5337.5781 & 5585.8555 & 5799.7148 & 5801.5977 & 5760.0781 & 5652.4258 \\
\hline Min End Plug & 821.8838 & 730.6895 & 743.0676 & 860.4067 & 1251.4243 & 1636.2646 & 2178.7661 & 3045.2324 \\
\hline All reentry angles u & L Lawrence Liv & rmore Thermo & hemistry tables & o obtain conse & vative ablation & esponse. & & \\
\hline All Time values are & en in seconds. & & & & & & & \\
\hline All Temperature va & are given in ${ }^{\circ} \mathrm{F}$ & & & & & & & \\
\hline Clad (Pt30Rh) melt & perature (eutect & $\left.-300^{\circ}\right): 279$ & ${ }^{\circ} \mathrm{F}$ & & & & & \\
\hline Fuel (238PuO2) me & mperature: 435 & ${ }^{\circ} \mathrm{F}$ (uncertaint & allowance 500 & $: 3850^{\circ} \mathrm{F}$ & & & & \\
\hline (1): Failure criteri & $t$ at stagnation $p$ & int node 1. & & & & & & \\
\hline (2): Failure criteri & by burn throug & h of outer aeros & hell edge. & & & & & \\
\hline (3): Failure criteri & by burn throus & hat end cap an & outer aeroshell & edge. & & & & \\
\hline & & & & & & & & \\
\hline
\end{tabular}




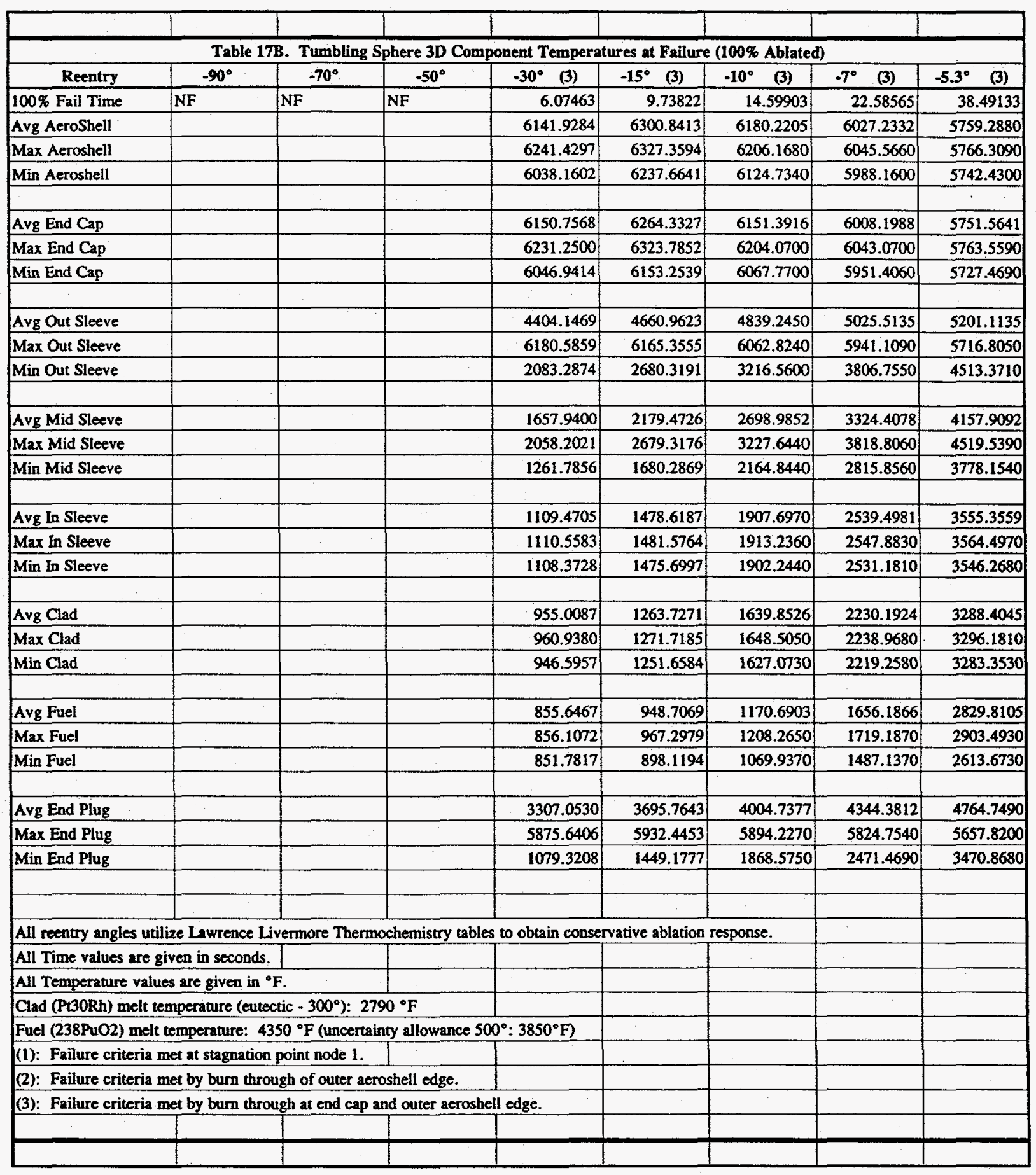


The Johns Hopkins University

Applied Physics Laboratory

Laurel, Maryland 20723-6099

\begin{tabular}{|c|c|c|c|c|c|c|c|c|}
\hline \multicolumn{9}{|c|}{ Table 18A. Random Tumbling LWRHU 3D Component Temperatures at Failure (75\% Ablated) } \\
\hline Reentry & $.90^{\circ}$ & $-70^{\circ}$ & $-50^{\circ}$ & $-30^{\circ}$ & $-15^{\circ}$ & $-10^{\circ}$ (3) & $.7^{\circ}(3)$ & $-5.2^{\circ}(3)$ \\
\hline 75\% Fail Time & NF & NF & NF & $\mathrm{NF}$ & $\mathrm{NF}$ & 24.98222 & 27.77724 & 65.18513 \\
\hline Avg AeroShell & & & & & & 4149.179643 & 5528.193583 & 4738.573538 \\
\hline Max Aeroshell & & & & & & 4269.4453 & 5615.3945 & 4801.0273 \\
\hline \multirow[t]{2}{*}{ VIII AETOSHCII } & & & & & & 4005.6265 & 5476.3789 & 4687.2305 \\
\hline & & & & & & & & \\
\hline Avg End Cap & & & & & & 4240.040132 & 5590.939221 & 4787.903471 \\
\hline Max End Cap & & & & & & 4383.6992 & 5605.582 & 4811.7891 \\
\hline \multirow[t]{2}{*}{ Min End Cap } & & & & & & 4112.1563 & 5562.457 & 4764.4023 \\
\hline & & & & & & & & \\
\hline Avg Out Sleeve & & & & & & 4113.14468 & 4903.120667 & 4731.093196 \\
\hline Max Out Sleeve & & & & & & 4320.3633 & 5506.043 & 4763.6289 \\
\hline \multirow[t]{2}{*}{ Min Out Sleeve } & & & & & & 3847.6538 & 4086.0796 & 4705.3281 \\
\hline & & & & & & & & \\
\hline Avg Mid Sleeve & & & & & & 3514.133507 & 3666.280469 & 4608.013581 \\
\hline Max Mid Sleeve & & & & & & 3854.8335 & 4097.0234 & 4705.6328 \\
\hline \multirow[t]{2}{*}{ Min Mid Sleeve } & & & & & & 3154.3435 & 3217.6753 & 4503.2422 \\
\hline & & & & & & & & \\
\hline Avg In Sleeve & & & & & & 2928.995543 & 2961.107357 & 4426.678286 \\
\hline Max In Sleeve & & & & & & 2937.0413 & 2970.394 & 4429.3555 \\
\hline \multirow[t]{2}{*}{ Min In Sleeve } & & & & & & 2921.0503 & 2951.8906 & 4424.0234 \\
\hline & & & & & & & & \\
\hline Avg Clad & & & & & & 2647.042205 & 2659.809124 & 4312.439548 \\
\hline Max Clad & & & & & & 2655.1936 & 2668.3528 & 4318.9883 \\
\hline \multirow[t]{2}{*}{ Min Clad } & & & & & & 2638.9338 & 2651.0188 & 4302.6797 \\
\hline & & & & & & & & \\
\hline Avg Fuel & & & & & & \begin{tabular}{|l|l|}
2082.162363 \\
\end{tabular} & 2084.312725 & 4225.287119 \\
\hline Max Fuel & & & & & & 2158.054 & 2160.2412 & 4237.3594 \\
\hline \multirow[t]{2}{*}{ Min Fuel } & & & & & & 1873.4236 & 1875.6672 & 4182.4766 \\
\hline & & & & & & & & \\
\hline Avg End Plug & & & & & & 3911.470053 & 4431.661936 & 4687.942015 \\
\hline Max End Plug & & & & & & 4425.3398 & 5501.4375 & 4817.3164 \\
\hline \multirow[t]{3}{*}{ Min End Plug } & & & & & & 2851.8689 & 2882.3777 & 4363.7656 \\
\hline & & & & & & & & \\
\hline & & & & & & & & \\
\hline \multicolumn{9}{|c|}{ All reentry angles utilize Lawrence Livermore Thermochemistry tables to obtain conservative ablation response. } \\
\hline \multicolumn{9}{|c|}{\begin{tabular}{|l|l|l|l|l|} 
All Time values are given in seconds. & & & & \\
\end{tabular}} \\
\hline \multicolumn{9}{|c|}{\begin{tabular}{|l|l|} 
All Temperature values are given in ${ }^{\circ} \mathrm{F}$. & \\
\end{tabular}} \\
\hline \multicolumn{9}{|c|}{\begin{tabular}{ll|l} 
Clad (Pt3ORh) melt temperature (eutectic $-300^{\circ}$ ): $2790^{\circ} \mathrm{F}$ & \\
\end{tabular}} \\
\hline \multicolumn{9}{|c|}{ Fuel (238PuO2) melt temperanure: $4350^{\circ} \mathrm{F}$ (uncertainty allowance $500^{\circ}: 3850^{\circ} \mathrm{F}$ ) } \\
\hline \multicolumn{9}{|c|}{\begin{tabular}{|l|l|l|} 
(1): Failure criteria met at stagnation point node 1. & & \\
\end{tabular}} \\
\hline \multicolumn{9}{|c|}{ (2): Failure criteria met by burn through of outer aeroshell edge. } \\
\hline \multicolumn{9}{|c|}{ (3): Failure criteria met by burn through at end cap and outer aeroshell edge. } \\
\hline & & & & & & & & \\
\hline & & & & & & & & \\
\hline
\end{tabular}




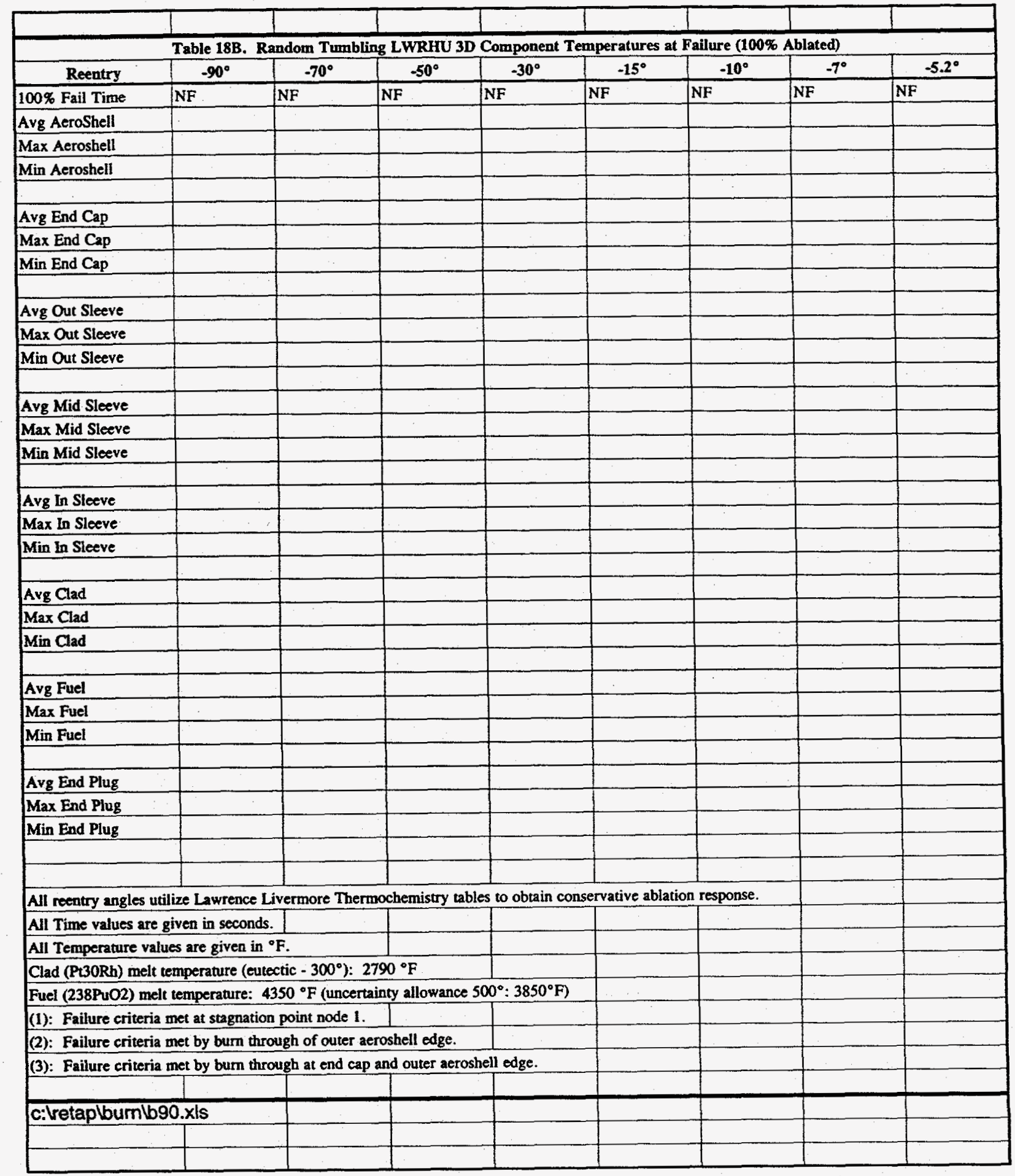




\begin{tabular}{|l|c|c|c|c|c|c|c|c||}
\hline \multicolumn{7}{|c|}{ TABLE 19.A Side-On Spinning LWRHU 3D CLAD Temperature Results for Cases not Demonstrating $100 \%$} \\
Ablation Failure.
\end{tabular}

c:lwp51leahmemlrhumelt.tab 
TABLE 19.B. Side-On Spinning LWRHU 3D Fuel Temperature Results for Cases not Demonstrating 100\% Ablation Failure.

\begin{tabular}{|c|c|c|c|c|c|c|c|c|}
\hline Reentry Angle & $-5.2^{\circ}$ & $-7.0^{\circ}$ & $-10^{\circ}$ & $-15^{\circ}$ & $-30^{\circ}$ & $-50^{\circ}$ & $-70^{\circ}$ & $-90^{\circ}$ \\
\hline Time of $\mathrm{M}=5$ & 218.4 & 59.0 & 33.1 & 19.8 & 9.7 & 6.2 & 5.1 & 4.7 \\
\hline $\begin{array}{l}\text { Initial } \\
\text { Temperature }\end{array}$ & 950.3 & 950.3 & 950.3 & 950.3 & 950.3 & 950.3 & 950.3 & 950.3 \\
\hline $\begin{array}{l}\text { Maximum } \\
\text { Temperature }\end{array}$ & 4260.4 & 3573.7 & 2711.5 & 1338.5 & 989.8 & 950.3 & 950.3 & 950.3 \\
\hline $\begin{array}{l}\text { Time at maximum } \\
\text { Temperature }\end{array}$ & 79.01 & 56.50 & 32.70 & 11.09 & 9.50 & 0.0 & 0.0 & 0.0 \\
\hline $\begin{array}{l}\text { Time at Melt }{ }^{1} \\
\text { Temperature }\end{array}$ & $\begin{array}{l}\text { No } \\
\text { Melt }\end{array}$ & $\begin{array}{l}\text { No } \\
\text { Melt }\end{array}$ & $\begin{array}{l}\text { No } \\
\text { Melt }\end{array}$ & $\begin{array}{l}\text { No } \\
\text { Melt }\end{array}$ & $\begin{array}{l}\text { No } \\
\text { Melt }\end{array}$ & $\begin{array}{l}\text { No } \\
\text { Melt }\end{array}$ & $\begin{array}{l}\text { No } \\
\text { Melt }\end{array}$ & $\begin{array}{l}\text { No } \\
\text { Melt }\end{array}$ \\
\hline & & & & & & & & \\
\hline \multicolumn{9}{|c|}{$\begin{array}{l}\text { All Reentry Angles utilize Lawrence Livermore Tables. } \\
\text { All Times are given in seconds } \\
\text { All Temperature are given in }{ }^{\circ} \mathrm{F} \\
{ }^{1} \text { Fuel Melt Temperature }=3850-4350^{\circ} \mathrm{F}\end{array}$} \\
\hline
\end{tabular}

c:lwp51VeahmemLrhumelt.tab 
TABLE 20.A Quasi-Tumbling Cylinder LWRHU 3D CLAD Temperature Results for Cases not Demonstrating $100 \%$ Ablation Failure.

\begin{tabular}{|c|c|c|c|c|c|c|c|c|}
\hline Reentry Angle & $-5.2^{\circ}$ & $-7.0^{\circ}$ & $-10^{\circ}$ & $-15^{\circ}$ & $-30^{\circ}$ & $-50^{\circ}$ & $-70^{\circ}$ & $-90^{\circ}$ \\
\hline Time of $M=5$ & 280.2 & 59.15 & 33.1 & 19.6 & 9.65 & 6.8 & 5.51 & 5.27 \\
\hline $\begin{array}{l}\text { Initial } \\
\text { Temperature }\end{array}$ & 945.3 & 945.3 & 945.3 & 945.3 & 945.3 & 945.3 & 945.3 & 945.3 \\
\hline $\begin{array}{l}\text { Maximum } \\
\text { Temperature }\end{array}$ & $\begin{array}{l}\text { Failed } \\
100 \%\end{array}$ & 3779.0 & 3140.1 & 2322.0 & 1349.8 & 1064.9 & 945.3 & 945.3 \\
\hline $\begin{array}{l}\text { Time at maximum } \\
\text { Temperature }\end{array}$ & & 51.01 & 32.70 & 19.63 & 9.50 & 6.80 & 0.0 & 0.0 \\
\hline $\begin{array}{l}\text { Time at Melt }{ }^{1} \\
\text { Temperature }\end{array}$ & & $\begin{array}{l}30.00- \\
59.15\end{array}$ & $\begin{array}{l}27.71- \\
33.1\end{array}$ & $\begin{array}{l}\text { No } \\
\text { Melt }\end{array}$ & $\begin{array}{l}\text { No } \\
\text { Melt }\end{array}$ & $\begin{array}{l}\text { No } \\
\text { Melt }\end{array}$ & $\begin{array}{l}\text { No } \\
\text { Melt }\end{array}$ & $\begin{array}{l}\text { No } \\
\text { Melt }\end{array}$ \\
\hline $\begin{array}{l}\text { Time at } \mathrm{Melt}^{2} \\
\text { Temperature }\end{array}$ & & $\begin{array}{l}34.50- \\
59.15\end{array}$ & $\begin{array}{l}\text { No } \\
\text { Melt }\end{array}$ & $\begin{array}{l}\text { No } \\
\text { Melt }\end{array}$ & $\begin{array}{l}\text { No } \\
\text { Melt }\end{array}$ & $\begin{array}{l}\text { No } \\
\text { Melt }\end{array}$ & $\begin{array}{l}\text { No } \\
\text { Melt }\end{array}$ & $\begin{array}{l}\text { No } \\
\text { Melt }\end{array}$ \\
\hline $\begin{array}{l}\text { Time at } \mathrm{Melt}^{3} \\
\text { Temperature }\end{array}$ & & $\begin{array}{l}39.00- \\
59.15\end{array}$ & $\begin{array}{l}\text { No } \\
\text { Melt }\end{array}$ & $\begin{array}{l}\text { No } \\
\text { Melt }\end{array}$ & $\begin{array}{l}\text { No } \\
\text { Melt }\end{array}$ & $\begin{array}{l}\text { No } \\
\text { Melt }\end{array}$ & $\begin{array}{l}\text { No } \\
\text { Melt }\end{array}$ & $\begin{array}{l}\text { No } \\
\text { Melt }\end{array}$ \\
\hline & & & & & & & & \\
\hline & & & & & & & & \\
\hline
\end{tabular}

All Reentry Angles utilize Lawrence Livermore Tables.

All Times are given in seconds

All Temperature are given in ${ }^{\circ} \mathrm{F}$

${ }^{1}$ Conservative Clad Melt Temperature (Upper Eutectic -500 ) $=2900^{\circ} \mathrm{F}$

${ }^{2}$ Clad Eutectic Temperature (lower bound) $=3090^{\circ} \mathrm{F}$

${ }^{3}$ Clad Melt Temperature (upper bound) $=3470^{\circ} \mathrm{F}$

c:lwp51 Veahmemlrhumelt.tab 
TABLE 20.B. Quasi-Tumbling Cylinder LWRHU 3D Fuel Temperature Results for Cases not Demonstrating 100\% Ablation Failure.

\begin{tabular}{||l|c|c|c|c|c|c|c|c||}
\hline Reentry Angle & $-5.2^{\circ}$ & $-7.0^{\circ}$ & $-10^{\circ}$ & $-15^{\circ}$ & $-30^{\circ}$ & $-50^{\circ}$ & $-70^{\circ}$ & $-90^{\circ}$ \\
\hline Time of M=5 & 280.2 & 59.15 & 33.1 & 19.6 & 9.65 & 6.8 & 5.51 & 5.27 \\
\hline $\begin{array}{l}\text { Initial } \\
\text { Temperature }\end{array}$ & 950.3 & 950.3 & 950.3 & 950.3 & 950.3 & 950.3 & 950.3 & 950.3 \\
\hline $\begin{array}{l}\text { Maximum } \\
\text { Temperature }\end{array}$ & $\begin{array}{c}\text { Failed } \\
100 \%\end{array}$ & 3742.6 & 2841.1 & 1819.0 & 1015.7 & 950.3 & 950.3 & 950.3 \\
\hline $\begin{array}{l}\text { Time at maximum } \\
\text { Temperature }\end{array}$ & 57.00 & 32.70 & 19.63 & 9.50 & 0.0 & 0.0 & 0.0 \\
\hline $\begin{array}{l}\text { Time at Melt } \\
\text { Temperature }\end{array}$ & & $\begin{array}{c}\text { No } \\
\text { Melt }\end{array}$ & $\begin{array}{c}\text { No } \\
\text { Melt }\end{array}$ & $\begin{array}{c}\text { No } \\
\text { Melt }\end{array}$ & $\begin{array}{c}\text { No } \\
\text { Melt }\end{array}$ & $\begin{array}{c}\text { No } \\
\text { Melt }\end{array}$ & $\begin{array}{c}\text { No } \\
\text { Melt }\end{array}$ & $\begin{array}{c}\text { No } \\
\text { Melt }\end{array}$ \\
\hline & & & & & & & \\
\hline
\end{tabular}

All Reentry Angles utilize Lawrence Livermore Tables.

All Times are given in seconds

All Temperature are given in ${ }^{\circ} \mathrm{F}$

' Fuel Melt Temperature $=3850-4350^{\circ} \mathrm{F}$

c:/wp51Veahmemtrhumelt.tab 


\begin{tabular}{|c|c|c|c|c|c|c|c|c|}
\hline \multicolumn{9}{|c|}{$\begin{array}{l}\text { TABLE 21.A Tumbling Sphere LWRHU 3D CLAD Temperature Results for Cases not Demonstrating 100\% } \\
\text { Ablation Failure. }\end{array}$} \\
\hline Reentry Angle & $-5.2^{\circ}$ & $-7.0^{\circ}$ & $-10^{\circ}$ & $-15^{\circ}$ & $-30^{\circ}$ & $-50^{\circ}$ & $-70^{\circ}$ & $-90^{\circ}$ \\
\hline Time of $M=5$ & 280.2 & 59.15 & 33.1 & 19.6 & 9.65 & 6.8 & 5.51 & 5.27 \\
\hline $\begin{array}{l}\text { Initial } \\
\text { Temperature }\end{array}$ & 945.3 & 945.3 & 945.3 & 945.3 & 945.3 & 945.3 & 945.3 & 945.3 \\
\hline $\begin{array}{l}\text { Maximum } \\
\text { Temperature }\end{array}$ & $\begin{array}{l}\text { Failed } \\
100 \%\end{array}$ & $\begin{array}{l}\text { Failed } \\
100 \%\end{array}$ & $\begin{array}{l}\text { Failed } \\
100 \%\end{array}$ & $\begin{array}{l}\text { Failed } \\
100 \%\end{array}$ & $\begin{array}{l}\text { Failed } \\
100 \%\end{array}$ & 971.0 & 945.3 & 945.3 \\
\hline \begin{tabular}{|l|} 
Time at maximum \\
Temperature
\end{tabular} & & & & & & 5.90 & 0.0 & 0.0 \\
\hline \begin{tabular}{|l|l|l} 
Time at Melt & \\
Temperature
\end{tabular} & & & & & & $\begin{array}{l}\text { No } \\
\text { Melt }\end{array}$ & $\begin{array}{l}\text { No } \\
\text { Melt }\end{array}$ & $\begin{array}{l}\text { No } \\
\text { Melt }\end{array}$ \\
\hline $\begin{array}{l}\text { Time at Melt }{ }^{2} \\
\text { Temperature }\end{array}$ & & & & & & $\begin{array}{l}\text { No } \\
\text { Melt }\end{array}$ & $\begin{array}{l}\text { No } \\
\text { Melt }\end{array}$ & $\begin{array}{l}\text { No } \\
\text { Melt }\end{array}$ \\
\hline $\begin{array}{l}\text { Time at } \text { Melt }^{3} \\
\text { Temperature }\end{array}$ & & & & & & $\begin{array}{l}\text { No } \\
\text { Melt }\end{array}$ & $\begin{array}{l}\text { No } \\
\text { Melt }\end{array}$ & $\begin{array}{l}\text { No } \\
\text { Melt }\end{array}$ \\
\hline & & & & & & & & \\
\hline & & & & & & & & \\
\hline & 'Cons & $\begin{array}{l}\text { ive Clad } \\
\text { Clad Eu } \\
{ }^{3} \text { Clad }\end{array}$ & $\begin{array}{l}\text { Igles uti } \\
1 \text { Times } \\
\text { Temper } \\
\text { it Temp } \\
\text { c Tempe } \\
\text { Tempera }\end{array}$ & $\begin{array}{l}\text { Lawreno } \\
\text { given in } \\
\text { are giv } \\
\text { re (Upp } \\
\text { (lowe } \\
\text { (upper }\end{array}$ & $\begin{array}{l}\text { vermore } \\
\text { nds } \\
{ }^{\circ} \mathrm{F} \\
\text { utectic } \\
\text { ind) }=3 \\
\text { d) }=34\end{array}$ & $\begin{array}{l}\text { les. } \\
=290 \\
{ }^{\circ} \mathrm{F} \\
F\end{array}$ & & \\
\hline
\end{tabular}

c:lwp51leahmemLrhumelt.tab 
TABLE 21.B. Tumbling Sphere LWRHU 3D Fuel Temperature Results for Cases not Demonstrating 100\% Ablation Failure.

\begin{tabular}{|c|c|c|c|c|c|c|c|c|}
\hline Reentry Angle & $-5.2^{\circ}$ & $-7.0^{\circ}$ & $-10^{\circ}$ & $-15^{\circ}$ & $-30^{\circ}$ & $-50^{\circ}$ & $-70^{\circ}$ & $-90^{\circ}$ \\
\hline Time of $M=5$ & 280.2 & 59.15 & 33.1 & 19.6 & 9.65 & 6.8 & 5.51 & 5.27 \\
\hline $\begin{array}{l}\text { Initial } \\
\text { Temperature }\end{array}$ & 950.3 & 950.3 & 950.3 & 950.3 & 950.3 & 950.3 & 950.3 & 950.3 \\
\hline $\begin{array}{l}\text { Maximum } \\
\text { Temperature }\end{array}$ & $\begin{array}{l}\text { Failed } \\
100 \%\end{array}$ & $\begin{array}{l}\text { Failed } \\
100 \%\end{array}$ & $\begin{array}{l}\text { Failed } \\
100 \%\end{array}$ & $\begin{array}{l}\text { Failed } \\
100 \%\end{array}$ & $\begin{array}{l}\text { Failed } \\
100 \%\end{array}$ & 950.3 & 950.3 & 950.3 \\
\hline $\begin{array}{l}\text { Time at maximum } \\
\text { Temperature }\end{array}$ & & & & & & 0.0 & 0.0 & 0.0 \\
\hline $\begin{array}{l}\text { Time at Melt }{ }^{1} \\
\text { Temperature }\end{array}$ & & & & & & $\begin{array}{l}\text { No } \\
\text { Melt }\end{array}$ & $\begin{array}{l}\text { No } \\
\text { Melt }\end{array}$ & $\begin{array}{l}\text { No } \\
\text { Melt }\end{array}$ \\
\hline & & & & & & & & \\
\hline & & & & & & & & \\
\hline \multicolumn{9}{|c|}{$\begin{array}{l}\text { All Reentry Angles utilize Lawrence Livermore Tables. } \\
\text { All Times are given in seconds } \\
\text { All Temperature are given in }{ }^{\circ} \mathrm{F} \\
\text { 'Fuel Melt Temperature }=3850-4350^{\circ} \mathrm{F}\end{array}$} \\
\hline
\end{tabular}

c:lwp51/leahmem Thumelt.tab 


\begin{tabular}{|l|c|c|c|c|c|c|c|c||}
\hline \multicolumn{2}{|c|}{ TABLE 22.A. Full Random Tumbling LWRHU 3D CLAD Temperature Results for Cases not Demonstrating } \\
$100 \%$ Ablation Failure.
\end{tabular}

c:Twp51Veahmemirhumelt.tab 


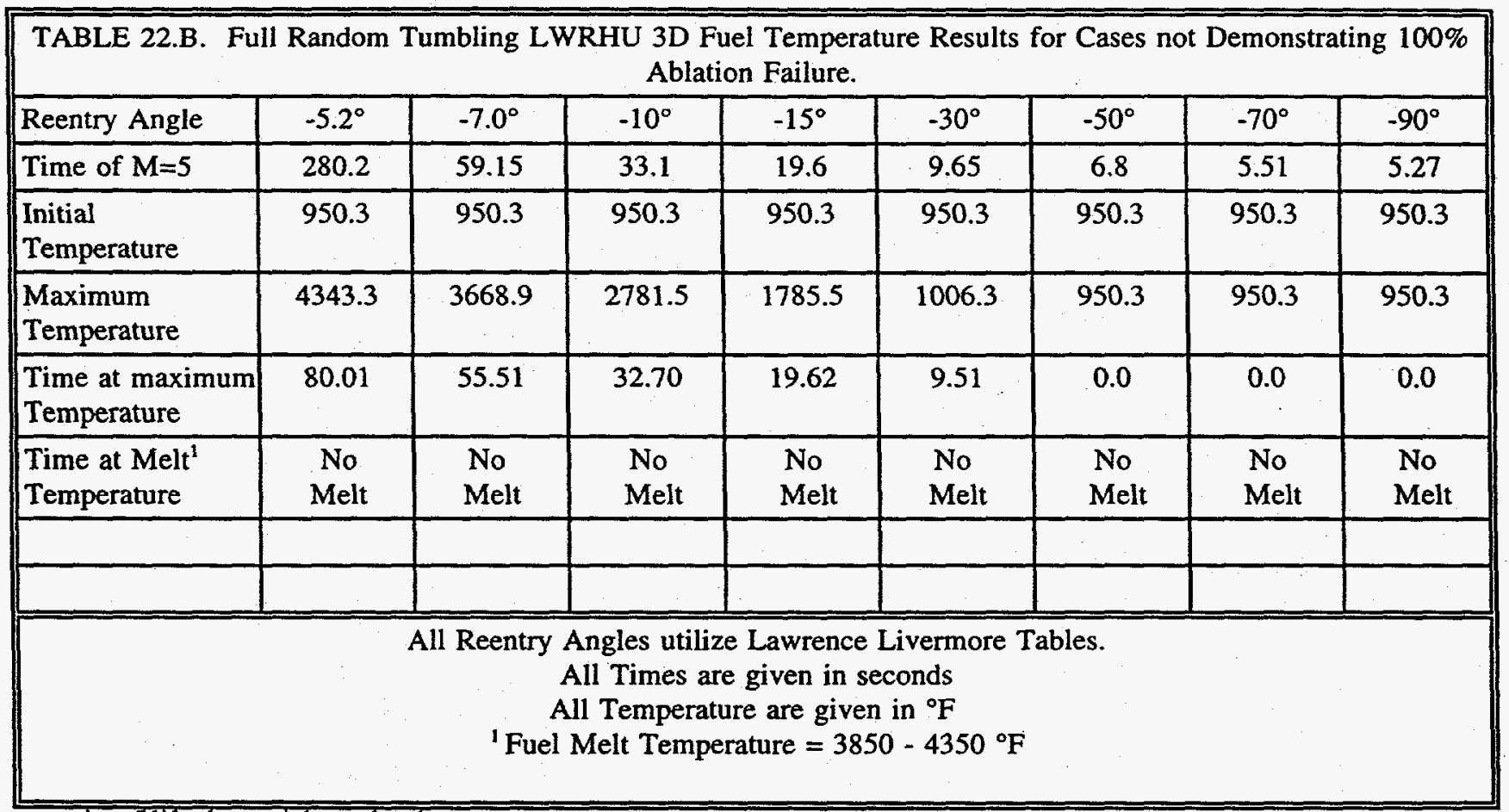

c:1wp51Ueahmemlrhumelt.tab 
Applied Physics Laboratory

Laurel, Maryland 20723.6099

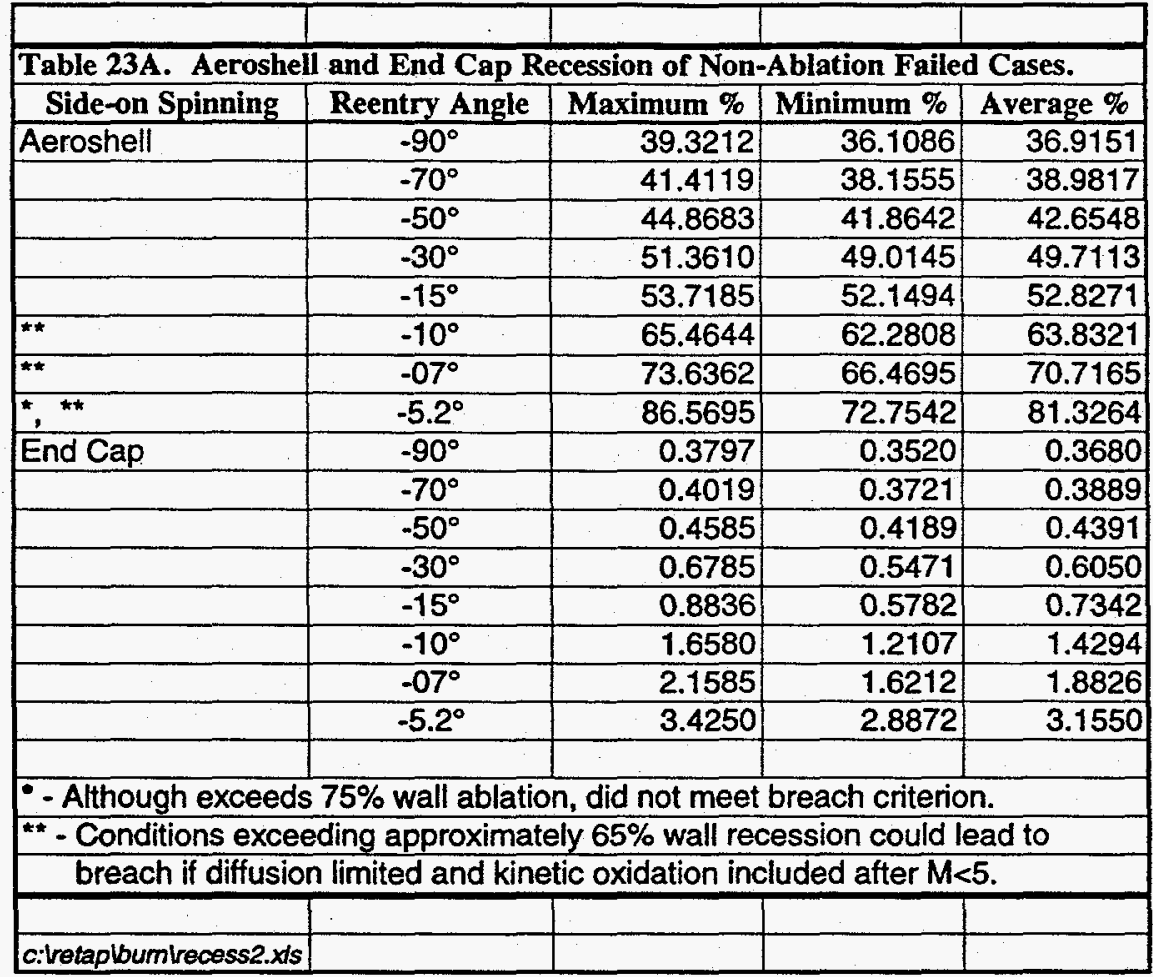




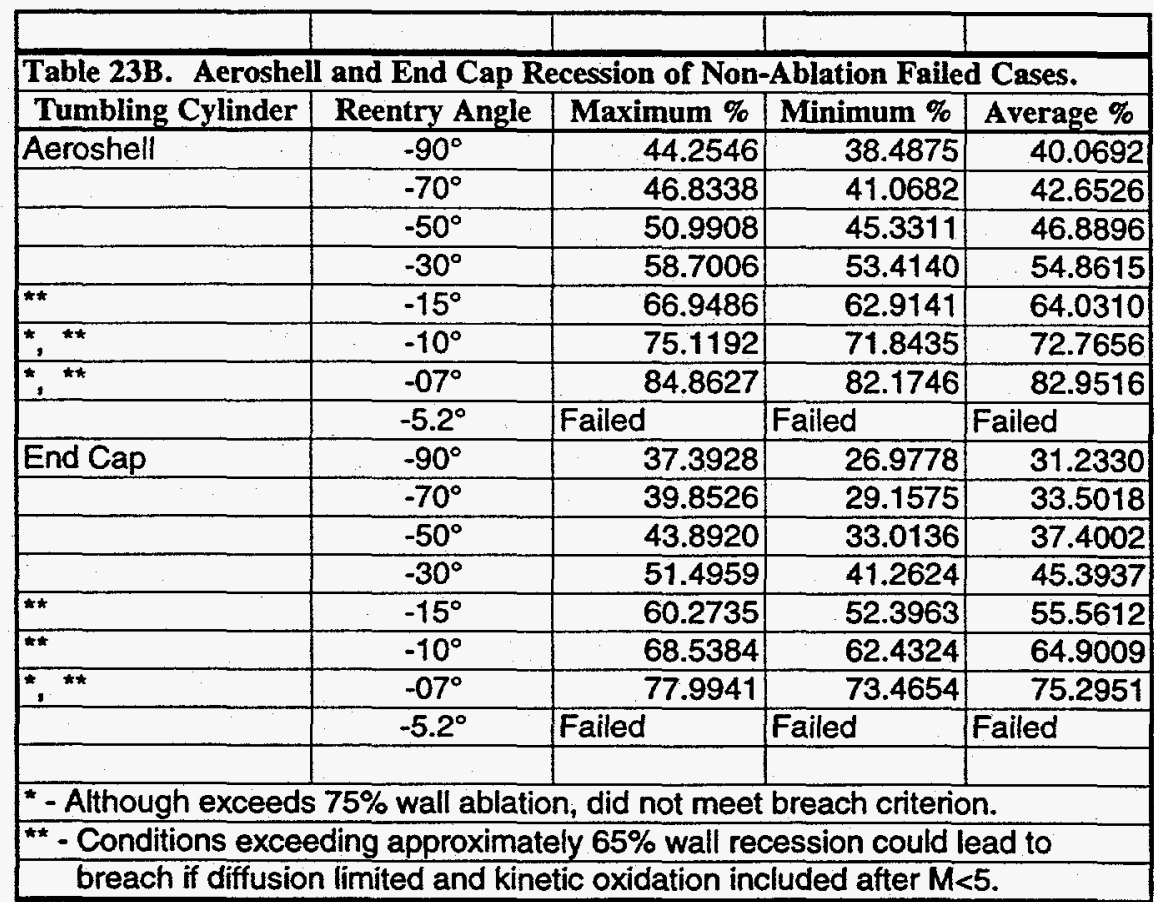




\begin{tabular}{|c|c|c|c|c|}
\hline \multicolumn{5}{|c|}{ Table 23C. Aeroshell and End Cap Recession of Non-Ablation Failed Cases. } \\
\hline Tumbling Sphere & Reentry Angle & Maximum \% & Minimum \% & Average \% \\
\hline Aeroshell ${ }^{\star}, \star \star$ & $-90^{\circ}$ & 80.3627 & 73.9492 & 75.6894 \\
\hline${ }^{*}, * *$ & $-70^{\circ}$ & 84.1096 & 77.7198 & 79.4555 \\
\hline \multirow[t]{6}{*}{ 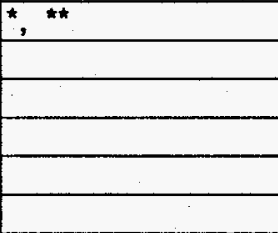 } & $-50^{\circ}$ & 92.4288 & 86.2203 & 87.9098 \\
\hline & $-30^{\circ}$ & Failed & Failed & Failed \\
\hline & $-15^{\circ}$ & Failed & Failed & Failed \\
\hline & $-10^{\circ}$ & Failed & Failed & Failed \\
\hline & $-07^{\circ}$ & Failed & Failed & Failed \\
\hline & $-5.2^{\circ}$ & Failed & Failed & Failed \\
\hline End Cap** & $-90^{\circ}$ & 71.3551 & 54.5022 & 61.3128 \\
\hline$\star \star$ & $-70^{\circ}$ & 74.9535 & 57.6741 & 64.6482 \\
\hline \multirow[t]{6}{*}{$*{ }^{* *}$} & $-50^{\circ}$ & 83.0465 & 65.3200 & 72.4182 \\
\hline & $-30^{\circ}$ & Failed & Failed & Failed \\
\hline & $-15^{\circ}$ & Failed & Failed & Failed \\
\hline & $-10^{\circ}$ & Failed & Failed & Failed \\
\hline & $-07^{\circ}$ & Failed & Failed & Failed \\
\hline & $-5.2^{\circ}$ & Failed & Failed & Failed \\
\hline \multicolumn{5}{|c|}{ * - Although exceeds $75 \%$ wall ablation, did not meet breach criterion. } \\
\hline \multicolumn{5}{|c|}{$\begin{array}{l}\text { - Conditions exceeding approximately } 65 \% \text { wall recession could lead to } \\
\text { breach if diffusion limited and kinetic oxidation included after } M<5 \text {. }\end{array}$} \\
\hline
\end{tabular}


Table 23D. Aeroshell and End Cap Recession of Non-Ablation Failed Cases.

\begin{tabular}{|l|l|l|l|l|}
\hline Random Tumbling & Reentry Angle & Maximum \% & Minimum \% & Average \% \\
\hline
\end{tabular}

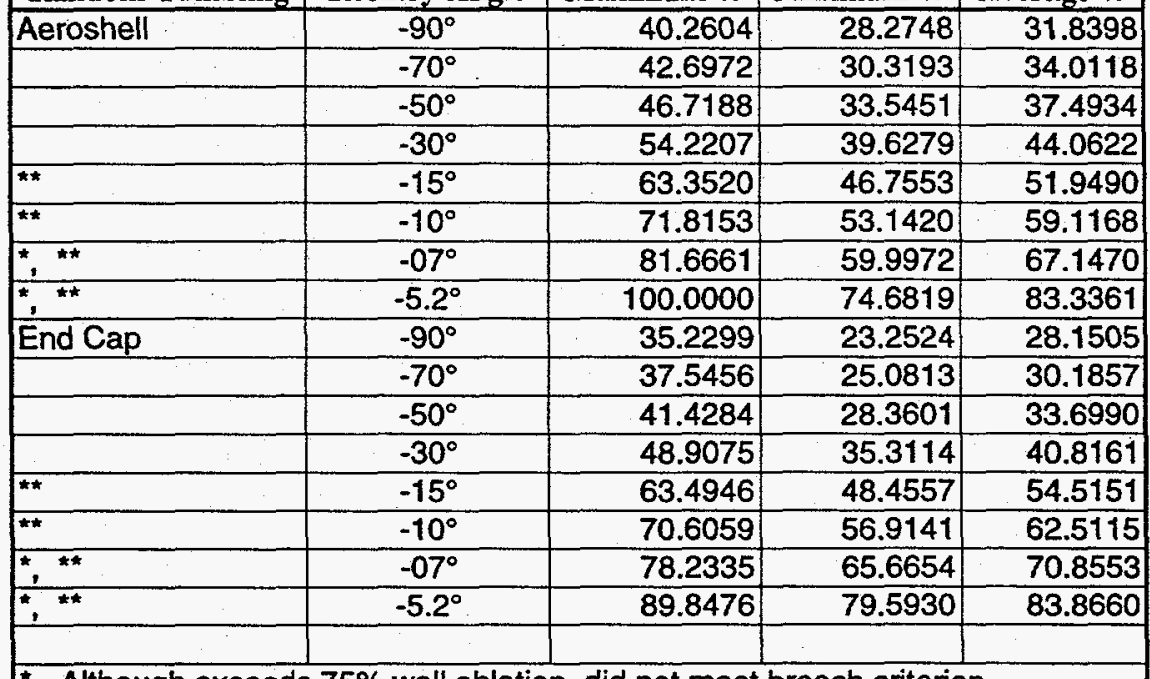

" - Although exceeds $75 \%$ wall ablation, did not meet breach criterion.

** - Conditions exceeding approximately $65 \%$ wall recession could lead to breach if diffusion limited and kinetic oxidation included after $M<5$. 
Table 24

Initial Trajectory Conditions for Fuel Pellet Release from Side-On Stable Reentry.

TDOF RUN 75\% FAILURE RELEASE STABLE/SPINNING AND TUMBLING:

$\begin{array}{lllllllll}\text { GAMMA } & -5.2^{\circ} & -7^{\circ} & -10^{\circ} & -15^{\circ} & -30^{\circ} & -50^{\circ} & -70^{\circ} & -90^{\circ} \\ \text { Time (sec) } & 22.71 & 14.46 & 9.42 & 6.03 & 3.40 & 2.42 & 2.08 & 1.93 \\ \text { Altitude (f) } & 242612 & 225948 & 210991 & 196168 & 167990 & 150716 & 141748 & 141359 \\ \text { Velocity (f/s) } & 59388 & 58897 & 58099 & 57281 & 53938 & 51380 & 49356 & 49666\end{array}$

TDOF RUN 100\% FAILURE RELEASE STABLE/SPINNING AND TUMBLING:

$\begin{array}{lllllllll}\text { GAMMA } & -5.2^{\circ} & -7^{\circ} & -10^{\circ} & -15^{\circ} & -30^{\circ} & -50^{\circ} & -70^{\circ} & -90^{\circ}\end{array}$

$\begin{array}{lllllllll}\text { Time (sec) } & 25.16 & 15.99 & 10.52 & 6.80 & 3.88 & 2.78 & 2.41 & 2.22\end{array}$

$\begin{array}{lllllllll}\text { Altitude (ft) } & 238007 & 218569 & 201817 & 185982 & 156208 & 137837 & 127925 & 128318\end{array}$

$\begin{array}{llllllllll}\text { Velocity (ft/s) } & 57388 & 56414 & 55060 & 53742 & 48538 & 44136 & 40462 & 41754\end{array}$ 
TABLE 25. Estimated Drag Coefficients and Bridging Parameters for Released Pellet

\begin{tabular}{|c|c|c|c|c|c|c|}
\hline Bridging Variable & \multicolumn{4}{|c|}{ Side-on Stable or Spinning Fuel } & \multicolumn{2}{|c|}{$\begin{array}{l}\text { Tumbling Fuel } \\
\text { Cylinder }\end{array}$} \\
\hline$R_{\mathrm{N}}$ & & & & & \multicolumn{2}{|c|}{$0.0354 \mathrm{ft}$} \\
\hline$S_{R E P}$ & \multicolumn{4}{|c|}{$0.000629 \mathrm{ft}^{2}$} & \multicolumn{2}{|c|}{$0.00033 \mathrm{ft}^{2}$} \\
\hline$D_{\text {REP }}$ & \multicolumn{4}{|c|}{$0.0205 \mathrm{ft}$} & \multicolumn{2}{|c|}{$0.02051 \mathrm{ft}$} \\
\hline SWMCH ** & \multicolumn{4}{|c|}{3.0} & \multicolumn{2}{|c|}{3.0} \\
\hline \multirow[t]{16}{*}{ Tabulated $C_{D}$} & $M$ & $C_{D}$ & Alt & $C_{D}$ & $\mathrm{M}$ & $\mathrm{C}_{\mathrm{D}}$ \\
\hline & 0.0 & 0.554 & 0.0 & 1.20 & 0.0 & 0.94 \\
\hline & 0.5 & 0.554 & 100000 & 1.20 & 0.2 & 0.94 \\
\hline & 0.6 & 1.26 & 200000 & 1.24 & 0.5 & 0.96 \\
\hline & 0.9 & 2.00 & 230000 & 1.32 & 0.6 & 1.53 \\
\hline & 1.0 & 2.10 & 235000 . & 1.33 & 0.8 & 2.04 \\
\hline & 1.1 & 2.00 & 250000 & 1.37 & 1.0 & 2.35 \\
\hline & 1.3 & 1.70 & 300000 . & 1.62 & 1.2 & 2.28 \\
\hline & 1.5 & 1.37 & 350000 . & 2.05 & 1.4 & 2.02 \\
\hline & 1.8 & 1.26 & 400000 & 2.10 & 1.6 & 1.95 \\
\hline & 2.0 & 1.24 & & & 1.8 & 1.92 \\
\hline & 2.3 & 1.21 & & . & 2.0 & 1.90 \\
\hline & 2.5 & 1.20 & & & 3.0 & 1.90 \\
\hline & 40.0 & 1.20 & & & 10.0 & 1.90 \\
\hline & 100.0 & 1.20 & & & 100.0 & 1.90 \\
\hline & & & & & & \\
\hline
\end{tabular}




\begin{tabular}{|c|c|c|c|c|c|}
\hline $\begin{array}{l}\text { Reentry } \\
\text { Angle } \\
\left(\gamma^{\circ}\right)\end{array}$ & $\begin{array}{c}\text { Orientation } \\
\text { (see key at end } \\
\text { of Table) }\end{array}$ & $\begin{array}{c}\text { Nodes } z \\
4812^{\circ} \mathrm{R}\end{array}$ & $\begin{array}{c}\text { Nodes } 2 \\
4312 \circ \mathrm{R}\end{array}$ & $\begin{array}{c}\text { Volume } \\
2 \\
4812^{\circ} \mathrm{R}\end{array}$ & $\begin{array}{c}\text { Volume } \\
2 \\
4312^{\circ} \mathrm{R}\end{array}$ \\
\hline 5.2 & 1: 758ablated & all & all & 1008 & $100 \%$ \\
\hline 7.0 & 1: 758ablated & all & all & $100 \%$ & $100 \%$ \\
\hline 10.0 & 1: 758ablated & all & aII & $100 \%$ & 1008 \\
\hline 15.0 & 1: 758ablated & al1 & all & $100 \%$ & $100 \%$ \\
\hline 30.0 & 1: 758ablated & all & al1 & 1008 & $100 \%$ \\
\hline 50.0 & 1: 758 ablated & $\begin{array}{c}\text { all except } \\
20,23,24,2 \\
6,27\end{array}$ & al1 & $698^{*}$ & $100 \%$ \\
\hline 70.0 & 1: 758ablated & $\begin{array}{c}\text { all except } \\
20-28\end{array}$ & $\begin{array}{c}\text { all except } \\
24,26,27\end{array}$ & $58 z^{*}$ & $878^{*}$ \\
\hline 90.0 & 1: 758ablated & $\begin{array}{c}\text { al1 except } \\
20-28\end{array}$ & $\begin{array}{c}\text { all except } \\
24,26,27 \\
\end{array}$ & $588^{*}$ & $87 z^{*}$ \\
\hline 5.2 & 1:1008ablated & 211 & all & $100 \%$ & 1008 \\
\hline 7.0 & $1: 100 z a b l a t e d$ & al1 & all & 1008 & 1008 \\
\hline 10.0 & 1:100zablated & all & all & $100 \%$ & $100 \%$ \\
\hline 15.0 & 1:1008ablated & all & al1 & 1008 & $100 \%$ \\
\hline 30.0 & 1:1008ablated & $\begin{array}{c}\text { all except } \\
21,23,24,2 \\
6,27 \\
\end{array}$ & all & $72 z^{*}$ & $100 \%$ \\
\hline 50.0 & 1:1008ablated & $1-17$ & $1-17$ & $518^{*}$ & $518^{*}$ \\
\hline 70.0 & 1:1008ablated & $1-9,11,14$ & $\begin{array}{c}1-9,12,14,1 \\
7\end{array}$ & $268^{*}$ & $41 q^{*}$ \\
\hline 90.0 & 1:1008ablated & $1-9,11,14$ & $\begin{array}{c}11,12,14,1 \\
7\end{array}$ & $26 q^{*}$ & $418^{*}$ \\
\hline 5.2 & 2: 758ablated & al1 & all & $100 \%$ & 1008 \\
\hline 7.0 & 2: 758ablated & al1 & all & $100 \%$ & 1008 \\
\hline 10.0 & 2: 758ablated & al1 & all & 1008 & 1008 \\
\hline 15.0 & 2:758ablated & a11 & al1 & $100 \%$ & 1008 \\
\hline 30.0 & 2: 758ablated & all & all & 1008 & 1008 \\
\hline 50.0 & 2: 758ablated & al1 & al1 & 1008 & 1008 \\
\hline 70.0 & 2: 758ablated & all & all & 1008 & 1008 \\
\hline 90.0 & 2: 758ablated & all & all & $100 \%$ & 1008 \\
\hline
\end{tabular}




\begin{tabular}{|c|c|c|c|c|c|}
\hline $\begin{array}{l}\text { Reentry } \\
\text { Angle } \\
\left(\gamma^{\circ}\right) \\
\end{array}$ & $\begin{array}{c}\text { Orientation } \\
\text { (see key at end } \\
\text { of Table) }\end{array}$ & $\begin{array}{l}\text { Nodes } 2 \\
4812^{\circ} \mathrm{R}\end{array}$ & $\begin{array}{c}\text { Nodes } 2 \\
4312^{\circ} \mathrm{R}\end{array}$ & $\begin{array}{c}\text { Volume } \\
2 \\
4812^{\circ} \mathrm{R} \\
\end{array}$ & $\begin{array}{c}\text { Volume } \\
2 \\
4312^{\circ} \mathrm{R} \\
\end{array}$ \\
\hline 5.2 & $2: 1008 a b l a t e d$ & al1 & all & 1008 & $100 \%$ \\
\hline 7.0 & $2: 100 \%$ ablated & all & all & 1008 & 1008 \\
\hline 10.0 & 2:1008ablated & all & all & 1008 & 1008 \\
\hline 15.0 & 2:1008ablated & all & all & $100 \%$ & 1008 \\
\hline 30.0 & 2:100zablated & all & all & 1008 & 1008 \\
\hline 50.0 & $2: 1008 a b l a t e d$ & $\begin{array}{c}1-8, \\
11,14,17,2 \\
0,23,26\end{array}$ & $\begin{array}{c}1-9, \\
11,12,14,1 \\
5,17,18,20 \\
21,23,24 \\
26,27 \\
\end{array}$ & $568^{*}$ & $898^{*}$ \\
\hline 70.0 & $2: 1008$ ablated & $\begin{array}{c}1-8, \\
11,14,17,2 \\
0,23,26 \\
\end{array}$ & $\begin{array}{c}1-8, \\
11,14,17,2 \\
0,23,26 \\
\end{array}$ & $568^{*}$ & $568^{*}$ \\
\hline 90.0 & $2: 1008 a b l a t e d$ & $\begin{array}{c}1-8, \\
11,14,17,2 \\
0,23,26 \\
\end{array}$ & $\begin{array}{c}1-8, \\
11,14,17,2 \\
0,23,26 \\
\end{array}$ & $568^{*}$ & $568^{*}$ \\
\hline 7.0 & 3: 758ablated & all & all & $100 \%$ & $100 \%$ \\
\hline 15.0 & 3: 758ablated & all & all & $100 \%$ & 1008 \\
\hline 90.0 & 3: 758ablated & all & all & 1008 & 1008 \\
\hline 7.0 & 3:1008ablated & all & al1 & 1008 & $100 \%$ \\
\hline 15.0 & 3:1008ablated & all & all & 1008 & $100 \%$ \\
\hline 90.0 & $3: 1008 a b l a t e d$ & $\begin{array}{l}1,2,5,6 \\
9,10,11 \\
13,14,15\end{array}$ & $\begin{array}{c}1,2,5,6 \\
9,10,11 \\
12,13,14,1 \\
5\end{array}$ & $668^{*}$ & $708^{*}$ \\
\hline 5.2 & 4: 758ablated & $\begin{array}{c}\text { only } \\
\text { surface } \\
\text { nodes }\end{array}$ & all & $-28^{* *}$ & 1008 \\
\hline 5.2 & 5: 758ablated & none & all & $\sim 08^{* *}$ & 1008 \\
\hline 5.2 & 6: 75\%ablated & none & al1 & $\sim 08^{* *}$ & 1008 \\
\hline 5.2 & 7: 758 ablated & all & all & 1008 & 1008 \\
\hline 7.0 & 7: 758 ablated & $\begin{array}{c}\text { al1 except } \\
5,6,7,20,2 \\
1,23,24,26 \\
.27\end{array}$ & all & 638 & $100 \%$ \\
\hline 10.0 & 7: 758 ablated & none & none & -08 & -08 \\
\hline 5.2 & 8: 758 ablated & al1 & all & 1008 & 1008 \\
\hline
\end{tabular}




\begin{tabular}{|c|c|c|c|c|c|}
\hline $\begin{array}{l}\text { Reentry } \\
\text { Angle } \\
\left(\gamma^{\circ}\right)\end{array}$ & $\begin{array}{c}\text { Orientation } \\
\text { (see key at end } \\
\text { of Table) } \\
\end{array}$ & $\begin{array}{c}\text { Nodes } z \\
4812^{\circ} \mathrm{R}\end{array}$ & $\begin{array}{c}\text { Nodes } 2 \\
4312^{\circ} \mathrm{R}\end{array}$ & $\begin{array}{c}\text { Volume } \\
2 \\
4812^{\circ} \mathrm{R}\end{array}$ & $\begin{array}{l}\text { Volume } \\
2 \\
4312^{\circ} \mathrm{R}\end{array}$ \\
\hline 7.0 & 8: 758 ablated & all & all & $100 \%$ & 1008 \\
\hline 10.0 & 8: $75 \%$ ablated & none & none & $-0 \%$ & $\sim 0 \%$ \\
\hline 5.2 & 9: $75 \%$ ablated & all & all & $100 \%$ & 1008 \\
\hline 7.0 & 9: 758 ablated & all & all & 1008 & $100 \%$ \\
\hline 10.0 & 9: 758 ablated & none & none & -08 & $-0 \%$ \\
\hline \multicolumn{6}{|c|}{$\begin{array}{l}\text { Expected to show } 1008 \text { melt: analysis conducted at less than } 1 / 2 \\
\text { expected convective heating rate (velocity gradient scaling factor } \\
\text { of } 3 \text { vice } 7.25 \text { ). } \\
\text { Although released pellet temperature does not reach melt, full } \\
\text { fuel melt was obtained prior to release; potential resolidification } \\
\text { prior to release, but release temperature exceeded } 4310^{\circ} \text {. These } \\
\text { conditions should be considered as full }\{100 \%\} \text { atmospheric source } \\
\text { terms. } \\
\text { c:imp51leahmemlfueimelt.tab }\end{array}$} \\
\hline \multicolumn{6}{|c|}{$\begin{array}{l}\text { Orientation Key: } \\
1 \\
2 \text { Side-on Stable LWRHU releasing Side-on Stable Fuel Pellet } \\
2 \text { Side-on Stable LWRHU releasing Side-on Spinning Fuel Pellet } \\
3 \text { Side-on Stable LWRHU releasing Random Tumbling Fuel Pellet } \\
4 \text { Side-on Spinning LWRHU releasing Side-on Stable Fuel Pellet } \\
5 \text { Side-on Spinning LWRHU releasing Side-on Spinning Fuel Pellet } \\
6 \text { Side-on Spinning LWRHU releasing Random Tumbling Fuel Pellet } \\
7 \text { Random Tumbling LWRHU releasing Side-on Stable Fuel Pellet } \\
8 \text { Random Tumbling LWRHU releasing Side-on Spinning Fuel Pellet } \\
9 \text { Random Tumbling LWRHU releasing Random Tumbling Fuel Pellet }\end{array}$} \\
\hline
\end{tabular}


Table 27 - Side-On Stable LWRHU Thermostructural Analysis Results (NF $=$ No Failure)

\begin{tabular}{|l|l|}
\hline Reentry Angle $\gamma$ & $-90^{\circ}$ \\
\hline Time of Initial Failure (.01 strain allowable) & $1.00 \mathrm{sec}$ \\
\hline Element No. & 3949 \\
\hline Factor of Safety-Direction & $.82-\mathrm{z}$ \\
\hline & \\
\hline Time of Lowest FS (.01 strain allowable) & $2.35 \mathrm{sec}$ \\
\hline Element No. & 3895 \\
\hline Factor of Safety-Direction & $.61-\mathrm{z}$ \\
\hline & \\
\hline Time of Initial Failure (.05 strain allowable) & $\mathrm{NF}$ \\
\hline Element No. & \\
\hline Factor of Safety-Direction & \\
\hline & \\
\hline
\end{tabular}

Table 28 - Side-on Spinning LWRHU Thermostructural Analysis Cases

\begin{tabular}{|l|l|}
\hline Reentry Angle $\gamma$ & Times (sec.) \\
\hline$-7^{\circ}$ & $3.00,9.00,13.00,16.00,19.00,20.00,21.00,22.00$ \\
\hline$-10^{\circ}$ & $12.41,14.00$ \\
\hline$-15^{\circ}$ & $7.90,9.02$ \\
\hline$-30^{\circ}$ & $4.20,4.60$ \\
\hline$-50^{\circ}$ & $0.60,1.40,2.70,2.90,3.10,4.05$ \\
\hline$-70^{\circ}$ & $2.25,2.50$ \\
\hline$-90^{\circ}$ & $0.40,0.60,0.80,1.00,1.40,1.80,2.15,2.35$ \\
\hline
\end{tabular}


Table 29 - Side-On Spinning LWRHU Thermostructural Analysis Results (NF = No Failure)

\begin{tabular}{|l|l|l|l|l|l|l|l|}
\hline Reentry Angle $\gamma$ & $-7^{\circ}$ & $-10^{\circ}$ & $-15^{\circ}$ & $-30^{\circ}$ & $-50^{\circ}$ & $-70^{\circ}$ & $-90^{\circ}$ \\
\hline Time of Initial Failure (.01 strain allowable) & 13.00 & 12.41 & 7.90 & 4.20 & 1.40 & 2.25 & 1.40 \\
\hline Element No. & 1865 & 1865 & 10 & 10 & 1865 & 1865 & 1865 \\
\hline Factor of Safety-Direction & $.97-\mathrm{z}$ & $.88-\mathrm{z}$ & $.84-\mathrm{z}$ & $.79-\mathrm{z}$ & $.86-\mathrm{z}$ & $.78-\mathrm{z}$ & $.92-\mathrm{z}$ \\
\hline & & & & & & & \\
\hline Time of Lowest FS (.01 strain allowable) & 20.00 & 12.41 & 7.90 & 4.20 & 2.90 & 2.50 & 2.35 \\
\hline Element No. & 1865 & 1865 & 10 & 10 & 10 & 1865 & 1865 \\
\hline Factor of Safety-Direction & $.93-\mathrm{z}$ & $.88-\mathrm{z}$ & $.84-\mathrm{z}$ & $.79-\mathrm{z}$ & $.77-\mathrm{z}$ & $.77-\mathrm{z}$ & $.77-\mathrm{z}$ \\
\hline & & & & & & & \\
\hline Time of Initial Failure (.05 strain allowable) & NF & NF & NF & NF & NF & NF & NF \\
\hline Element No. & & & & & & & \\
\hline Factor of Safety-Direction & & & & & & & \\
\hline & & & & & & & \\
\hline
\end{tabular}

Table 30 - Random Tumbling LWRHU Thermostructural Analysis Cases

\begin{tabular}{|l|l|}
\hline Reentry Angle $\gamma$ & Times (sec.) \\
\hline$-15^{\circ}$ & $2.00,4.00,6.00,7.90,8.50,9.00,9.50$ \\
\hline$-30^{\circ}$ & $4.10,4.60$ \\
\hline$-50^{\circ}$ & $0.40,1.00,1.80,2.80,3.00,3.10,3.50$ \\
\hline$-70^{\circ}$ & $2.25,2.50$ \\
\hline$-90^{\circ}$ & $0.40,0.80,1.80,2.15,2.35,2.60$ \\
\hline
\end{tabular}


Table 31 - Random Tumbling LWRHU Thermostructural Analysis Results (NF = No Failure)

\begin{tabular}{|l|l|l|l|l|l|}
\hline Reentry Angle $\gamma$ & $-15^{\circ}$ & $-30^{\circ}$ & $-50^{\circ}$ & $-70^{\circ}$ & $-90^{\circ}$ \\
\hline Time of Initial Failure (.01 strain allowable) & 4.00 & 4.10 & 1.00 & 2.25 & .80 \\
\hline Element No. & 3610 & 3960 & 2934 & 3948 & 3103 \\
\hline Factor of Safety-Direction & $1.00-\mathrm{z}$ & $.80-\mathrm{z}$ & $.98-\mathrm{z}$ & $.76-\mathrm{z}$ & $.86-\mathrm{z}$ \\
\hline & & & & & \\
\hline Time of Lowest FS (.01 strain allowable) & 7.90 & 4.10 & 2.80 & 2.25 & 2.15 \\
\hline Element No. & 3966 & 3960 & 3954 & 3948 & 3948 \\
\hline Factor of Safety-Direction & $.86-\mathrm{z}$ & $.80-\mathrm{z}$ & $.77-\mathrm{z}$ & $.76-\mathrm{z}$ & $.75-\mathrm{z}$ \\
\hline & & & & & \\
\hline Time of Initial Failure (.05 strain allowable) & NF & NF & NF & NF & NF \\
\hline Element No. & & & & & \\
\hline Factor of Safety-Direction & & & & & \\
\hline & & & & & \\
\hline
\end{tabular}


The Johns Hopkins University

\section{Applied Physics Laboratory}

Laurel, Maryland 20723.6099

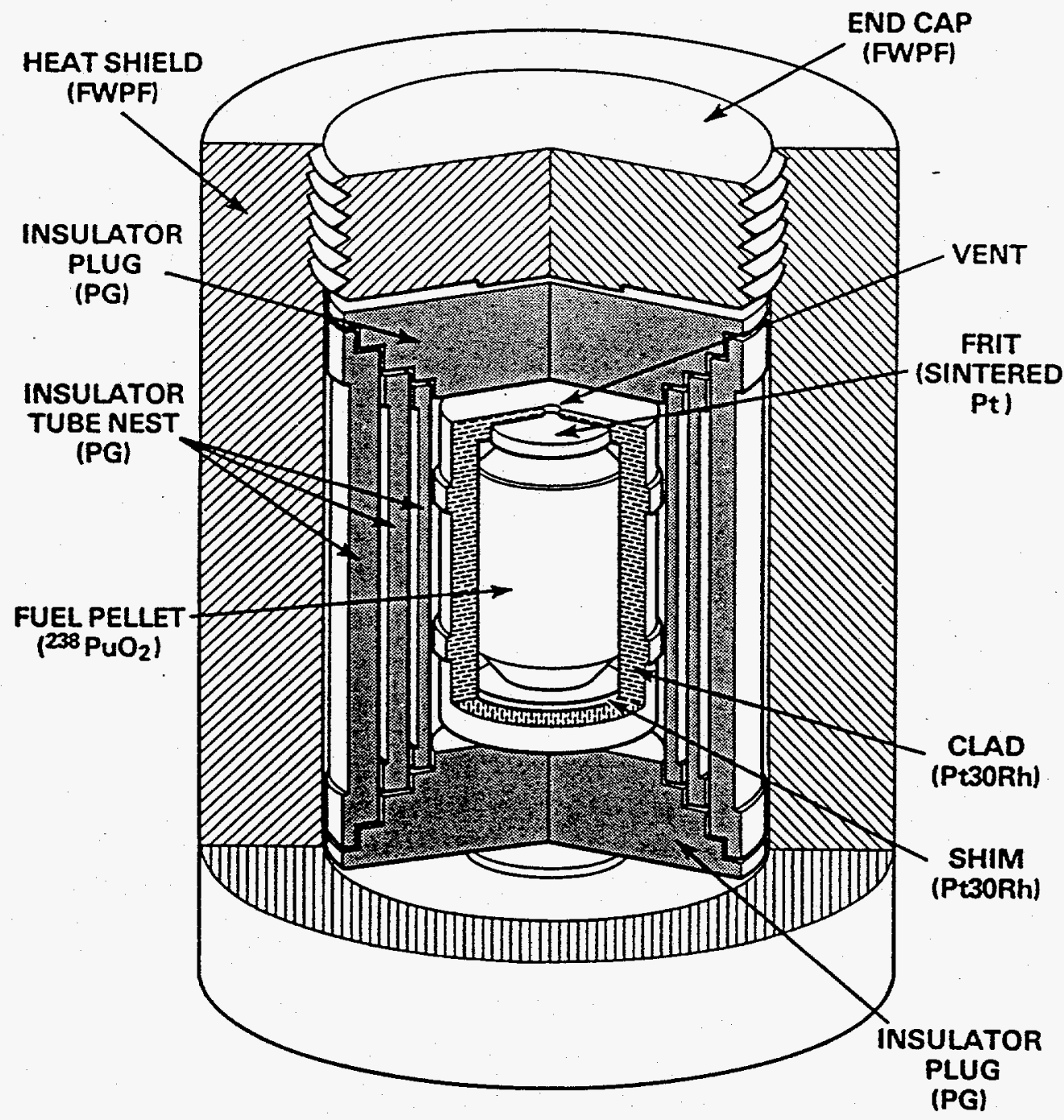

Figure 1 - Cross-sectional view of LWRHU assembly (from Ref. 1) 


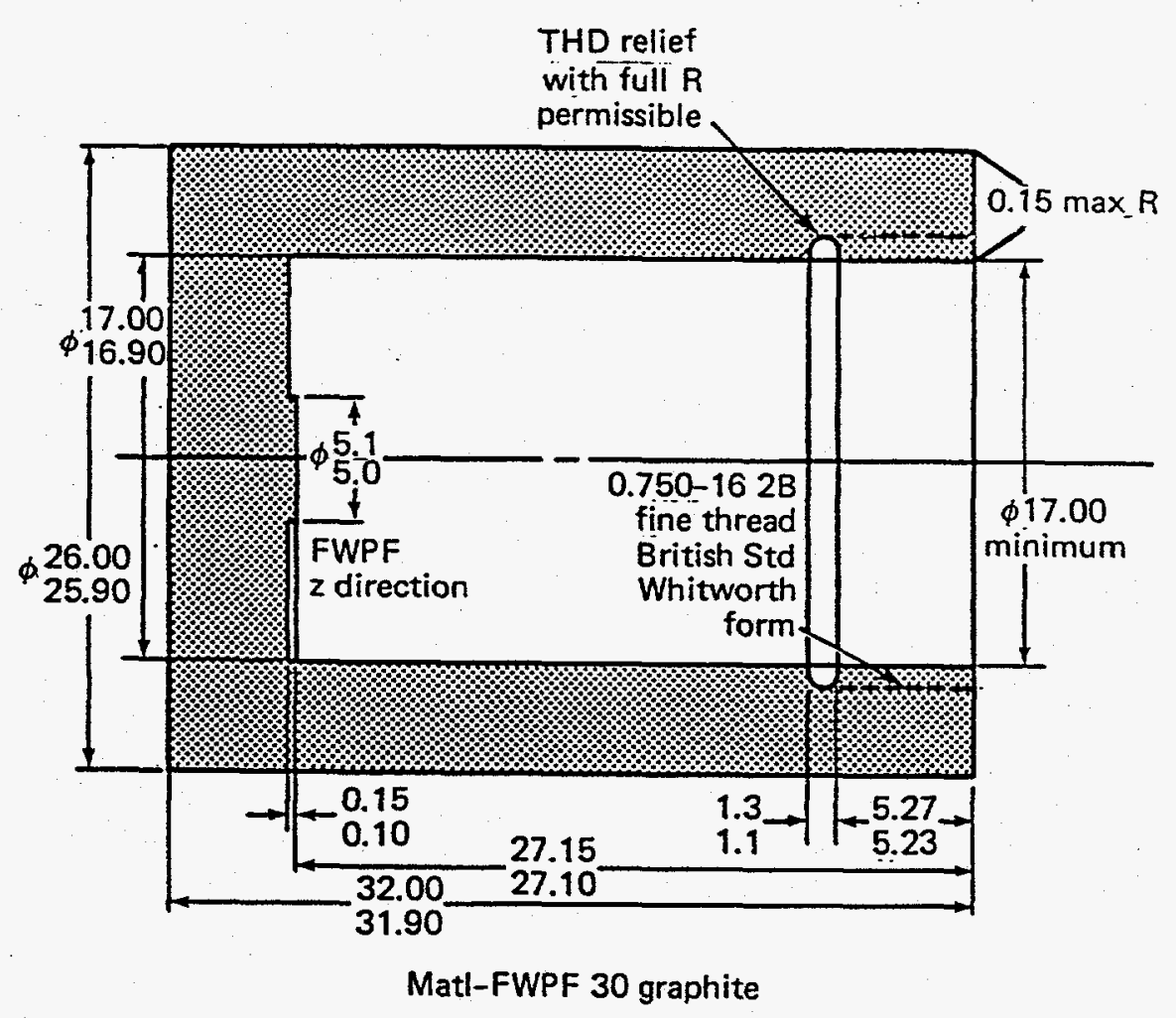

Dimensions in mm: $W=39.787 \mathrm{~g}$ (TYP) for LWRHU assembly

Figure 2- LWRHU aeroshell body (from Ref. 1) 


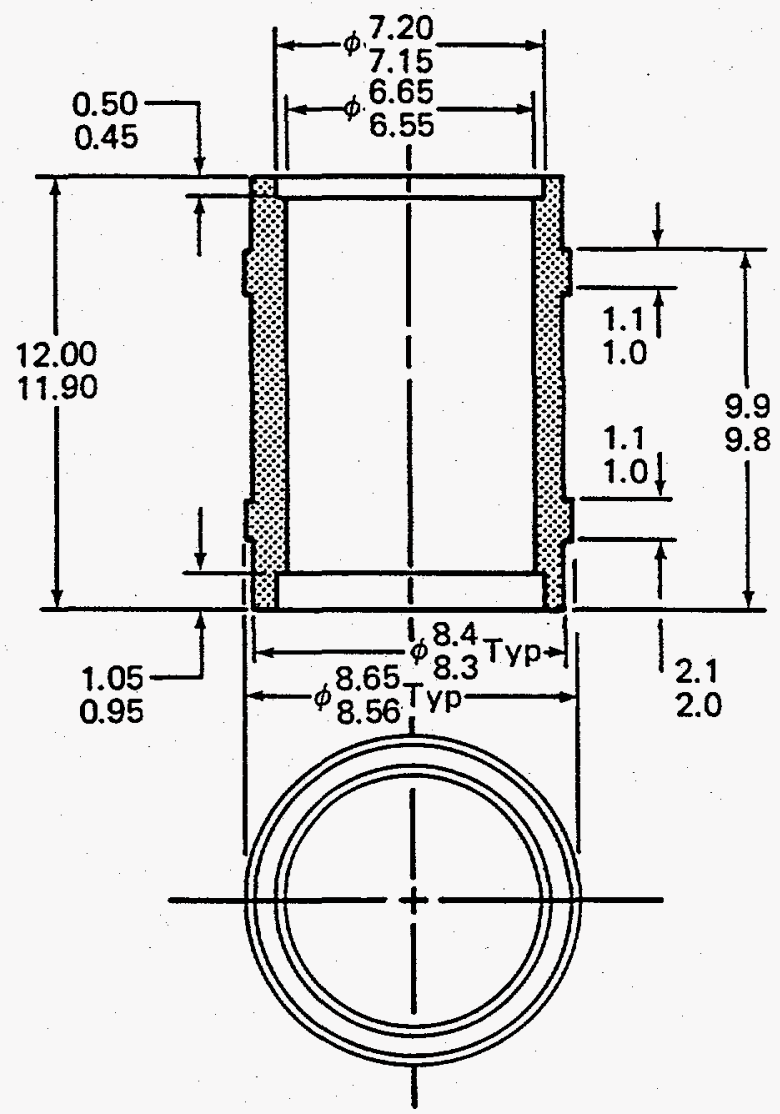

Dimensions in mm: $W=8.366 \mathrm{~g}$ (TYP) for CLAD/FUEL assembly

Figure 3- LWRHU clad body (from Ref. 1) 


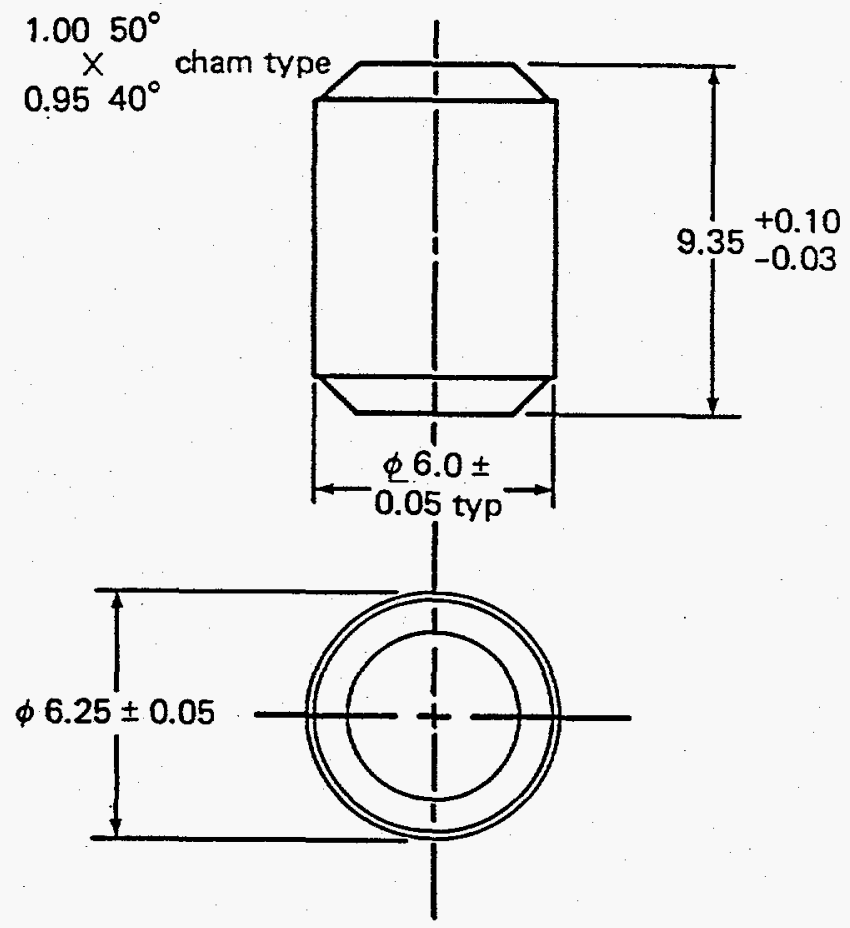

Dimensions in $\mathrm{mm}: \mathrm{W}=2.664 \mathrm{~g}$ (TYP)

Figure 4 - LWRHU fuel pellet (from Ref. 1) 


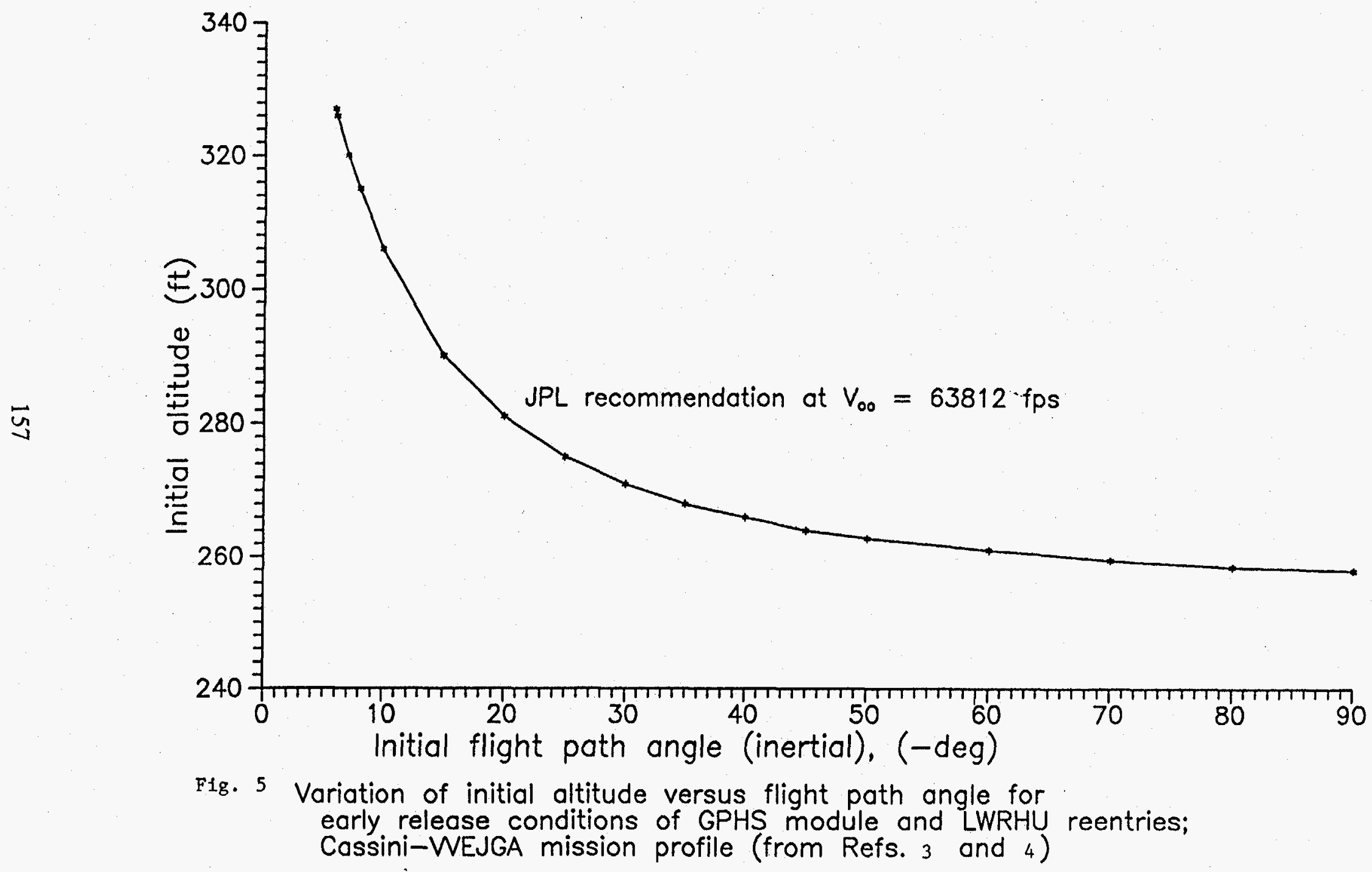


Figure 6. Cassini Mission TDOF Results

LWRHU Side-on Stable and Side-on Spinning

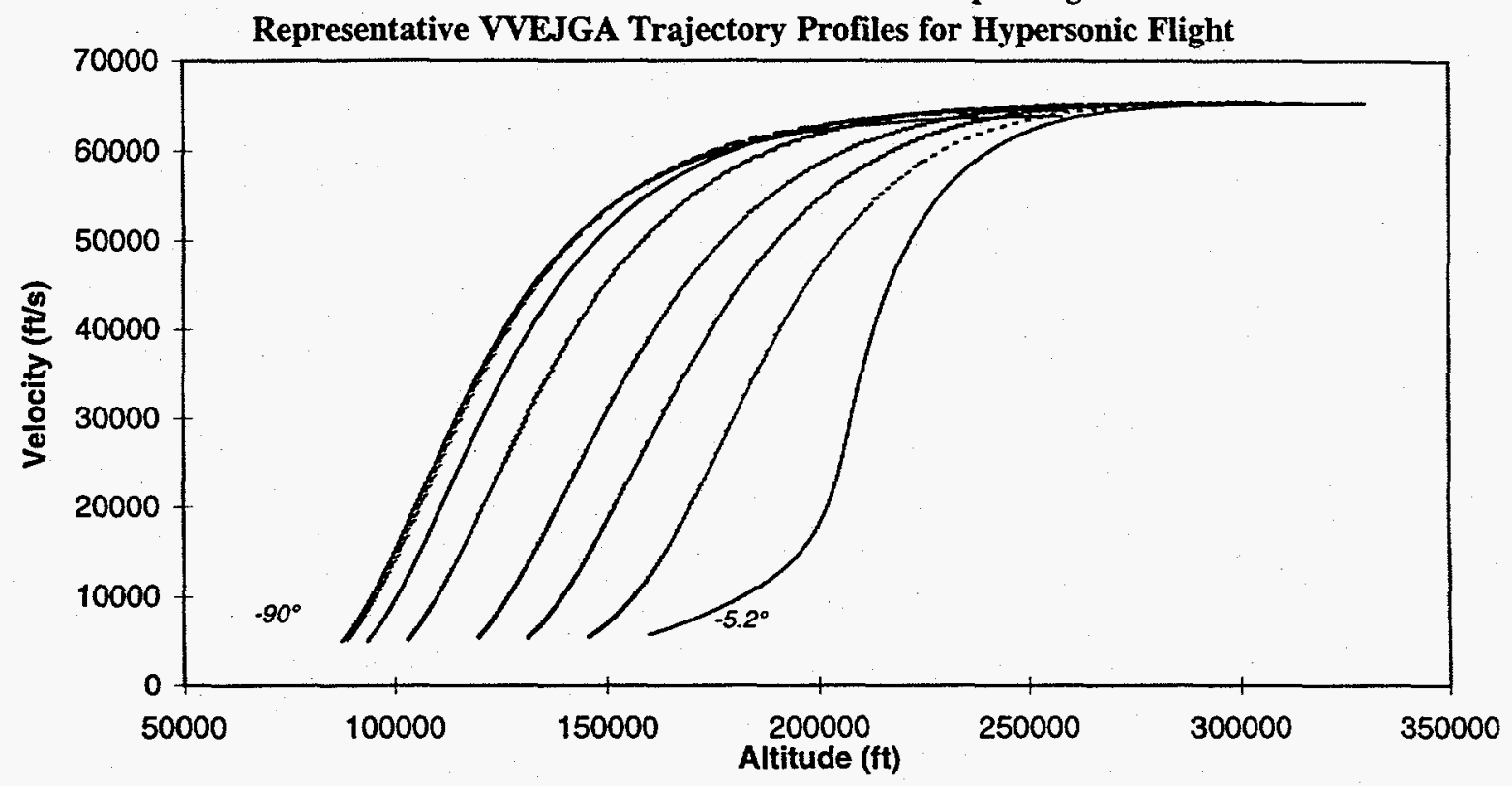

Reentry angles shown in descending order left to night: $-90^{\circ},-70^{\circ},-50^{\circ},-30^{\circ},-15^{\circ},-10^{\circ},-7^{\circ},-5.2^{\circ}$ TDOF Run through $M=5$

Figure 7. Cassini Mission TDOF Results

LWRHU Side-on Stable and Side-on Spinning

Representative VVEJGA Reentry Altitude History

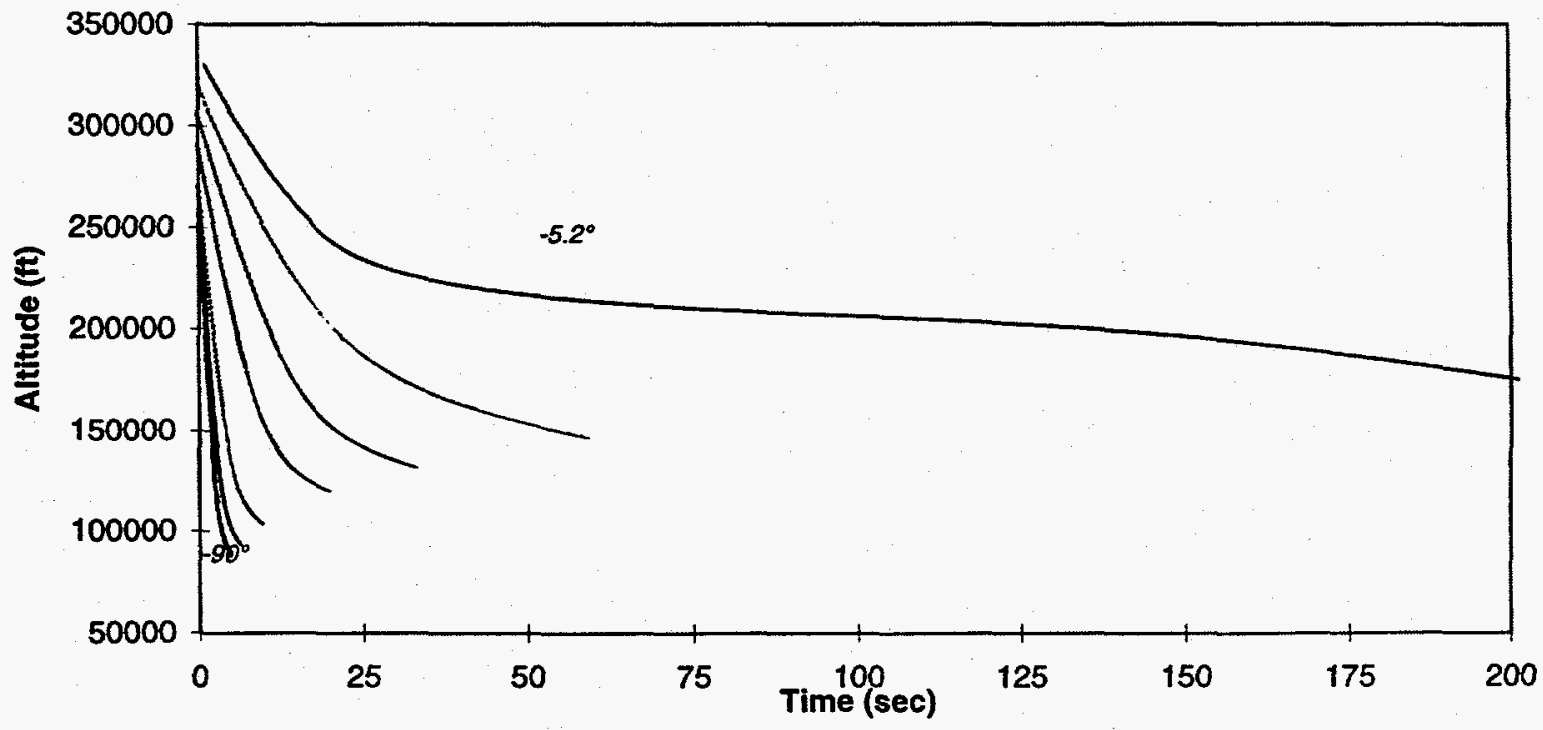

Reentry angles shown in descending order left to right: $-90^{\circ},-70^{\circ},-50^{\circ},-30^{\circ},-15^{\circ},-10^{\circ},-7^{\circ},-5.2^{\circ}$ 
Figure 8 Cassini Mission TDOF Results

LWRHU Tumbling Cylinder Reentry

Representative VVEJGA Trajectories for Hypersonic Flight

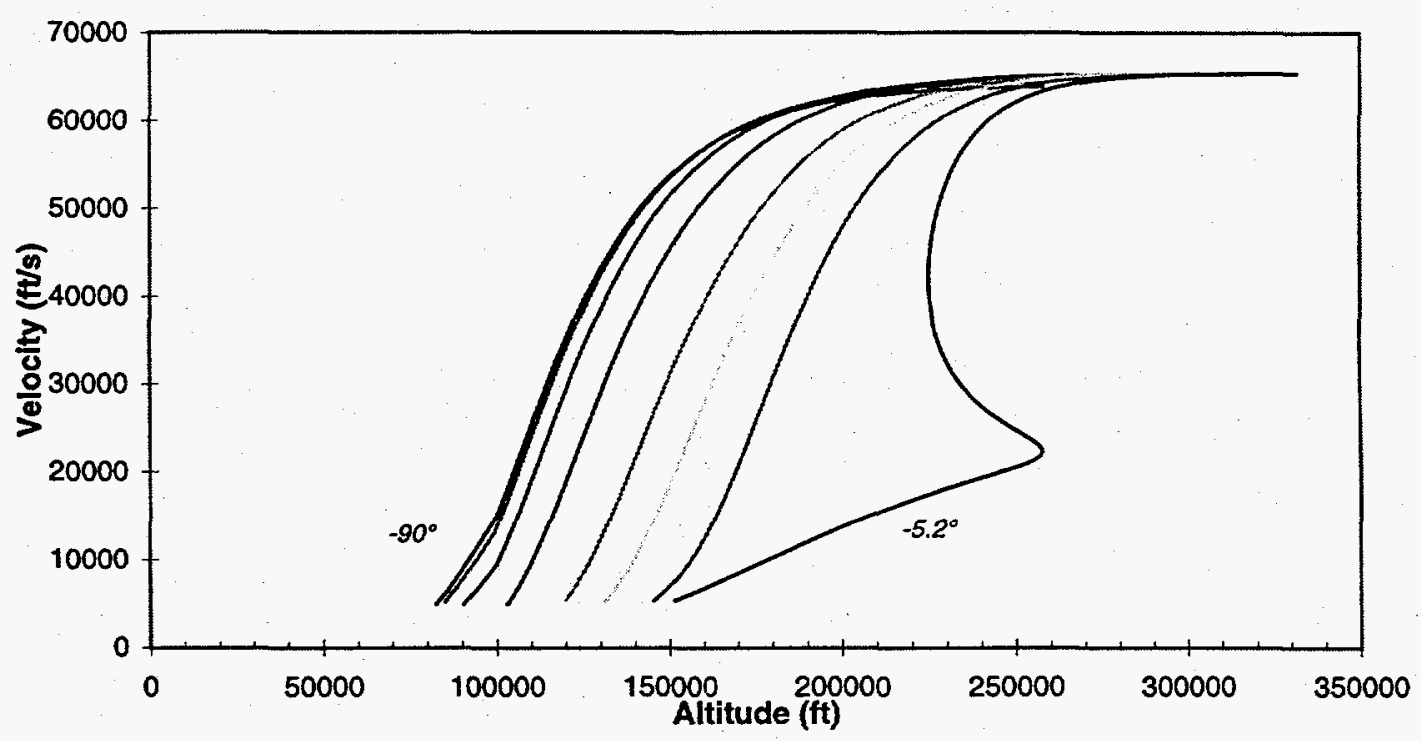

Reentry angles shown in descending order left to right: $-90^{\circ},-70^{\circ},-50^{\circ},-30^{\circ},-15^{\circ},-10^{\circ},-7^{\circ},-5.2^{\circ}$ TDOF Run through $M=5$

Figure 9. Cassini Mission TDOF Results LWRHU Tumbling Cylinder Reentry Representative VVEJGA Altitude History

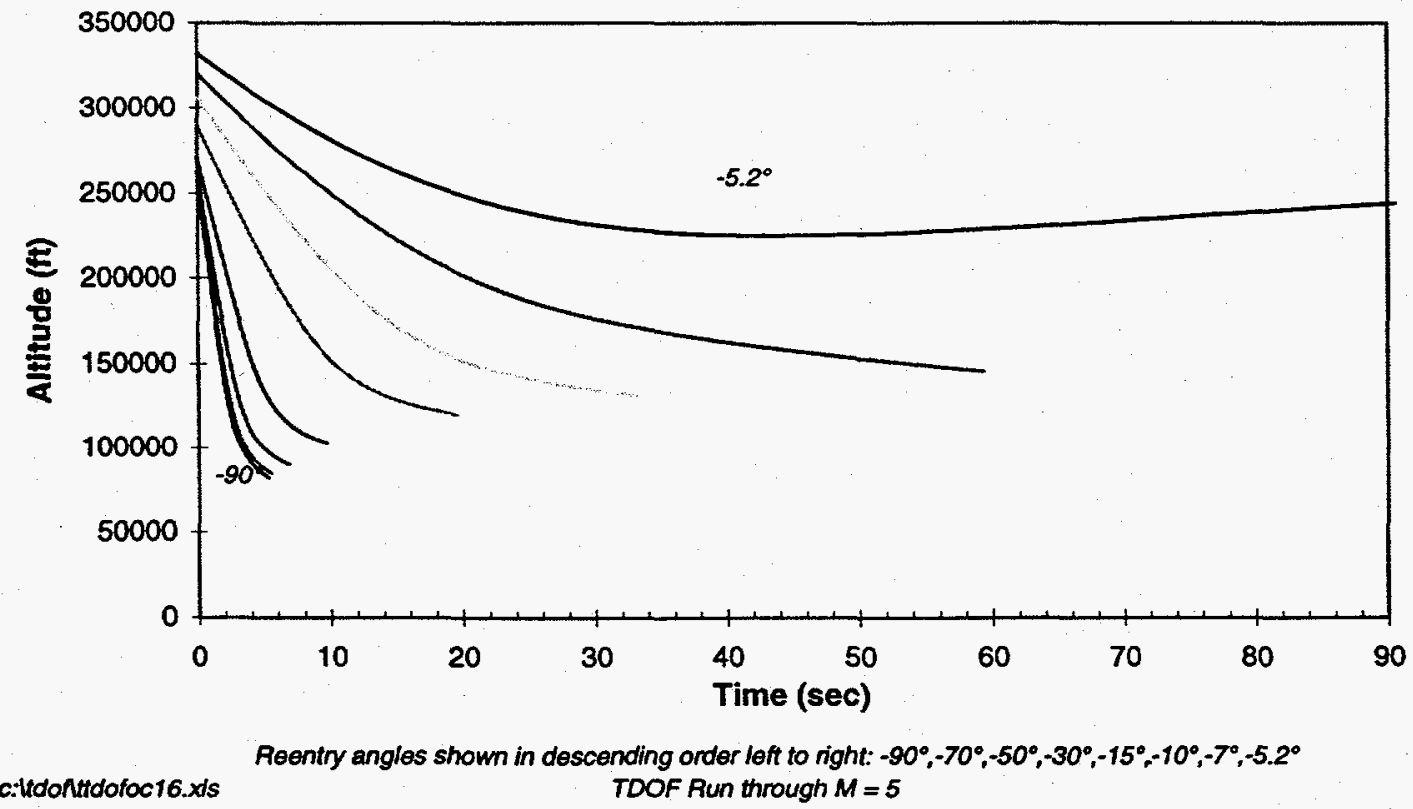


Figure 10. Cassini Mission TDOF Results LWRHU Tumbling Sphere

Representative VVEJGA Trajectories for Hypersonic Flight

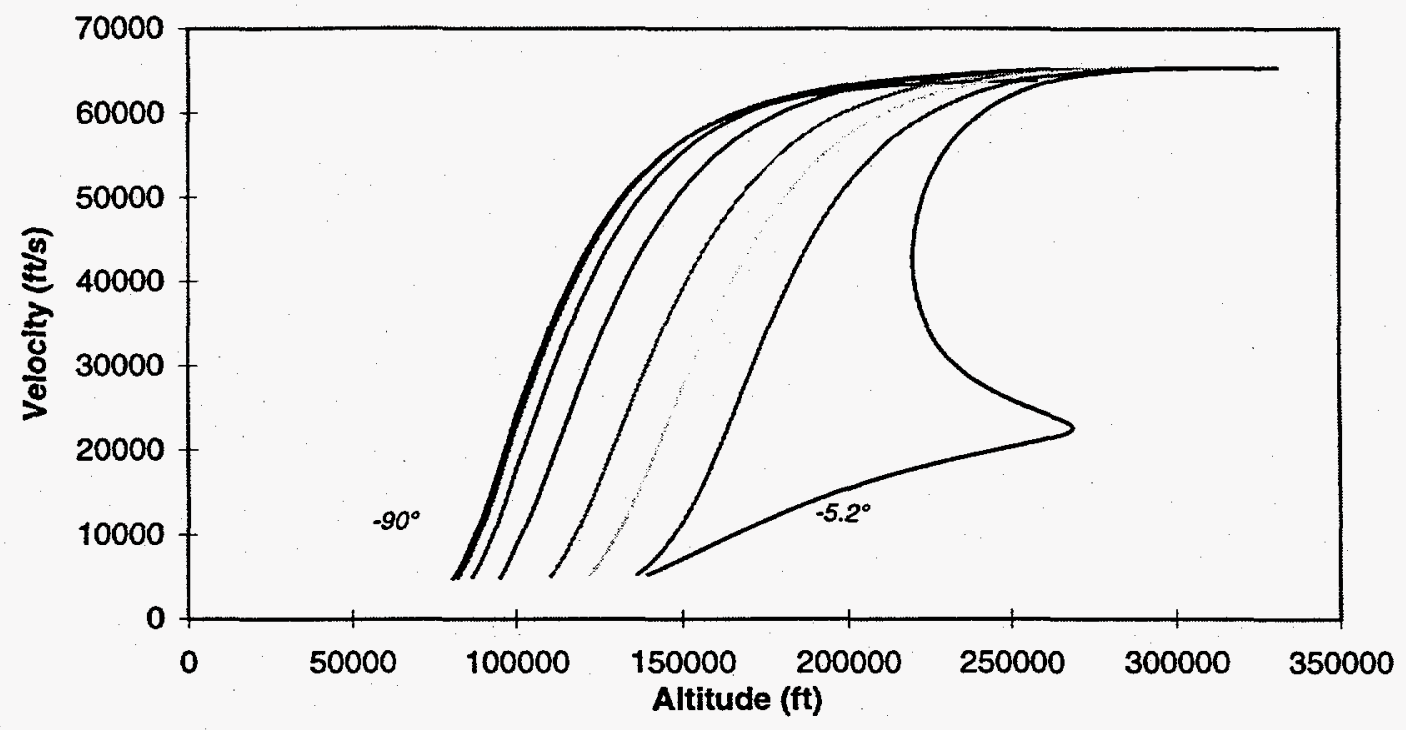

Reentry angles shown in descending order left to right: $-90^{\circ},-70^{\circ},-50^{\circ},-30^{\circ},-15^{\circ},-10^{\circ},-7^{\circ},-5.2^{\circ}$ TDOF Run through $M=5$

Figure 11. Cassini Mission TDOF Results LWRHU Tumbling Sphere Representative VVEJGA Altitude History

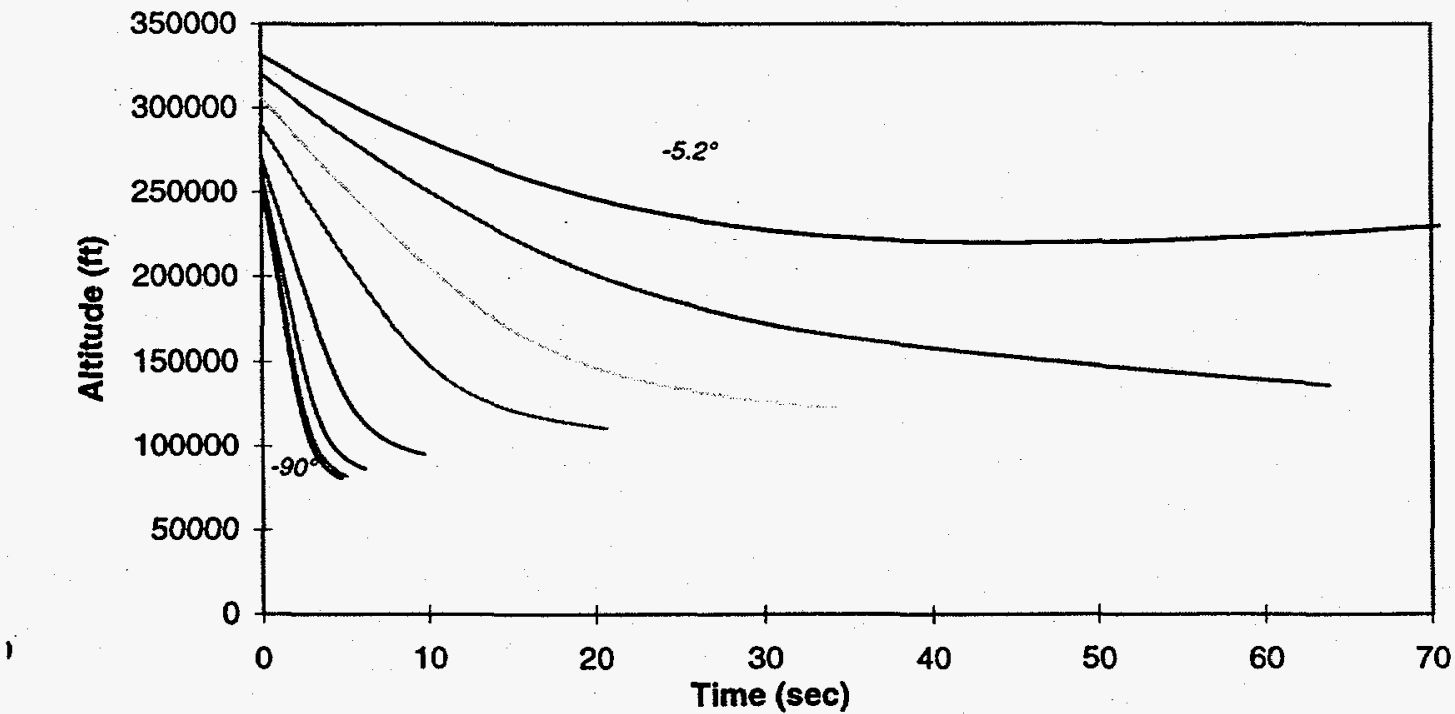

Reentry angles shown in descending order left to right: $-90^{\circ},-70^{\circ},-50^{\circ},-30^{\circ},-15^{\circ},-10^{\circ},-7^{\circ},-5.2^{\circ}$ c.trdoftdofoc16.xts TDOF Run through $M=5$ 
The Johns Hopkins University

Applied Physics Laboratory

Laurel, Maryland 20723-6099

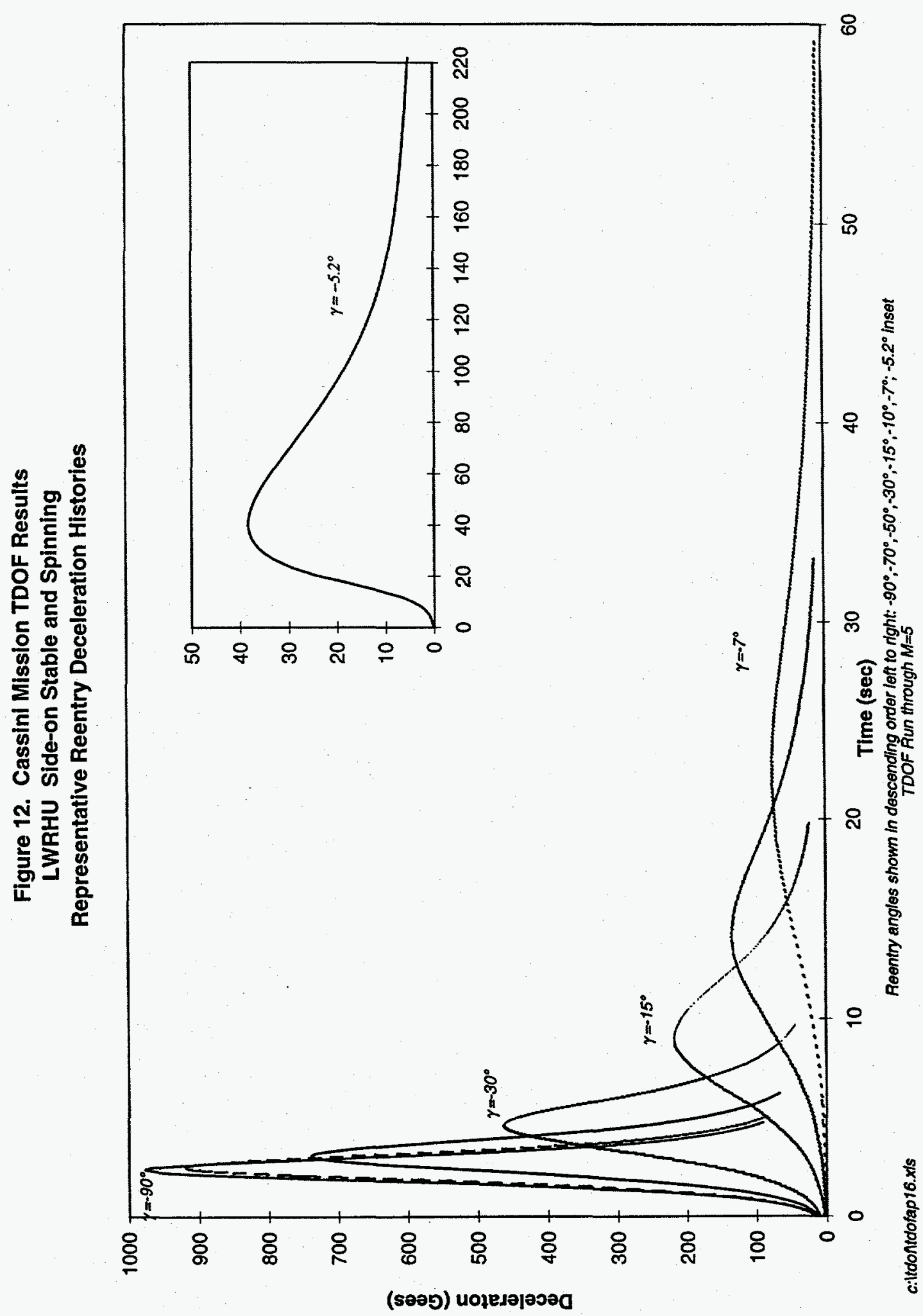


Figure 13. Cassini Mission TDOF Results

LWRHU Tumbling Cylinder Reentry

Representative Reentry Deceleration Histories

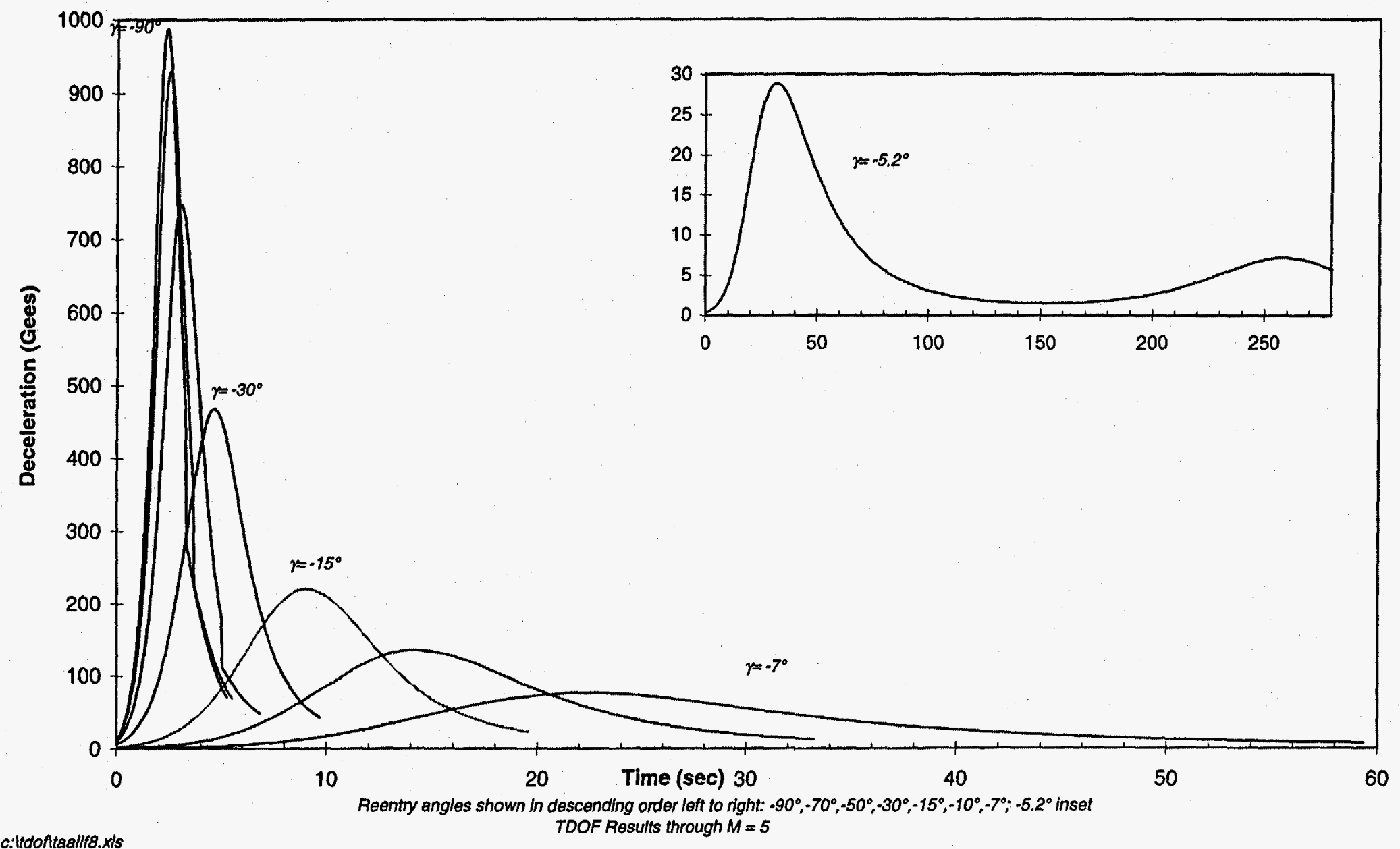


The Johns Hopkins University

Applied Physics Laboratory

Laurel, Maryland 20723-6099

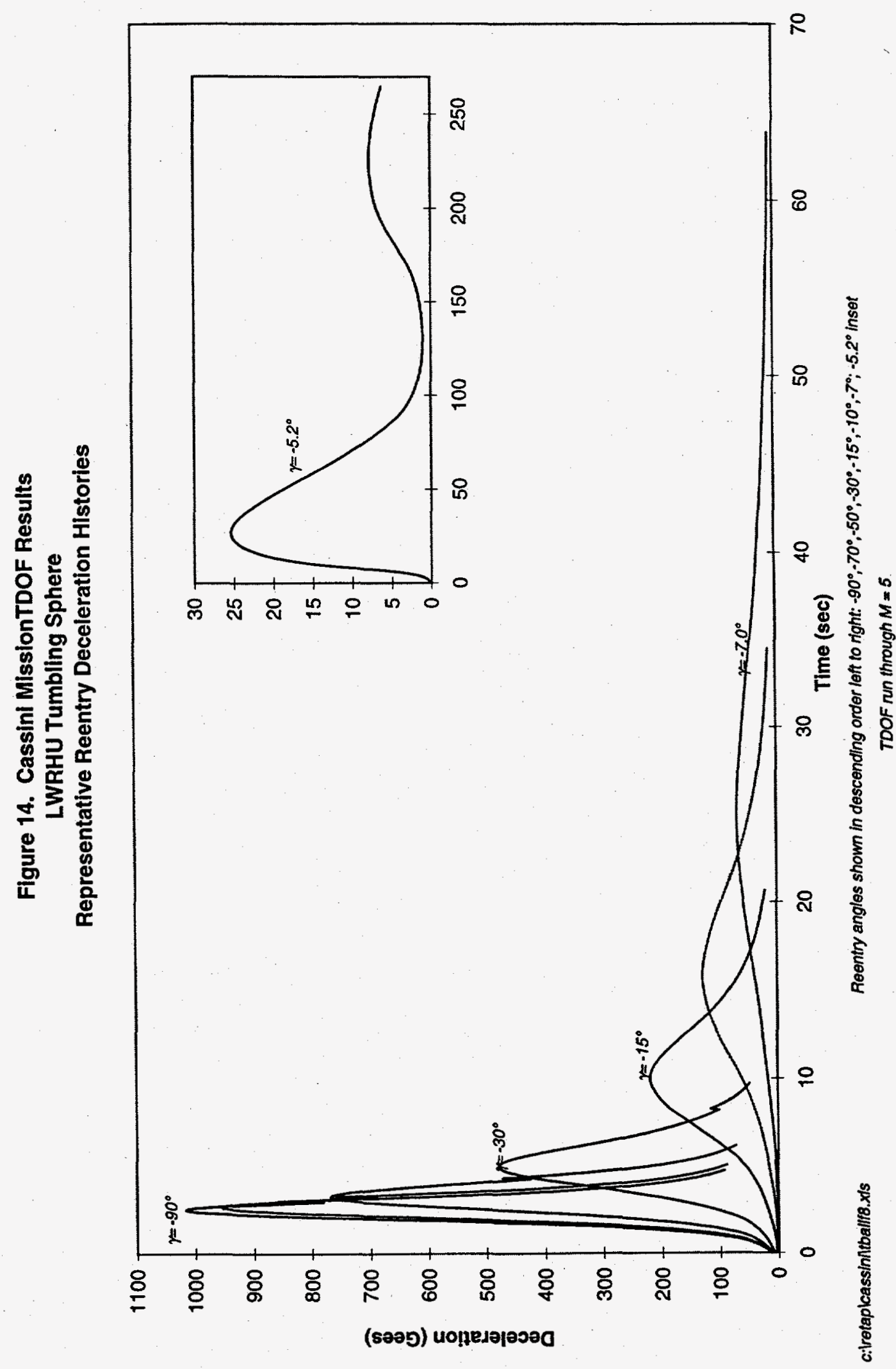


Figure 15. Cassini Mission TDOF Results

LWRHU Side-on Stable and Spinning

Steep Reentry Reference Convective Heating Histories

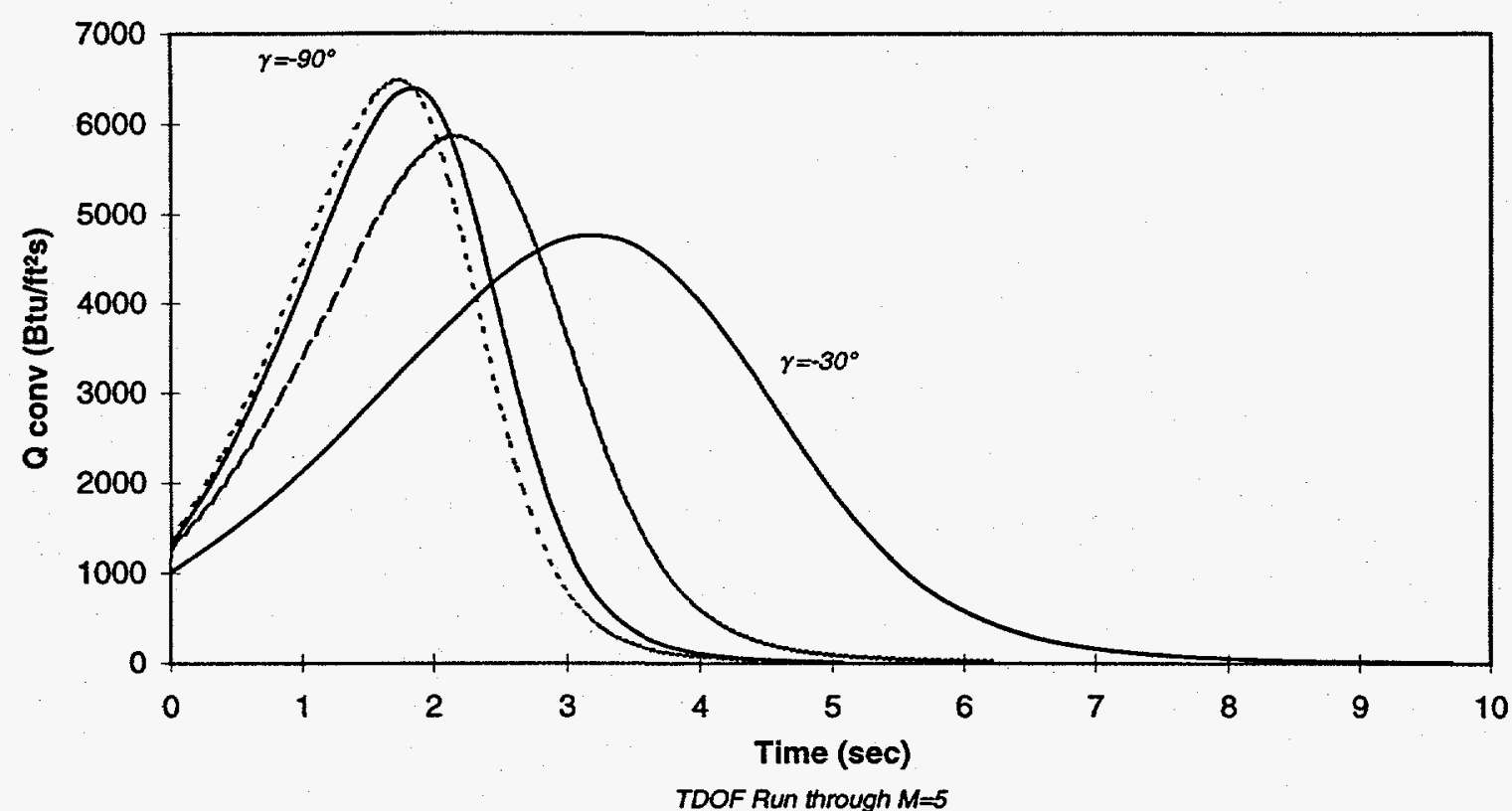

Reentry angles shown in descending order left to right: $-90^{\circ},-70^{\circ},-50^{\circ},-30^{\circ}$

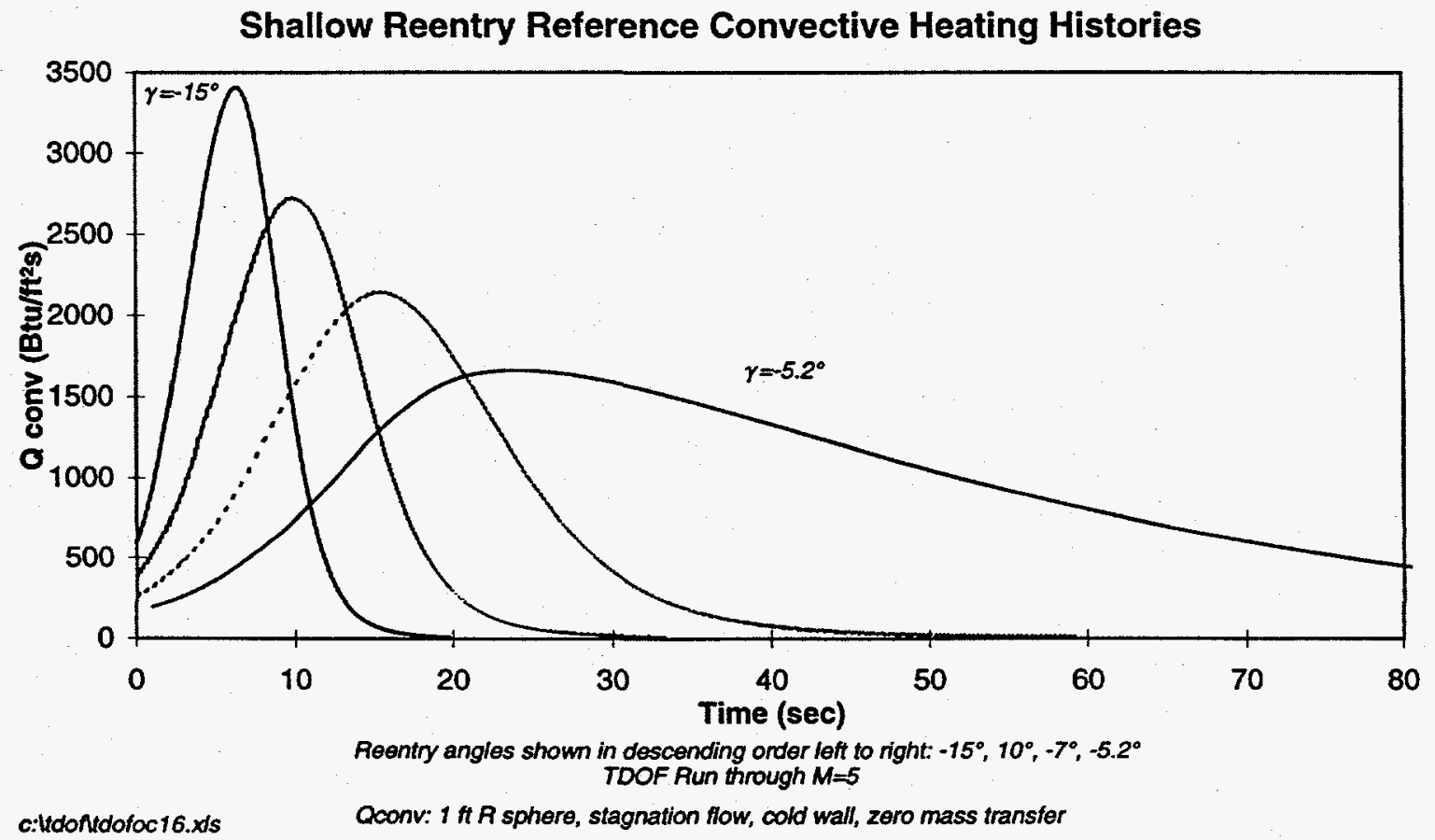


Figure 16. Cassini Mission TDOF Results LWRHU Tumbling Cylinder

Steep Reentry Reference Convective Heating Histories

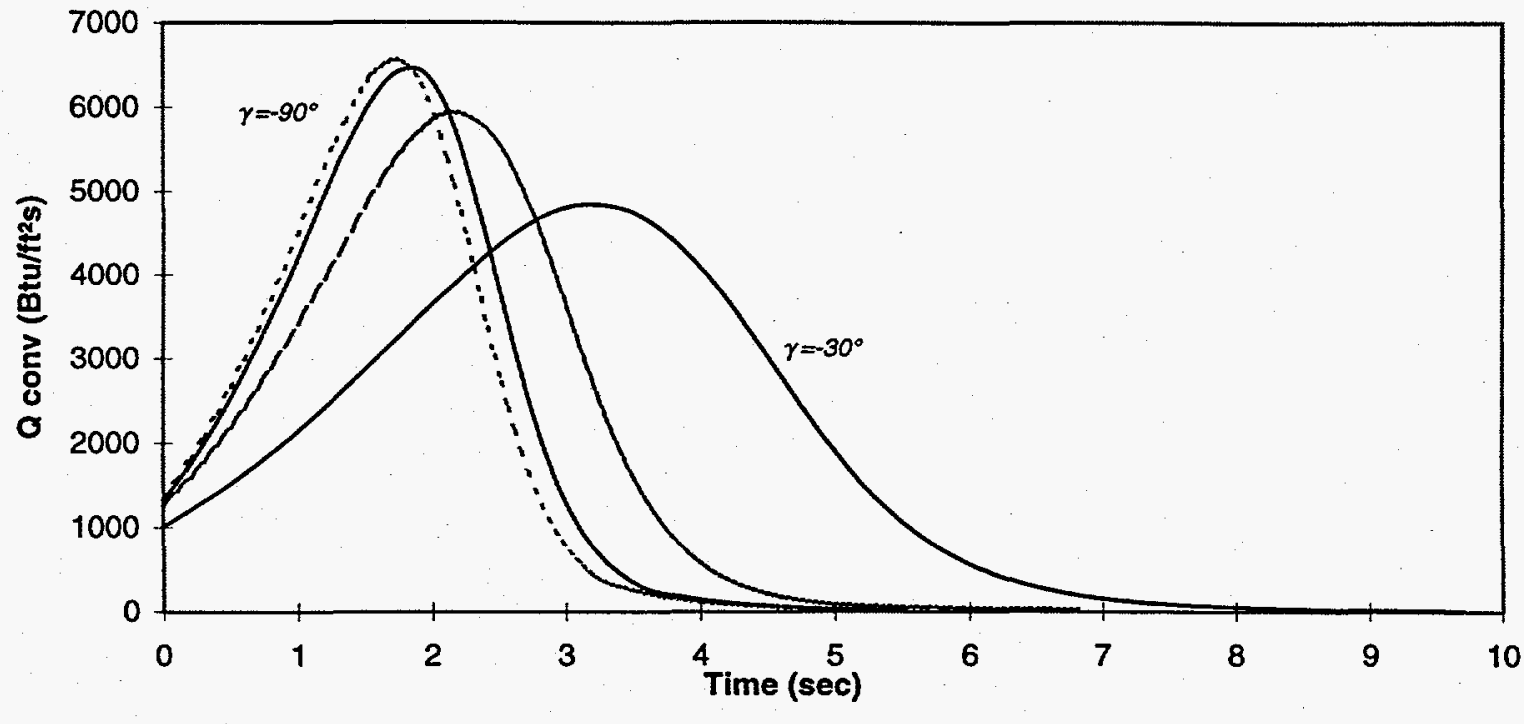

TDOF Run through $M=5$

Reentry angles shown in descending onder left to right: $-90^{\circ},-70^{\circ},-50^{\circ},-30^{\circ}$

Shallow Reentry Reference Convective Heating Histories

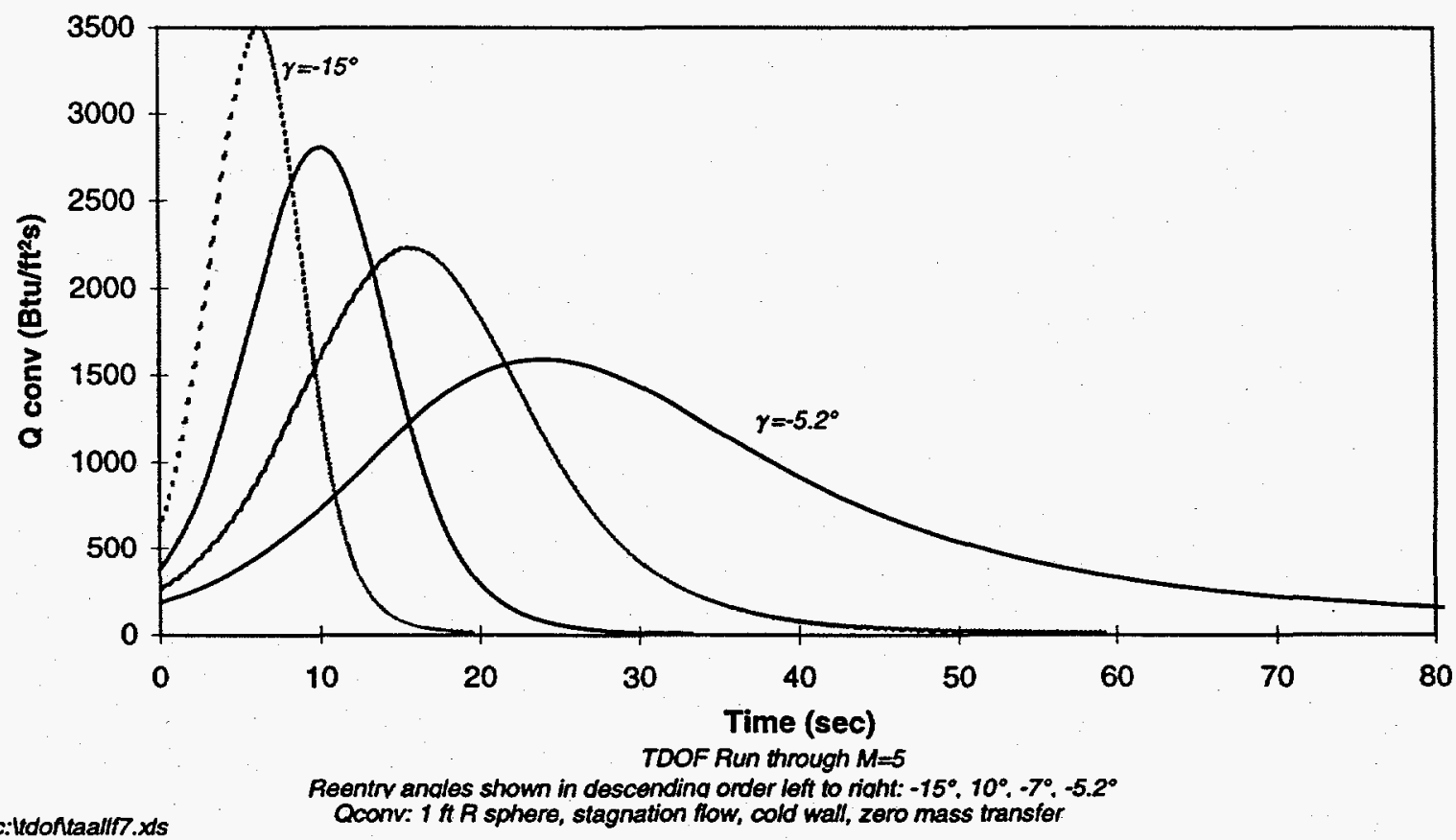


Figure 17. Cassini Mission TDOF Results LWRHU Tumbling Sphere

Steep Reentry Reference Convective Heating Histories

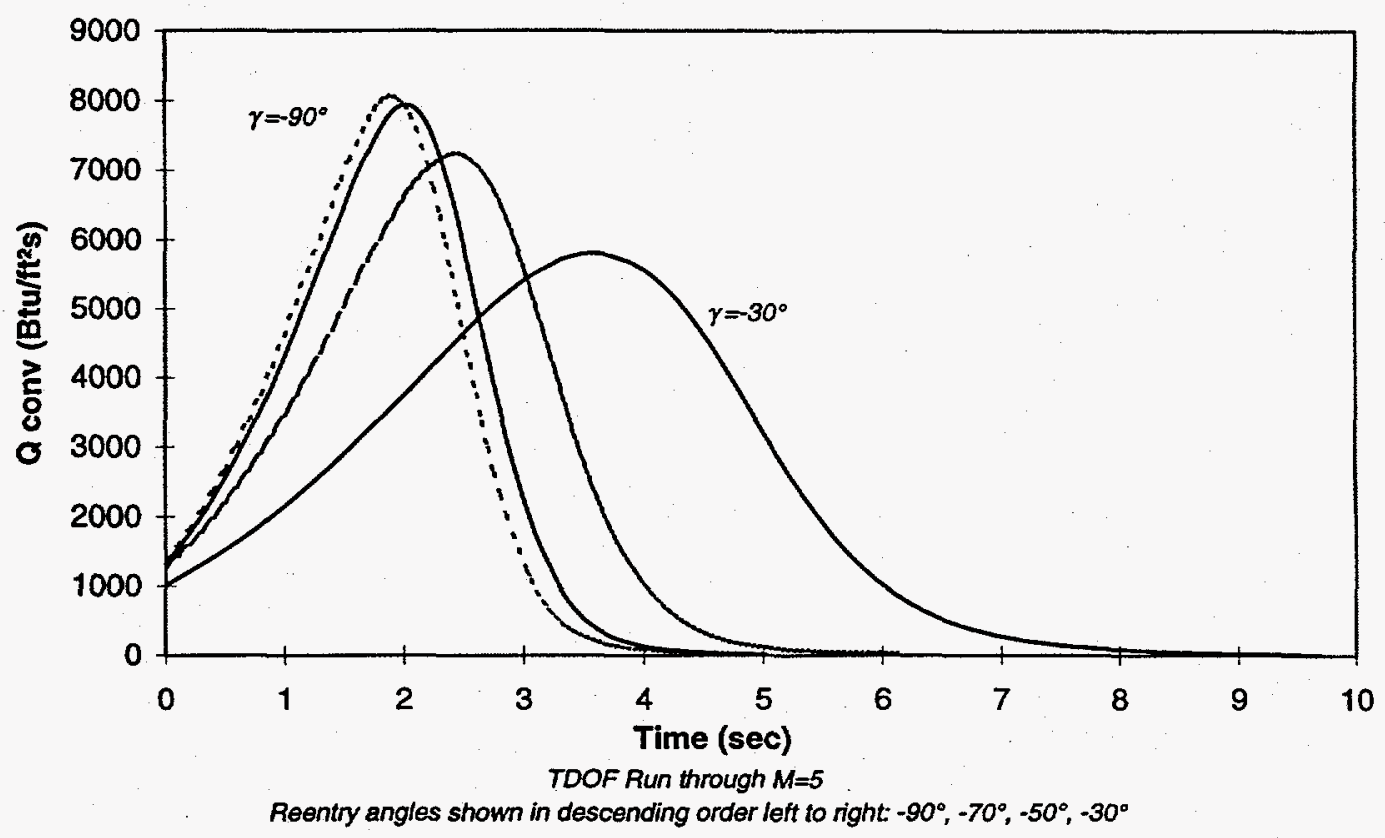

Shallow Reentry Reference Convective Heating Histories

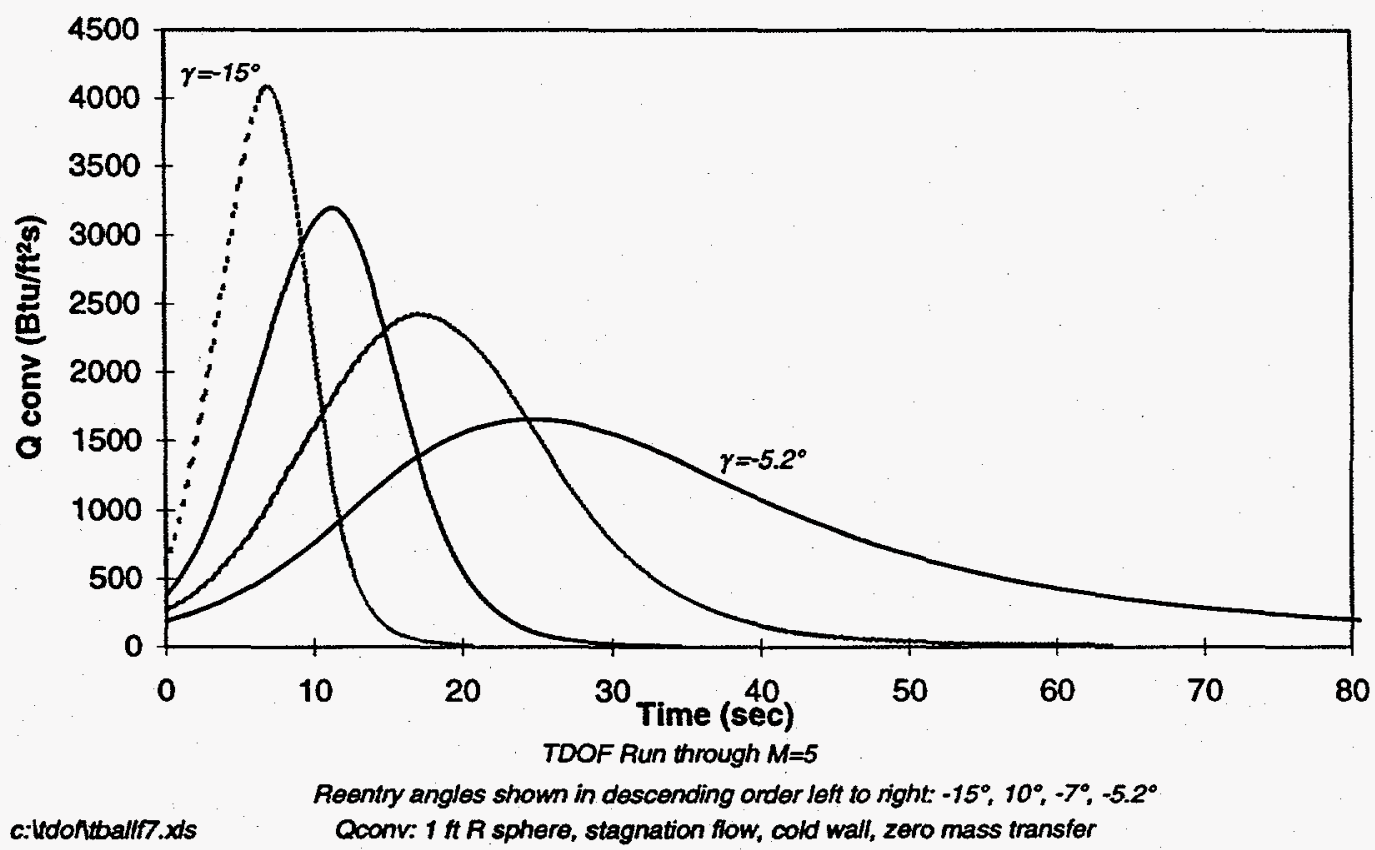


Figure 18. Cassini Mission TDOF Results

LWRHU Side-on Stable and Spinning Steep Reentry Reference Radiative Heating Histories

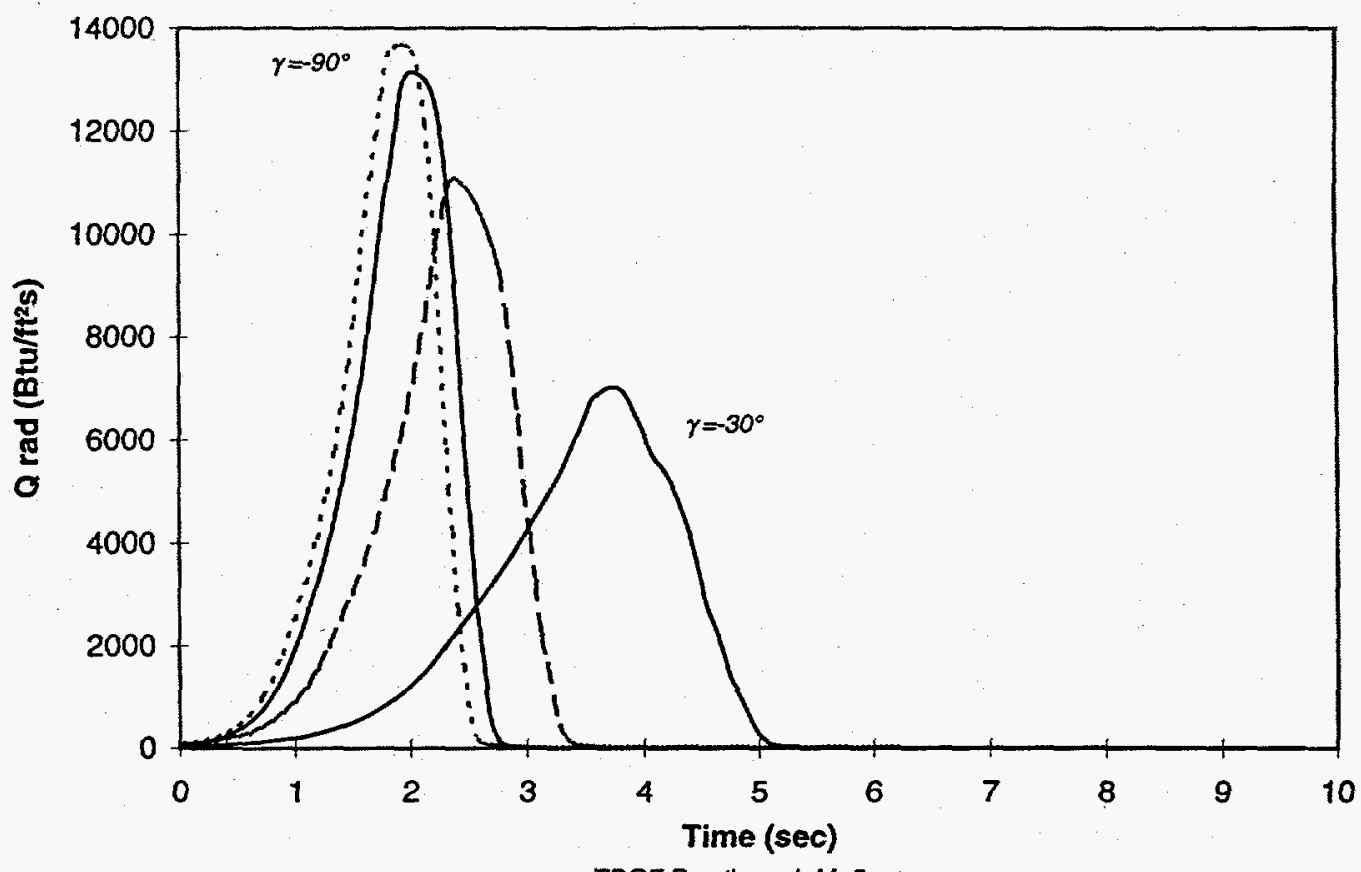

TDOF Run through $M=5$

keentry angies snown in cescenang oraer ien to ngnt: $-4 U^{-},-1 U^{-},-\infty U^{-},-3 U^{-}$ Shallow Reentry Reference Radiative Heating Histories

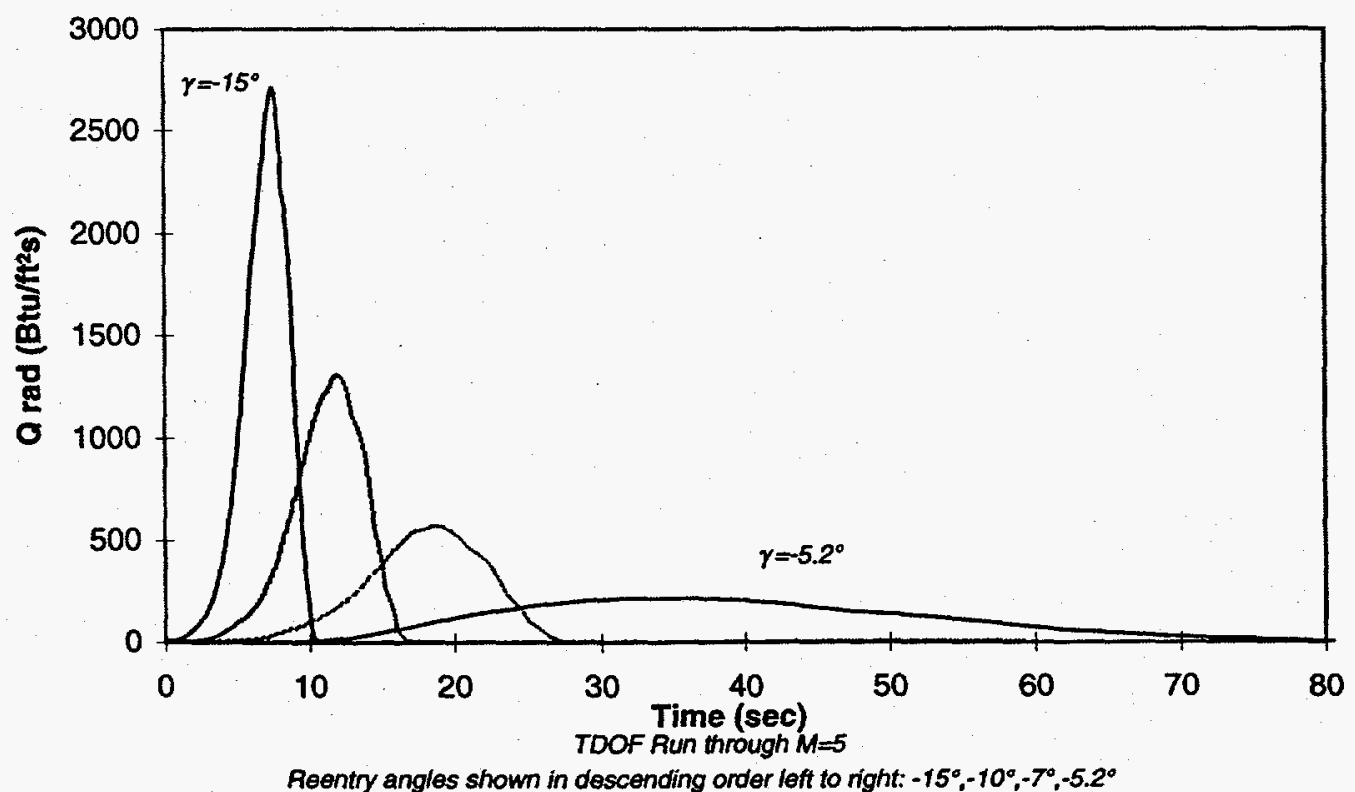

Orad: RAO/EQUIL, inviscid stagnation streamline flow, black body, effective spherical radius c.ttdoftdofoc $16 . x d s$ 
Applied Physics Laboratory

Laurel. Maryland 20723-6099

Figure 19. Cassini Mission TDOF Results

LWRHU Tumbling Cylinder

Steep Reentry Reference Radiative Heating Histories

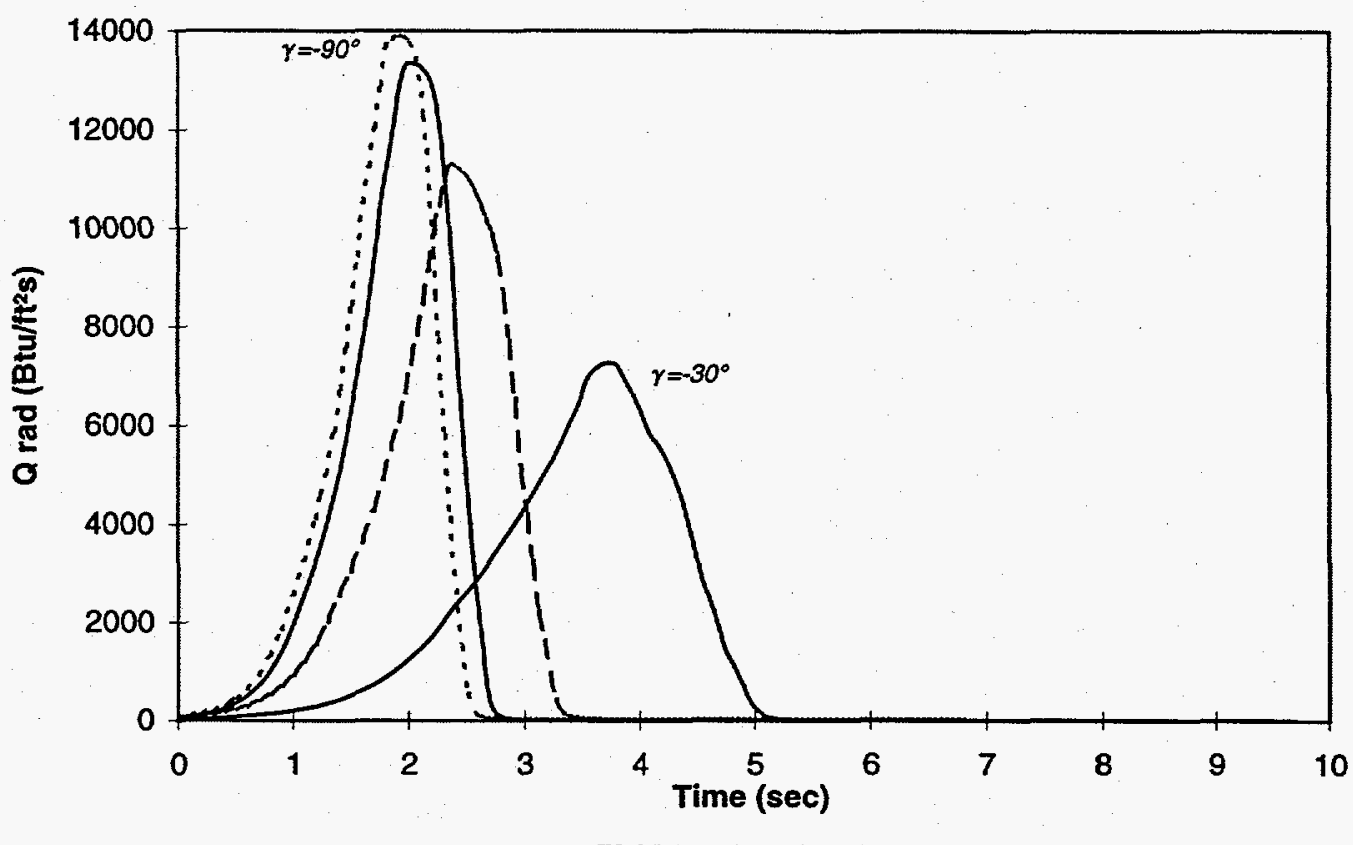

TDOF Run through $M=5$

Reentry angles shown in descending order left to right: $-90,-70^{\circ},-50^{\circ},-30^{\circ}$

Shallow Reentry Reference Radiative Heating Histories

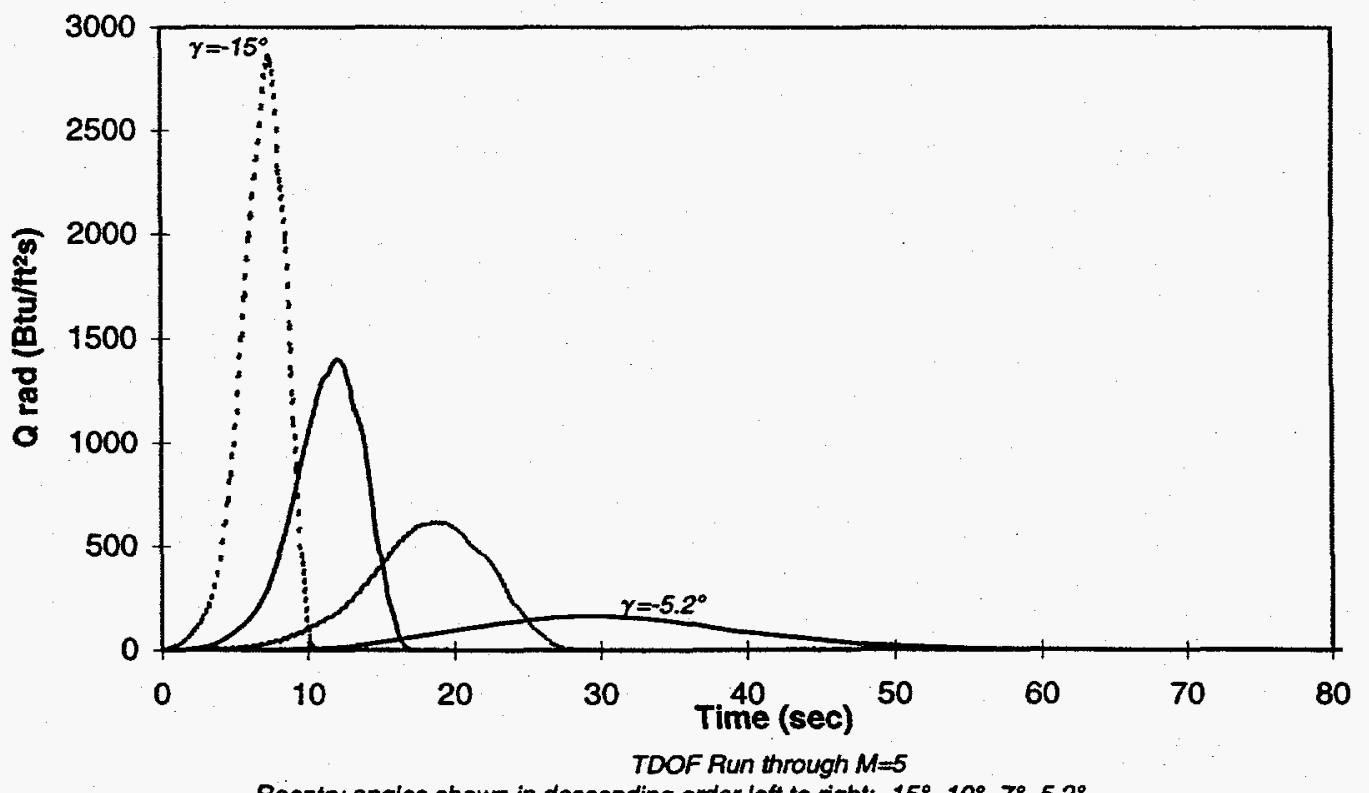

Reentry angles shown in descending order left to right: $-15^{\circ},-10^{\circ},-7^{\circ},-5.2^{\circ}$

Qrad: RAD/EQUIL, inviscid stagnation streamline flow, black body, effective spherical radius 
Figure 20. Cassini Mission TDOF Results

\section{LWRHU Tumbling Sphere}

Steep Reentry Reference Radiative Heating Histories

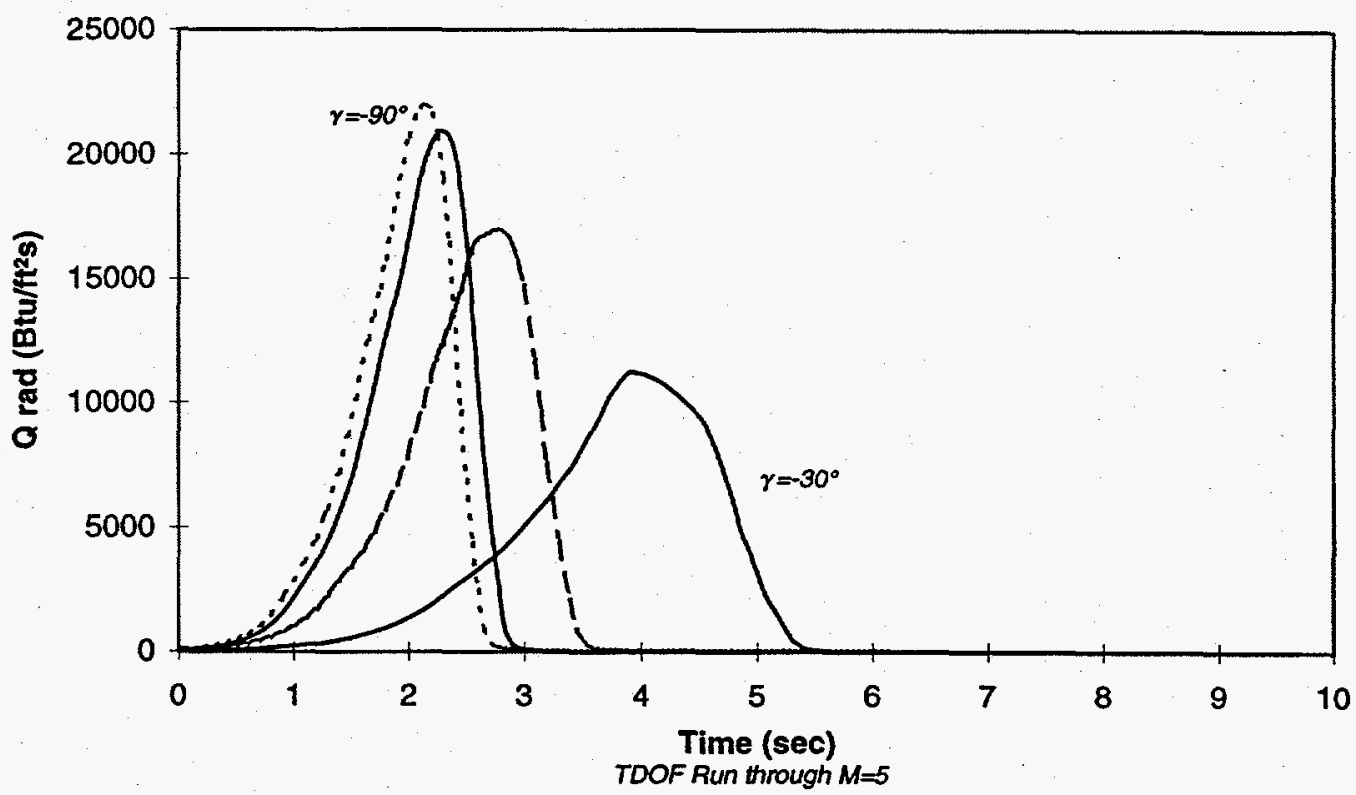

Reentry angles shown in descending order left to right: $-90,-70^{\circ},-50^{\circ},-30^{\circ}$

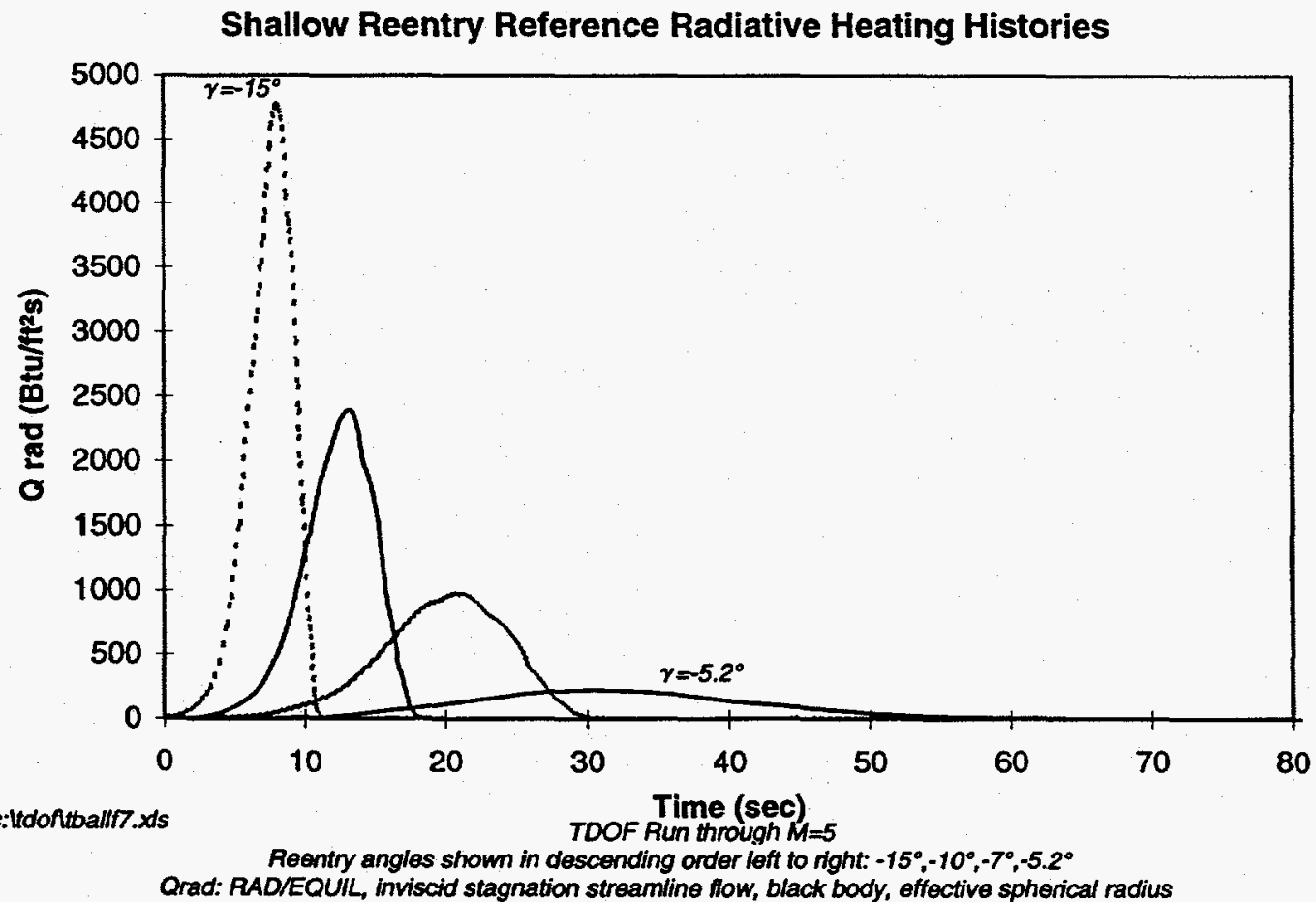


Applied Physics Laboratory

Laurel, Maryland 20723-6099

FIGURE 21. Cassini Mission TDOF Results

LWRHU $-90^{\circ}$ VVEJGA Reentry

Sample Steep Trajectory for Different Orientations
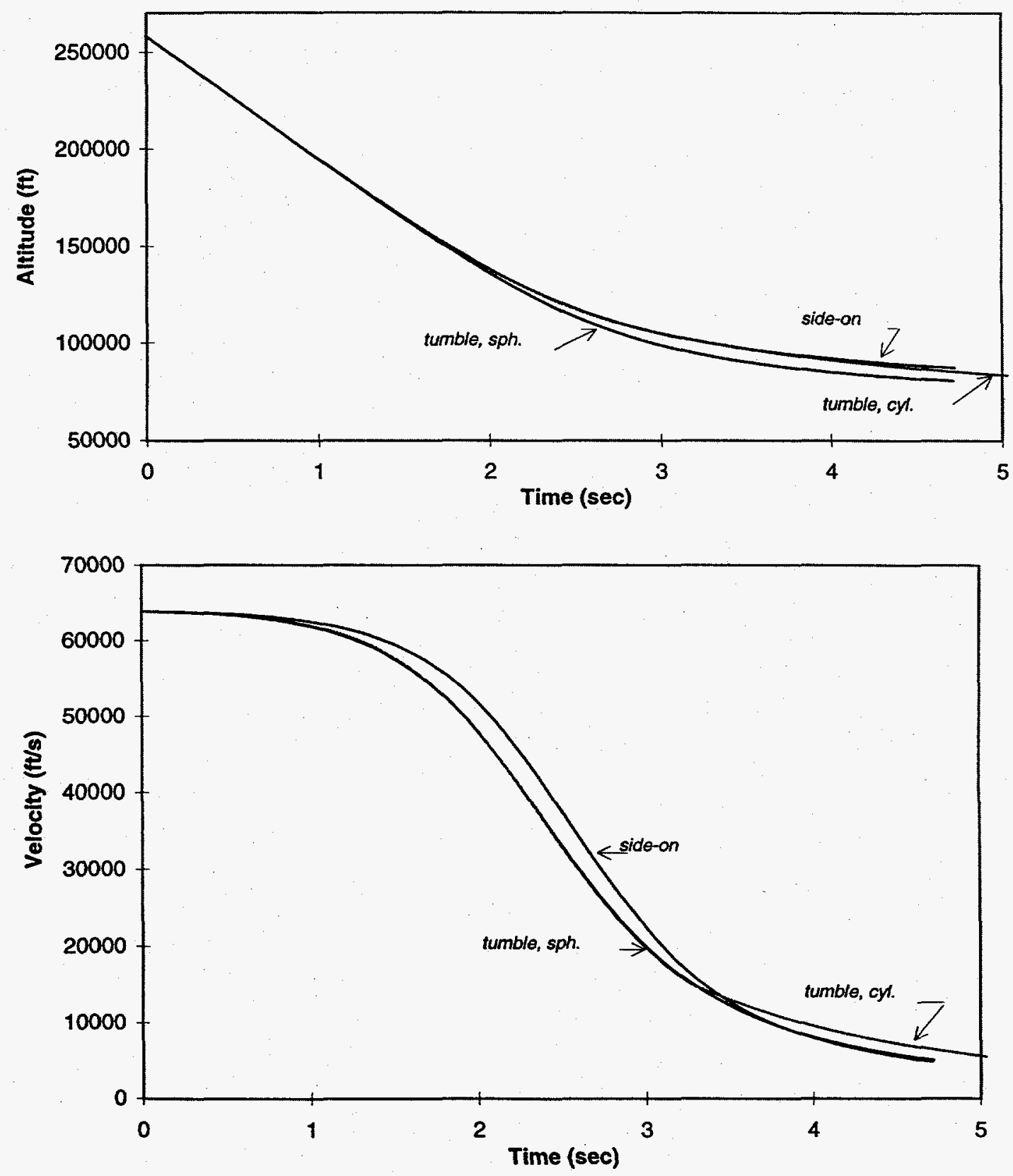

c:ttontumble.xts

TDOF Results Through Mach $=5$ 
Applied Physics Laboratory

Laurel, Maryland 20723.60)94

Figure 22. Cassini Mission TDOF

LWRHU $-07^{\circ}$ Reentry

Sample Shallow Altitude History for Different Orientations

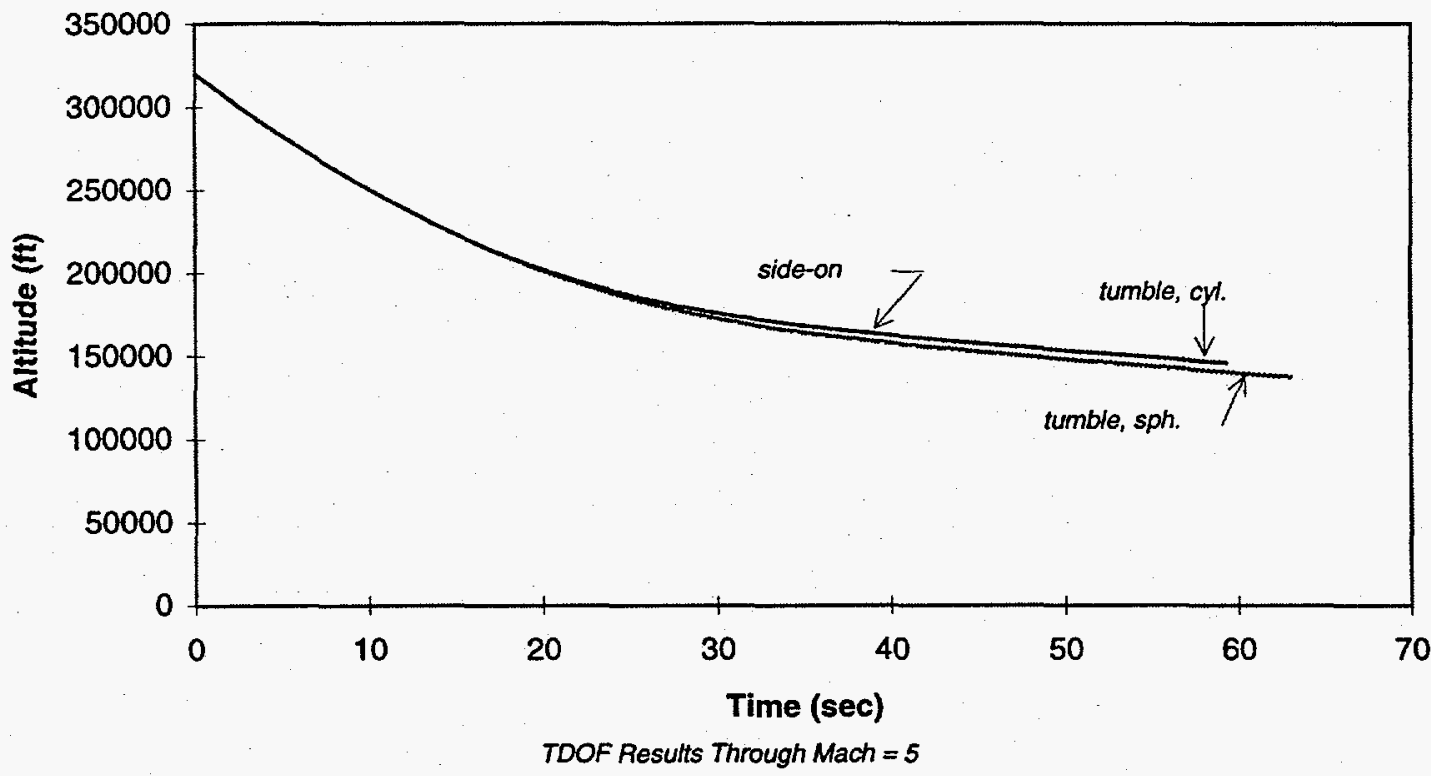

Sample Shallow Velocity History for Different Orientations

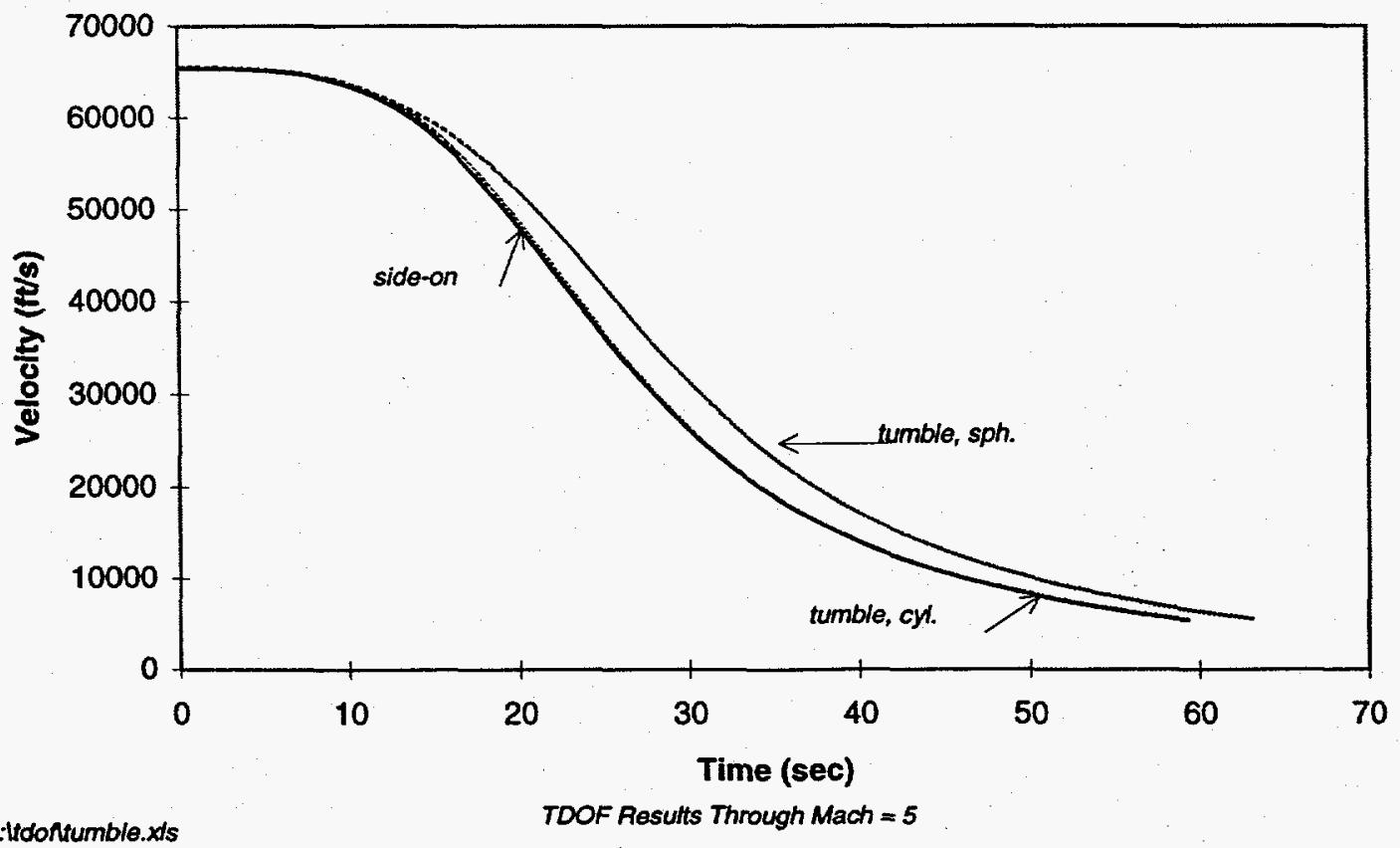


The Johns Hopkins University

Applied Physics Laboratory

Laurel, Maryland 20723.6099

Figure 23. Cassini Mission TDOF Results LWRHU

Sample Shallow Reentry Deceleration Forces for Different

Orientations

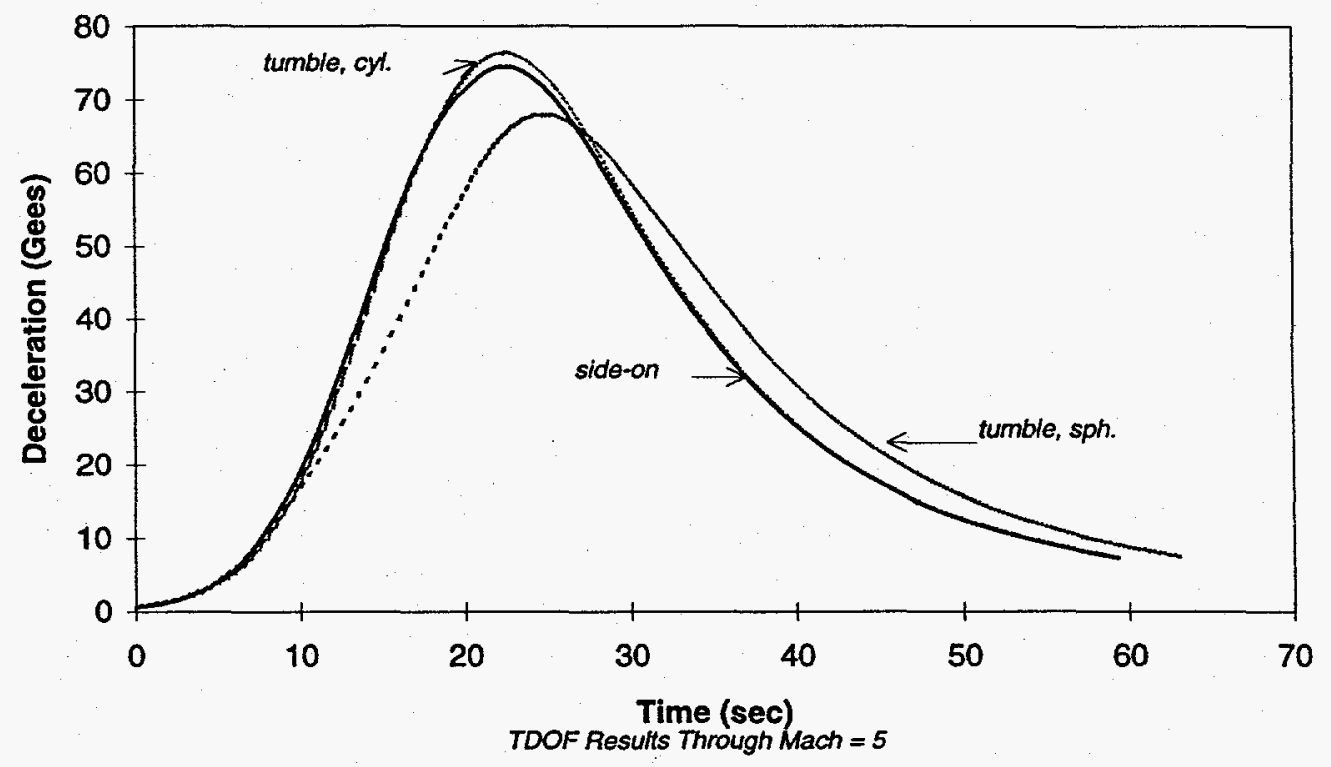

Sample Steep Reentry Deceleration Forces for Different Orientations

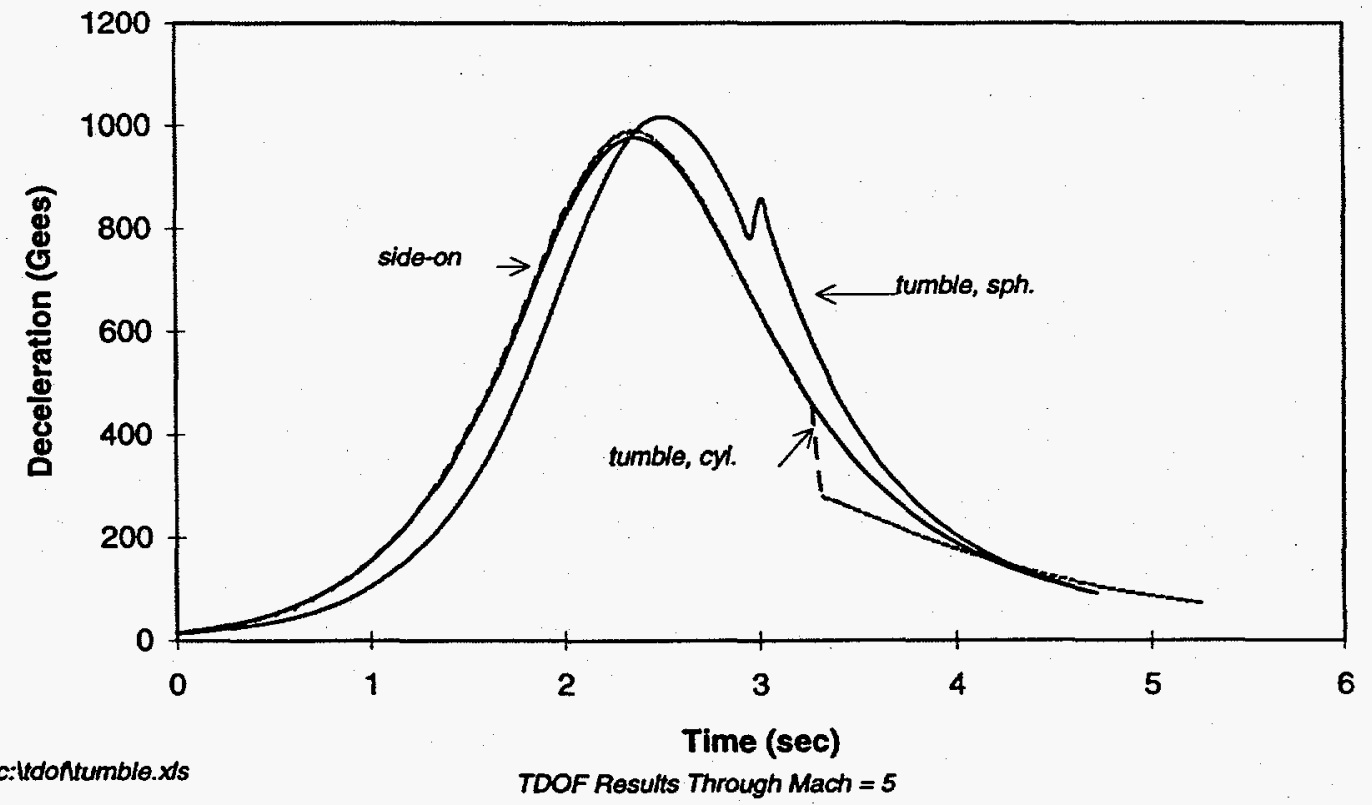


Applied Physics Laboratory

Laurel. Maryland 20723-6099

FIGURE 24. Cassini Mission TDOF Results

LWRHU $-90^{\circ}$ Reentry

Sample Steep Radiative Heating History for Different Orientations

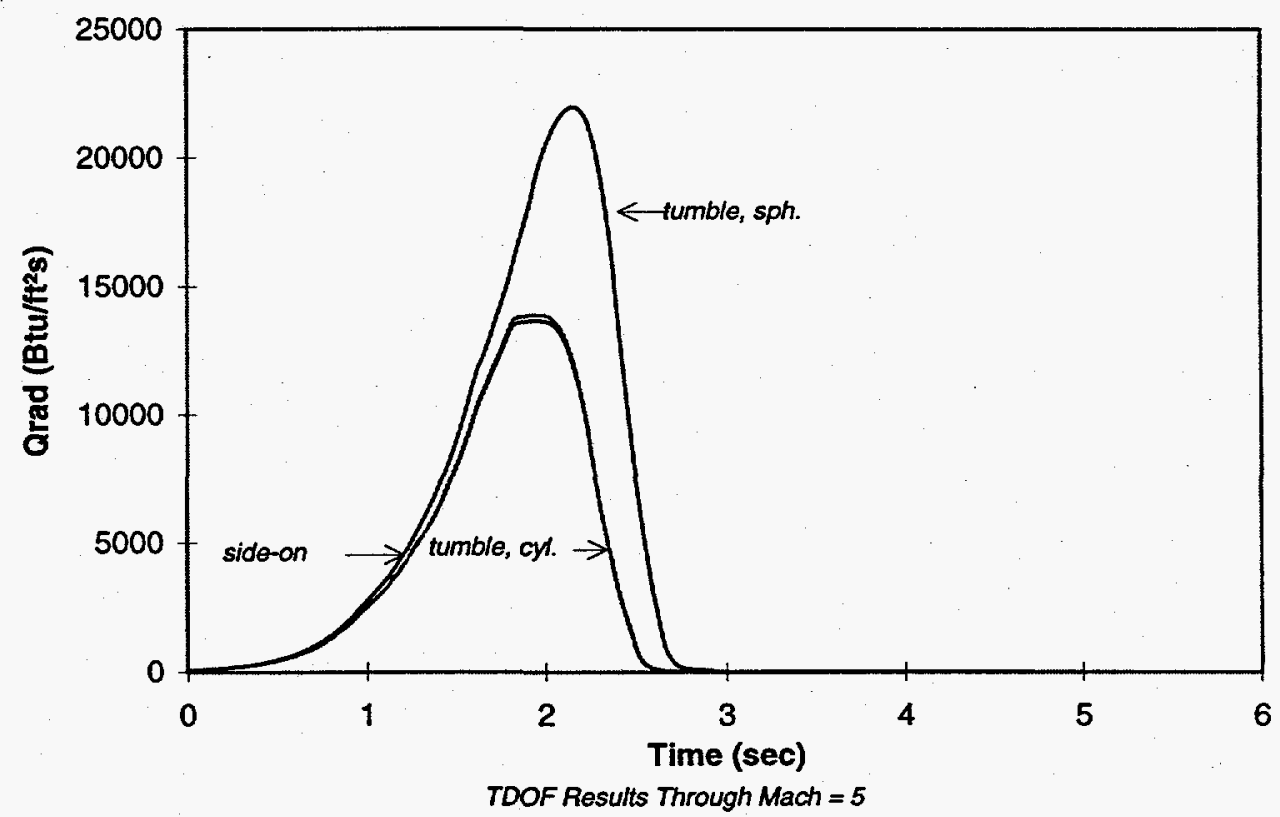

Sample Steep Convective Heating History

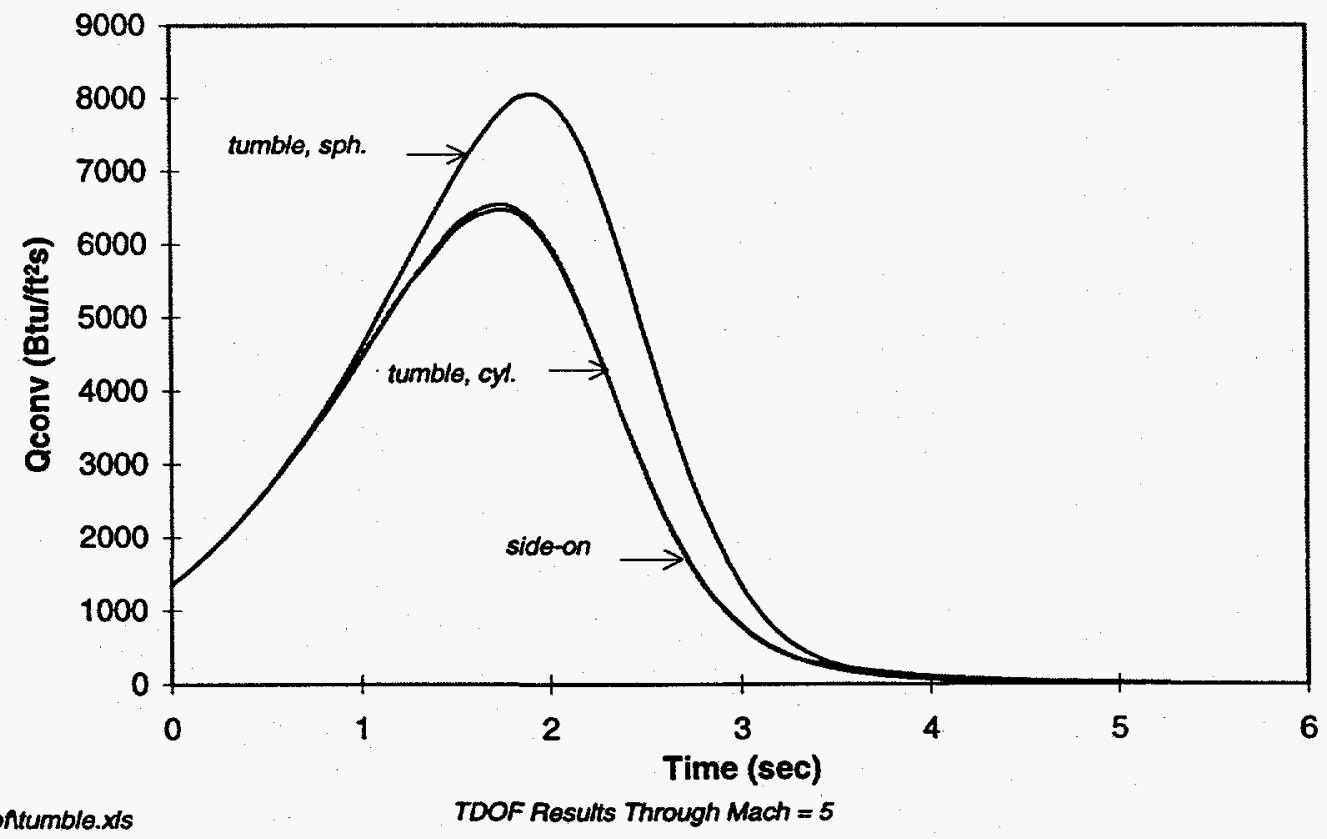


Applied Physics Laboratory

Laurel, Maryland 20723-6099

Figure 25. Cassini Mission TDOF Results

LWRHU $-7^{\circ}$ Reentry

Sample Shallow Radiative Heating History for Different Orientations
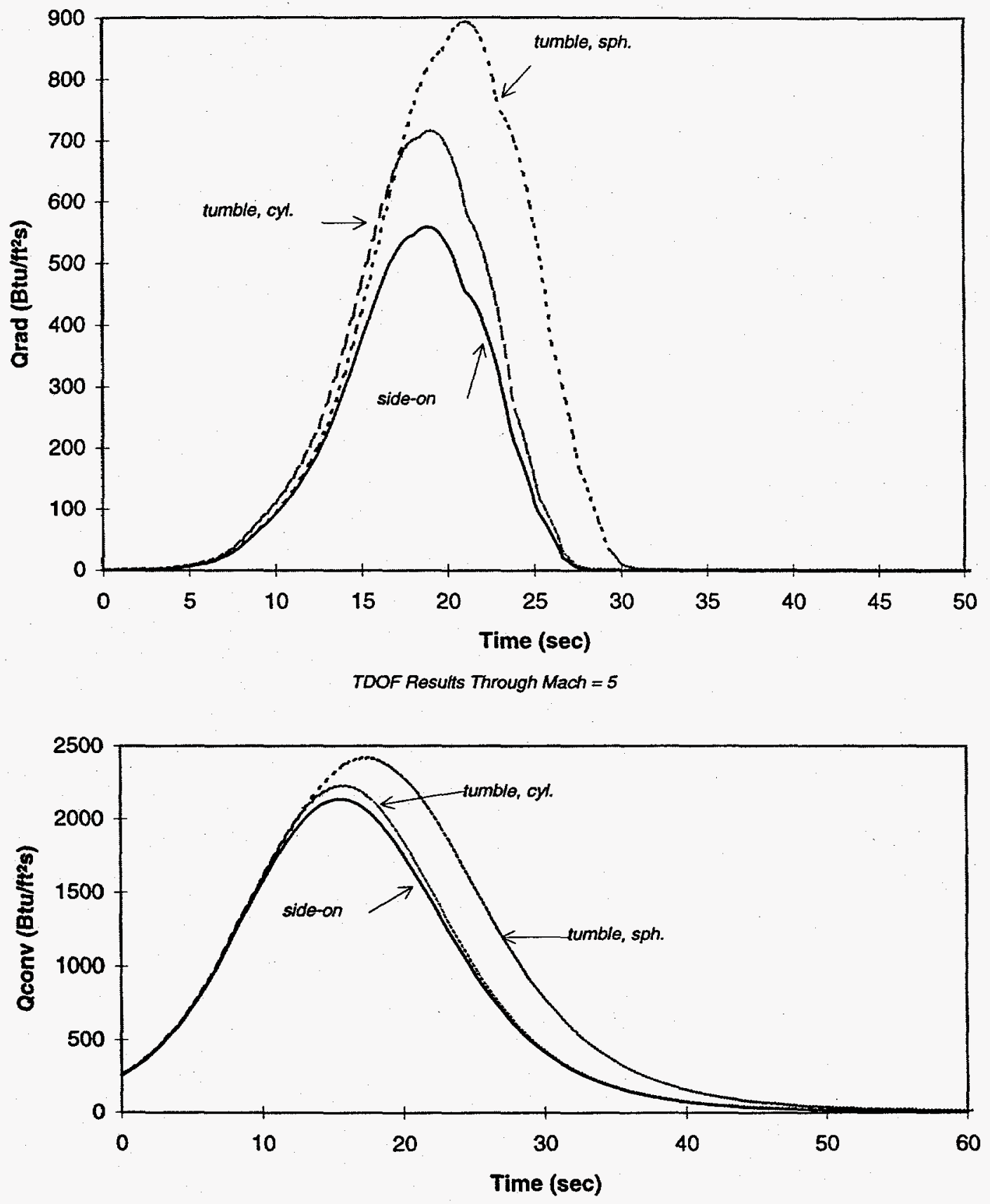

c:ltdofitumble.xds

TDOF Results Through Mach $=5$ 
Figure 26. Cassini Mission TDOF Results

LWRHU Side-on

Shallow Reentry Total Pressure Behind Normal Shock

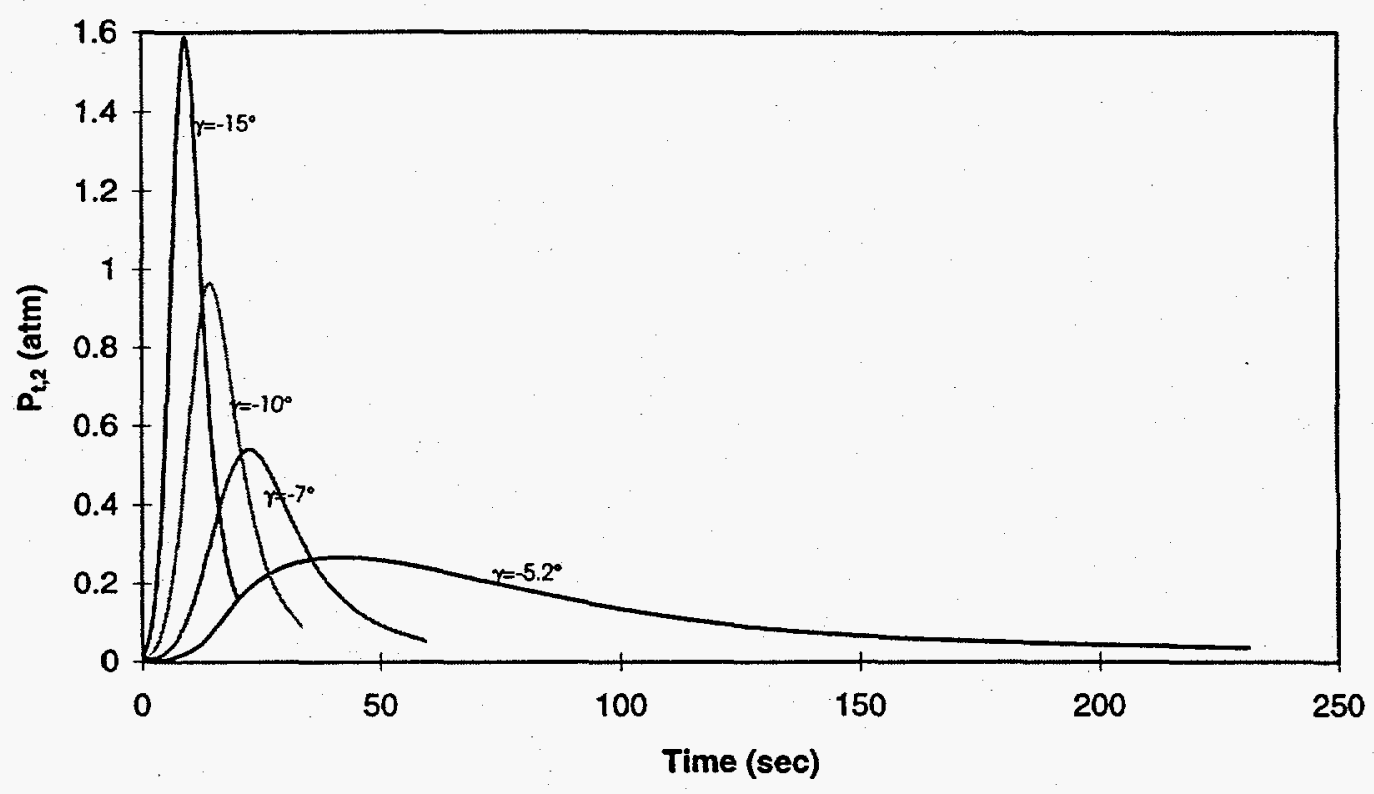

Steep Reentry Total Pressure Behind Normal Shock

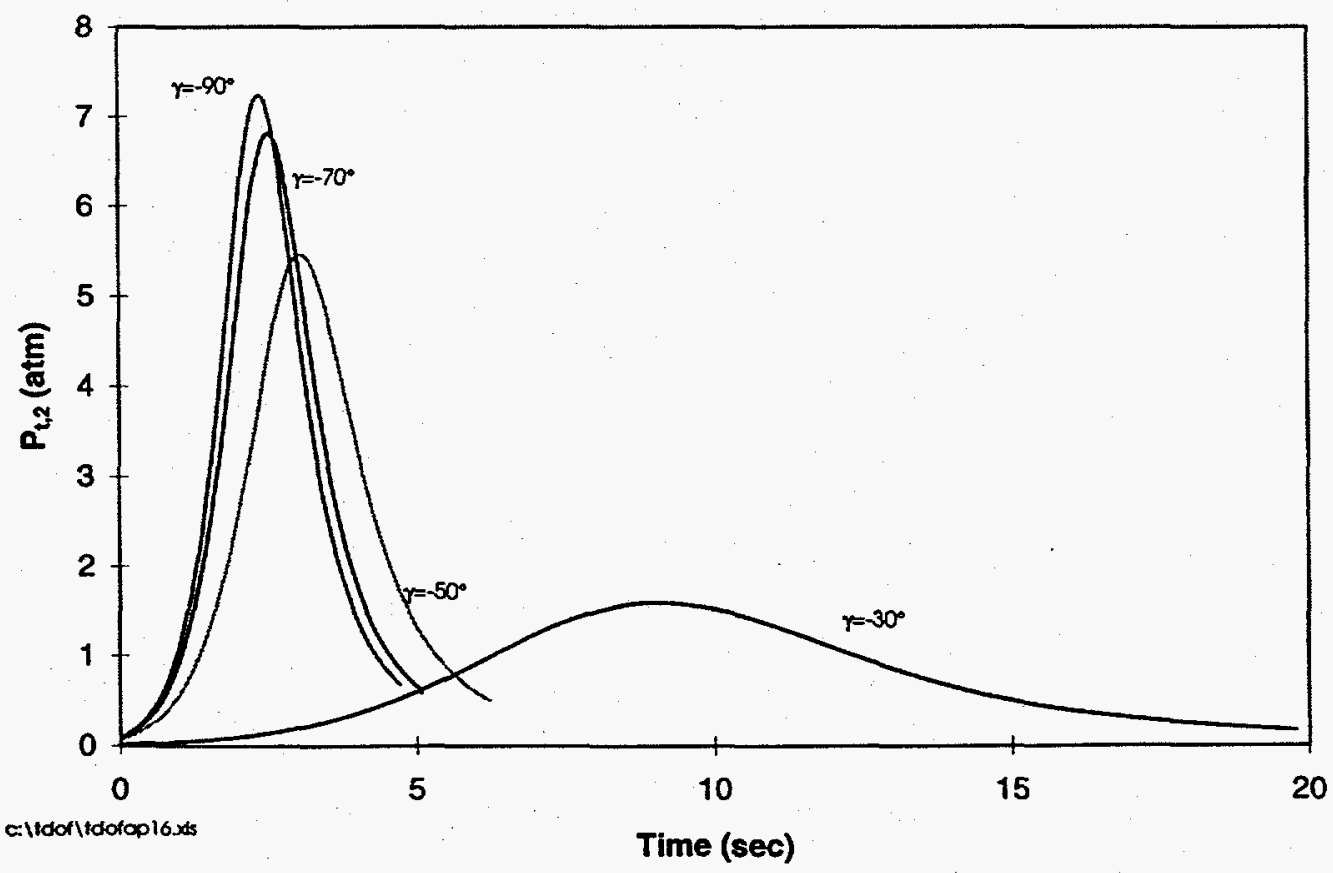


Figure 27. Cassini Mission TDOF Results

LWRHU Side-on

Shallow Reentry Total Enthalpy

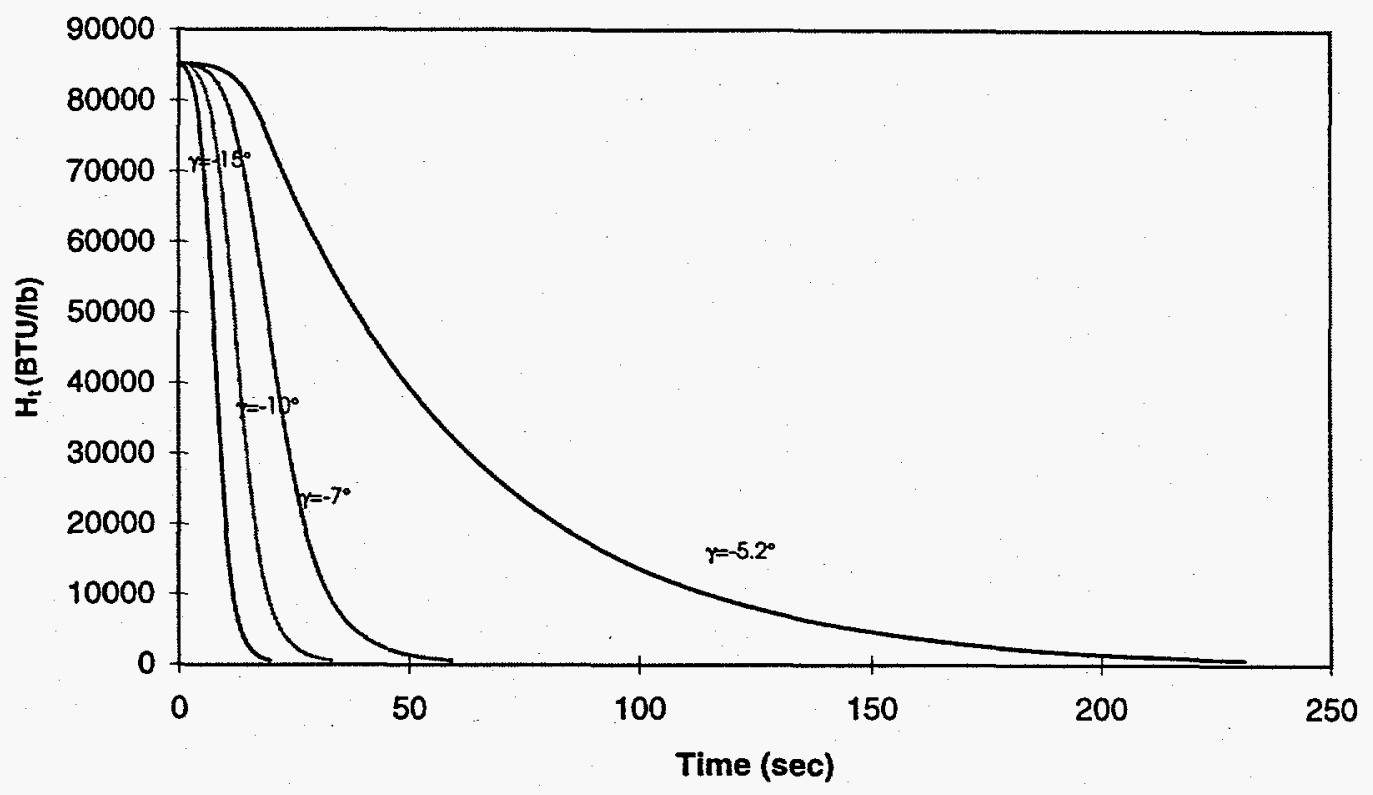

Steep Reentry Total Enthalpy

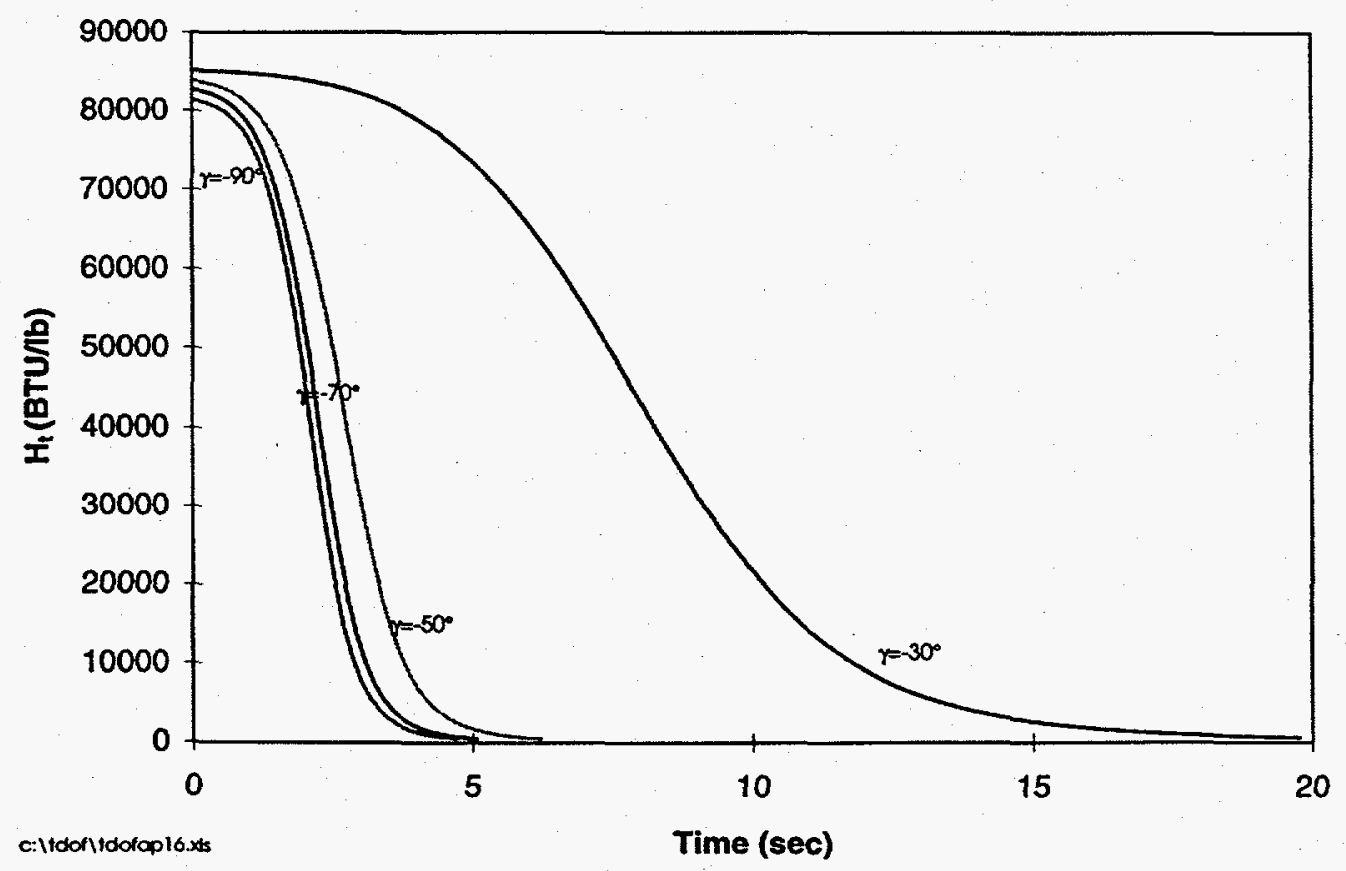


Figure 28. Cassini Mission TDOF Results

LWRHU Tumbling Cylinder

Steep Reentry Total Pressure Behind Normal Shock

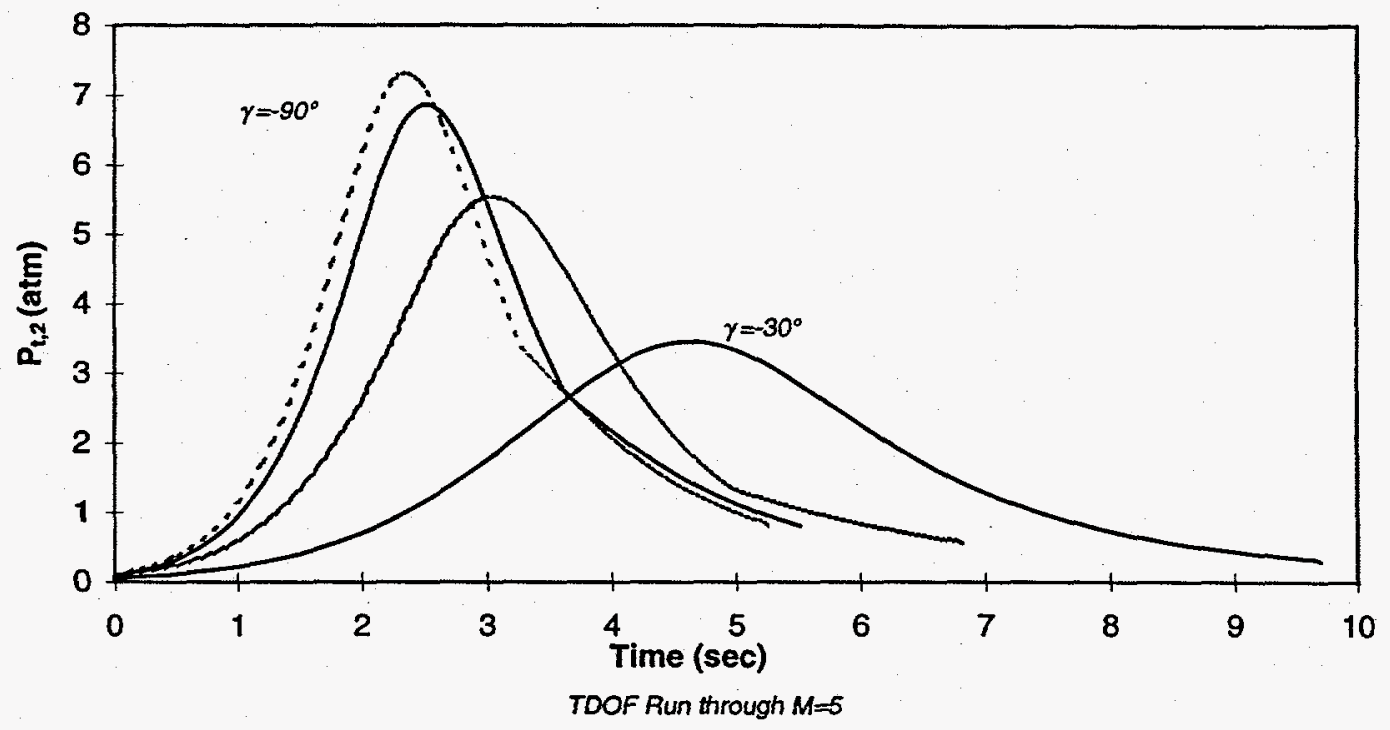

Reentry angles shown in descending order left to right: $-90^{\circ},-70^{\circ},-50^{\circ},-30^{\circ}$

Shallow Reentry Total Pressure Behind Normal Shock

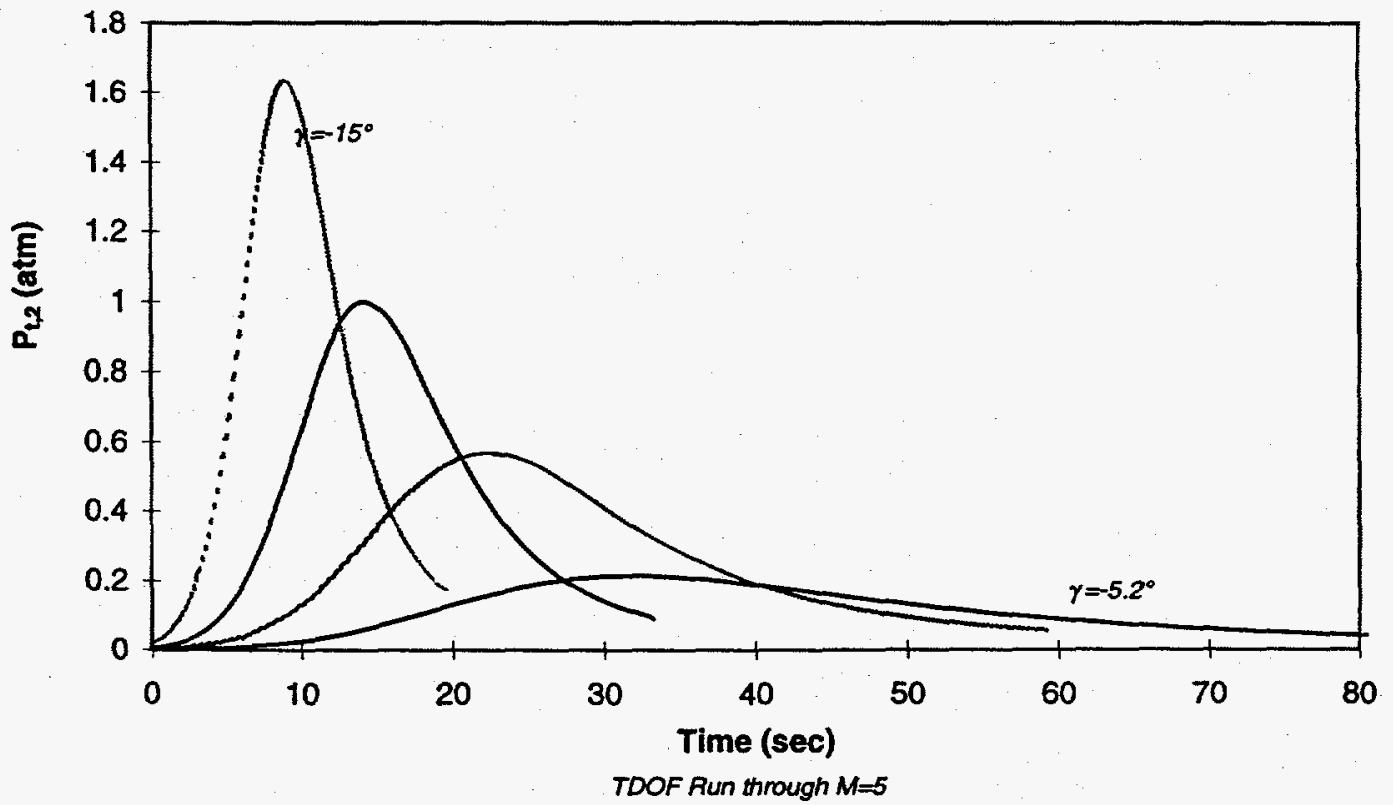

c:Utontaalli7.xds

Reentry angles shown in descending order left to right: $-15^{\circ}, 10^{\circ},-7^{\circ},-5.2^{\circ}$ 
Figure 29. Cassini Mission TDOF Results LWRHU Tumbling Cylinder

Steep Reentry Total Enthalpy

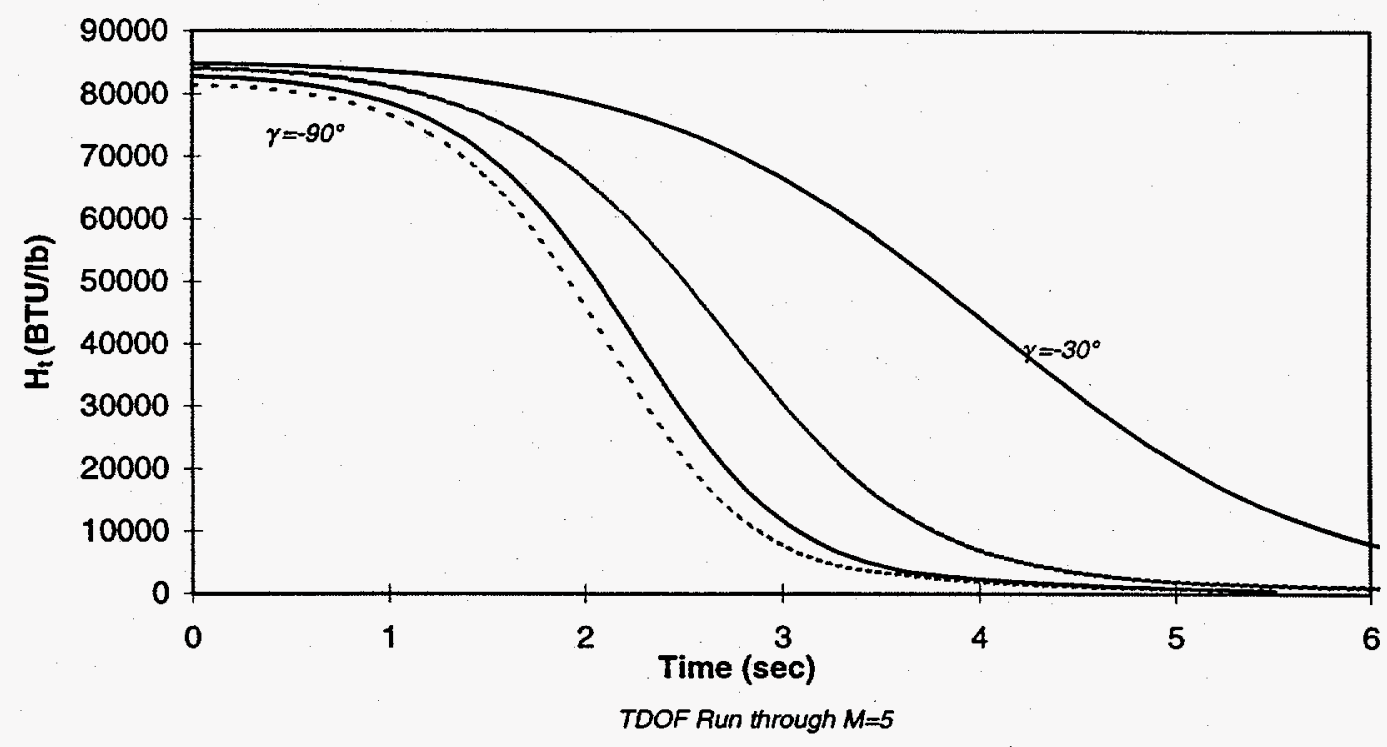

Reentry angles shown in descending order left to right: $-90^{\circ},-70^{\circ},-50^{\circ},-30^{\circ}$

\section{Shallow Reentry Total Enthalpy}

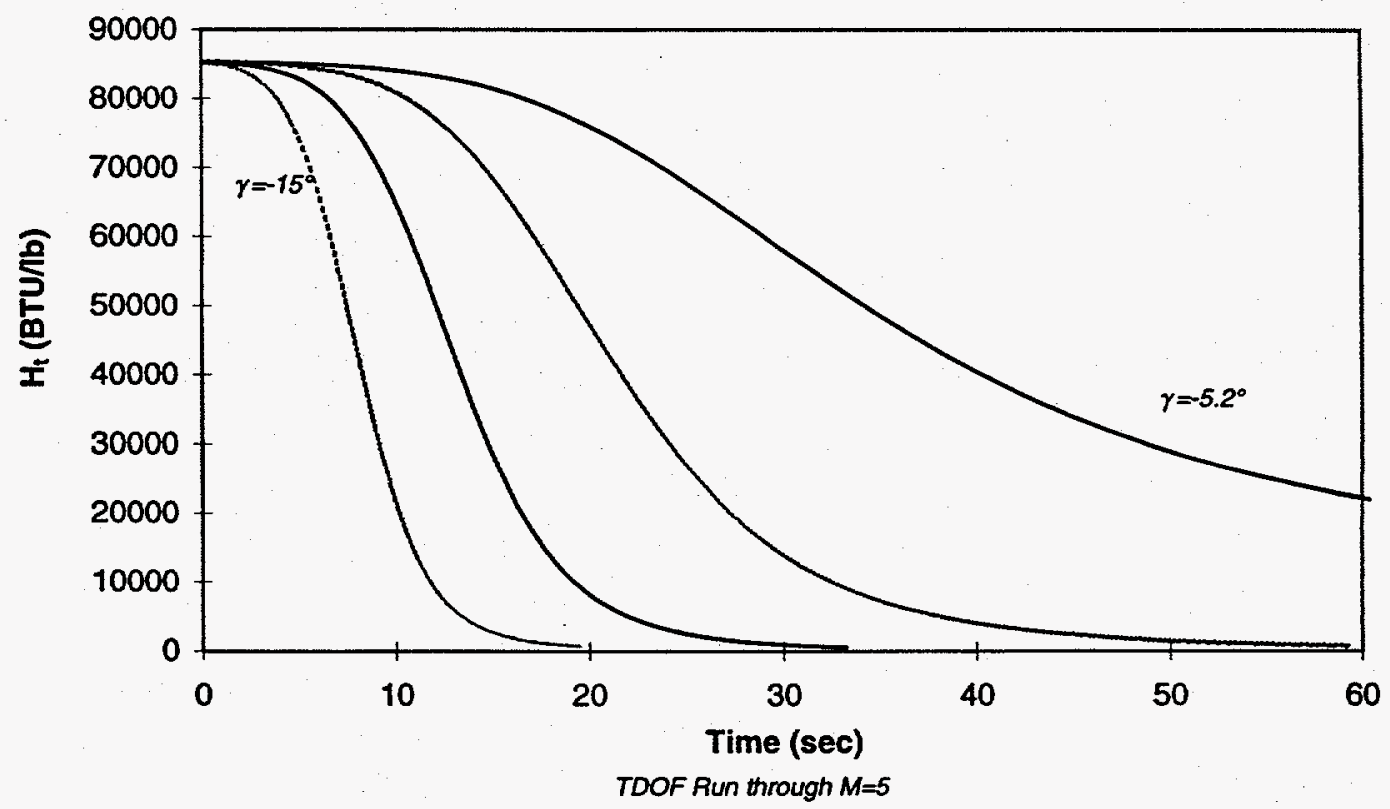

c: Urdoftaallf7.xds 
Figure 30. Cassini Mission TDOF Results LWRHU Tumbling Sphere Steep Reentry Total Pressure Behind Normal Shock

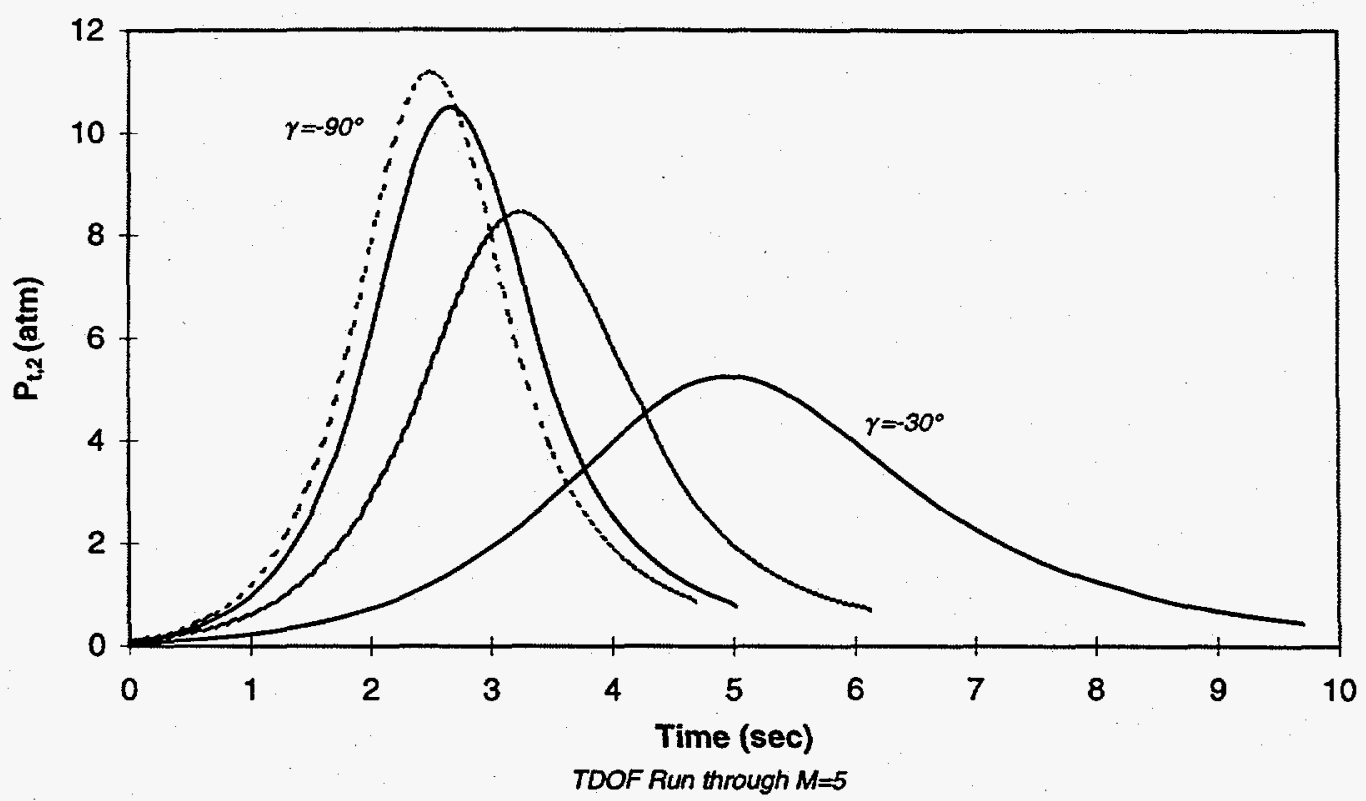

Shallow Reentry Total Pressure Behind Normal Shock

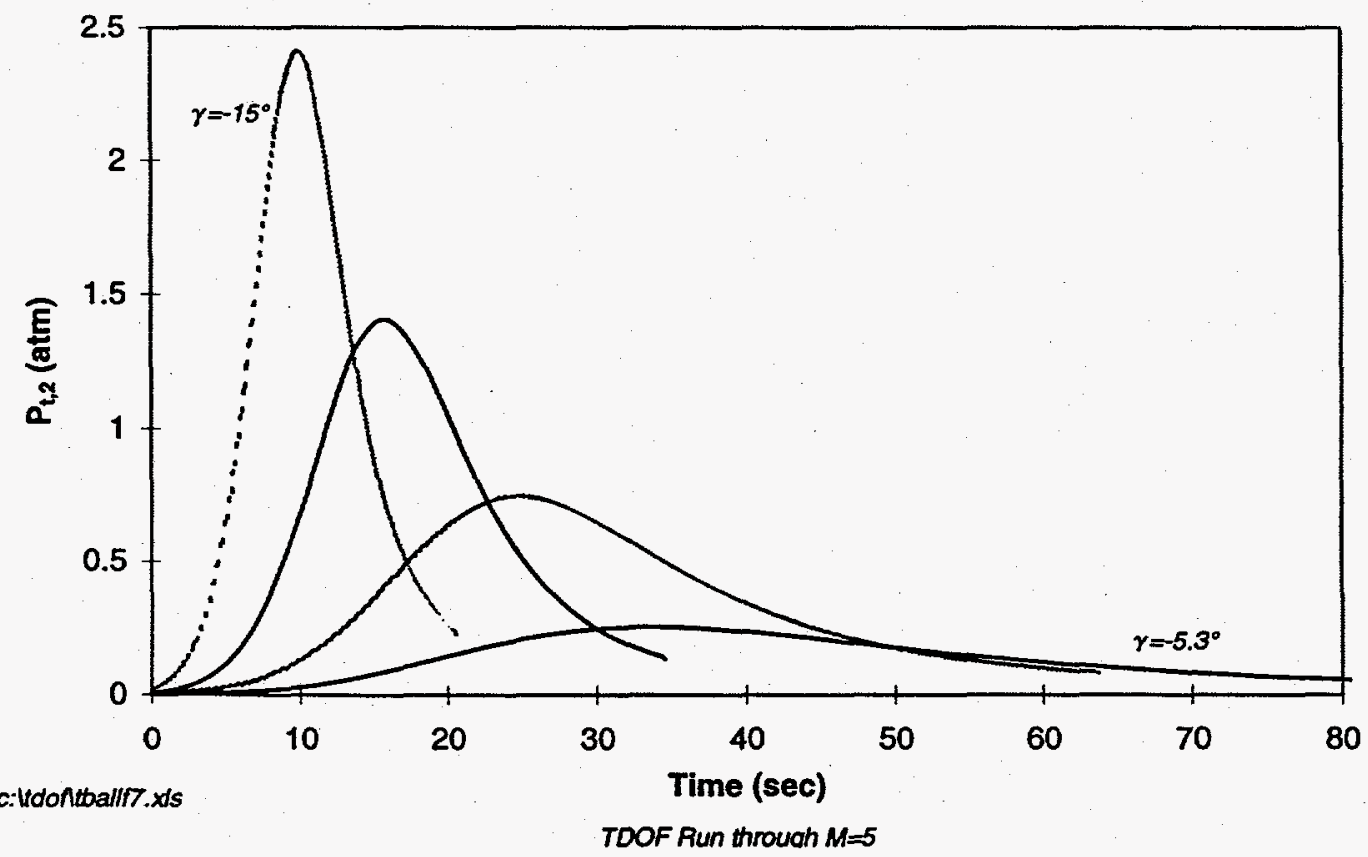


Figure 31. Cassini Mission TDOF Results LWRHU Tumbling Sphere

Steep Reentry Total Enthalpy

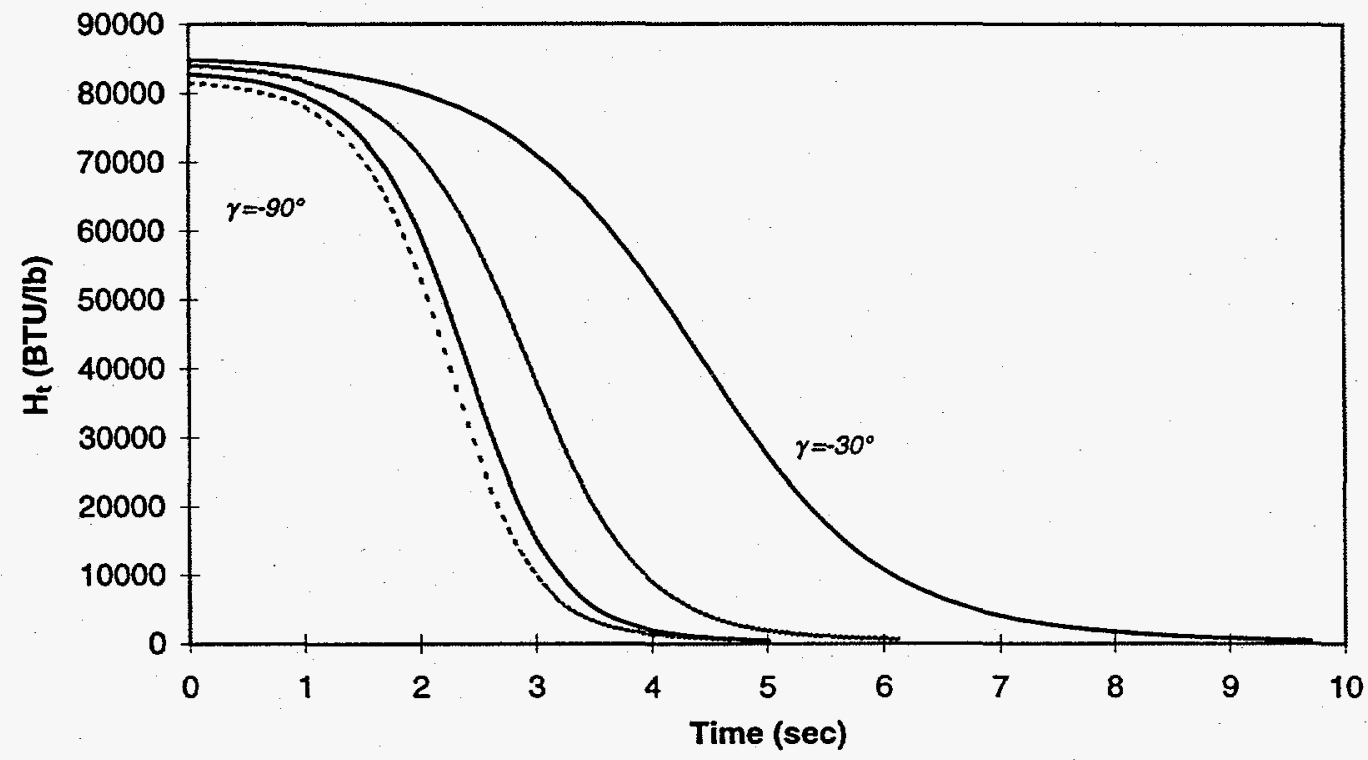

TDOF Run through $M=5$

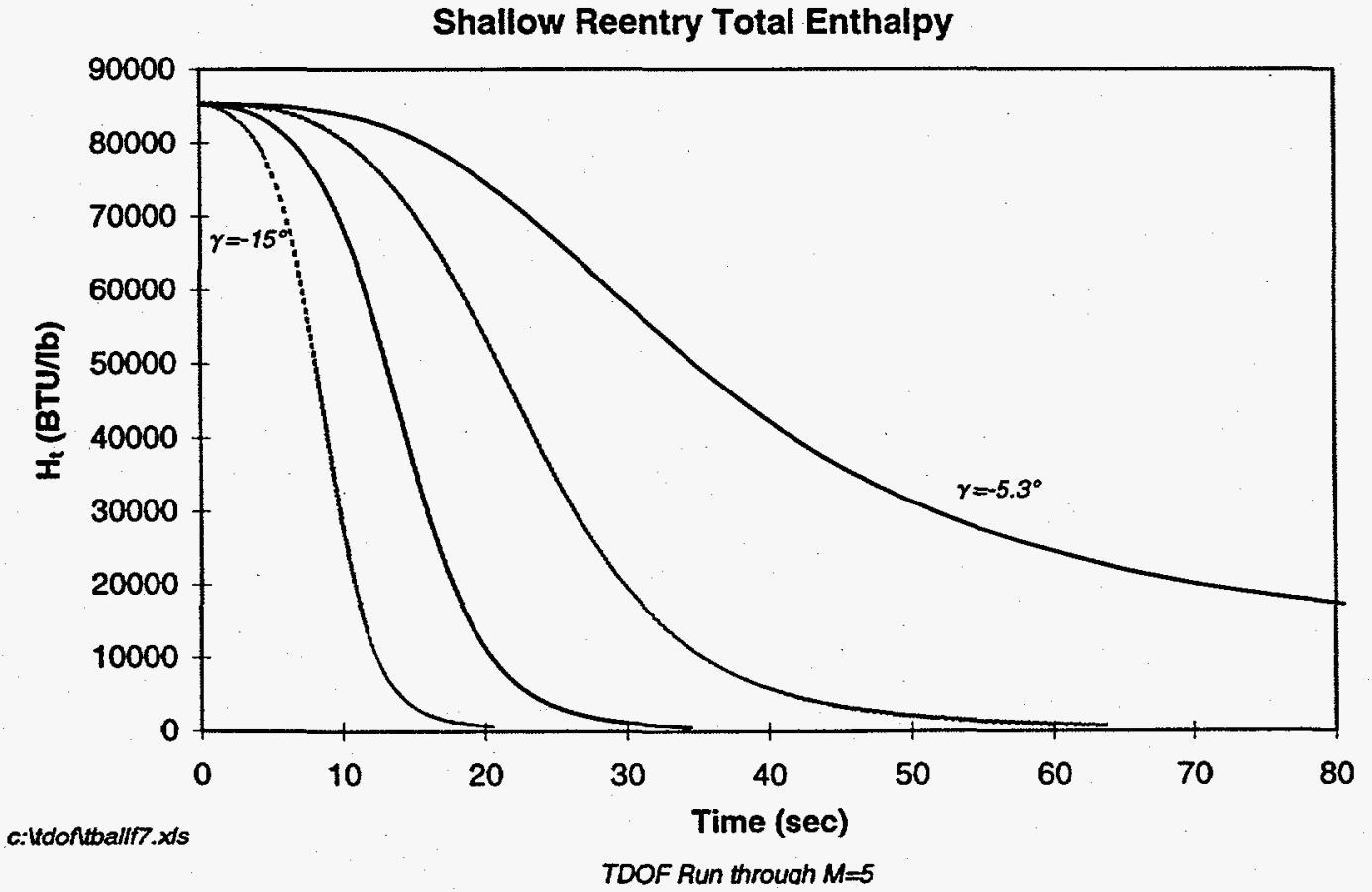


The Johns Hopkins University

Applied Physics Laboratory

Laurel, Maryland 20723-6099

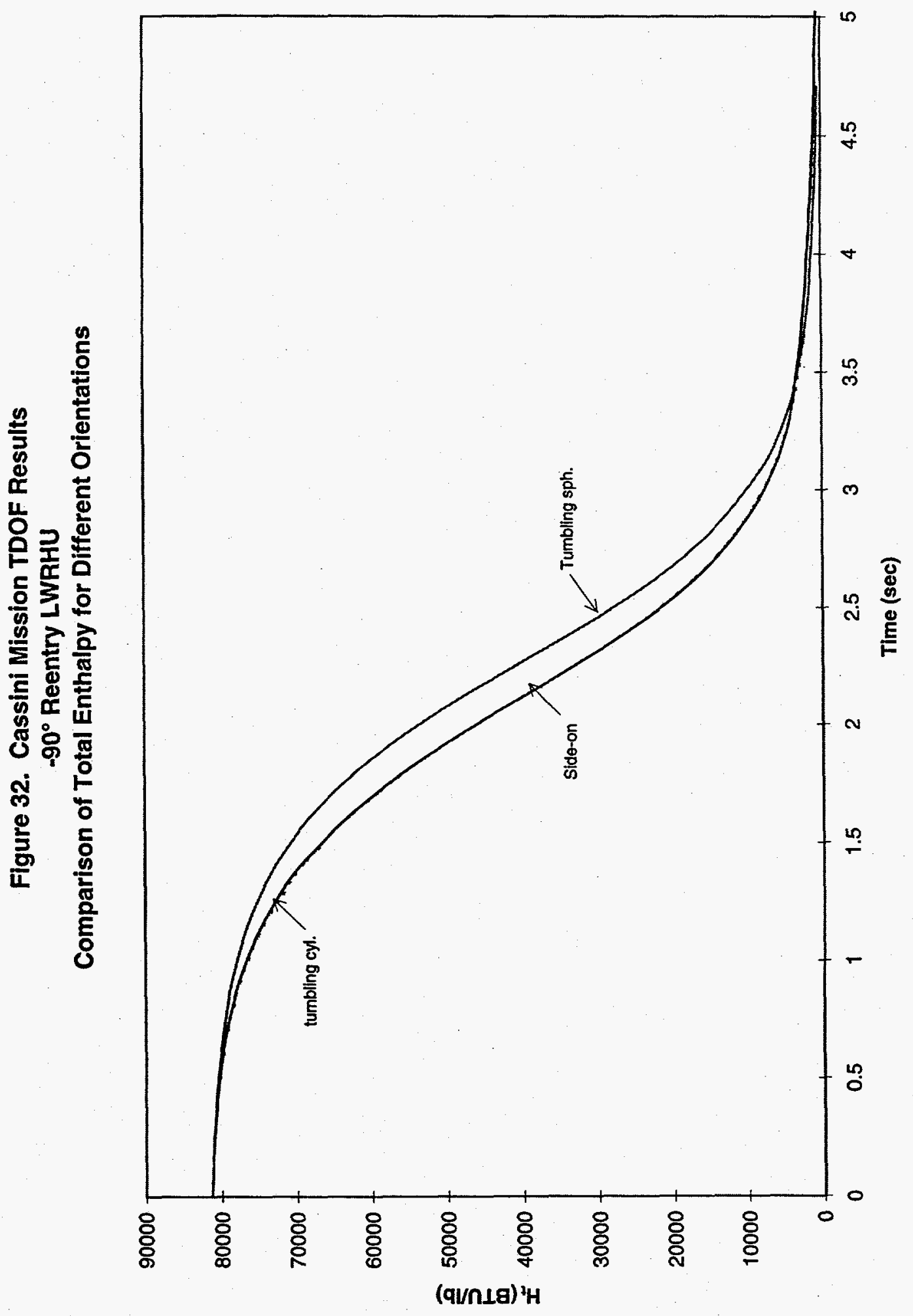


The Johns Hopkins University

Applied Physics Laboratory

Laurel. Maryland 20723-6099

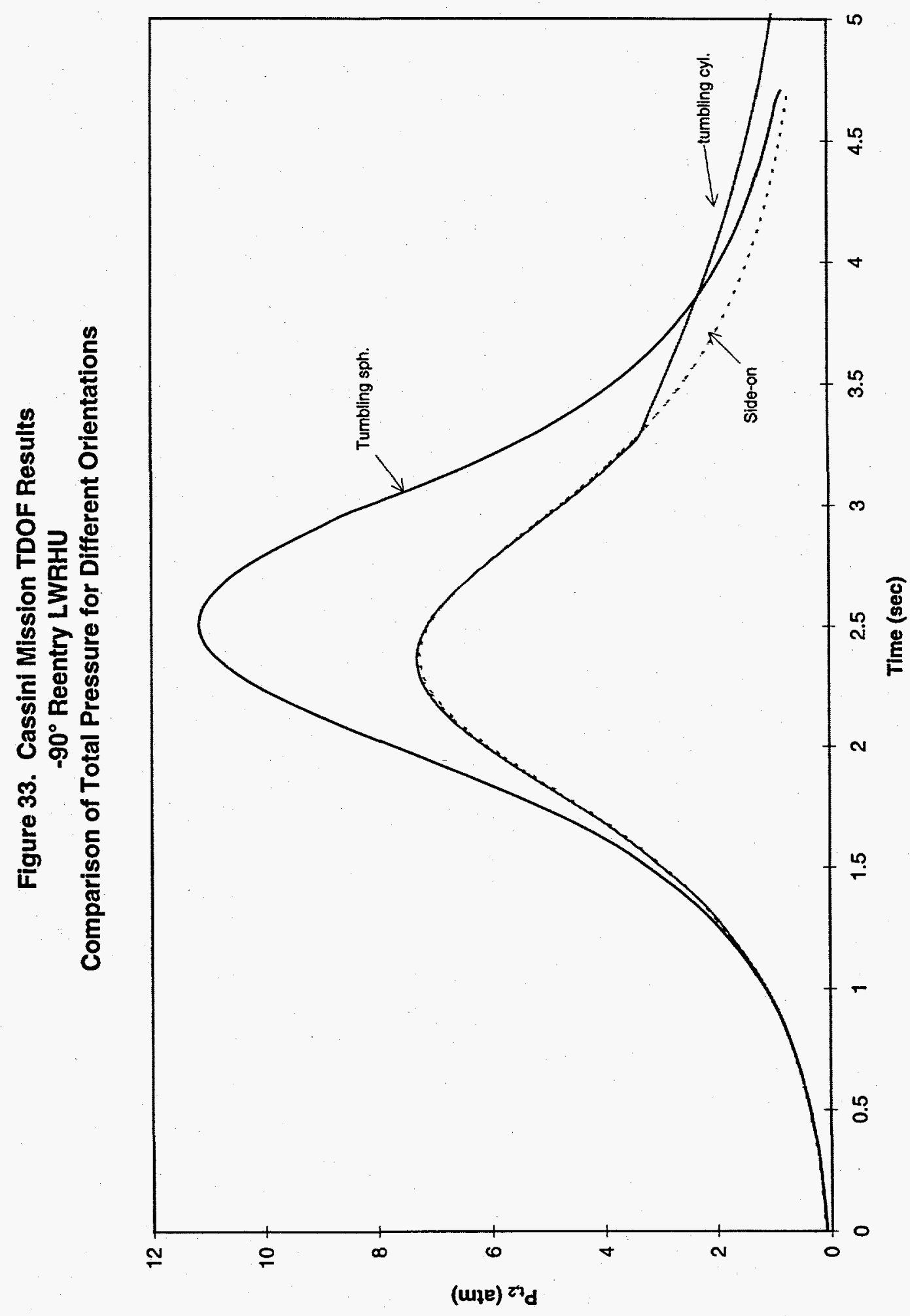




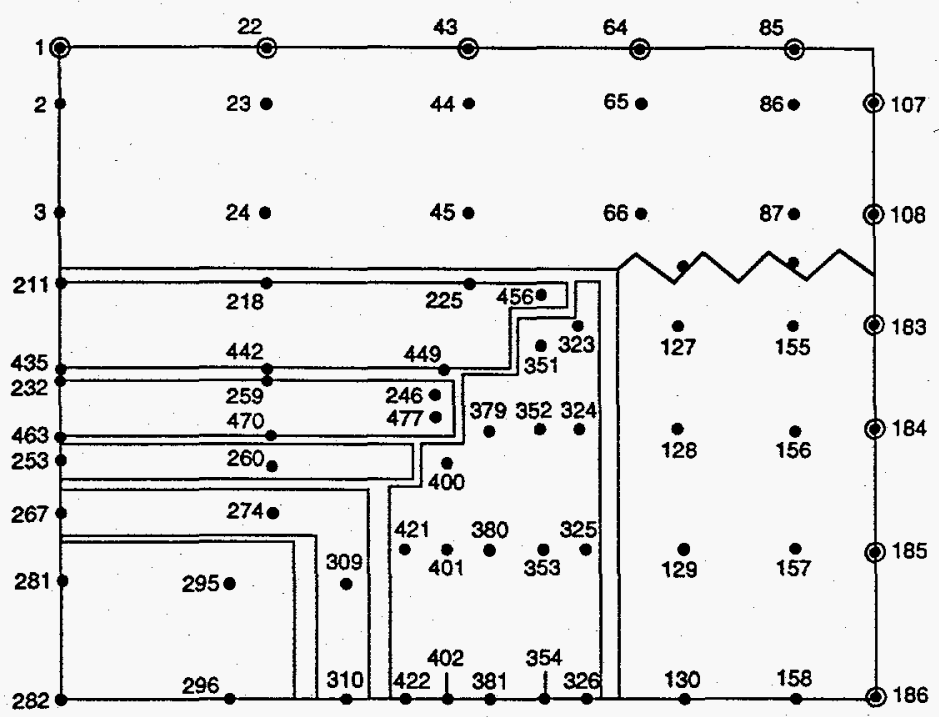

R-Z Cross-section of stagnation sector $\left(\theta=0^{\circ}-15^{\circ}\right)$ LWRHU assembly

Windward

stagnation

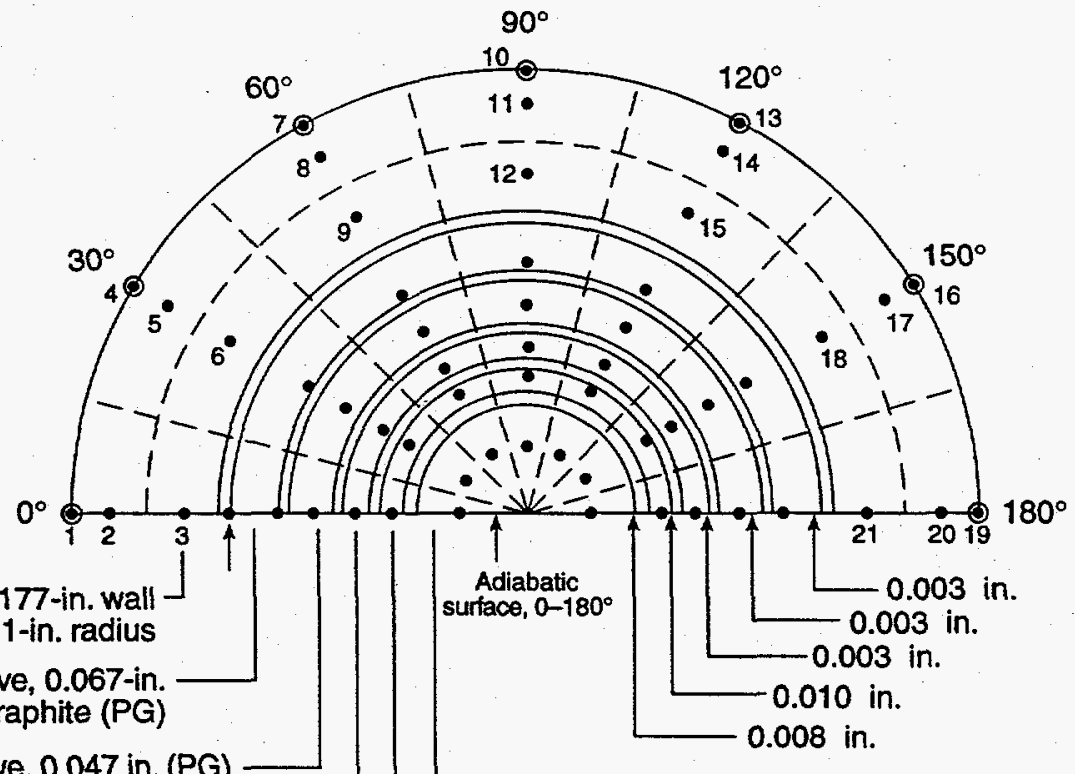

Aeroshell, 0.177 -in wall FWPF C/C, $0.511-$ in. radius

Outer sleeve, 0.067-in

pyrolytic graphite (PG)

Middle sleeve, 0.047 in. (PG)

Inner sleeve, 0.027 in. (PG)

Interfaces; gap thickness

Clad, 0.035 in. /Pt 30 Rh

Fuel pellet, $0.118 \mathrm{in}$.

$$
\mathrm{PuO}_{2}, 0.118 \text { in. }
$$

R- $\boldsymbol{\theta}$ cross-section at midspan

Component; wall thickness or radius

- Capacitance (mass) nodes material; outer radius

- Surface (zero mass) nodes

Note: All internal surfaces assigned surface nodes; omitted for clarity purposes.

Figure 34. LWRHU thermal model, quarter section 
(a)

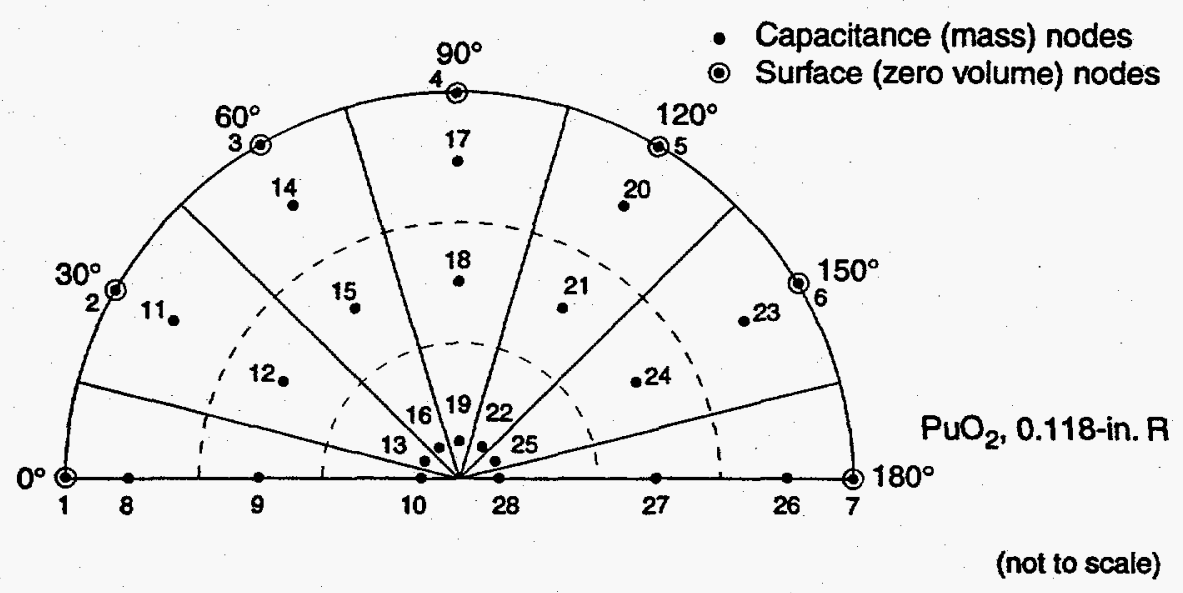

(b)

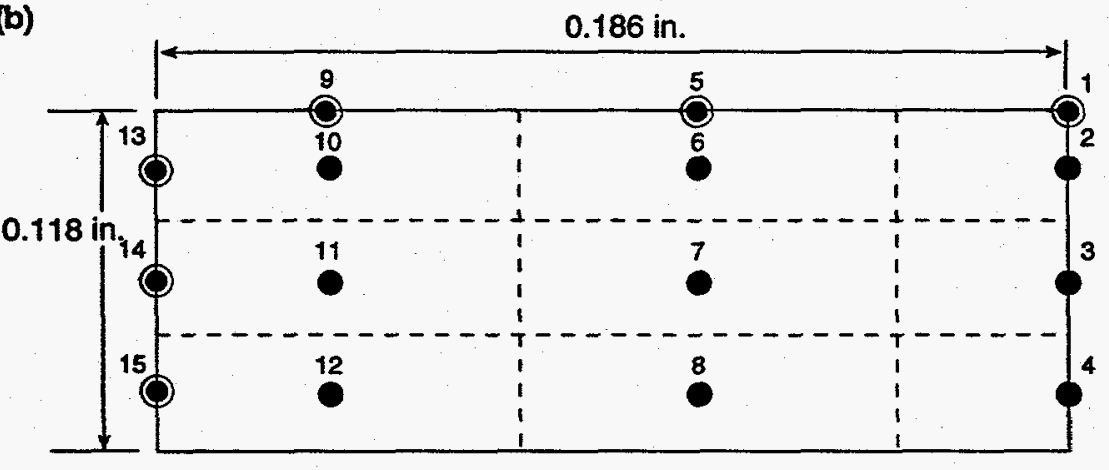

(not to scale)

Figure 35. (a) R- $\theta$ thermal model, side-on flight orientation (adapted from Galileo/VEEGA) (b) Fuel pellet $R-Z$ thermal model, random tumbling flight orientation. 
(a) Helium at 1 atmosphere

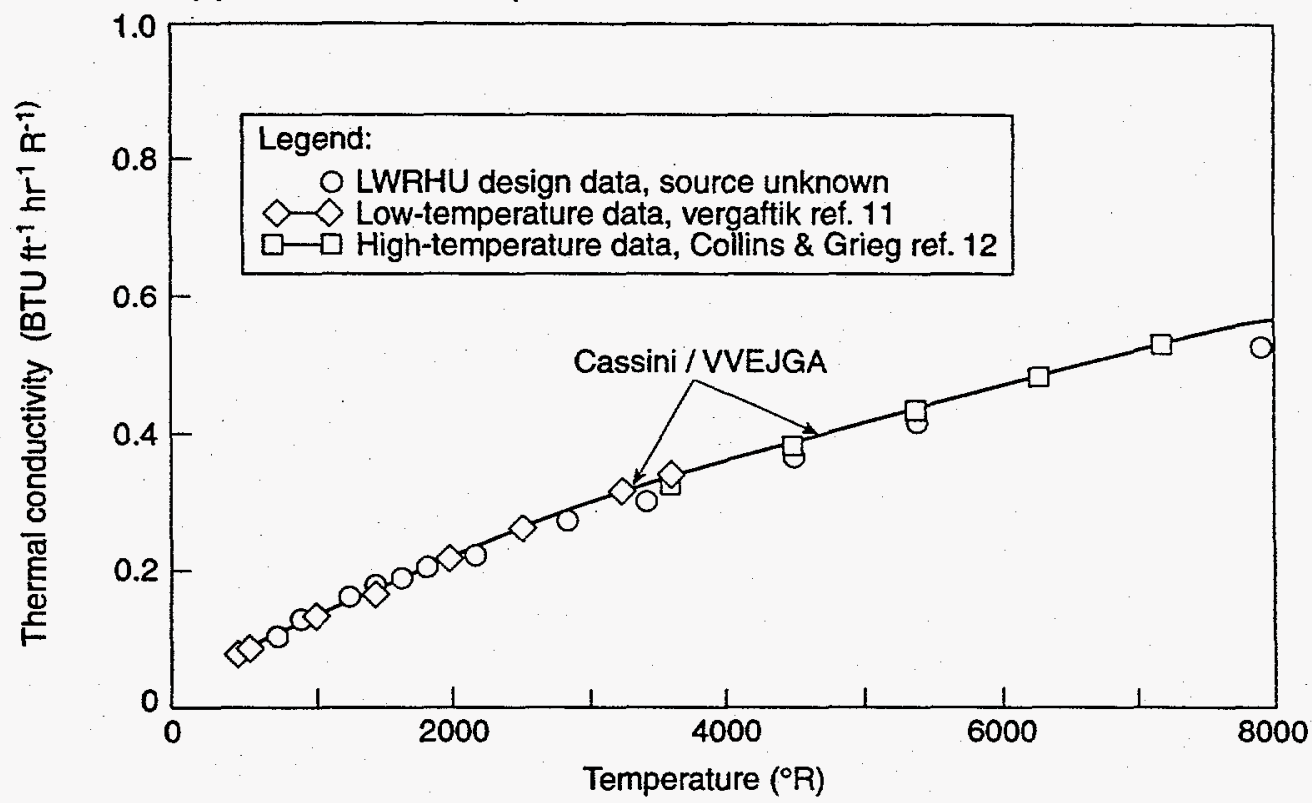

(b) Air at 1 atmosphere

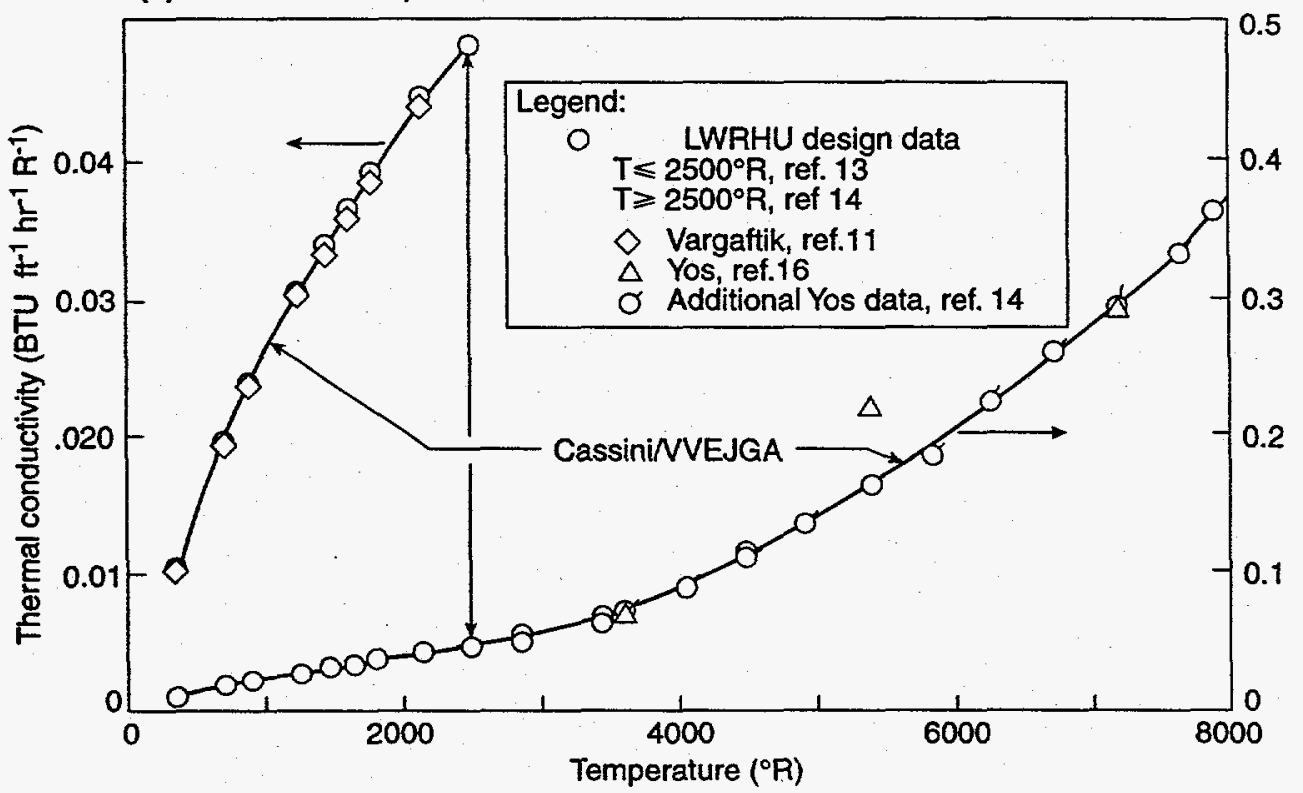

Figure 36. Thermal conductivity schedules for LWRHU interface gas fills. 
The Johns Hopkins University

Applied Physics Laboratory

Laurel, Maryland 20723.6099
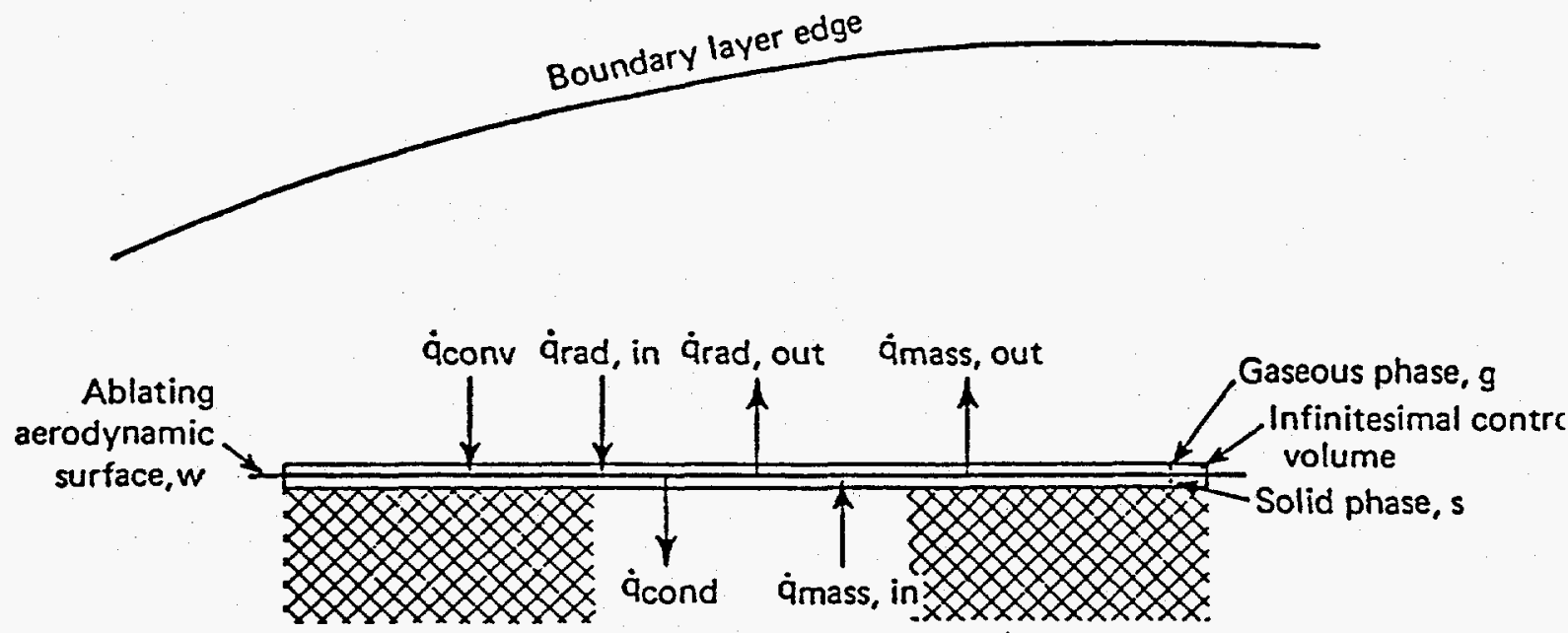

Boundary condition: $\sum_{i} \dot{q}_{i}=0$ where

- $\dot{\mathrm{q}}_{\mathrm{conv}}=$ Boundary layer convective tranisfer comprised of gaseous conduction

- $\dot{q}_{\mathrm{rad}, \text { in }} \quad$ transfer $\left(-k_{\left.\mathrm{t}_{\mathrm{g}} \partial \mathrm{T} / \partial \mathrm{r}\right) \mathrm{g}, r=w}\right.$

- $\dot{a}_{\text {rad, out }}=$ Surface emissive transfer, $\sigma \epsilon_{\mathrm{h}} \mathrm{T}_{W}^{4}$

- $\dot{q}_{\text {cond }} \quad=$ Solid conduction (Fourier) transfer, $-k_{t_{s}}(\partial T / \partial r)_{s, r}=w$

- $\dot{\mathrm{q}}_{\mathrm{mass}}$ in = Mass flux transfer-solid phase, $\dot{\mathrm{m}} \mathrm{H}_{\mathrm{w}_{\mathrm{s}}}$

- $\dot{q}_{\text {mass, out }}=$ Mass flux transfer-gaseous phase, $\left.\dot{m} H_{w_{s}}\right\}$ ablation

Figure $37^{\circ}$ - Surface Energy Balance - Reentry Thermal Analysis 


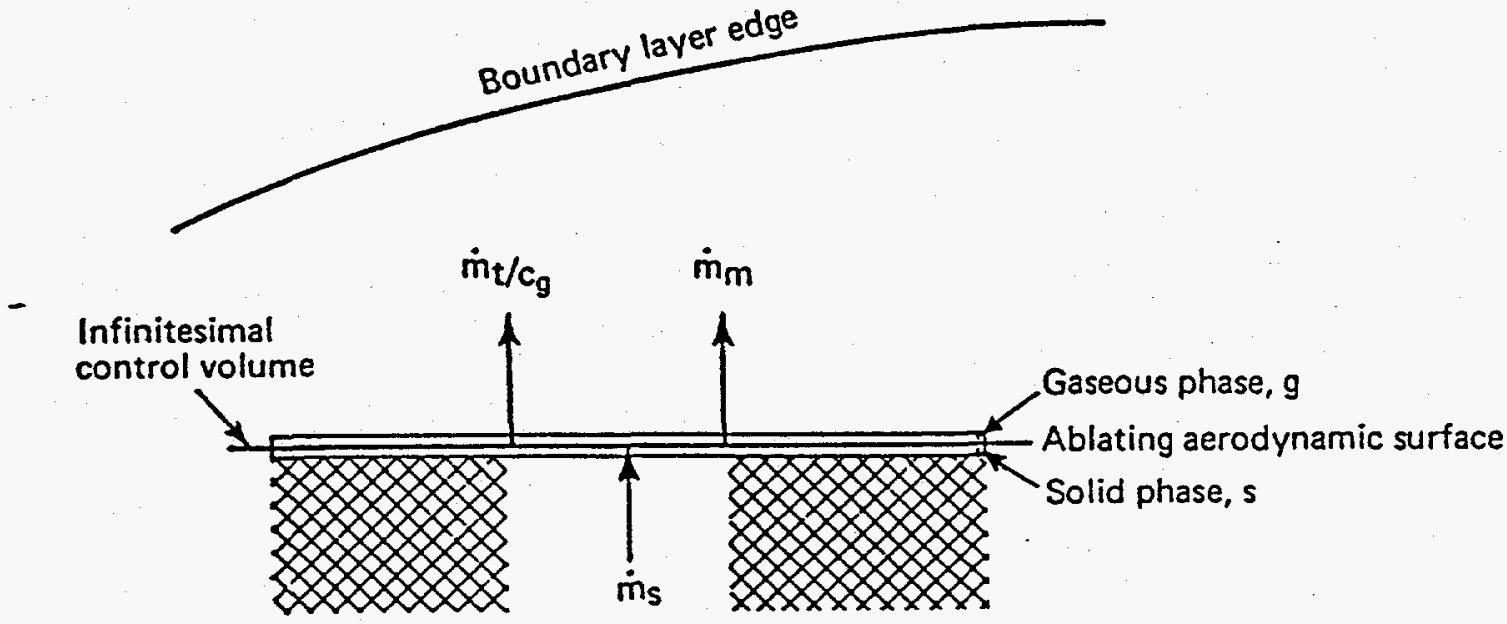

Boundary condition: $\sum_{i} \dot{m}_{i}=0$

- $\dot{m}_{\mathrm{s}} \sim$ Mass flux due to ablation, solid phase

- $\dot{m}_{t} / c_{g} \sim$ Mass flux due to thermochemical ablation, gaseous phase

- $\dot{\mathrm{m}}_{\mathrm{m}} \sim$ Mass flux due to mechanical ablation, solid phase

Figure 38- Surface Mass Balance - Reentry Thermal Analysis 
The Johns Hopkins University

Applied Physics Laboratory

Laurel, Maryland 20723.6099

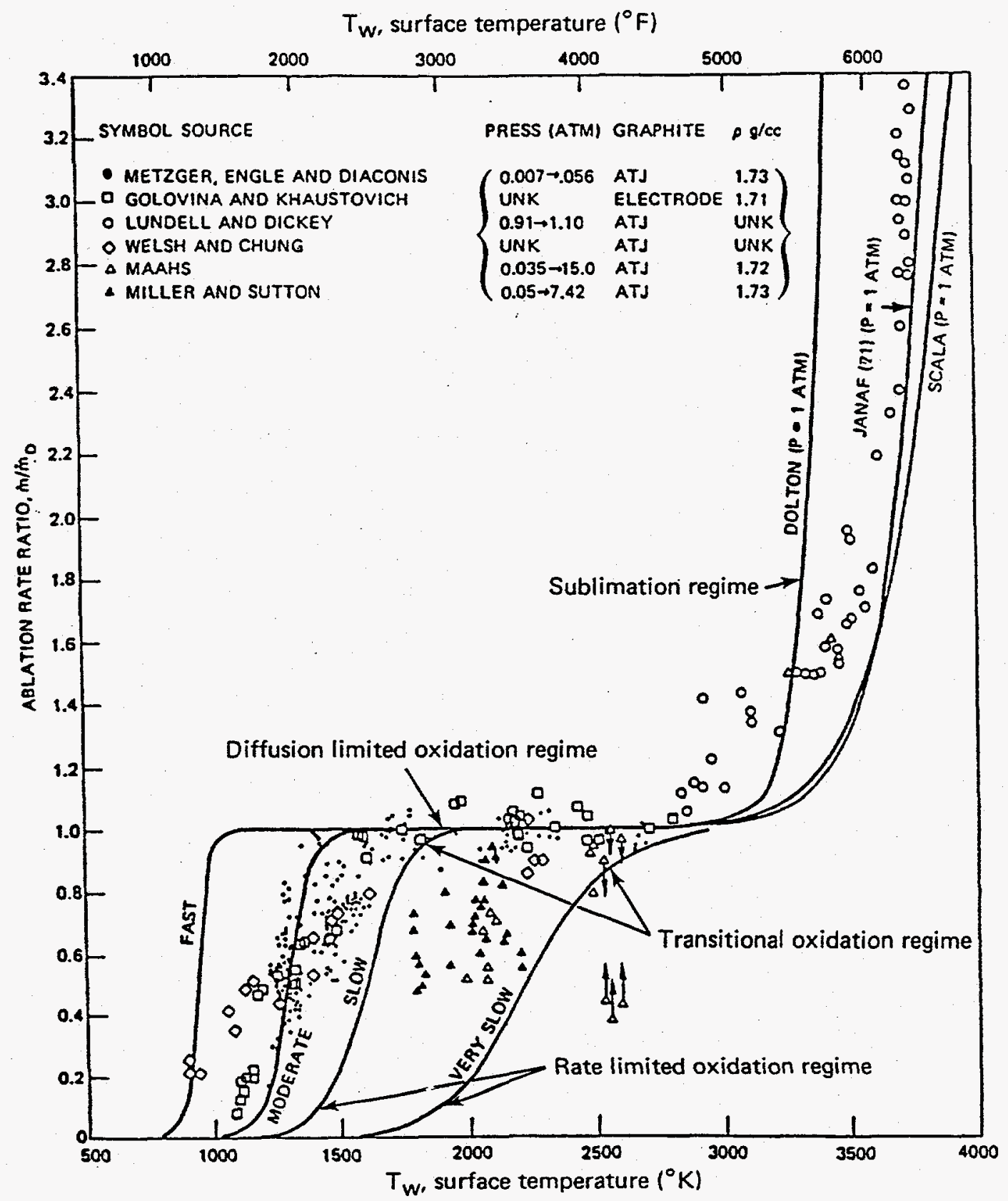

Figure 39 - Thermochemical Ablation Regimes for Graphite 
(a) Thermal conductivity, $x$ and $y$ axis

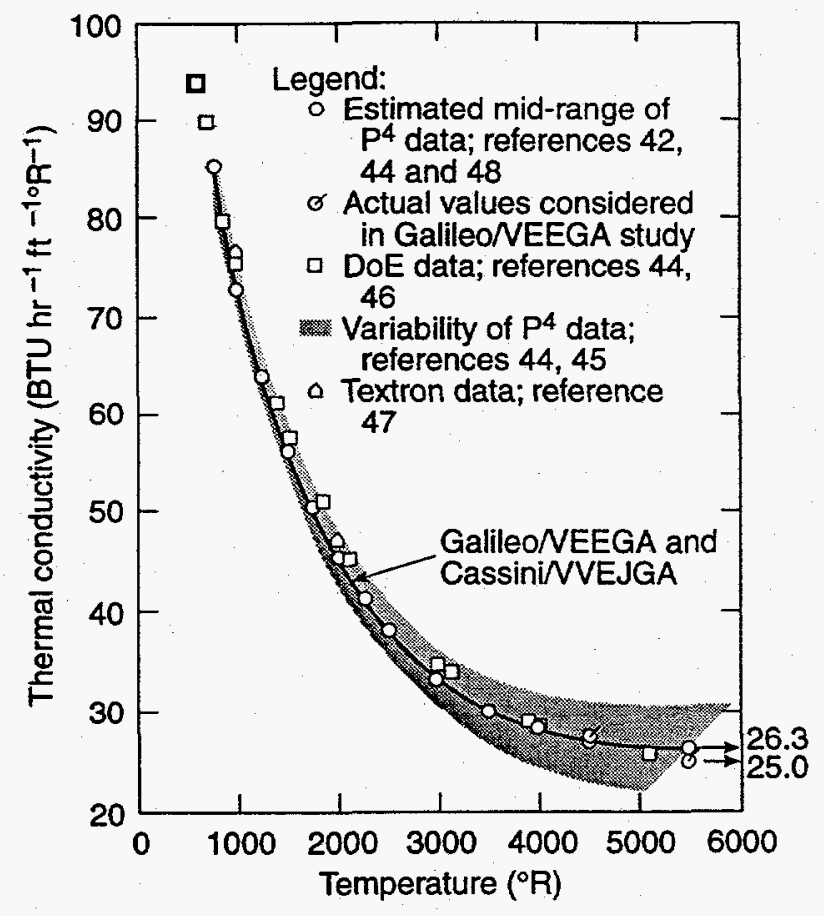

(b) Thermal conductivity, $45^{\circ}$ off $x-y$ axis

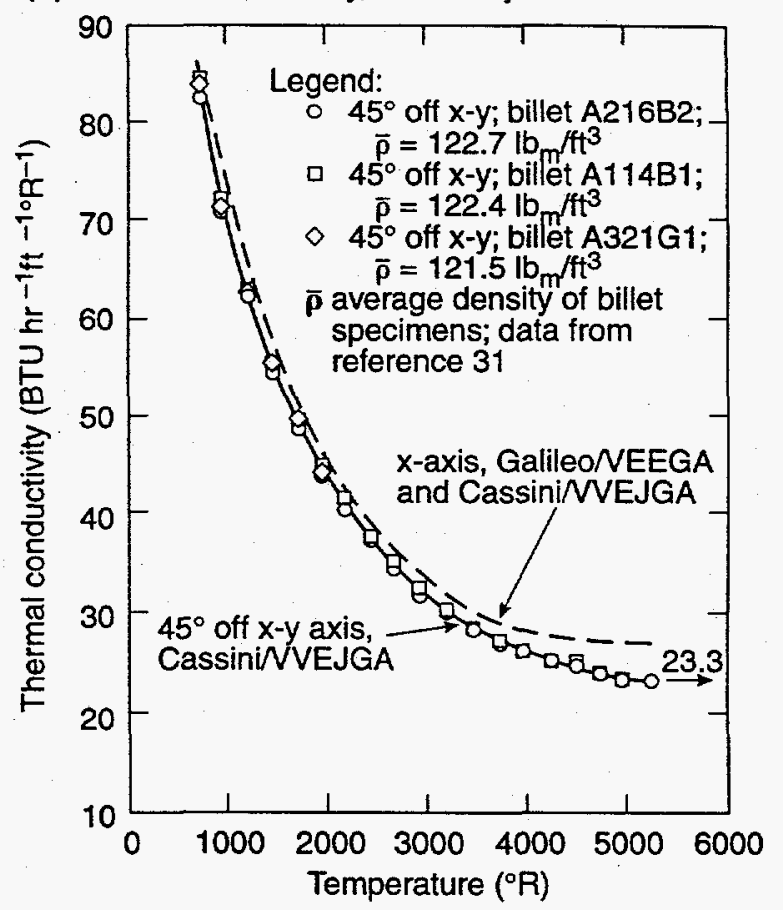

(c) Thermal conductivity, $z$ axis

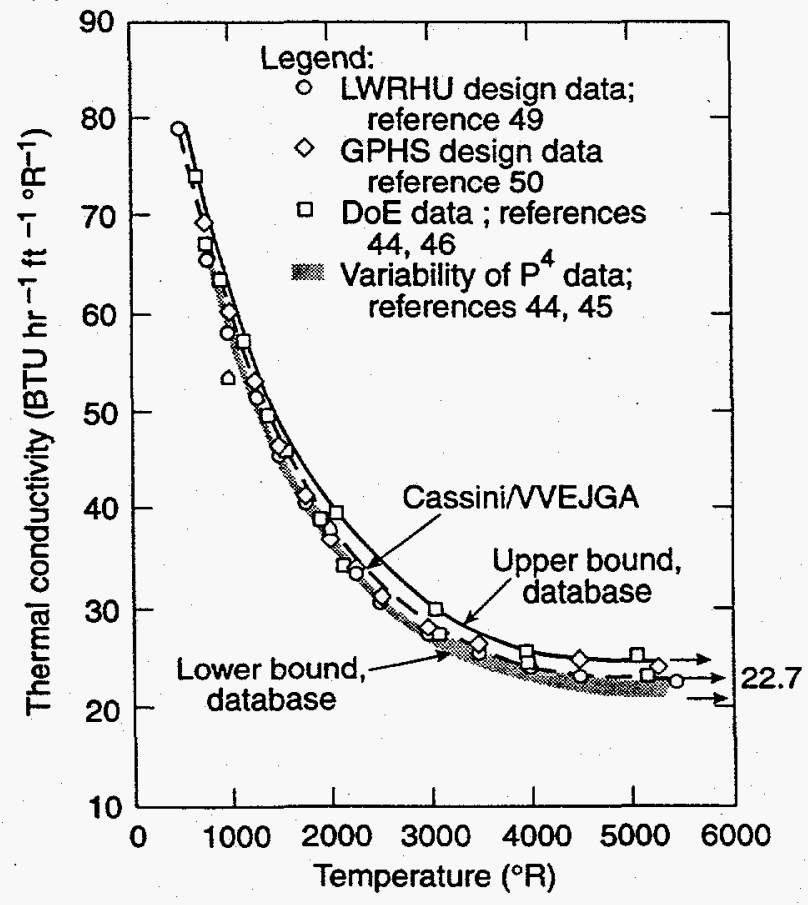

Figure 40 Thermal conductivity schedules for Fine Weave Pierced Fabric $^{\text {tm }}$ (FWPF ${ }^{\mathrm{tm}}$ ) carbon-carbon composite; FWPFtm: product of AVCO Systems Division. 


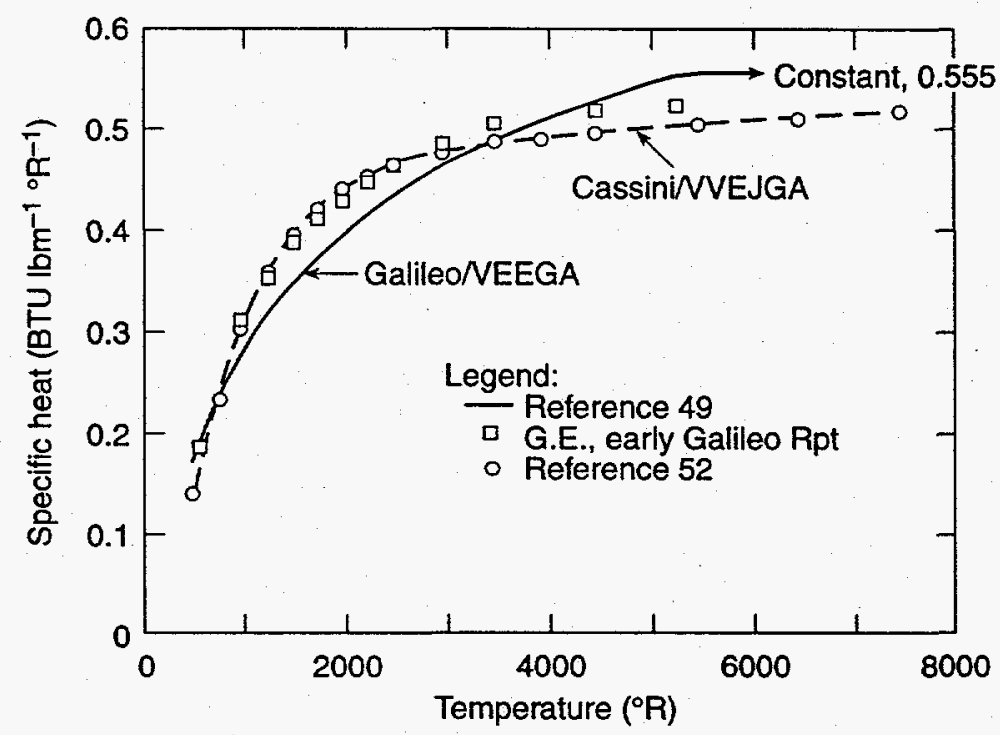

Figure 41 Specific heat schedules for Fine Weave Pierced Fabrictm (FWPFtm) carbon-carbon composite; FWPFtm: product of AVCO Systems Division.

$96-6374$ 
(a) Thermal conductivity schedules; perpendicular

to deposition plane.
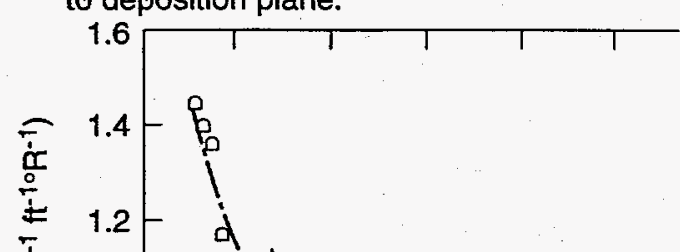

놀

$$
\text { 蛋 }
$$

CassiniMVEJGA

insulator caps

$\circ$
Legend:

Recommended schedule, Cassini/NVEJGA insulator sleeves, reference 42

- - - Extrapolated region

-.- Recommended schedule, CassiniMVEJGA

I $\begin{aligned} & \text { insulator caps } \\ & \text { LWRHU acceptance specification, reference }\end{aligned}$ 59

* Insulator sleeve, Cassini/LWRHU conformance datum, reference 54

$\varangle \quad$ Insulator cap, Cassini/LWRHU conformance datum, reference 53

- 1966 LASL plate data, reference 55

$\underset{a}{a} \rightarrow \begin{array}{ccc}\text { Constant } \\ 0.76 & 1966 \text { LASL sleeve data, reference } 55 \\ 0 & & 1966 \text { SORI sleeve data, reference } 55\end{array}$

$\underset{a}{a} \rightarrow \begin{array}{ccc}\text { Constant } \\ 0.76 & 1966 \text { LASL sleeve data, reference } 55 \\ 0 & & 1966 \text { SORI sleeve data, reference } 55\end{array}$

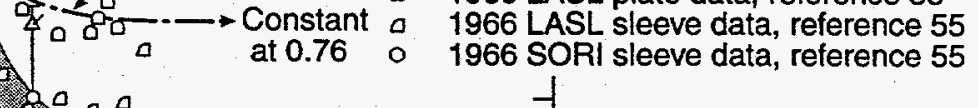

$\begin{array}{ccc}- & \text { Constant } & 1966 \text { LASL sleeve data, reference } 55 \\ a & \text { at } 0.76 & 0 \\ 0 & 1966 \text { SORI sleeve data, reference } 55\end{array}$

Constant 0.2

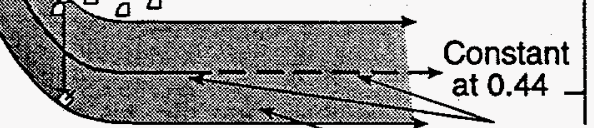

Nominal, Galileo/NEEGA and Cassini/ WEJGA insulator sleeves $\pm 25 \%$ nominal

(b) Thermal conductivity schedules; parallel to deposition plane.

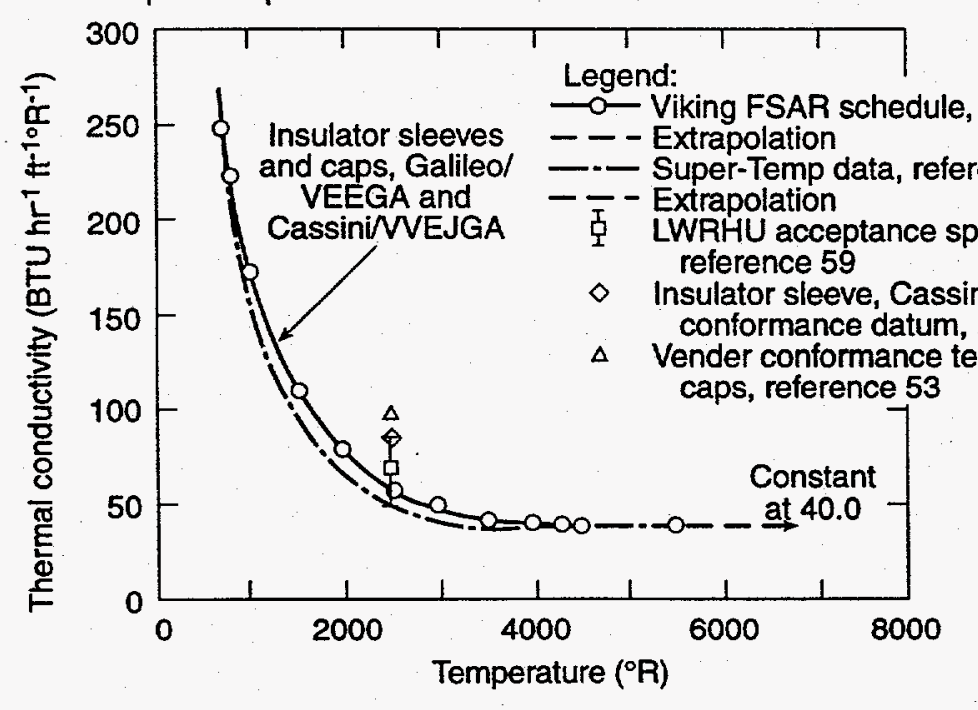

Figure 42 Thermal conductivity properties for pyrolytic graphite. 


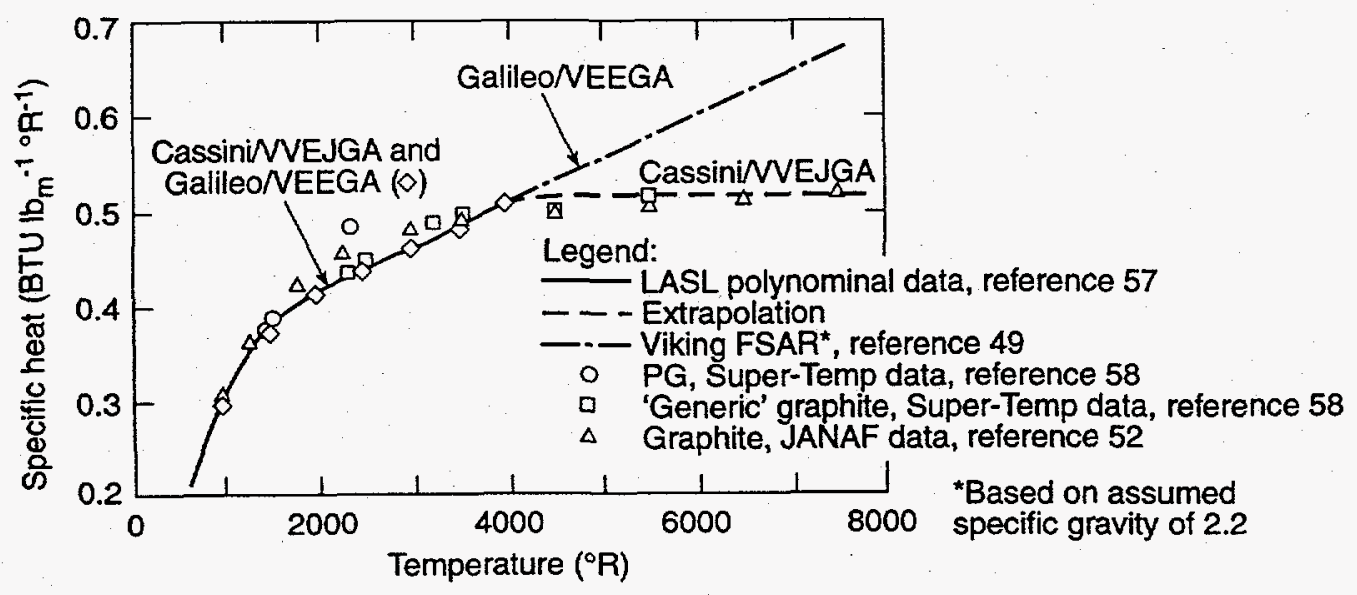

Figure 43 Specific heat schedules for pyrolytic graphite.

$96-6374$ 
The Johns Hopkins University

Applied Physics Laboratory

Laurel. Maryland 20723-6099

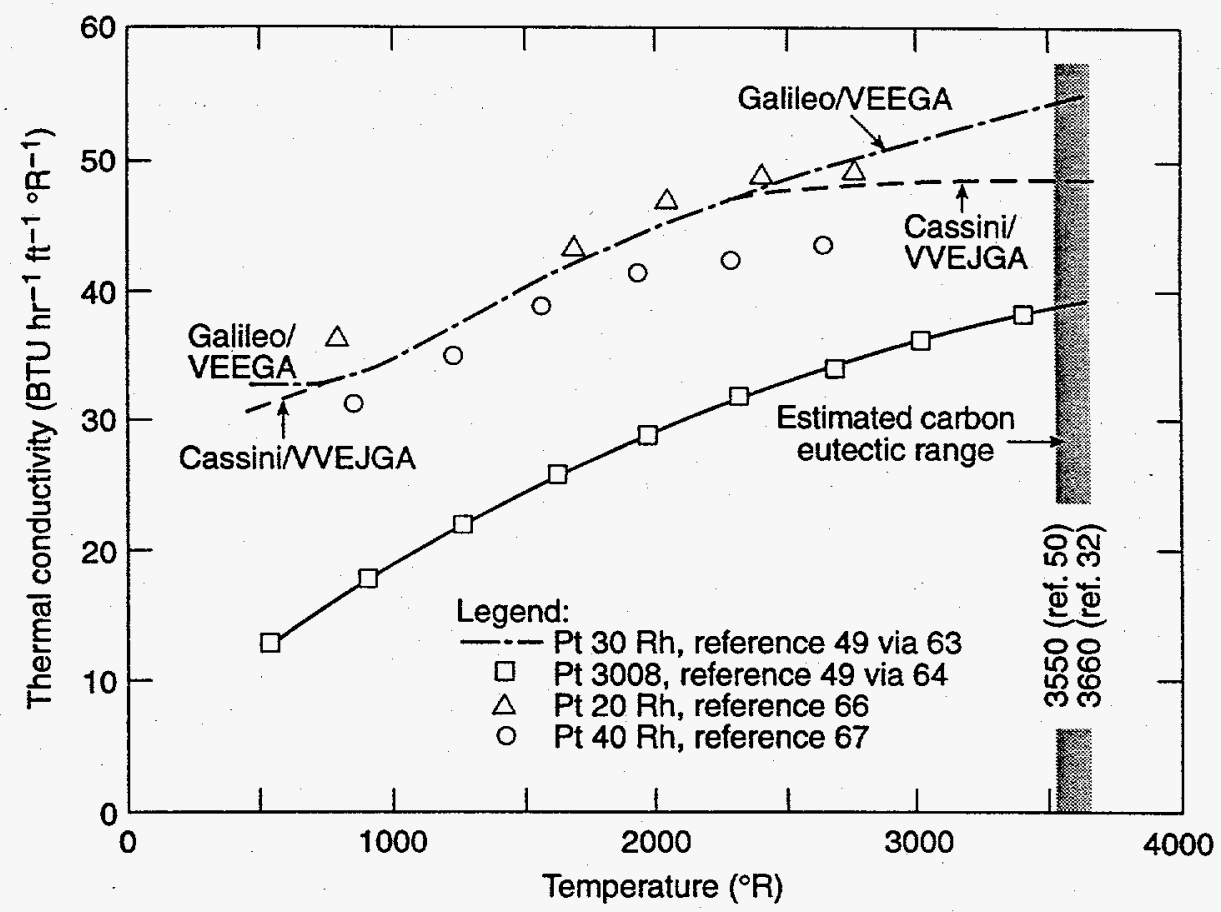

Figure 44 Thermal conductivity schedules for Pt-30 Rh.

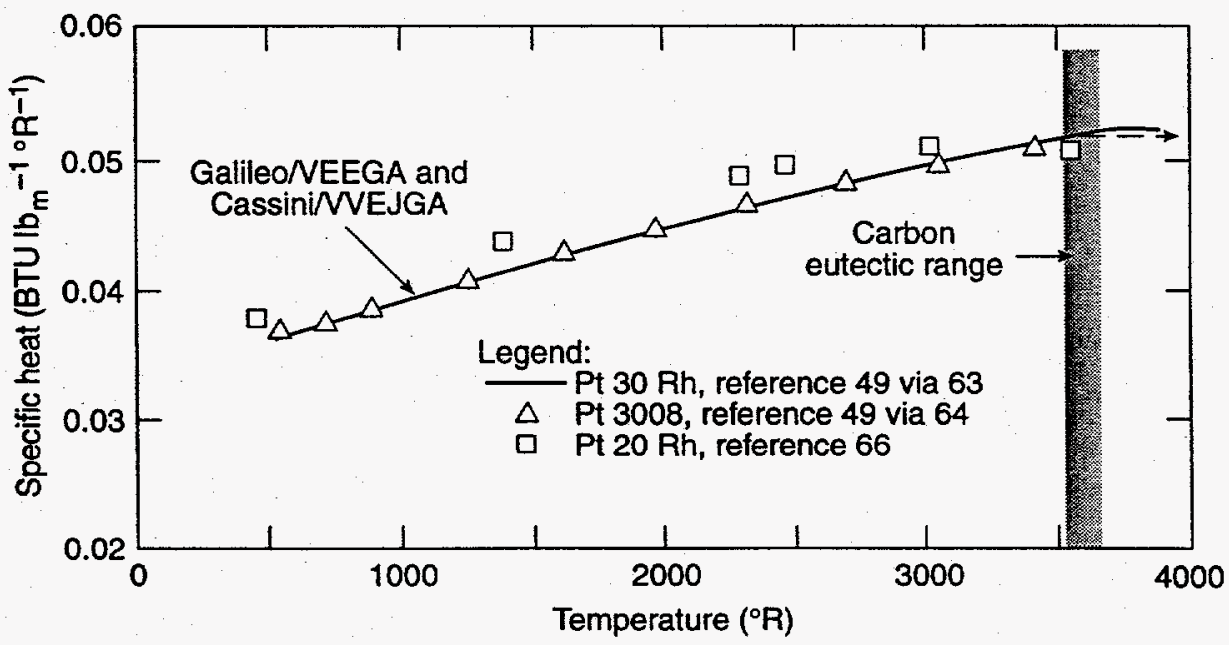

Figure 45 Specific heat schedules for Pt-30 Rh. 
The Johns Hopkins University

Applied Physics Laboratory

Laurel, Maryland 20723.6099

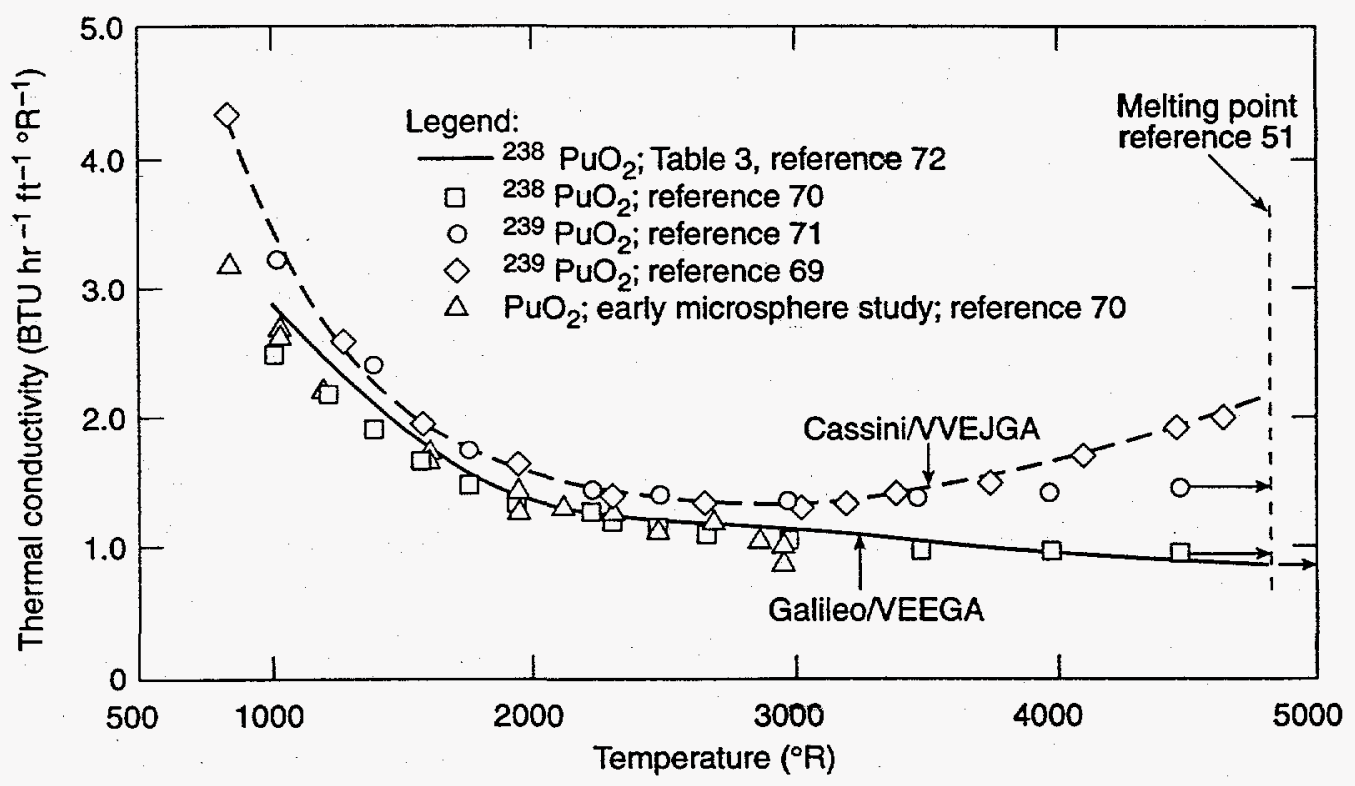

Figure 46 Thermal conductivity schedules for $\mathrm{PuO}_{2}$.

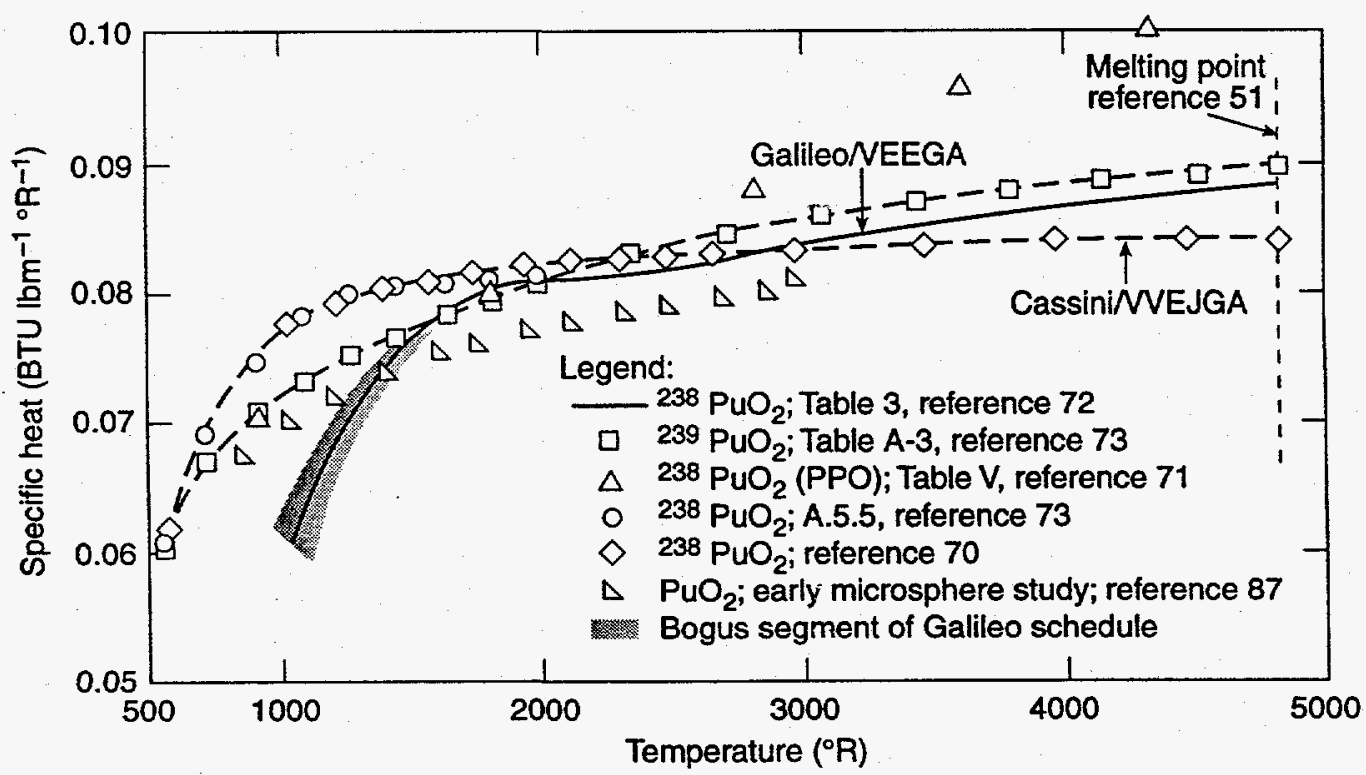

Figure 47 Specific heat schedules for $\mathrm{PuO}_{2}$. 
(a) Directional emittance, FWPFtm carbon-carbon

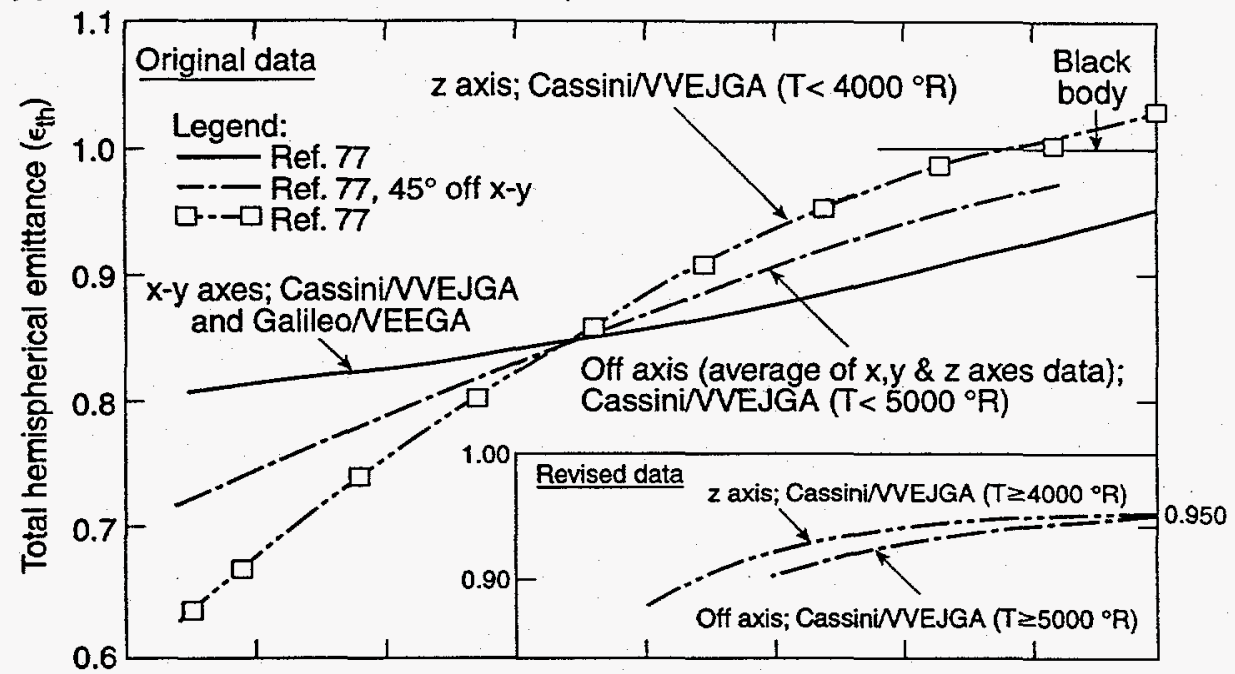

(b) Perpendicular to deposition plane, pyrolytic graphite

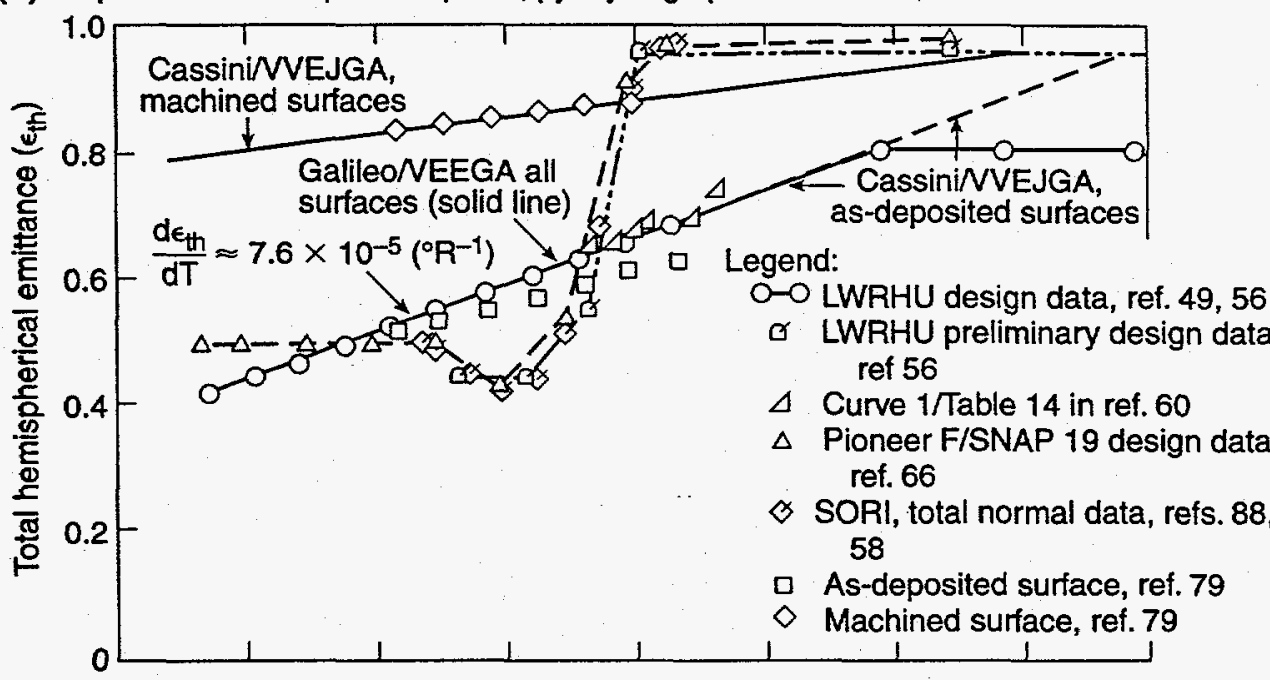

(c) Parallel to deposition plane, pyrolytic graphite

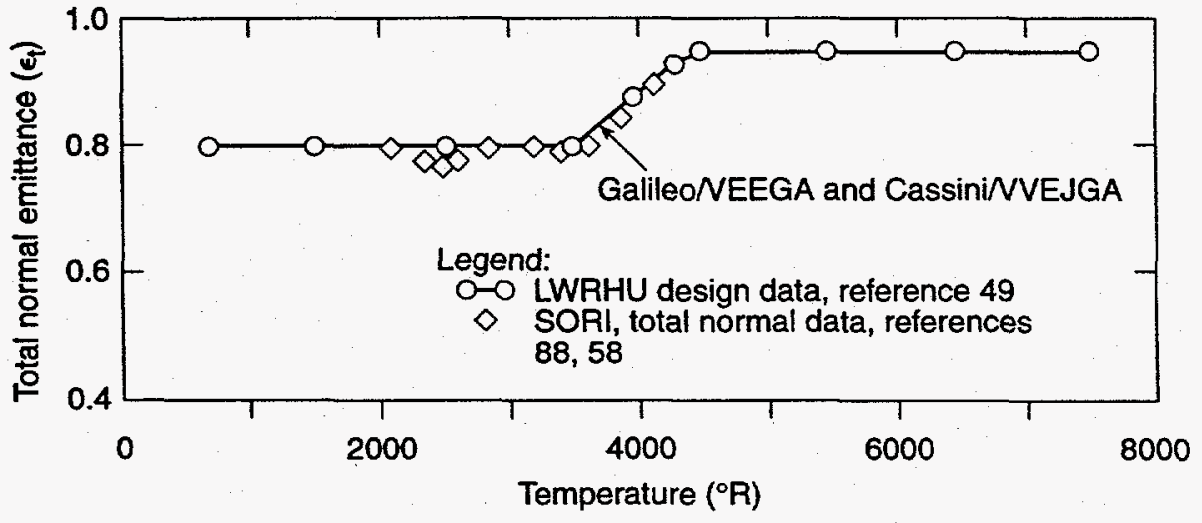

Figure 48 Thermal radiative properties for LWRHU's constituent materials. 
Applied Physics Laboratory

Laurel, Maryland 20723-60999

(d) Platinum and platinum alloys

Legend:

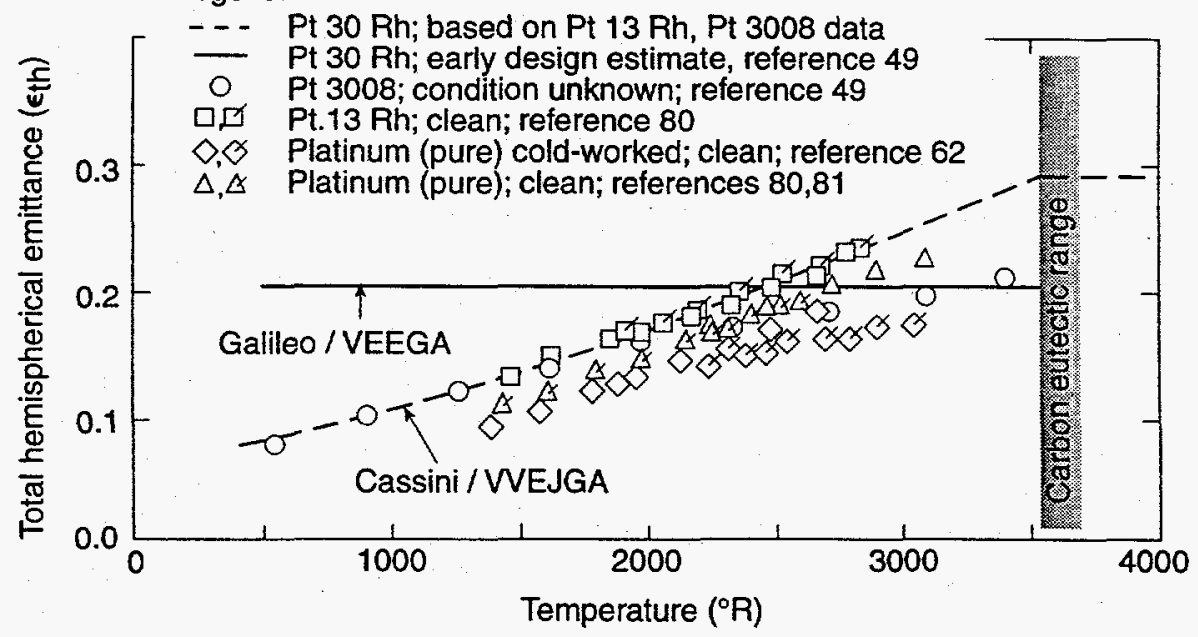

(e) Plutonium dioxide

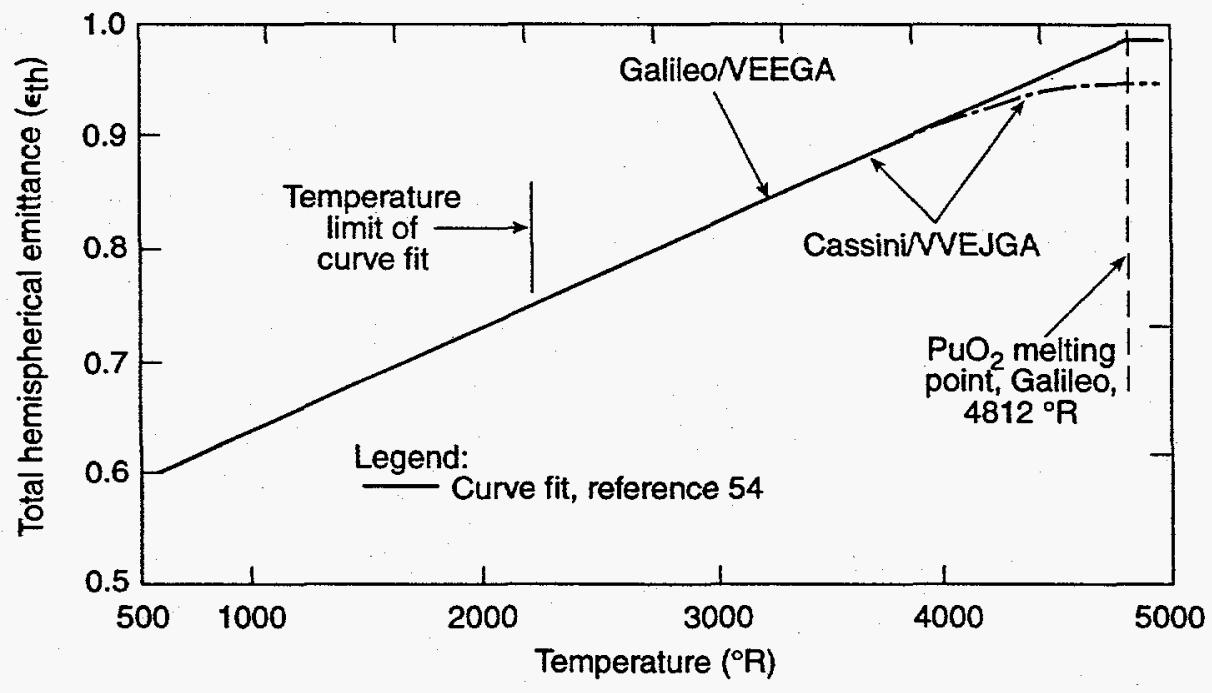

Figure 48 (continued) Thermal radiative properties for LWRHU's constituent materials.

86-6374 
The Johns Hopkins University

Applied Physics Laboratory

Laurel, Maryland 20723-6099

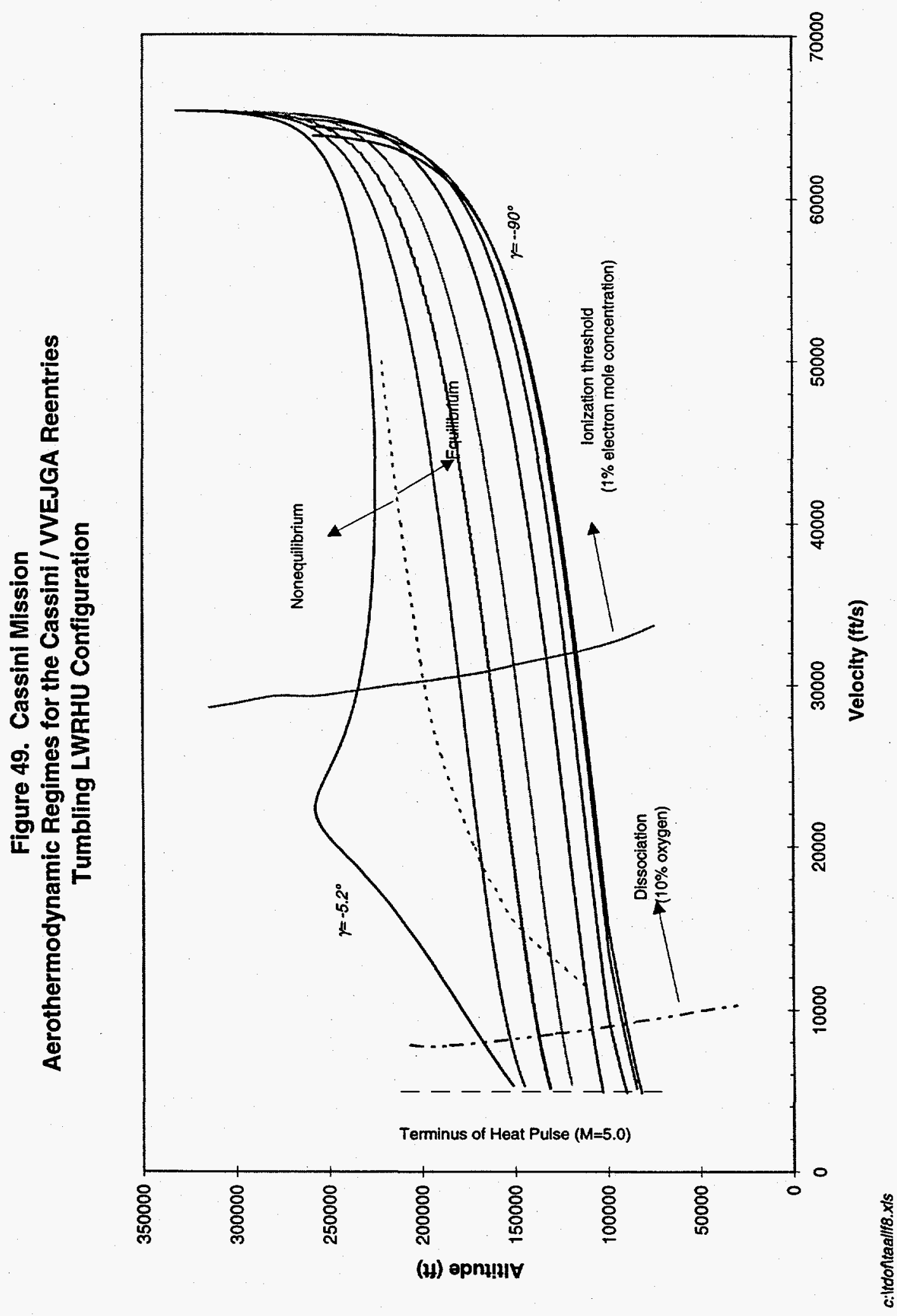




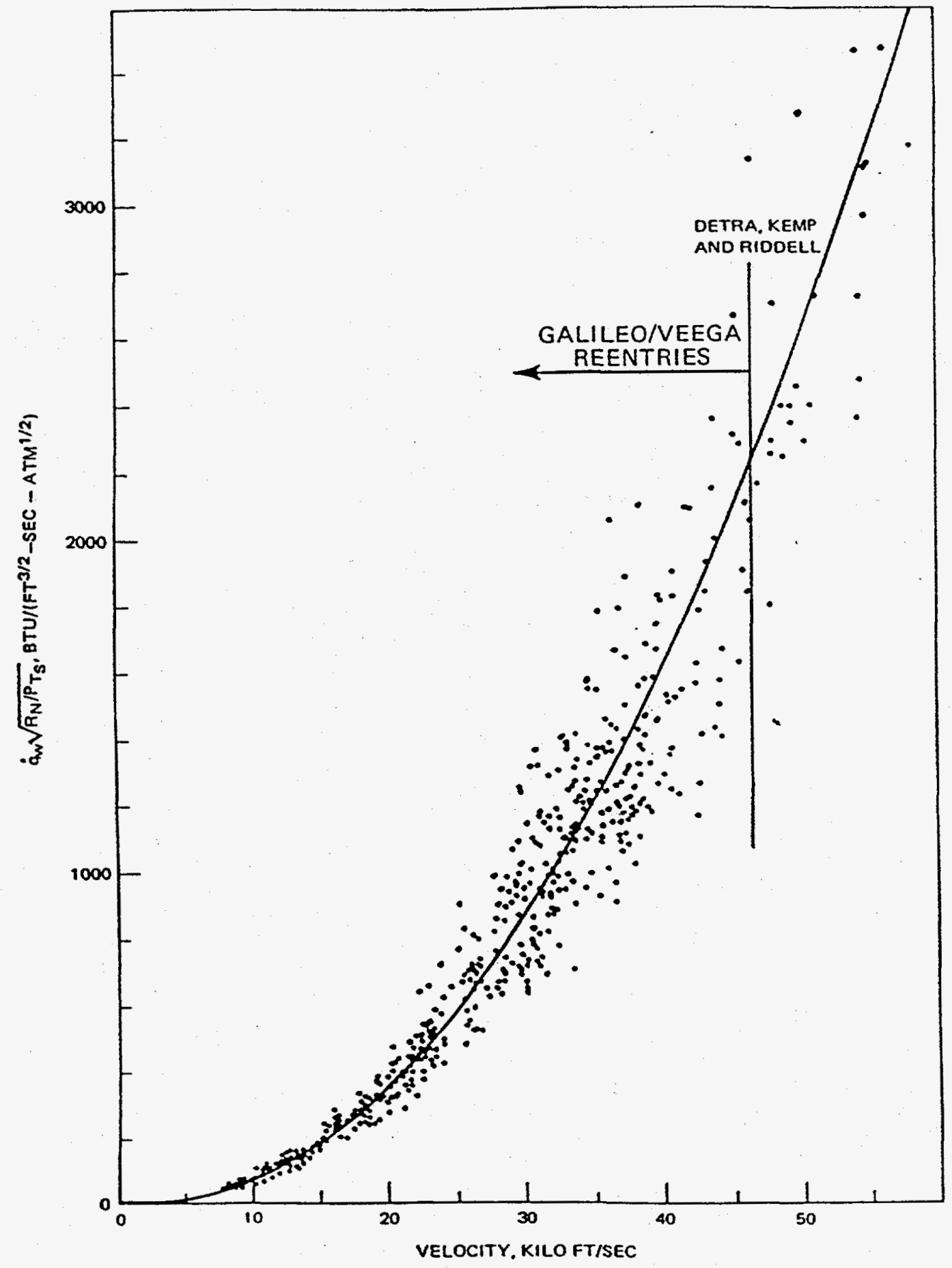

Figure 50 Composite Plot Comparing Experimental Data With the Detra, Kemp, and Riddell Correlation 94 


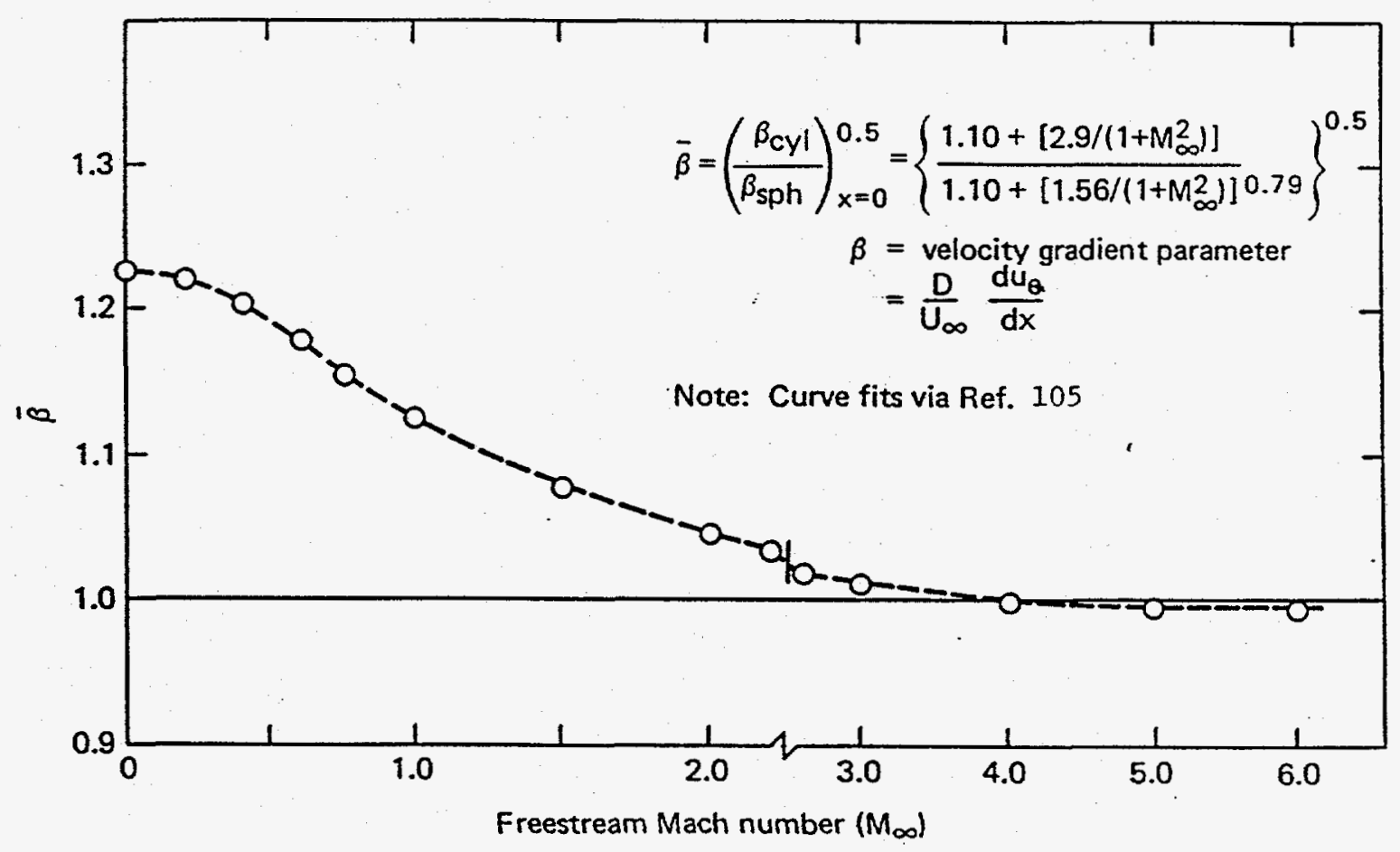

Figure 51 Stagnation Velocity Gradient Correction on the Side-on LWRHU Stagnation Convective Heating Rate (Without Geometry Scaling) 

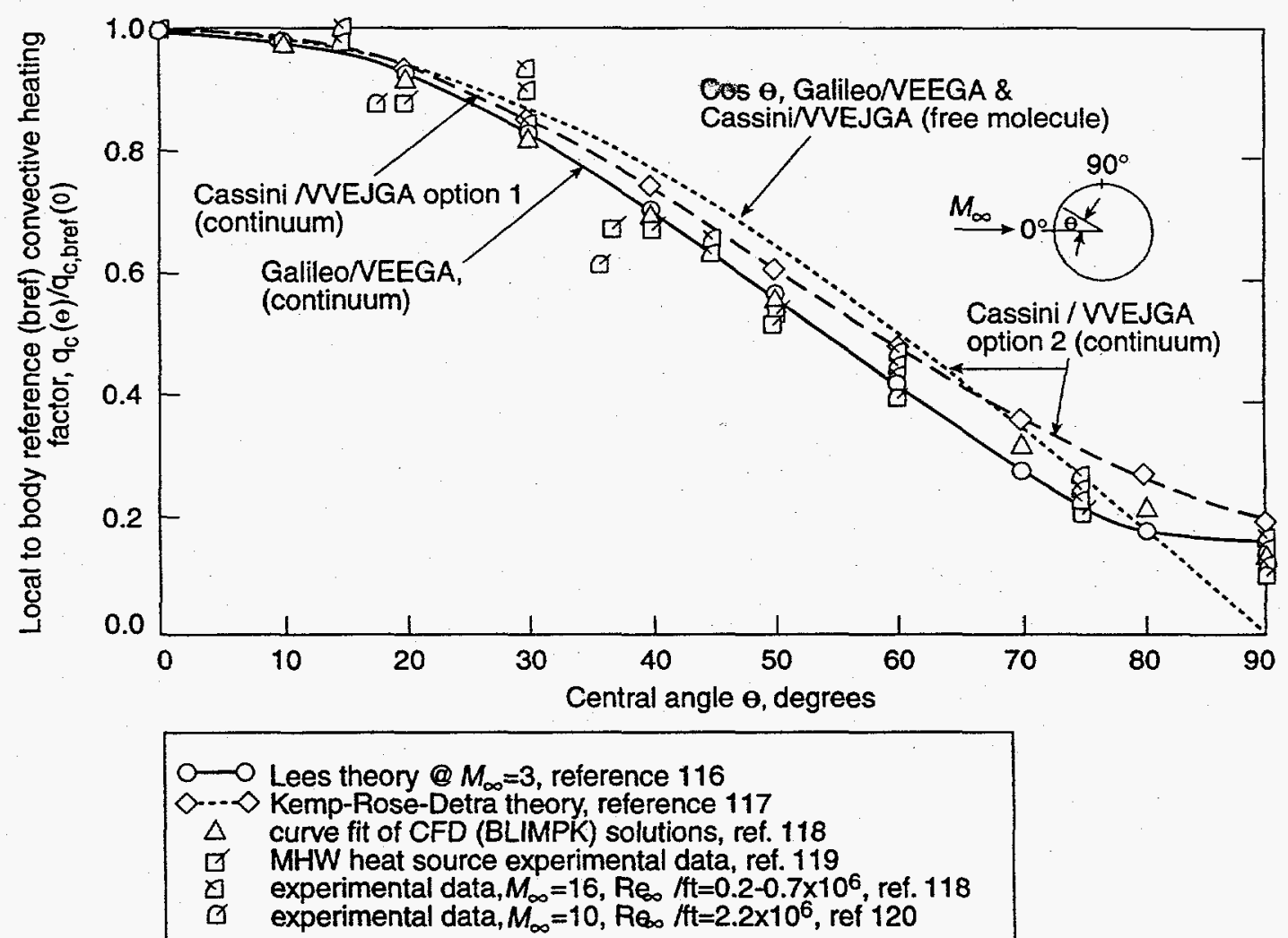

Figure 52 Hypersonic, windward circumferential convective heating factor distributions; cold-wall, non-ablating LWRHU; side-on stable flight orientation. 
Applied Physics Laboratory

Laurel, Maryland 20723.6099

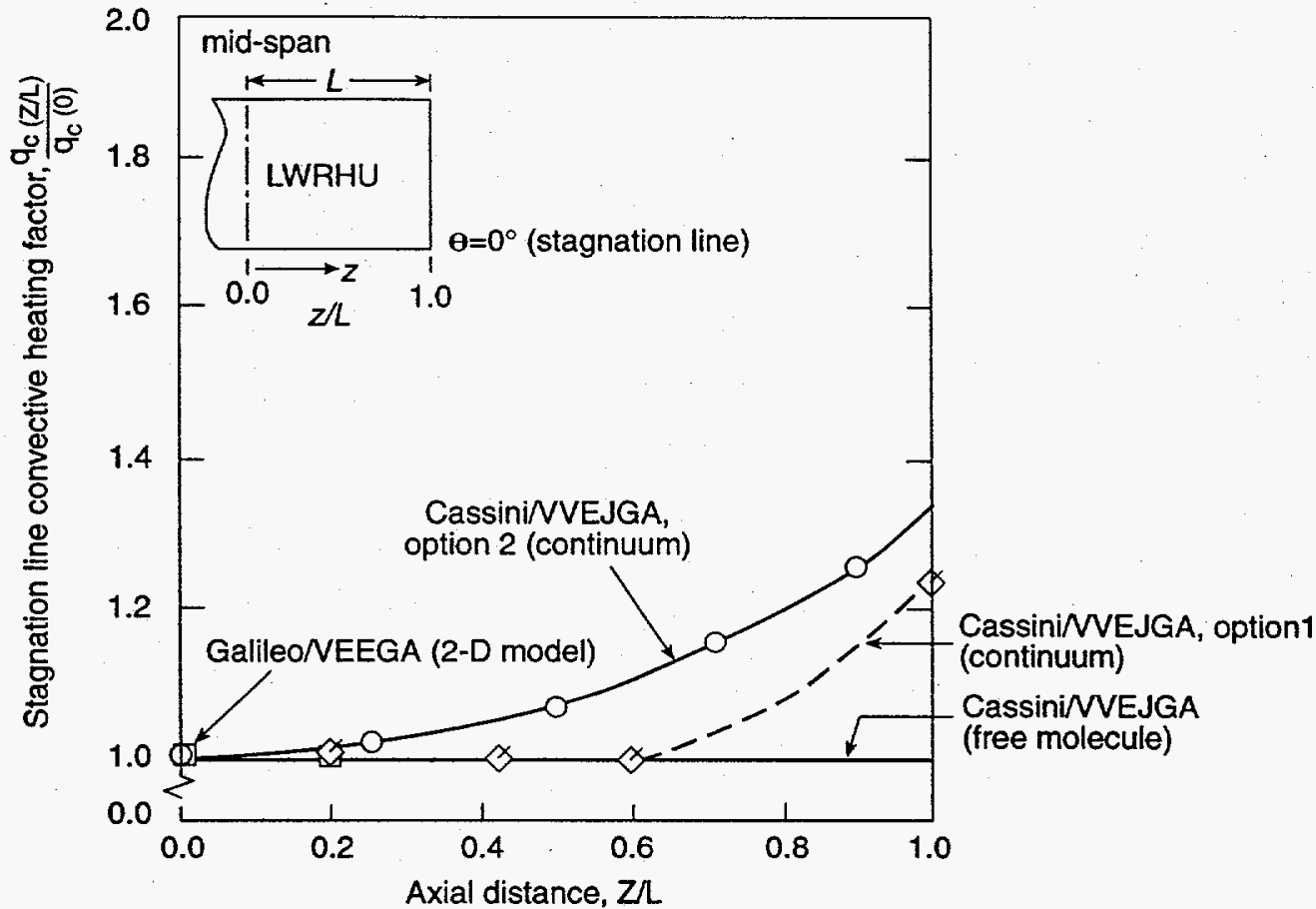

O LWRHU design curve-estimated, reference 121

$\diamond$ experimental data - Multi Hundred Watt (MHW) heat source, reference 119

Figure 53 Hypersonic stagnation line convective heating factor distributions; cold wall, non-ablating LWRHU; side-on stable flight orientation. 


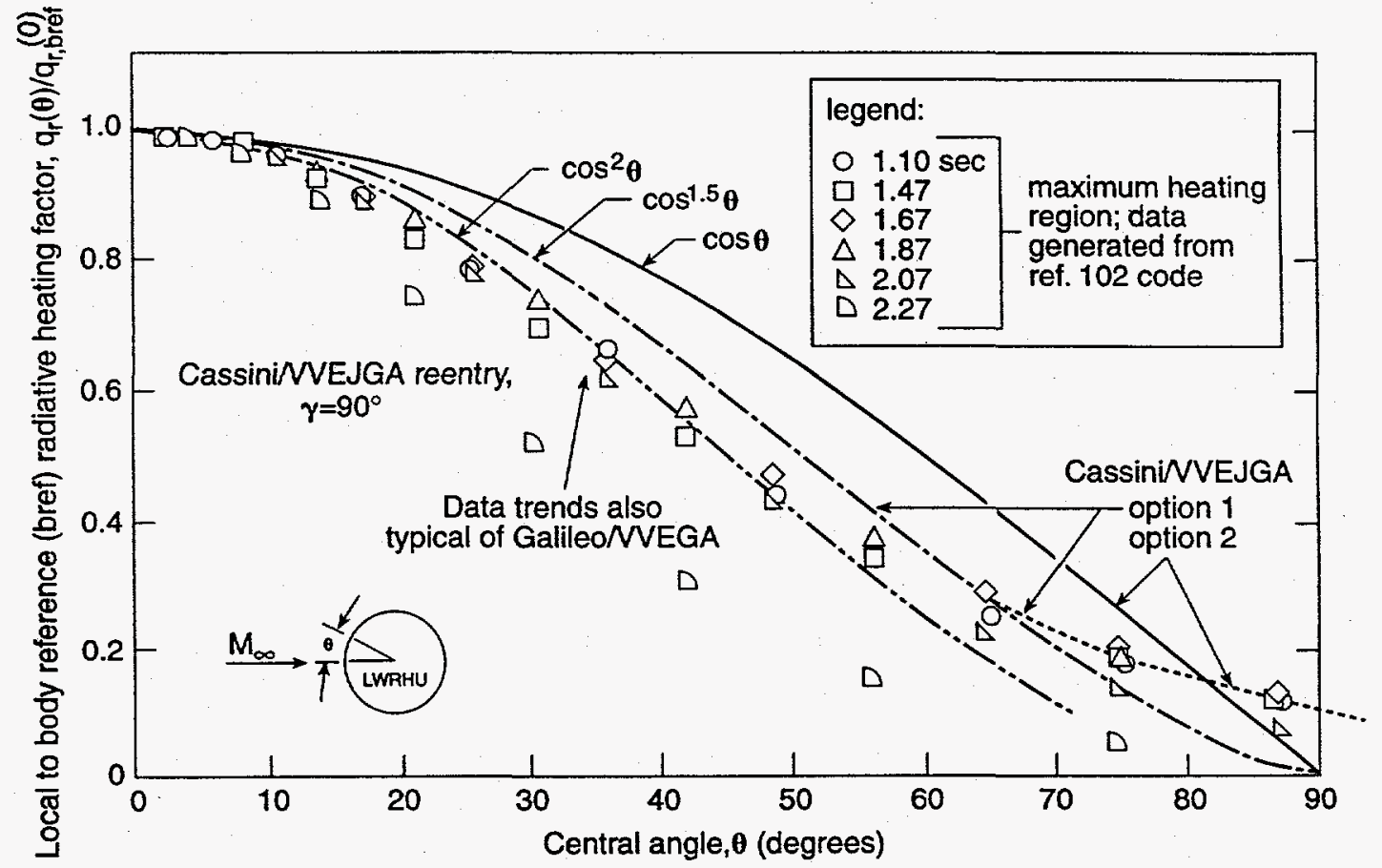

Figure 54 Hypersonic, windward circumferential radiative heating factor distributions; black body, non-ablating LWRHU; side-on stable flight orientation.

$96-6374$ 
Applied Physics Laboratory

Laurel. Maryland 20723-6099

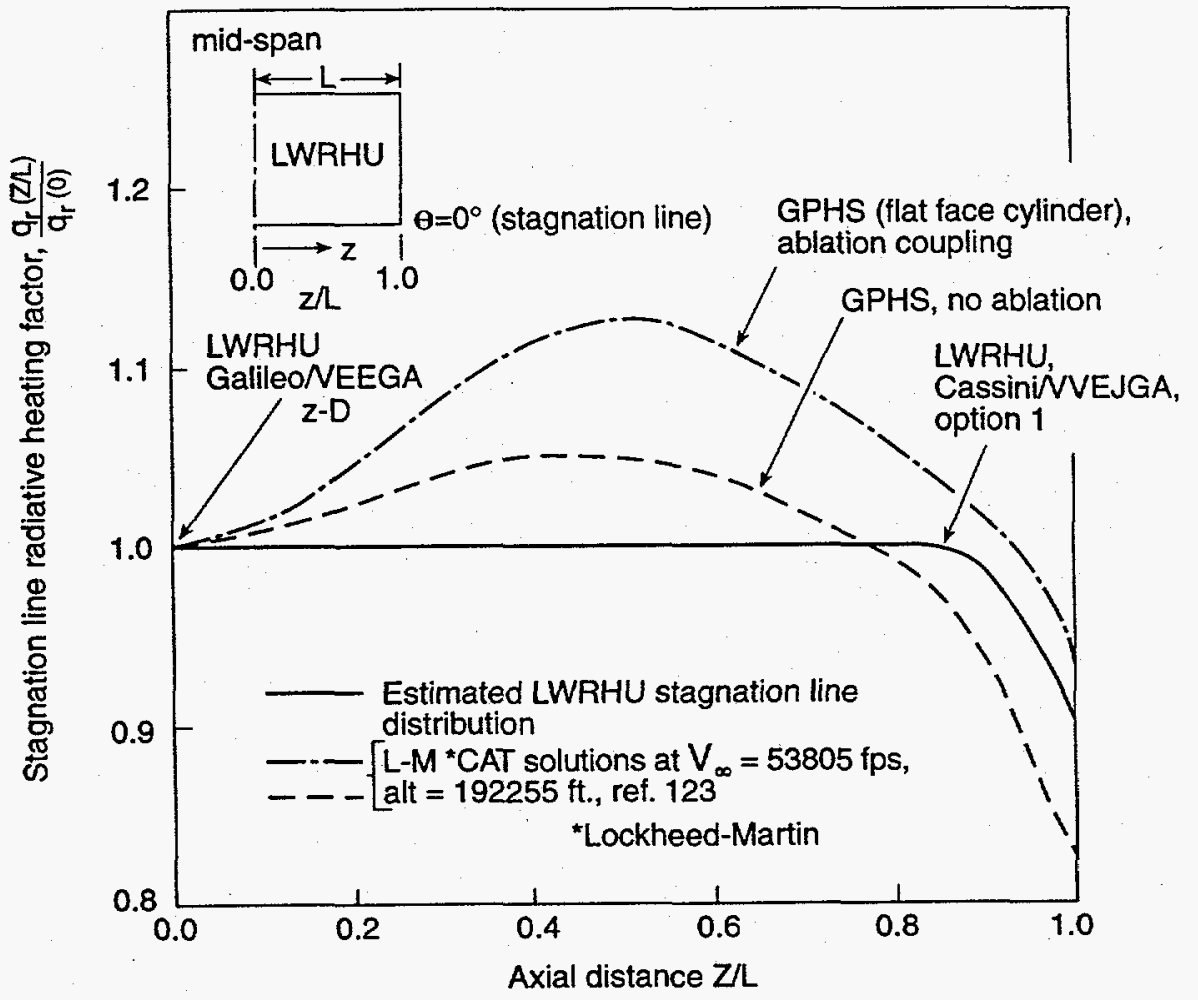

Figure 55 Hypersonic, stagnation line radiative heating factor distributions; LWRHU side-on stable flight orientation.

$96-6374$ 


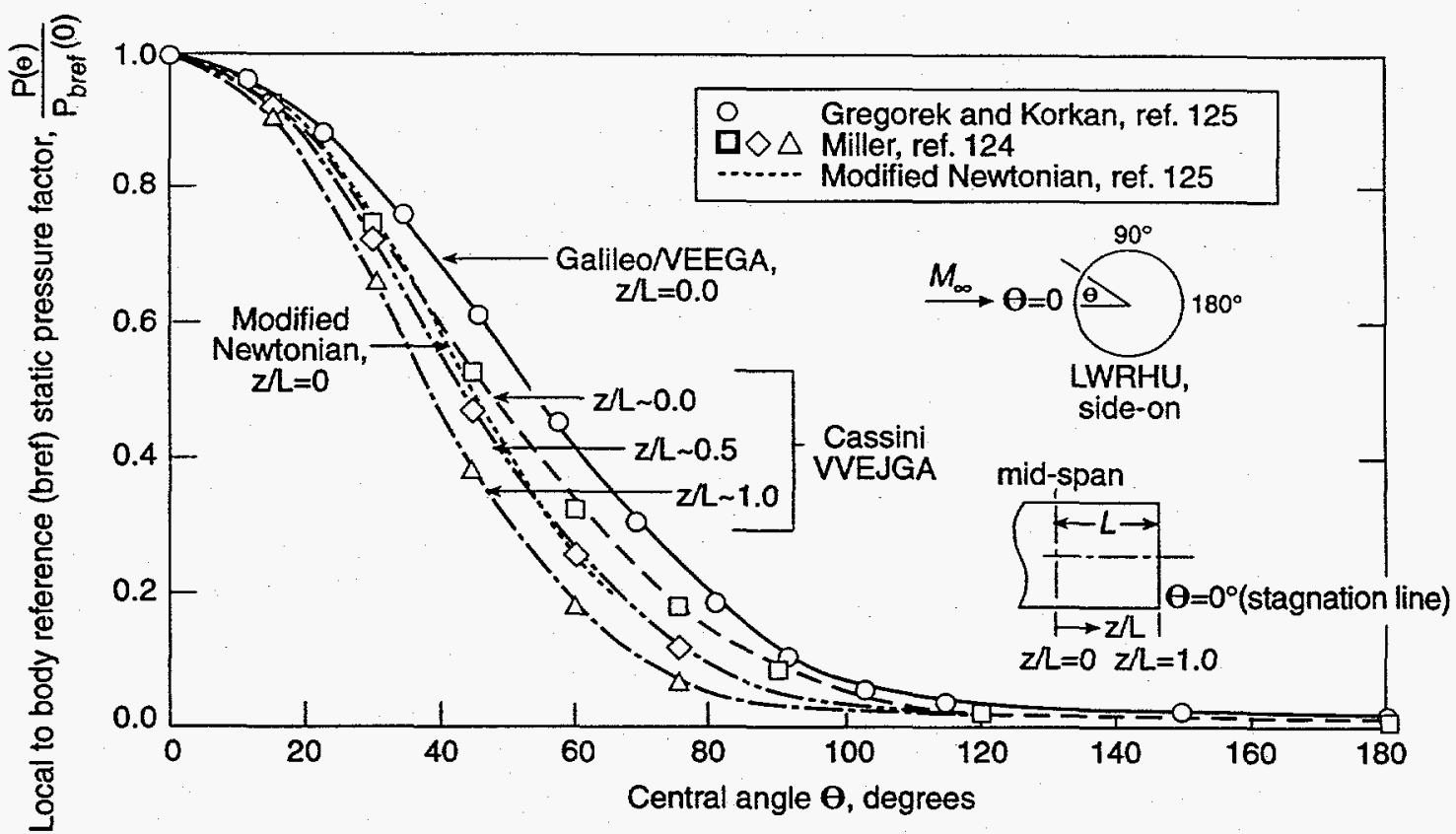

Figure 56 Hypersonic circumferential static pressure factor distribution as a function of axial location; LWRHU side-on flight orientation.

$96-6374$ 
Enclosure (1) to: AlC-97-095

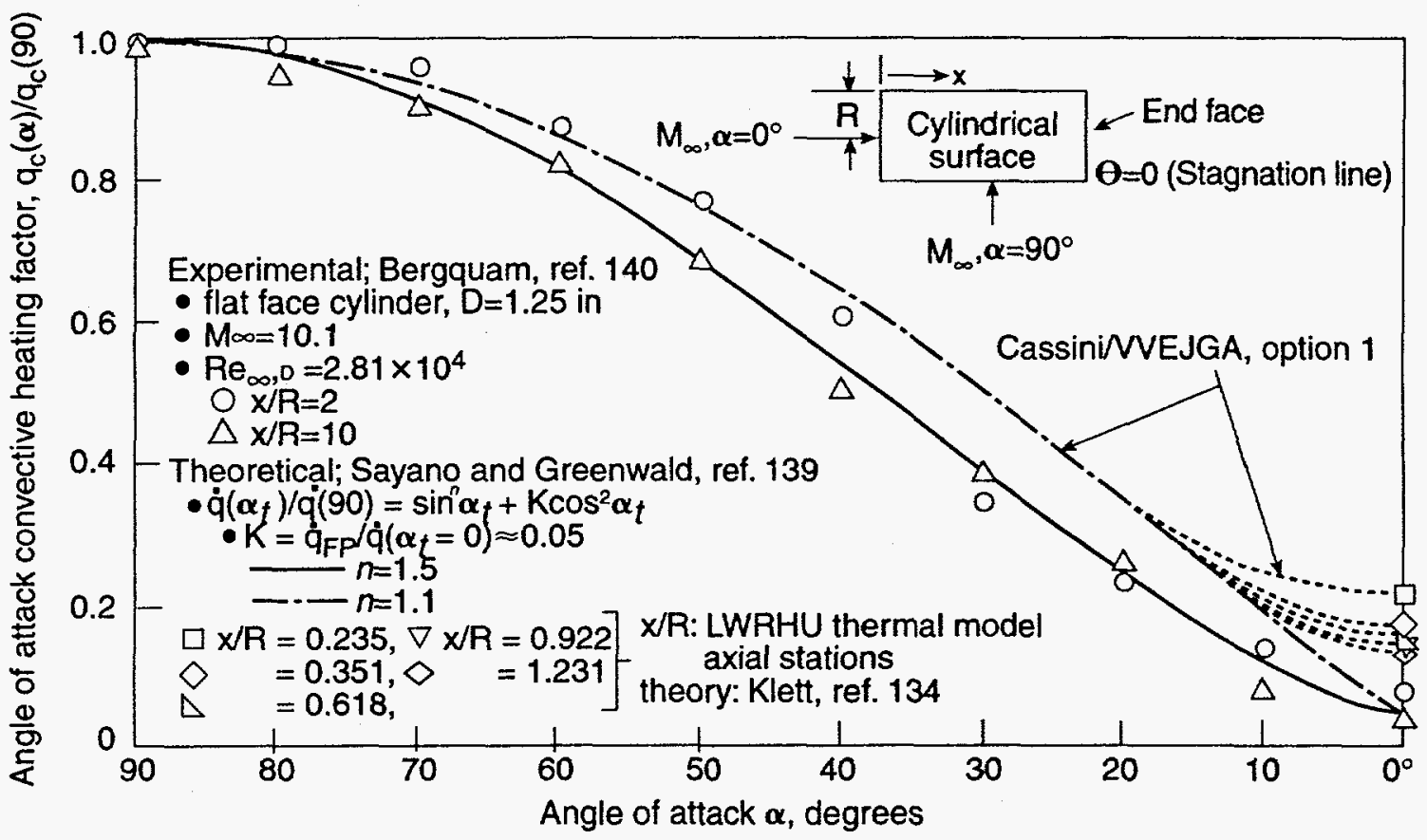

Figure 57 Hypersonic, angle of attack convective heating factor, stagnation line of LWRHU's cylindrical surface; random tumbling flight orientation. 
Applied Physics Laboratory

Laurel. Maryland 20723.6099

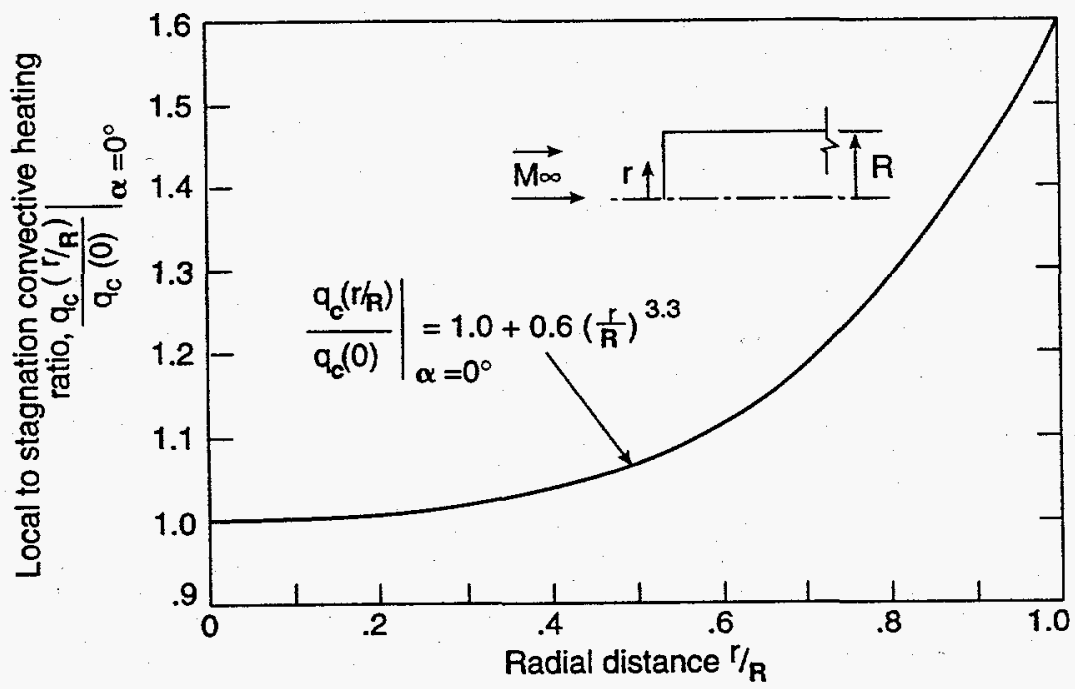

Figure 58 Local convective heating ratio on LWRHU's end face; zero angle of attack; random tumbling flight orientation. (Curve from Figure 10, Klett, ref. 134).

$96-6374$ 
Applied Physics Laboratory

Laurel, Maryland 20723.6099

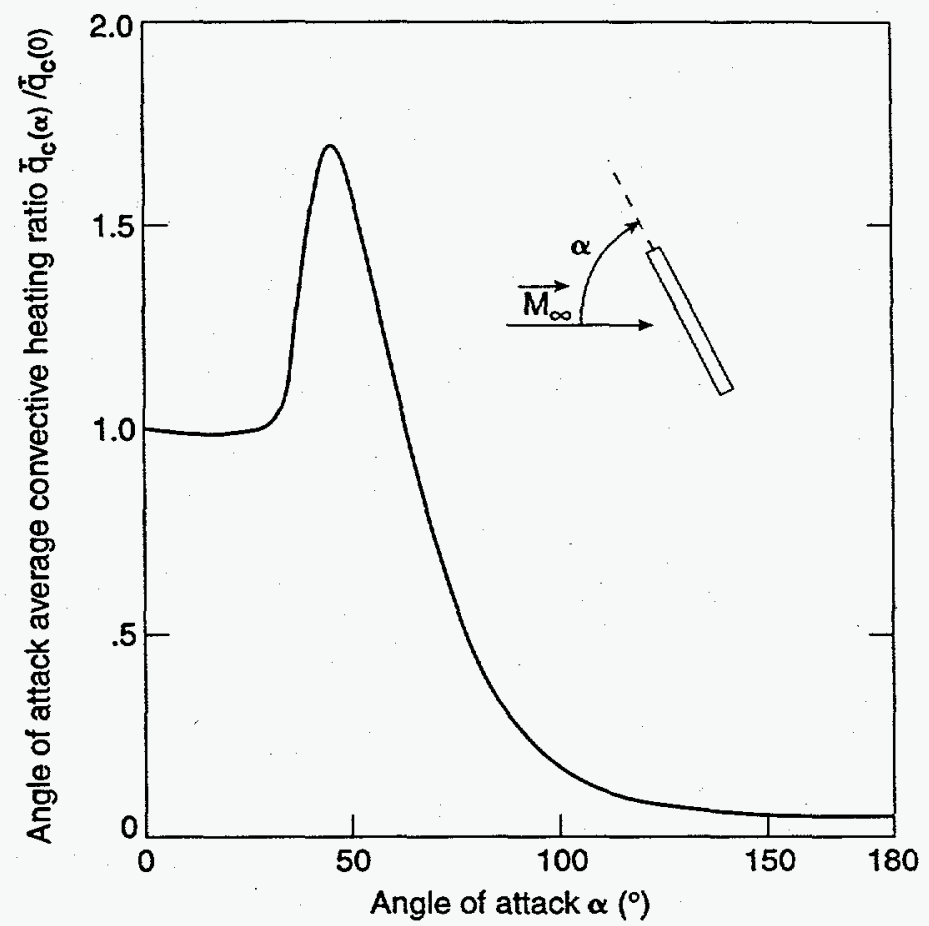

Figure 59 Average convective heating ratio to LWRHU's end face as a function of angle of attack; random tumbling flight orientation (curve from figure 11, Klett, ref. 134).

86-6374 
The Johns Hopkins Lniversity

Applied Physics Laboratory

Laurel, Maryland 20723-6099

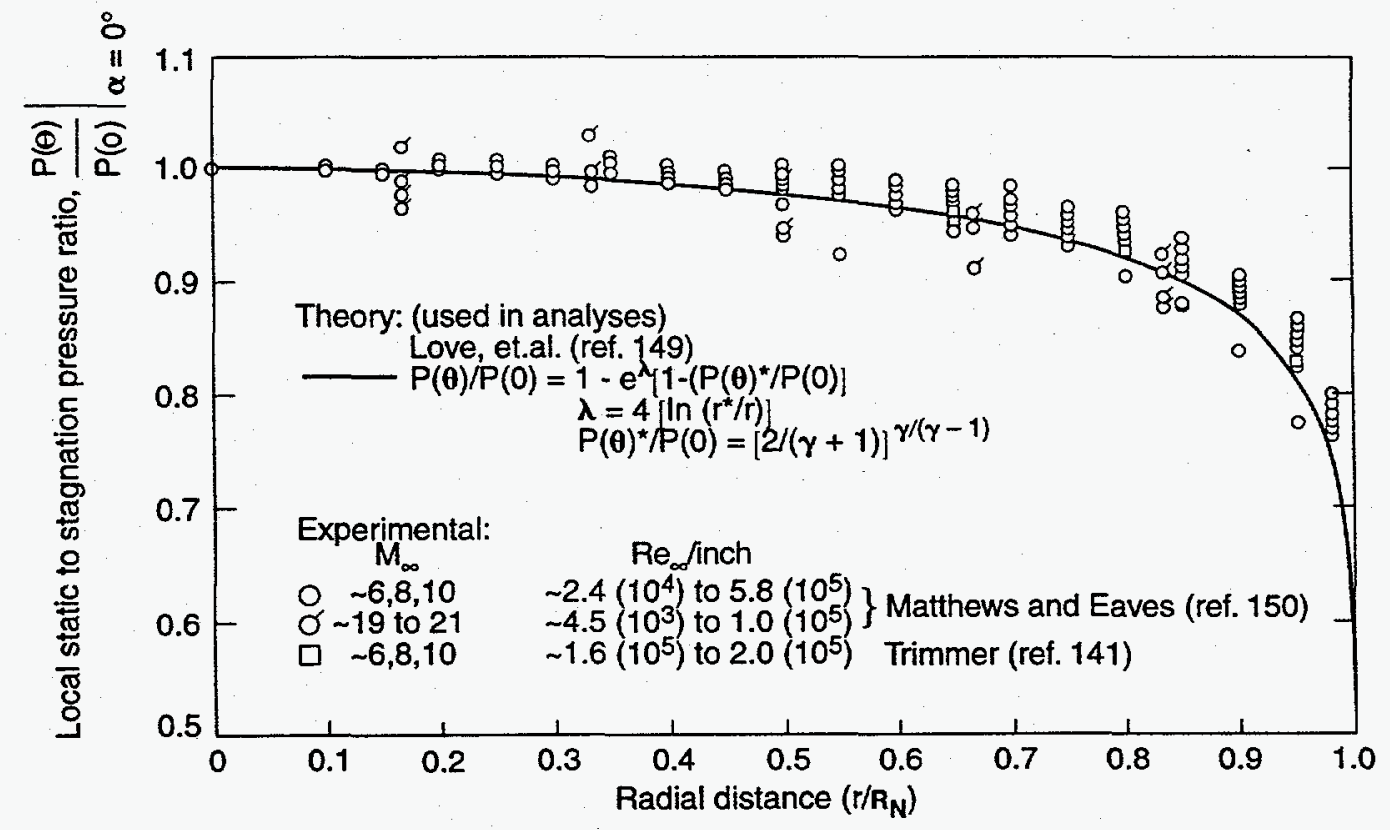

Figure 60 Comparison of correlated and experimental static pressure distributions over the LWRHU's end face; zero angle of attack; random tumbling flight orientation. 
The Johns Hopkins University

Applied Physics Laboratory

Laurel. Maryland 20723-6099

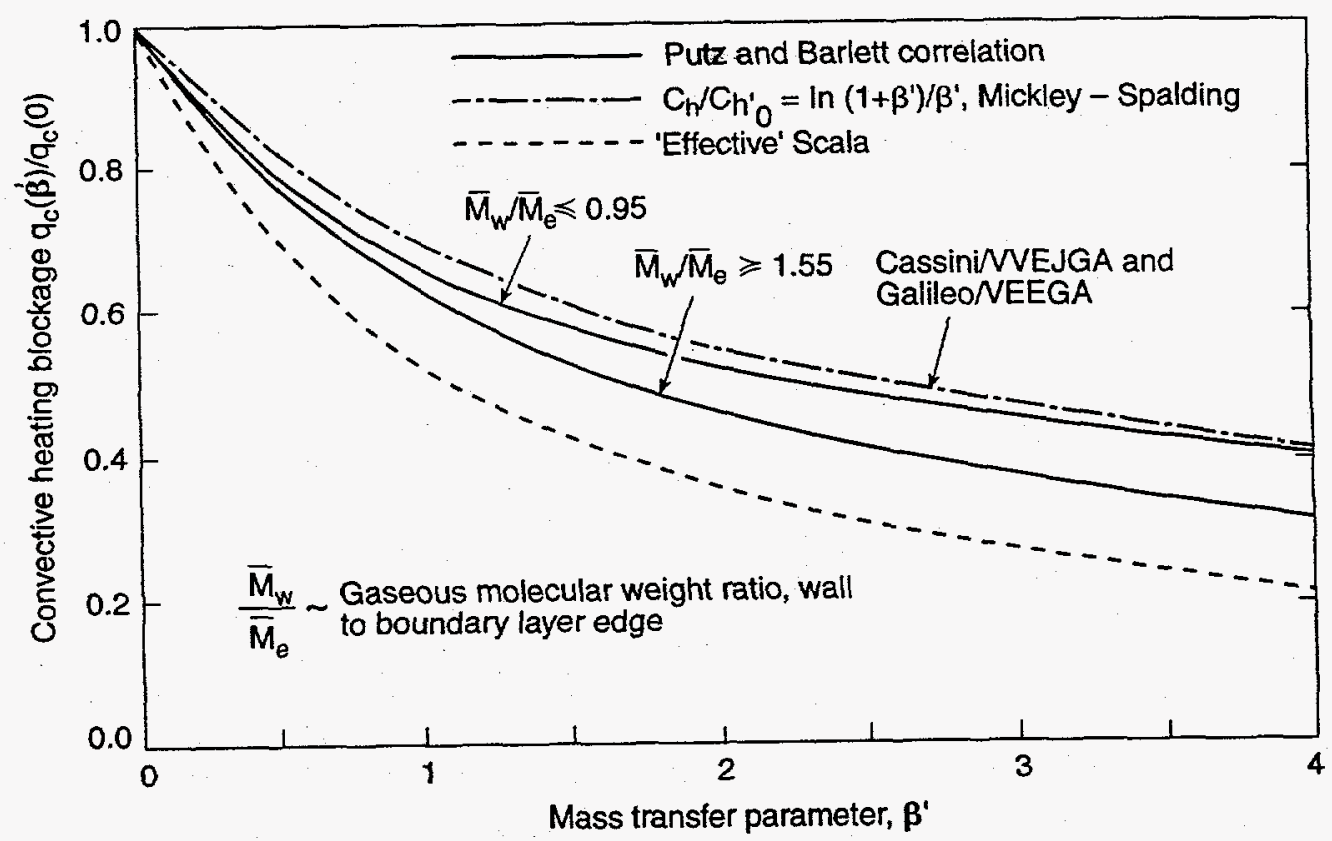

Figure 61 Blockage of convective heating rate as a function of the mass transfer parameter, $\beta^{\prime}$, continuum flow regimes (reproduced from ref. 160).

$96-6374$ 
The Johns Hopkins University

Applied Physics Laboratory

Laurel, Maryland 20723-6090

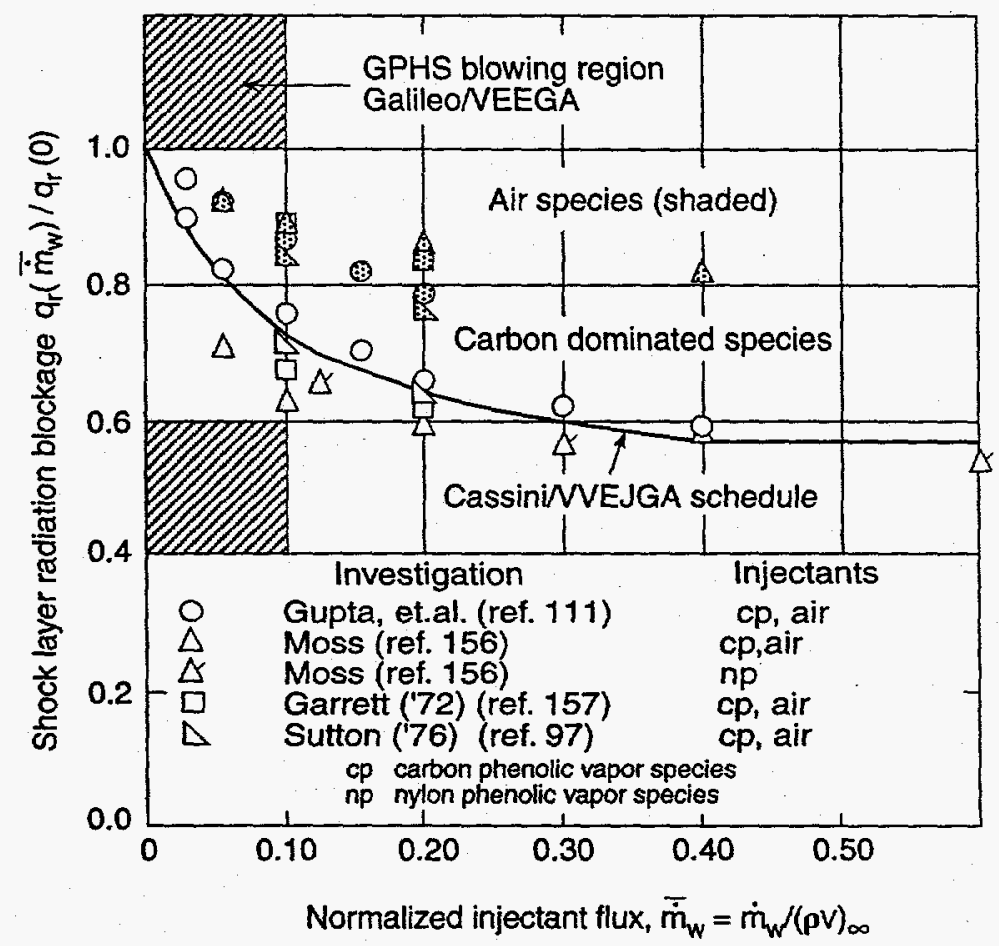

Figure 62 Blockage of stagnation radiative heating rates versus injectant mass flux and chemical constituency; continuum flow regimes.

$96-6374$ 


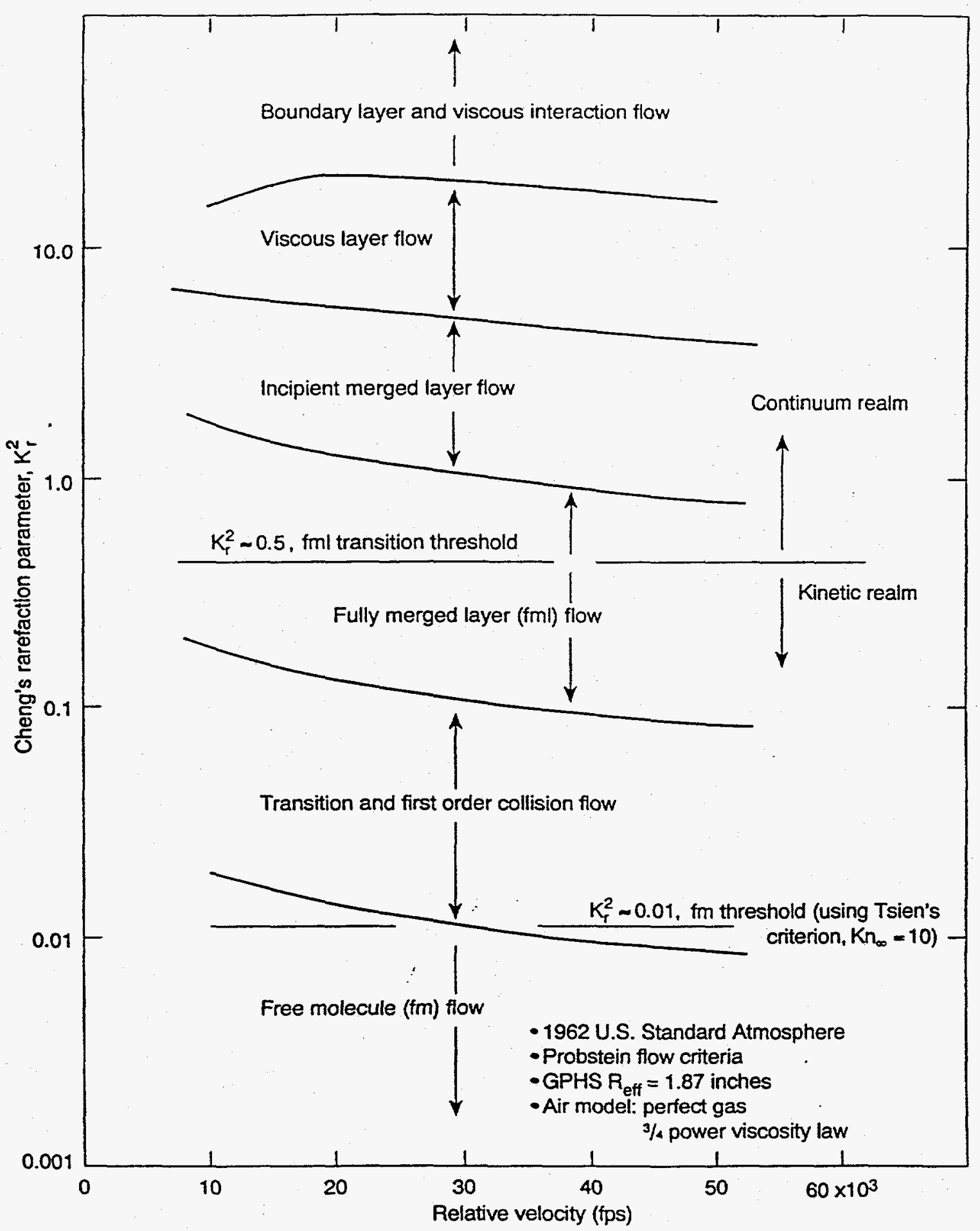

Figure 63 Continuum and Kinetic Flow Regimes with Threshold Values for Radiation Blockage 
Figure 64. Cassini Mission RETAP Results

Comparison of Fuel Release Conditions with

Sandia Melt Boundaries and FOILS EIS Studies

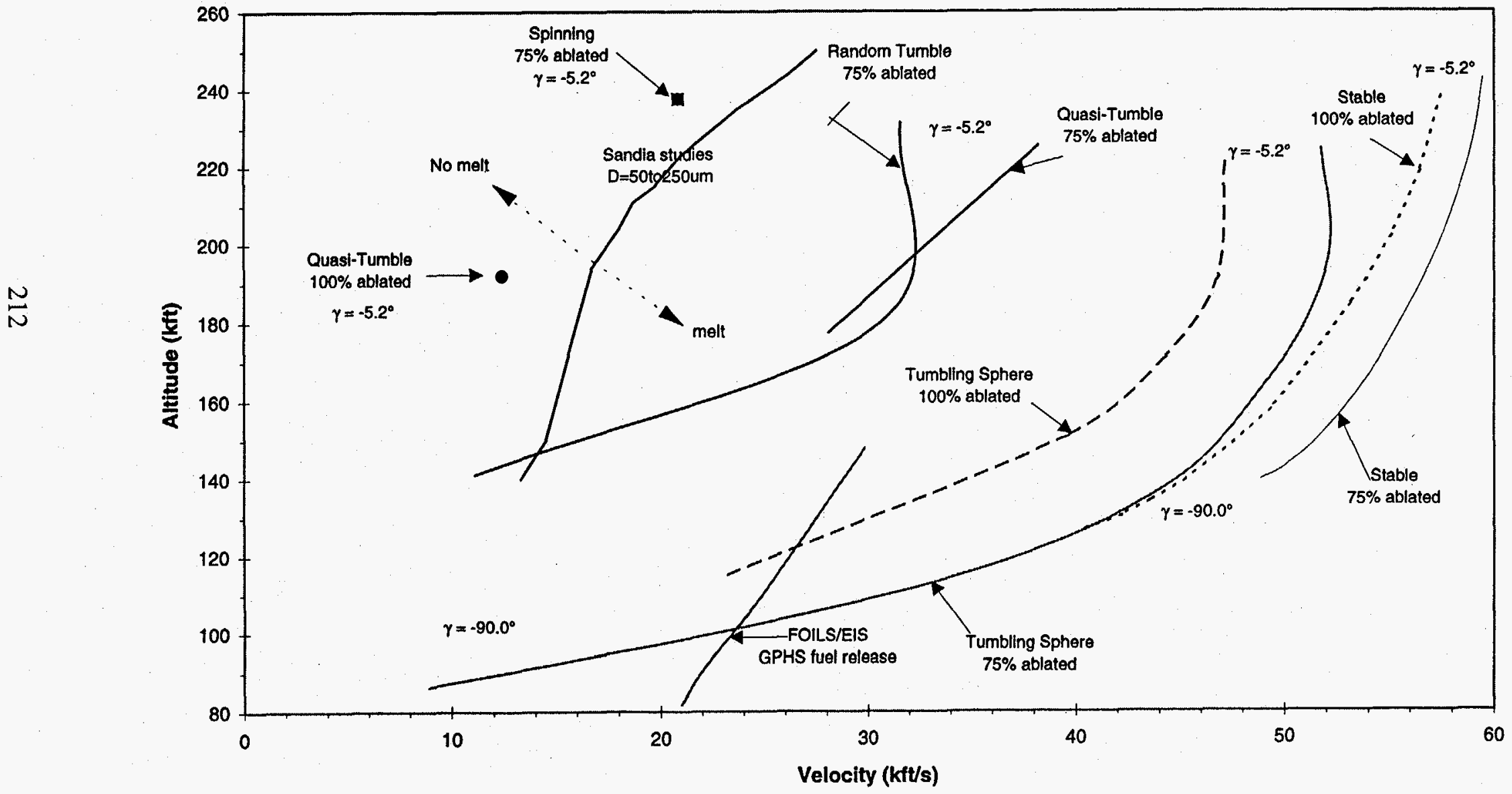


Figure 65. Cassini Mission RETAP Results LWRHU $-90^{\circ}$ Side-on Stable VVEJGA Reentry

\section{A. Component Thermal Response}

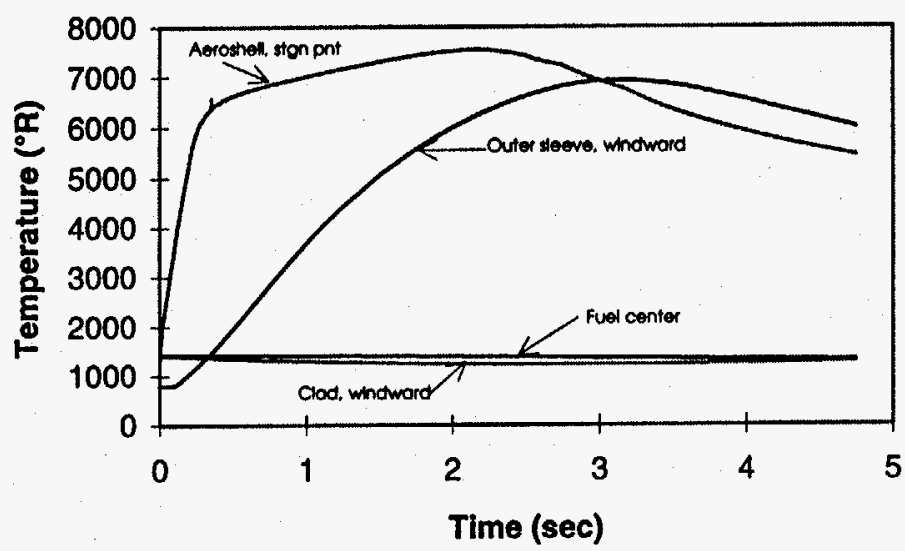

Analysis utlizes Lawrence Livermore Thermochemistry Data

C. Surface Node Recession Response

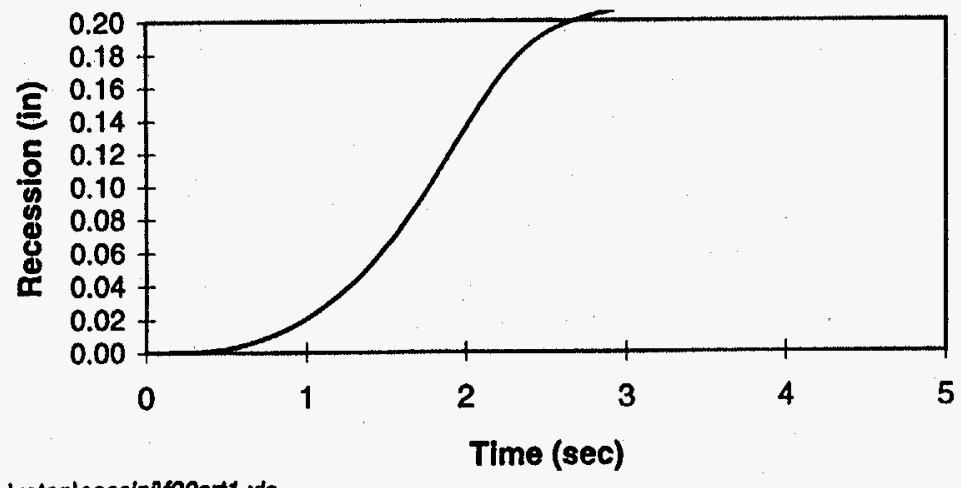

B. Surface Node Mass Transfer Parameter Response

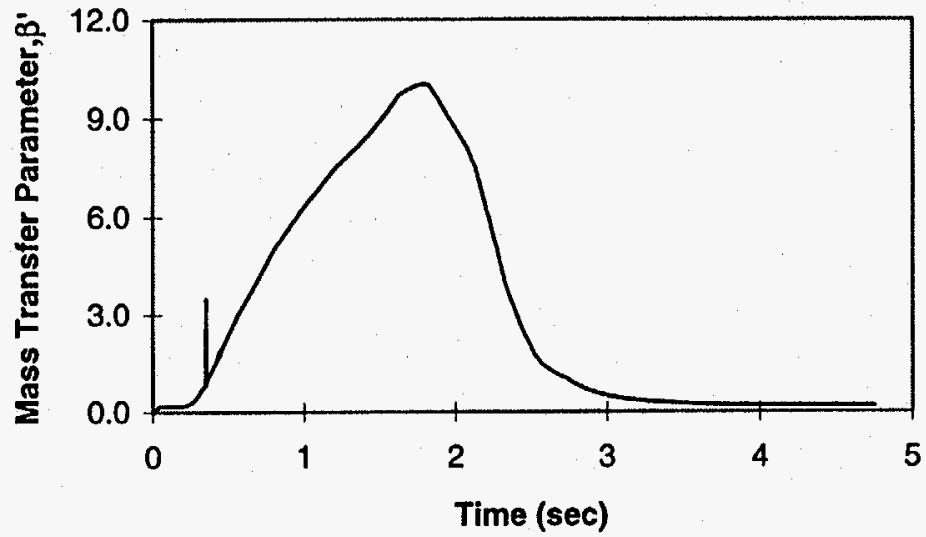

D. Recession Failure For Modeled Surface Nodes

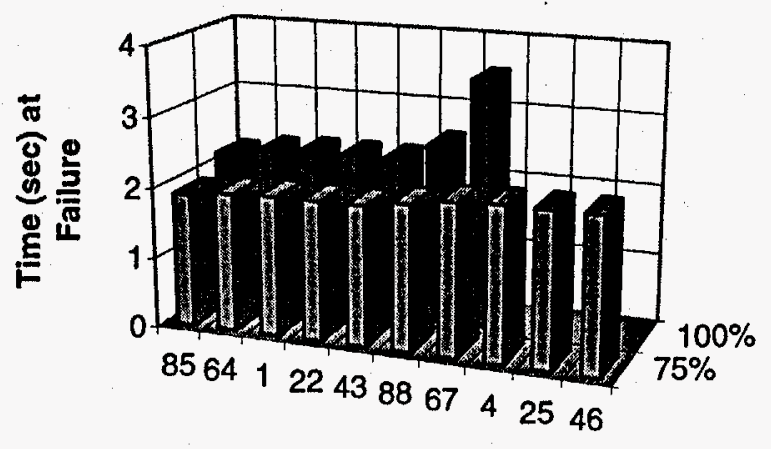

Surface Node Number re: flo.34 for node location 
Figure 66. Cassini Mission RETAP Results

LWRHU 3D $-70^{\circ}$ Side-on Stable VVEJGA Reentry

A. Component Thermal Response

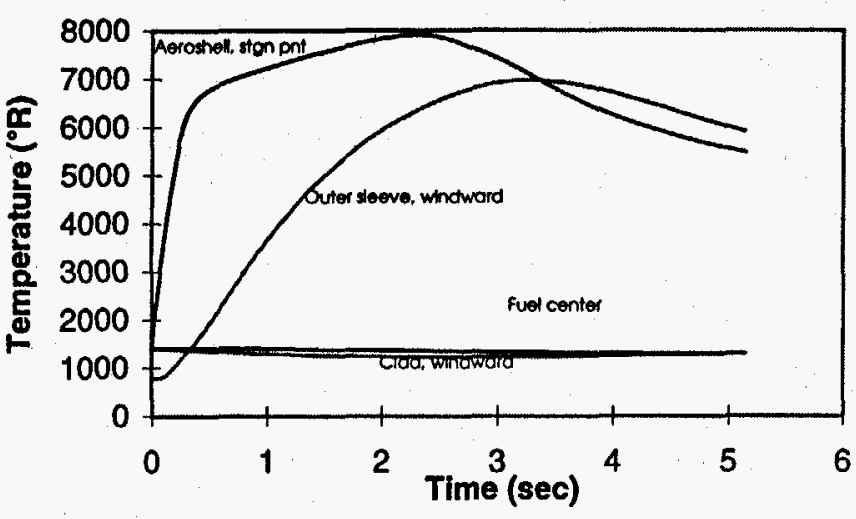

Analysis utilizes Lawrence Livermore Thermochemistry Tables

\section{Surface Node Recession}

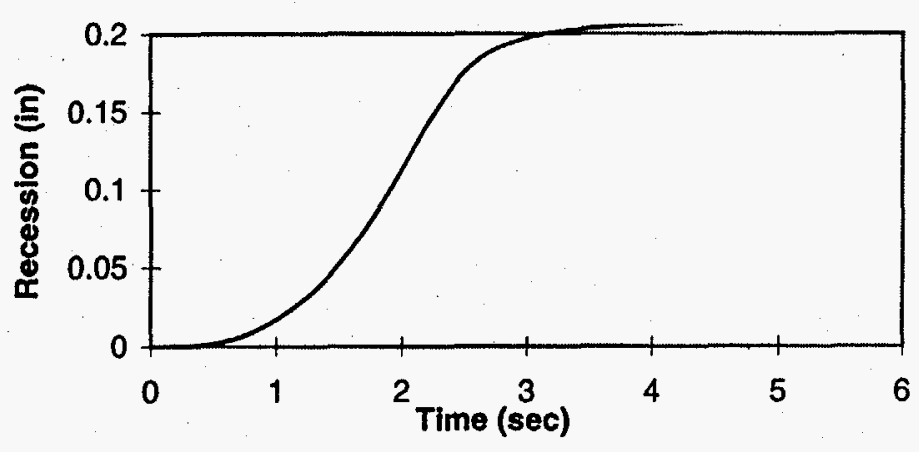

c:Iretaplcassinila70srti.xls
B. Surface Node Mass Transfer Parameter Response

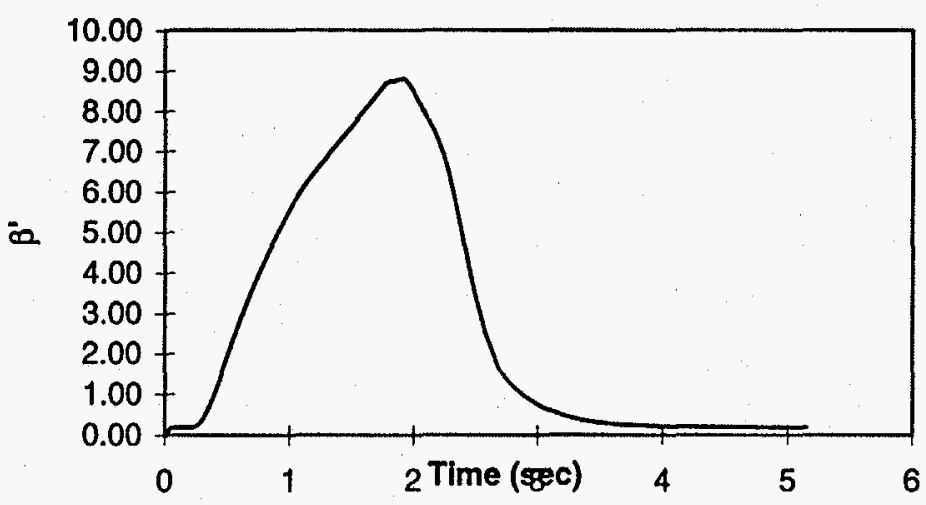

D. Recession Failure for Modeled Surface Nodes

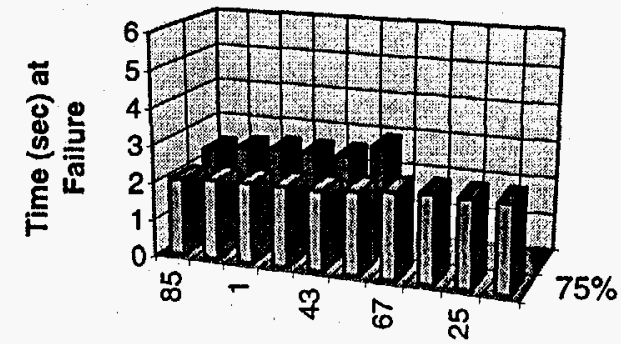

Surface Node Number 
Applied Physics Laboratory

Laurel, Maryland 20723-60199

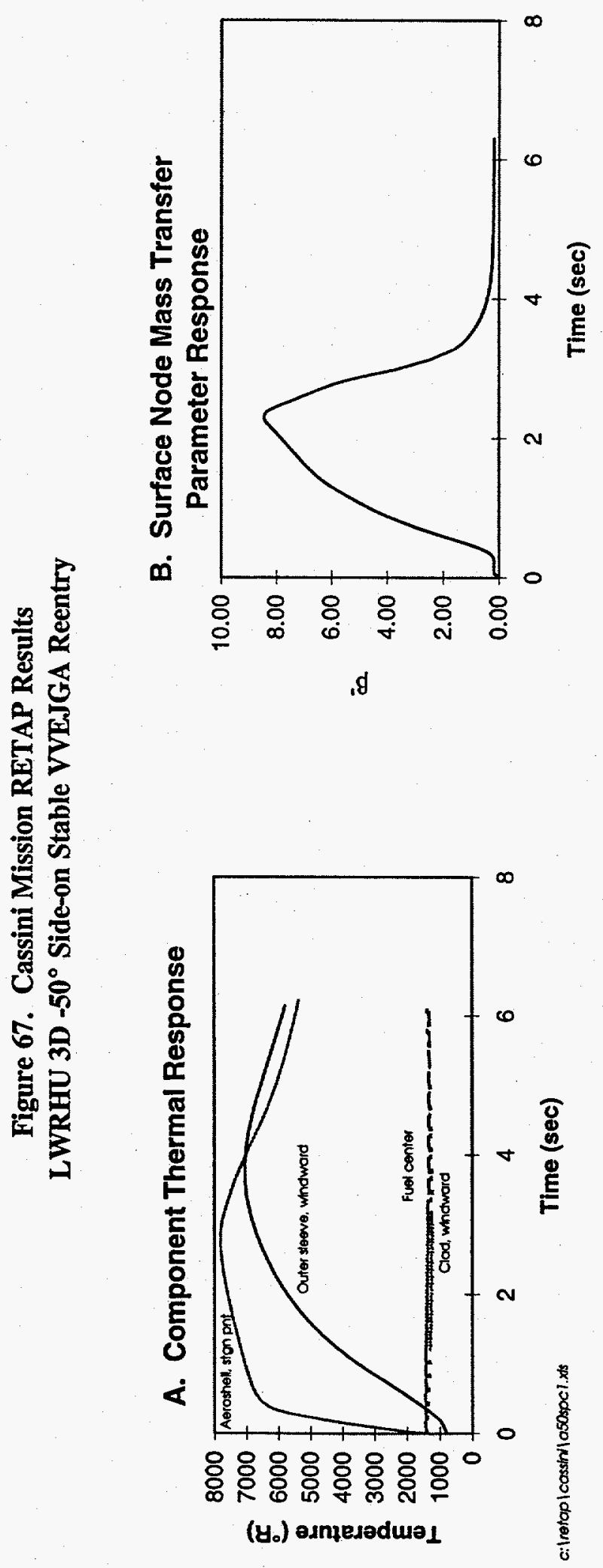

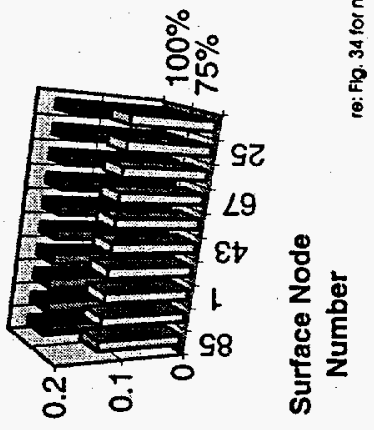

(u!) $u$

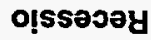

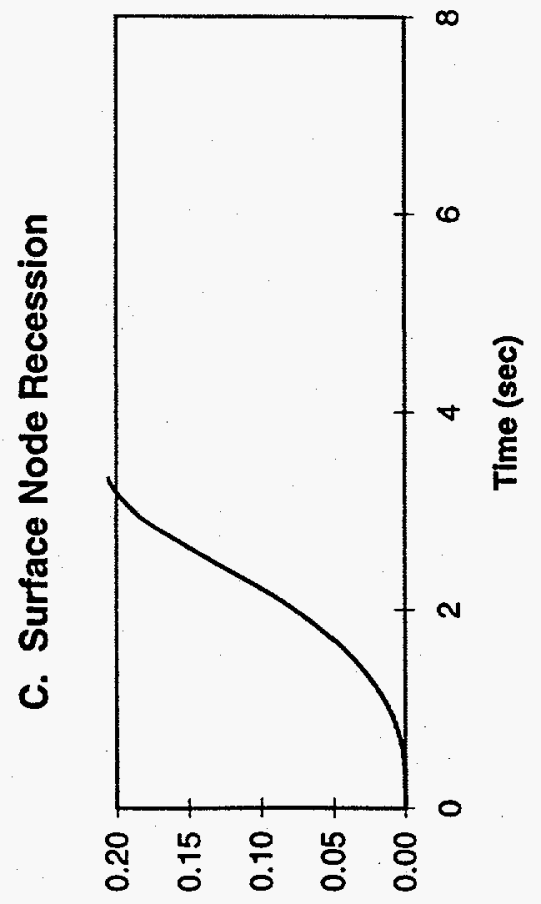

(u!) uo!ssejey 
Figure 68. Cassini Mission RETAP Results LWRHU 3D $-30^{\circ}$ Side-on Stable VVEJGA Reentry

A. Component Thermal Response

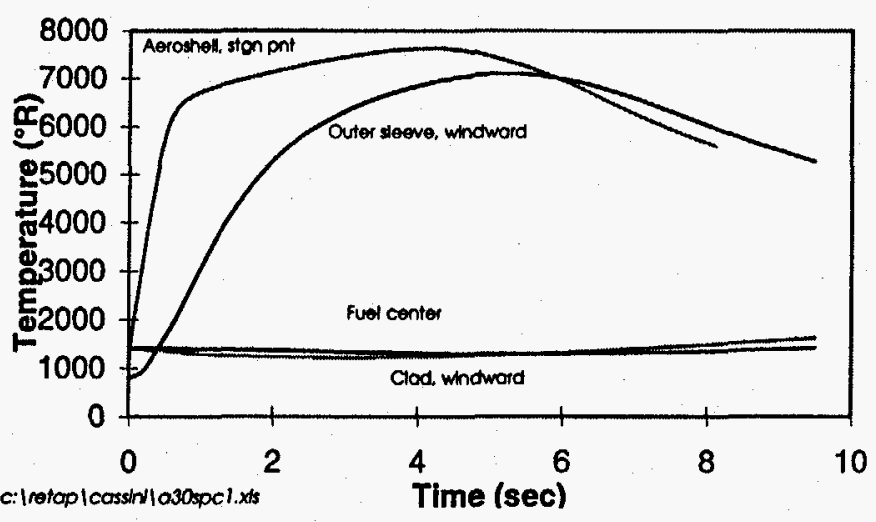

C. Surface Node Recession

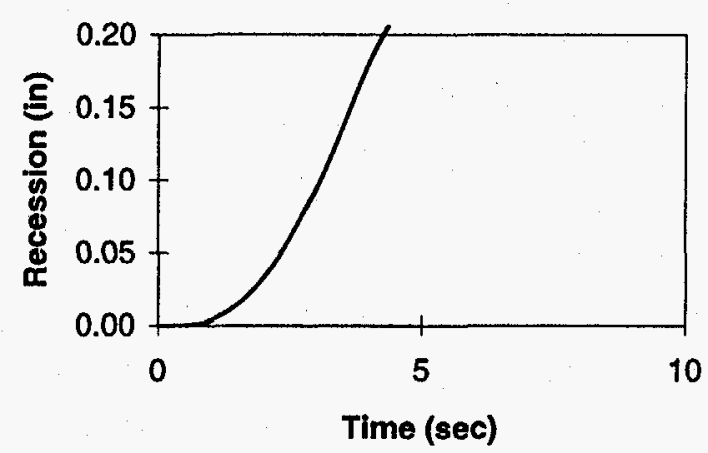

B. Surface Node Mass Transfer Parameter

Response

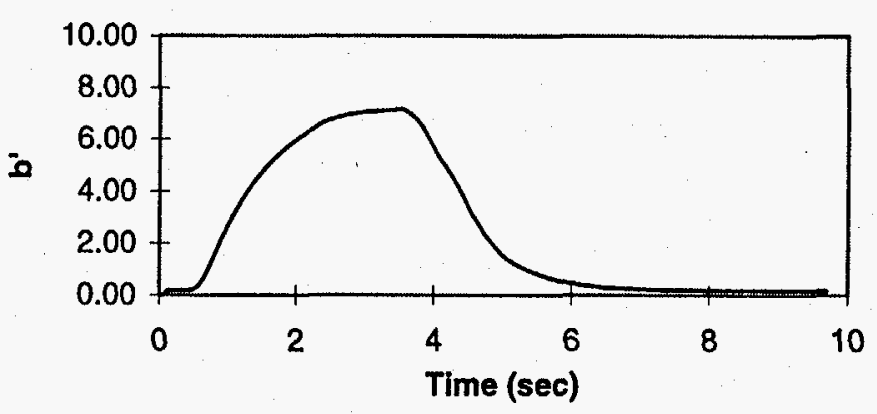

D. Recession Failure for Modeled Surface Nodes

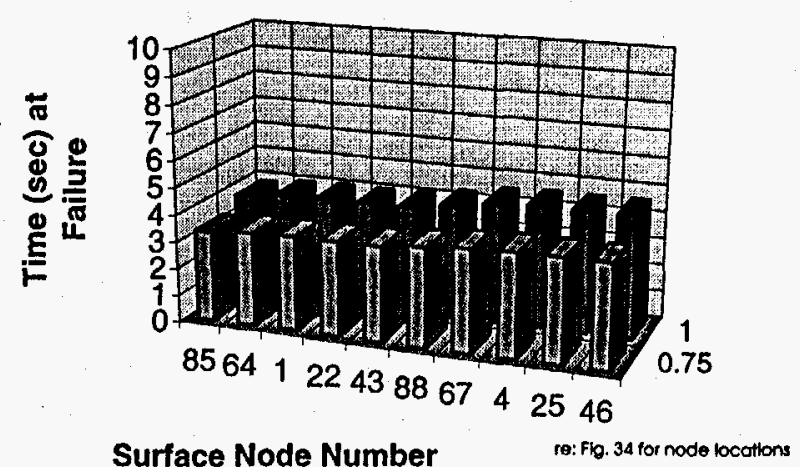



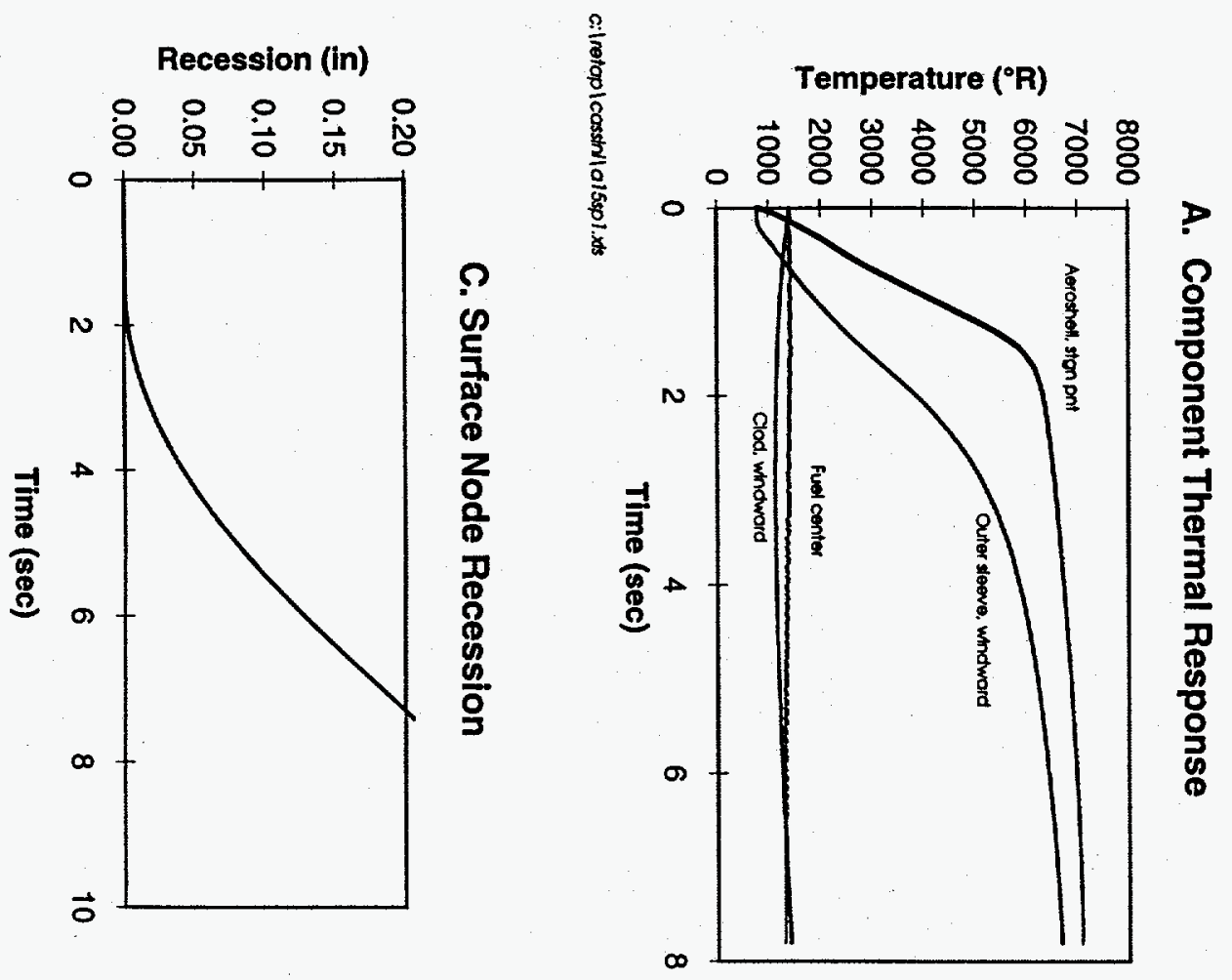

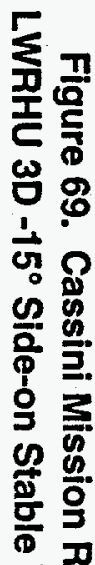
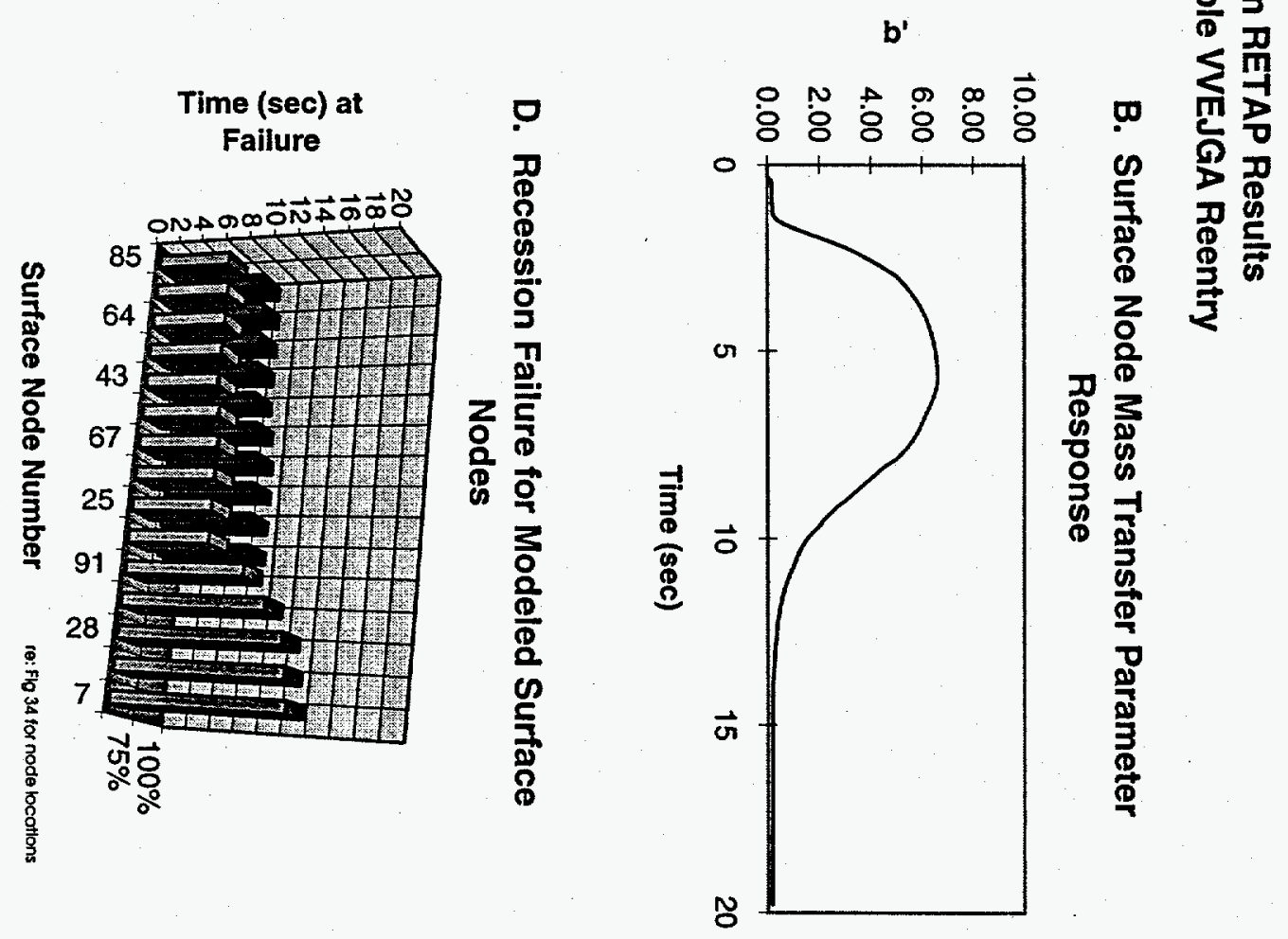
The Johns Hopkins University

Applied Physics Laboratory

Laurel, Maryland 20723-60999
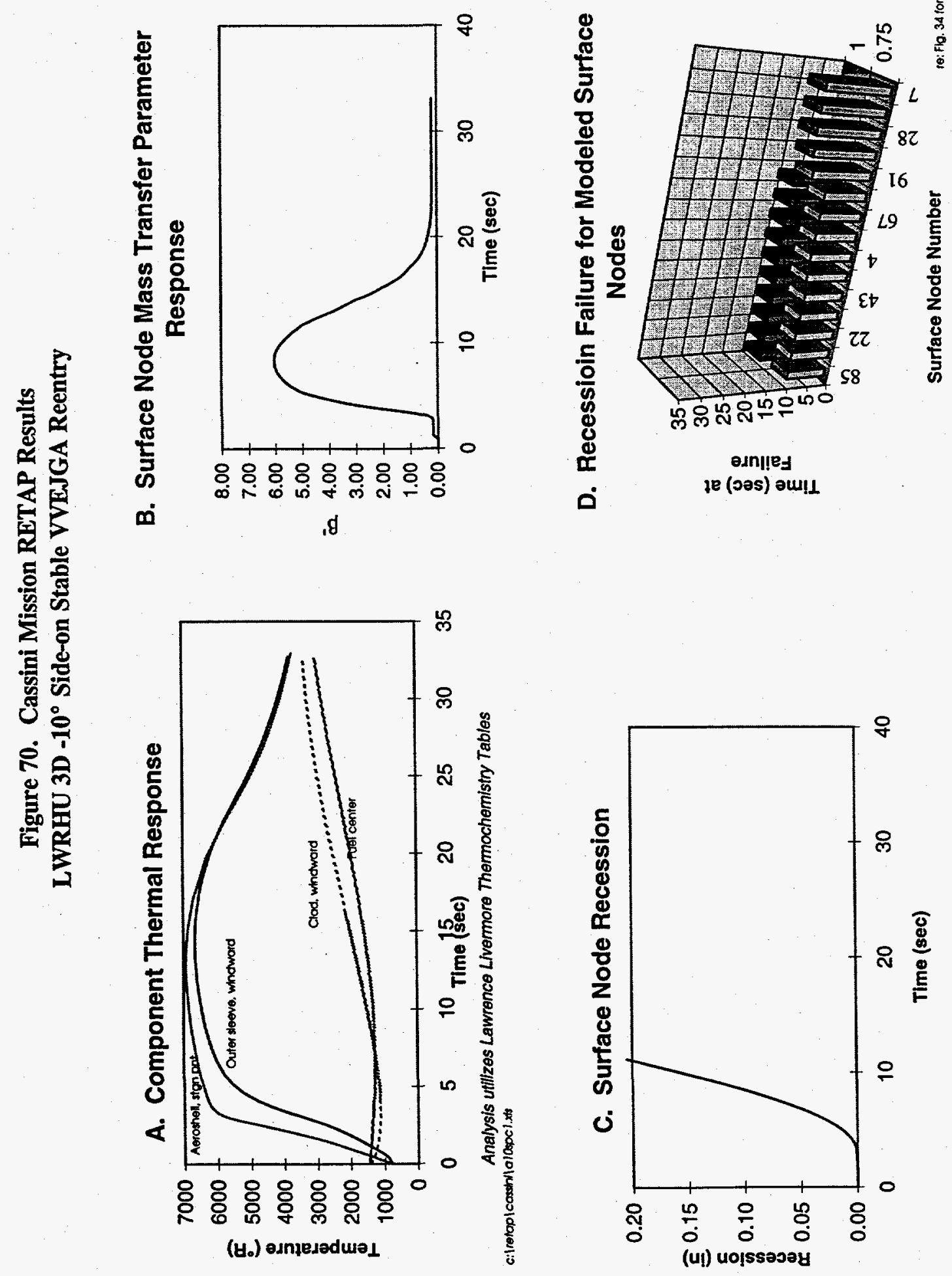

ญ्.

D je (oos) ou!n

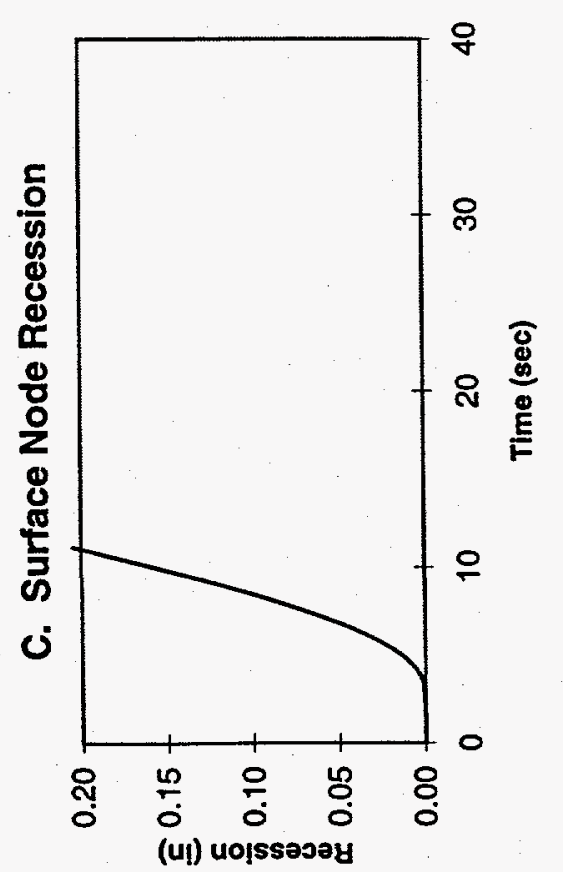


Figure 71. Cassini Mission RETAP Results

LWRHU 3D $-7^{\circ}$ Side-on Stable VVEJGA Reentry

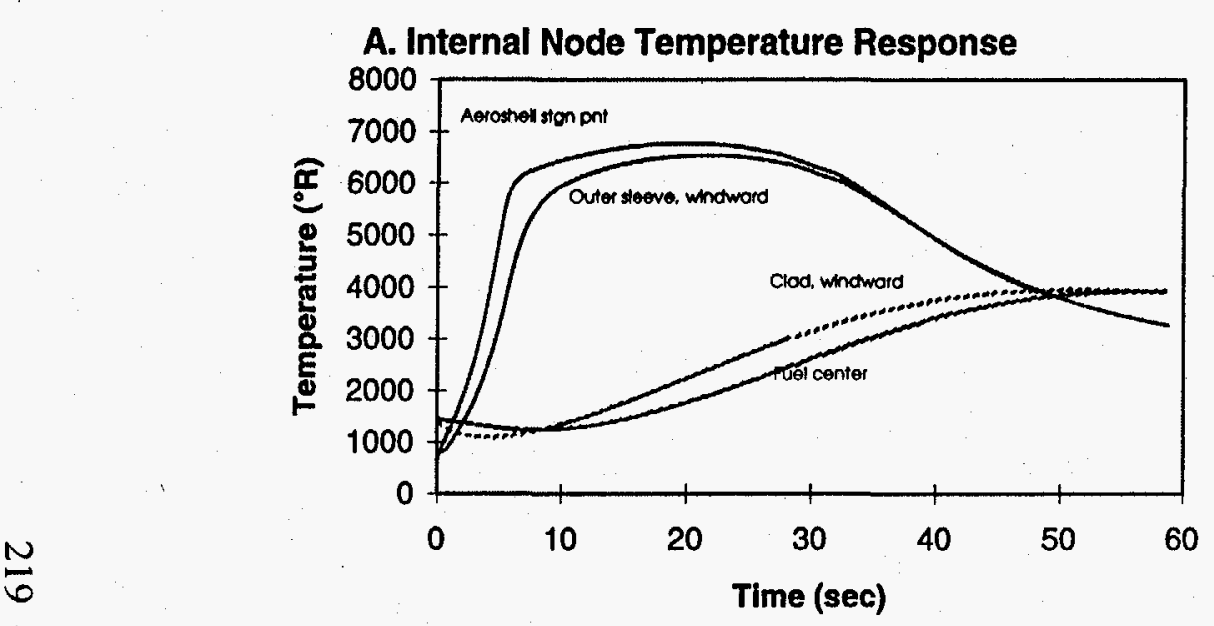

B. Surface NodeMass Transfer Response

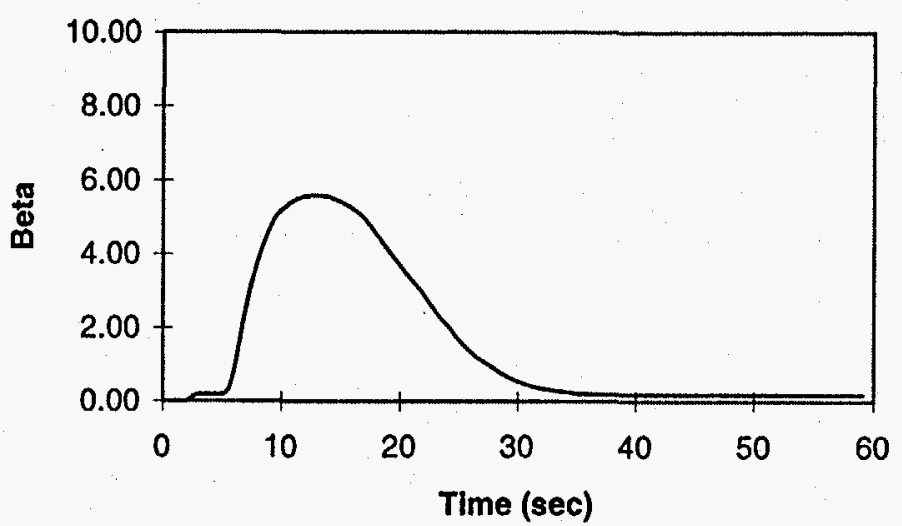

c:Iretaplcosstil|c07soc1.xs

\section{Surface Node Recession}

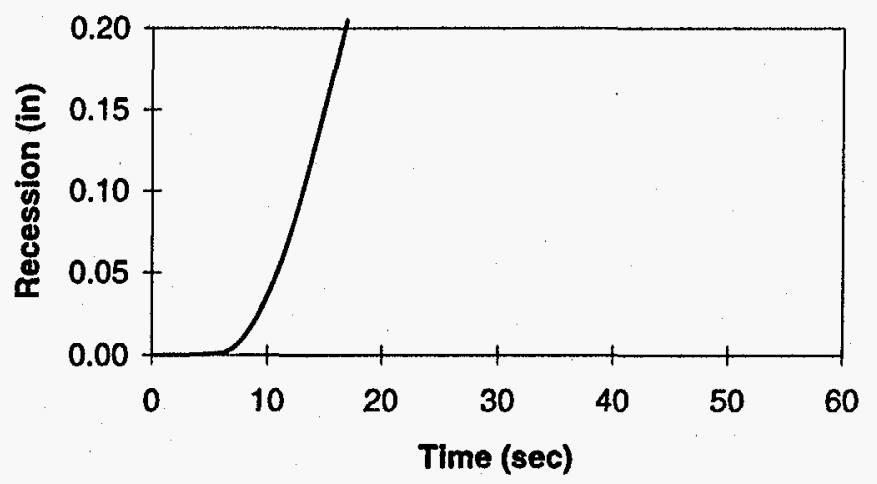

D. Recession Failure for Modeled Surface Nodes

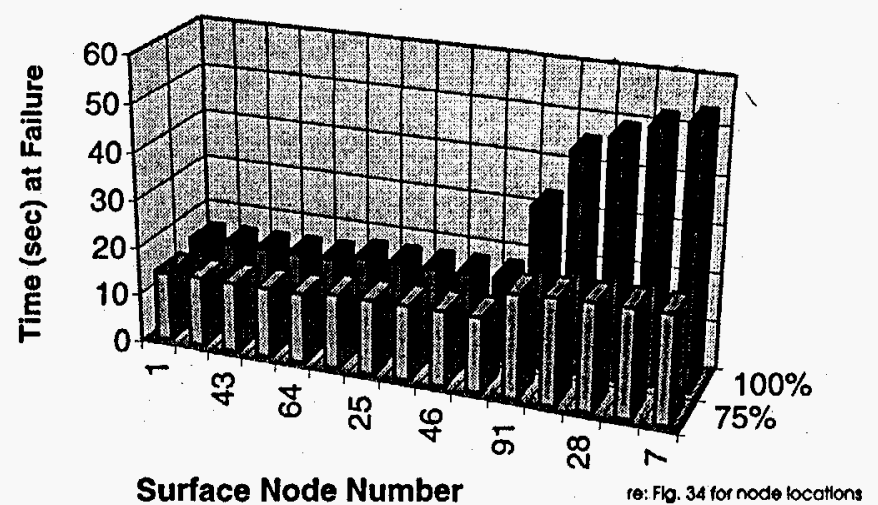


Figure 72. Cassini Mission RETAP Results LWRHU 3D $-5.2^{\circ}$ Side-on Stable VVEJGA Reentry

A. Component Thermal Response

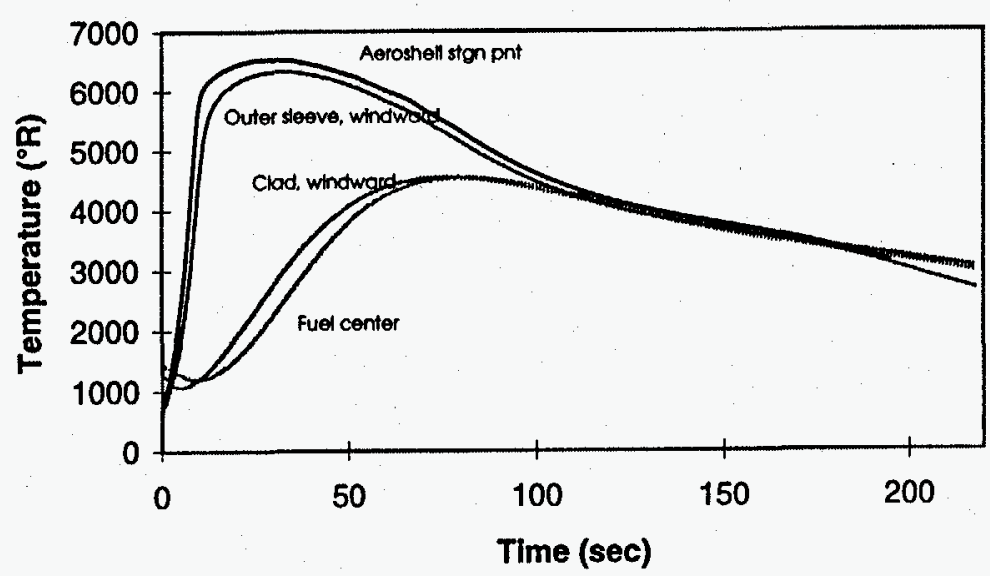

C. Surface Node Recession

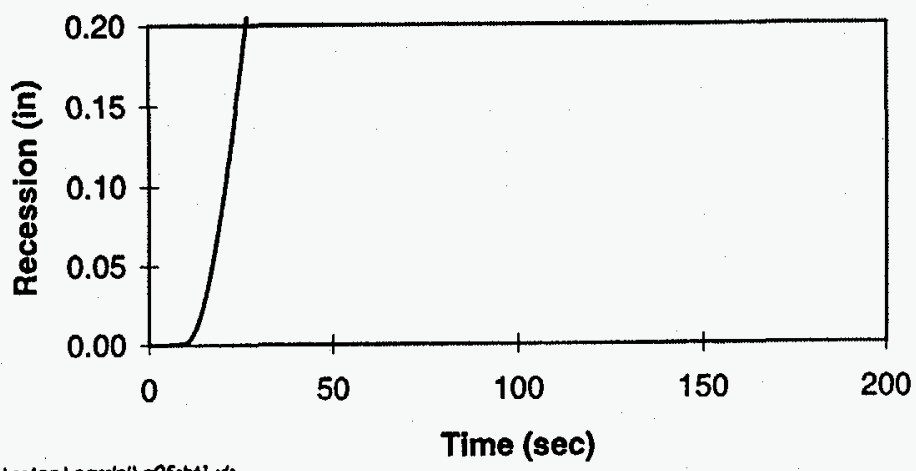

B. Surface Node Mass Transfer Response

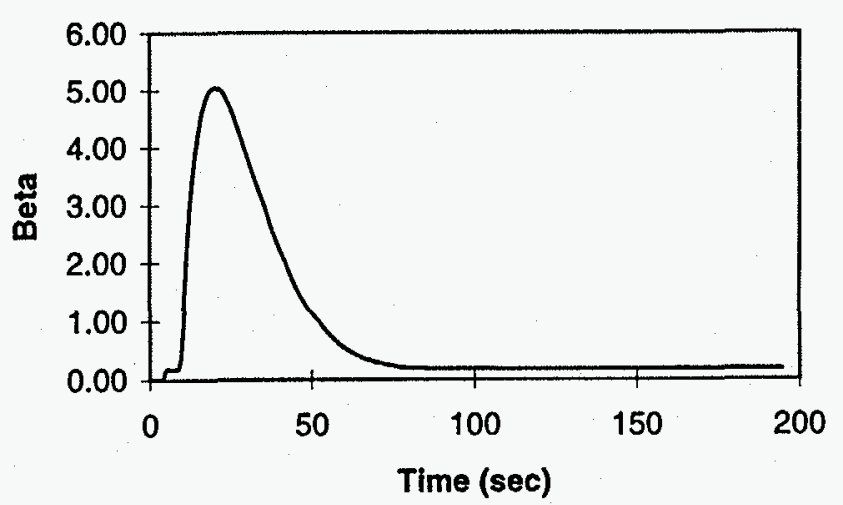

D. Recession Failure at Modeled Surface Nodes

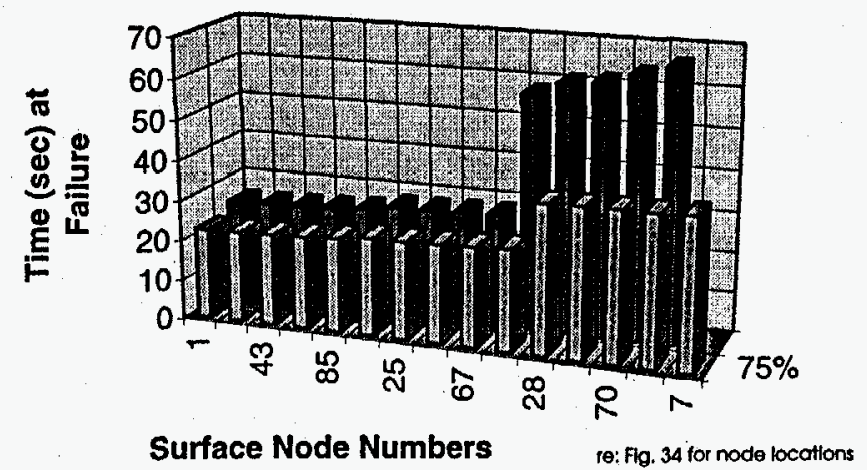


Figure 73. Cassini Mission RETAP Results Side-on Stable LWRHU 3D -90 $0^{\circ}$ VVEJGA Reentry Ablation Failure Relative to Heat Pulse

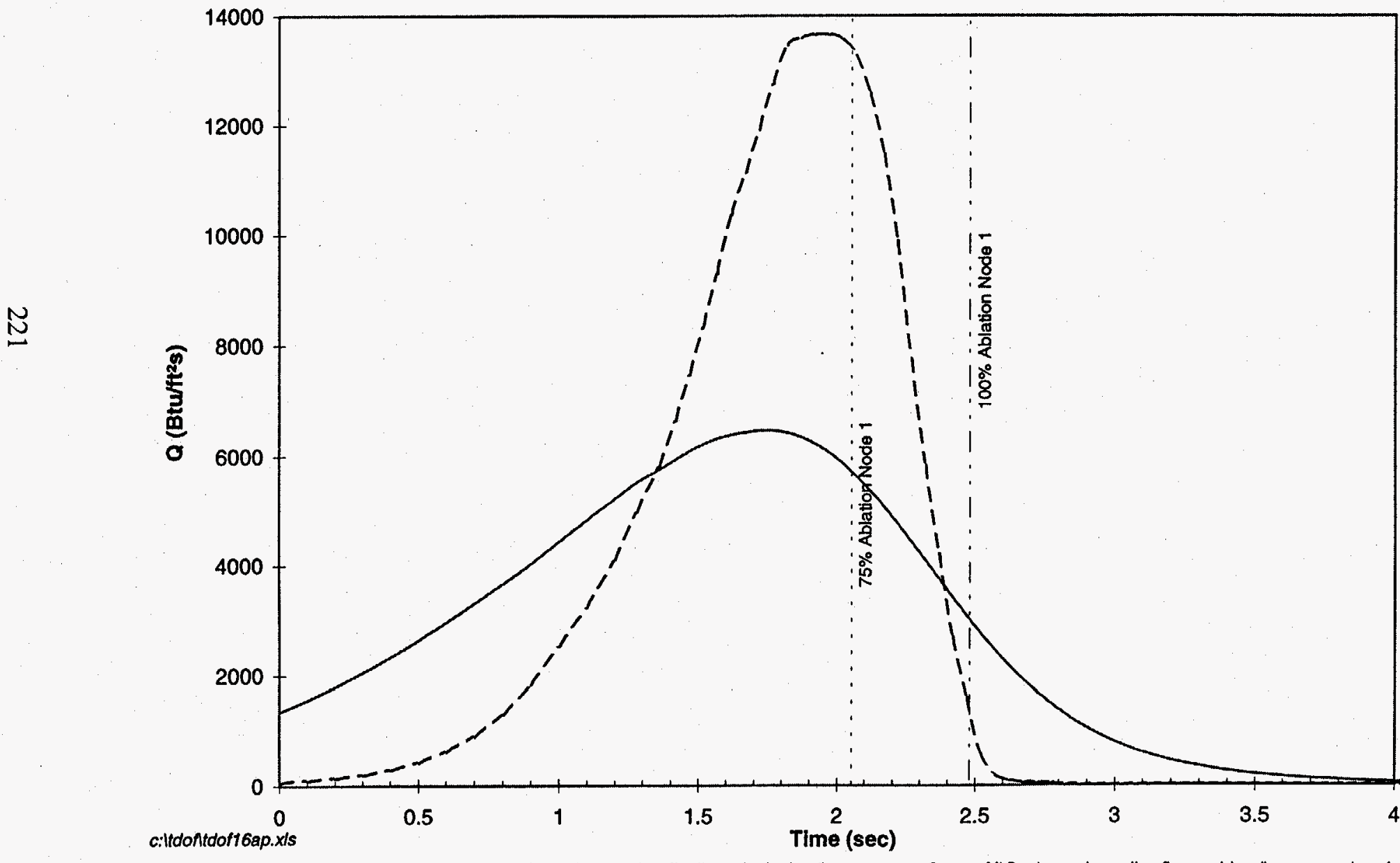


Figure 74. Cassini Mission RETAP Results

Side-on Stable LWRHU 3D -70 ${ }^{\circ}$ VEJGA Reentry

Ablation Failure Relative to Heat Pulse

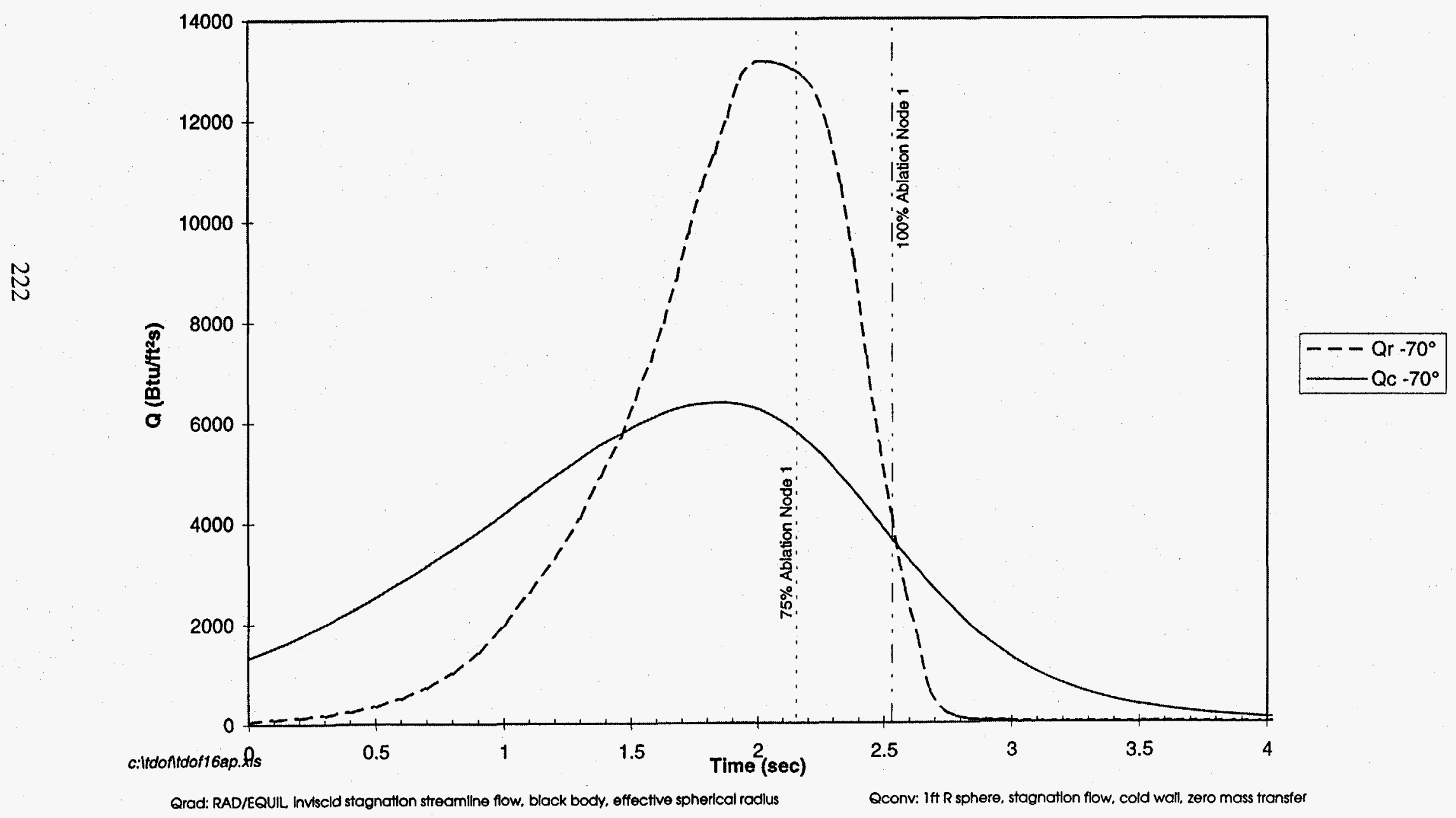


Figure 75. Cassini Mission RETAP Results Side-on Stable LWRHU 3D -50 VVEJGA Reentry Ablation Failure Relative to Heat Pulse

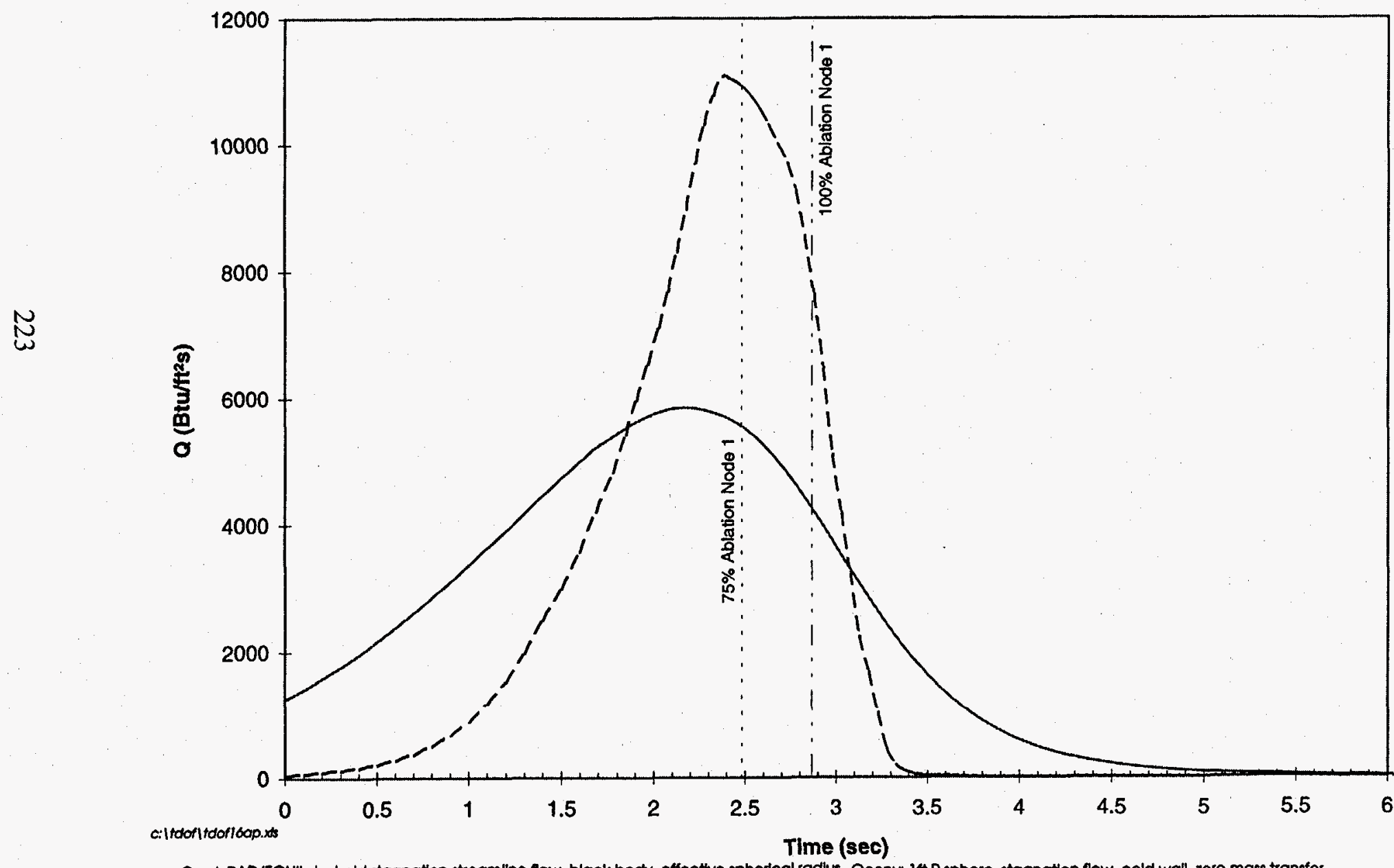


Figure 76. Cassini Mission RETAP Results

Side-on Stable LWRHU 3D -30 VVEJGA Reentry

Ablation Failure Relative to Heat Pulse

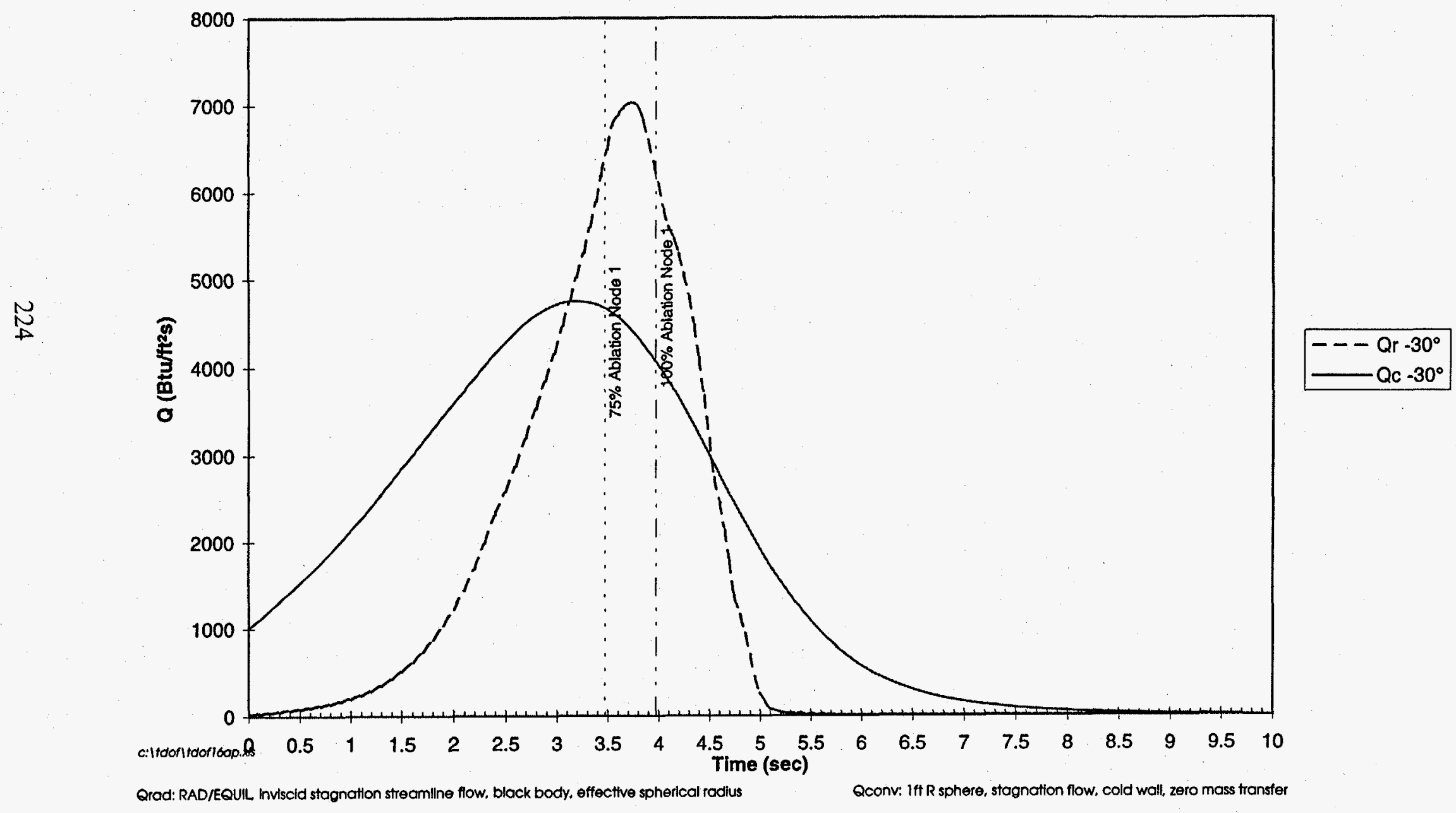


Figure 77. Cassini Mission RETAP Results

Side-on Stable LWRHU 3D -15 ${ }^{\circ}$ VVEJGA Reentry

Ablation Failure Relative to Heat Pulse

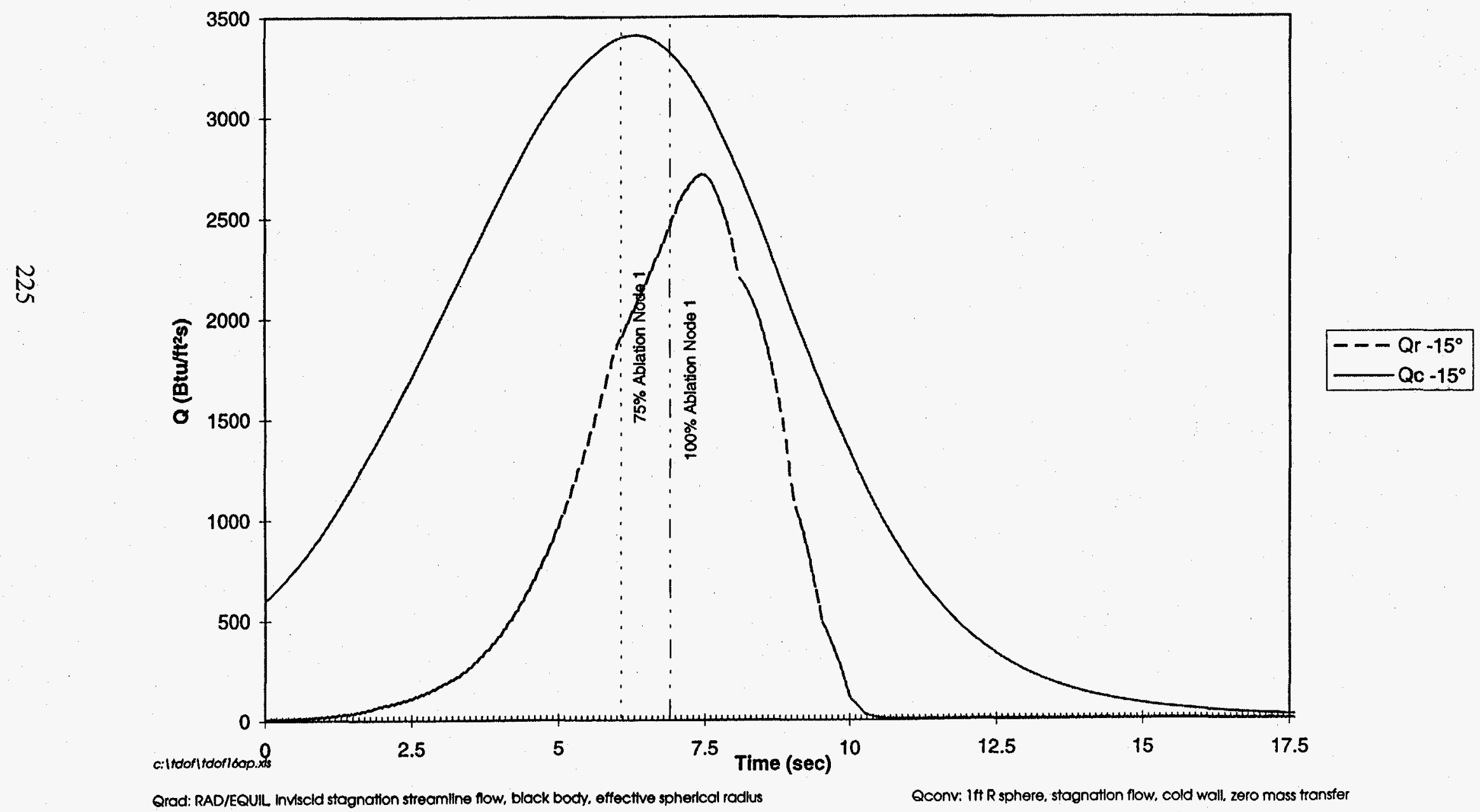


Figure 78. Cassini Mission RETAP Results Side-on Stable LWRHU 3D - $10^{\circ}$ VVEJGA Reentry Ablation Failure Relative to Heat Pulse

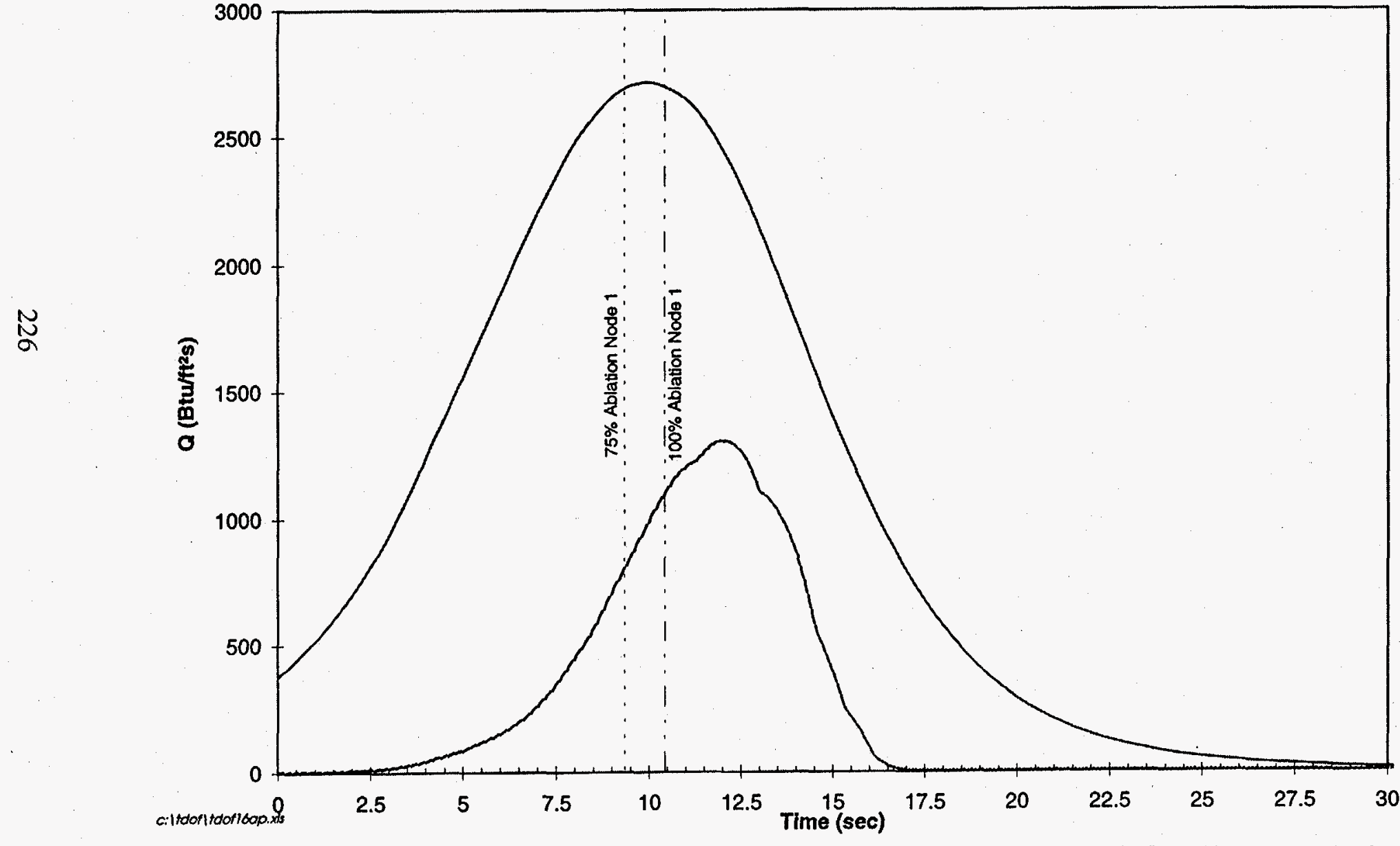


Figure 79. Cassini Mission RETAP Results Side-on Stable LWRHU 3D $-7^{\circ}$ VVEJGA Reentry Ablation Failure Relative to Heat Pulse

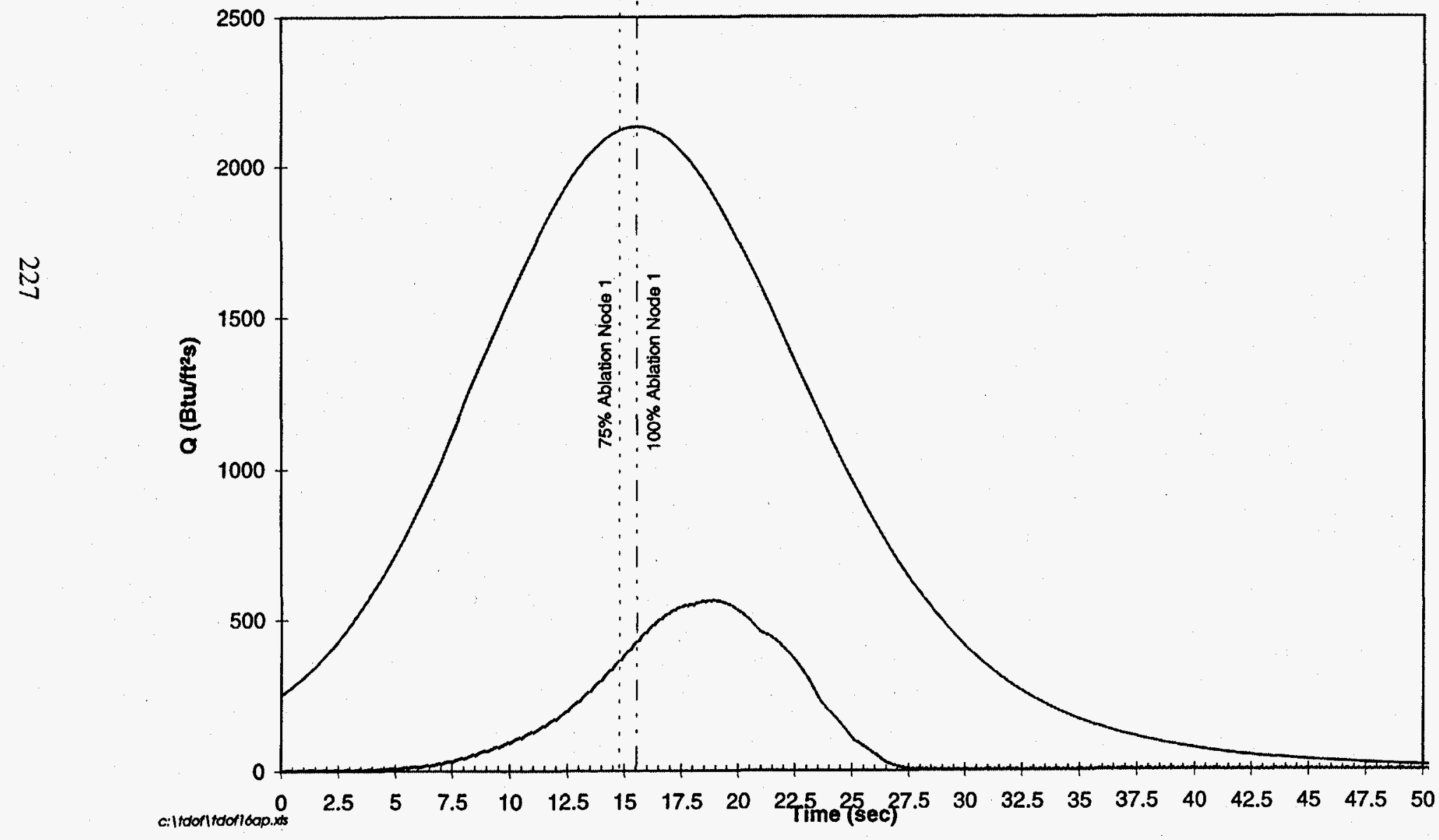


Figure 80. Cassini Mission RETAP Results

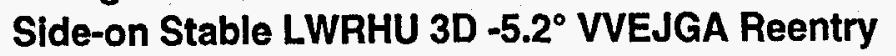

Ablation Failure Relative to Heat Pulse

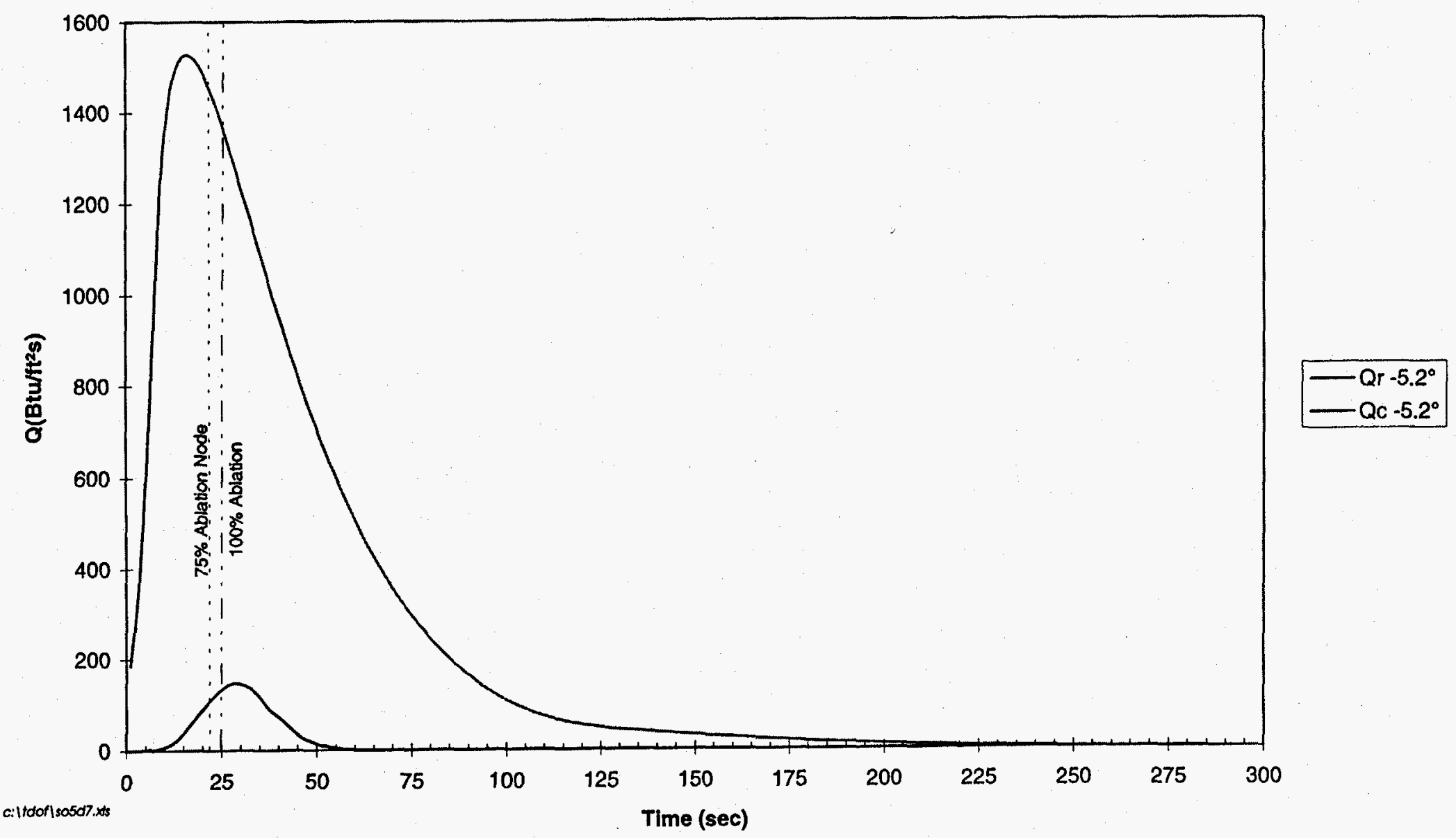


Figure 81. Cassini Mission RETAP Results Side-on Spinning LWRHU 3D $-05.2^{\circ}$ VVEJGA Reentry Recession Failure at Modeled Surface Nodes

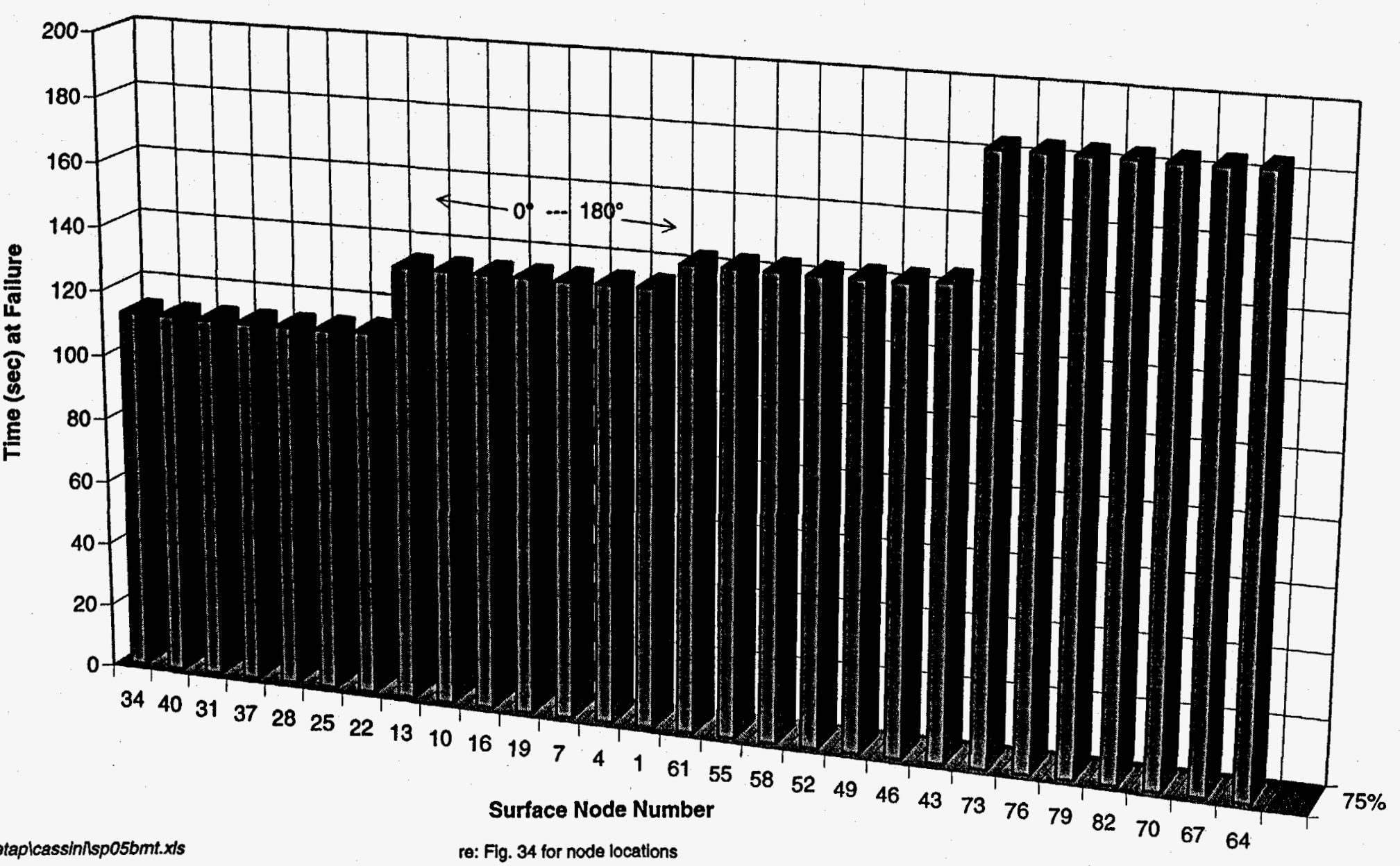


Applied Physics Laboratory

Laurel, Maryland 20723-6099

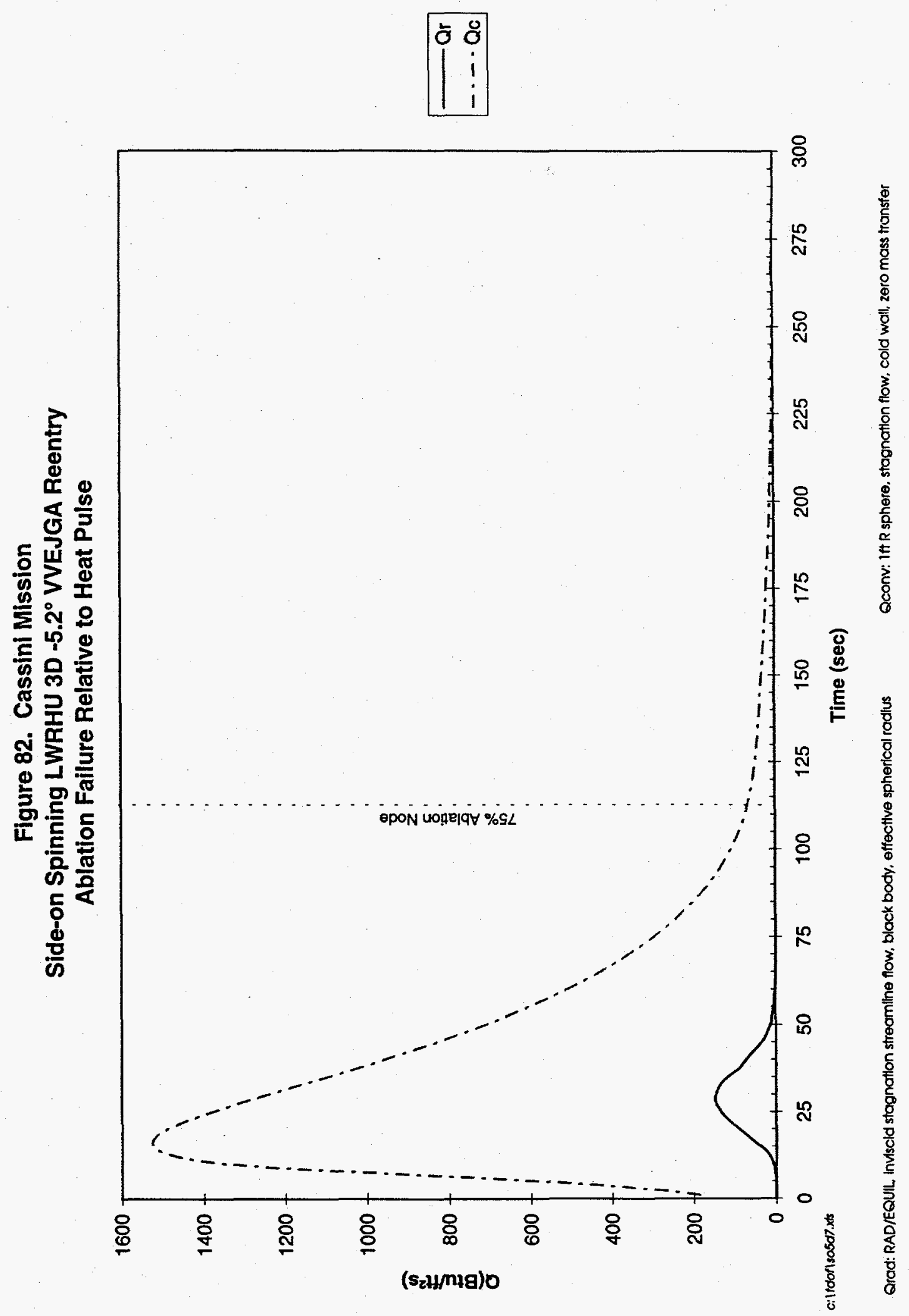


Figure 83. Cassini Mission RETAP Results LWRHU 3D -07 Side-on Spinning VVEJGA Reentry Component Thermal Response

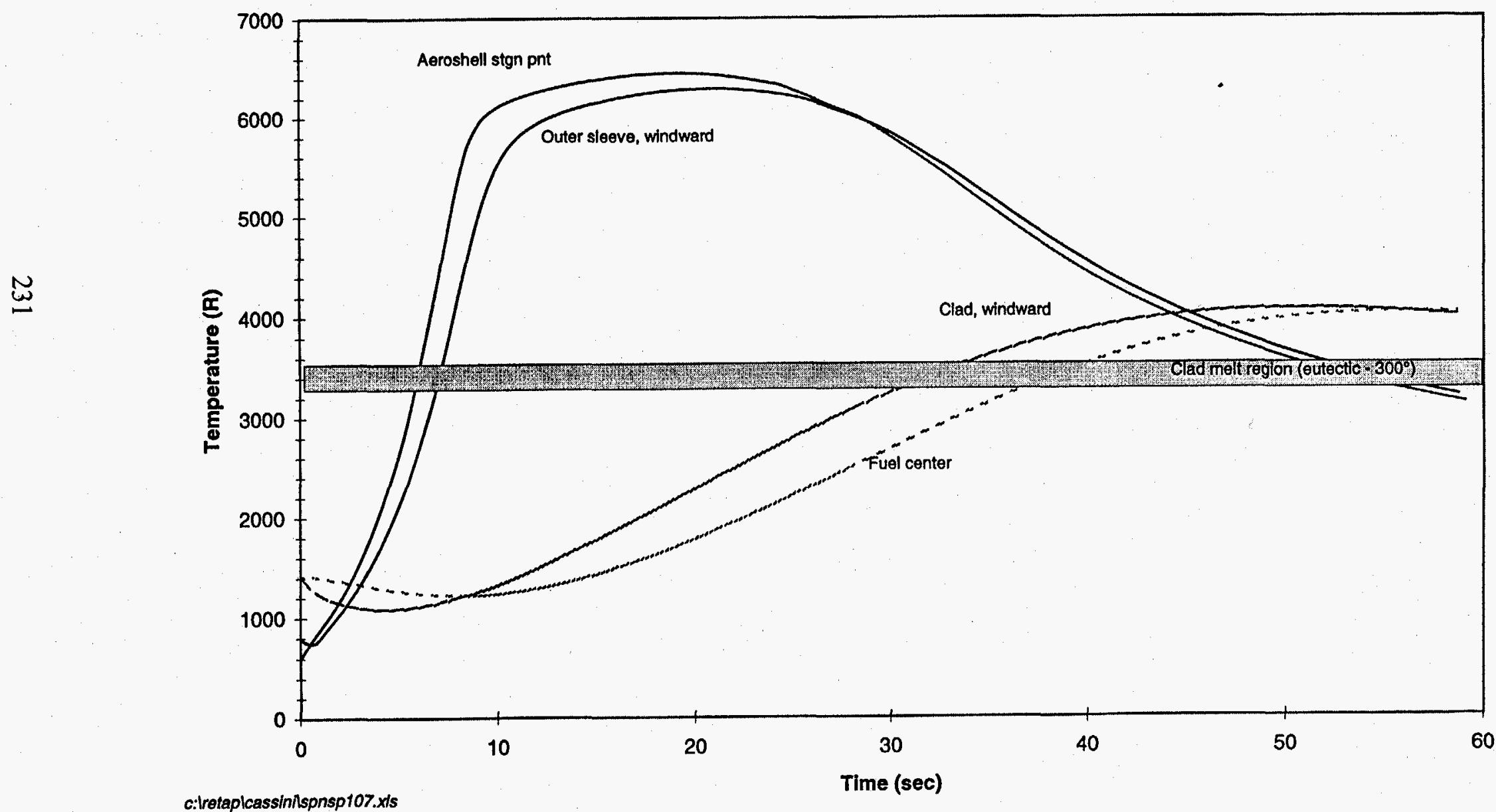


Figure 84. Cassini Mission RETAP Results Recession Failure at Modeled Surface Nodes

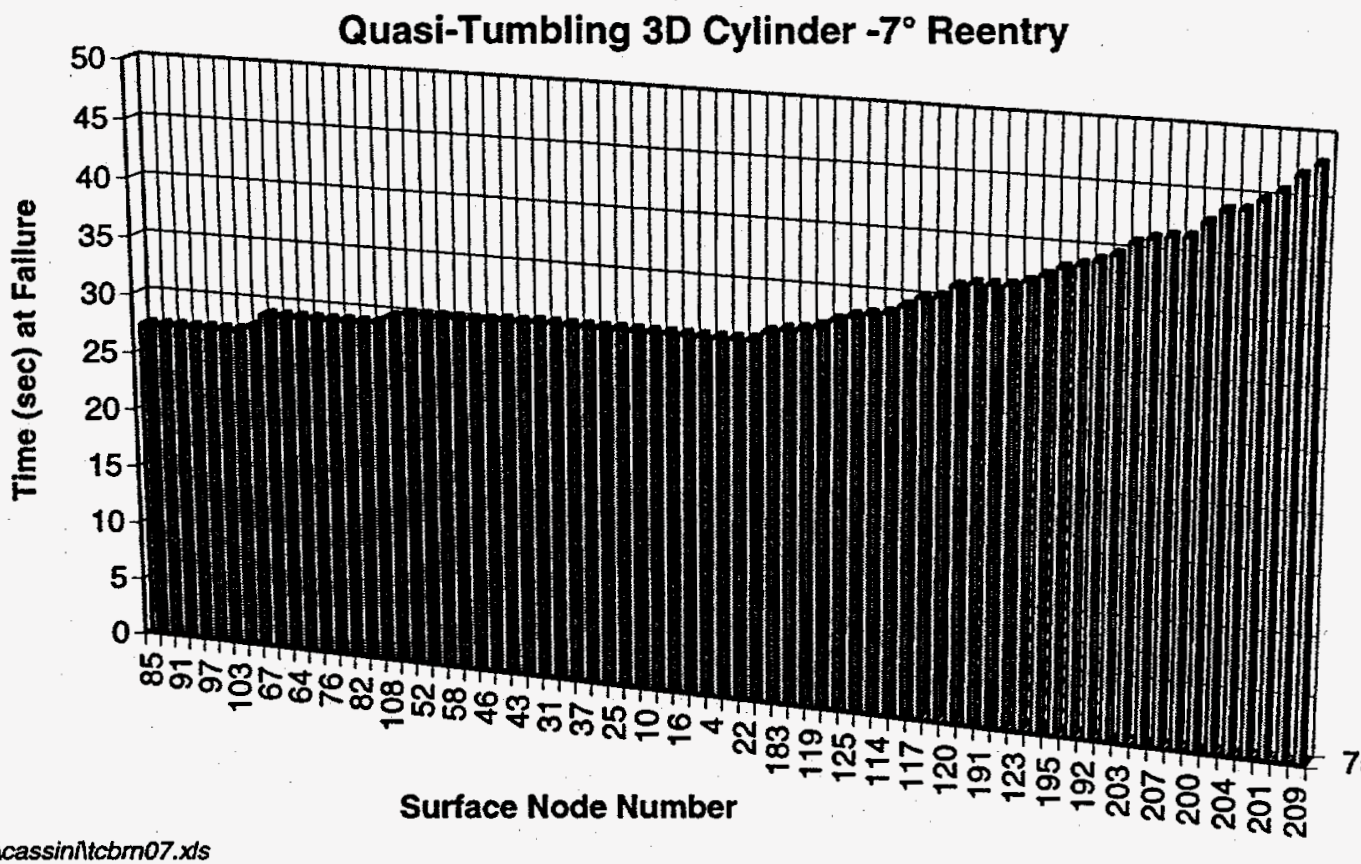

\section{Quasi-Tumbling Cylinder LWRHU 3D $-5.2^{\circ}$ Reentry}

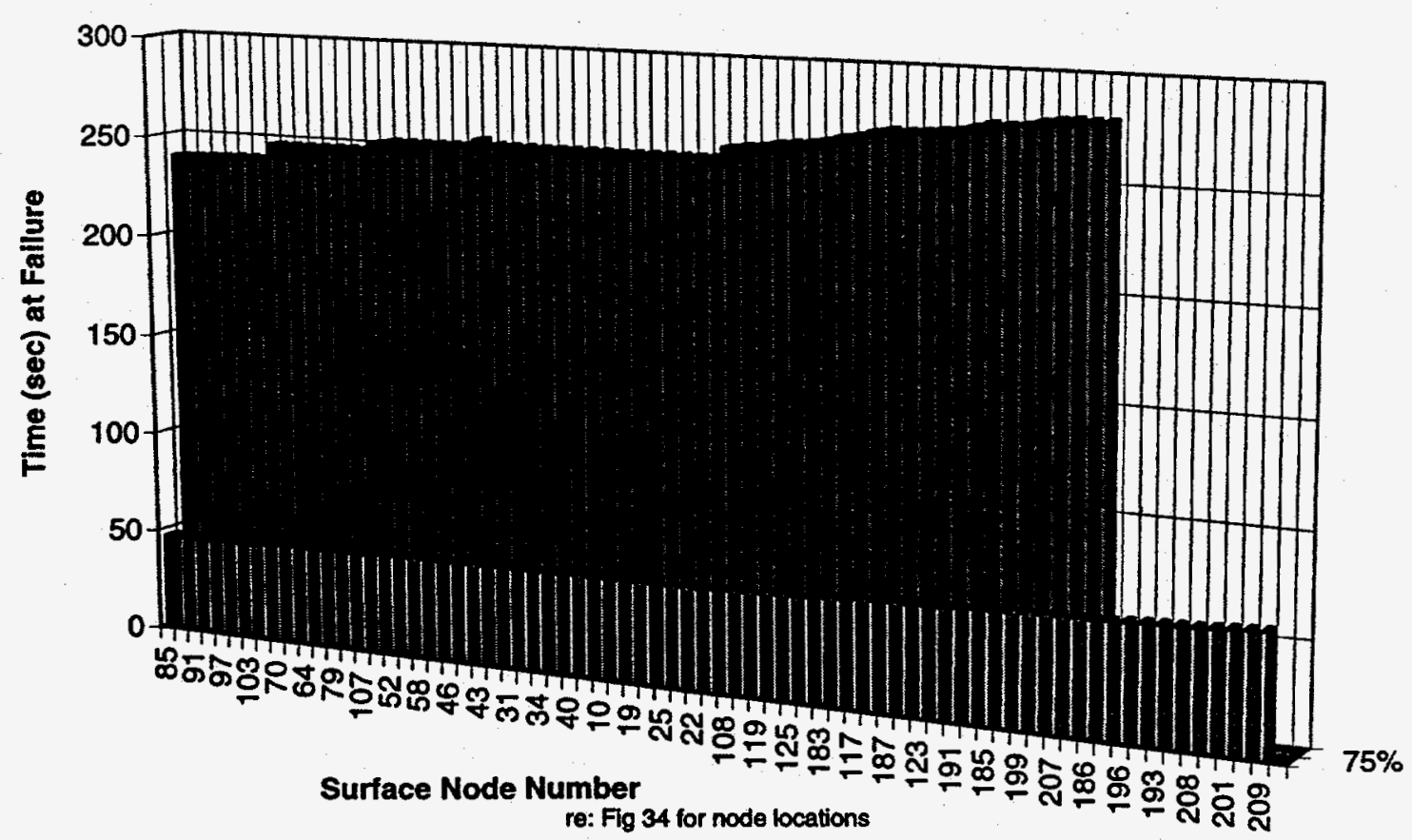


Figure 85. Cassini Mission RETAP Results Ablation Failure Heat Pulse Relative to Heat Pulse

Quasi-Tumbling Cylinder $-07^{\circ}$

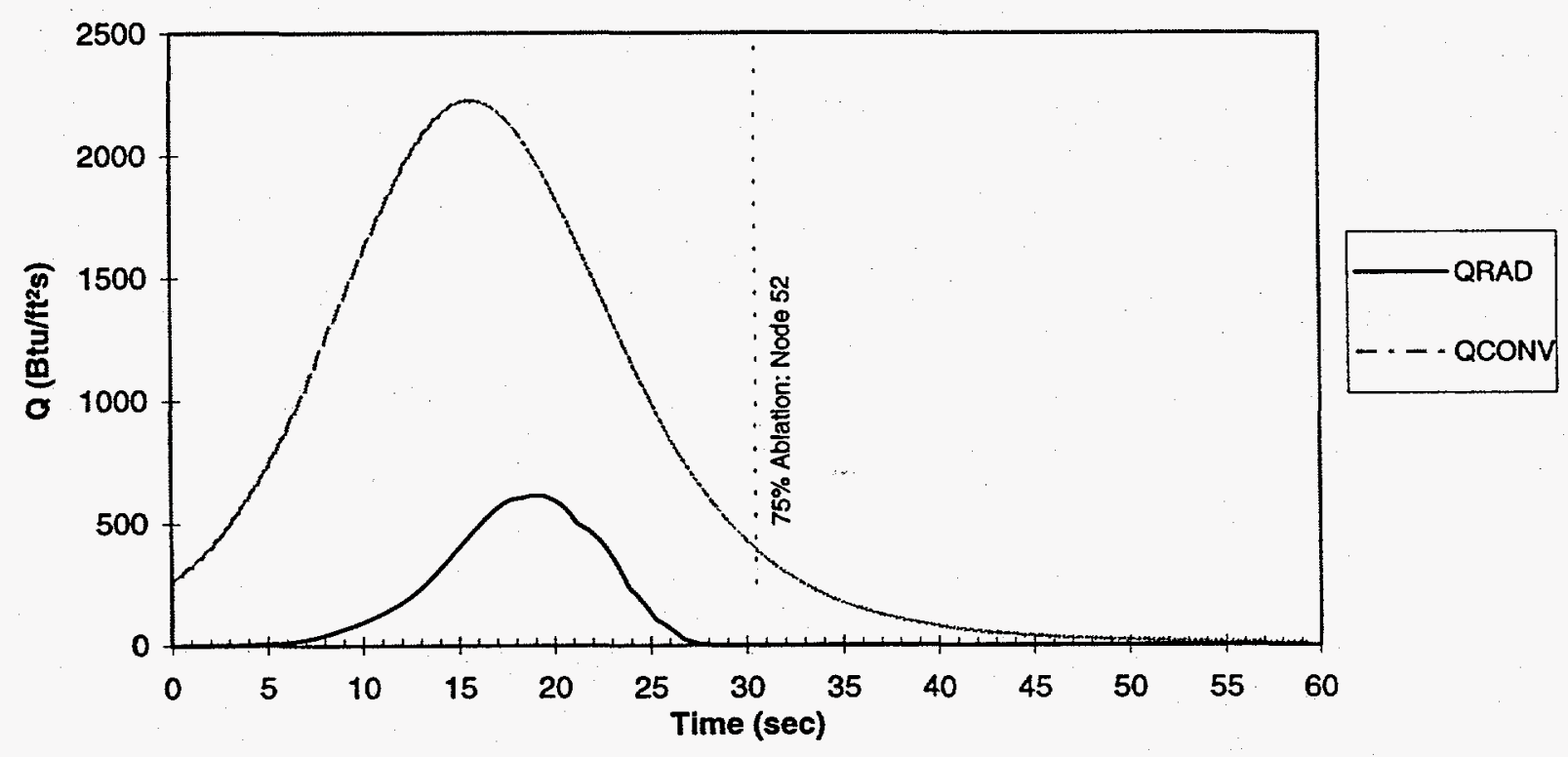

Quasi-Tumbling Cylinder $-05.2^{\circ}$ Reentry

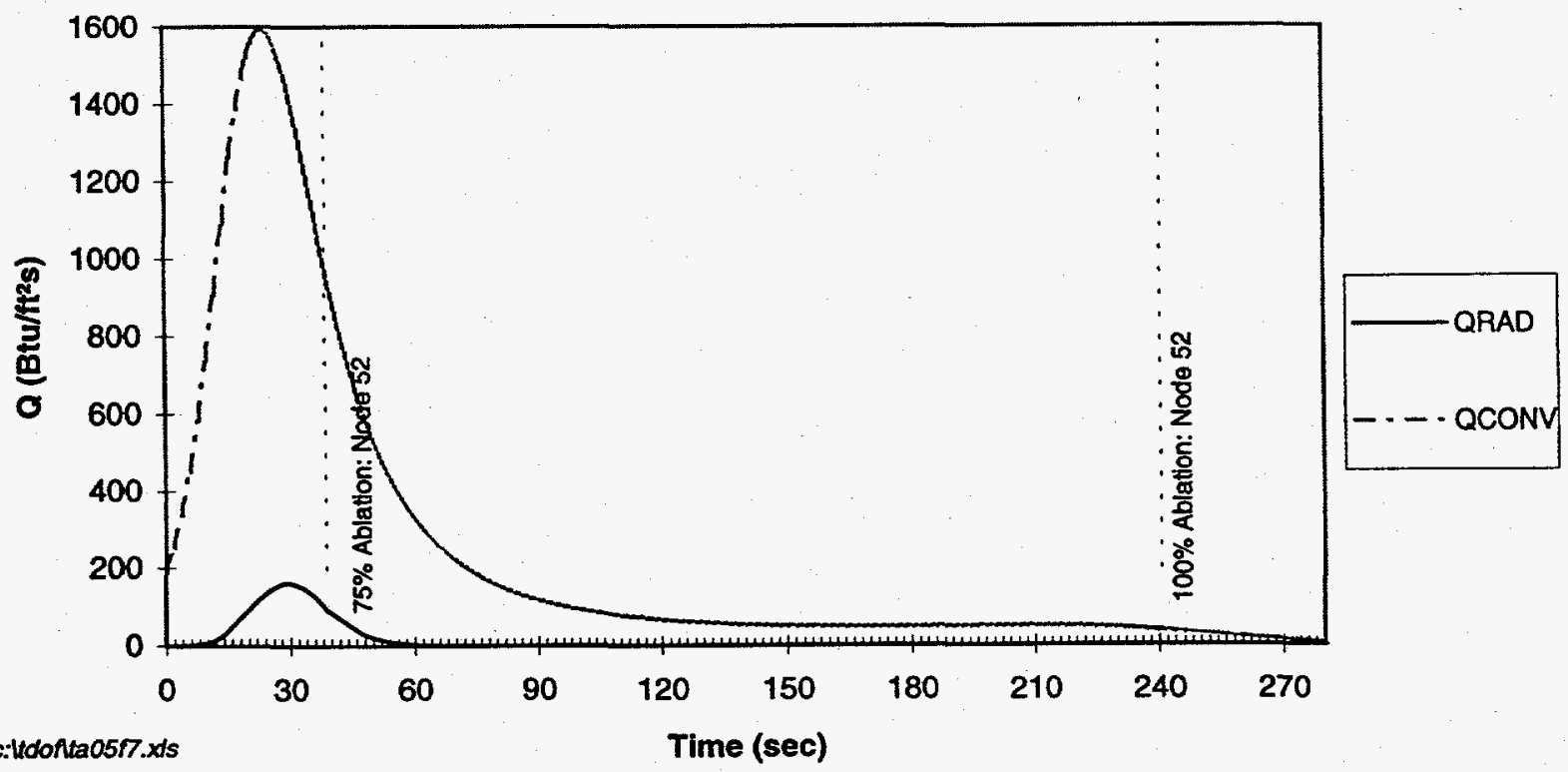

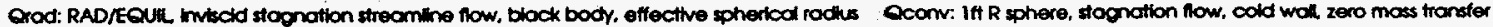


Figure 86. Cassini Mission RETAP Results Random Tumbling 3D LWRHU VVEJGA Reentry Recession Failure at Modeled Surface Nodes $-5.2^{\circ}$ Reentry
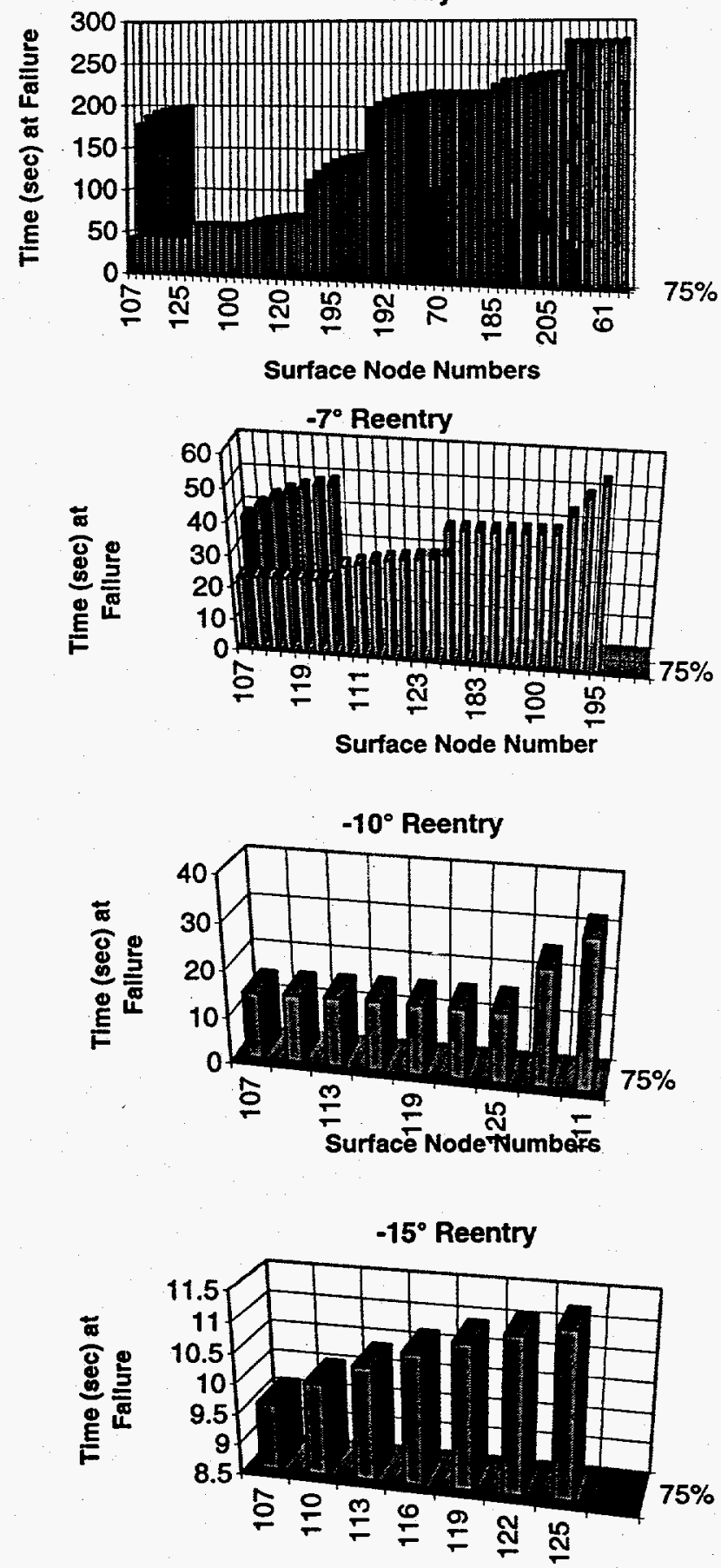

Surface Node Numbers

re: Fig 34 for node locations 
Figure 87. Cassini Mission RETAP Results LWRHU 3D Ablation Failure Relative to Heat Pulse

$-05.2^{\circ}$ Random Tumbling Reentry

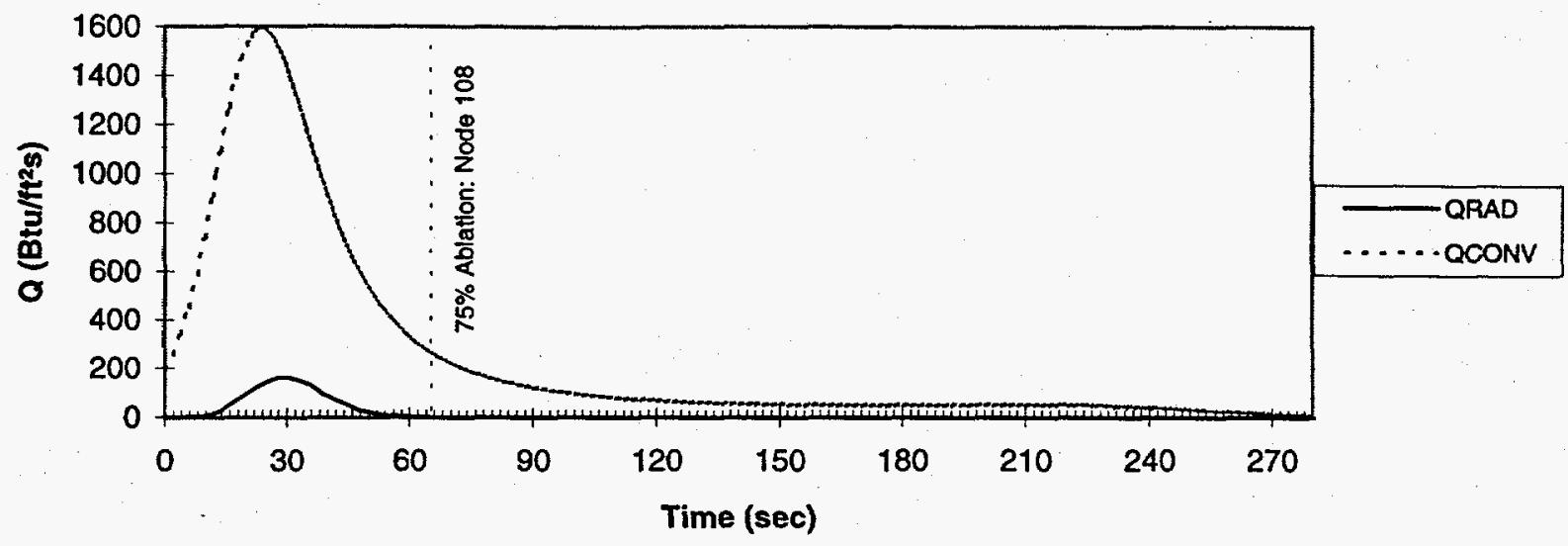

$-07^{\circ}$ Random Tumbling Reentry

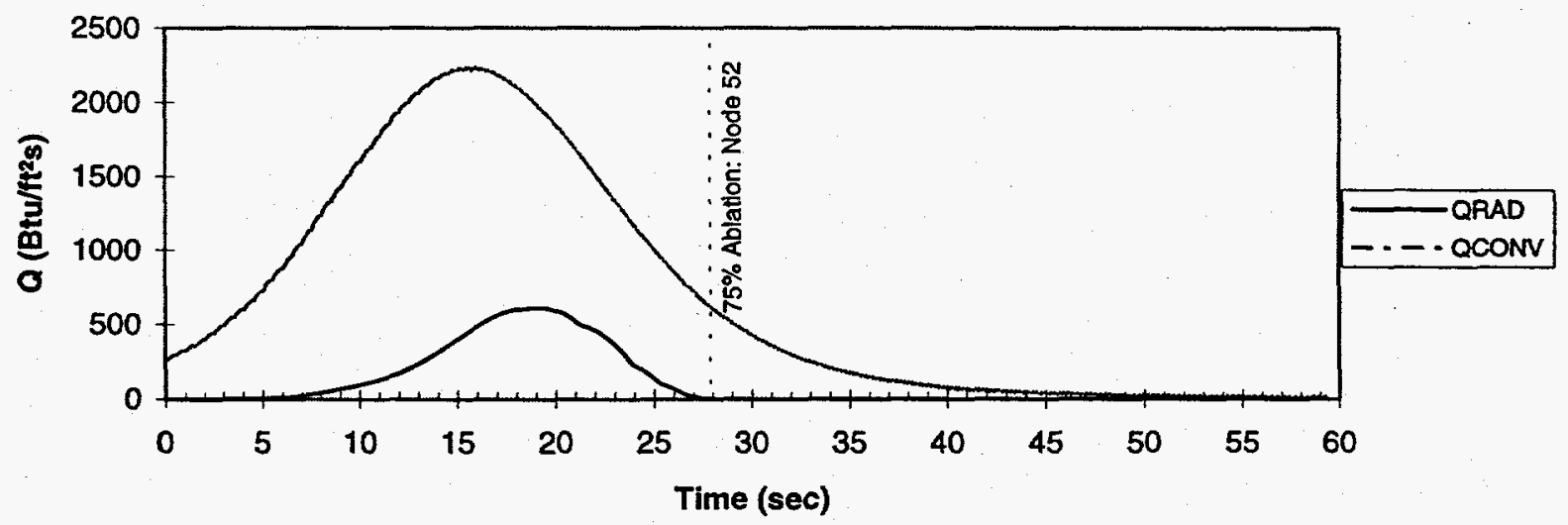

$-10^{\circ}$ Random Tumbling Reentry

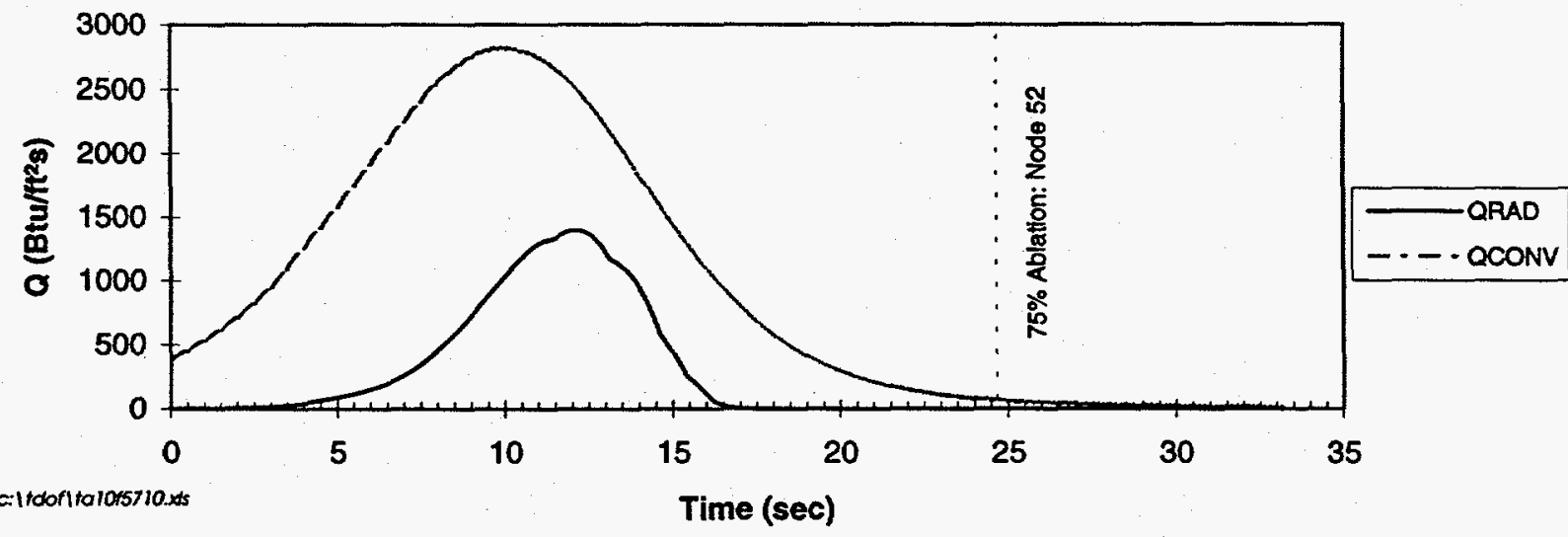

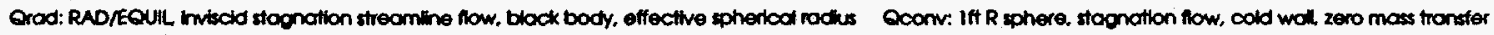


Figure 88. Cassini Mission RETAP Results

Tumbling Sphere VVEJGA Reentry

Recession Failure at Modeled Surface Nodes $-90^{\circ}$ Reentry

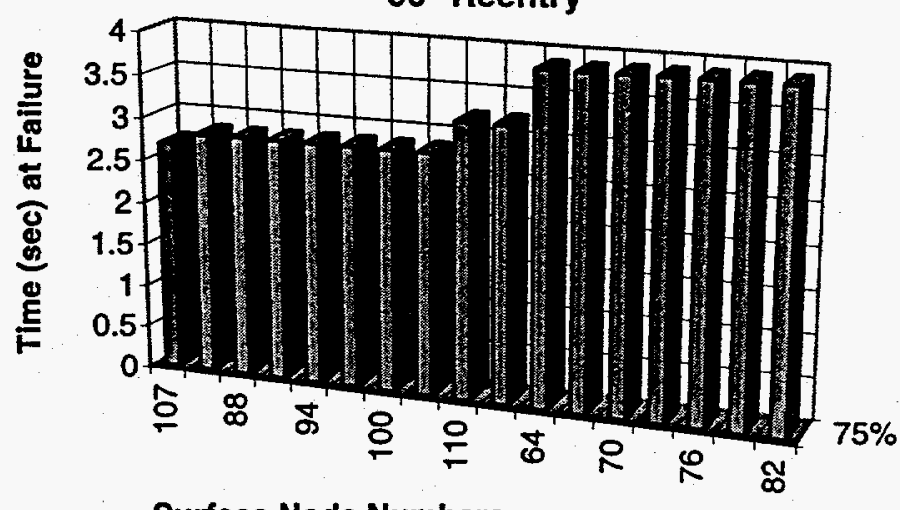

Surface Node Numbers

$-70^{\circ}$ Reentry

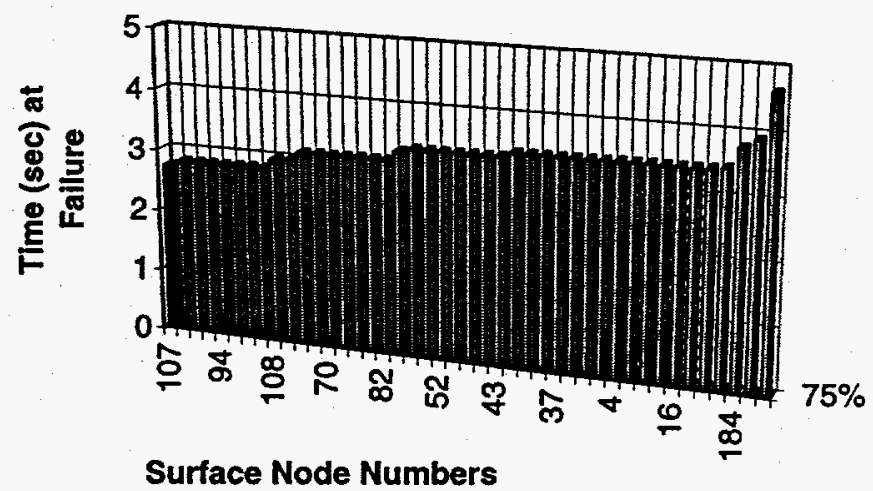

$-50^{\circ}$ Reentry

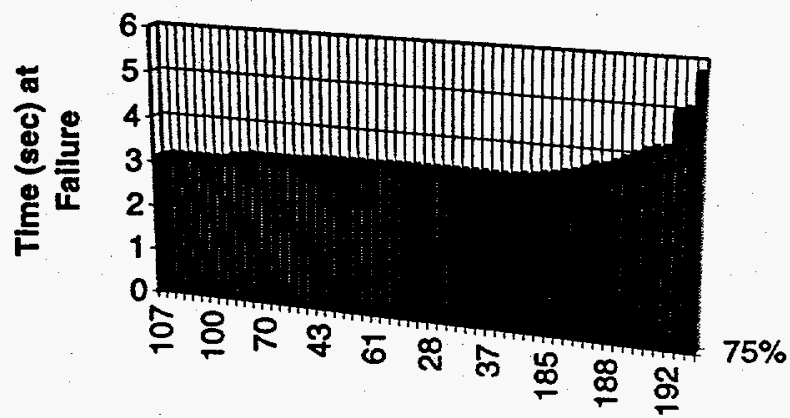

Surface Node Numbers 
Figure 88. (Continued) Cassini Mission RETAP Results Tumbling Sphere VVEJGA Reentry Recession Failure at Modeled Surface Nodes $-30^{\circ}$ Reentry

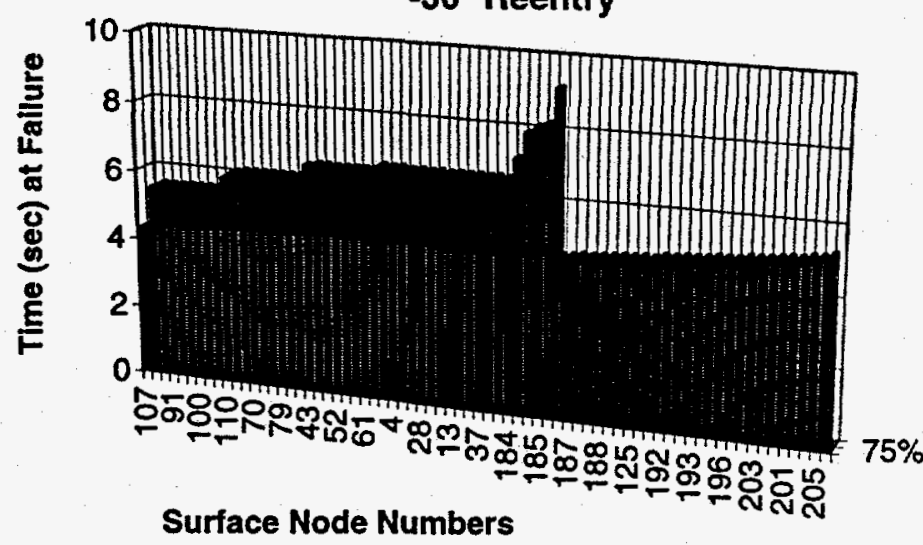

$-15^{\circ}$ Reentry

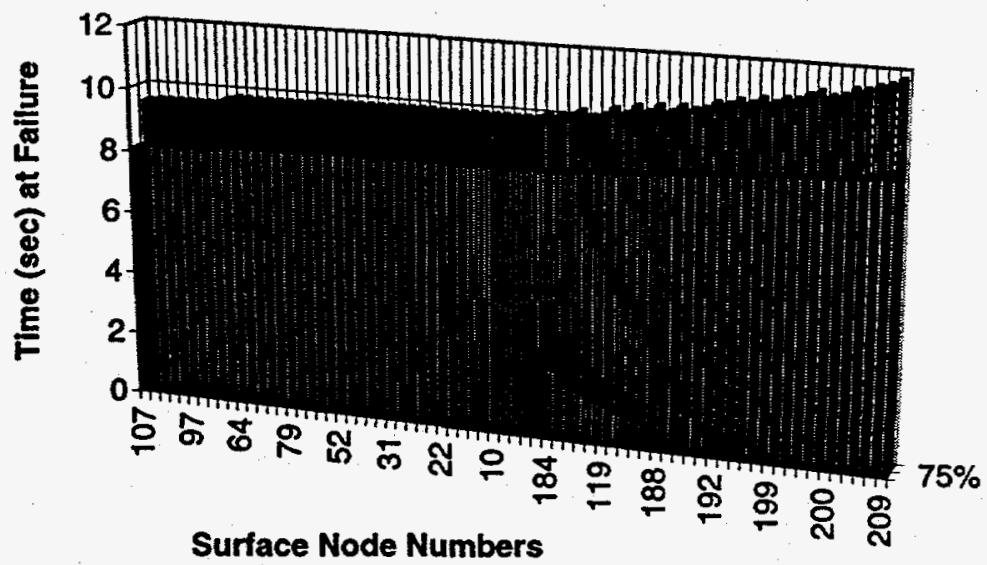

$-10^{\circ}$ Reentry

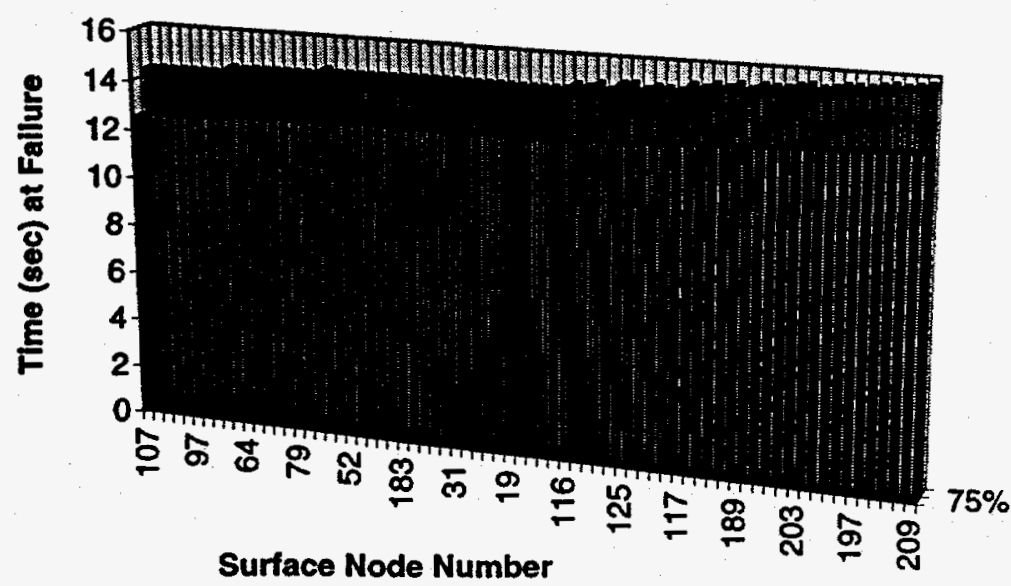


Figure 88.(Continued) Cassini Mission RETAP Results Tumbling Sphere VVEJGA Reentry

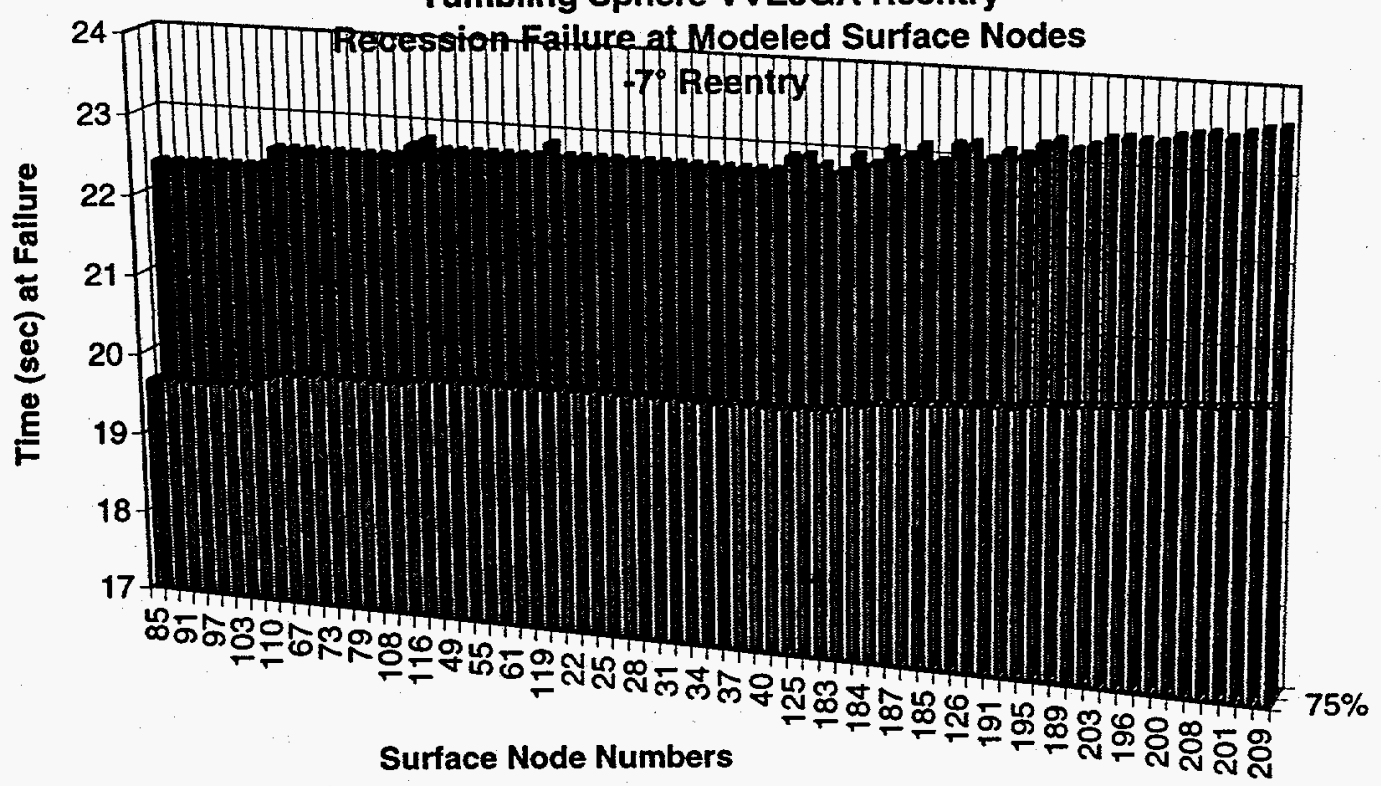

$-5.2^{\circ}$ Reentry

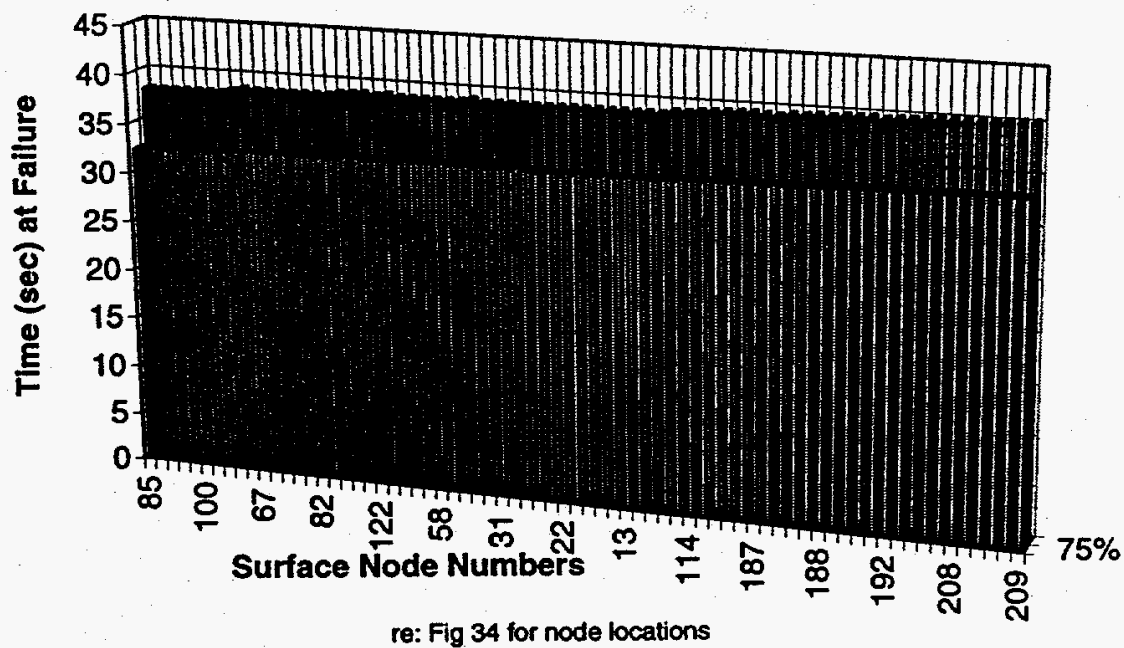

re: Fig 34 for node locations 
Figure 89. Cassini Mission RETAP Results

Ablation Failure Relative to Heat Pulse

$-90^{\circ}$ Tumbling Sphere Reentry

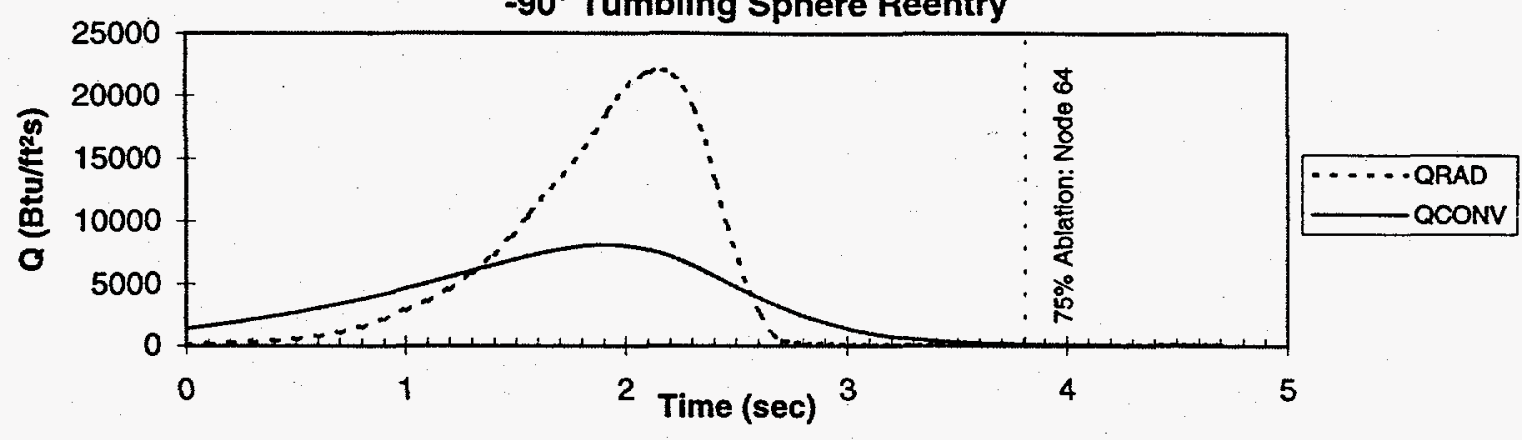

$-70^{\circ}$ Tumbling Sphere Reentry
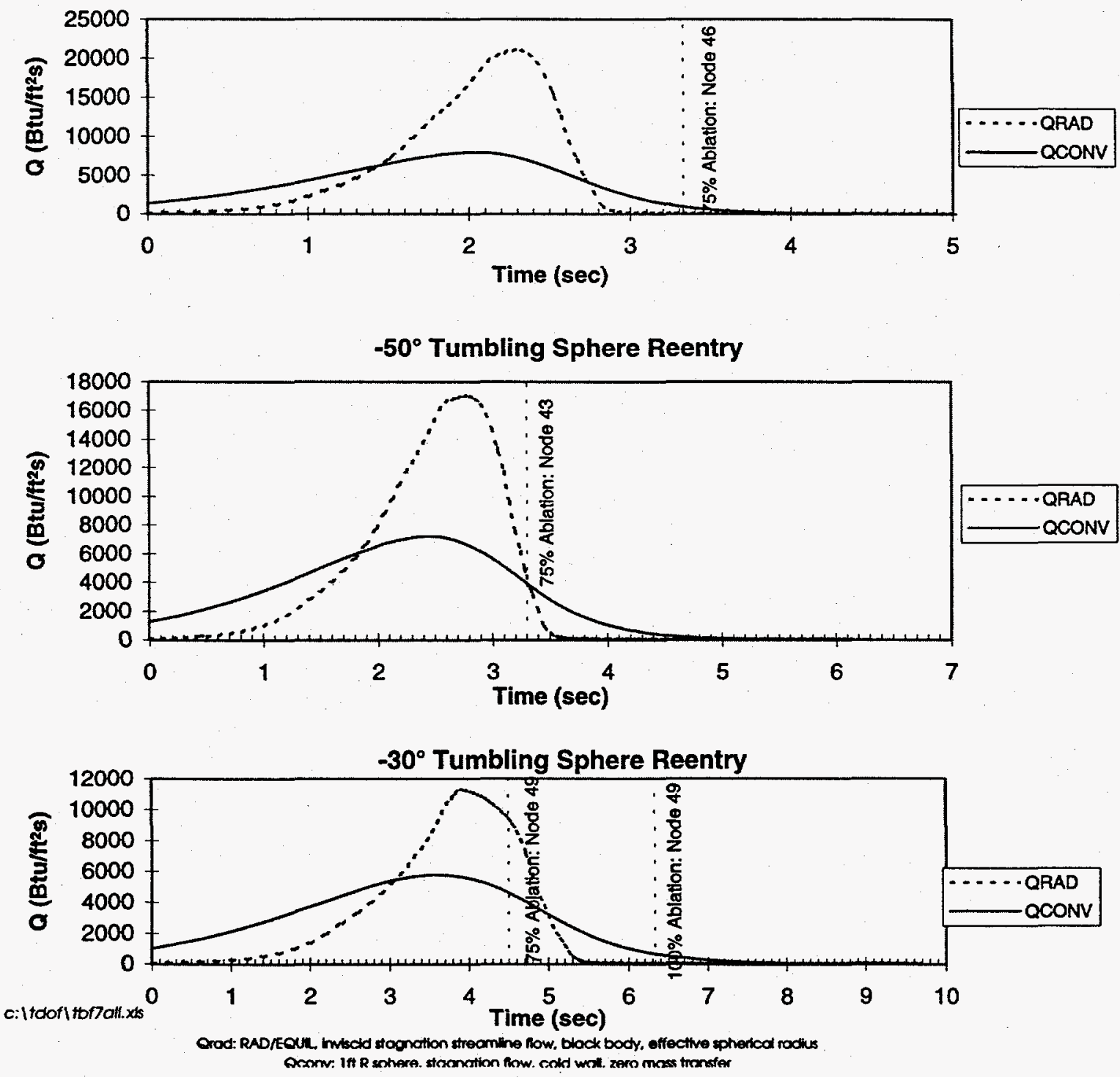
Figure 89.(Continued) Cassini Mission RETAP Results Ablation Failure Relative to Heat Pulse

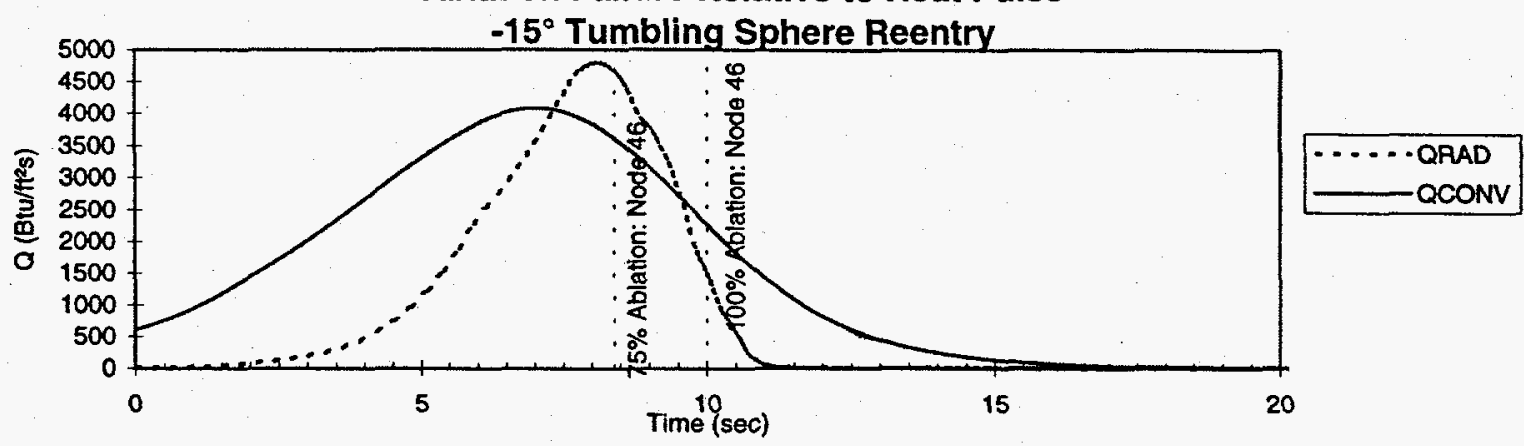

$-10^{\circ}$ Tumbling Sphere Reentry

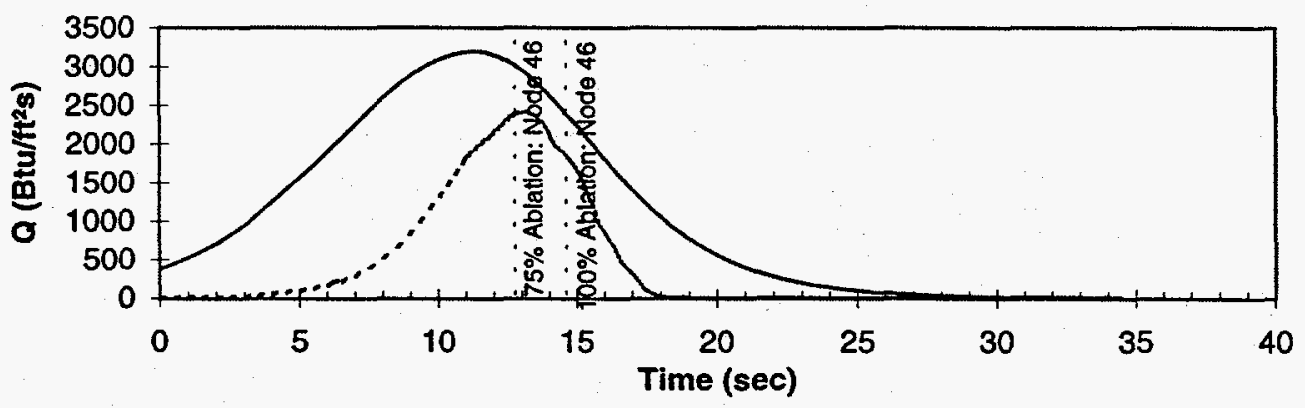

$-07^{\circ}$ Tumbling Sphere Reentry
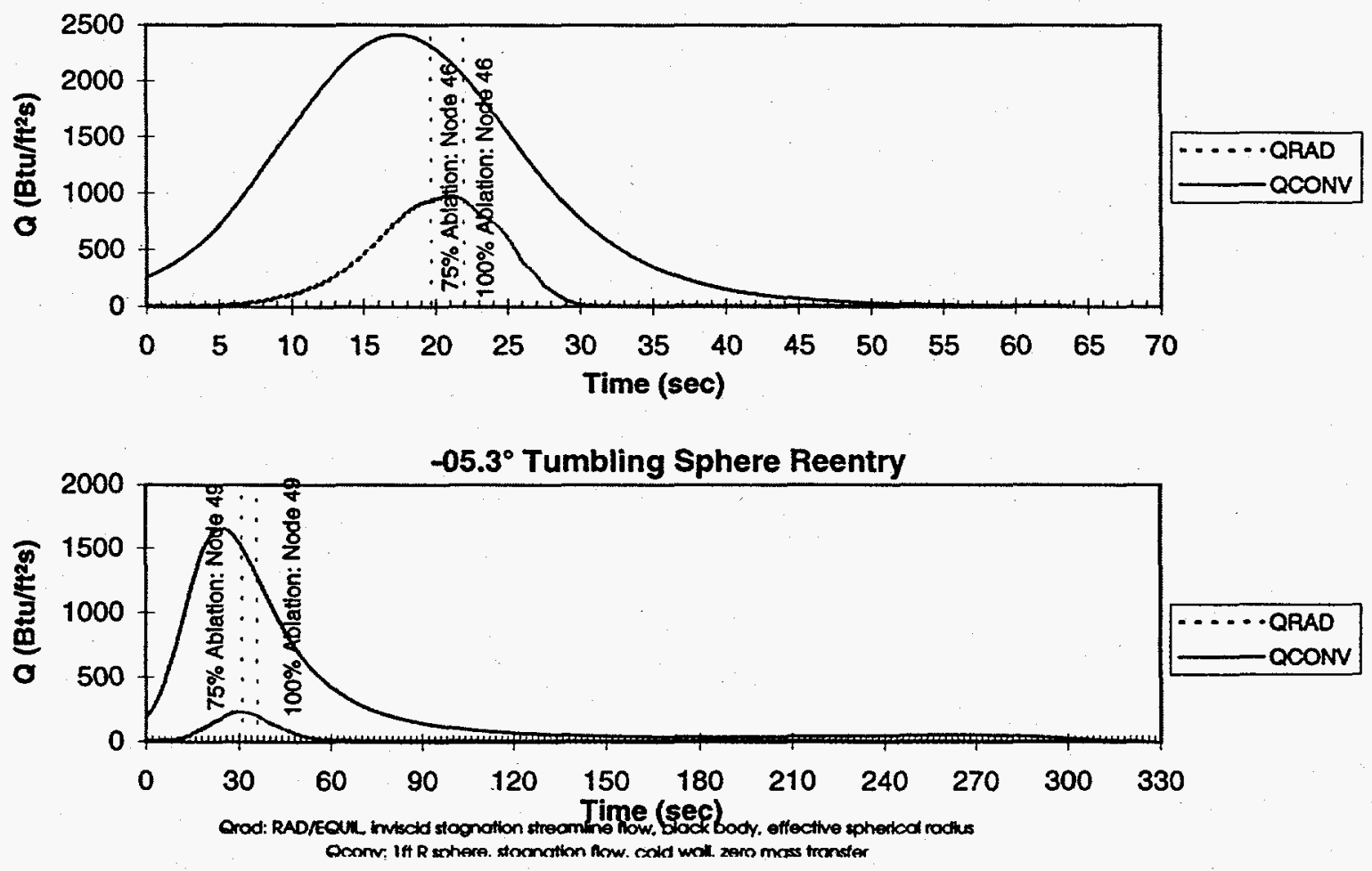
Figure 90 Cassini Mission RETAP Results

Recession Failure Comparison: JANNAF 89 and Lawrence Livermore

Side-on Stable 3D LWRHU $-90^{\circ}$ VVEJGA Reentry

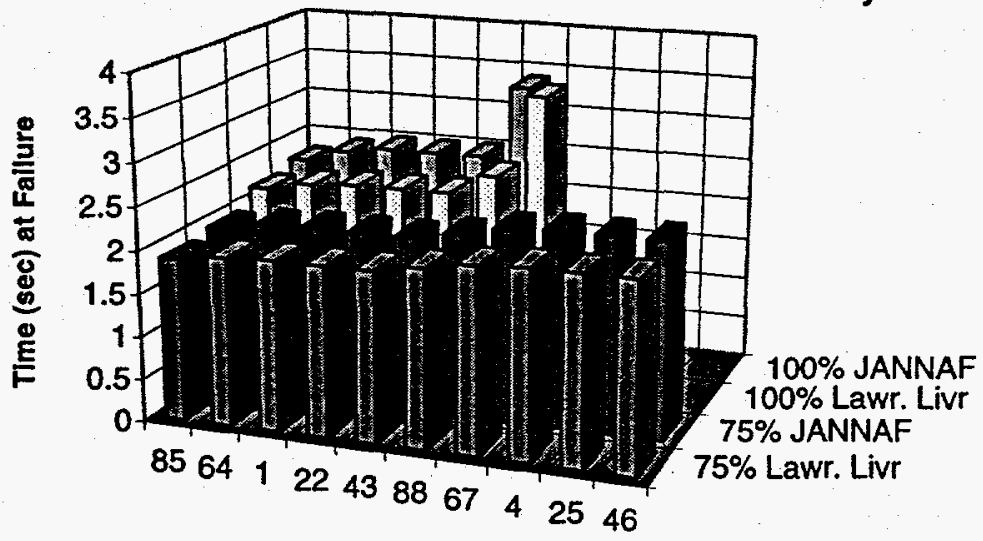

Stagnation Surface Node Numbers

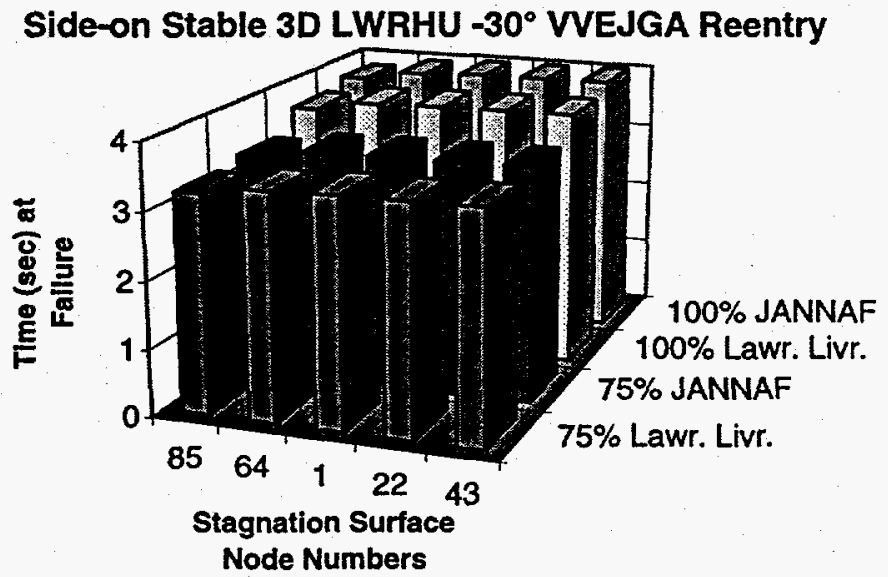

Side-on Stable 3D LWRHU $-15^{\circ}$ VVEJGA Reentry

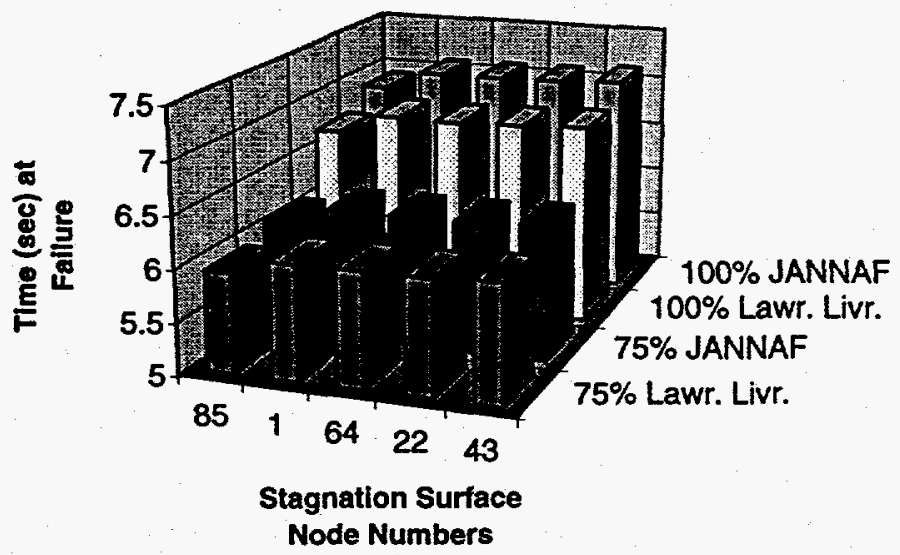


Figure ${ }^{91}$ Cassini Mission RETAP Results Side-on Stable LWRHU Comparison of Ablation Failure Relative to Heat Pulse
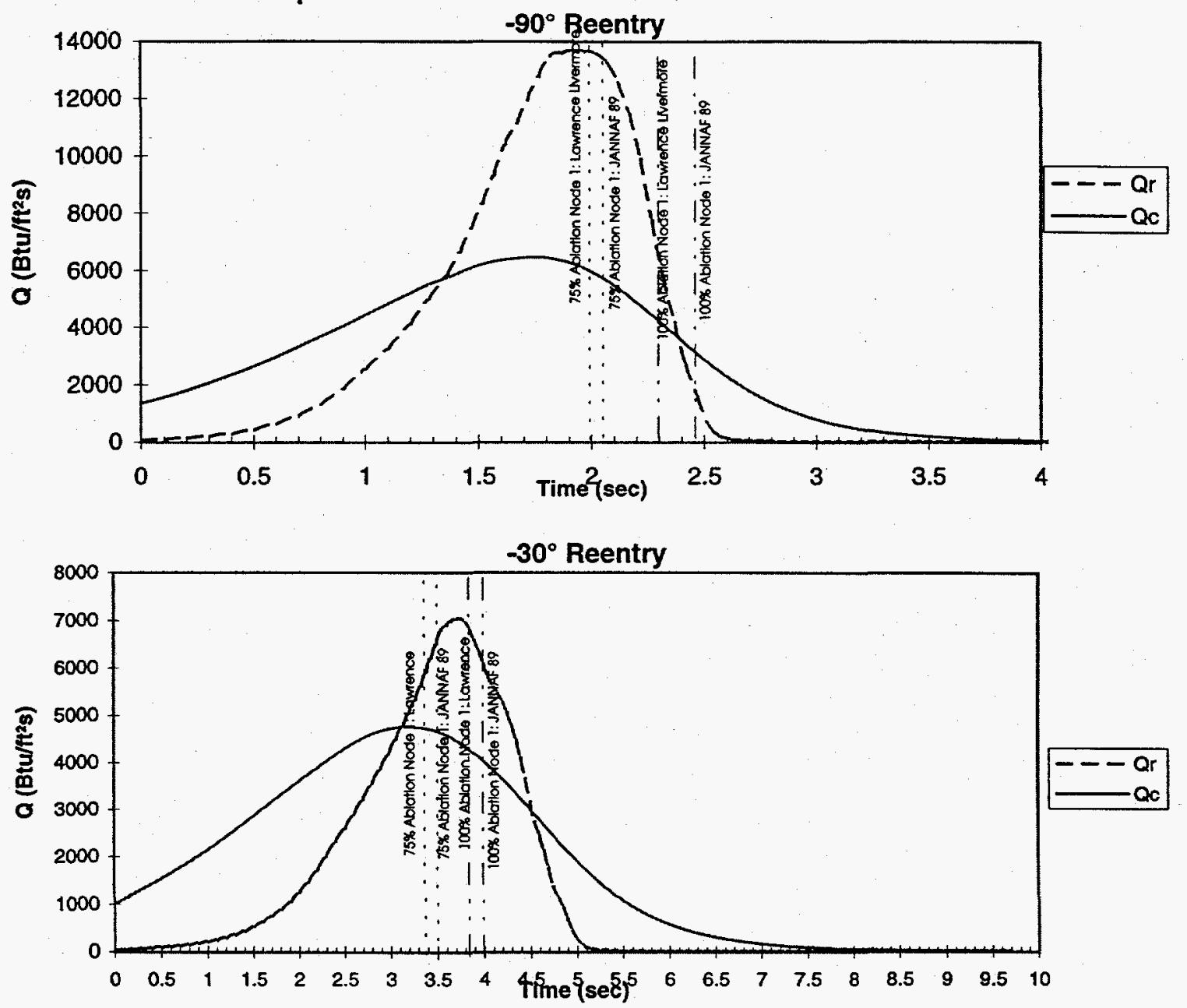

$-15^{\circ}$ Reentry

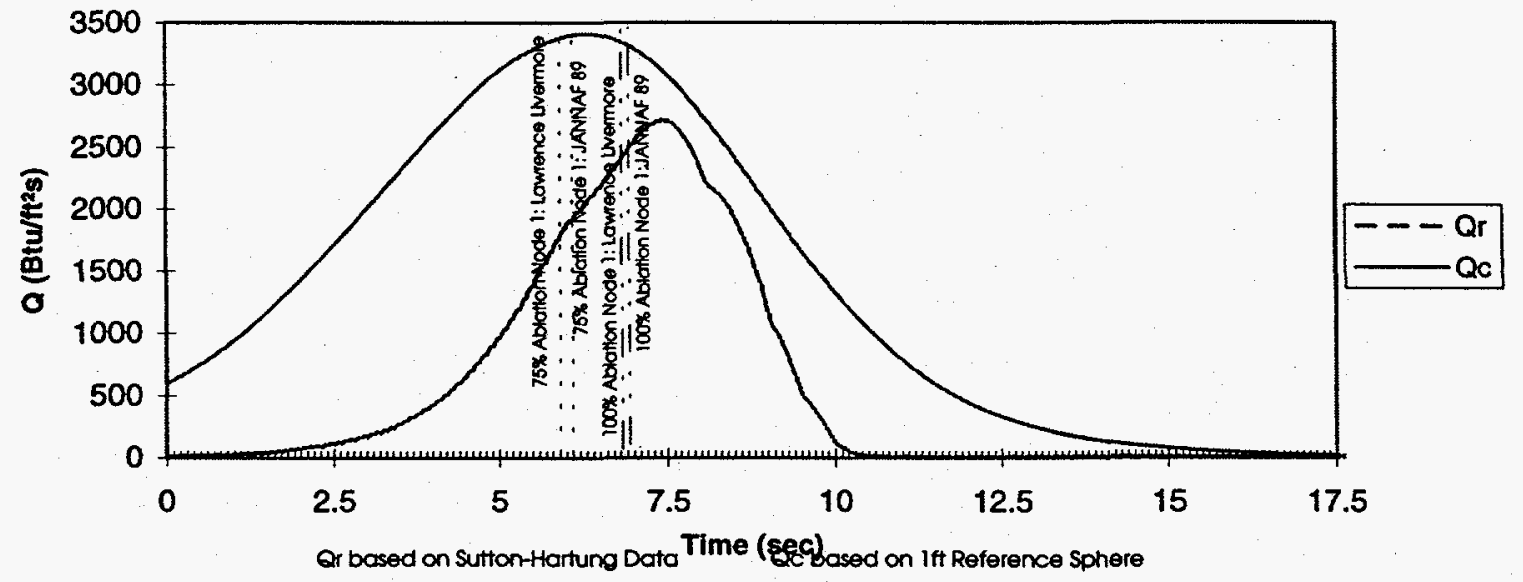


Figure 92 Cassini Mission RETAP Results

Side-on Stable 3D LWRHU 3D $-90^{\circ}$ Reentry Recession Failure Comparison: Gas-Radiation Cooling Affect
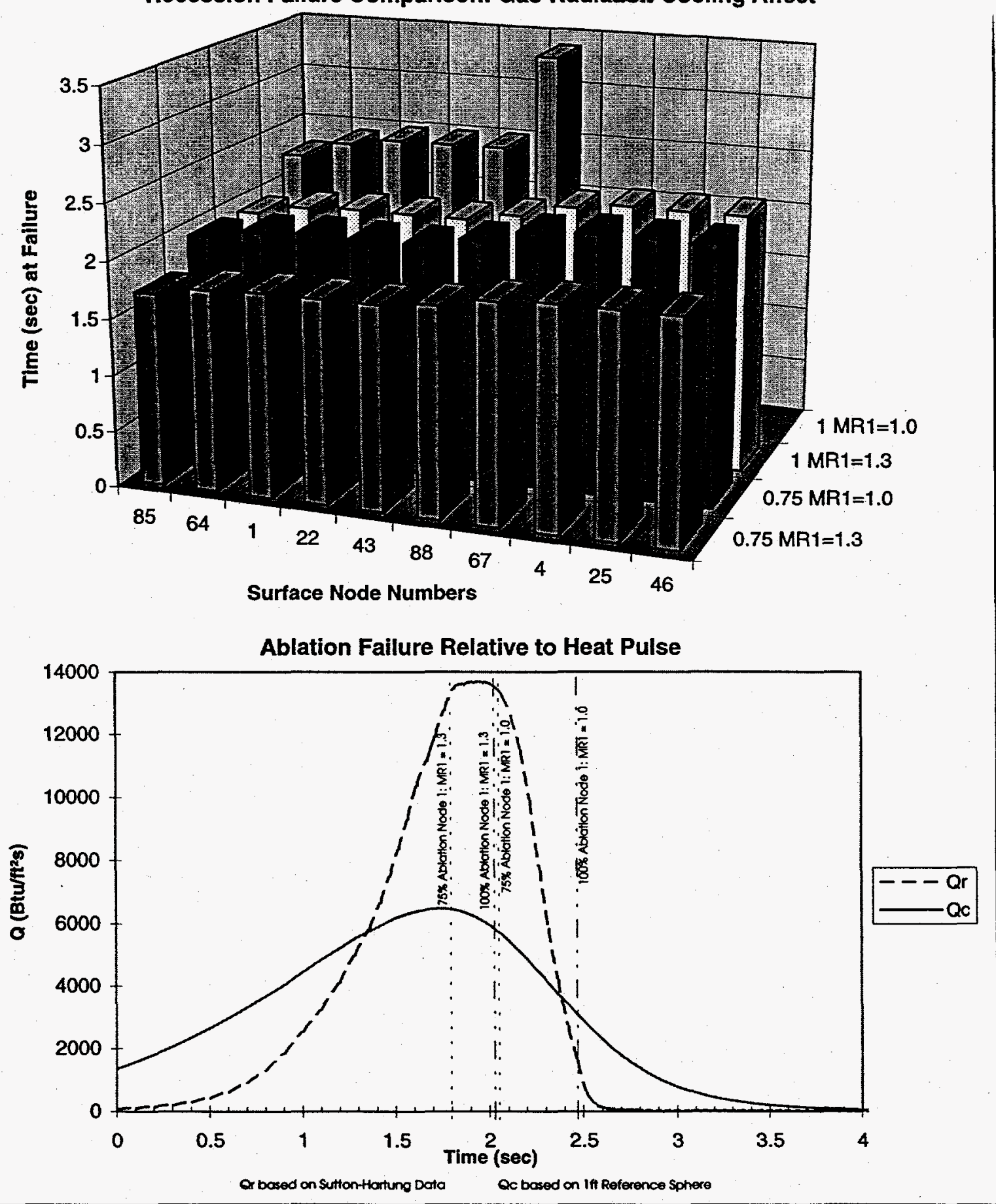


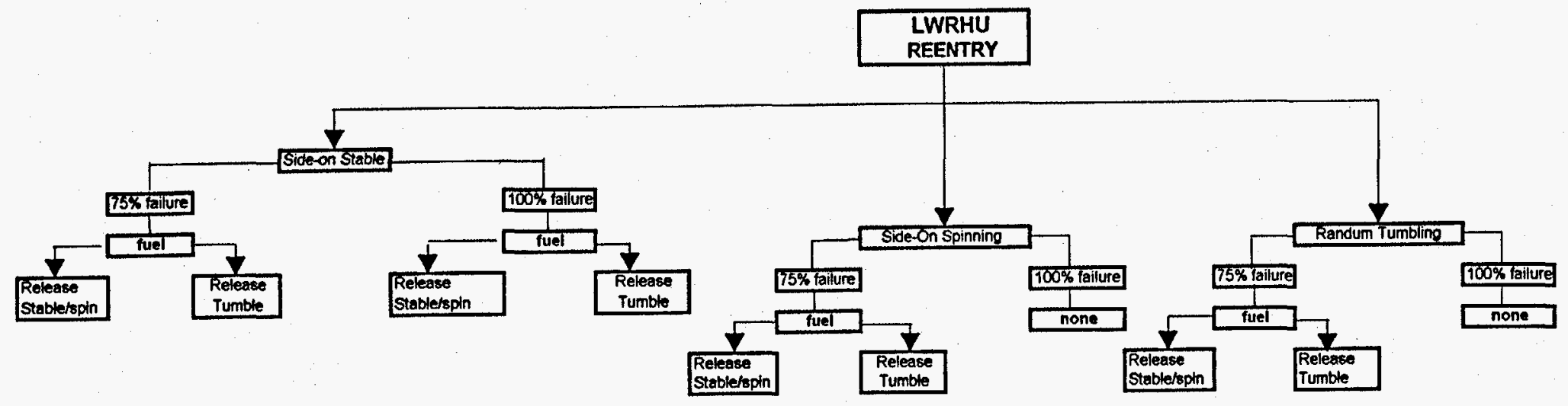

INITIAL CONDITIONS FOR TDOF RUNS

\begin{tabular}{|c|c|c|c|c|c|c|c|c|c|c|}
\hline \multirow[b]{3}{*}{ Angles $\rightarrow>$} & \multirow{2}{*}{\multicolumn{2}{|c|}{$75 \%$}} & \multicolumn{3}{|c|}{ For Gammas -5.2 to $-90^{\circ}$} & & \multirow[t]{2}{*}{ Only for $-5.2^{\circ}$} & \multicolumn{3}{|c|}{ Only for $-5.2,-7,-10^{\circ}$} \\
\hline & & & & & $100 \%$ & & & & & \\
\hline & $-5.2^{\circ}$ & $\ldots$ & $.90^{\circ}$ & $-5.2^{\circ}$ & $\ldots$ & $-90^{\circ}$ & $-5.2^{\circ}$ & $-5.2^{\circ}$ & .7 & $-10^{\circ}$ \\
\hline $\begin{array}{l}\text { Time(s) } \\
\text { Vetrel } \\
\text { Ant (n) }\end{array}$ & $\begin{array}{r}22.7 \\
59388 \\
242612\end{array}$ & & $\begin{array}{l}1.93 \\
49666 \\
141359\end{array}$ & $\begin{array}{r}25.16 \\
57388 \\
238007\end{array}$ & & $\begin{array}{l}2.22 \\
41753 \\
128318\end{array}$ & $\begin{array}{r}112.8 \\
20850 \\
237633\end{array}$ & $\begin{array}{r}65.18 \\
31548 \\
231373\end{array}$ & $\begin{array}{r}27.78 \\
30587 \\
179975\end{array}$ & $\begin{array}{r}24.98 \\
11094 \\
141124\end{array}$ \\
\hline
\end{tabular}


Ihe Johns Hopkins University

Applied Physics Laboratory

Laurel. Maryland 20723-6099

Figure 94 CASSINI MISSION LWRHU FUEL TDOF RESULTS

SIDE ON STABLE LWRHU 75\% AEROSHELL BURNTHROUGH

RELEASING FUEL PELLET SIDE-ON STABLE OR SPINNING

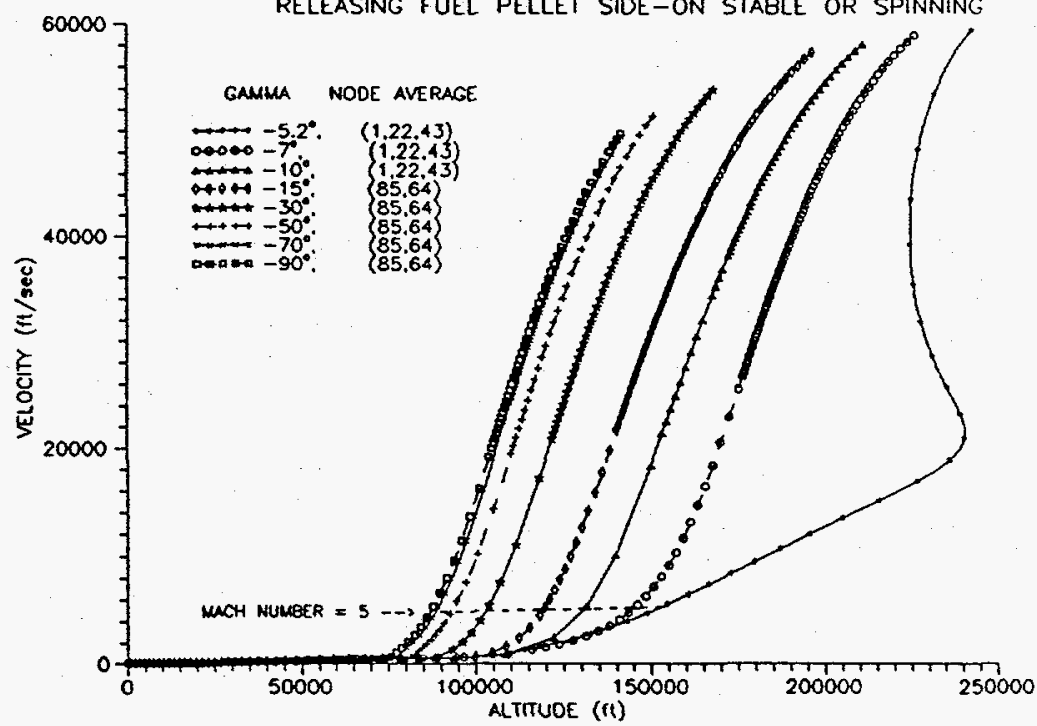

Figure 95 CASSINI MISSION, LWRHU FUEL TDOF RESULTS SIDE ON STABLE LWRHU 75\% AEROSHELL BURNTHROUGH RELEASING FUEL PELLET SIDE-ON STABLE OR SPINNING

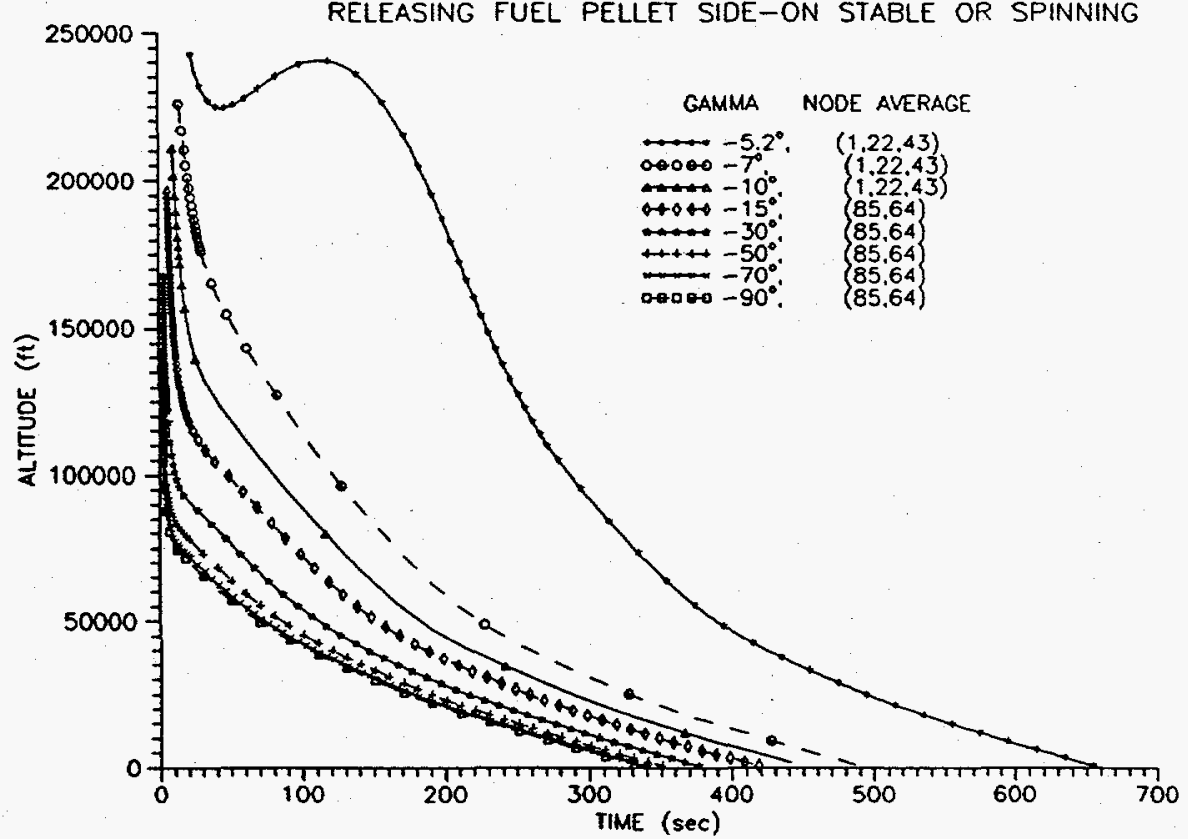


Figure 96A. Cassini Mission TDOF Fuel Results

Reference Convective Heating Rate History for Side-on Stable 75\%

Breach Releasing Side-on Fuel Pellet
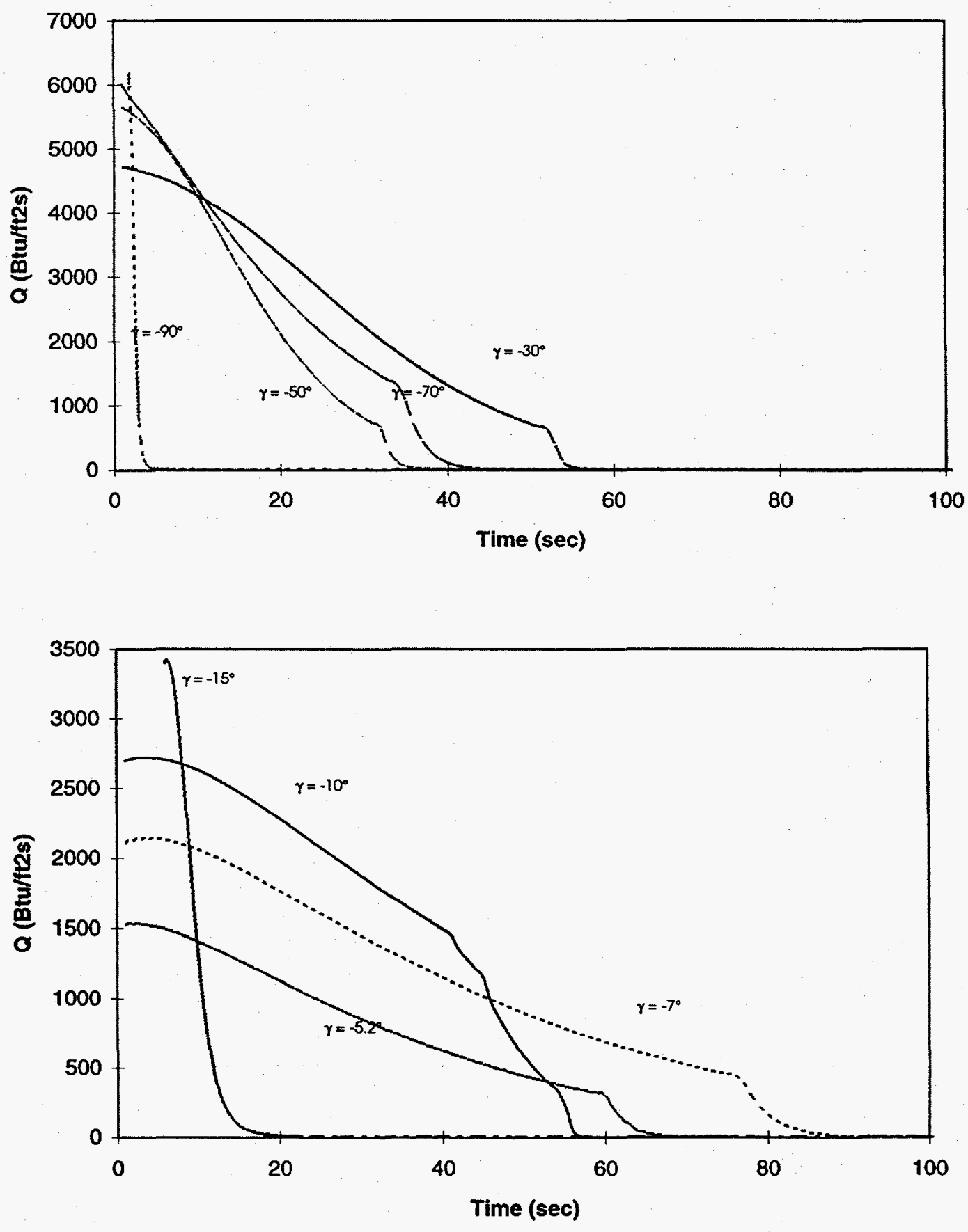
Figure 96B. Cassini Mission TDOF Fuel Results Radiative Heating Rate History for Side-on Stable 75\% Breach Releasing Side-on Fuel
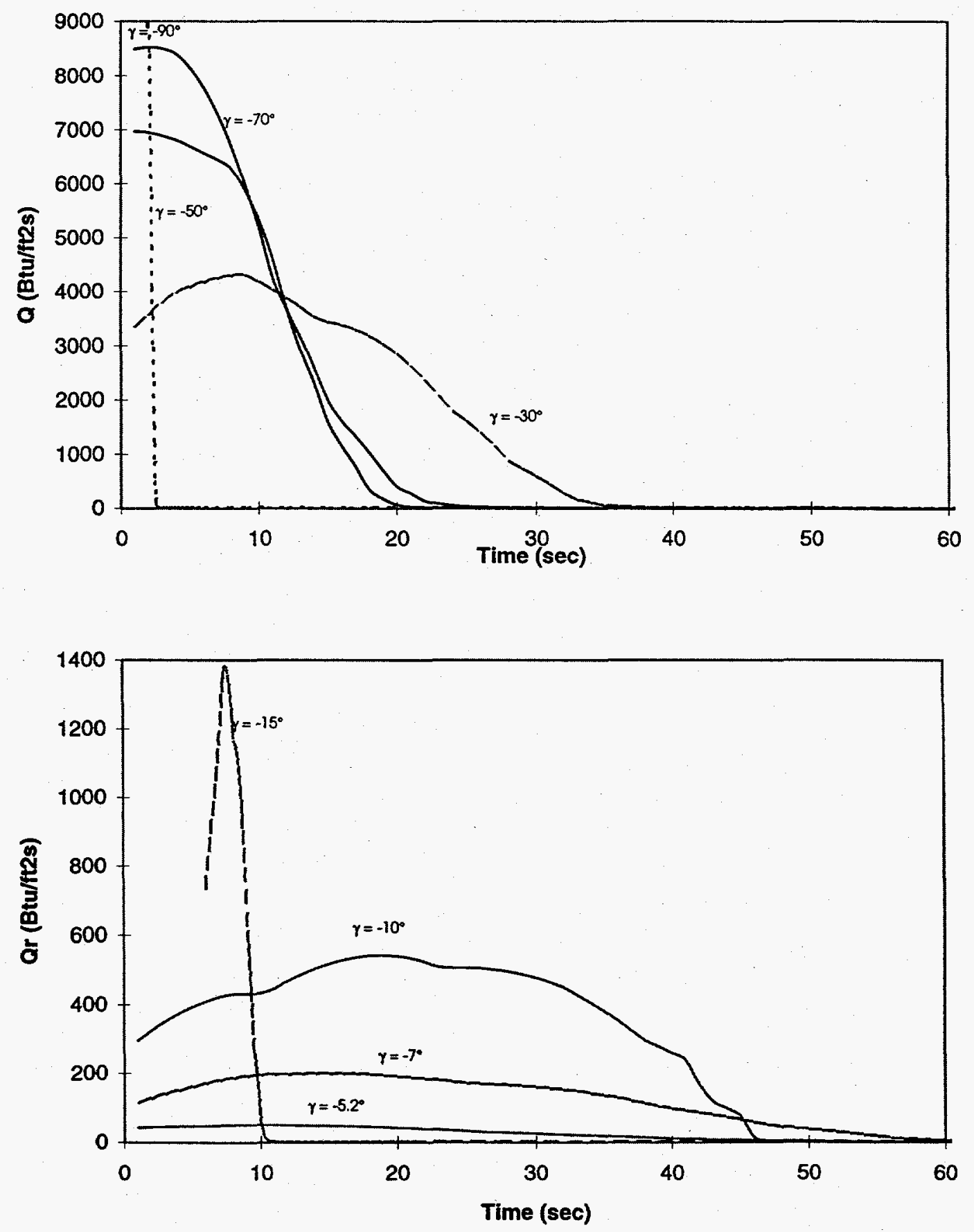
Figure 96C. Cassini Mission TDOF Fuel Results

Reference Convective Heating Rate History for Side-on

Stable $100 \%$ Breach Releasing Side-on Fuel Pellet
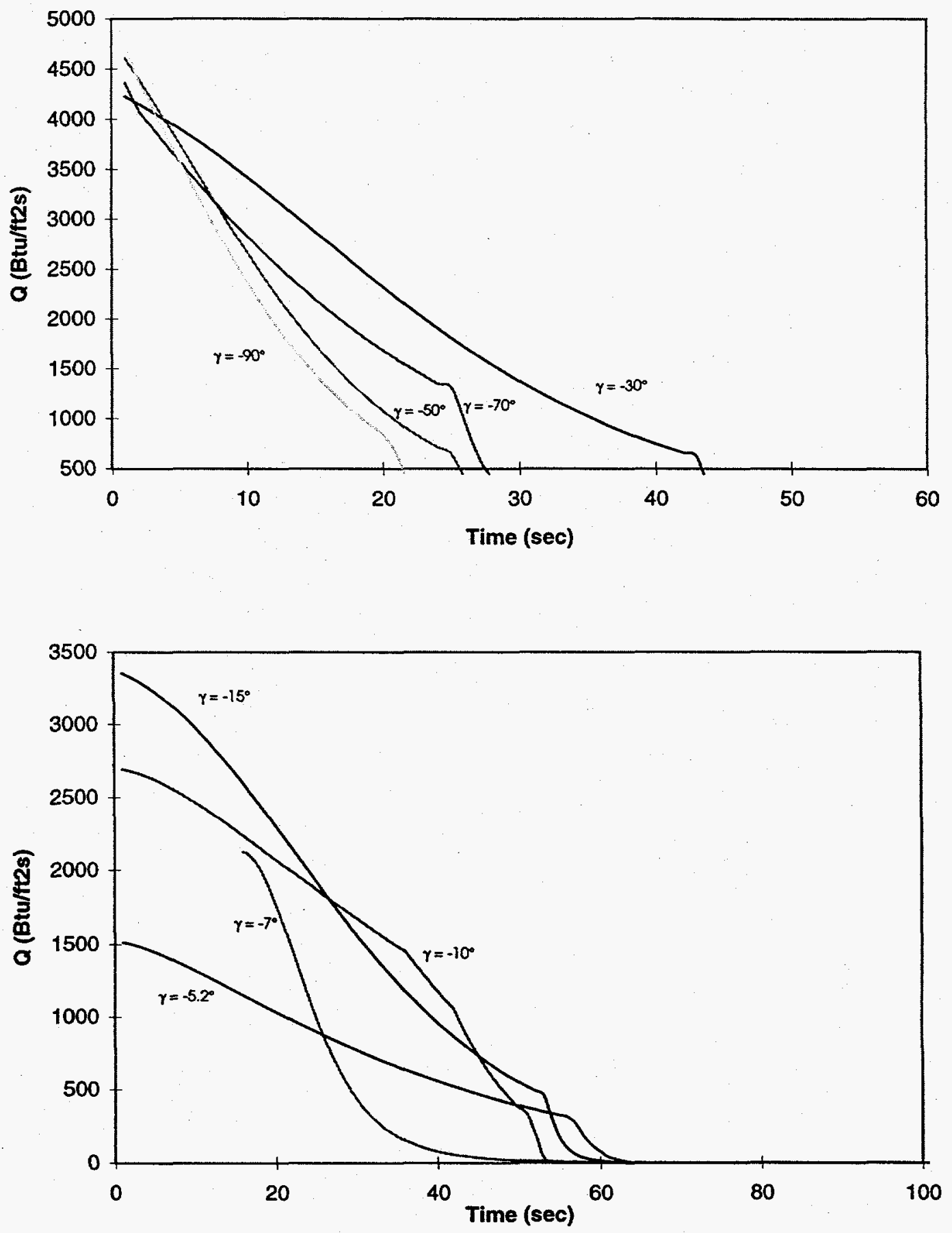
Figure 96D. Cassini Mission TDOF Fuel Results

Reference Radiative Heating Rate History for Side-on Stable 100\% Breach Releasing Side-on Fuel Pellet
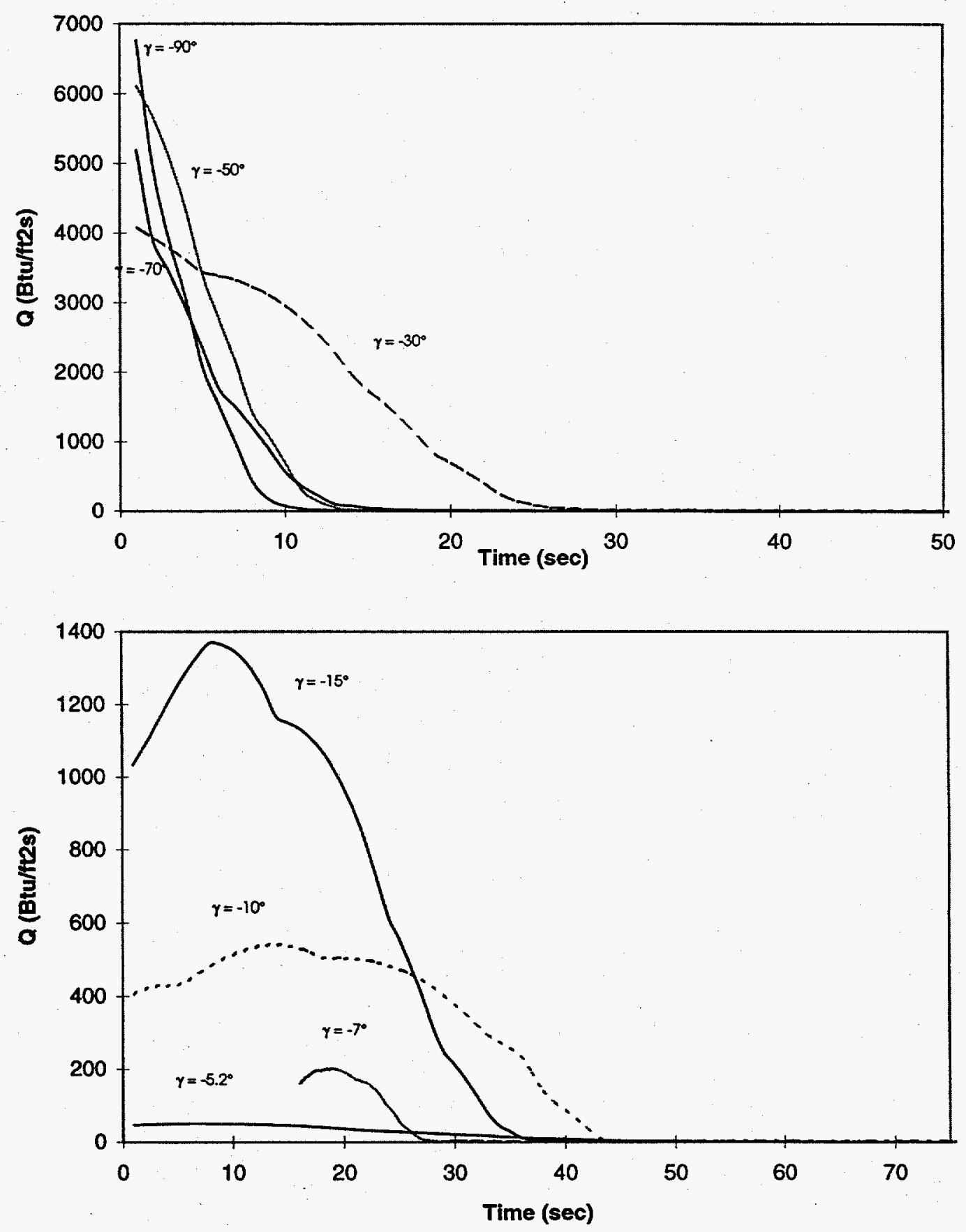
Figure 97. Cassini Mission TDOF Fuel Results Heating Rate History for Side-on Stable 75\% Breach Releasing

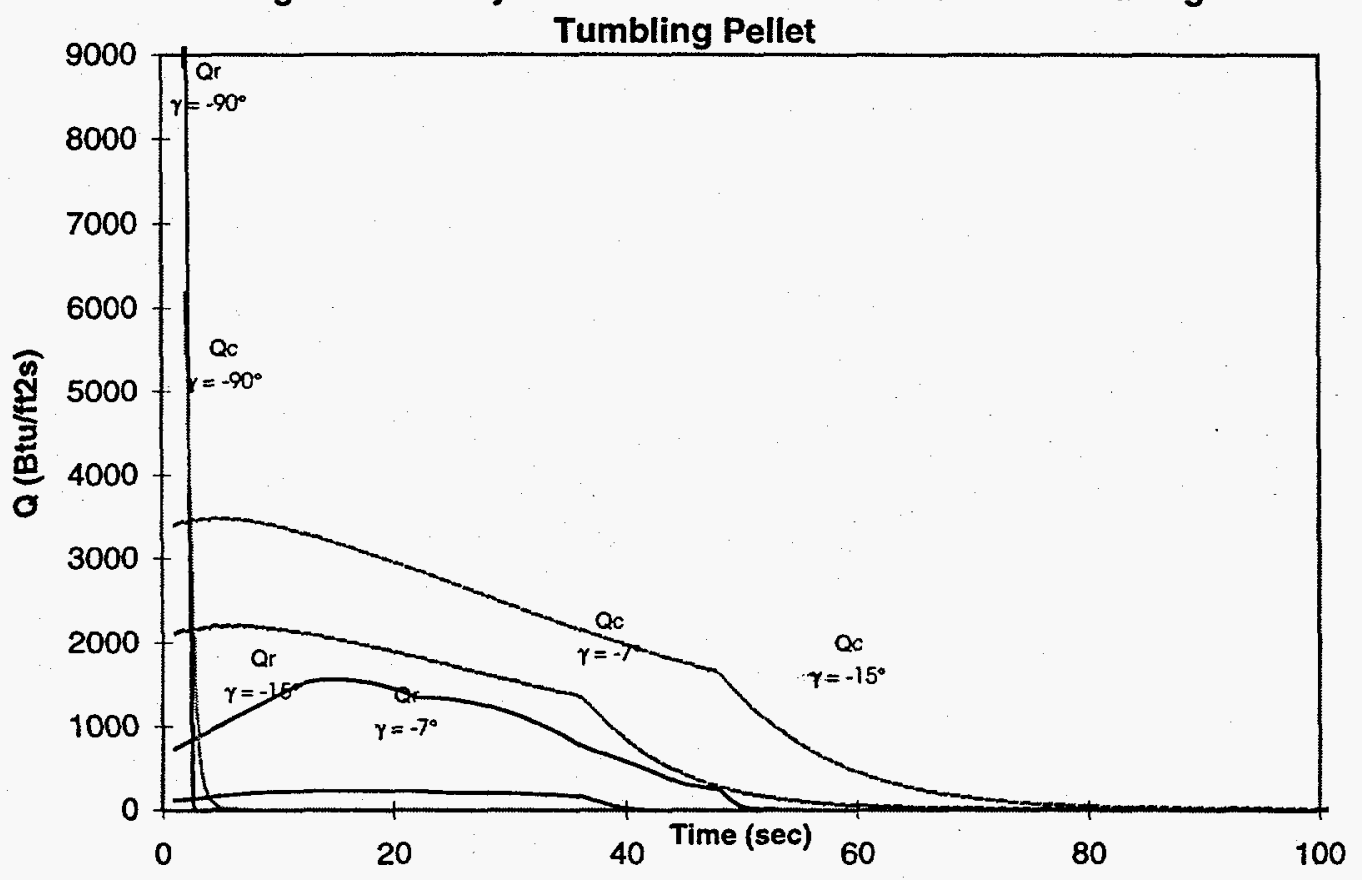

Heating Rate History for Side-on Stable 100\% Breach Releasing Tumbling Pellet

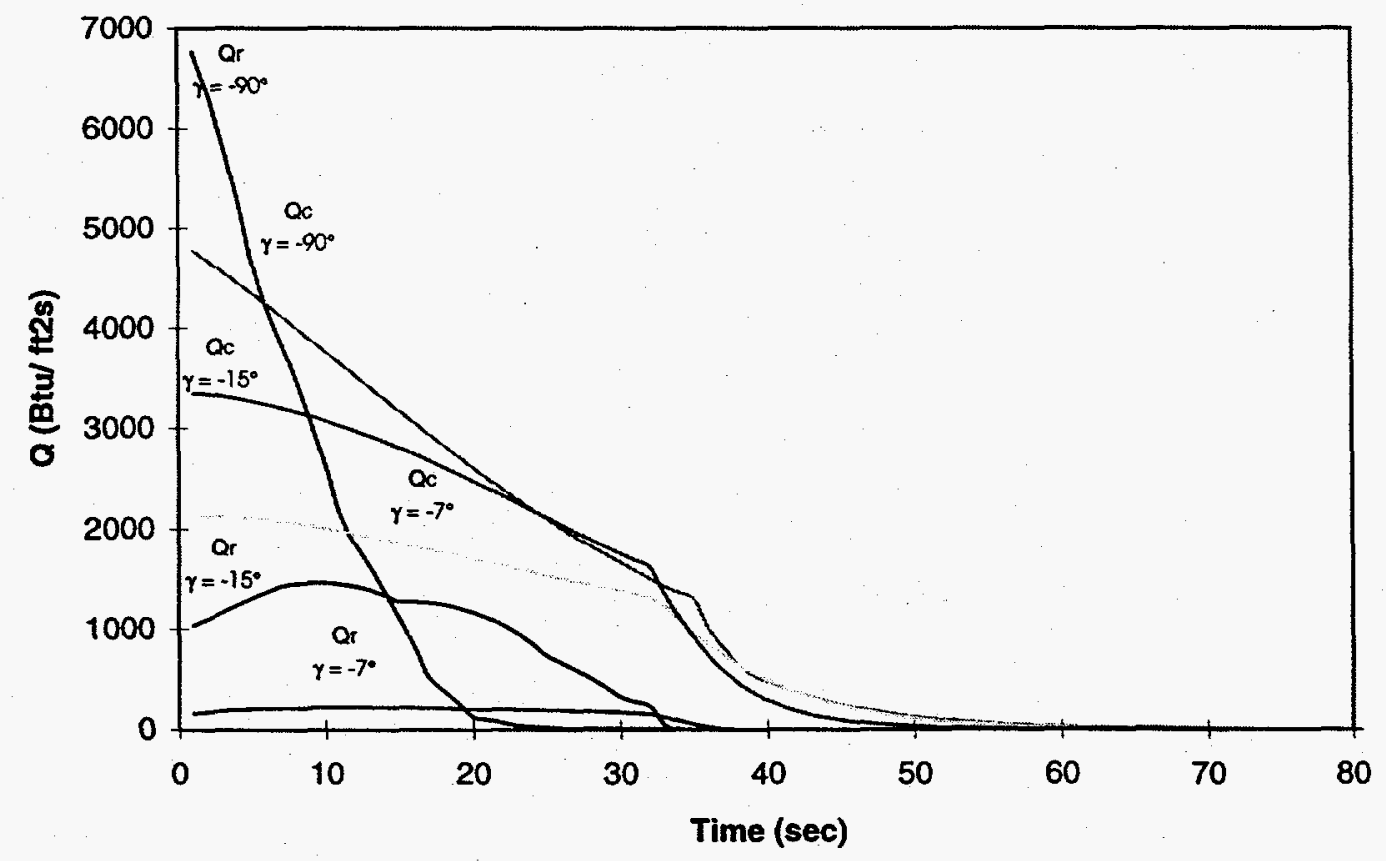

c.itdofifuelisostoroo.xts 
Figure 98. Cassini Mission TDOF Fuel Results

Reference Heating Rate History for Side-on Spinning 75\% Breach Releasing Side-on Fuel Pellet

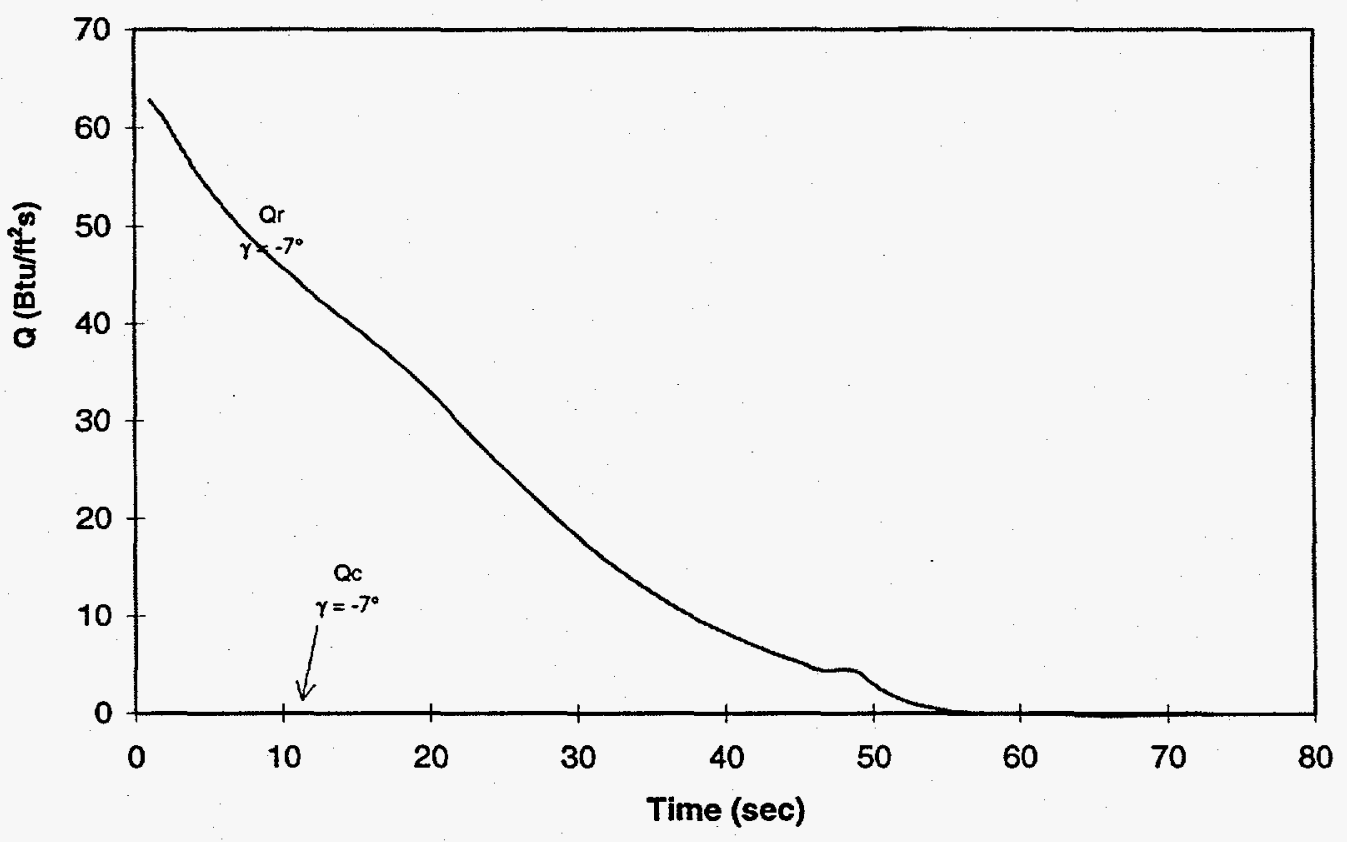

Figure 99. Cassini Mission TDOF Fuel Results

Reference Heating Rate History for Side-on Spinning 75\% Breach Releasing Tumbling Fuel Pellet

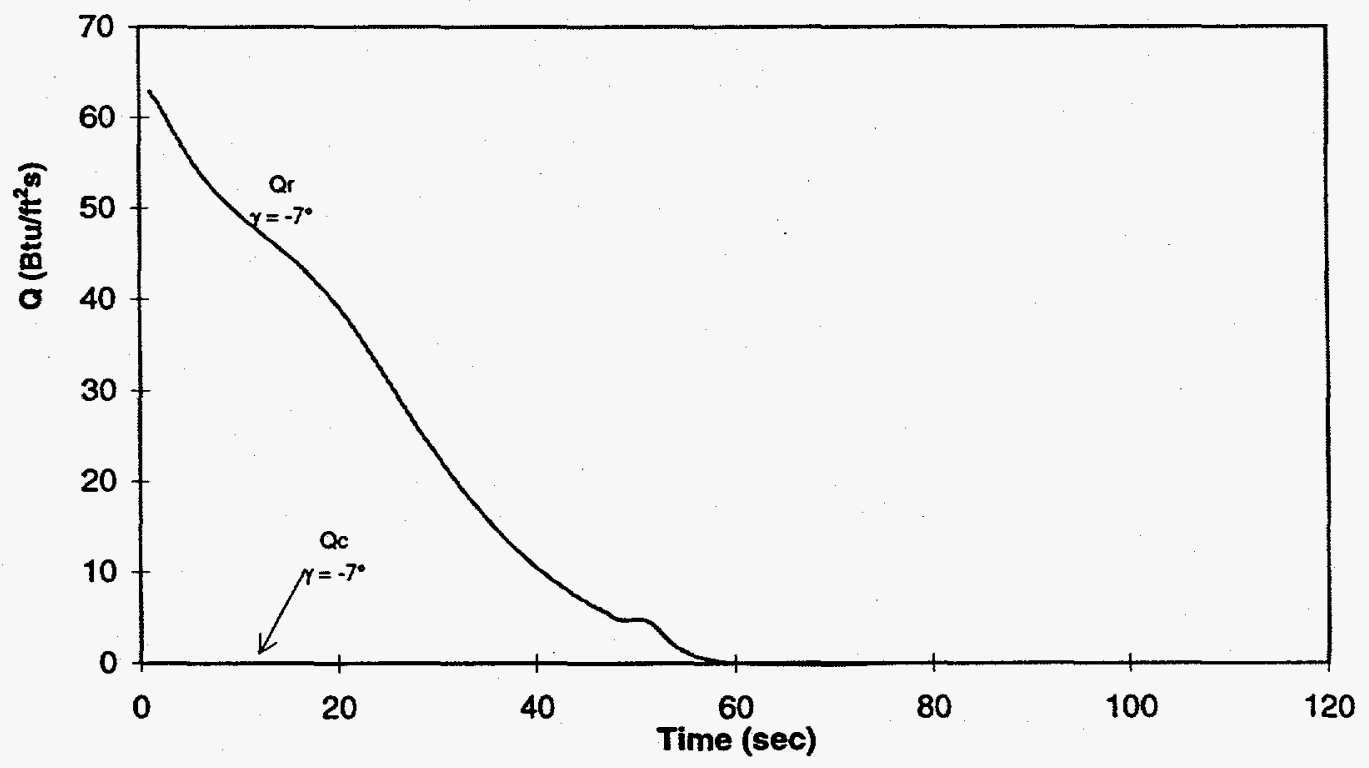




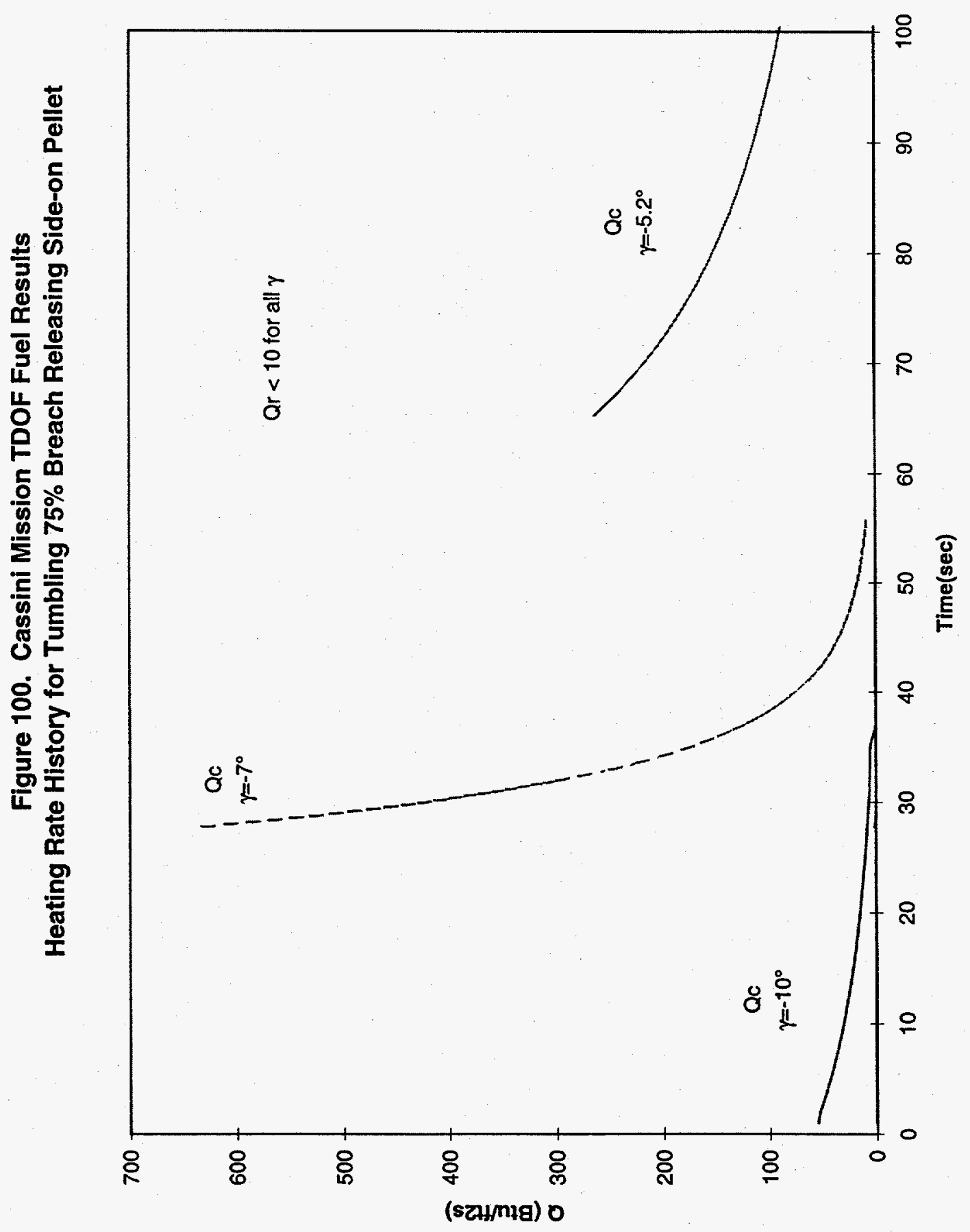


The Johns Hopkins University

Applied Physics Laboratory

Laurel. Maryland 20723-6099

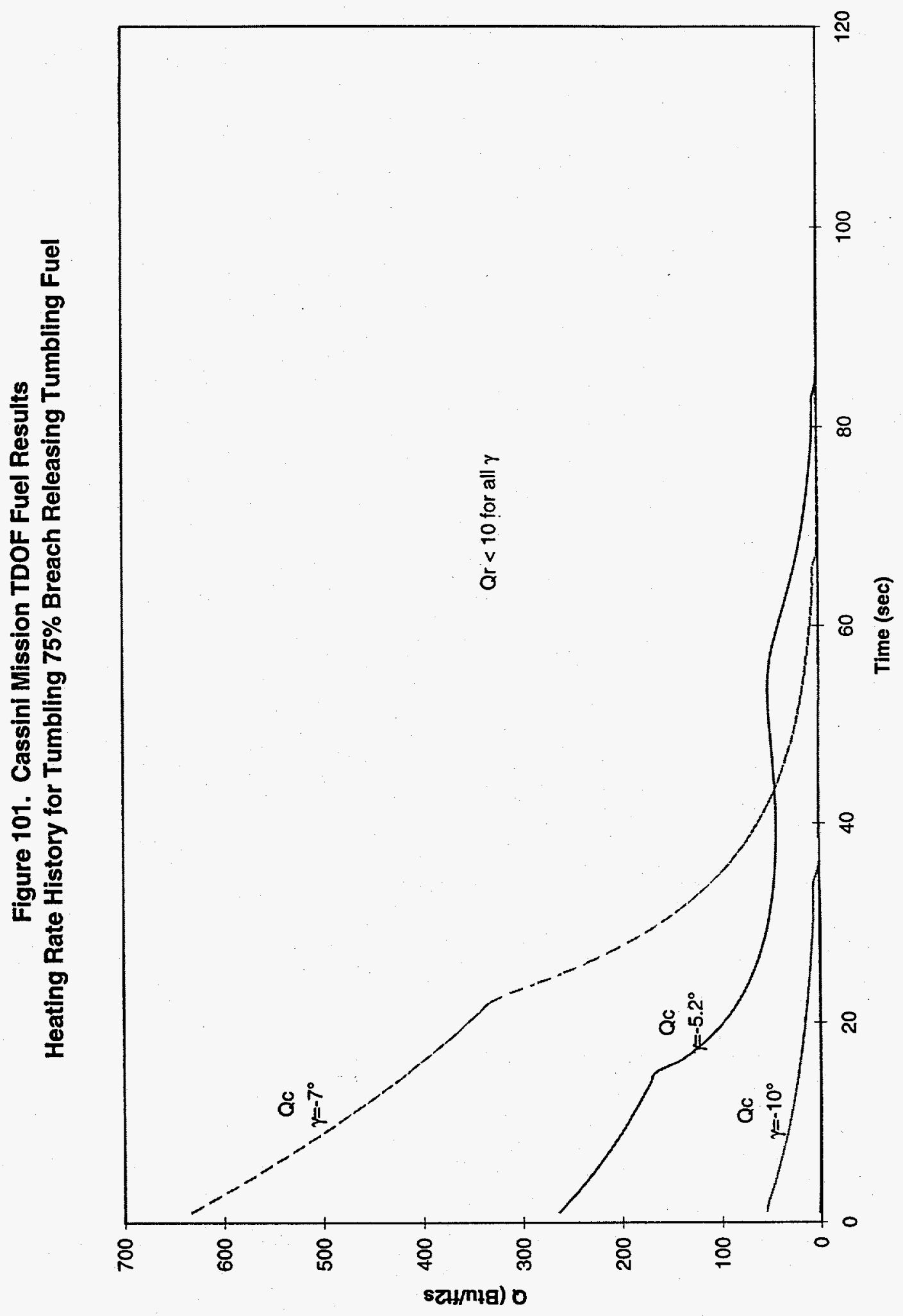


The Johns Hopkins University

Applied Physics Laboratory

Laurel, Maryland 20723-6094

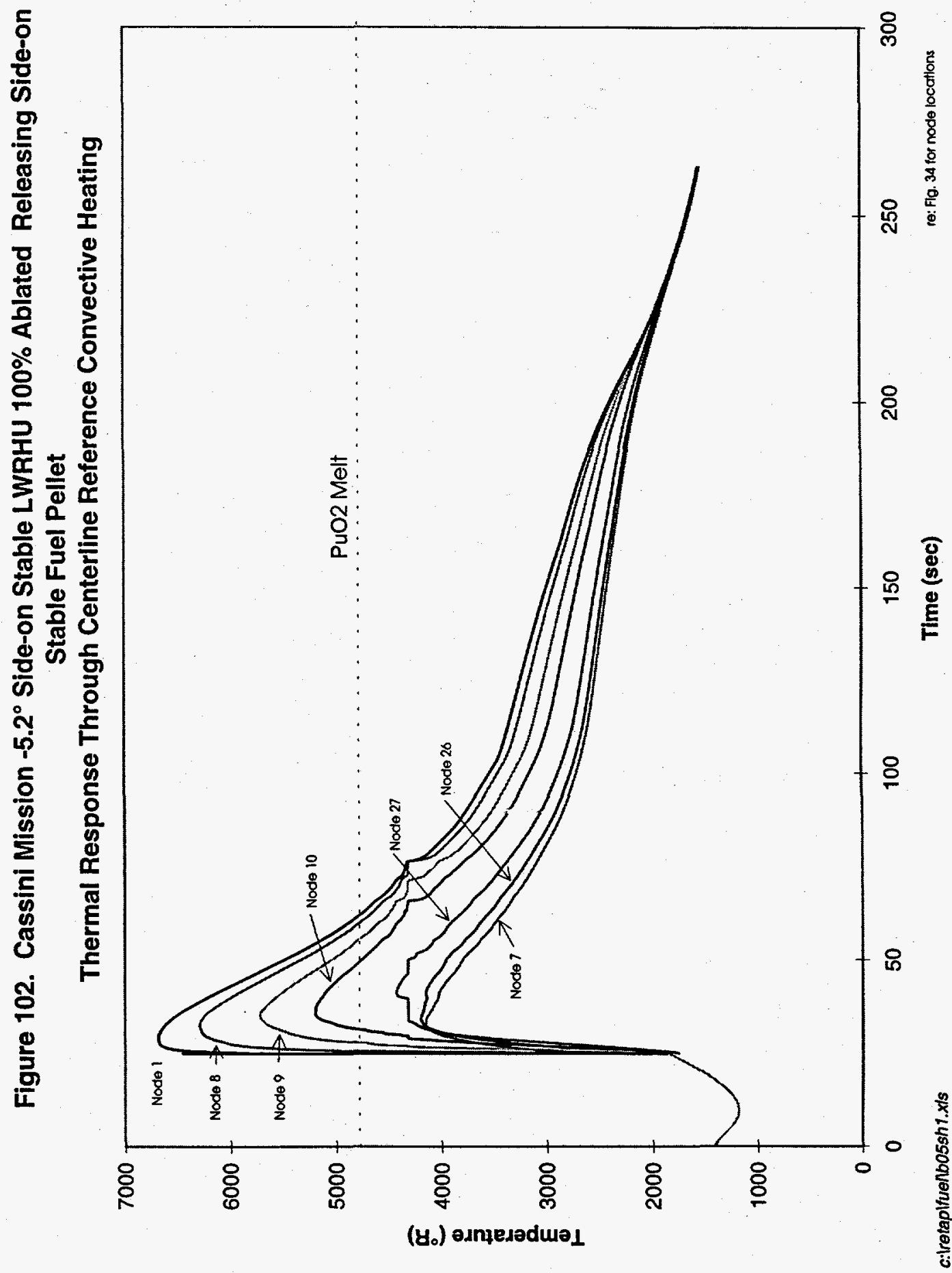


Figure 103. Cassini Mission LWRHU Fuel Results Comparison of $-5.2^{\circ}$ Stable LWRHU Releasing Stable Fuel

Response at $1 X$ and $3 X$ Reference Convective Heating $75 \%$ Ablated

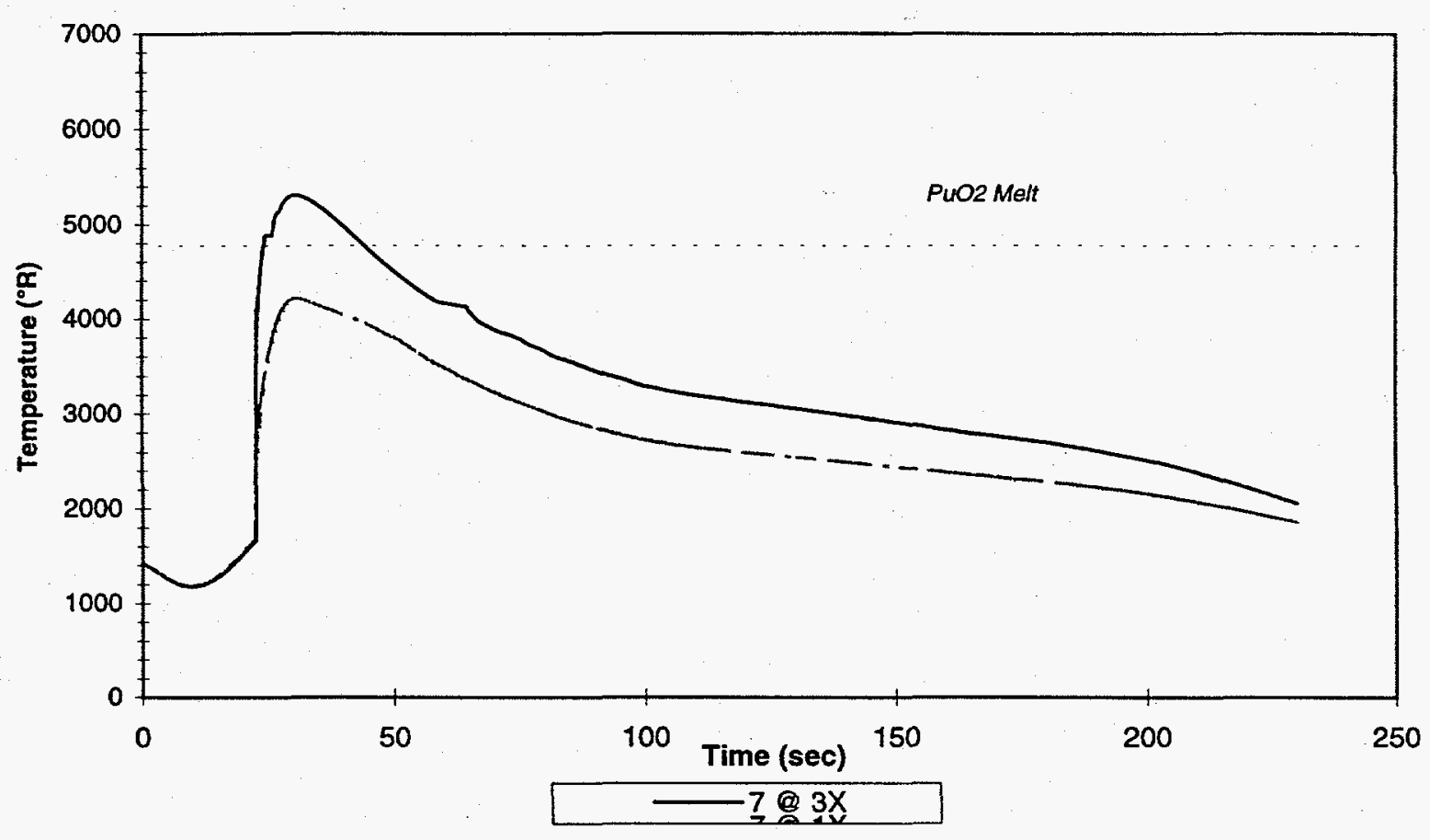

$100 \%$ Ablated

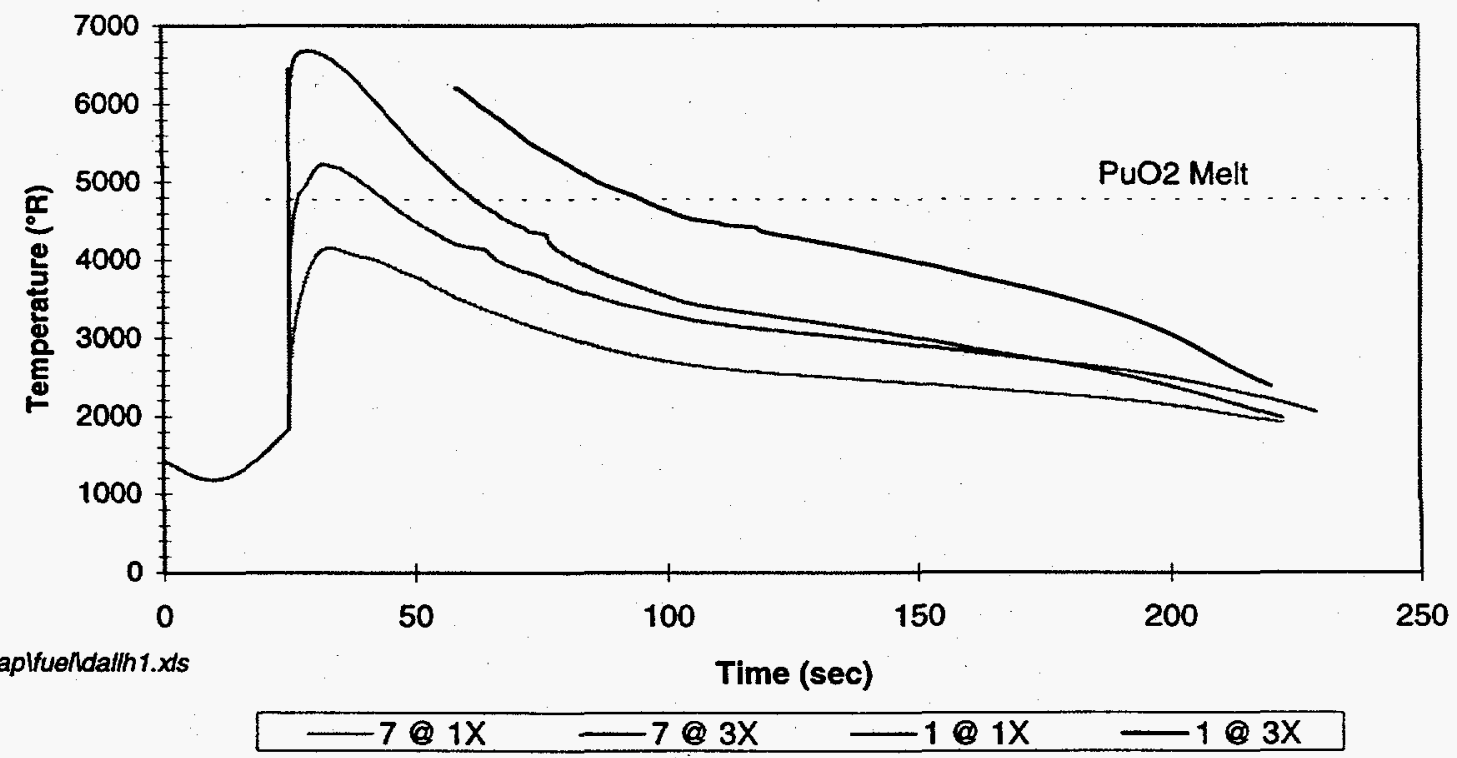


Applied Physics Laboratory

Laurel, Maryland 20723.6099

Figure 104. Cassini Mission LWRHU Fuel Results

Comparison of $-7.0^{\circ}$ Stable LWRHU Releasing Stable Fuel

Response at $1 \mathrm{X}$ and $3 \mathrm{X}$ Reference Convective Heating

$75 \%$ Ablated

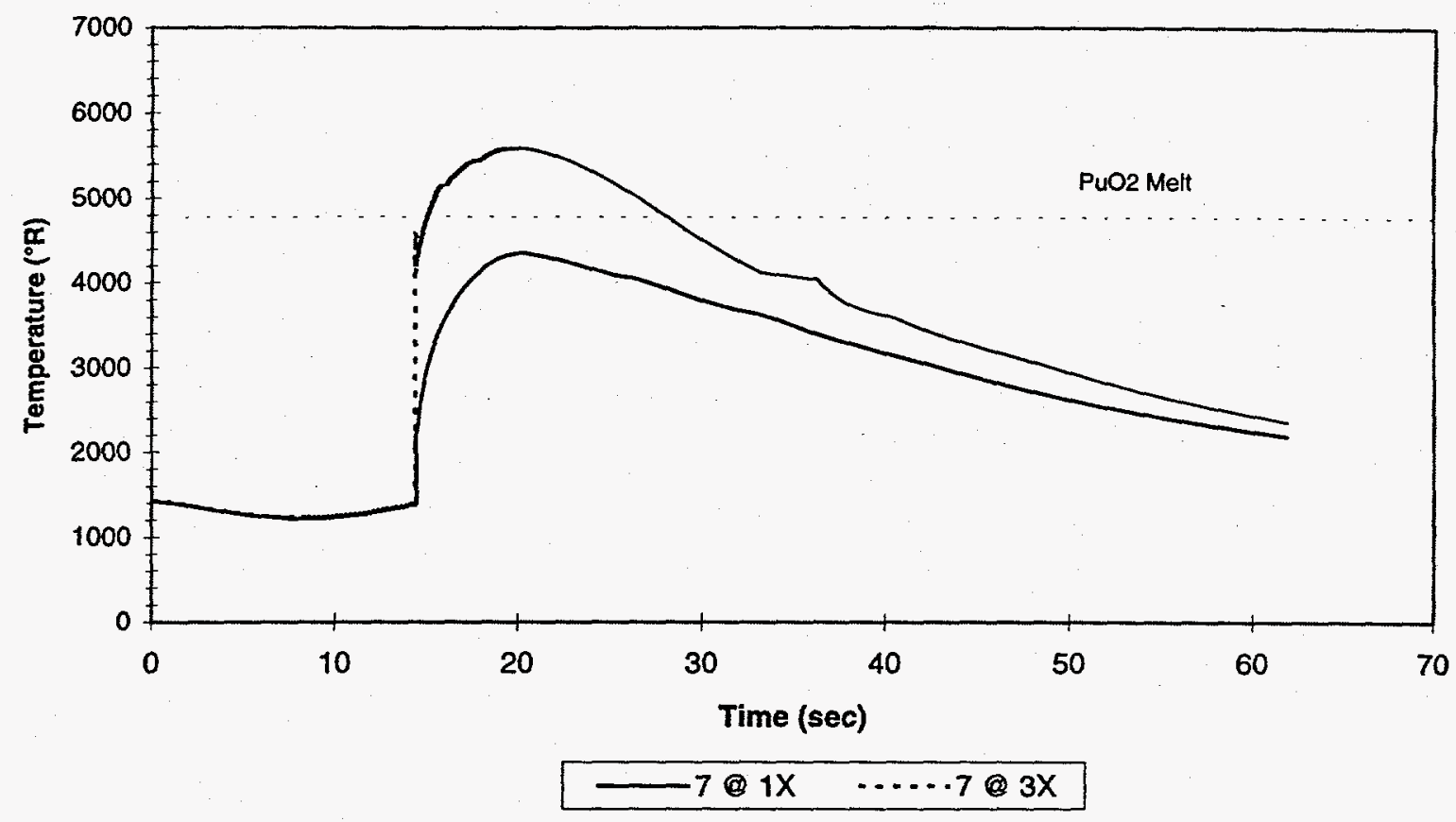

$100 \%$ Ablated

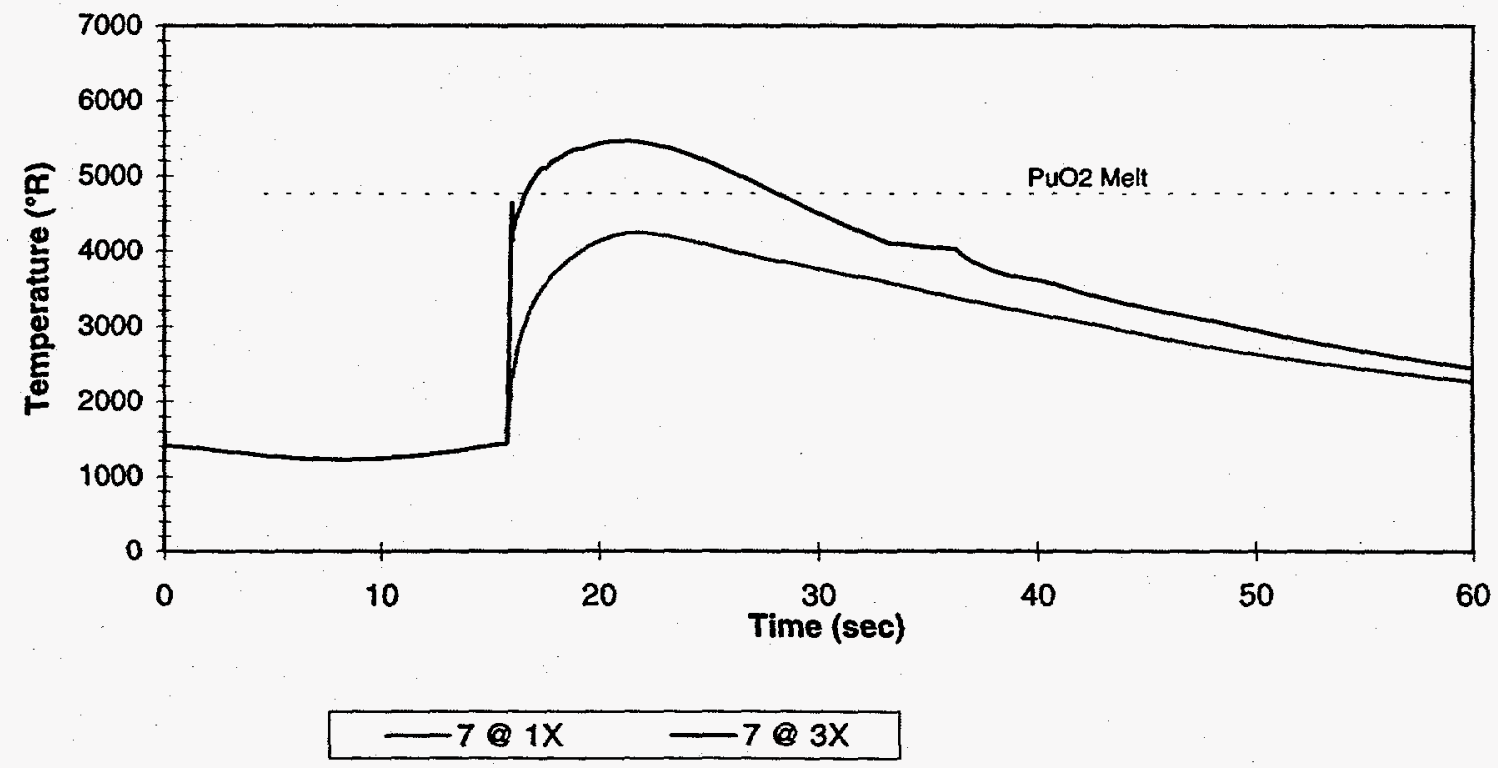


Figure 105. Cassini Mission LWRHU Fuel Results

Comparison of $-10^{\circ}$ Stable LWRHU Releasing Stable Fuel

Response at $1 \mathrm{X}$ and $3 \mathrm{X}$ Reference Convective Heating $75 \%$ Ablated

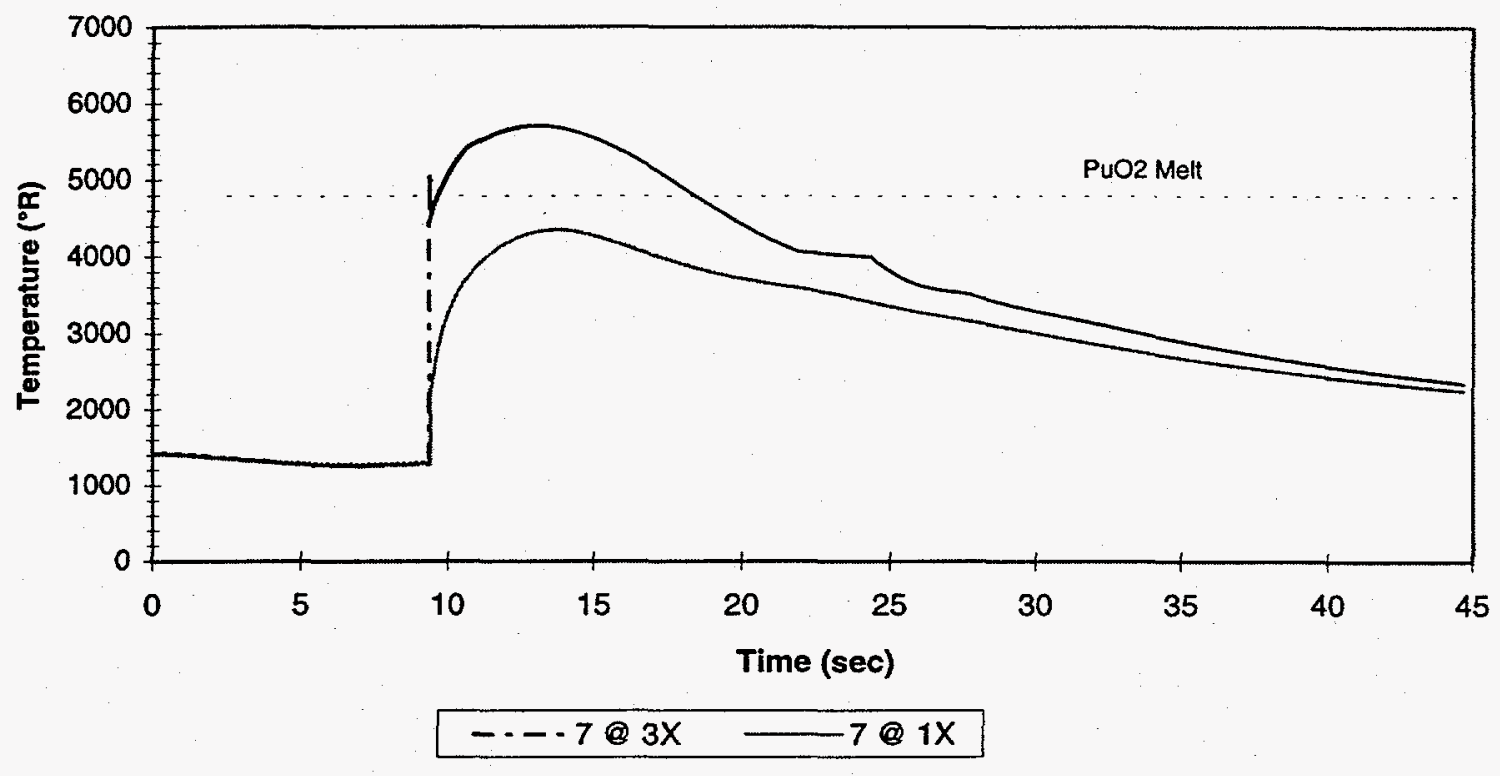

$100 \%$ Ablated

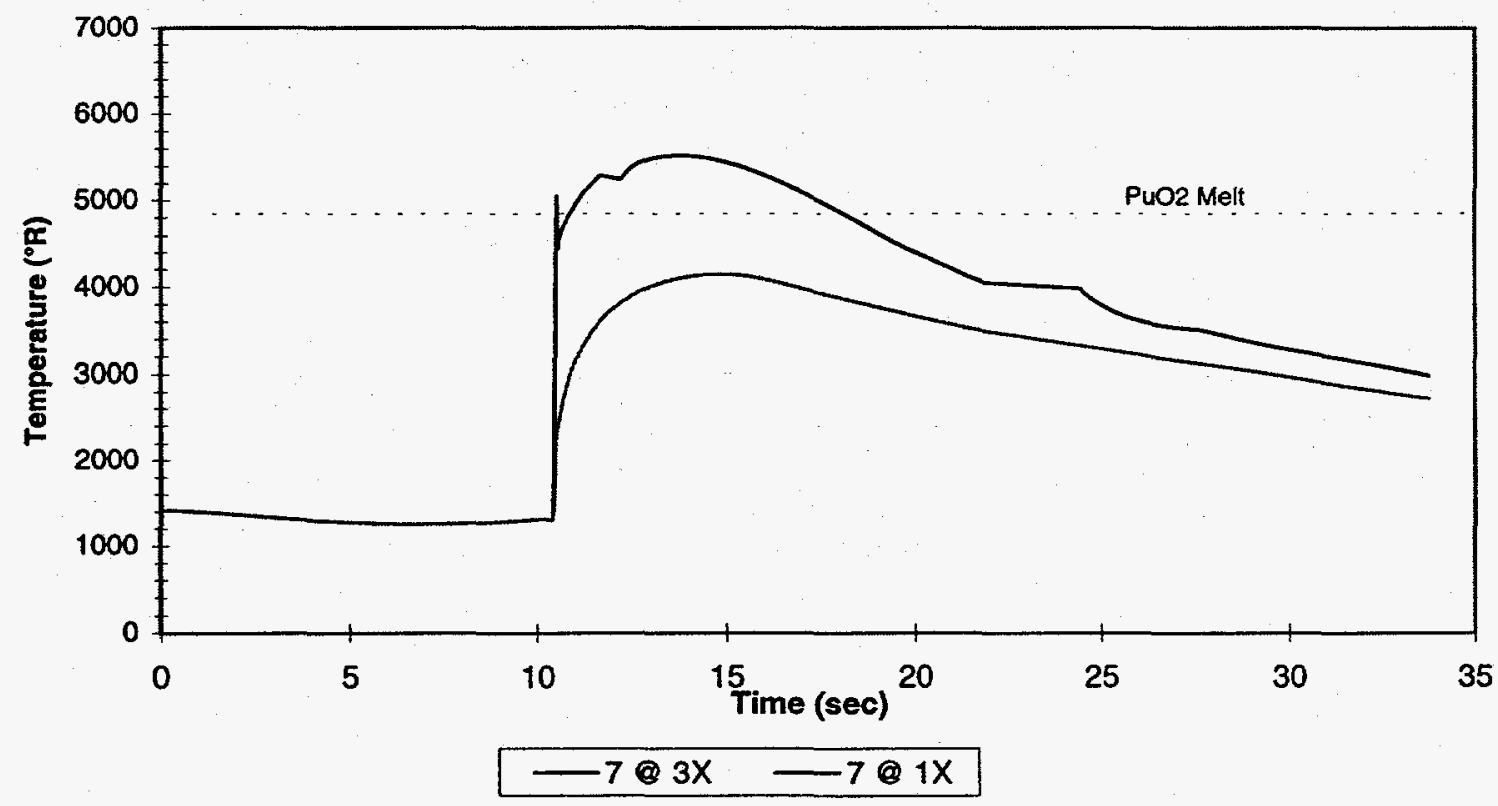


Figure 106. Cassini Mission LWRHU Fuel Results Comparison of $-15^{\circ}$ Stable LWRHU Releasing Stable Fuel Response at $1 X$ and $3 X$ Reference Convective Heating 75\% Ablated

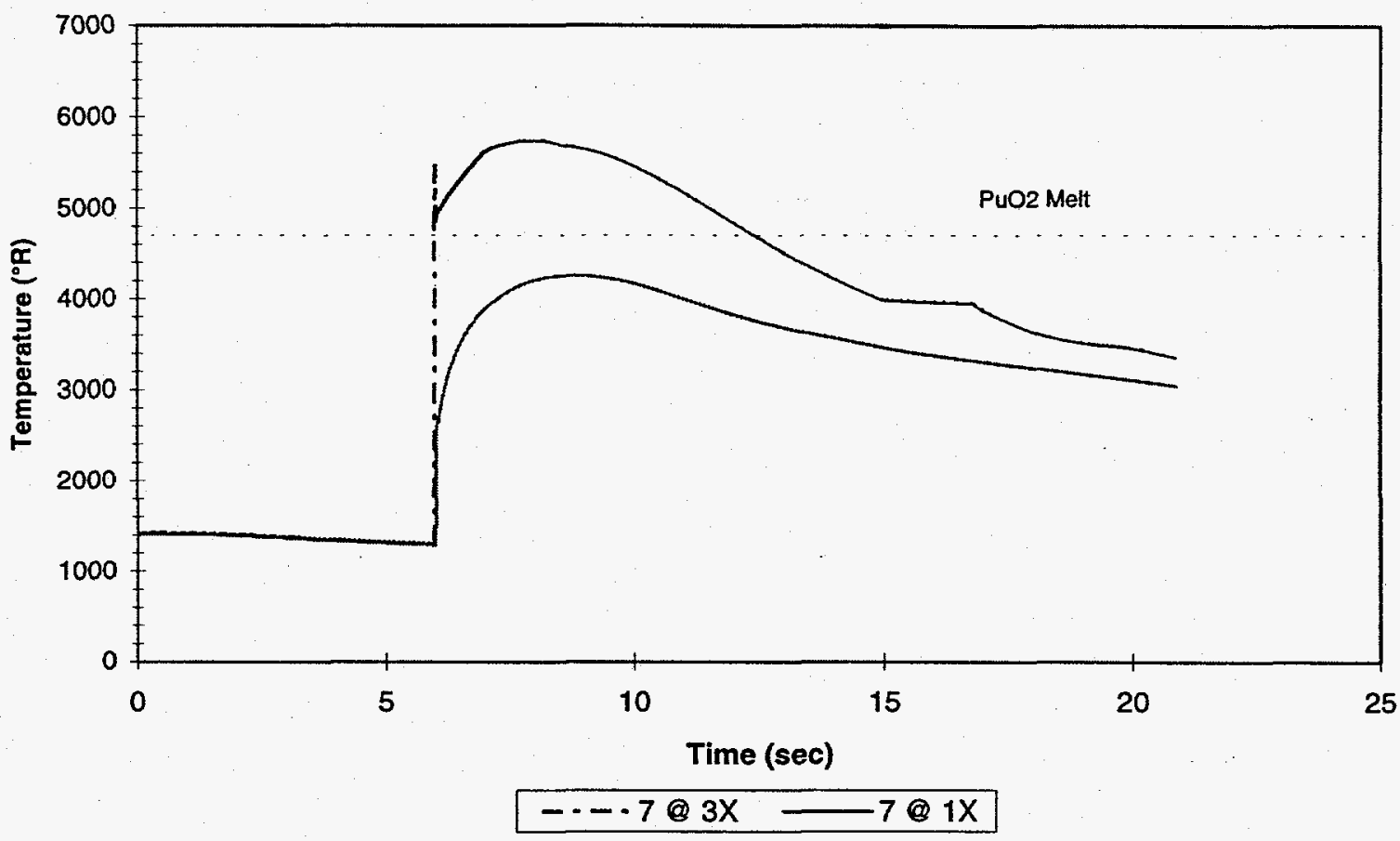

$100 \%$ Ablated

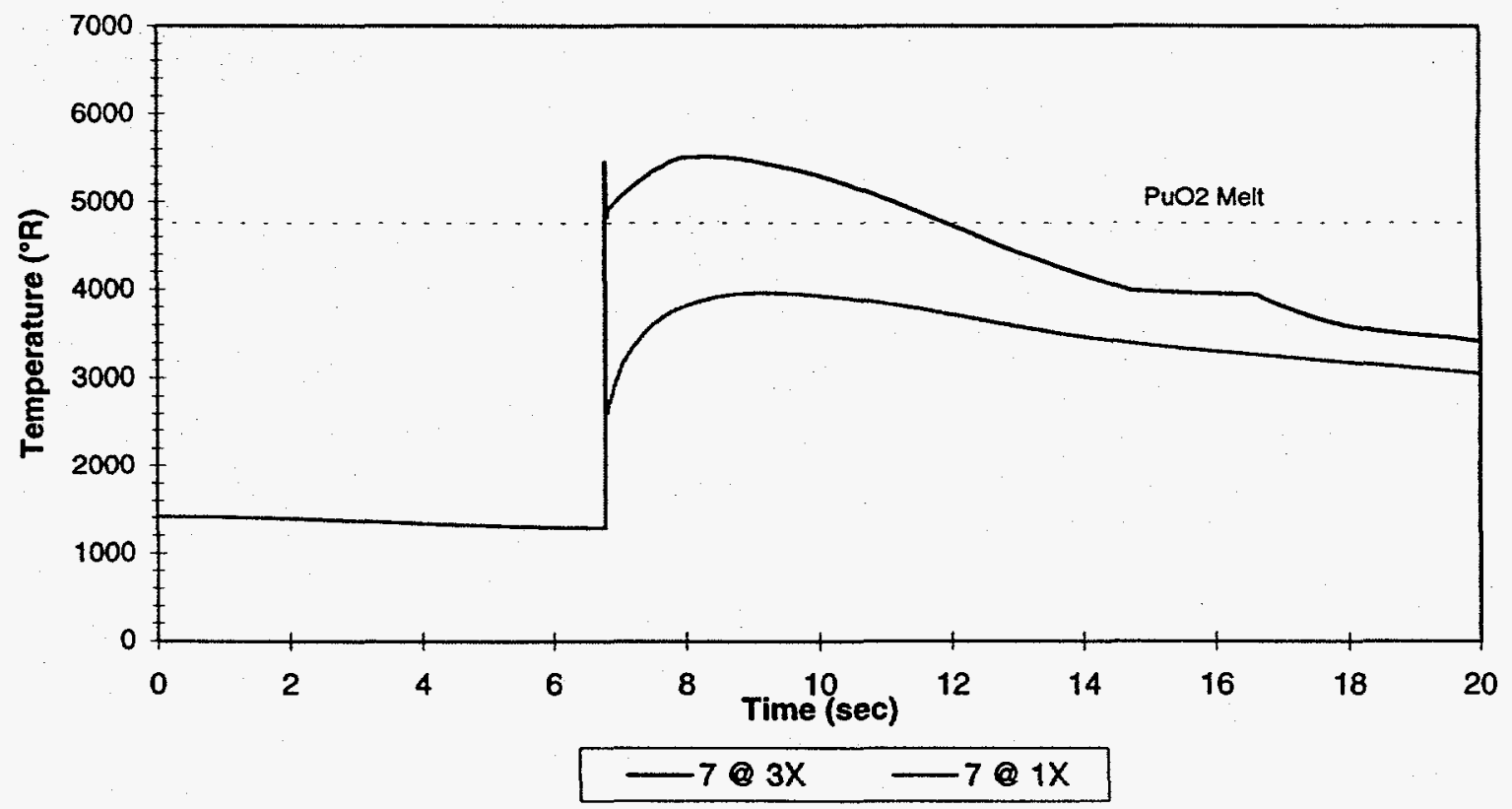


Figure 107. Cassini Mission LWRHU Fuel Results

Comparison of $-30^{\circ}$ Stable LWRHU Releasing Stable Fuel

Response at $1 X$ and $3 X$ Reference Convective Heating 75\% Ablated

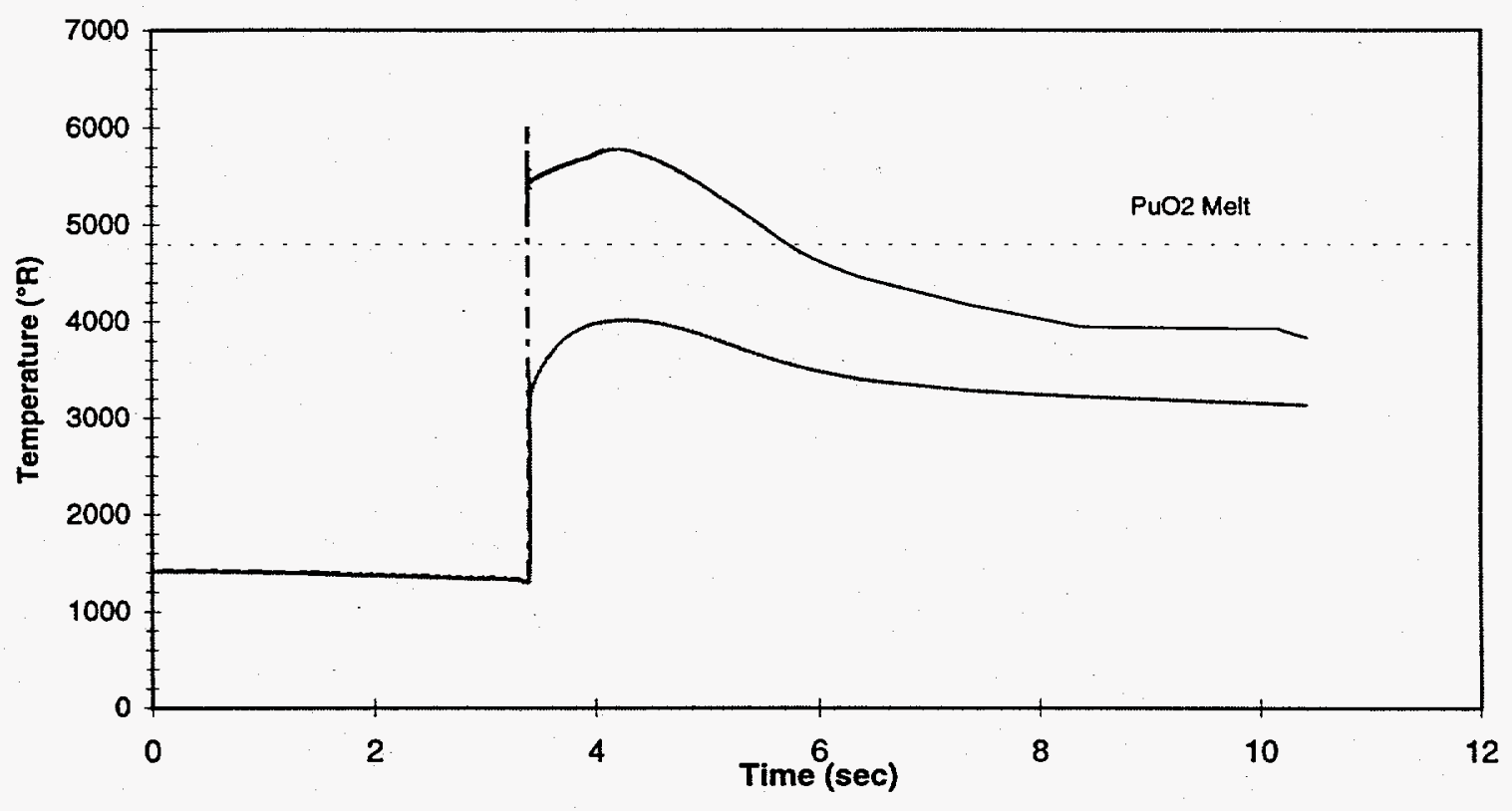

$100 \%$ Ablated

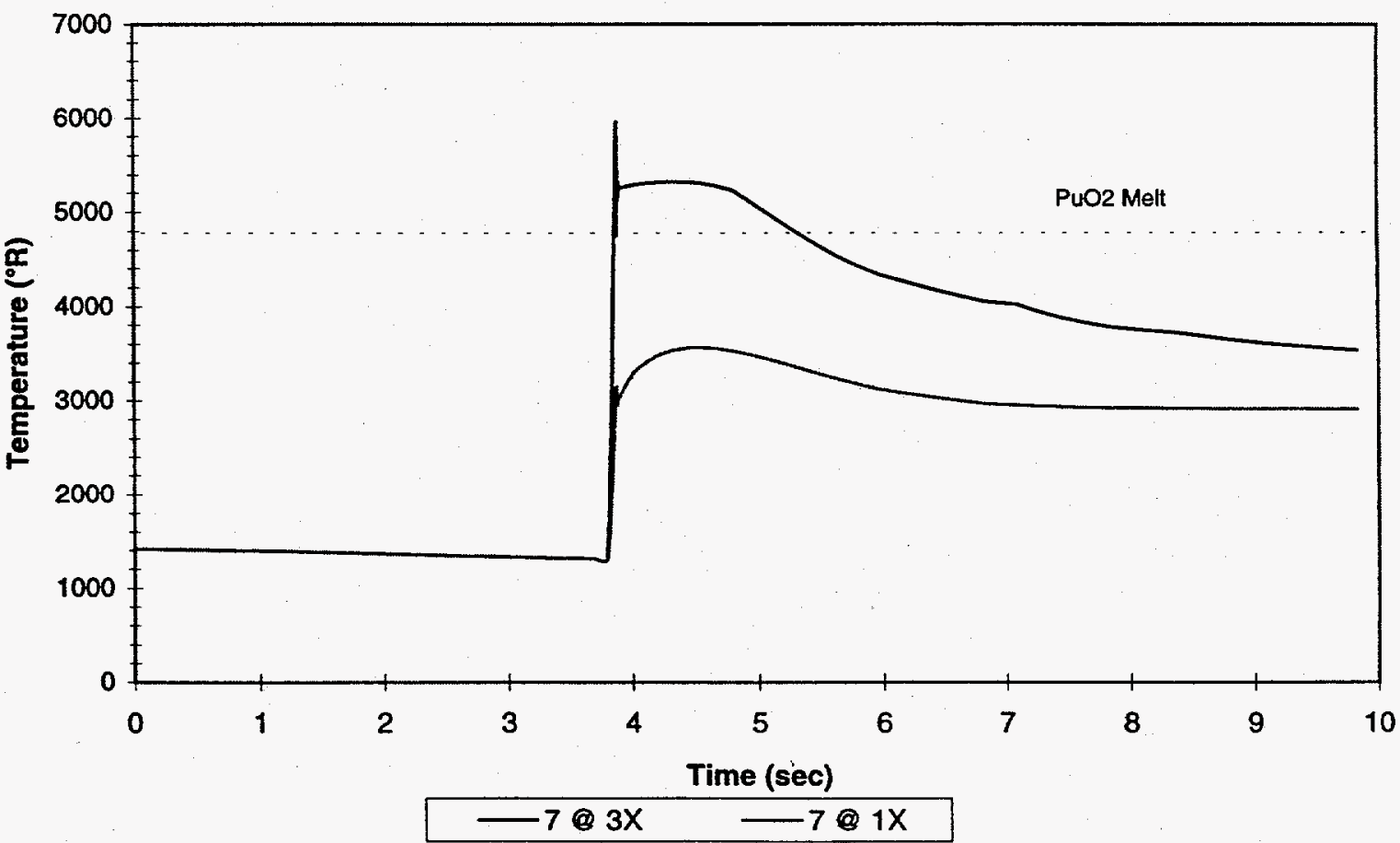


Figure 108. Cassini Mission LWRHU Fuel Results

Comparison of $-50^{\circ}$ Stable LWRHU Releasing Stable Fuel

Response at $1 X$ and $3 X$ Reference Convective Heating $75 \%$ Ablated

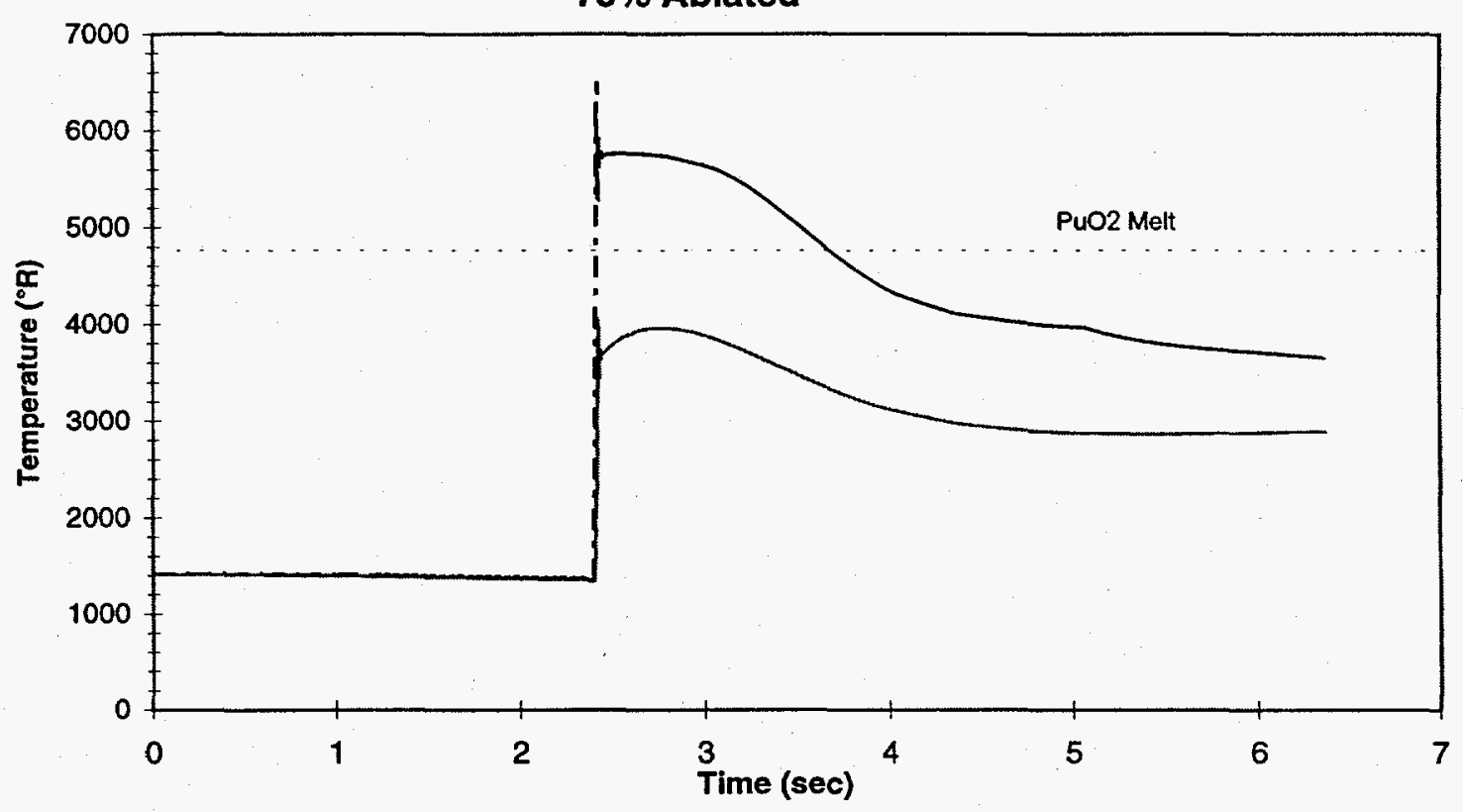

$100 \%$ Ablated

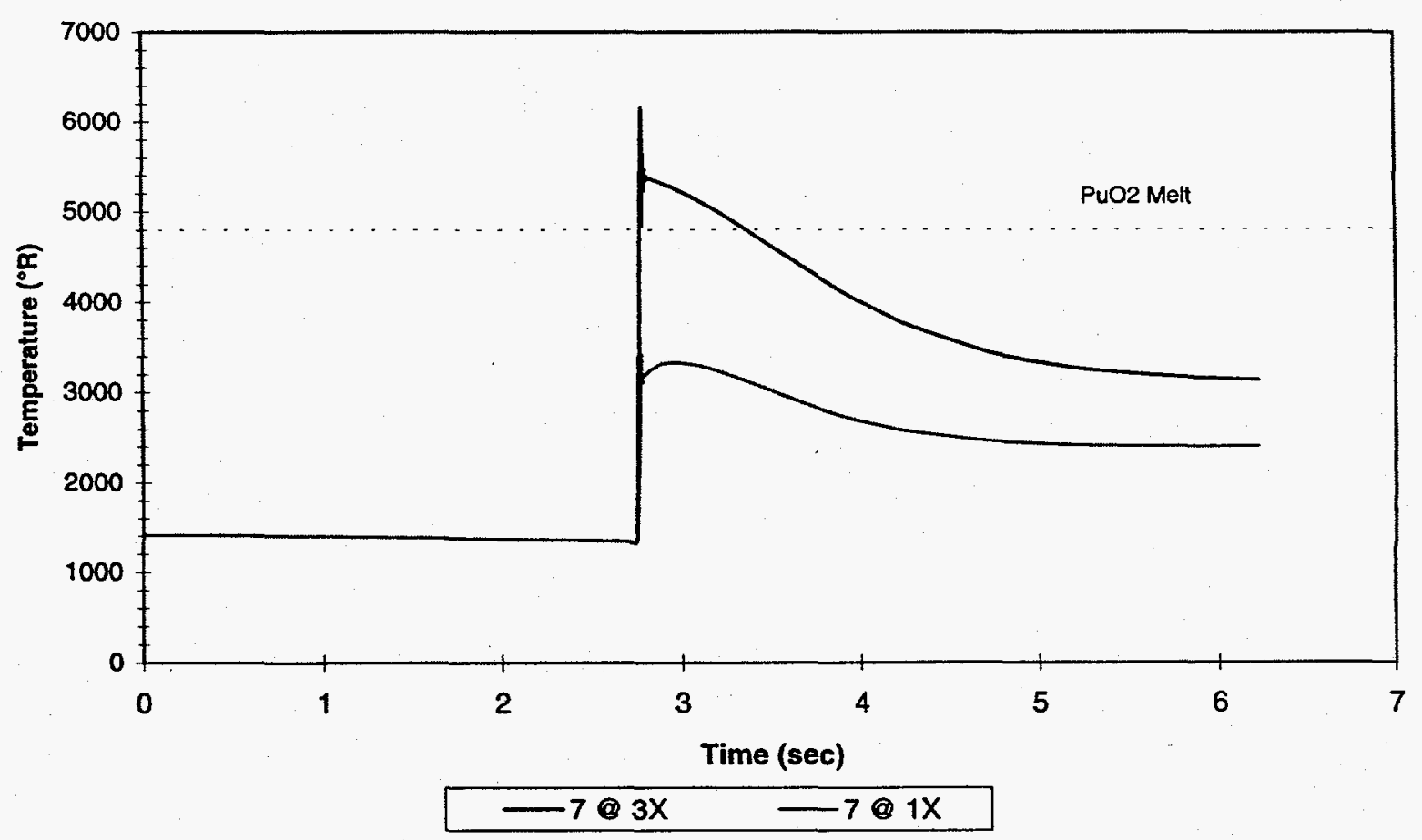


Applied Physics Laboratory

Laurel. Maryland 20723-60999

Figure 109. Cassini Mission LWRHU Fuel Results Comparison of $-70^{\circ}$ Stable LWRHU Releasing Stable Fuel Response at $1 \mathrm{X}$ and $3 \mathrm{X}$ Reference Convective Heating $75 \%$ Ablated

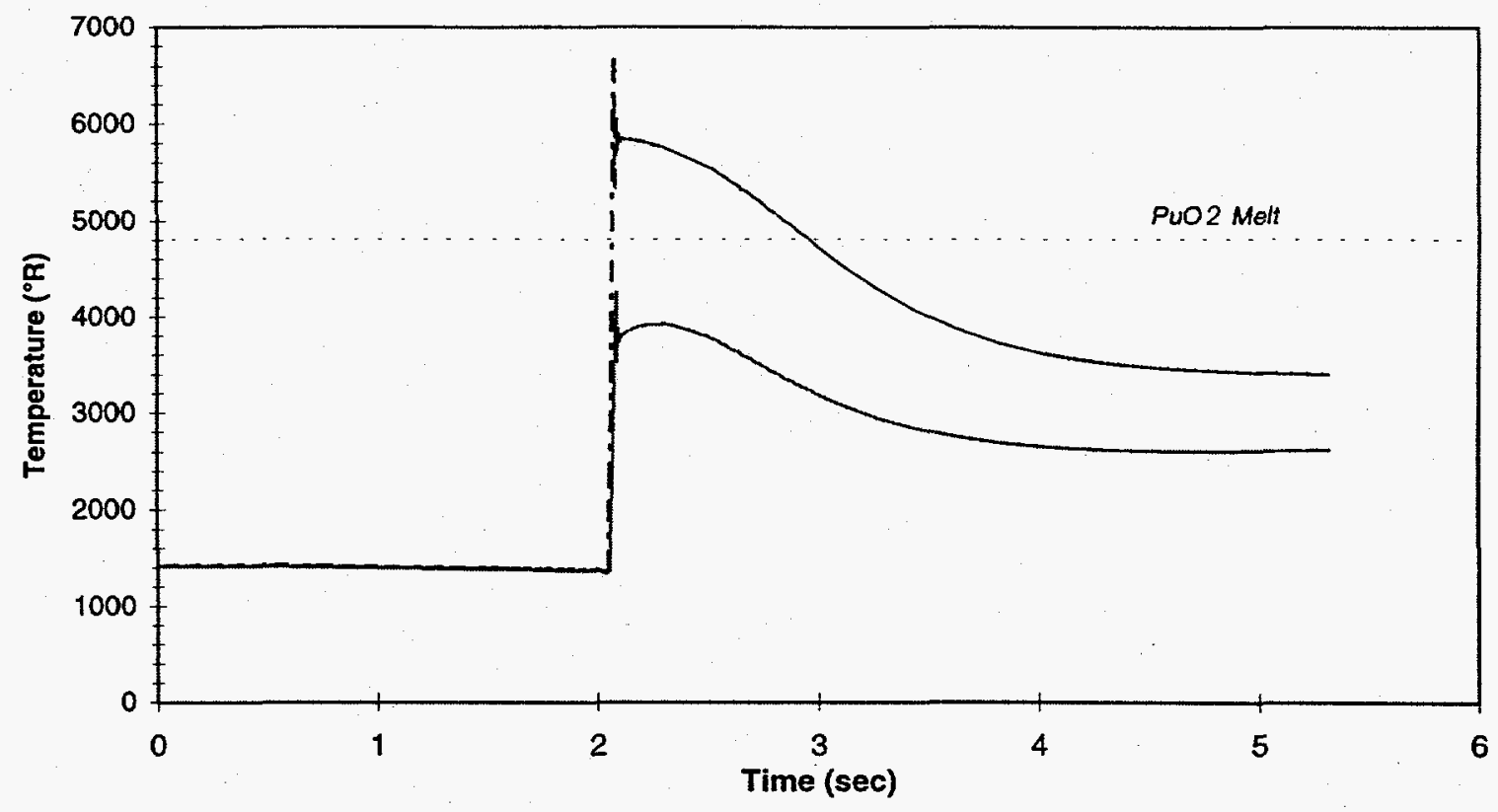

$100 \%$ Ablated

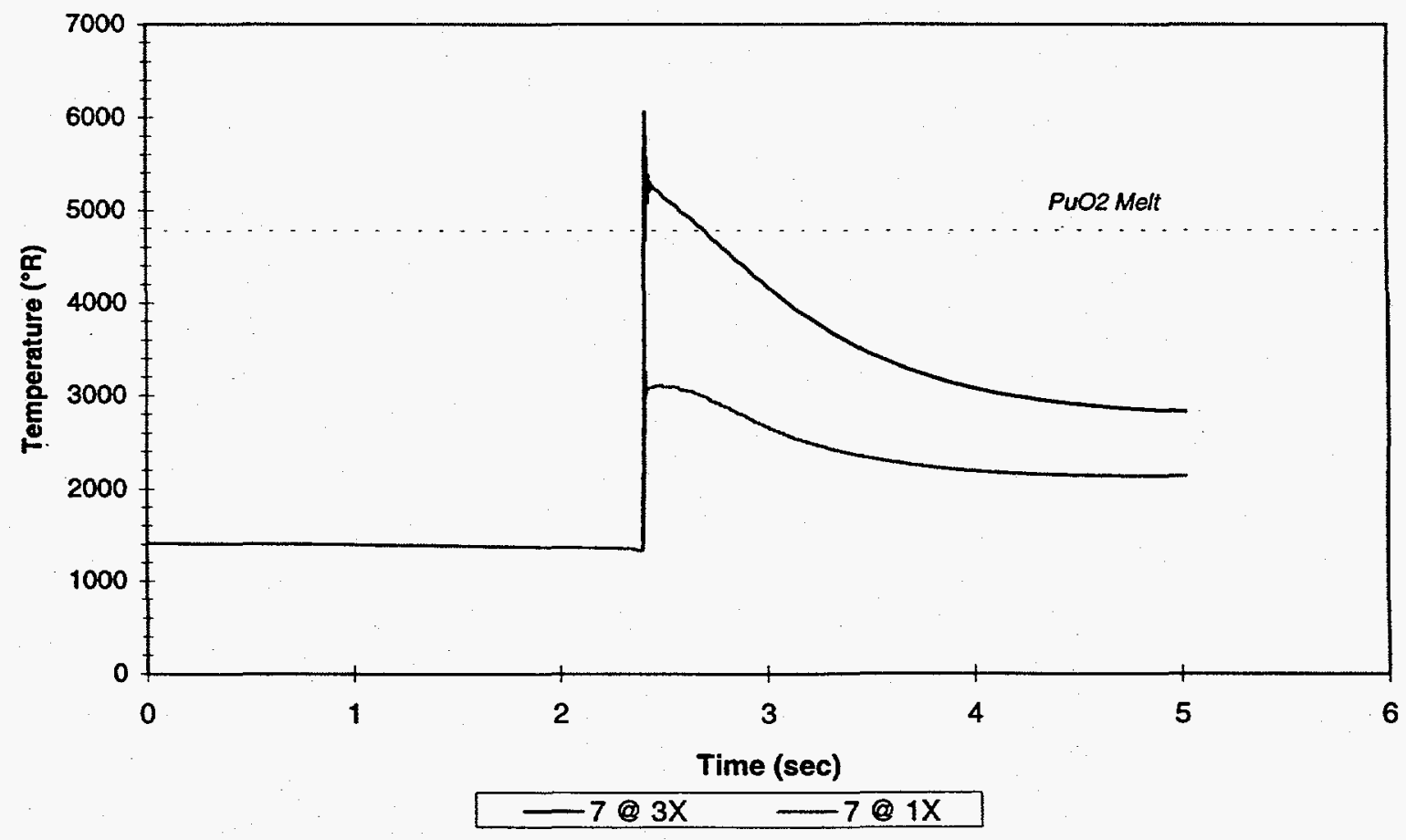


Figure 110. Cassini Mission LWRHU Fuel Results Comparison of $-90^{\circ}$ Stable LWRHU Releasing Stable Fuel Response at $1 X$ and $3 X$ Reference Convective Heating $75 \%$ Ablated

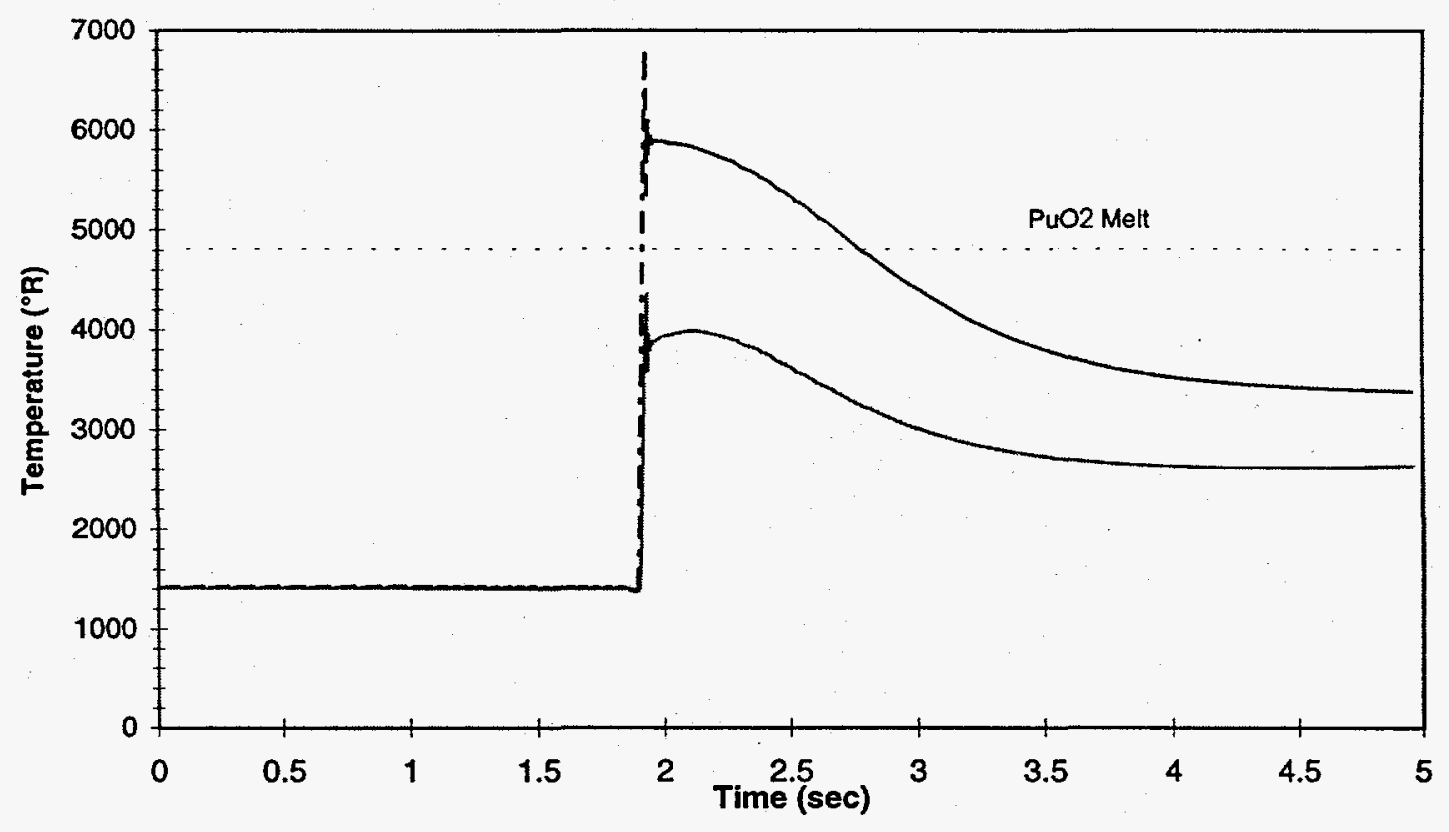

$100 \%$ Ablated

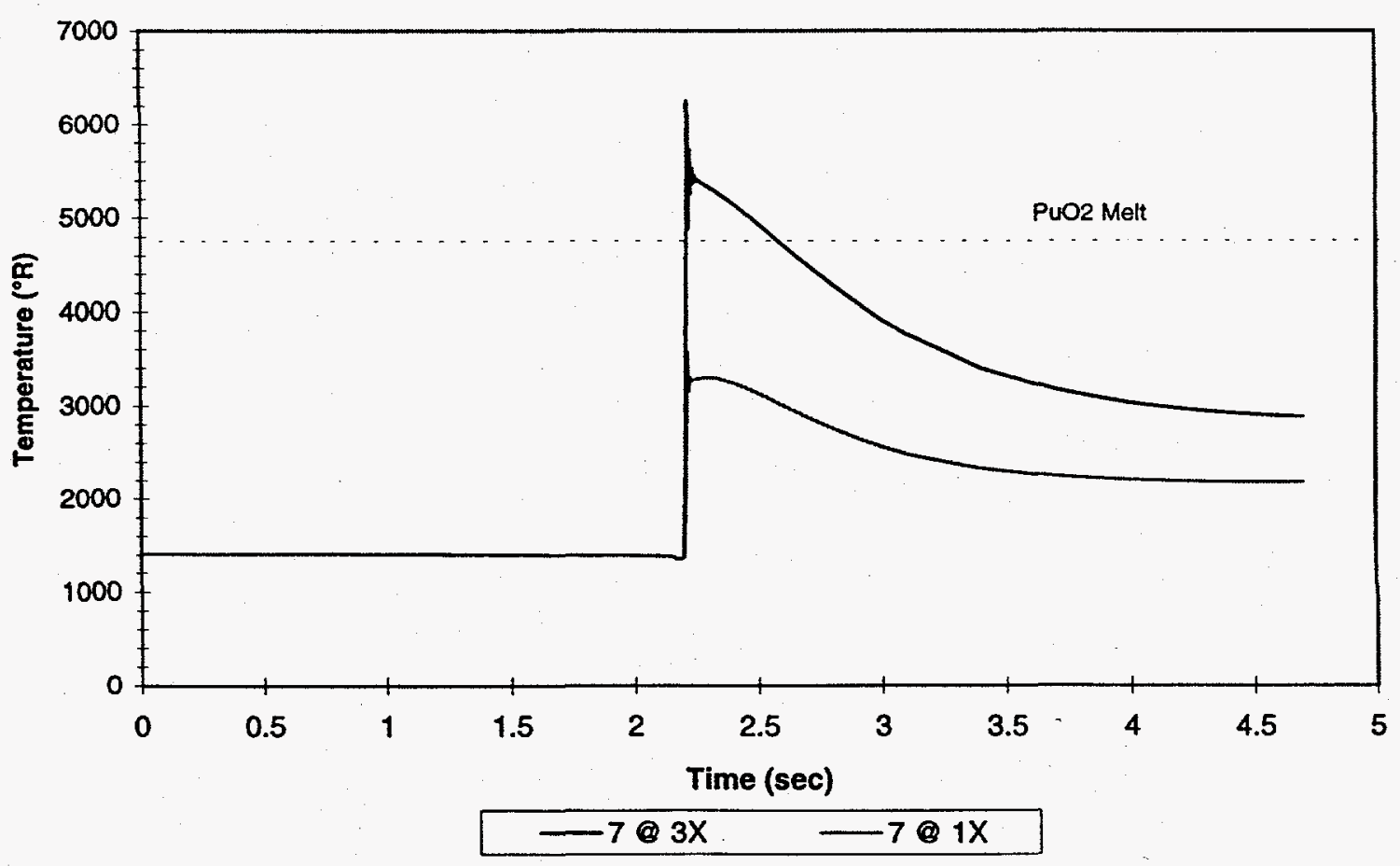


Figure 111. Cassini Mission LWRHU Fuel Results

75\% Ablated Side-on Stable LWRHU Releasing Spinning Fuel

$-7^{\circ}$ Reentry at Reference Convective Heating

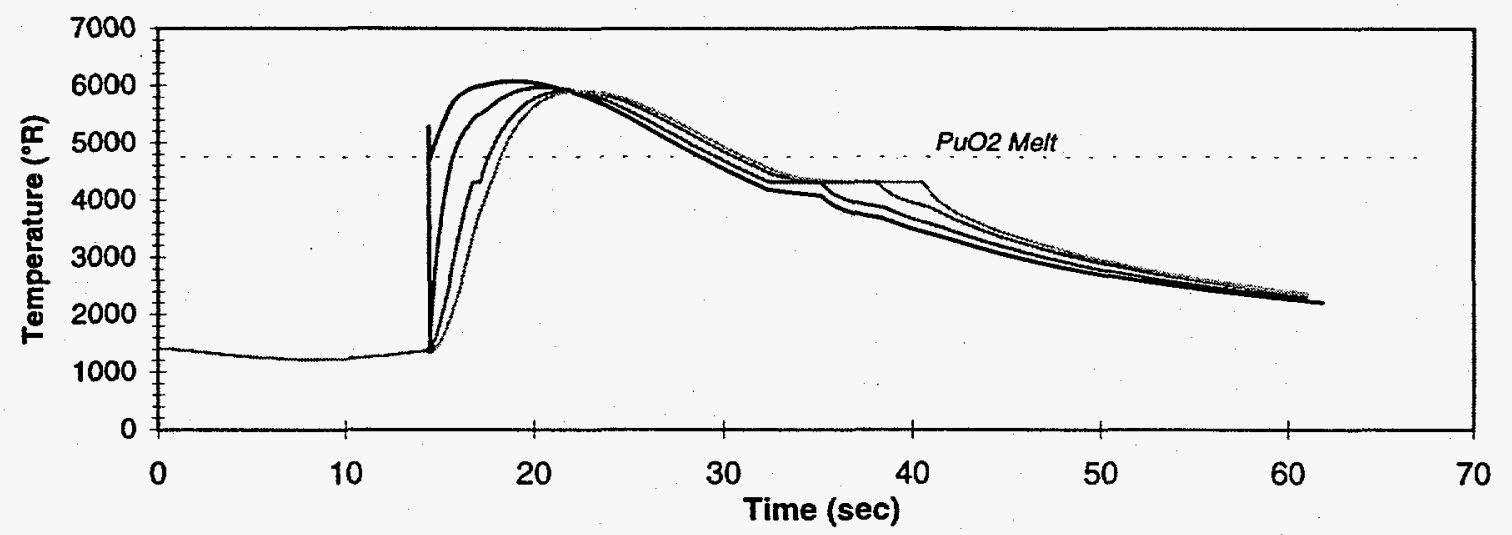

$-15^{\circ}$ Reentry at Reference Convective Heating
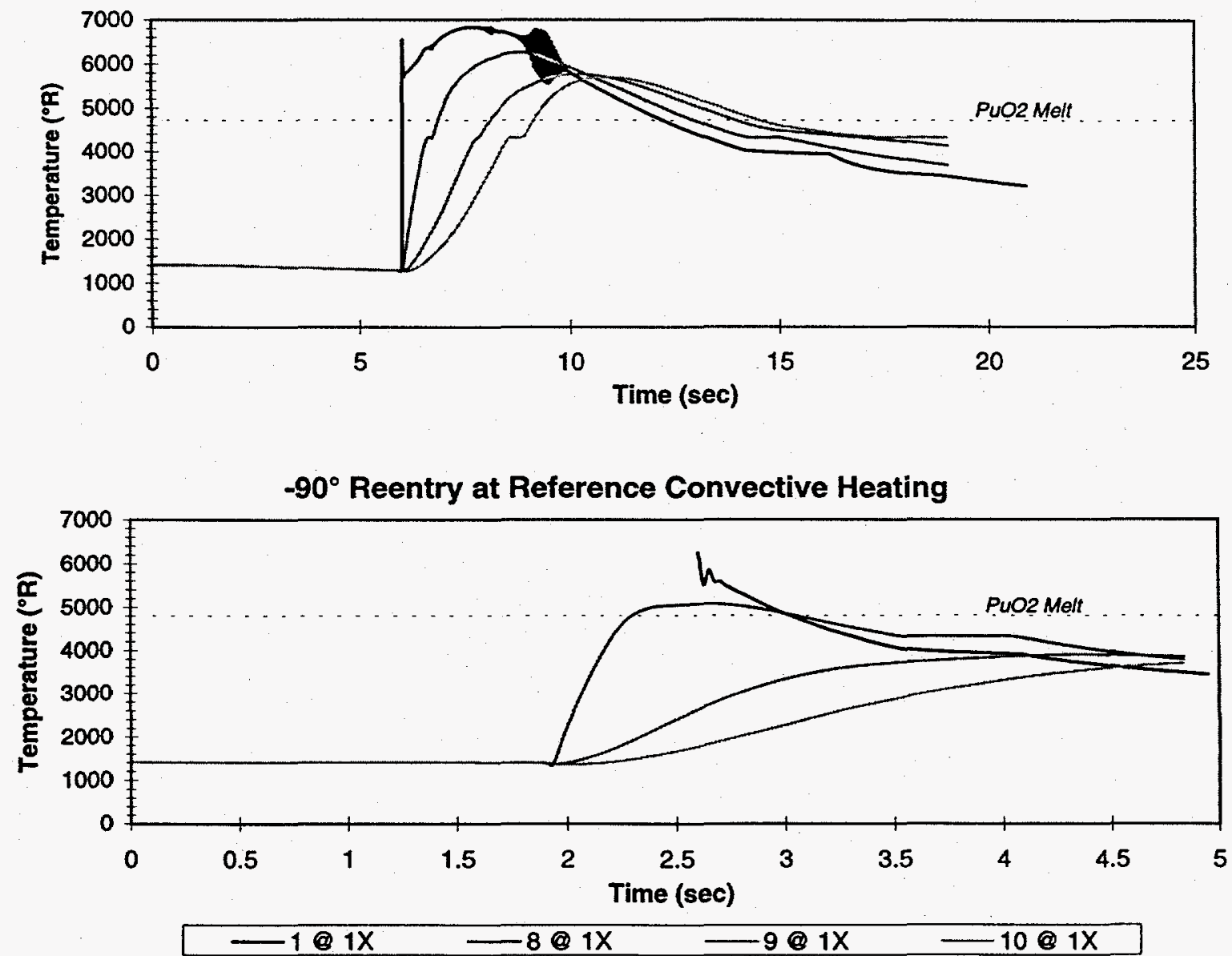
Figure 112. Cassini Mission LWRHU Fuel Results $100 \%$ Ablated Side-on Stable LWRHU Releasing Spinning Fuel $-7^{\circ}$ Reentry at Reference Convective Heating

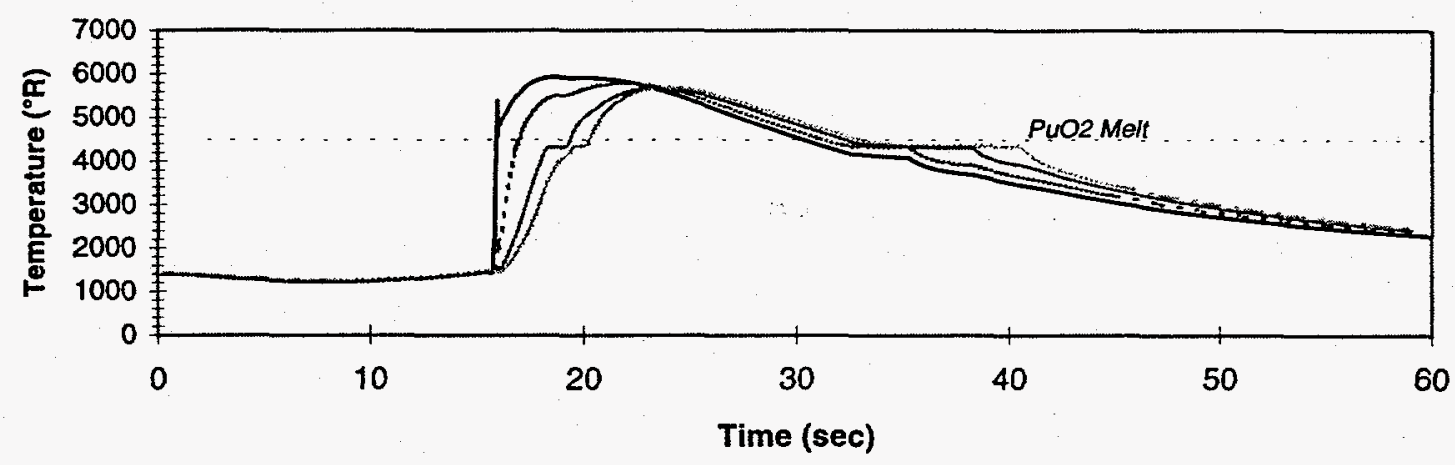

$-15^{\circ}$ Reentry at Reference Convective Heating

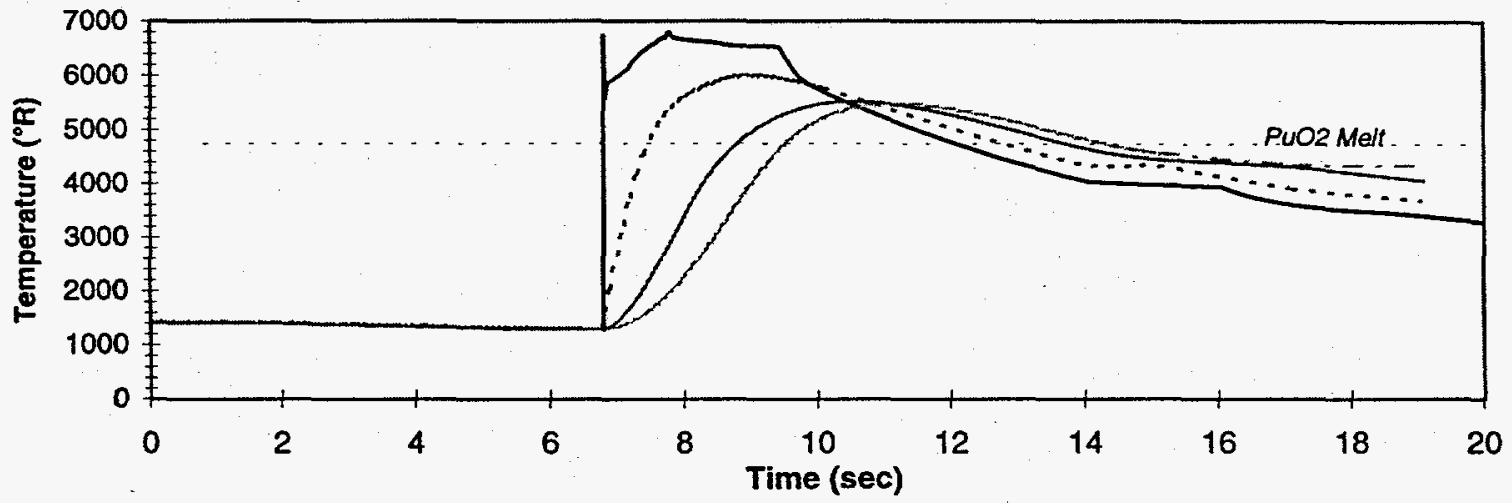

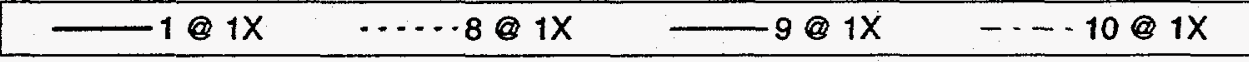

$-90^{\circ}$ Reentry at $3 \times$ Reference Convective Heating

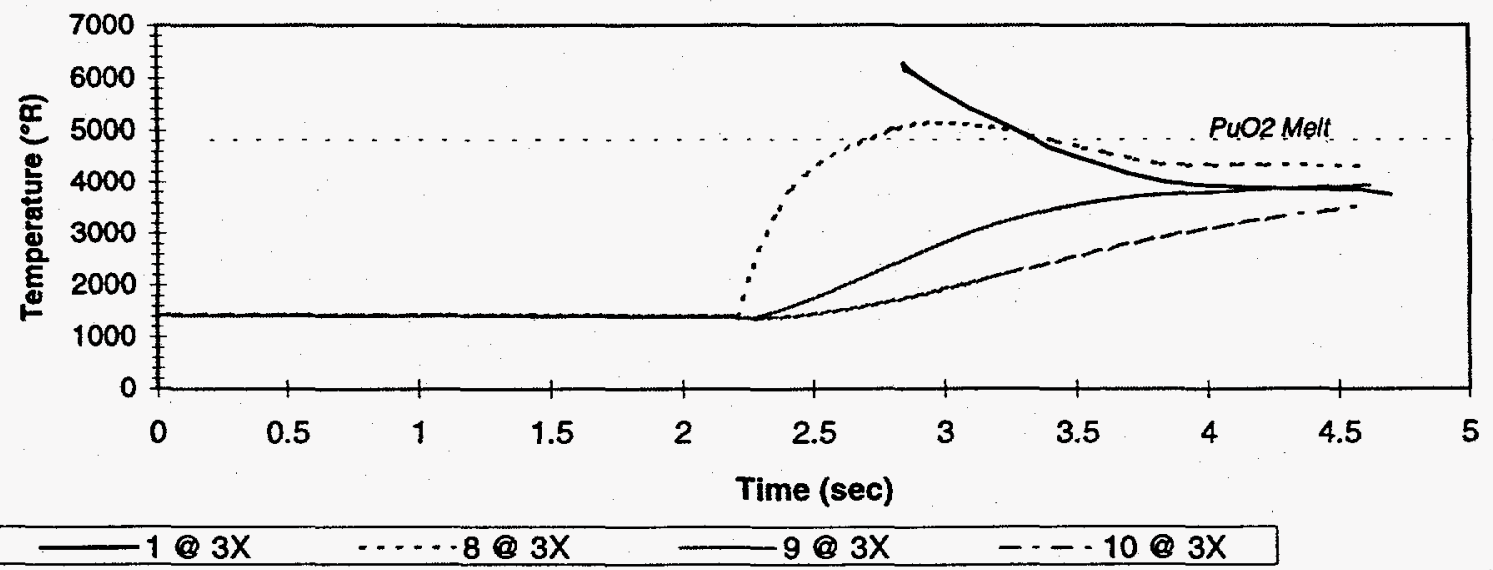


Figure 113. Cassini Mission LWRHU Fuel Results 75\% Ablated Stable LWRHU Releasing Tumbling Fuel

$-7^{\circ}$ Reentry at Reference Convective Heating

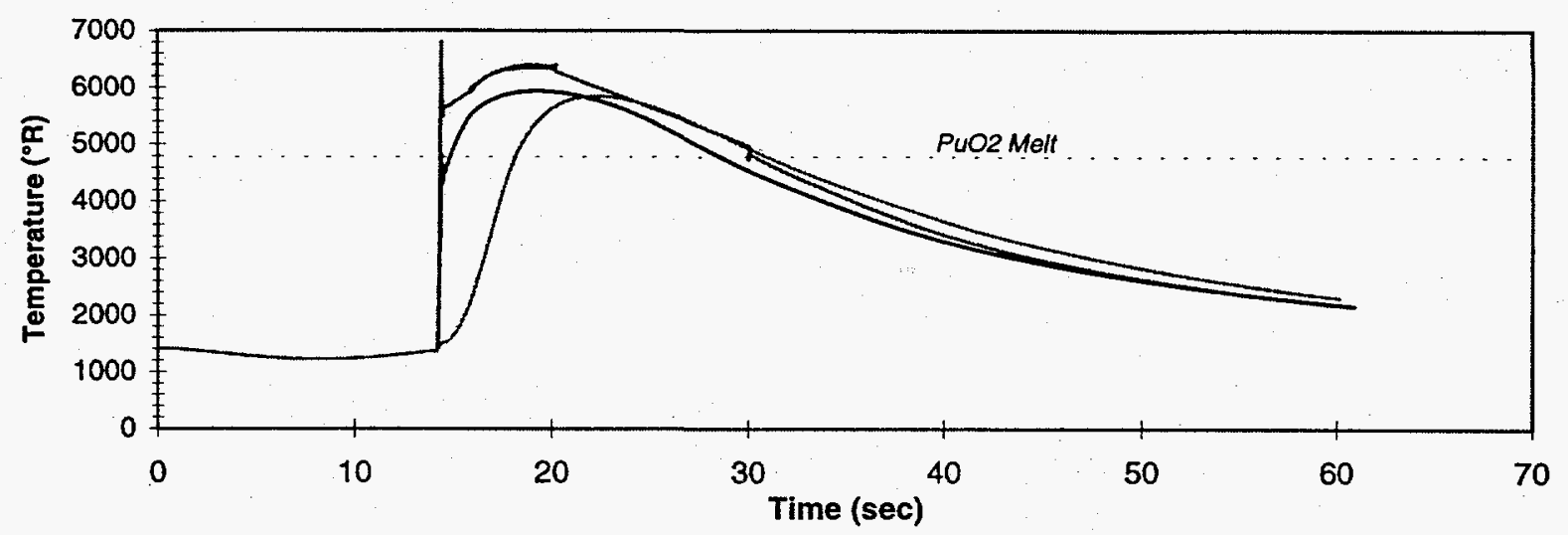

$-15^{\circ}$ Reentry at Reference Convective Heating
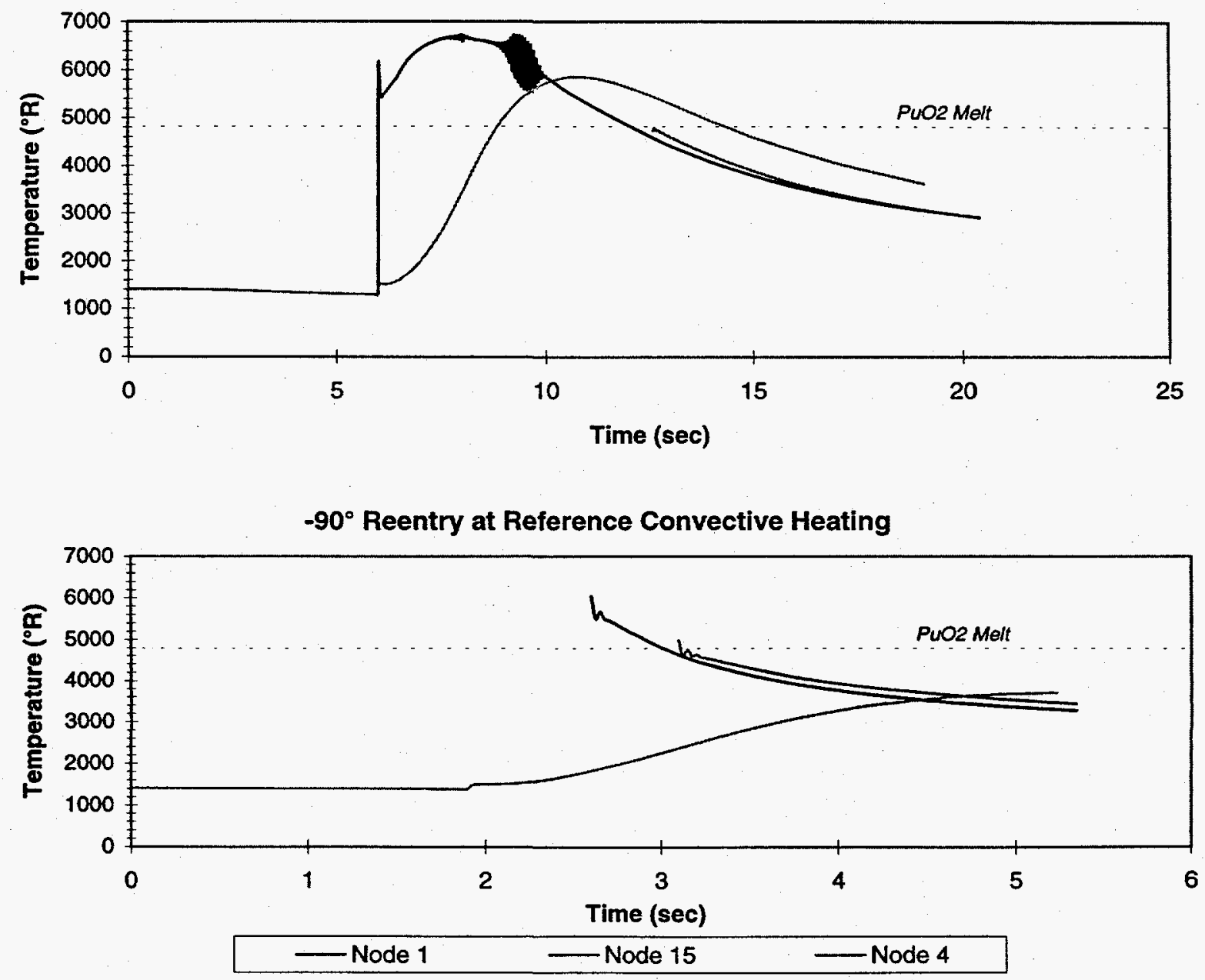
Figure 114. Cassini Mission LWRHU Fuel Results $100 \%$ Ablated Stable LWRHU Releasing Tumbling Fuel $-7^{\circ}$ Reentry at Reference Convective Heating

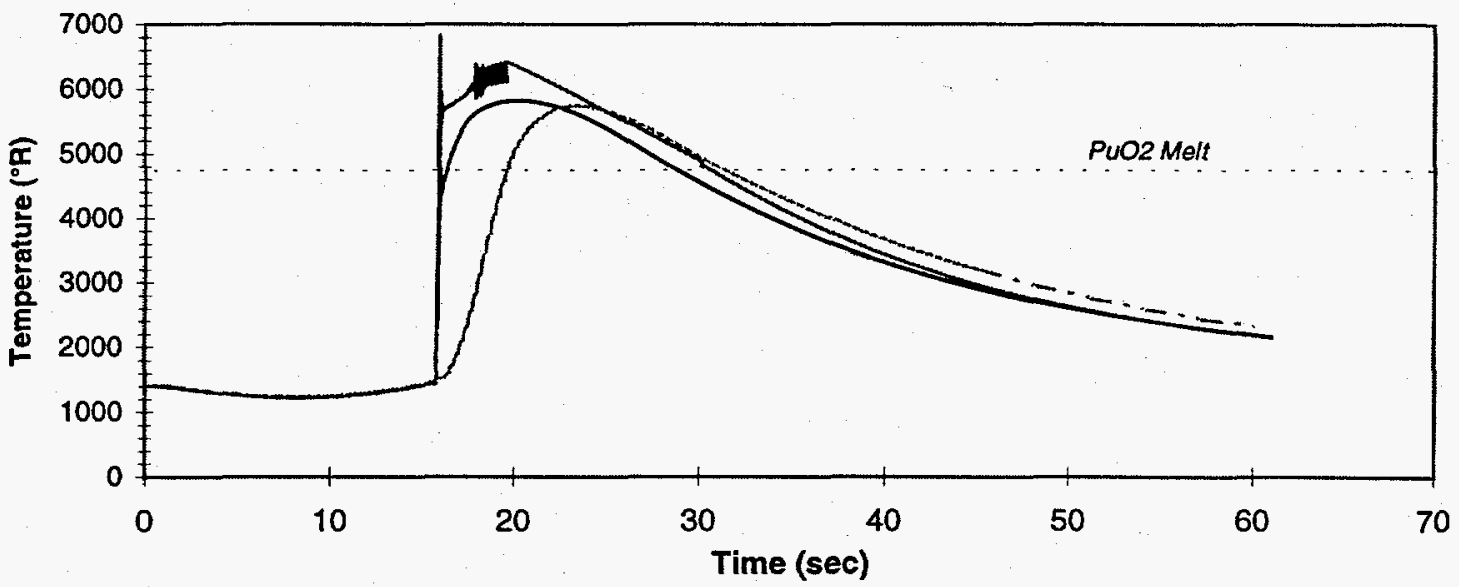

$-15^{\circ}$ Reentry at Reference Convective Heating

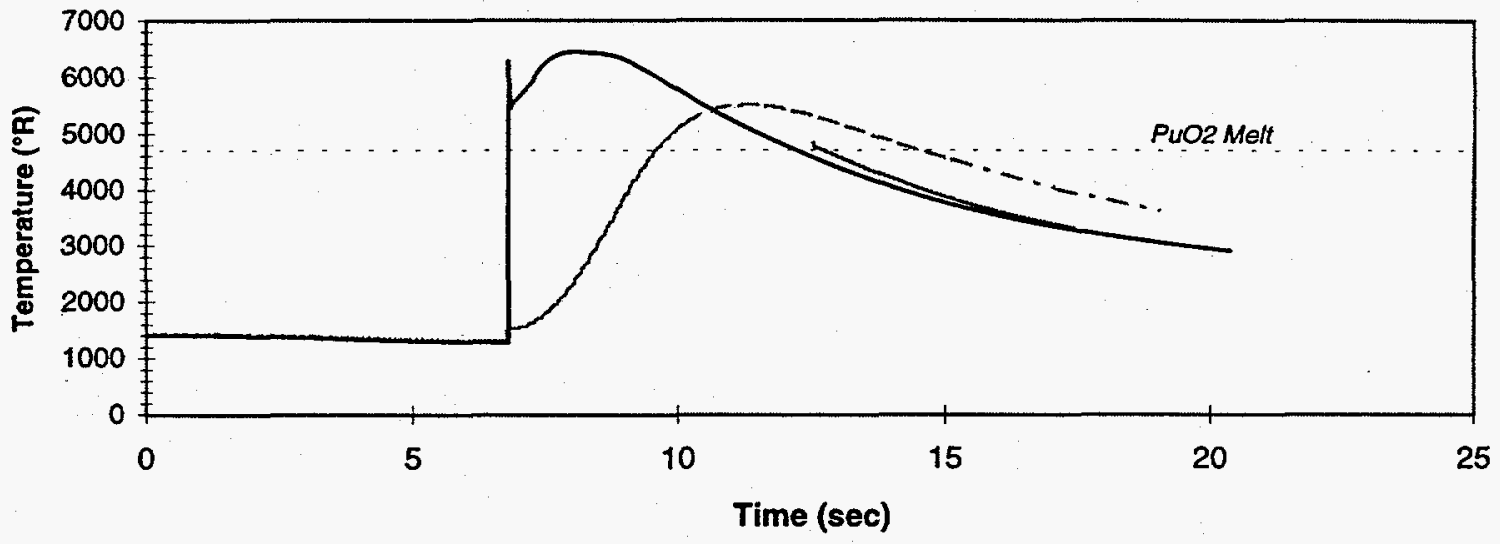

$-90^{\circ}$ Reentry at Reference Convective Heating

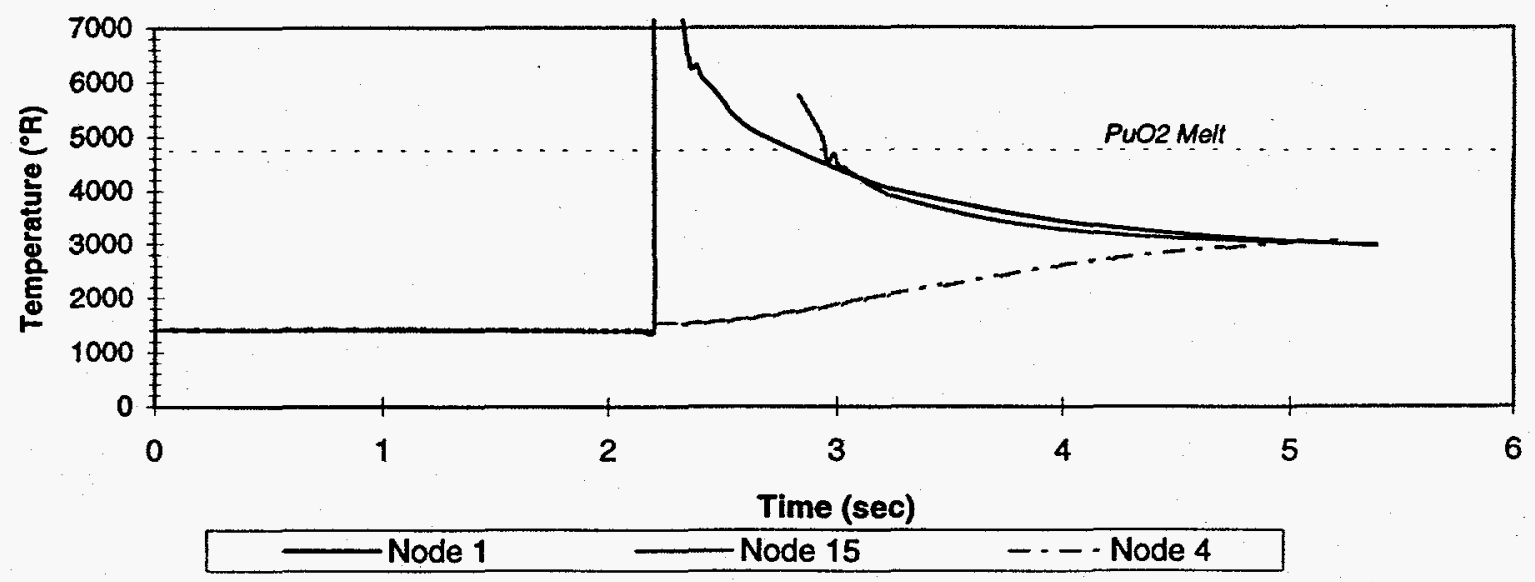


Figure 115. Cassini Mission LWRHU Fuel Results Full Convective Heating

75\% Ablated $-5.2^{\circ}$ Spinning LWRHU Releasing Stable Fuel

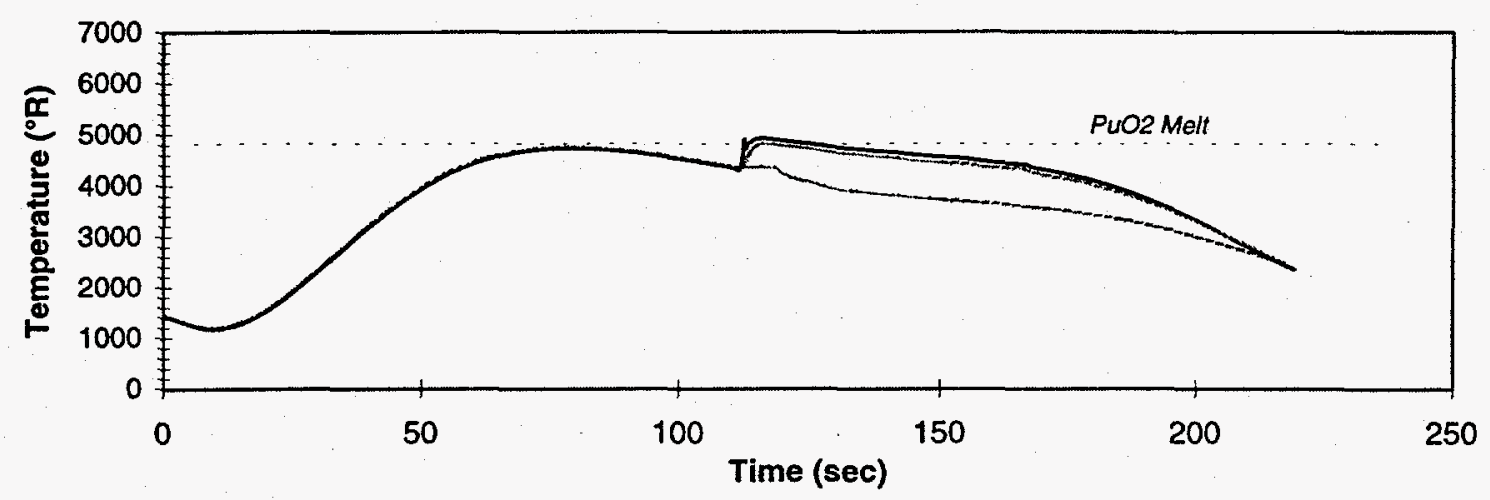

75\% Ablated $-5.2^{\circ}$ Spinning LWRHU Releasing Spinning Fuel

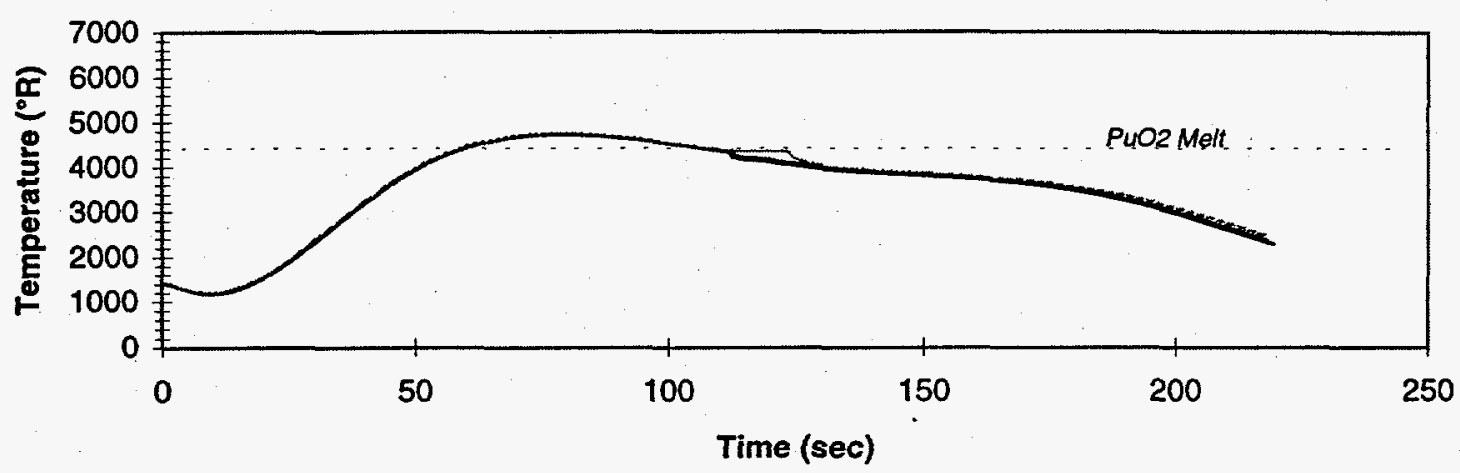

75\% Ablated $-5.2^{\circ}$ Spinning LWRHU Releasing Tumbling Fuel

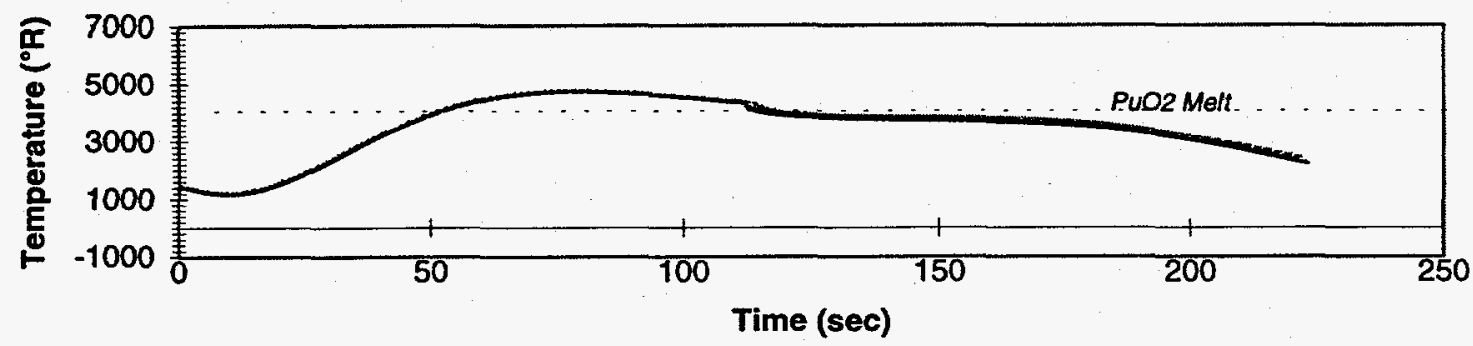

c:Iretaplfuelvallsh2.xls Node $1-$ Node $15-\cdots-$ Node 4 
Figure 116. Cassini Mission LWRHU Fuel Results Full Convective Heating

$75 \%$ Ablated $-10^{\circ}$ Tumbling LWRHU Releasing Stable Fuel

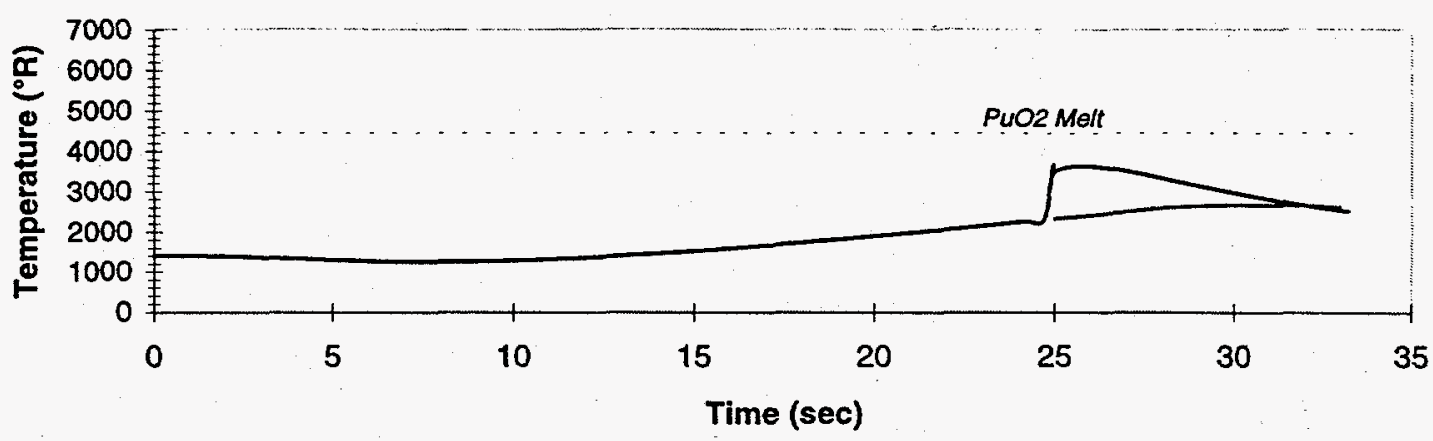

75\% Ablated $-7.0^{\circ}$ Tumbling LWRHU Releasing Stable Fuel

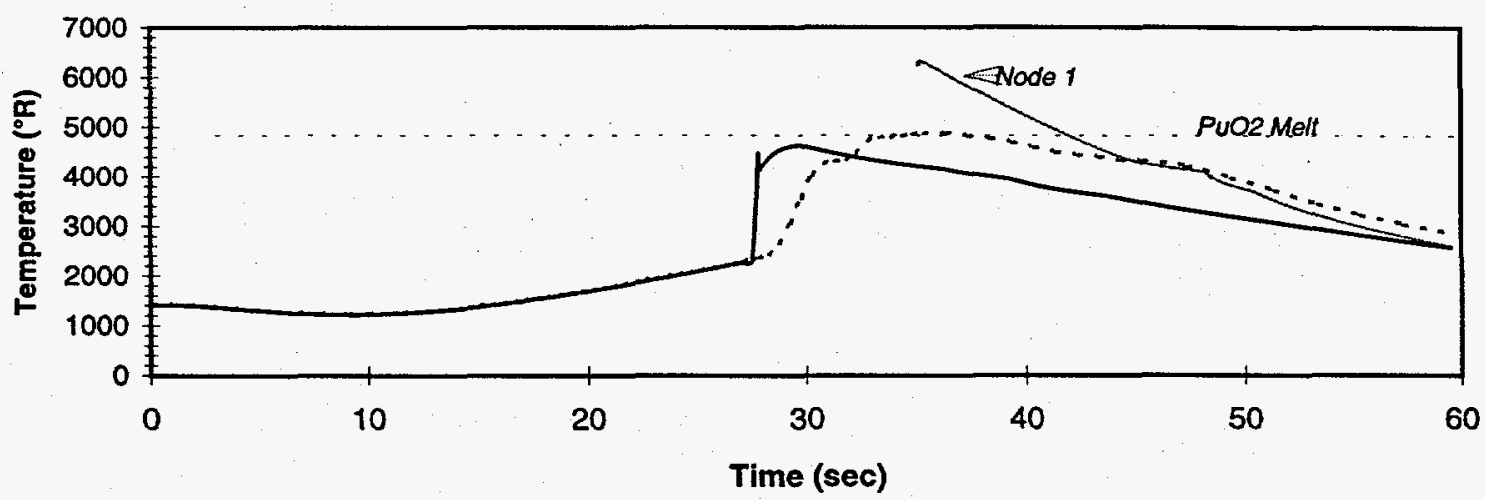

75\% Ablated $-5.2^{\circ}$ Tumbling LWRHU Releasing Stable Fuel

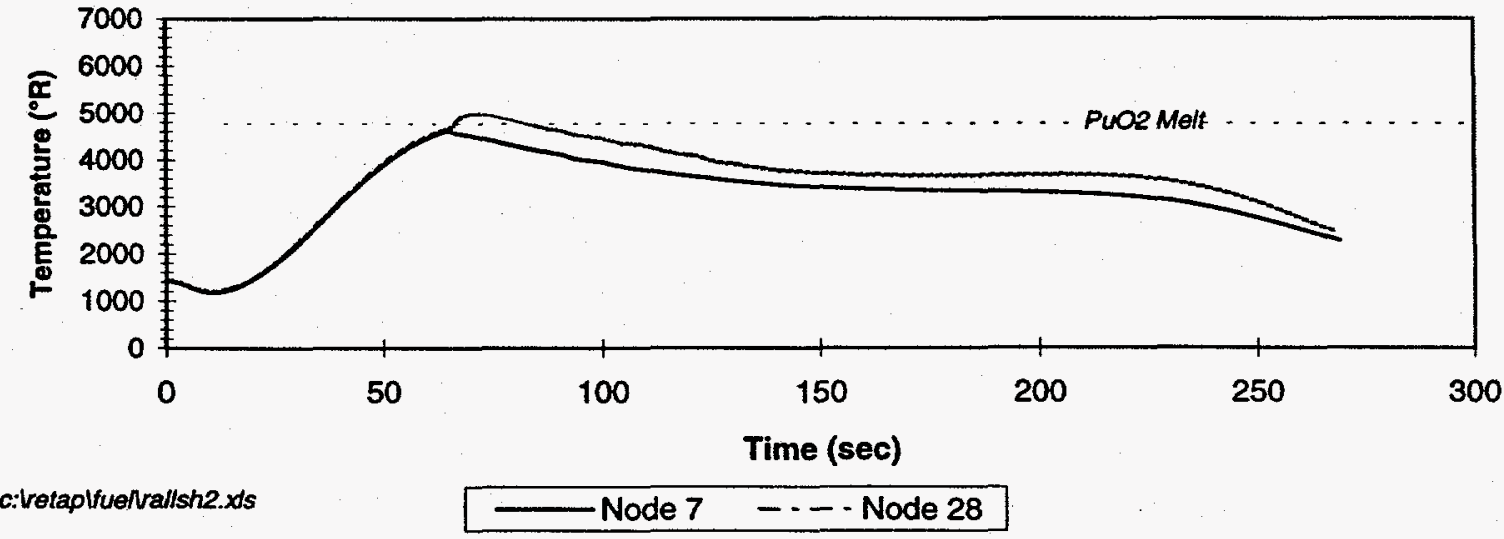


Figure 117. Cassini Mission LWRHU Fuel Results Full Convectlve Heating $75 \%$ Ablated $-10^{\circ}$ Tumbling LWRHU Releasing Spinning Fuel

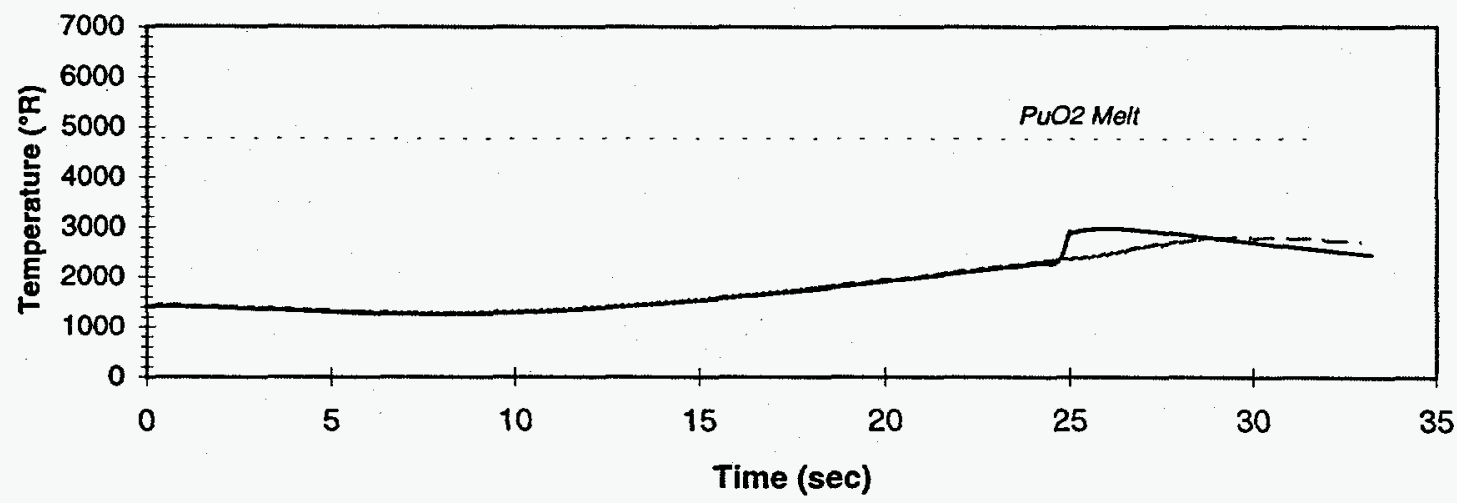

75\% Ablated $-7.0^{\circ}$ Tumbling LWRHU Releasing Spinning Fuel

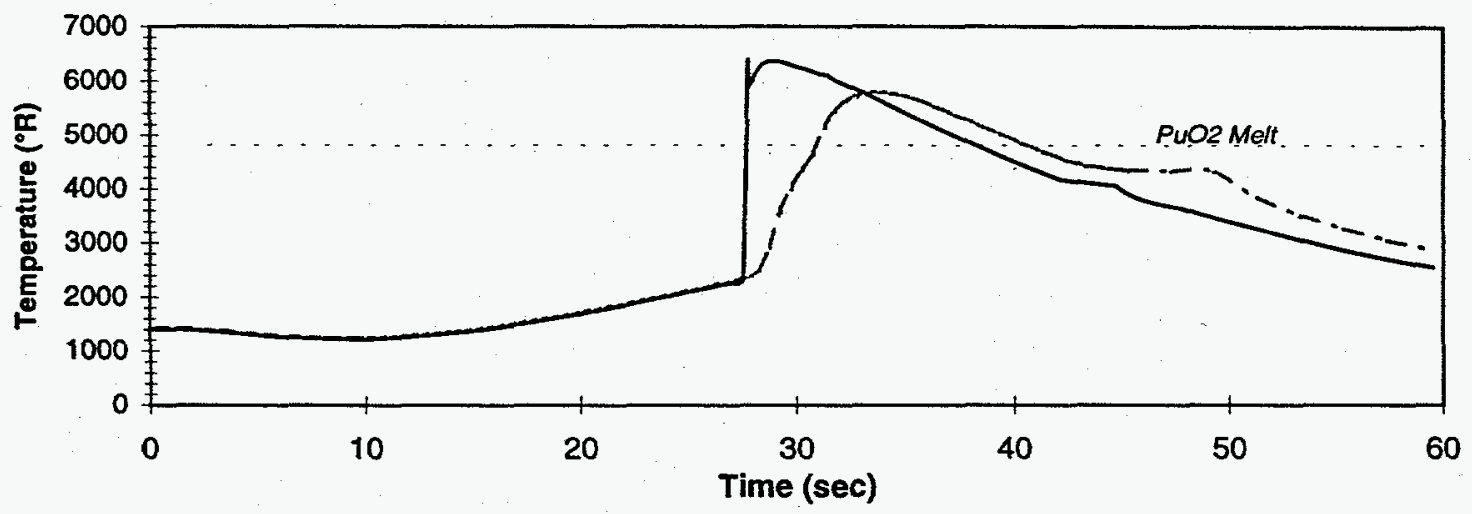

75\% Ablated $-5.2^{\circ}$ Tumbling LWRHU Releasing Spinning Fuel

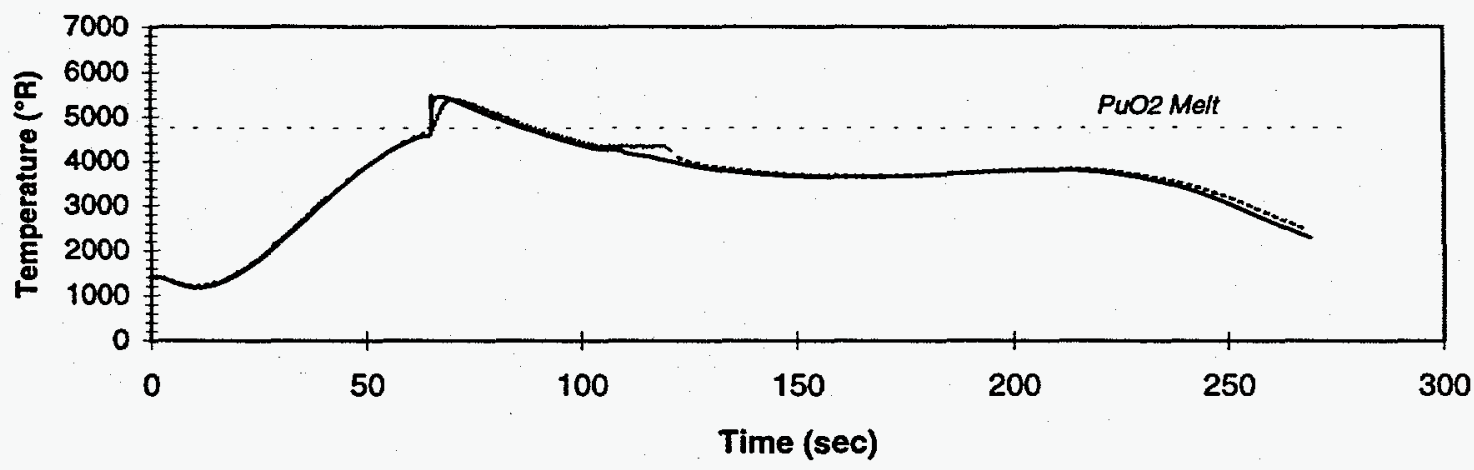

c:Vetaplfuenrallsh2.xis

Node 1 .... Node 28 
Figure 118. Cassini Mission LWRHU Fuel Results

Full Convective Heating

$75 \%$ Ablated $-10^{\circ}$ Tumbling LWRHU Releasing Tumbling Fuel

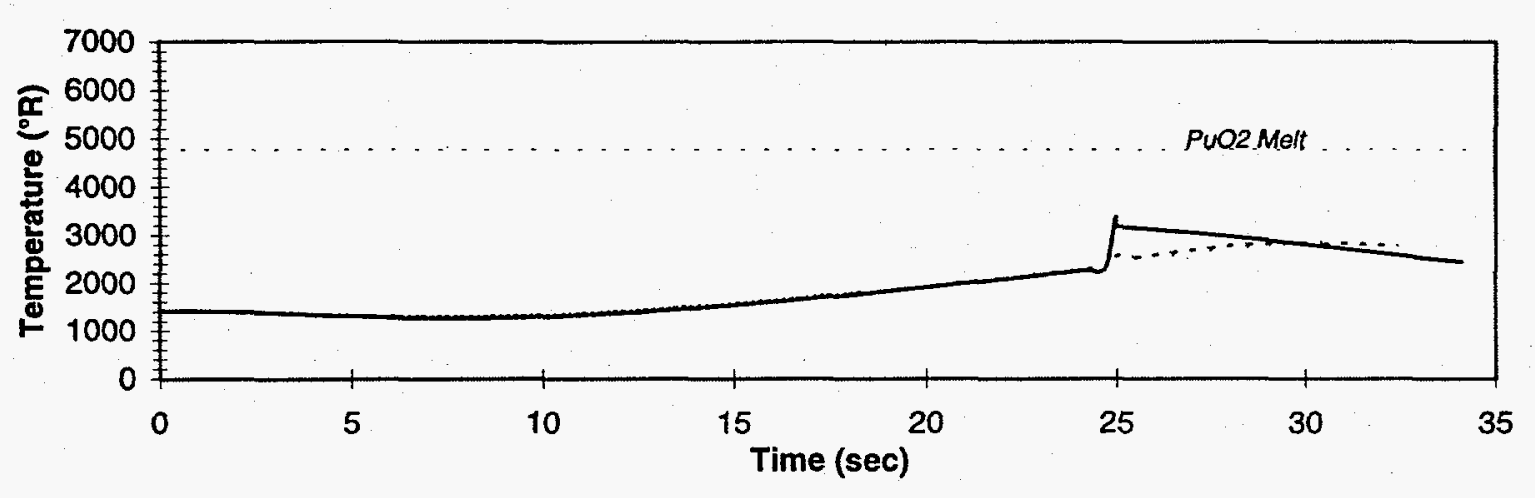

75\% Ablated $-7^{\circ}$ Tumbling LWRHU Releasing Tumbling Fuel

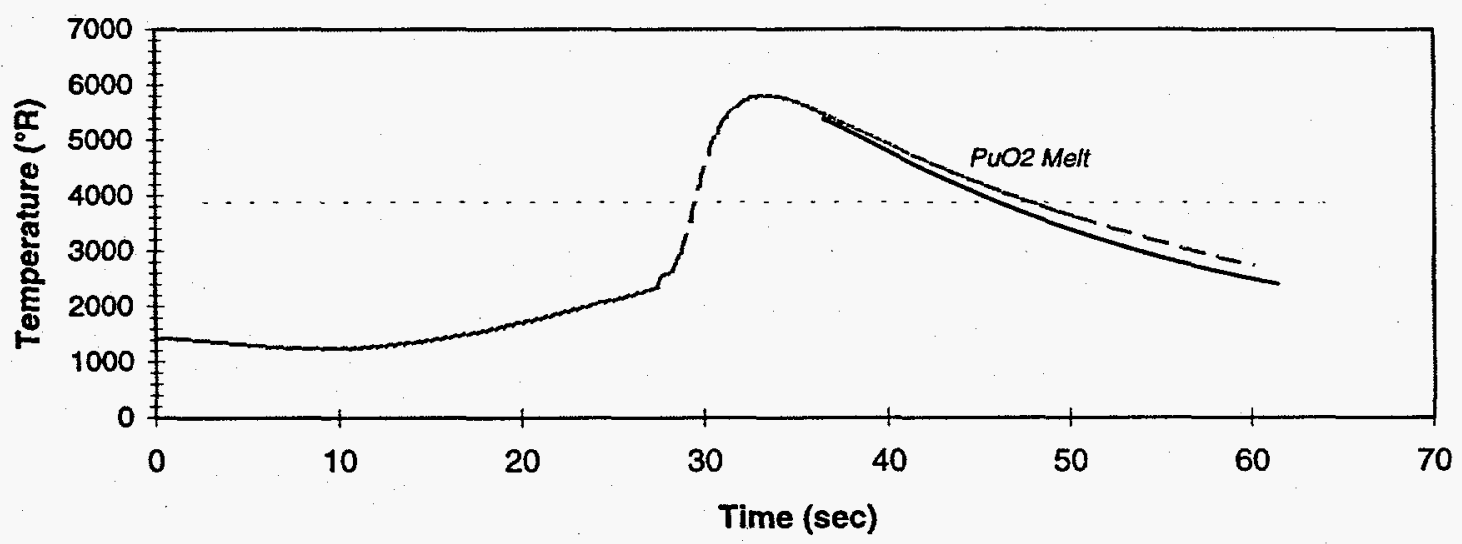

75\% Ablated $-5.2^{\circ}$ Tumbling LWRHU Releasing Tumbling Fuel

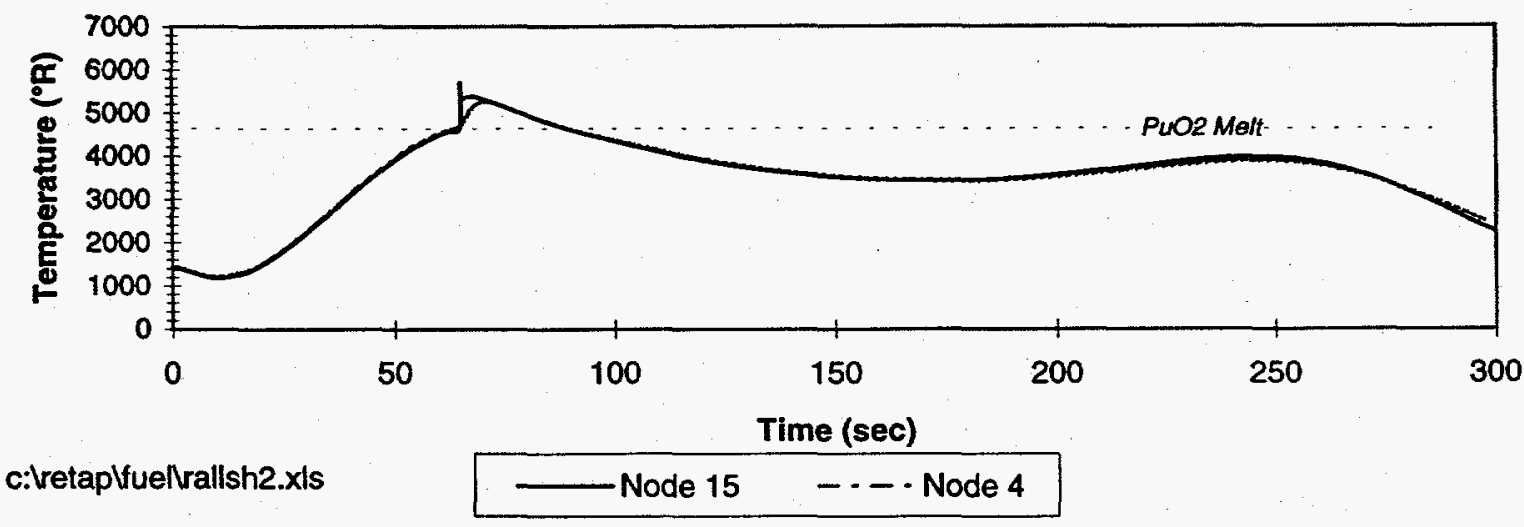




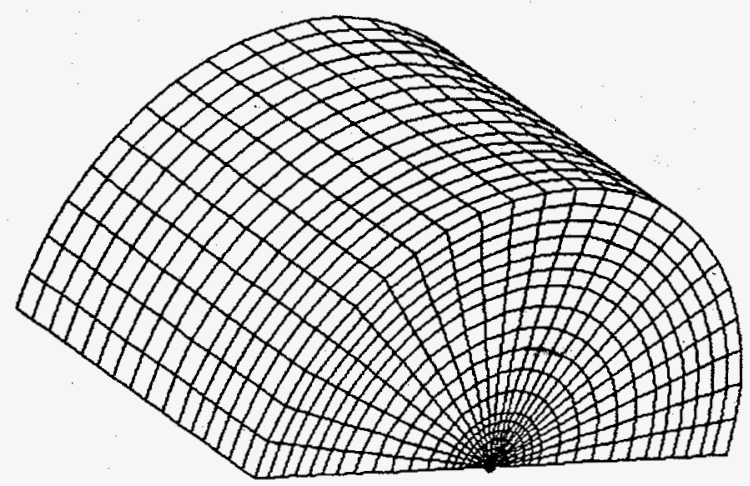

$x_{x}^{2}$
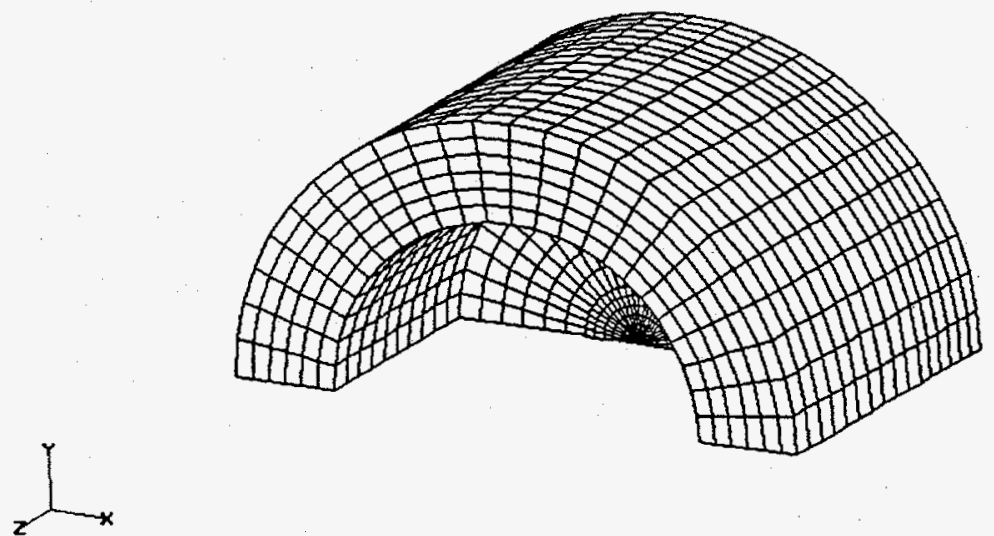

4932 Nodes

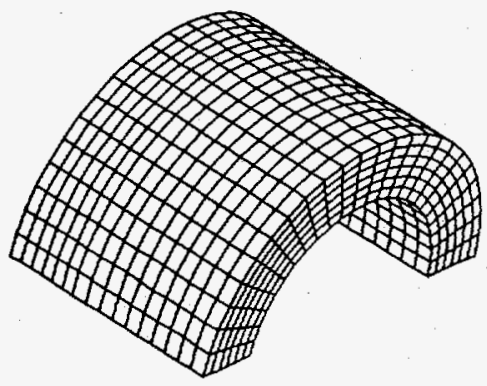
4056 Elements

Figure 119

Thermostructural Model for the LWRHU 

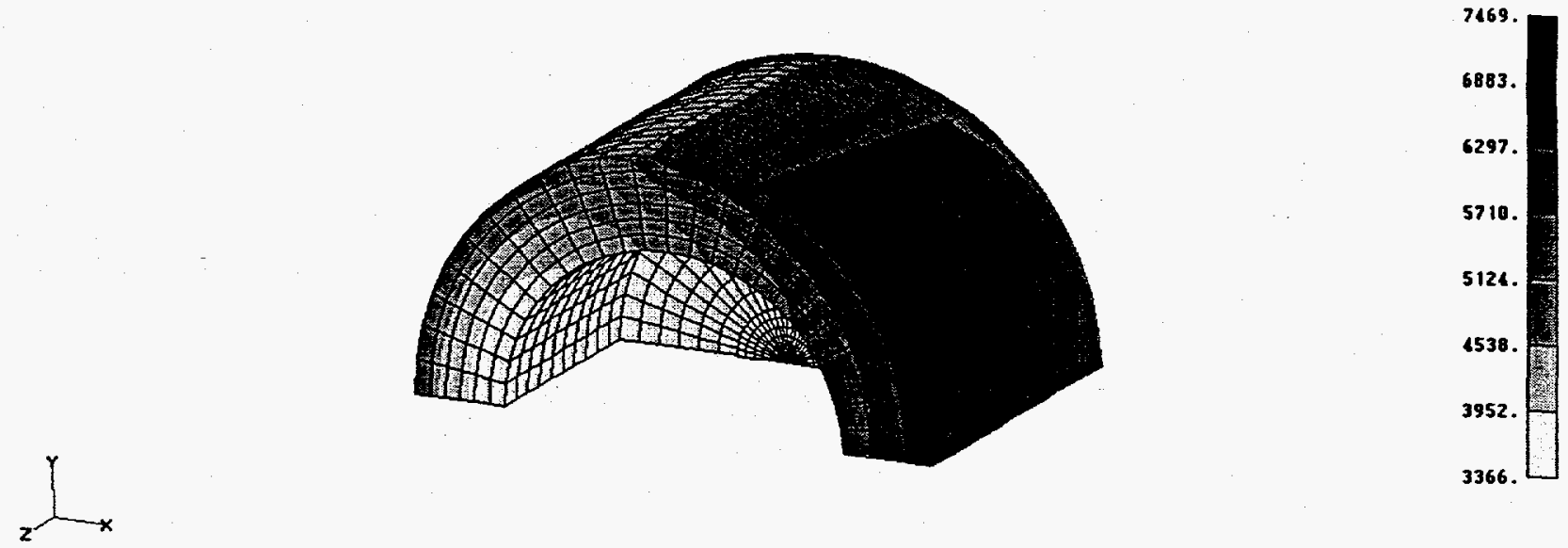

Output Set: Temps -90 stable $2.15 \mathrm{Sec}$ Contour: Temp load set 200
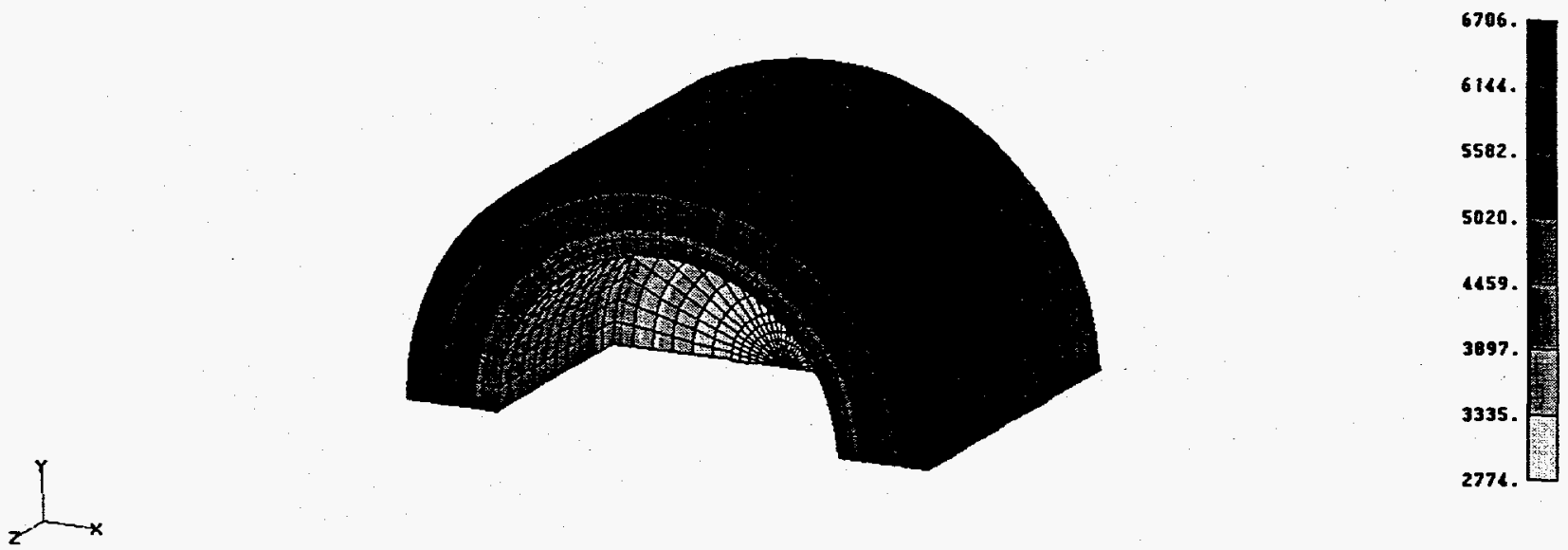

output Set: Tenps $-90 \mathrm{Spin} 2.15 \mathrm{sec}$ Contour: Tenp Load Set 200

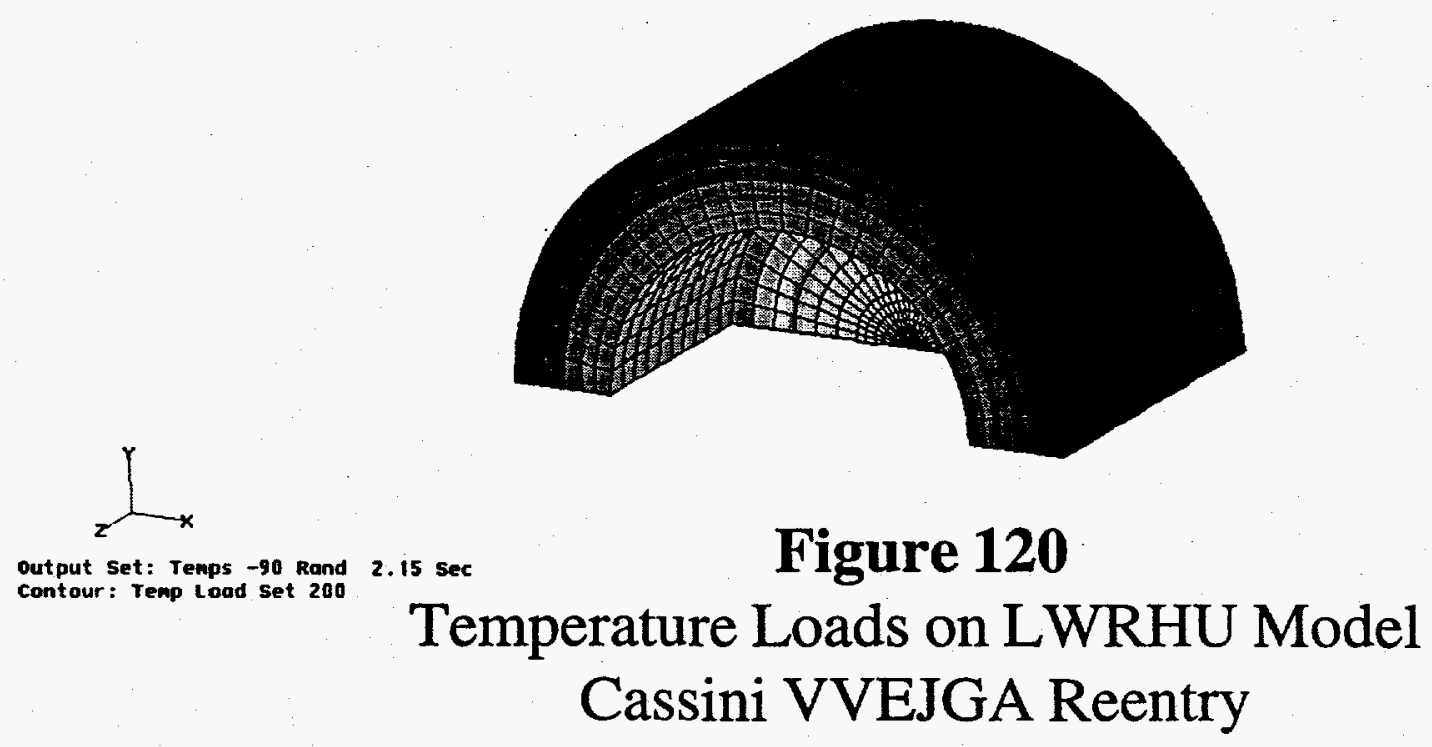



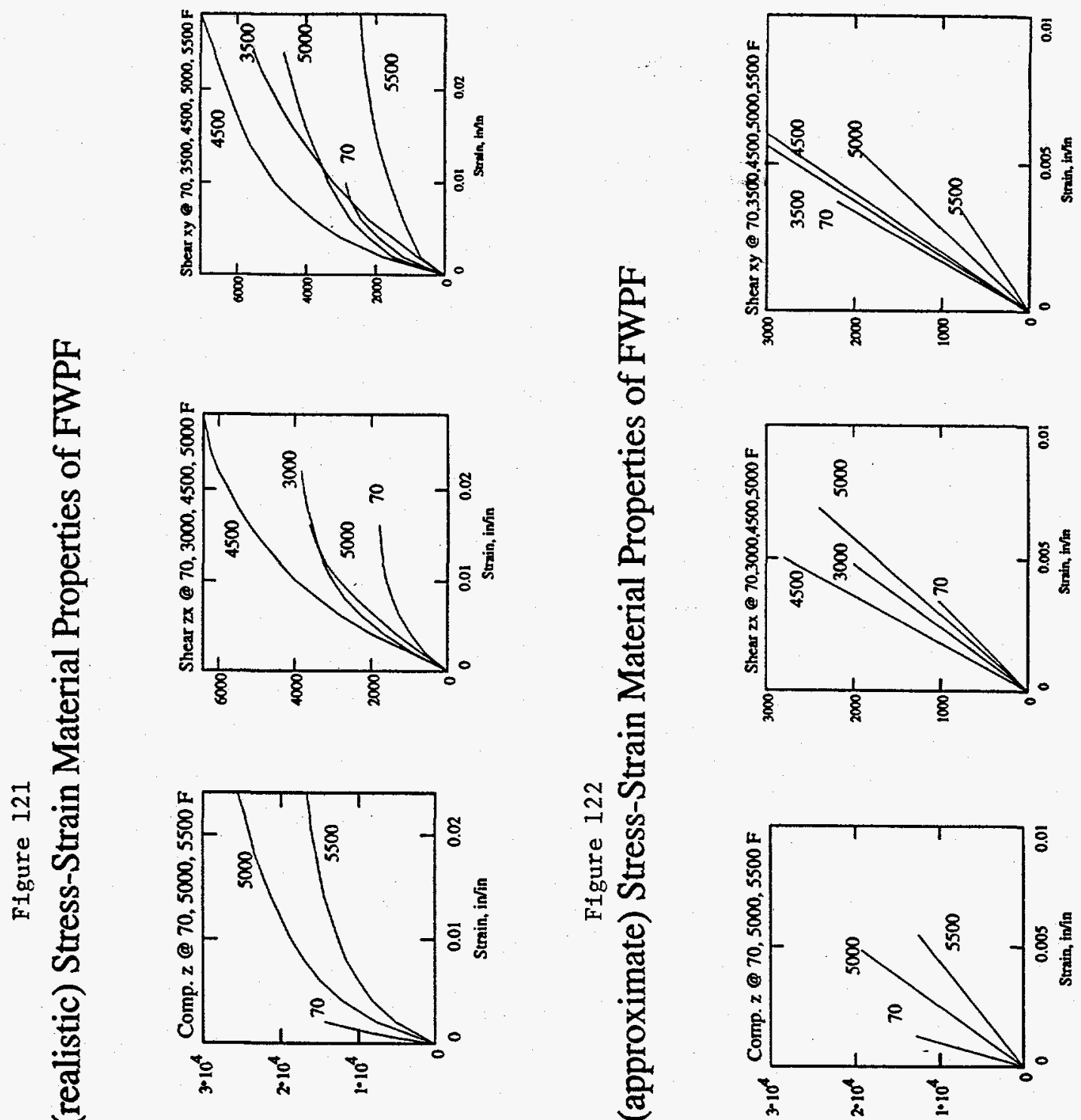

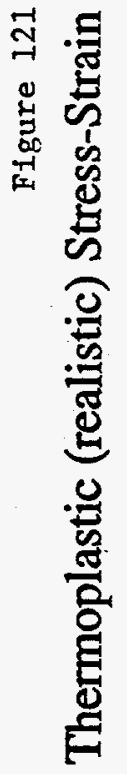
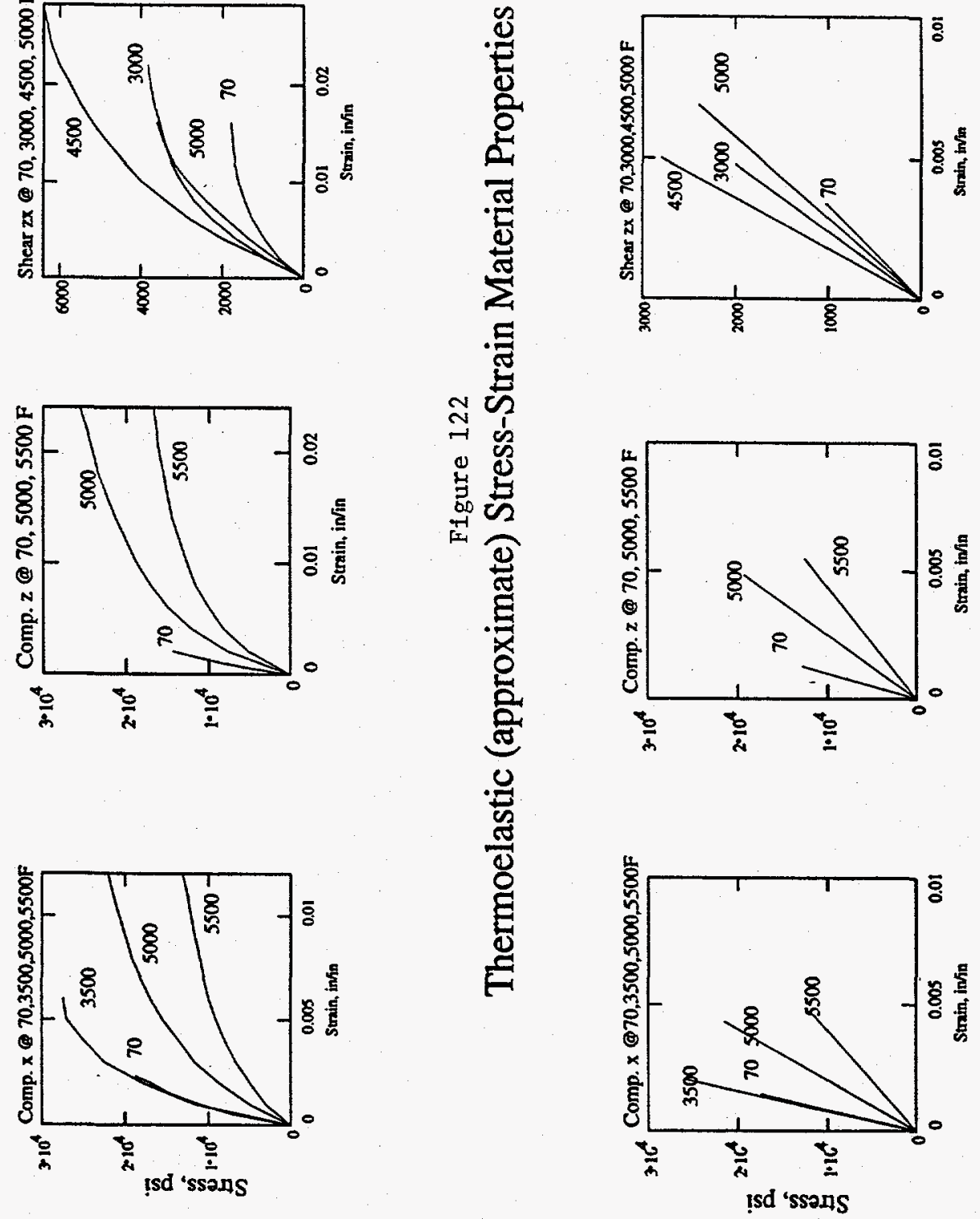
The Johns Hopkins University

Applied Physics Laboratory

Laurel, Maryland 20723-6099
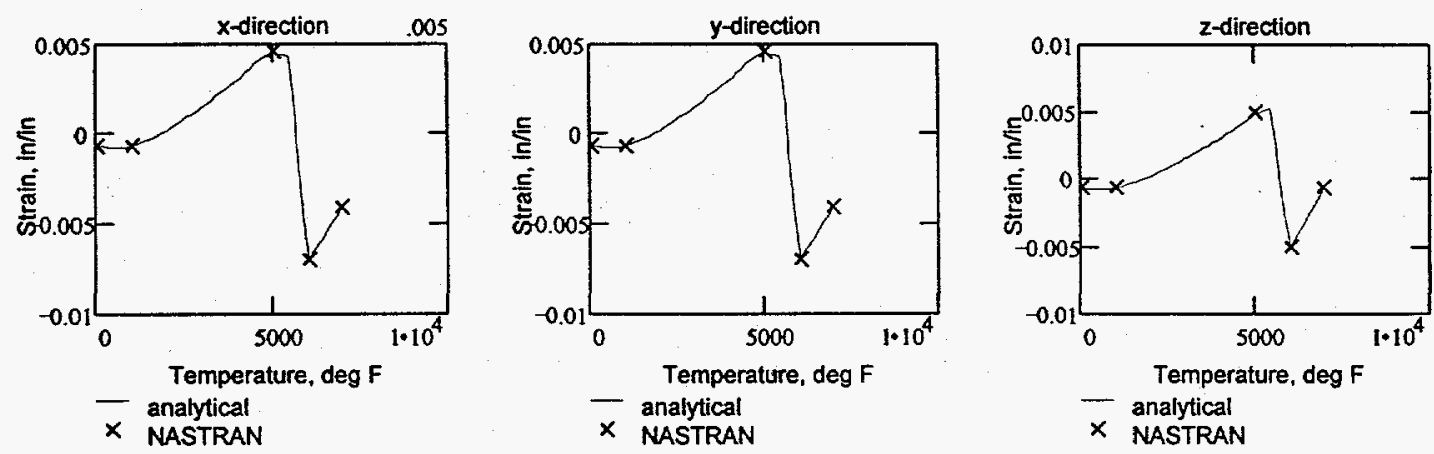

Figure 123

Normal strains of a FWPF Unit Cell Model with Thermoelastic Material Properties Under Hydrostatic Compressive Loads, at Different Temperatures 
The Johns Hopkins University

Applied Physics Laboratory

Laurel, Maryland 20723.6(1)99

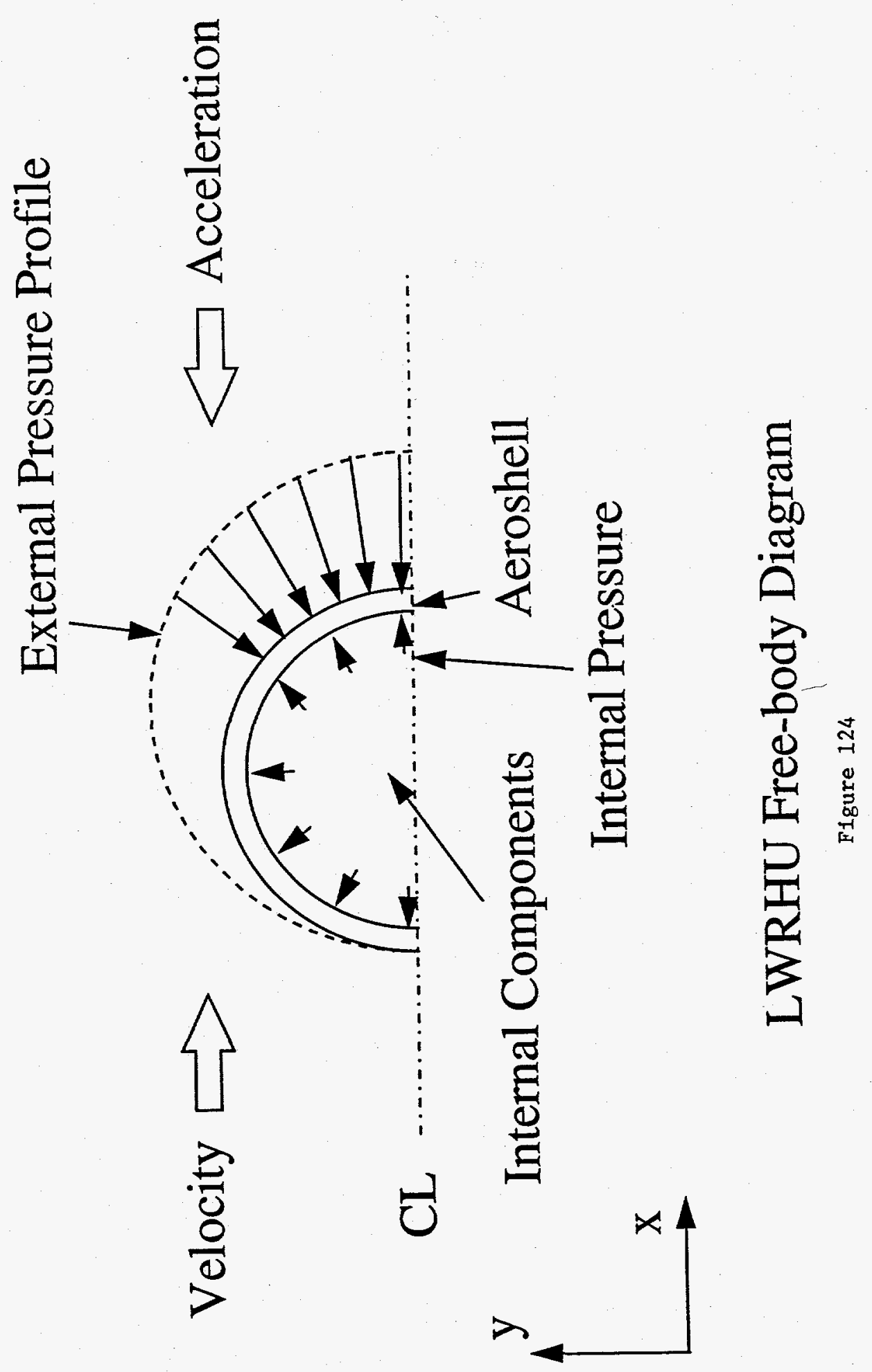




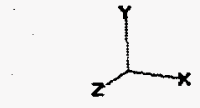

Output Set: -90 stable $2.35 \mathrm{sec}$ Contour: STRAIK FS_X

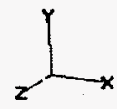

outact set: -90 stable $2.35 \mathrm{sec}$ contour: STRAIK FS_Y

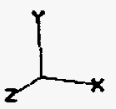

Output Set: -90 stoble $2.35 \mathrm{sec}$ contour: straIs Fs_z

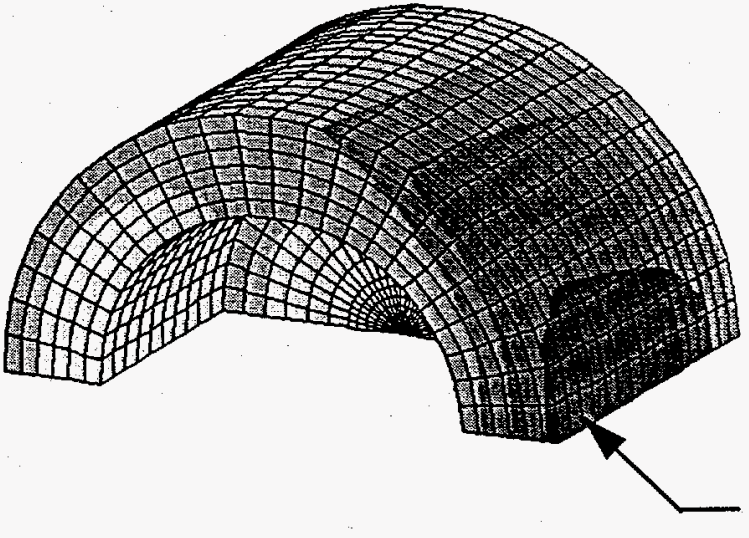

Minimum FS_X=.78 @ Elem 3895
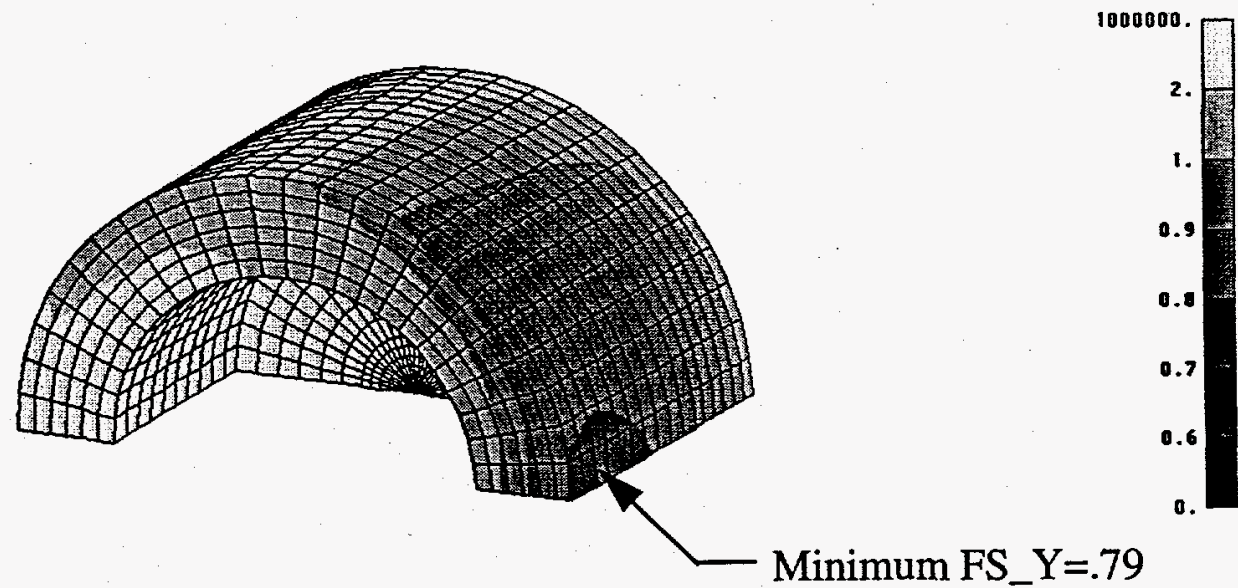

Minimum FS_Y=.79 @ Elem 3895

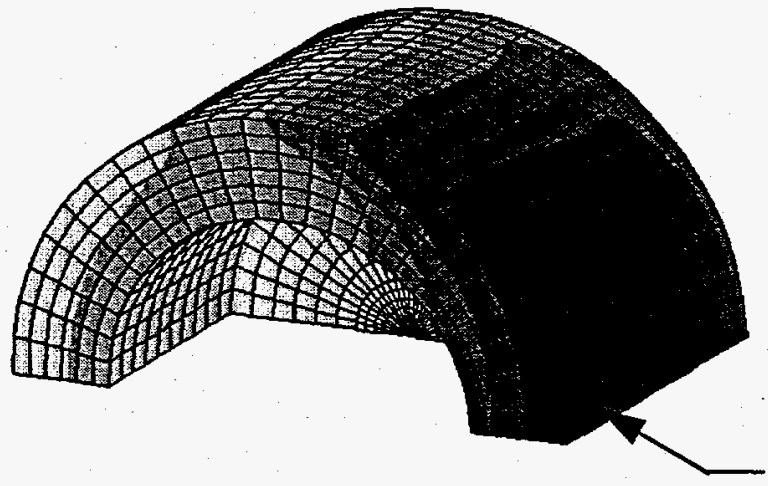

Figure 124 Minimum FS_Z=.61 1000000

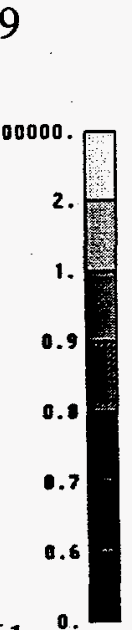
@ Elem 3895

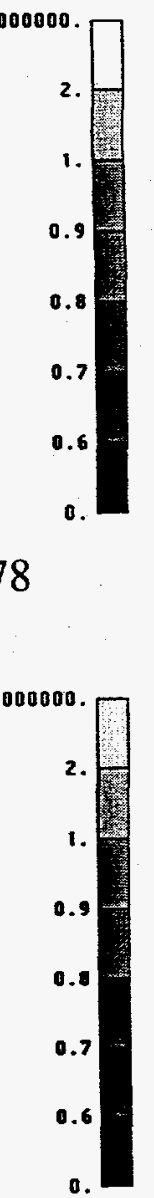




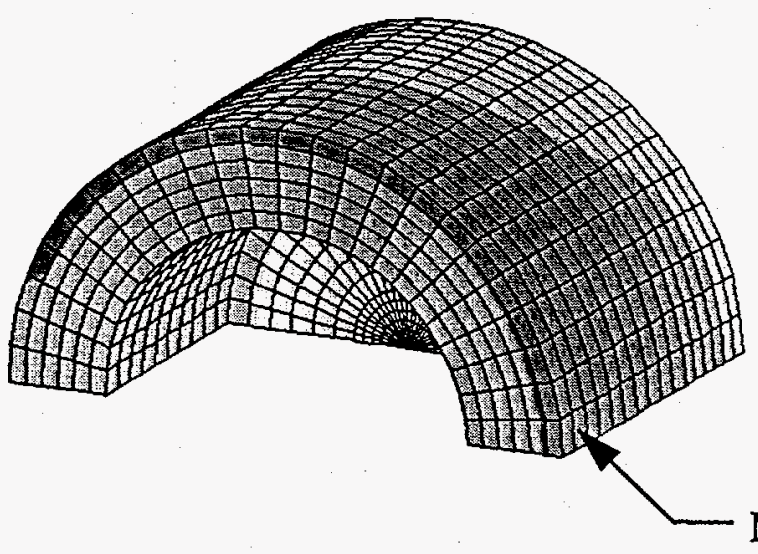

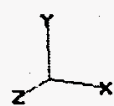

Output Set: -90 Spinning 2.35 5e Contour: STRAIH FS_ $x$
Minimum FS_X $=.95$ @ Elem 3897
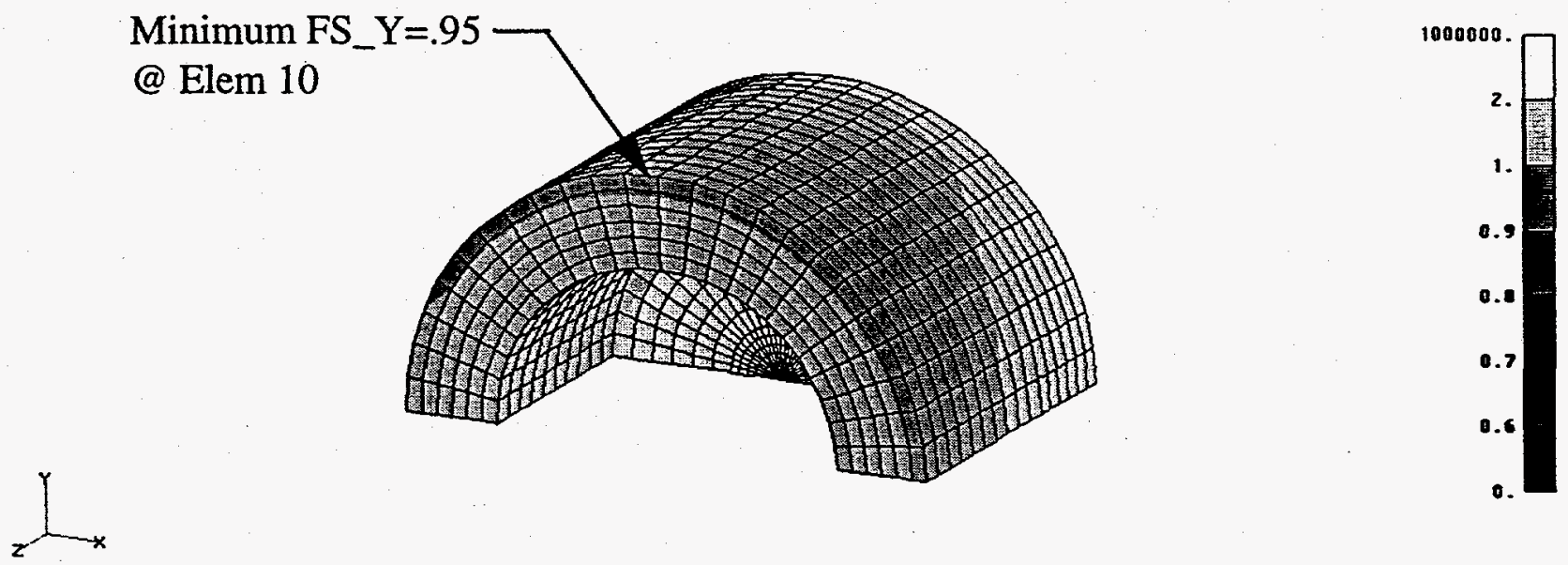

output Set: -90 spinning 2.35 Sec Contour: STRAIH FS_Y
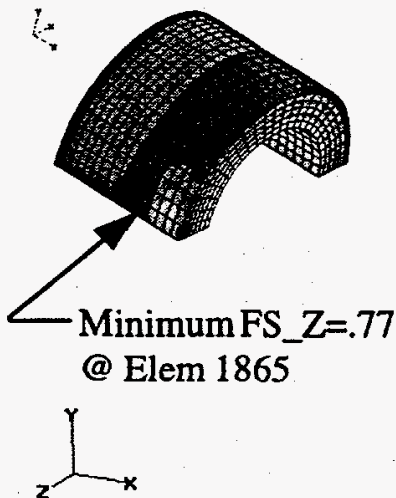
Output Set: -90 spinning 2.35 5e LWRHU Side-on Spinning Gamma $=-90^{\circ}$ Strain Factors of Safety

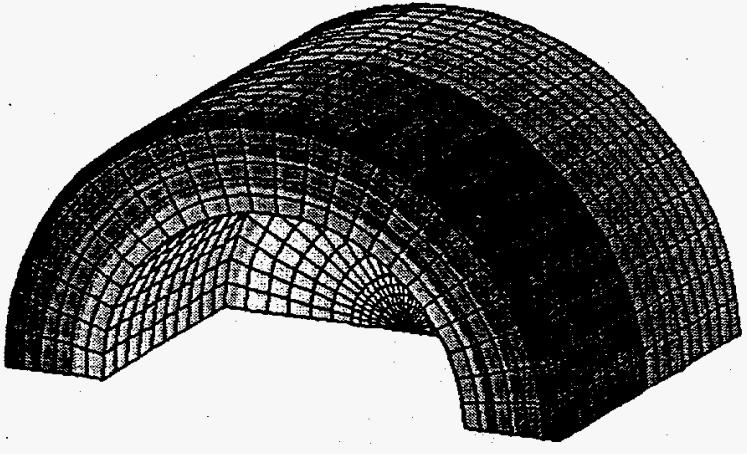

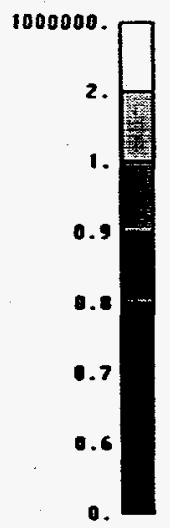

Figure 125 


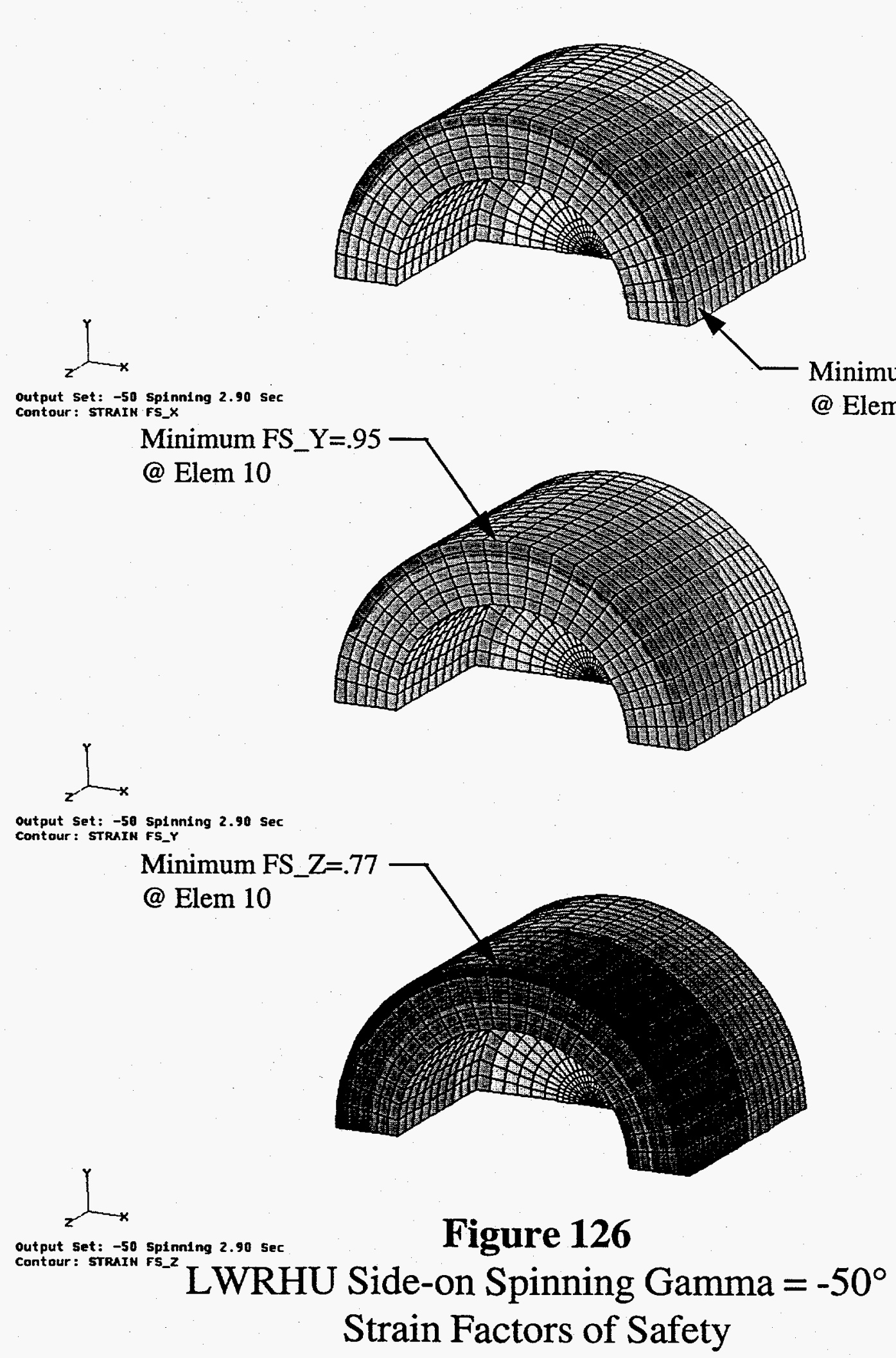



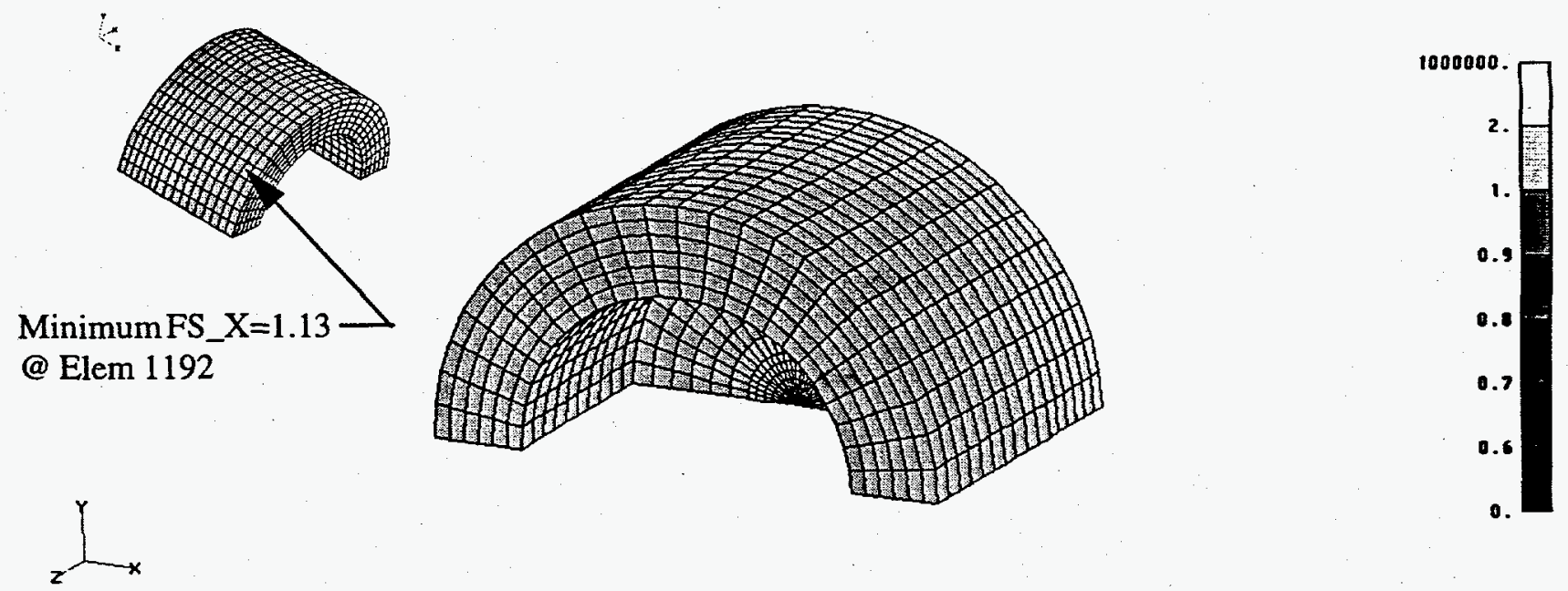

output Set: -7 Spinning $20.00 \mathrm{Sec}$

contour: STRAIN FS_X

Minimum FS_Y=1.13

@ Elem 10
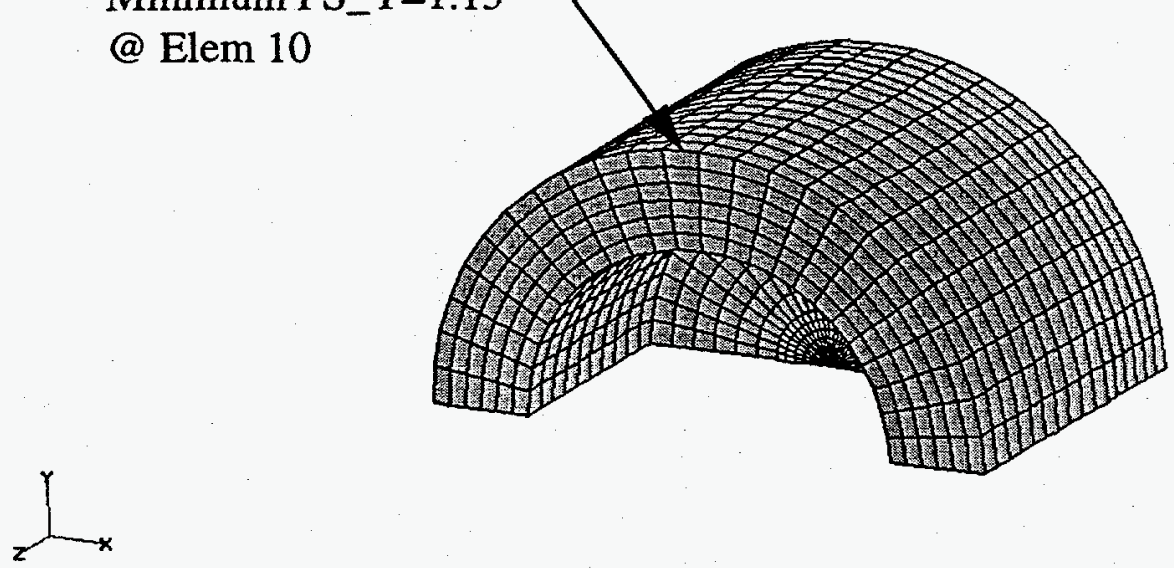

output Set: -7 Spinning $20.00 \mathrm{Sec}$

Contour: STRAIK FS_r
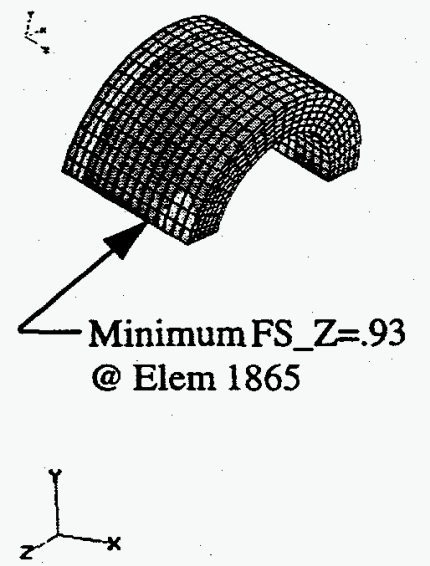

output Set: -7 Spinning $20.00 \mathrm{sec}$ Contour: STRAIN Fs

\section{LWRHU Side-on Spinning Gamma $=-7^{\circ}$ Strain Factors of Safety}

Figure 127

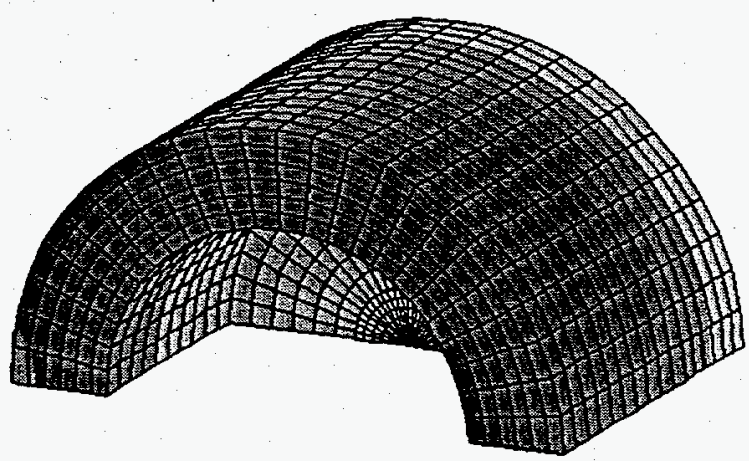

. 
The Johns Hopkins University

Applied Physics Laboratory

Laurel, Maryland 20723-6099

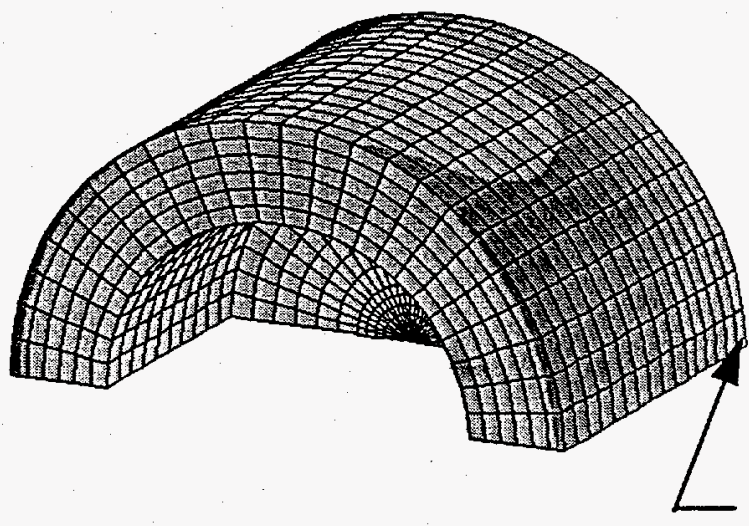

Minimum FS_X=.94

1000000

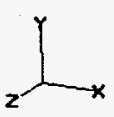

@ Elem 3948

output Set: -90 Rand Tumb $2.15 \mathrm{sec}$

Minimum FS_Y $=.95$

@ Elem 2089

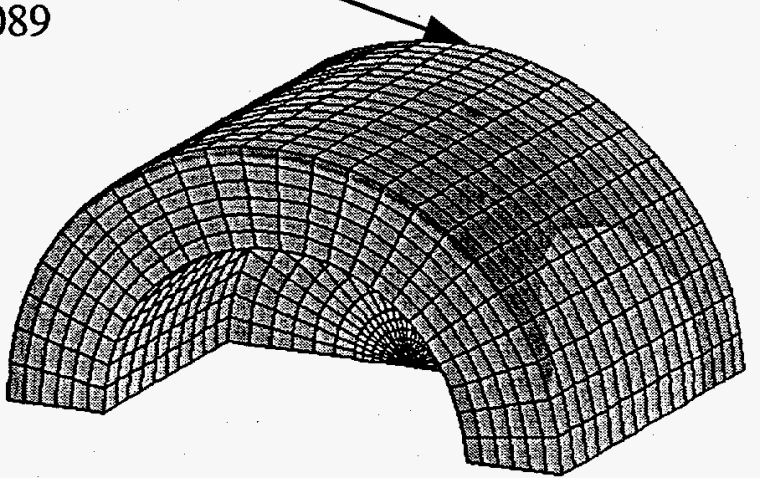

1000000

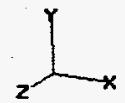

output Set: -90 Rand Tunb $2.15 \mathrm{sec}$ Contuur: STRAIH FS_Y

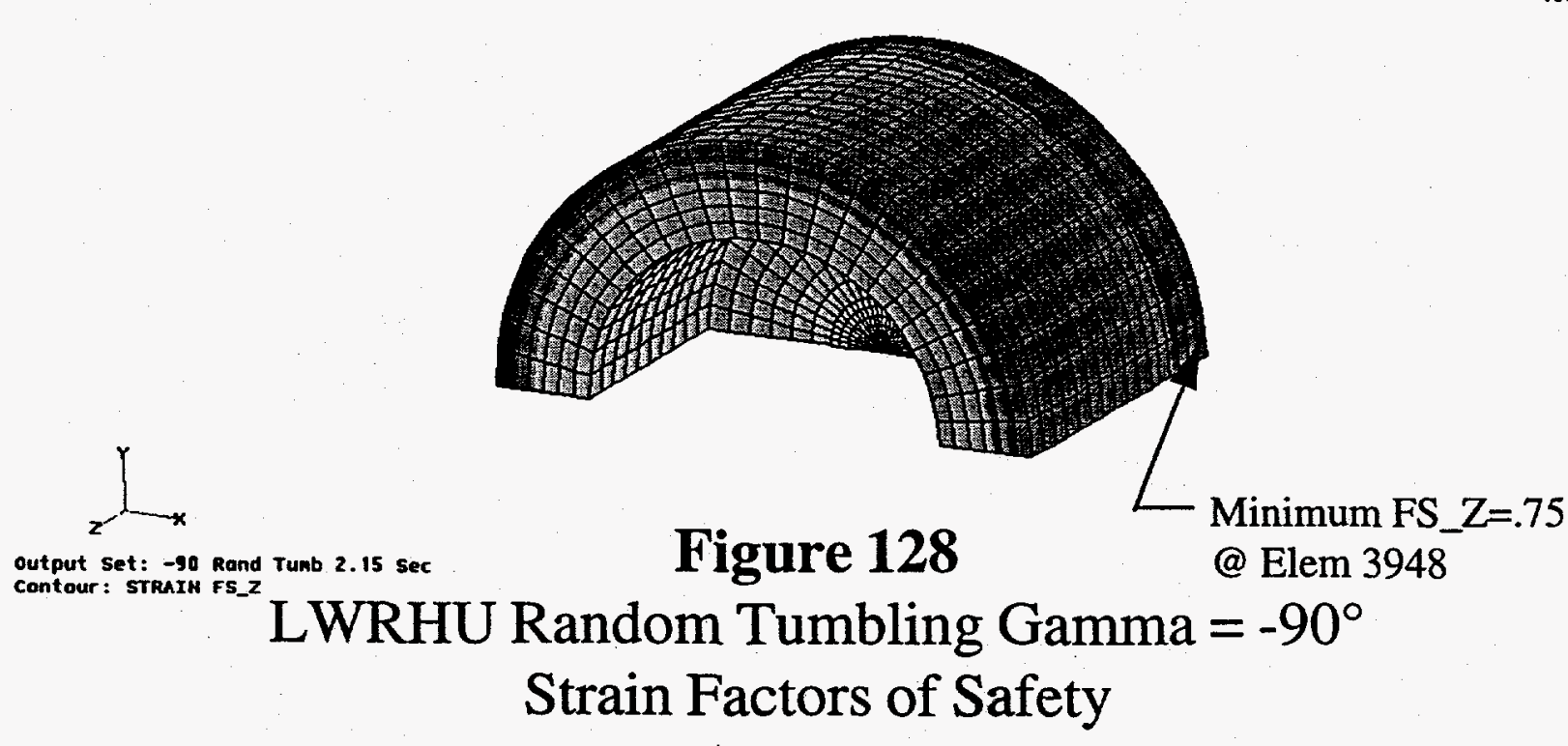

1000000

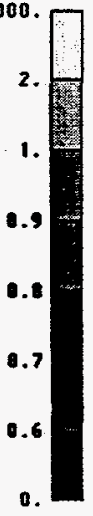

Figure 128 @ Elem 3948
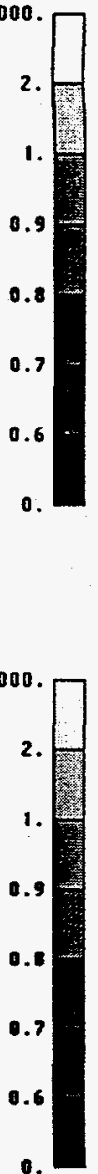

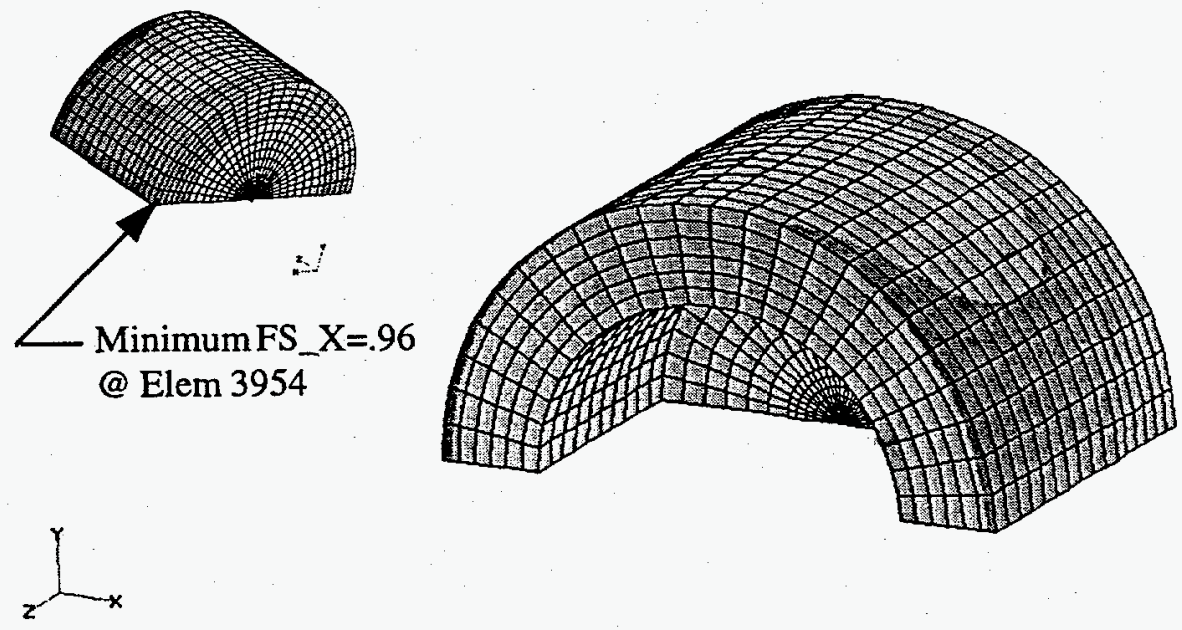

output set: -50 Rand Tumb $2.80 \mathrm{sec}$

Contour: STRAIH FS_X

Minimum FS_Y $=.97$

@ Elem 2089
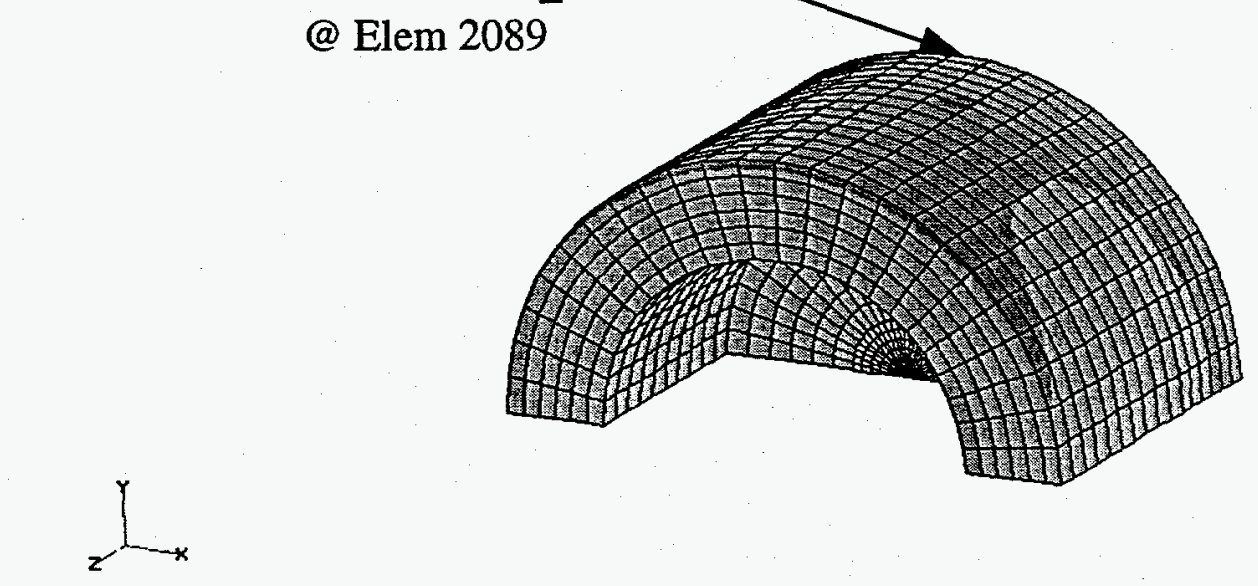

Output Set: -50 Rand Tumb $2.80 \mathrm{sec}$

contour: STRAIK FS_Y

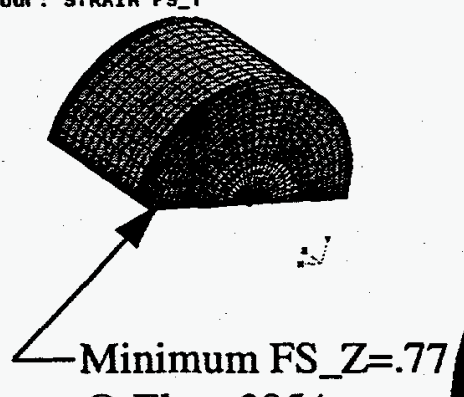

@ Elem 3954

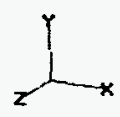

output Set: -50 Rand runb $2.00 \mathrm{sec}$

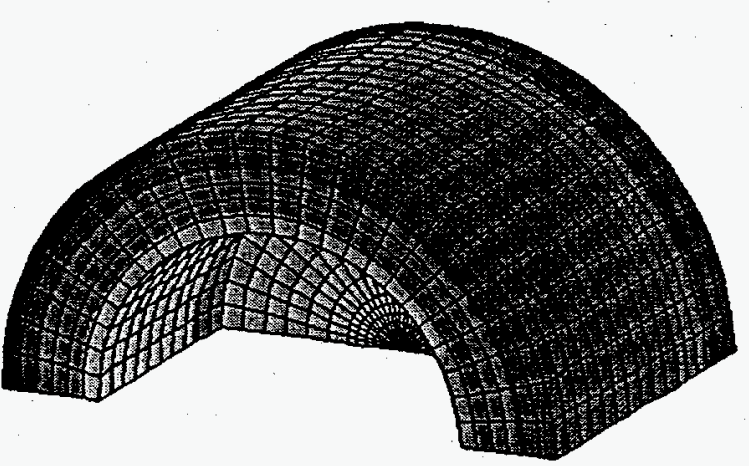

Figure 129

LWRHU Random Tumbling Gamma $=-50^{\circ}$ Strain Factors of Safety
1000800

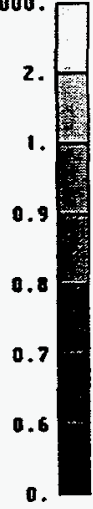

100000.

1000000

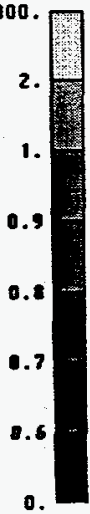



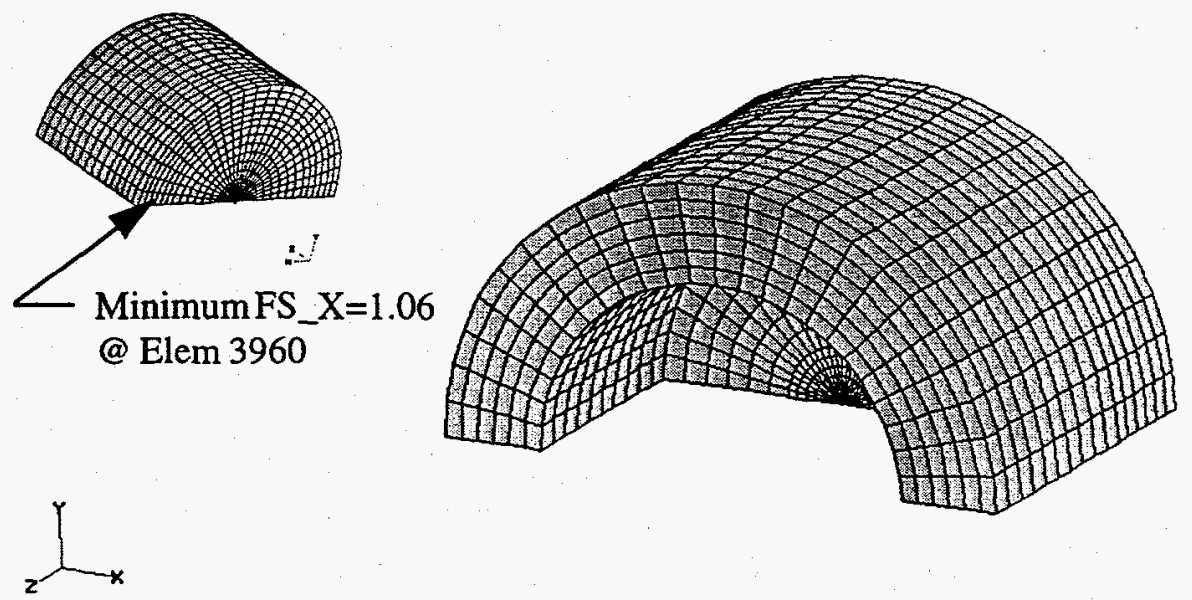

Output Set: -15 Rand Tumb $7.90 \mathrm{sec}$ Contour: straiH Fs_ $x$
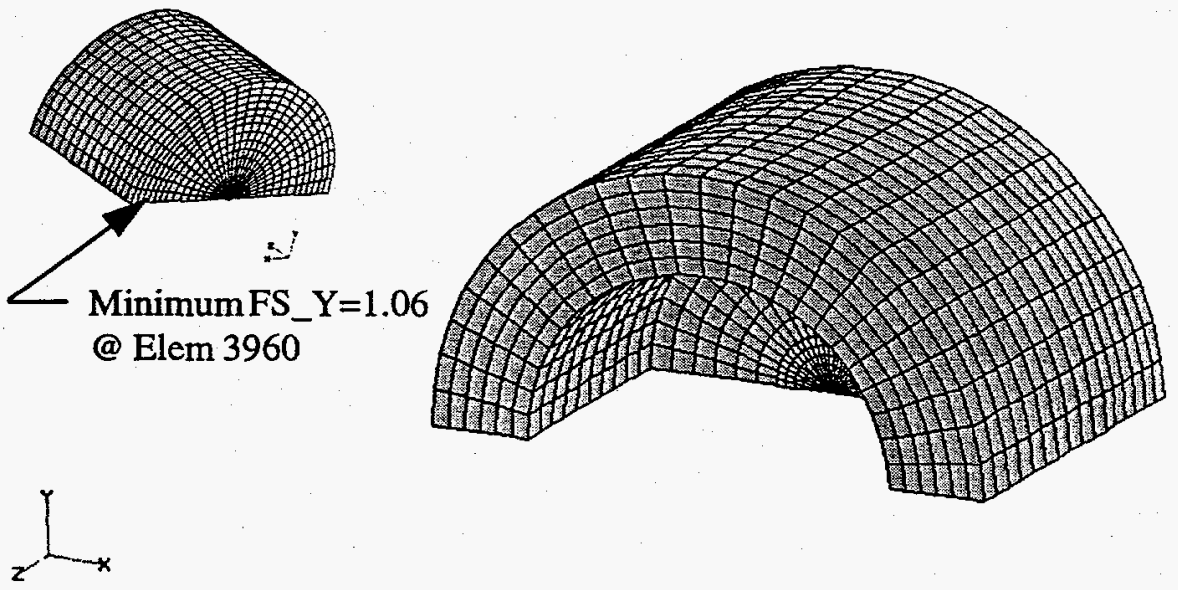

output Set: - IS Rand Tunb 7.90 Sec Cantour: STRAIN FS_r

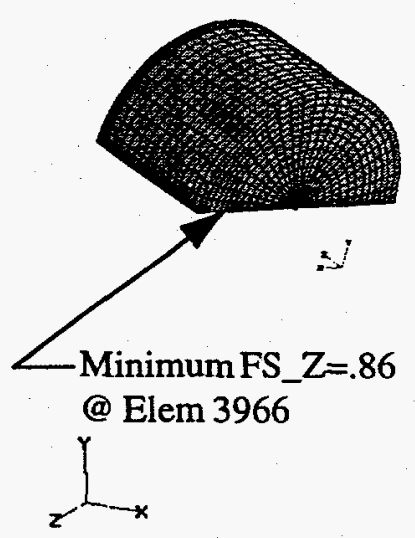

Output set: -15 Rand Tunb $7.90 \mathrm{sec}$ Contour: STRAIN FS_Z

\section{LWRHU Random Tumbling Gamma $=-15^{\circ}$ Strain Factors of Safety}


Initial Distribution External to the Applied Physics Laboratory ANSP-M-24

The Aerospace Corporation

P. O. Box 92957

Los Angeles, CA 90009

Haliburton NUS Corporation

910 Clopper Road

Gaithersburg, MD 20878

Lockheed Martin Missiles \& Space

Astro-Space Division

Building $\mathrm{B}$

720 Vandenberg Road

King of Prussia, PA 19406

Jet Propulsion Laboratory

California Institute of Technology

4800 Oak Grove Drive

Pasadena, CA 91103

Los Alamos National Laboratory

P. O. Box 1663

Los Alamos, NM 87545

LAI/EG\&G Mound Applied Technologies

P. O. Box 3000

Maimisburg, OH 45342

NASA Headquarters

300 E. St. SW

Washington, DC 20546

Oak Ridge National Laboratory

P. O. Box 2008

Oak Ridge, TN 37831-6285

Orbital Sciences Corporation

20301 Century Blvd

Germantown, MD 20874
William H. Ailor

M1-023

B. W. Bartram

H. Firstenburg

L. E. DeFillipo, 29B12

R. J. Hemler, 29B12-KB

W. Tobery, 20B41

A. D. McRonald, MS 157-102

L. Reinhart

R. Wilcox, MS 264-450

T. George

E. W. Johnson

E. Huckins

Code $\mathrm{S}$

J. P. Moore

Bldg 4508MS6080

Robert T. Carpenter

E. A. Skrabek 


\section{Initial Distribution External to the Applied Physics Laboratory \\ ANSP-M-24 \\ (Continued)}

U. S. Department of Energy

Office of Scientific and Technical Information (OSTI)

Special Assistant for Reproduction and Processing

P. O. Box 62

Oak Ridge, TN 37830

U. S. Department of Energy/GTN

Space and National Security Programs

Office of Nuclear Energy, Science and Technology

19901 Germantown Road

Germantown, MD 20874

U. S. Department of Energy

Office of Procurement Operations

1000 Independence Avenue, SW

Washington, DC 20585
W. J. Barnett, NE-50

B. A. Cook, NE-50

R. R. Furlong, NE-50

A. Mehner, NE-50

W. D. Owings, NE-50

L. L. Rutger, NE-50

Contracting Officer, MA-453.2 
Applied Physics Laboratory Laurel. Marvland 20723-6099

\section{Internal Distribution}

ANSP-M-24

The Johns Hopkins University Applied Physics Laboratory Johns Hopkins Road

Laurel, MD 20723
RW Constantine

WH Zinger

TR Betzer

DC Mitchell

RT Lundy

WJ Tropf

JA Krill

WG Bath

JE Whitely, Jr.

JJ Wozniak

PT Brenza

Y Chang

DW Conn

JA Ecker

LG Everhart

JK Frazer

EF Lucero

JS O'Connor

$\mathrm{RC}$ Sharbaugh

JT Stadter 